\title{
Highly Enantio- and Diastereoselective Synthesis of 1,2,3-Trisubstituted Cyclopropanes from $\alpha, \beta$-Unsaturated Amides and Stabilized Sulfur Ylides Catalyzed by a Chiral Copper(I) Complex
}

\author{
Santosh K. Pagire, ${ }^{+}$Naoya Kumagai, ${ }^{+, \neq * *}$ and Masakatsu Shibasaki ${ }^{+, *}$
}

'Institute of Microbial Chemistry (BIKAKEN), 3-14-23 Kamiosaki, Shinagawa-ku, Tokyo 141-0021, Japan.

‡Faculty of Pharmacy, Keio University, 1-5-30 Shibakoen, Minato-ku, Tokyo 105-8512, Japan.

\section{Table of Contents}

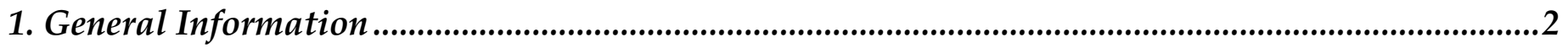

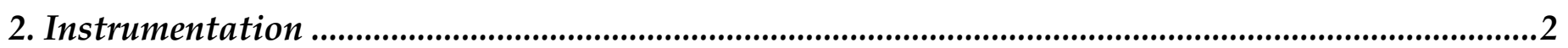

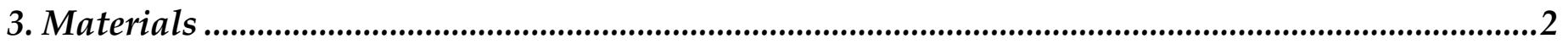

4. General procedures for the synthesis of required starting materials ..............................................3

4.1. Generation of $\alpha$-keto-stabilized sulfur ylides (General Procedure 1) .................................................................................................................

4.2. Preparation of amides from crotonoyl chloride (General Procedure 2) .................................................................................................................

4.3. Preparation of amides from carboxylic acids (General Procedure 3) .........................................................................................................11

4.4. Preparation of amides from 9 using Horner-Wadsworth-Emmons (HWE) reaction (General Procedure 4) ......................................................16

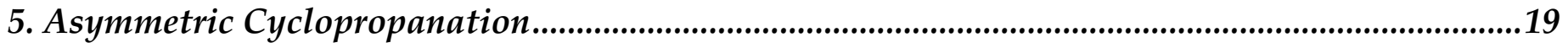

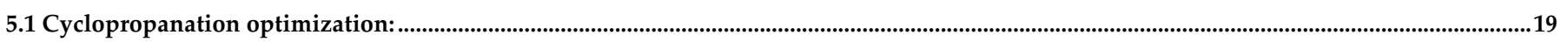

5.2: Standard Procedure 1 (SP-1) for asymmetric cyclopropanation: Variation of 7 -azaindolines ..........................................................................24

5.3: Standard Procedure 2 (SP-2) for asymmetric cyclopropanation: Variation of ylides .........................................................................................31

5.4: Standard Procedure 3 (SP-3) for asymmetric cyclopropanation: Variation at the $\beta$-position ..............................................................................39

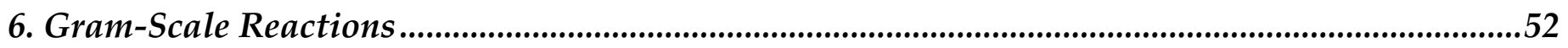

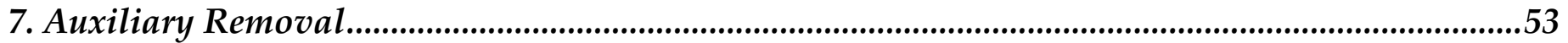

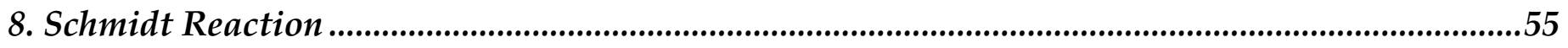

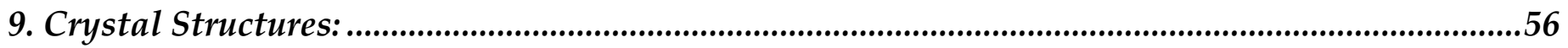

9.1. Solid-state structure of $2 \mathrm{p}$

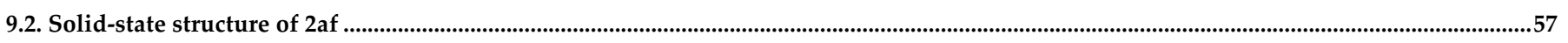

9.3. Solid-state structure of 4ap: (determination of the absolute configuration) ..............................................................................................................58

9.4. Solid-state structure of 4ap': (confirmation of the diastereomer structure) …….............................................................................................59

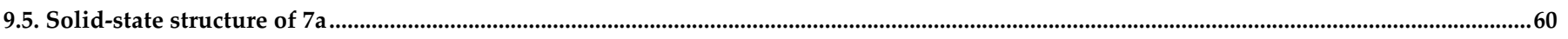

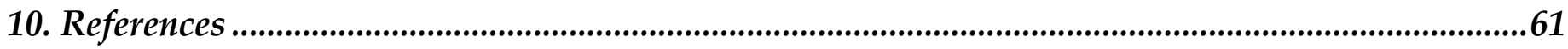

11. Representative 7-azaindolines and their copper complexes in $\mathrm{CD}_{2} \mathrm{Cl}_{2}$ :........................................62

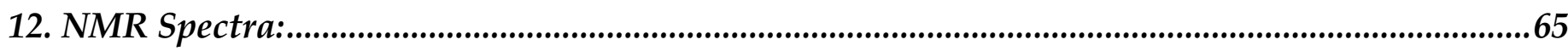




\section{General Information}

All the reactions were performed in a flame-dried $4 / 10 \mathrm{~mL}$ glass Schenk test tube with a Teflon-coated magnetic stirring bar unless otherwise noted. The reactions were run under inert (Argon) atmosphere. Airand moisture-sensitive liquids were transferred via a gas-tight syringe and a stainless-steel needle. All workup and purification procedures were carried out with reagent-grade solvents under an ambient atmosphere. Flash column chromatography was performed using the Biotage Isolera One system.

\section{Instrumentation}

Unless otherwise stated, all the NMR spectra were recorded in $\mathrm{CDCl}_{3}$ on Bruker AVANCE III HD400 NMR spectrometer. Chemical shifts for proton are reported in parts per million (ppm) downfield from tetramethylsilane (TMS) and are referenced to residual protium in the NMR solvent ( $\left.\mathrm{CDCl}_{3}: \delta 7.26 \mathrm{ppm}\right)$. For ${ }^{13} \mathrm{C} \mathrm{NMR}$, chemical shifts were reported in the scale relative to $\mathrm{NMR}$ solvent $\left(\mathrm{CDCl}_{3}: \delta 77.16 \mathrm{ppm}\right)$ as an internal reference. NMR data are reported as follows: chemical shifts, multiplicity (s: singlet, d: doublet, $\mathrm{t}$ : triplet, q: quartet, m: multiplet, bs: broad signal), coupling constants (Hz), and integration. Infrared (IR) spectra were recorded on a HORIBA FT210 Fourier transform infrared spectrophotometer. Single-crystal Xray data were collected on a Rigaku R-AXIS RAPID II imaging plate area detector with graphitemonochromated $\mathrm{Cu}-\mathrm{K} \alpha$ radiation. Optical rotation was measured using a $1 \mathrm{~mL}$ cell with a $1.0 \mathrm{dm}$ path length on a JASCO polarimeter P-1030. High-resolution mass spectra (ESI TOF (+)) were measured on Thermo Fisher Scientific LTQ Orbitrap XL. Preparative HPLC was conducted on a JASCO HPLC system equipped with Daicel chiral-stationary-phase columns ( $\phi 20 \mathrm{~mm}$ x $250 \mathrm{~mm}$ ).

\section{Materials}

Unless otherwise noted, all the required materials and chemicals were purchased from commercial suppliers and were used without further purification. Dichloromethane $\left(\mathrm{DCM} / \mathrm{CH}_{2} \mathrm{Cl}_{2}\right)$ was dried and purified by passing through a solvent purification system (Glass Contour). Metals and Ligands were purchased from Tokyo Chemical Industry (TCI)/Strem Chemical, Inc./Sigma-Aldrich and used as received (opened and handled in the glove box). Column chromatography was performed with silica gel Merck 60 (230-400 mesh ASTM), silica gel $60 \mathrm{~N}$ (spherical, neutral, 40-50 $\mu \mathrm{m}$ ) from Kanto Chemical Co., Inc.

Preparative Thin Layer Chromatography (PTLC) plates (Silica gel 60, F254, 0.5 mm, 20x20 cm, 1.05744.0001) were purchased from Merck, Germany. 


\section{General procedures for the synthesis of required starting materials}

\subsection{Generation of $\alpha$-keto-stabilized sulfur ylides (General Procedure 1)}

Step-I: To a $0.5 \mathrm{M}$ solution of bromoketone A ( $5 \mathrm{mmol}, 1.0$ equiv.) in acetone dimethyl sulfide $(440 \mu \mathrm{L}, 6.0$ mmol, 1.2 equiv.) was added and the mixture was stirred for $48 \mathrm{~h}$ at rt. Then, the precipitate of sulfonium salt $\mathbf{B}$ was separated from the solution, washed with acetone and dried under reduced pressure to give pure sulfonium salt $\mathbf{B}$, which was readily converted to the corresponding sulfonium ylides $\mathbf{1}$ (Step-II).

Step-II: To a suspension of sulfonium salt $\mathbf{B}$ (1.7 mmol, 1 equiv.) in $5 \mathrm{~mL} \mathrm{CH}_{2} \mathrm{Cl}_{2}$ a solution of $\mathrm{NaOH}(8.5$ mmol, 5 equiv.) in $5 \mathrm{~mL} \mathrm{H}_{2} \mathrm{O}$ was added and the mixture was intensively stirred for $1 \mathrm{~h}$ at room temperature. Then the reaction mixture was filtered through cotton wool. The organic layer was separated and the aqueous layer was washed with $\mathrm{CH}_{2} \mathrm{Cl}_{2}(5 \times 15 \mathrm{~mL})$. The combined organic layer was dried over anhydrous sodium sulfate, filtered, and concentrated under reduced pressure. The residue was dried in a vacuum until constant weight to give sulfonium ylides $\mathbf{1}$ (as unstable solid or oil), which was immediately characterized and used in the next stage.

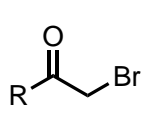

A
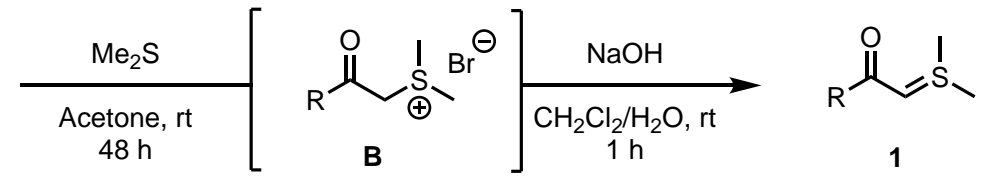

[Note: Except for B1, the subsequent sulfonium bromide ylide derivatives (B) were directly converted to their corresponding stabilized sulfur ylide forms (1) and immediately used for the next step after confirming by NMR, IR, and HRMS].

The obtained data of these ylides is in correspondence with the literature data. ${ }^{1}$

Dimethyl(2-oxo-2-phenylethyl)sulfoniumbromide (B1):

The reaction performed according to general procedure 1 (step-I) afforded $975 \mathrm{mg}$ (75\%).<smiles>C[Se](C)(C)CC(=O)c1ccccc1</smiles>

Colorless solid.

${ }^{1}$ H NMR (400 MHz, 300 K, DMSO-d $)$ : $\delta 8.07$ - 7.99 (m, 2H), 7.82 - 7.71 (m, 1H), 7.67 - 7.57

$(\mathrm{m}, 2 \mathrm{H}), 5.71(\mathrm{~s}, 2 \mathrm{H}), 3.06(\mathrm{~s}, 6 \mathrm{H})$.

${ }^{13}$ C NMR (101 MHz, 300 K, DMSO-d $)$ : $\delta$ 191.4, 135.0, 133.9, 129.1, 128.6, 52.8, 24.5.

2-(dimethyl- $\lambda^{4}$-sulfaneylidene)-1-phenylethan-1-one (1a):

The reaction performed according to general procedure 1 (step-II) afforded $291 \mathrm{mg}(66 \%)$.

Yellow solid.

11

${ }^{1} \mathrm{H}$ NMR (400 MHz, 300 K, CDCl 3$)$ : $\delta 7.80-7.72(\mathrm{~m}, 2 \mathrm{H}), 7.37-7.29(\mathrm{~m}, 3 \mathrm{H}), 4.29(\mathrm{~s}, 1 \mathrm{H}), 2.95$ $(\mathrm{s}, 6 \mathrm{H})$.

${ }^{13} \mathrm{C}$ NMR (101 MHz, 300 K, $\left.\mathrm{CDCl}_{3}\right): \delta$ 183.3, 141.0, 129.6, 128.0, 126.4, 50.9, 28.6.

IR (thin film): $\widetilde{v} 3385,3055,3015,2921,1671,1584,1512,1487,1391,1293,1204,1097,1066$, $1026,991,926,854,829,791,705 \mathrm{~cm}^{-1}$.

HRMS (ESI): $m / z$ calculated for $\mathrm{C}_{10} \mathrm{H}_{13} \mathrm{OS}[\mathrm{M}+\mathrm{H}]^{+}: 181.0682$, found: 181.0681 . 
2-(dimethyl- $\lambda^{4}$-sulfaneylidene)-1-( $p$-tolyl)ethan-1-one (1b):

The reaction performed according to general procedure 1 afforded $234 \mathrm{mg}$ (71\%).

Yellow solid.

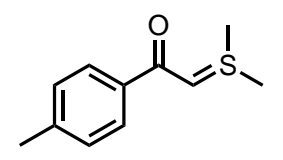

${ }^{1} \mathrm{H}$ NMR $\left(400 \mathrm{MHz}, 300 \mathrm{~K}, \mathrm{CDCl}_{3}\right): \delta 7.68-7.59(\mathrm{~m}, 2 \mathrm{H}), 7.14-7.06(\mathrm{~m}, 2 \mathrm{H}), 4.24(\mathrm{~s}, 1 \mathrm{H})$, $2.90(\mathrm{~s}, 6 \mathrm{H}), 2.30(\mathrm{~s}, 3 \mathrm{H})$.

${ }^{13} \mathrm{C}$ NMR (101 MHz, $\left.300 \mathrm{~K}, \mathrm{CDCl}_{3}\right): \delta 183.0,139.4,138.2,128.5,126.3,50.5,28.6,21.3$.

IR (thin film): $\widetilde{v} 3377,3018,2919,1668,1607,1578,1514,1498,1388,1276,1197,1179,1092$, $1034,991,913,859,744 \mathrm{~cm}^{-1}$.

HRMS (ESI): $m / z$ calculated for $\mathrm{C}_{11} \mathrm{H}_{15} \mathrm{OS}[\mathrm{M}+\mathrm{H}]^{+}:$195.0838, found: 195.0836.

2-(dimethyl- $\lambda^{4}$-sulfaneylidene)-1-(4-methoxyphenyl)ethan-1-one (1c):

The reaction performed according to general procedure 1 afforded $250 \mathrm{mg}$ (70\%).

Yellow solid.

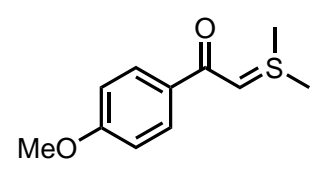

${ }^{1} \mathrm{H}$ NMR (400 MHz, 300 K, $\left.\mathrm{CDCl}_{3}\right): \delta 7.74-7.66(\mathrm{~m}, 2 \mathrm{H}), 6.85-6.77(\mathrm{~m}, 2 \mathrm{H}), 4.20(\mathrm{~s}, 1 \mathrm{H})$,

$3.76(\mathrm{~s}, 3 \mathrm{H}), 2.90(\mathrm{~s}, 6 \mathrm{H})$.

${ }^{13} \mathrm{C}$ NMR (101 MHz, 300 K, $\left.\mathrm{CDCl}_{3}\right): \delta$ 182.6, 160.8, 133.7, 127.9, 113.0, 55.2, 49.7, 28.7.

IR (thin film): $\widetilde{v} 3375,3072,3004,2931,2836,1663,1602,1583,1502,1391,1301,1250$, $1208,1170,1091,1029,990,848,775,756,690 \mathrm{~cm}^{-1}$.

HRMS (ESI): $m / z$ calculated for $\mathrm{C}_{11} \mathrm{H}_{15} \mathrm{O}_{2} \mathrm{~S}[\mathrm{M}+\mathrm{H}]^{+}: 211.0787$, found: 211.0785.

2-(dimethyl- $\lambda^{4}$-sulfaneylidene)-1-(4-nitrophenyl)ethan-1-one (1d):

The reaction performed according to general procedure 1 afforded $260 \mathrm{mg}(68 \%)$.

Yellow solid.

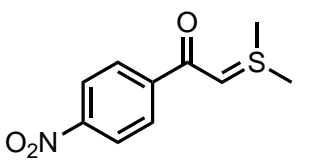

${ }^{1} \mathrm{H}$ NMR (400 MHz, 300 K, $\left.\mathrm{CDCl}_{3}\right)$ : $\delta 8.21-8.13(\mathrm{~m}, 2 \mathrm{H}), 7.93-7.83(\mathrm{~m}, 2 \mathrm{H}), 4.38(\mathrm{~s}, 1 \mathrm{H})$, $3.02(\mathrm{~s}, 6 \mathrm{H})$.

${ }^{13}$ C NMR (101 MHz, 300 K, CDCl $)$ : $\delta$ 180.2, 148.4, 146.9, 127.3, 123.4, 53.6, 28.2.

IR (thin film): $\widetilde{v} 3104,3069,3031,3003,2926,1588,1536,1509,1481,1424,1402,1385$, $1345,1319,1308,1293,1203,1107,1092,1038,998,926,870,835,716,698,664 \mathrm{~cm}^{-1}$. HRMS (ESI): $m / z$ calculated for $\mathrm{C}_{10} \mathrm{H}_{12} \mathrm{O}_{3} \mathrm{NS}[\mathrm{M}+\mathrm{H}]^{+}: 226.0532$, found: 226.0534 .

2-(dimethyl- $\lambda 4$-sulfaneylidene)-1-(2-methoxyphenyl)ethan-1-one (1e):

The reaction performed according to general procedure 1 afforded $261 \mathrm{mg}(73 \%)$.

Yellow solid.

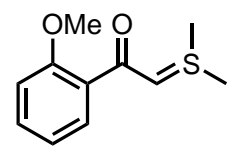

${ }^{1}$ H NMR (400 MHz, 300 K, $\left.\mathrm{CDCl}_{3}\right)$ : $\delta 7.68(\mathrm{dd}, J=7.6,1.9 \mathrm{~Hz}, 1 \mathrm{H}), 7.20(\mathrm{ddd}, J=8.2,7.3$, $1.8 \mathrm{~Hz}, 1 \mathrm{H}), 6.88(\mathrm{td}, J=7.4,1.1 \mathrm{~Hz}, 1 \mathrm{H}), 6.81(\mathrm{dd}, J=8.3,1.1 \mathrm{~Hz}, 1 \mathrm{H}), 4.38(\mathrm{~s}, 1 \mathrm{H}), 3.77(\mathrm{~s}$, $3 \mathrm{H}), 2.87(\mathrm{~s}, 6 \mathrm{H})$.

${ }^{13}$ C NMR (101 MHz, 300 K, $\left.\mathrm{CDCl}_{3}\right): \delta$ 181.5, 157.0, 130.9, 129.8, 129.5, 120.2, 111.2, 56.1, $55.6,28.6$.

IR (thin film): $\widetilde{v} 3393,3068,3002,2919,2834,1659,1596,1579,1515,1483,1463,1433$, $1376,1293,1273,1239,1180,1161,1116,1087,1048,1023,991,956,854,832,756,638,580$ $\mathrm{cm}^{-1}$.

HRMS (ESI): $m / z$ calculated for $\mathrm{C}_{11} \mathrm{H}_{15} \mathrm{O}_{2} \mathrm{~S}[\mathrm{M}+\mathrm{H}]^{+}: 211.0787$, found: 211.0790. 
2-(dimethyl- $\lambda^{4}$-sulfaneylidene)-1-(naphthalen-2-yl)ethan-1-one (1f):

The reaction performed according to general procedure 1 afforded $281 \mathrm{mg}$ (72\%).

Yellow solid.

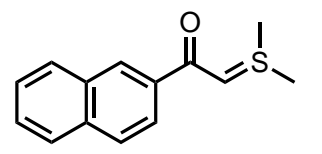

${ }^{1} \mathrm{H}$ NMR $\left(400 \mathrm{MHz}, 300 \mathrm{~K}, \mathrm{CDCl}_{3}\right): \delta 8.31-8.24(\mathrm{~m}, 1 \mathrm{H}), 7.89-7.82(\mathrm{~m}, 2 \mathrm{H}), 7.77(\mathrm{q}, J=$ 4.6, $4.1 \mathrm{~Hz}, 2 \mathrm{H}), 7.45-7.38(\mathrm{~m}, 2 \mathrm{H}), 4.39(\mathrm{~s}, 1 \mathrm{H}), 2.86(\mathrm{~s}, 6 \mathrm{H})$.

${ }^{13} \mathrm{C}$ NMR (101 MHz, 300 K, $\left.\mathrm{CDCl}_{3}\right)$ : $\delta$ 182.5, 138.2, 134.0, 132.9, 128.8, 127.4, 127.3, 126.3, $125.9,125.9,124.2,52.0,28.4$.

IR (thin film): $\widetilde{v} 3382,3054,3014,2920,1670,1626,1596,1576,1521,1494,1466,1428$, 1392, 1339, 1304, 1272, 1180, 1152, 1124, 1080, 1030, 991, 952, 910, 865, 846, 826, 774, 758, $727 \mathrm{~cm}^{-1}$.

HRMS (ESI): $m / z$ calculated for $\mathrm{C}_{14} \mathrm{H}_{15} \mathrm{OS}[\mathrm{M}+\mathrm{H}]^{+}: 231.0838$, found: 231.0838 .

1-(benzofuran-2-yl)-2-(dimethyl- $\lambda^{4}$-sulfaneylidene)ethan-1-one (1g):

The reaction performed according to general procedure 1 afforded $261 \mathrm{mg}$ (70\%).

Yellow solid.

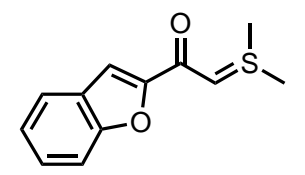

${ }^{1}$ H NMR (400 MHz, 300 K, $\left.\mathbf{C D C l}_{3}\right): \delta 7.56(\mathrm{ddd}, J=7.7,1.4,0.7 \mathrm{~Hz}, 1 \mathrm{H}), 7.42(\mathrm{dq}, J=8.2$, $0.9 \mathrm{~Hz}, 1 \mathrm{H}), 7.26(\mathrm{ddd}, J=8.4,7.2,1.3 \mathrm{~Hz}, 1 \mathrm{H}), 7.20-7.16(\mathrm{~m}, 1 \mathrm{H}), 7.14(\mathrm{dd}, J=4.0,1.0 \mathrm{~Hz}$, $1 \mathrm{H}), 4.54(\mathrm{~s}, 1 \mathrm{H}), 2.95(\mathrm{~s}, 6 \mathrm{H})$.

${ }^{13} \mathrm{C}$ NMR (101 MHz, 300 K, CDCl $)$ : $\delta 173.4,156.3,154.7,128.3,125.3,122.8,121.9,111.5$, 105.4, 53.3, 28.4.

IR (thin film): $\widetilde{v} 3372,3064,3013,2921,1784,1668,1612,1579,1519,1472,1449,1391$, 1343, 1305, 1259, 1245, 1157, 1141, 1109, 1080, 1033, 993, 941, 885, 848, $744 \mathrm{~cm}^{-1}$.

HRMS (ESI): $m / z$ calculated for $\mathrm{C}_{12} \mathrm{H}_{13} \mathrm{O}_{2} \mathrm{~S}[\mathrm{M}+\mathrm{H}]^{+}: 221.0631$, found: 221.0633.

2-(dimethyl- $\lambda^{4}$-sulfaneylidene)-1-(thiophen-2-yl)ethan-1-one (1h):

The reaction performed according to general procedure 1 afforded $234 \mathrm{mg}$ (74\%).

Yellow solid.

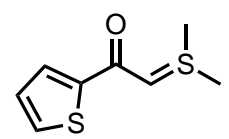

${ }^{1} \mathrm{H}$ NMR (400 MHz, 300 K, $\left.\mathrm{CDCl}_{3}\right)$ : $\delta 7.35$ (dd, J = 3.6, $\left.1.2 \mathrm{~Hz}, 1 \mathrm{H}\right), 7.27(\mathrm{dd}, J=5.0,1.2 \mathrm{~Hz}$, $1 \mathrm{H}), 6.97(\mathrm{dd}, J=5.0,3.6 \mathrm{~Hz}, 1 \mathrm{H}), 4.21(\mathrm{~s}, 1 \mathrm{H}), 2.97(\mathrm{~s}, 6 \mathrm{H})$.

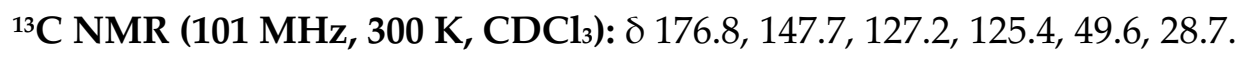

IR (thin film): $\widetilde{v} 3388,3068,3011,2918,1649,1530,1508,1425,1383,1230,1186,1088$, $1069,1030,988,854,728 \mathrm{~cm}^{-1}$.

HRMS (ESI): $m / z$ calculated for $\mathrm{C}_{8} \mathrm{H}_{11} \mathrm{OS}_{2}[\mathrm{M}+\mathrm{H}]^{+}:$187.0246, found: 187.0244.

1-((1S,3s)-adamantan-1-yl)-2-(dimethyl- $\lambda^{4}$-sulfaneylidene)ethan-1-one (1i):

The reaction performed according to general procedure 1 afforded $234 \mathrm{mg}$ (58\%).

Colorless solid.

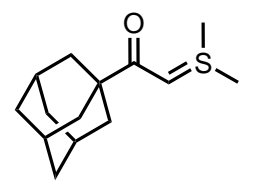

${ }^{1} \mathrm{H}$ NMR (400 MHz, $\left.300 \mathrm{~K}, \mathrm{CDCl}_{3}\right)$ : $\delta 3.61$ (s, 1H), 2.76 (s, 6H), $1.94-1.84$ (m, 3H), 1.75 $1.67(\mathrm{~m}, 6 \mathrm{H}), 1.65-1.53(\mathrm{~m}, 6 \mathrm{H})$.

${ }^{13}$ C NMR (101 MHz, 300 K, CDCl $)$ ): $\delta$ 197.6, 48.0, 42.5, 40.1, 37.0, 28.7, 28.7.

IR (thin film): $\widetilde{v} 2991,2928,2905,2845,1509,1451,1425,1390,1378,1365,1311,1251,1184$, $1162,1098,1071,1035,991,967,934,923,908,849,814,729,653,564 \mathrm{~cm}^{-1}$.

HRMS (ESI): $m / z$ calculated for $\mathrm{C}_{14} \mathrm{H}_{23} \mathrm{OS}[\mathrm{M}+\mathrm{H}]^{+}:$239.1464, found: 239.1462. 
1-(dimethyl- $\lambda^{4}$-sulfaneylidene)-3,3-dimethylbutan-2-one (1j):

The reaction performed according to general procedure 1 afforded $128 \mathrm{mg}$ (47\%).

Colorless solid.

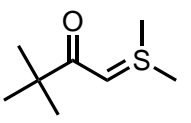

${ }^{1} \mathrm{H}$ NMR (400 MHz, 300 K, CDCl $)$ ): $\delta 3.73$ (s, 1H), 2.85 (s, 6H), 1.09 (s, 9H).

${ }^{13} \mathrm{C}$ NMR (101 MHz, 300 K, CDCl $)$ : $\delta$ 198.3, 47.7, 40.6, 28.8, 28.5.

IR (thin film): $\widetilde{v} 3488,3015,2955,1680,1493,1455,1427,1397,1352,1220,1136,1036,991$, $914,847,748,604,539 \mathrm{~cm}^{-1}$.

HRMS (ESI): $\mathrm{m} / z$ calculated for $\mathrm{C}_{8} \mathrm{H}_{17} \mathrm{OS}[\mathrm{M}+\mathrm{H}]^{+}:$161.0995, found: 161.0993 . 


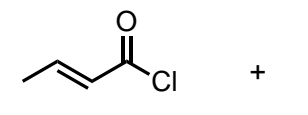

crotonyl chloride<smiles>C1CCPNC1</smiles>

$2^{\circ}$ amines

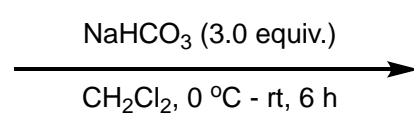

$\mathrm{CH}_{2} \mathrm{Cl}_{2}, 0^{\circ} \mathrm{C}-\mathrm{rt}, 6 \mathrm{~h}$

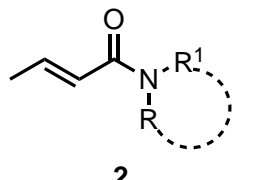

2

To a solution of the corresponding $2^{\circ}$ amines ( $3 \mathrm{mmol}, 1.0$ equiv) and $\mathrm{NaHCO}_{3}(9 \mathrm{mmol}, 3$ equiv) in anhydrous $\mathrm{CH}_{2} \mathrm{Cl}_{2}(30 \mathrm{~mL})$ was added crotonyl chloride $\left(193 \mu \mathrm{L}, 6 \mathrm{mmol}, 2.0\right.$ equiv) at $0{ }^{\circ} \mathrm{C}$ and the mixture was stirred at RT for $6 \mathrm{~h}$. The reaction mixture was diluted with water $(50 \mathrm{~mL})$ and the product was extracted with $\mathrm{CH}_{2} \mathrm{Cl}_{2}$ $(3 \times 20 \mathrm{~mL})$. Combined organic layers were dried over $\mathrm{Na}_{2} \mathrm{SO}_{4}$ and concentrated under reduced pressure. The crude residue was purified by automated flash column chromatography (Biotage Isolera One) with a prepacked silica gel column using Hexane/Ethyl acetate ( $~ 8 / 2)$ eluents. All the solid products were subsequently recrystallized from hot Hexane or $\mathrm{CH}_{2} \mathrm{Cl}_{2} / \mathrm{Hexane}$.

\section{(E)-3-(but-2-enoyl)oxazolidin-2-one (2a):}

The reaction performed according to general procedure 2 afforded $283 \mathrm{mg}(61 \%)$.

Colorless oil (solidifies upon cooling).

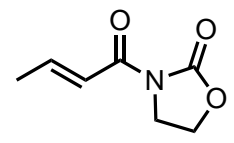

${ }^{1} \mathrm{H}$ NMR (400 MHz, $\left.300 \mathrm{~K}, \mathrm{CDCl}_{3}\right): \delta 7.28-7.11(\mathrm{~m}, 2 \mathrm{H}), 4.41(\mathrm{t}, J=7.5 \mathrm{~Hz}, 2 \mathrm{H}), 4.06(\mathrm{t}, 7.5$ $\mathrm{Hz}, 2 \mathrm{H}), 1.96(\mathrm{~d}, J=6.5 \mathrm{~Hz}, 3 \mathrm{H})$.

${ }^{13} \mathrm{C}$ NMR (101 MHz, 300 K, $\left.\mathrm{CDCl}_{3}\right)$ : $\delta$ 165.3, 153.6, 147.0, 121.6, 62.1, 42.8, 18.6.

The obtained data is in accordance with the literature data. ${ }^{2}$

(E)-1-(2,3-dihydro-1H-pyrrolo[2,3-b]pyridin-1-yl)but-2-en-1-one (2b):

The reaction performed according to general procedure 2 afforded $395 \mathrm{mg}$ (70\%).

Colorless solid (m.p. $65-67^{\circ} \mathrm{C}$ ).

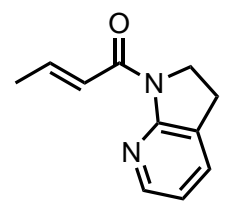

${ }^{1} \mathrm{H}$ NMR (400 MHz, $\left.300 \mathrm{~K}, \mathrm{CDCl}_{3}\right)$ : $\delta 8.12$ (d, J = 5.2, $\left.1.3 \mathrm{~Hz}, 1 \mathrm{H}\right), 7.79(\mathrm{dq}, J=15.3,1.7 \mathrm{~Hz}, 1 \mathrm{H})$, $7.44(\mathrm{dq}, J=7.4,1.4 \mathrm{~Hz}, 1 \mathrm{H}), 7.17-7.04(\mathrm{~m}, 1 \mathrm{H}), 6.85(\mathrm{dd}, J=7.3,5.1 \mathrm{~Hz}, 1 \mathrm{H}), 4.19-4.09(\mathrm{~m}$, $2 \mathrm{H}), 3.08-3.00(\mathrm{~m}, 2 \mathrm{H}), 1.97(\mathrm{dd}, J=7.0,1.7 \mathrm{~Hz}, 3 \mathrm{H})$.

${ }^{13} \mathrm{C}$ NMR (101 MHz, 300 K, $\left.\mathrm{CDCl}_{3}\right)$ : $\delta$ 165.6, 156.4, 146.4, 142.9, 133.7, 126.6, 124.8, 118.1, 46.1, 24.5, 18.6.

The obtained data is in accordance with the literature data. ${ }^{2}$

(E)-1-(1H-pyrrolo[2,3-b]pyridin-1-yl)but-2-en-1-one (2c):

The reaction performed according to general procedure 2 afforded $424 \mathrm{mg}$ (76\%).

Colorless solid (m.p. $54-56{ }^{\circ} \mathrm{C}$ ).

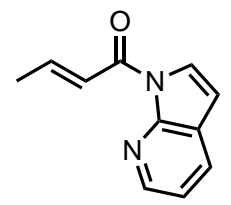

${ }^{1} \mathrm{H}$ NMR (400 MHz, $\left.300 \mathrm{~K}, \mathrm{CDCl}_{3}\right): \delta 8.35(\mathrm{dd}, J=4.8,1.7 \mathrm{~Hz}, 1 \mathrm{H}), 8.25(\mathrm{dq}, J=15.3,1.7 \mathrm{~Hz}$, $1 \mathrm{H}), 8.03(\mathrm{~d}, J=4.1 \mathrm{~Hz}, 1 \mathrm{H}), 7.84(\mathrm{dt}, J=7.8,1.2 \mathrm{~Hz}, 1 \mathrm{H}), 7.39(\mathrm{dq}, J=15.3,7.0 \mathrm{~Hz}, 1 \mathrm{H}), 7.15$ $(\mathrm{ddd}, J=7.8,4.8,0.7 \mathrm{~Hz}, 1 \mathrm{H}), 6.57(\mathrm{dd}, J=4.1,0.7 \mathrm{~Hz}, 1 \mathrm{H}), 2.07(\mathrm{dd}, J=7.0,1.7 \mathrm{~Hz}, 3 \mathrm{H})$.

${ }^{13} \mathrm{C}$ NMR (101 MHz, 300 K, $\left.\mathrm{CDCl}_{3}\right): \delta$ 163.9, 147.9, 147.4, 143.6, 129.3, 126.1, 124.6, 124.1, 118.6, 105.9, 18.9.

IR (thin film): $\widetilde{v} 3073,2971,1692,1643,1595,1579,1530,1471,1443,1406,1373,1331,1294$, $1282,1262,1210,1129,1114,1100,1060,1045,1024,971,925,889,832,803,777,754,735,680$, $661,597,501 \mathrm{~cm}^{-1}$.

HRMS (ESI): $m / z$ calculated for $\mathrm{C}_{11} \mathrm{H}_{11} \mathrm{~N}_{2} \mathrm{O}[\mathrm{M}+\mathrm{H}]^{+}:$: 187.0866 , found: 187.0867 . 
(E)-1-(2,3-dihydro-1H-pyrrolo[3,2-b]pyridin-1-yl)but-2-en-1-one (2d):

The reaction performed according to general procedure 2 afforded $445 \mathrm{mg}(79 \%)$.

Colorless solid (m.p. $65-67^{\circ} \mathrm{C}$ ).

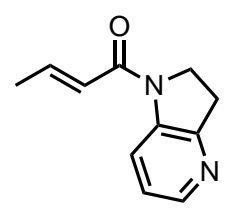

${ }^{1} \mathrm{H}$ NMR (400 MHz, DMSO- $\left.d_{6}\right): \delta 8.12(\mathrm{dd}, J=5.2,1.5 \mathrm{~Hz}, 1 \mathrm{H}), 7.79(\mathrm{dd}, J=15.3,1.7 \mathrm{~Hz}, 1 \mathrm{H})$, $7.63(\mathrm{dd}, J=7.4,1.5 \mathrm{~Hz}, 1 \mathrm{H}), 6.97(\mathrm{dd}, J=7.4,5.1 \mathrm{~Hz}, 1 \mathrm{H}), 6.91(\mathrm{dt}, J=15.3,6.9 \mathrm{~Hz}, 1 \mathrm{H}), 4.00$ $(\mathrm{dd}, J=9.1,7.8 \mathrm{~Hz}, 2 \mathrm{H}), 3.08-2.99(\mathrm{~m}, 2 \mathrm{H}), 1.91(\mathrm{dd}, J=6.9,1.7 \mathrm{~Hz}, 3 \mathrm{H})$.

${ }^{13}$ C NMR (101 MHz, DMSO-d $\left.d_{6}\right): \delta 163.8,155.6,145.7,141.5,134.0,126.7,124.6,118.1,45.7$, 23.6, 18.0 .

IR (thin film): $\widetilde{v} 2909,1660,1615,1600,1587,1475,1443,1419,1383,1352,1323,1289,1240$, $1165,972,916,773,692 \mathrm{~cm}^{-1}$.

HRMS (ESI): $m / z$ calculated for $\mathrm{C}_{11} \mathrm{H}_{13} \mathrm{~N}_{2} \mathrm{O}[\mathrm{M}+\mathrm{H}]^{+}:$189.1022, found: 189.1025 .

(E)-1-(indolin-1-yl)but-2-en-1-one (2e):

The reaction performed according to general procedure 2 afforded $449 \mathrm{mg}(80 \%)$.

Colorless solid.

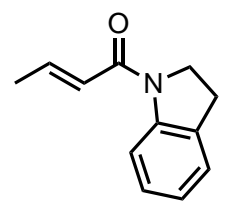

${ }^{1} \mathrm{H}$ NMR (400 MHz, $\left.300 \mathrm{~K}, \mathrm{CDCl}_{3}\right)$ ) $\delta 8.27$ (bs, 1H), $7.23-7.12(\mathrm{~m}, 2 \mathrm{H}), 7.14-6.95(\mathrm{~m}, 2 \mathrm{H})$, $6.25(\mathrm{~d}, J=15.1 \mathrm{~Hz}, 1 \mathrm{H}), 4.13(\mathrm{dd}, J=9.0,8.0 \mathrm{~Hz}, 2 \mathrm{H}), 3.17(\mathrm{~s}, 2 \mathrm{H}), 1.94(\mathrm{dd}, J=6.9,1.7 \mathrm{~Hz}, 3 \mathrm{H})$. ${ }^{13} \mathrm{C}$ NMR (101 MHz, $\left.300 \mathrm{~K}, \mathrm{CDCl}_{3}\right):{ }^{13} \mathrm{C}$ NMR (101 MHz, $\left.\mathrm{CDCl}_{3}\right) \delta$ 164.4, 143.2, 142.8, 131.6, 127.6, 124.6, 123.7, 117.5, 48.1, 28.1, 18.4.

The obtained data is in accordance with the literature data. ${ }^{2}$

(E)-N-methyl- $N$-(pyridin-2-yl)but-2-enamide (2f):

The reaction performed according to general procedure 2 afforded $386 \mathrm{mg}$ (73\%).

Colorless oil.

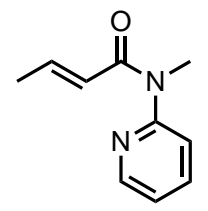

${ }^{1} \mathrm{H}$ NMR (400 MHz, 300 K, $\mathrm{CDCl}_{3}$ ): $\delta 8.51$ (ddd, J = 4.7, 2.0, $\left.1.0 \mathrm{~Hz}, 1 \mathrm{H}\right), 7.73$ (ddd, J = 8.0, 7.4, $2.0 \mathrm{~Hz}, 1 \mathrm{H}), 7.19(\mathrm{ddd}, J=7.4,5.3,1.0 \mathrm{~Hz}, 2 \mathrm{H}), 6.97(\mathrm{dt}, J=15.0,6.9 \mathrm{~Hz}, 1 \mathrm{H}), 5.93(\mathrm{dt}, J=15.0$, $1.7 \mathrm{~Hz}, 1 \mathrm{H}), 3.44(\mathrm{~s}, 3 \mathrm{H}), 1.80(\mathrm{dd}, J=6.9,1.7 \mathrm{~Hz}, 3 \mathrm{H})$.

${ }^{13} \mathrm{C}$ NMR (101 MHz, 300 K, $\left.\mathrm{CDCl}_{3}\right)$ : $\delta$ 166.6, 156.2, 149.2, 142.0, 138.1, 123.4, 121.6, 121.2, 35.4, 18.2 .

The obtained data is in accordance with the literature data. ${ }^{2}$

(E)-N-methoxy- $N$-methylbut-2-enamide (2g):

The reaction performed according to general procedure 2 afforded $290 \mathrm{mg}$ (75\%).

Colorless oil.

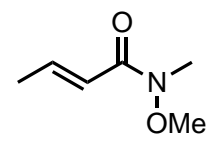

${ }^{1} \mathrm{H}$ NMR $\left(400 \mathrm{MHz}, 300 \mathrm{~K}, \mathrm{CDCl}_{3}\right): \delta 6.91(\mathrm{ddt}, J=15.5,6.8,3.1 \mathrm{~Hz}, 1 \mathrm{H}), 6.36(\mathrm{dq}, J=15.4,2.3$, $1.8 \mathrm{~Hz}, 1 \mathrm{H}), 3.69-3.61(\mathrm{~m}, 3 \mathrm{H}), 3.22-3.14(\mathrm{~m}, 3 \mathrm{H}), 1.89-1.82(\mathrm{~m}, 3 \mathrm{H})$.

${ }^{13} \mathrm{C}$ NMR (101 MHz, 300 K, $\left.\mathrm{CDCl}_{3}\right)$ : $\delta$ 167.0, 142.9, 120.2, 61.7, 32.3, 18.2.

IR (thin film): $\widetilde{v} 2968,2938,2915,1669,1637,1448,1412,1381,1297,1179,1152,1124,1083$, $1045,1009,966,913,826,815,675,620,516,441 \mathrm{~cm}^{-1}$.

HRMS (ESI): $\mathrm{m} / z$ calculated for $\mathrm{C}_{6} \mathrm{H}_{12} \mathrm{NO}_{2}[\mathrm{M}+\mathrm{H}]^{+}:$130.0863, found: 130.0863 . 
(E)- $N, N$-dimethylbut-2-enamide (2h):

The reaction performed according to general procedure 2 afforded $227 \mathrm{mg}(67 \%)$.

$\overbrace{N^{\prime}}$

Colorless solid.

${ }^{1} \mathrm{H}$ NMR (400 MHz, 300 K, $\left.\mathrm{CDCl}_{3}\right): \delta 6.79(\mathrm{dq}, J=15.0,6.8 \mathrm{~Hz}, 1 \mathrm{H}), 6.21(\mathrm{dq}, J=15.0,1.7 \mathrm{~Hz}$, $1 \mathrm{H}), 3.00(\mathrm{~s}, 3 \mathrm{H}), 2.92(\mathrm{~s}, 3 \mathrm{H}), 1.81(\mathrm{dd}, J=6.9,1.7 \mathrm{~Hz}, 3 \mathrm{H})$.

${ }^{13}$ C NMR (101 MHz, 300 K, $\left.\mathrm{CDCl}_{3}\right)$ : $\delta$ 166.8, 141.0, 121.8, 37.3, 35.6, 18.1.

The obtained data is in accordance with the literature data. ${ }^{2}$

(E)-1-(6-methyl-2,3-dihydro-1H-pyrrolo[2,3-b]pyridin-1-yl)but-2-en-1-one (2i):

The reaction performed according to general procedure 2 afforded $406 \mathrm{mg}$ (67\%).

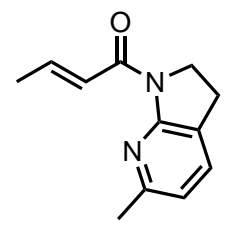

Colorless solid (m.p. $67-69^{\circ} \mathrm{C}$ ).

${ }^{1} \mathrm{H}$ NMR (400 MHz, $\left.300 \mathrm{~K}, \mathrm{CDCl}_{3}\right)$ : $\delta 7.90\left(\mathrm{dq}_{1} J=15.3,1.7 \mathrm{~Hz}, 1 \mathrm{H}\right), 7.32(\mathrm{dd}, J=7.6,1.3 \mathrm{~Hz}$, $1 \mathrm{H}), 7.17-7.04(\mathrm{~m}, 1 \mathrm{H}), 6.71(\mathrm{~d}, J=7.5 \mathrm{~Hz}, 1 \mathrm{H}), 4.17-4.09(\mathrm{~m}, 2 \mathrm{H}), 3.03-2.94(\mathrm{~m}, 2 \mathrm{H}), 2.47$ $(\mathrm{s}, 3 \mathrm{H}), 1.97(\mathrm{dd}, J=6.9,1.7 \mathrm{~Hz}, 3 \mathrm{H})$.

${ }^{13} \mathrm{C}$ NMR (101 MHz, 300 K, CDCl 3 ): $\delta$ 165.5, 155.8, 155.6, 142.3, 133.7, 125.0, 123.0, 117.2, 46.2, 24.3, 24.1, 18.5.

IR (thin film): $\widetilde{v} 2913,1660,1624,1592,1447,1415,1385,1341,1290,1255,1221,1085,974$, $899,771,678,640 \mathrm{~cm}^{-1}$.

HRMS (ESI): $m / z$ calculated for $\mathrm{C}_{12} \mathrm{H}_{14} \mathrm{~N}_{2} \mathrm{ONa}$ [M+Na] $]^{+}$225.0998, found: 225.1003.

(E)-1-(6-methoxy-2,3-dihydro-1H-pyrrolo[2,3-b]pyridin-1-yl)but-2-en-1-one (2j):

The reaction performed according to general procedure 2 afforded $405 \mathrm{mg}$ (62\%).

Colorless solid (m.p. $\left.104-106^{\circ} \mathrm{C}\right)$.

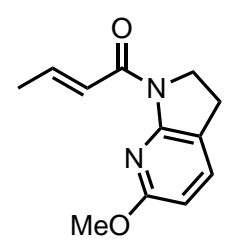

${ }^{1} \mathrm{H}$ NMR (400 MHz, 300 K, $\left.\mathrm{CDCl}_{3}\right): \delta 7.74(\mathrm{dd}, J=15.3,1.7 \mathrm{~Hz}, 1 \mathrm{H}), 7.29(\mathrm{dd}, J=8.1,1.0 \mathrm{~Hz}$, $1 \mathrm{H}), 7.04-6.92(\mathrm{~m}, 1 \mathrm{H}), 6.26(\mathrm{~d}, J=8.1 \mathrm{~Hz}, 1 \mathrm{H}), 4.11-4.01(\mathrm{~m}, 2 \mathrm{H}), 3.85(\mathrm{~s}, 3 \mathrm{H}), 2.94-2.84$ $(\mathrm{m}, 2 \mathrm{H}), 1.88(\mathrm{dd}, J=6.9,1.7 \mathrm{~Hz}, 3 \mathrm{H})$.

${ }^{13} \mathrm{C}$ NMR (101 MHz, 300 K, $\left.\mathrm{CDCl}_{3}\right)$ : $\delta$ 164.9, 162.9, 153.6, 141.3, 136.0, 125.0, 116.9, 103.4, 53.5, $46.5,23.5,18.5$.

IR (thin film): $\widetilde{v} 2954,1659,1609,1590,1465,1440,1419,1386,1343,1319,12191,1254,1224$, 1192, 1160, 1100, 1024, 962, 902, 822, 777, $668 \mathrm{~cm}^{-1}$.

HRMS (ESI): $m / z$ calculated for $\mathrm{C}_{12} \mathrm{H}_{14} \mathrm{~N}_{2} \mathrm{O}_{2} \mathrm{Na}$ [M+Na] $]^{+}: 241.0947$, found: 241.0951.

(E)-1-(6-phenyl-2,3-dihydro-1H-pyrrolo[2,3-b]pyridin-1-yl)but-2-en-1-one (2k):

The reaction performed according to general procedure 2 afforded $657 \mathrm{mg}(83 \%)$.

Colorless solid (m.p. $\left.161-163^{\circ} \mathrm{C}\right)$.

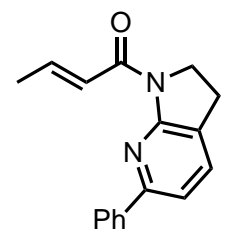

${ }^{1} \mathrm{H}$ NMR $\left(400 \mathrm{MHz}, 300 \mathrm{~K}, \mathrm{CDCl}_{3}\right): \delta 8.06(\mathrm{dd}, J=15.3,1.7 \mathrm{~Hz}, 1 \mathrm{H}), 8.04-7.97(\mathrm{~m}, 2 \mathrm{H}), 7.55$

$-7.43(\mathrm{~m}, 3 \mathrm{H}), 7.45-7.36(\mathrm{~m}, 1 \mathrm{H}), 7.36(\mathrm{~d}, J=7.7 \mathrm{~Hz}, 1 \mathrm{H}), 7.19-7.06(\mathrm{~m}, 1 \mathrm{H}), 4.26-4.14(\mathrm{~m}$, $2 \mathrm{H}), 3.12-2.99(\mathrm{~m}, 2 \mathrm{H}), 2.02(\mathrm{dd}, J=6.9,1.7 \mathrm{~Hz}, 3 \mathrm{H})$.

${ }^{13} \mathrm{C}$ NMR (101 MHz, 300 K, $\left.\mathrm{CDCl}_{3}\right): \delta$ 165.7, 156.2, 154.5, 142.0, 139.1, 134.2, 129.0, 128.9, 126.7, $125.5,125.0,114.5,46.2,24.2,18.7$.

IR (thin film): $\widetilde{v} 2965,1661,1628,1590,1575,1499,1483,1456,1446,1433,1415,1376,1342$, $1295,1242,1222,1186,1112,1030,976,916,870,851,838,774,743,695,671,638 \mathrm{~cm}^{-1}$.

HRMS (ESI): $m / z$ calculated for $\mathrm{C}_{17} \mathrm{H}_{16} \mathrm{~N}_{2} \mathrm{ONa}$ [M+Na] $]^{+}$287.1155, found: 287.1159 . 
(E)-1-(6-chloro-2,3-dihydro-1H-pyrrolo[2,3-b]pyridin-1-yl)but-2-en-1-one (21):

The reaction performed according to general procedure 2 afforded $425 \mathrm{mg}(64 \%)$.

Colorless solid (m.p. $\left.108-110^{\circ} \mathrm{C}\right)$.

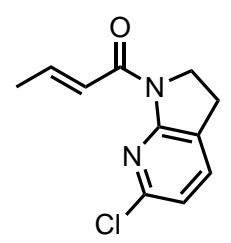

${ }^{1} \mathrm{H}$ NMR (400 MHz, 300 K, CDCl CD $_{3}$ : $7.61(\mathrm{dd}, J=15.2,1.7 \mathrm{~Hz}, 1 \mathrm{H}), 7.45-7.24(\mathrm{~m}, 1 \mathrm{H}), 7.22$ $-6.92(\mathrm{~m}, 1 \mathrm{H}), 6.80(\mathrm{~d}, J=7.7 \mathrm{~Hz}, 1 \mathrm{H}), 4.19-4.00(\mathrm{~m}, 2 \mathrm{H}), 3.11-2.84(\mathrm{~m}, 2 \mathrm{H}), 1.92(\mathrm{dd}, J=$ $6.9,1.7 \mathrm{~Hz}, 3 \mathrm{H})$.

${ }^{13} \mathrm{C}$ NMR (101 MHz, 300 K, $\left.\mathrm{CDCl}_{3}\right): \delta$ 165.1, 155.7, 147.8, 143.3, 135.4, 124.7, 124.1, 117.4, 46.4, 23.6, 18.4 .

IR (thin film): $\widetilde{v} 2914,1661,1624,1600,1575,1475,1422,1379,1339,1290,1250,1196,1113$, $1071,970,918,875,809,772,716,677,638 \mathrm{~cm}^{-1}$.

HRMS (ESI): $m / z$ calculated for $\mathrm{C}_{11} \mathrm{H}_{11} \mathrm{~N}_{2} \mathrm{OClNa}[\mathrm{M}+\mathrm{Na}]^{+}: 245.0452$, found: 245.0457 .

(E)-1-(but-2-enoyl)-2,3-dihydro-1H-pyrrolo[2,3-b]pyridine-6-carbonitrile (2m):

The reaction performed according to general procedure 2 afforded $492 \mathrm{mg}$ (77\%).

Colorless solid (m.p. $156-158^{\circ} \mathrm{C}$ ).

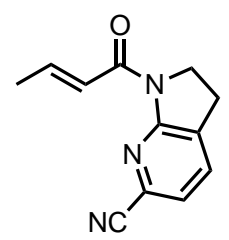

${ }^{1} \mathrm{H}$ NMR (400 MHz, $\left.300 \mathrm{~K}, \mathrm{CDCl}_{3}\right)$ : $\delta 7.66(\mathrm{dq} J=15.2,1.7 \mathrm{~Hz}, 1 \mathrm{H}), 7.53(\mathrm{dt}, J=7.4,1.4 \mathrm{~Hz}$, $1 \mathrm{H}), 7.28(\mathrm{~d}, J=7.5 \mathrm{~Hz}, 1 \mathrm{H}), 7.17(\mathrm{dq}, J=15.2,6.9 \mathrm{~Hz}, 1 \mathrm{H}), 4.19(\mathrm{dd}, J=9.2,8.0 \mathrm{~Hz}, 2 \mathrm{H}), 3.13$ $(\mathrm{ddd}, J=9.5,7.9,1.4 \mathrm{~Hz}, 2 \mathrm{H}), 2.00(\mathrm{dd}, J=6.9,1.7 \mathrm{~Hz}, 3 \mathrm{H})$.

${ }^{13}$ C NMR (101 MHz, 300 K, CDCl 3 ): $\delta$ 165.6, 157.0, 144.7, 133.8, 132.0, 129.7, 124.1, 123.4, 117.6, 46.1, 24.5, 18.6.

IR (thin film): $\widetilde{v} 3073,2969,2942,2231,1629,1601,1576,1478,1438,1381,1341,1288,1268$, 1250, 1233, 1216, 1080, 1032, 975, 921, 893, 849, 832, 772, 749, 679, 655, $501 \mathrm{~cm}^{-1}$.

HRMS (ESI): $m / z$ calculated for $\mathrm{C}_{12} \mathrm{H}_{12} \mathrm{~N}_{3} \mathrm{O}[\mathrm{M}+\mathrm{H}]^{+}: 214.0975$, found: 214.0975 .

(E)-1-(4-chloro-2,3-dihydro-1H-pyrrolo[2,3-b]pyridin-1-yl)but-2-en-1-one (2n):

The reaction performed according to general procedure 2 afforded $453 \mathrm{mg}(68 \%)$.

Colorless solid (m.p. $124-126^{\circ} \mathrm{C}$ ).

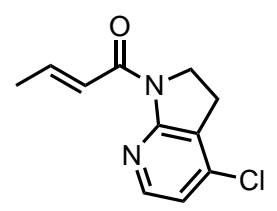

${ }^{1} \mathrm{H}$ NMR $\left(400 \mathrm{MHz}, 300 \mathrm{~K}, \mathrm{CDCl}_{3}\right): \delta 7.98(\mathrm{~d}, J=5.6 \mathrm{~Hz}, 1 \mathrm{H}), 7.72-7.65(\mathrm{~m}, 1 \mathrm{H}), 7.14-7.03$ $(\mathrm{m}, 1 \mathrm{H}), 6.82(\mathrm{~d}, J=5.6 \mathrm{~Hz}, 1 \mathrm{H}), 4.17-4.05(\mathrm{~m}, 2 \mathrm{H}), 3.11-2.95(\mathrm{~m}, 2 \mathrm{H}), 1.93(\mathrm{dd}, J=7.0,1.7$ $\mathrm{Hz}, 3 \mathrm{H})$.

${ }^{13}$ C NMR (101 MHz, 300 K, CDCl $): \delta$ 165.3, 157.1, 147.2, 143.3, 140.8, 125.1, 124.3, 118.2, 45.6, 23.5, 18.4.

IR (thin film): $\widetilde{v} 2907,1661,1624,1597,1484,1434,1409,1349,1240,1081,974,804,640$ $\mathrm{cm}^{-1}$.

HRMS (ESI): $m / z$ calculated for $\mathrm{C}_{11} \mathrm{H}_{11} \mathrm{~N}_{2} \mathrm{OClNa}[\mathrm{M}+\mathrm{Na}]^{+}: 245.0452$, found: 245.0457 .

(E)-1-(3,3-dimethyl-2,3-dihydro-1H-pyrrolo[2,3-b]pyridin-1-yl)but-2-en-1-one (2o):

The reaction performed according to general procedure 2 afforded $382 \mathrm{mg}$ (59\%).

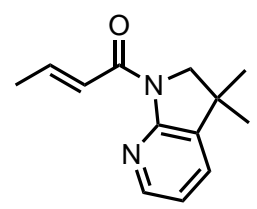

Colorless solid (m.p. $64-66^{\circ} \mathrm{C}$ ).

${ }^{1} \mathrm{H}$ NMR (400 MHz, 300 K, $\left.\mathrm{CDCl}_{3}\right)$ : $\delta 8.15(\mathrm{dd}, J=5.1,1.6 \mathrm{~Hz}, 1 \mathrm{H}), 7.79(\mathrm{dd}, J=15.3,1.7 \mathrm{~Hz}$, $1 \mathrm{H}), 7.41(\mathrm{dd}, J=7.4,1.7 \mathrm{~Hz}, 1 \mathrm{H}), 7.20-7.06(\mathrm{~m}, 1 \mathrm{H}), 6.90(\mathrm{dd}, J=7.4,5.1 \mathrm{~Hz}, 1 \mathrm{H}), 3.89(\mathrm{~s}$, $2 \mathrm{H}), 1.98(\mathrm{dd}, J=6.9,1.7 \mathrm{~Hz}, 3 \mathrm{H}), 1.32(\mathrm{~s}, 6 \mathrm{H})$.

${ }^{13} \mathrm{C}$ NMR (101 MHz, $\left.300 \mathrm{~K}, \mathrm{CDCl}_{3}\right)$ : $\delta$ 165.5, 154.9, 146.5, 142.9, 135.6, 131.1, 124.8, 118.4, 60.5, $36.7,28.5,18.5$.

IR (thin film): $\widetilde{v} 2960,1663,1624,1598,1418,1377,1349,1294,1221,1138,1065,972,916$, $777,694,641 \mathrm{~cm}^{-1}$.

HRMS (ESI): $m / z$ calculated for $\mathrm{C}_{13} \mathrm{H}_{17} \mathrm{~N}_{2} \mathrm{O}[\mathrm{M}+\mathrm{H}]^{+}:$217.1335, found: 217.1338. 


\subsection{Preparation of amides from carboxylic acids (General Procedure 3)}

To a solution of the corresponding carboxylic acid (1.2 mmol, 1.2 equiv.), 2,3-dihydro-7-azaindole (120 mg, $1 \mathrm{mmol}, 1$ equiv.) and 4-dimethylaminopyridine (DMAP: $61 \mathrm{mg}, 0.5 \mathrm{mmol}, 0.5$ equiv.) in anhydrous $\mathrm{CH}_{2} \mathrm{Cl}_{2}$ $(20 \mathrm{~mL})$ was added solid EDCI.HCl (230 mg, $1.2 \mathrm{mmol}, 1.2$ equiv) in one portion and the mixture was stirred at room temperature for $24 \mathrm{~h}$. The reaction mixture was diluted with saturated $\mathrm{NaHCO}_{3}(30 \mathrm{~mL})$ and the product was extracted with $\mathrm{CH}_{2} \mathrm{Cl}_{2}(3 \times 20 \mathrm{~mL})$. The combined organic layer was dried over $\mathrm{Na}_{2} \mathrm{SO}_{4}$ and concentrated under reduced pressure. The crude residue was purified by automated flash column chromatography (Biotage Isolera One) with a pre-packed silica gel column using Hexane/Ethyl acetate $(\sim 8 / 2)$ eluents. All the solid products were subsequently recrystallized from hot Hexane or $\mathrm{CH}_{2} \mathrm{Cl}_{2} / \mathrm{Hexane}$.

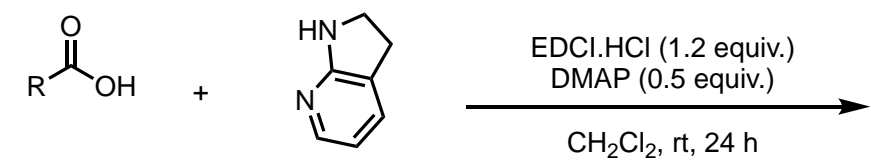

carboxylic acid

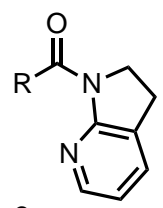

2

(E)-1-(2,3-dihydro-1H-pyrrolo[2,3-b]pyridin-1-yl)-4,4,4-trifluorobut-2-en-1-one (2p):

The reaction performed according to general procedure 3 afforded $184 \mathrm{mg}(76 \%)$.

Colorless solid (m.p. $72-73^{\circ} \mathrm{C}$ ).

${ }^{1} \mathrm{H}$ NMR (400 MHz, 300 K, $\mathrm{CDCl}_{3}$ ): $\delta 8.59$ (dq, $\left.J=15.6,2.1 \mathrm{~Hz}, 1 \mathrm{H}\right), 8.14$ (ddt, $J=5.1,1.8$, $1.0 \mathrm{~Hz}, 1 \mathrm{H}), 7.51(\mathrm{dq}, J=7.4,1.4 \mathrm{~Hz}, 1 \mathrm{H}), 6.94(\mathrm{dd}, J=7.4,5.1 \mathrm{~Hz}, 1 \mathrm{H}), 6.92-6.82(\mathrm{~m}, 1 \mathrm{H})$,

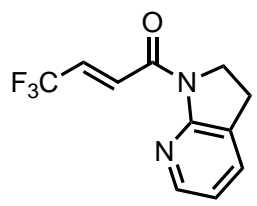
$4.18(\mathrm{dd}, J=9.1,7.8 \mathrm{~Hz}, 2 \mathrm{H}), 3.14-3.07(\mathrm{~m}, 2 \mathrm{H})$.

${ }^{13} \mathrm{C}$ NMR (101 MHz, 300 K, CDCl 3 ): $\delta$ 162.2, 155.4, 146.6, 134.1, 130.9 (q, J = 6.2 Hz), 128.9 $(q, J=34.8 \mathrm{~Hz}), 126.31,123.1(q, J=269.9 \mathrm{~Hz}), 119.1,46.1,24.4$.

${ }^{19} \mathrm{~F}$ NMR (376 MHz, $\left.300 \mathrm{~K}, \mathrm{CDCl}_{3}\right)$ : $\delta-64.66$.

IR (thin film): $\widetilde{v} 3264,3188,3091,3060,3019,2972,2914,2858,1680,1650,1634,1590$, $1479,1447,1433,1358,1309,1240,1116,1075,1039,1015,977,886,863,790,728,700,647$, $634,600,557 \mathrm{~cm}^{-1}$.

HRMS (ESI): $m / z$ calculated for $\mathrm{C}_{11} \mathrm{H}_{10} \mathrm{~N}_{2} \mathrm{OF}_{3}[\mathrm{M}+\mathrm{H}]^{+}: 243.0740$, found: 243.0741 .

(E)-1-(2,3-dihydro-1H-pyrrolo[2,3-b]pyridin-1-yl)-3-phenylprop-2-en-1-one (2u):

The reaction performed according to general procedure 3 afforded $213 \mathrm{mg}(85 \%)$.

Colorless solid (m.p. $\left.101-103^{\circ} \mathrm{C}\right)$.

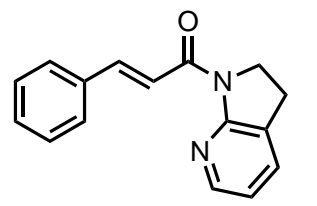

${ }^{1} \mathrm{H}$ NMR $\left(400 \mathrm{MHz}, 300 \mathrm{~K}, \mathrm{CDCl}_{3}\right): \delta 8.57(\mathrm{~d}, J=15.7 \mathrm{~Hz}, 1 \mathrm{H}), 8.25-8.12(\mathrm{~m}, 1 \mathrm{H}), 7.85(\mathrm{~d}$, $J=15.8 \mathrm{~Hz}, 1 \mathrm{H}), 7.72-7.59(\mathrm{~m}, 2 \mathrm{H}), 7.48(\mathrm{dd}, J=7.3,1.5 \mathrm{~Hz}, 1 \mathrm{H}), 7.46-7.31(\mathrm{~m}, 3 \mathrm{H}), 6.89$ (dd, $J=7.3,5.0 \mathrm{~Hz}, 1 \mathrm{H}), 4.34-4.05(\mathrm{~m}, 2 \mathrm{H}), 3.16-2.98(\mathrm{~m}, 2 \mathrm{H})$.

${ }^{13} \mathrm{C}$ NMR (101 MHz, $\left.300 \mathrm{~K}, \mathrm{CDCl}_{3}\right)$ : $\delta$ 165.6, 156.4, 146.5, 142.9, 135.9, 133.7, 129.7, 128.8, $128.4,126.5,121.0,118.2,46.2,24.4$.

IR (thin film): $\widetilde{v} 3080,1650,1600,1587,1476,1442,1419,1381,1355,1300,1240,1222$, $1020,983,763,703,567 \mathrm{~cm}^{-1}$.

HRMS (ESI): $m / z$ calculated for $\mathrm{C}_{16} \mathrm{H}_{15} \mathrm{~N}_{2} \mathrm{O}[\mathrm{M}+\mathrm{H}]^{+}: 251.1179$, found: 251.1180 . 
(E)-1-(2,3-dihydro-1H-pyrrolo[2,3-b]pyridin-1-yl)-3-(2-(trifluoromethyl)phenyl)prop-2-en-1-one (2v):

The reaction performed according to general procedure 3 afforded $308 \mathrm{mg}(97 \%)$.

Colorless solid (m.p. $112-114^{\circ} \mathrm{C}$ ).

${ }^{1} \mathrm{H}$ NMR (400 MHz, 300 K, $\left.\mathrm{CDCl}_{3}\right): \delta 8.55(\mathrm{~d}, J=15.5 \mathrm{~Hz}, 1 \mathrm{H}), 8.21(\mathrm{dq}, J=15.6,2.4 \mathrm{~Hz}$,

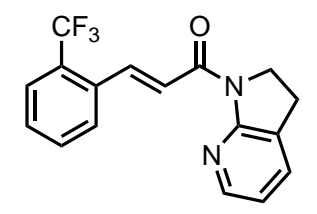
$1 \mathrm{H}), 8.15(\mathrm{ddt}, J=5.1,1.8,1.0 \mathrm{~Hz}, 1 \mathrm{H}), 7.91(\mathrm{~d}, J=7.8 \mathrm{~Hz}, 1 \mathrm{H}), 7.72-7.67(\mathrm{~m}, 1 \mathrm{H}), 7.63-$ $7.56(\mathrm{~m}, 1 \mathrm{H}), 7.49(\mathrm{dq}, J=7.5,1.5 \mathrm{~Hz}, 1 \mathrm{H}), 7.47-7.42(\mathrm{~m}, 1 \mathrm{H}), 6.90(\mathrm{dd}, J=7.3,5.1 \mathrm{~Hz}$, $1 \mathrm{H}), 4.24(\mathrm{dd}, J=9.2,7.7 \mathrm{~Hz}, 2 \mathrm{H}), 3.10(\mathrm{tt}, J=8.6,1.2 \mathrm{~Hz}, 2 \mathrm{H})$.

${ }^{13} \mathrm{C}$ NMR (101 MHz, $\left.300 \mathrm{~K}, \mathrm{CDCl}_{3}\right): \delta$ 164.7, 156.2, 146.4, 138.1 (d, $\left.J=2.2 \mathrm{~Hz}\right), 134.9$ (q, $J$ $=1.8 \mathrm{~Hz}), 133.8,132.1,129.1,129.0(\mathrm{q}, J=30.3 \mathrm{~Hz}), 128.5,126.6,126.1(\mathrm{q}, J=5.6 \mathrm{~Hz}), 125.1$, $124.2(\mathrm{q}, J=274.0 \mathrm{~Hz}), 118.3,46.1,24.4$.

${ }^{19}$ F NMR (376 MHz, $\left.300 \mathrm{~K}, \mathrm{CDCl}_{3}\right)$ : $\delta-58.90$.

IR (thin film): $\widetilde{v} 3085,3011,2967,2912,1654,1617,1600,1589,1575,1485,1444,1421$, 1384, 1356, 1314, 1295, 1280, 1240, 1226, 1160, 1121, 1060, 1036, 1021, 979, 877, 766, 694, $649,595,578 \mathrm{~cm}^{-1}$.

HRMS (ESI): $m / z$ calculated for $\mathrm{C}_{17} \mathrm{H}_{13} \mathrm{ON}_{2} \mathrm{~F}_{3} \mathrm{Na}[\mathrm{M}+\mathrm{Na}]^{+}: 341.0872$, found: 341.0877 .

(E)-1-(2,3-dihydro-1H-pyrrolo[2,3-b]pyridin-1-yl)-3-(3-(trifluoromethyl)phenyl)prop-2-en-1-one (2w):

The reaction performed according to general procedure 3 afforded $280 \mathrm{mg}(88 \%)$.

Colorless solid (m.p. $116-118^{\circ} \mathrm{C}$ ).

${ }^{1}$ H NMR (400 MHz, 300 K, $\left.\mathrm{CDCl}_{3}\right): \delta 8.62(\mathrm{~d}, J=15.7 \mathrm{~Hz}, 1 \mathrm{H}), 8.22-8.15(\mathrm{~m}, 1 \mathrm{H}), 7.89$ $-7.78(\mathrm{~m}, 3 \mathrm{H}), 7.60(\mathrm{~d}, J=7.8 \mathrm{~Hz}, 1 \mathrm{H}), 7.55-7.46(\mathrm{~m}, 2 \mathrm{H}), 6.91(\mathrm{ddd}, J=7.2,5.0,1.6 \mathrm{~Hz}$,<smiles>O=C(/C=C/c1cccc(C(F)(F)F)c1)N1CCc2cccnc21</smiles>
$1 \mathrm{H}), 4.27-4.18(\mathrm{~m}, 2 \mathrm{H}), 3.15-3.05(\mathrm{~m}, 2 \mathrm{H})$.

${ }^{13}$ C NMR (101 MHz, 300 K, CDCl 3 ): $\delta$ 165.0, 156.2, 146.5, 140.9, 136.7, 133.8, 131.4, 131.3 $(\mathrm{q}, J=32.3 \mathrm{~Hz}), 129.4,126.5,126.1(\mathrm{q}, J=3.8 \mathrm{~Hz}), 124.9(\mathrm{q}, J=3.9 \mathrm{~Hz}), 124.1(\mathrm{q}, J=272.4$ Hz), 122.9, 118.4, 46.1, 24.4.

${ }^{19} \mathrm{~F}$ NMR (376 MHz, $\left.300 \mathrm{~K}, \mathrm{CDCl}_{3}\right)$ : $\delta-62.80$.

IR (thin film): $\widetilde{v} 3078,3015,2965,2912,1655,1618,1601,1589,1480,1440,1421,1383$, $1357,1332,1299,1242,1223,1197,1164,1124,1096,1076,1020,984,922,902,864,799$, $783,745,692,657,594 \mathrm{~cm}^{-1}$.

HRMS (ESI): $m / z$ calculated for $\mathrm{C}_{17} \mathrm{H}_{13} \mathrm{ON}_{2} \mathrm{~F}_{3} \mathrm{Na}[\mathrm{M}+\mathrm{Na}]^{+}: 341.0872$, found: 341.0876 .

(E)-1-(2,3-dihydro-1H-pyrrolo[2,3-b]pyridin-1-yl)-3-(4-(trifluoromethyl)phenyl)prop-2-en-1-one (2x):

The reaction performed according to general procedure 3 afforded $254 \mathrm{mg}(80 \%)$.

Colorless solid (m.p. $133-135^{\circ} \mathrm{C}$ ).

${ }^{1} \mathrm{H}$ NMR (400 MHz, 300 K, CDCl CD $_{3}$ : $8.65(\mathrm{~d}, J=15.8 \mathrm{~Hz}, 1 \mathrm{H}), 8.19(\mathrm{dd}, J=5.1,1.5 \mathrm{~Hz}$, $1 \mathrm{H}), 7.84(\mathrm{~d}, J=15.8 \mathrm{~Hz}, 1 \mathrm{H}), 7.77-7.62(\mathrm{~m}, 4 \mathrm{H}), 7.50(\mathrm{dq}, J=7.5,1.5 \mathrm{~Hz}, 1 \mathrm{H}), 6.92(\mathrm{dd}$,<smiles>O=C(/C=C/c1ccc(C(F)(F)F)cc1)N1CCc2cccnc21</smiles>
$J=7.3,5.1 \mathrm{~Hz}, 1 \mathrm{H}), 4.23(\mathrm{dd}, J=9.1,7.9 \mathrm{~Hz}, 2 \mathrm{H}), 3.11(\mathrm{t}, J=8.4 \mathrm{~Hz}, 2 \mathrm{H})$.

${ }^{13} \mathrm{C}$ NMR (101 MHz, $\left.300 \mathrm{~K}, \mathrm{CDCl}_{3}\right): \delta$ 165.0, 156.2, 146.5, 140.8, $139.3(\mathrm{~d}, J=1.5 \mathrm{~Hz})$, $133.8,131.2(q, J=32.6 \mathrm{~Hz}), 128.5,126.6,125.8(\mathrm{q}, J=3.8 \mathrm{~Hz}), 124.1(\mathrm{q}, J=272.0 \mathrm{~Hz})$, 123.5, 118.4, 46.2, 24.4 .

${ }^{19} \mathrm{~F}$ NMR (376 MHz, $\left.300 \mathrm{~K}, \mathrm{CDCl}_{3}\right)$ : $\delta-62.72$.

IR (thin film): $\widetilde{v} 3090,3050,1654,1618,1601,1586,1475,1441,1416,1381,1356,1336$, 1323, 1299, 1284, 1239, 1157, 1118, 1107, 1067, 1024, 1016, 1001, 848, 835, 790, 745, 694, $618,593 \mathrm{~cm}^{-1}$.

HRMS (ESI): $m / z$ calculated for $\mathrm{C}_{17} \mathrm{H}_{13} \mathrm{ON}_{2} \mathrm{~F}_{3} \mathrm{Na}$ [M+Na] $]^{+}$341.0872, found: 341.0874 . 
(E)-1-(2,3-dihydro-1H-pyrrolo[2,3-b]pyridin-1-yl)-3-(2-methoxyphenyl)prop-2-en-1-one (2y):

The reaction performed according to general procedure 3 afforded $255 \mathrm{mg}(91 \%)$.

Colorless solid (m.p. $117-119^{\circ} \mathrm{C}$ ).

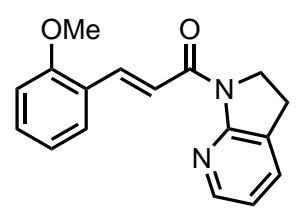

${ }^{1} \mathrm{H}$ NMR $\left(400 \mathrm{MHz}, 300 \mathrm{~K}, \mathrm{CDCl}_{3}\right)$ : $\delta 8.64-8.15(\mathrm{~m}, 3 \mathrm{H}), 7.69(\mathrm{dd}, J=7.6,1.7 \mathrm{~Hz}, 1 \mathrm{H})$, $7.47(\mathrm{dq}, J=7.3,1.4 \mathrm{~Hz}, 1 \mathrm{H}), 7.33(\mathrm{ddd}, J=8.2,7.3,1.7 \mathrm{~Hz}, 1 \mathrm{H}), 6.99(\mathrm{td}, J=7.5,1.1 \mathrm{~Hz}$, $1 \mathrm{H}), 6.94-6.85(\mathrm{~m}, 2 \mathrm{H}), 4.27-4.19(\mathrm{~m}, 2 \mathrm{H}), 3.91(\mathrm{~s}, 3 \mathrm{H}), 3.09(\mathrm{ddt}, J=9.7,7.6,1.1 \mathrm{~Hz}$, $2 \mathrm{H})$.

${ }^{13} \mathrm{C}$ NMR (101 MHz, 300 K, CDCl 3 ): $\delta$ 166.1, 158.5, 156.6, 146.4, 138.2, 133.6, 130.9, 129.1, 126.6, 125.0, 121.3, 120.7, 118.0, 111.2, 55.7, 46.2, 24.5.

IR (thin film): $\widetilde{v} 3079,3005,2960,2836,1649,1600,1487,1462,1441,1418,1381,1354$, 1296, 1278, 1243, 1222, 1196, 1162, 1106, 1050, 1023, 992, 869, 782, 751, 689, 596, 588, 566 $\mathrm{cm}^{-1}$.

HRMS (ESI): $m / z$ calculated for $\mathrm{C}_{17} \mathrm{H}_{17} \mathrm{O}_{2} \mathrm{~N}_{2}[\mathrm{M}+\mathrm{H}]^{+}:$281.1285, found: 281.1289 .

(E)-1-(2,3-dihydro-1H-pyrrolo[2,3-b]pyridin-1-yl)-3-(3-methoxyphenyl)prop-2-en-1-one (2z):

The reaction performed according to general procedure 3 afforded $254 \mathrm{mg}(91 \%)$.

Colorless solid (m.p. $68-70^{\circ} \mathrm{C}$ ).

${ }^{1} \mathrm{H}$ NMR (400 MHz, 300 K, $\left.\mathbf{C D C l}_{3}\right): \delta 8.55(\mathrm{~d}, J=15.8 \mathrm{~Hz}, 1 \mathrm{H}), 8.18$ (ddt, J = 5.2, 1.9,<smiles>COc1cccc(/C=C/C(=O)N2CCc3cccnc32)c1</smiles>
$1.0 \mathrm{~Hz}, 1 \mathrm{H}), 7.84-7.77(\mathrm{~m}, 1 \mathrm{H}), 7.48(\mathrm{dt}, J=6.9,1.4 \mathrm{~Hz}, 1 \mathrm{H}), 7.32(\mathrm{t}, J=7.8 \mathrm{~Hz}, 1 \mathrm{H})$, $7.28-7.23(\mathrm{~m}, 1 \mathrm{H}), 7.19(\mathrm{t}, J=2.2 \mathrm{~Hz}, 1 \mathrm{H}), 6.94-6.86(\mathrm{~m}, 2 \mathrm{H}), 4.23(\mathrm{td}, J=8.1,1.6 \mathrm{~Hz}$, $2 \mathrm{H}), 3.86(\mathrm{~s}, 3 \mathrm{H}), 3.13-3.05(\mathrm{~m}, 2 \mathrm{H})$.

${ }^{13} \mathrm{C}$ NMR (101 MHz, $\left.300 \mathrm{~K}, \mathrm{CDCl}_{3}\right): \delta$ 165.5, 159.9, 156.3, 146.5, 142.7, 137.3, 133.7, $129.8,126.5,121.3,121.1,118.2,115.3,113.7,55.4,46.1,24.4$.

IR (thin film): $\widetilde{v} 3082,2959,2834,1651,1600,1587,1486,1441,1418,1381,1354,1316$, $1290,1266,1241,1219,1162,1046,1021,984,850,780,735,678 \mathrm{~cm}^{-1}$.

HRMS (ESI): $m / z$ calculated for $\mathrm{C}_{17} \mathrm{H}_{17} \mathrm{O}_{2} \mathrm{~N}_{2}[\mathrm{M}+\mathrm{H}]^{+}: 281.1285$, found: 281.1289.

(E)-1-(2,3-dihydro-1H-pyrrolo[2,3-b]pyridin-1-yl)-3-(4-methoxyphenyl)prop-2-en-1-one (2aa):

The reaction performed according to general procedure 3 afforded $255 \mathrm{mg}$ (91\%).

Colorless solid (m.p. $113-115^{\circ} \mathrm{C}$ ).

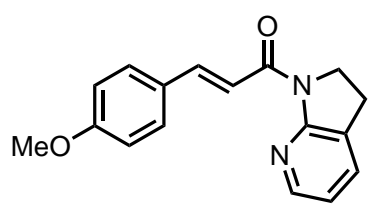

${ }^{1} \mathrm{H}$ NMR (400 MHz, 300 K, $\left.\mathrm{CDCl}_{3}\right): \delta 8.44(\mathrm{~d}, J=15.7 \mathrm{~Hz}, 1 \mathrm{H}), 8.18$ (ddt, J = 5.2, 1.8, $1.0 \mathrm{~Hz}, 1 \mathrm{H}), 7.81(\mathrm{~d}, J=15.7 \mathrm{~Hz}, 1 \mathrm{H}), 7.63-7.57(\mathrm{~m}, 2 \mathrm{H}), 7.46(\mathrm{dq}, J=7.3,1.4 \mathrm{~Hz}, 1 \mathrm{H})$, $6.94-6.89(\mathrm{~m}, 2 \mathrm{H}), 6.89-6.85(\mathrm{~m}, 1 \mathrm{H}), 4.24-4.17(\mathrm{~m}, 2 \mathrm{H}), 3.83(\mathrm{~d}, J=1.0 \mathrm{~Hz}, 3 \mathrm{H}), 3.11$ $-3.02(\mathrm{~m}, 2 \mathrm{H})$.

${ }^{13} \mathrm{C}$ NMR (101 MHz, 300 K, $\left.\mathrm{CDCl}_{3}\right): \delta 165.9,161.0,156.5,146.4,142.6,133.6,130.0$, $128.7,126.5,118.5,118.0,114.2,55.4,46.1,24.4$.

IR (thin film): $\widetilde{v} 3082,2959,2834,1649,1574,1510,1477,1441,1419,1380,1356,1305$, $1288,1249,1223,1171,1026,986,827,782,554,535,518 \mathrm{~cm}^{-1}$.

HRMS (ESI): $m / z$ calculated for $\mathrm{C}_{17} \mathrm{H}_{17} \mathrm{O}_{2} \mathrm{~N}_{2}[\mathrm{M}+\mathrm{H}]^{+}:$281.1285, found: 281.1291. 
(E)-1-(2,3-dihydro-1H-pyrrolo[2,3-b]pyridin-1-yl)-3-(o-tolyl)prop-2-en-1-one (2ab):

The reaction performed according to general procedure 3 afforded $232 \mathrm{mg}(88 \%)$.

Colorless solid (m.p. $\left.114-116^{\circ} \mathrm{C}\right)$.

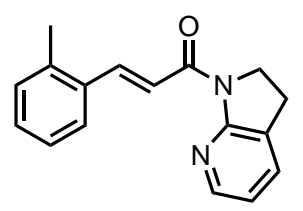

${ }^{1} \mathrm{H}$ NMR (400 MHz, 300 K, $\left.\mathrm{CDCl}_{3}\right): \delta 8.47(\mathrm{~d}, J=15.6 \mathrm{~Hz}, 1 \mathrm{H}), 8.17$ (ddt, $J=5.1,1.8,1.0$ $\mathrm{Hz}, 1 \mathrm{H}), 8.13(\mathrm{~d}, J=15.6 \mathrm{~Hz}, 1 \mathrm{H}), 7.77-7.71(\mathrm{~m}, 1 \mathrm{H}), 7.48(\mathrm{dq}, J=7.3,1.4 \mathrm{~Hz}, 1 \mathrm{H}), 7.29-$ $7.23(\mathrm{~m}, 2 \mathrm{H}), 7.23-7.18(\mathrm{~m}, 1 \mathrm{H}), 6.89(\mathrm{dd}, J=7.3,5.1 \mathrm{~Hz}, 1 \mathrm{H}), 4.28-4.20(\mathrm{~m}, 2 \mathrm{H}), 3.10$ $(\mathrm{ddt}, J=9.7,7.5,1.1 \mathrm{~Hz}, 2 \mathrm{H}), 2.49(\mathrm{~d}, J=0.7 \mathrm{~Hz}, 3 \mathrm{H})$.

${ }^{13}$ C NMR (101 MHz, 300 K, $\left.\mathrm{CDCl}_{3}\right)$ : $\delta$ 165.7, 156.4, 146.5, 140.6, 137.8, 134.8, 133.6, 130.8, 129.5, 127.1, 126.5, 126.3, 122.0, 118.1, 46.2, 24.5, 20.1.

IR (thin film): $\widetilde{v} 3081,3016,2964,1650,1599,1587,1480,1441,1419,1381,1353,1295$, $1281,1240,1222,1164,1020,984,868,781,759,727,687,595 \mathrm{~cm}^{-1}$.

HRMS (ESI): $m / z$ calculated for $\mathrm{C}_{17} \mathrm{H}_{16} \mathrm{ON} 2 \mathrm{Na}[\mathrm{M}+\mathrm{Na}]^{+}: 287.1155$, found: 287.1158.

(E)-1-(2,3-dihydro-1H-pyrrolo[2,3-b]pyridin-1-yl)-3-( $p$-tolyl)prop-2-en-1-one (2ac):

The reaction performed according to general procedure 3 afforded $237 \mathrm{mg}(90 \%)$.

Colorless solid (m.p. $116-118^{\circ} \mathrm{C}$ ).

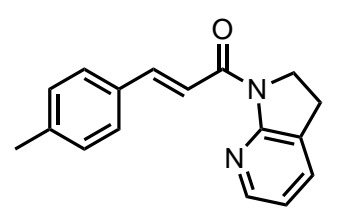

${ }^{1} \mathrm{H}$ NMR (400 MHz, $300 \mathrm{~K}, \mathrm{CDCl}_{3}$ ): $\delta 8.51(\mathrm{~d}, J=15.7 \mathrm{~Hz}, 1 \mathrm{H}), 8.19$ (ddt, $J=5.1,1.8,1.0$ $\mathrm{Hz}, 1 \mathrm{H}), 7.83(\mathrm{~d}, J=15.7 \mathrm{~Hz}, 1 \mathrm{H}), 7.58-7.52(\mathrm{~m}, 2 \mathrm{H}), 7.47(\mathrm{dq}, J=7.3,1.4 \mathrm{~Hz}, 1 \mathrm{H}), 7.20$ $(\mathrm{d}, J=7.9 \mathrm{~Hz}, 2 \mathrm{H}), 6.89(\mathrm{dd}, J=7.3,5.1 \mathrm{~Hz}, 1 \mathrm{H}), 4.22(\mathrm{dd}, J=9.1,7.9 \mathrm{~Hz}, 2 \mathrm{H}), 3.09(\mathrm{tt}, J=$ 8.6, $1.1 \mathrm{~Hz}, 2 \mathrm{H}), 2.38(\mathrm{~s}, 3 \mathrm{H})$.

${ }^{13} \mathrm{C}$ NMR (101 MHz, 300 K, CDCl $\mathrm{CD}_{3}$ : $\delta$ 165.8, 156.5, 146.5, 142.9, 140.0, 133.6, 133.2, 129.6, $128.4,126.5,119.9,118.1,46.2,24.4,21.6$.

IR (thin film): $\widetilde{v} 3084,2916,1650,1601,1586,1511,1477,1442,1419,1381,1354,1297$, $1241,1224,1211,1180,1164,1020,986,811,781,683 \mathrm{~cm}^{-1}$.

HRMS (ESI): $m / z$ calculated for $\mathrm{C}_{17} \mathrm{H}_{17} \mathrm{ON}_{2}[\mathrm{M}+\mathrm{H}]^{+}: 265.1335$, found: 265.1339 .

(E)-1-(2,3-dihydro-1H-pyrrolo[2,3-b]pyridin-1-yl)-3-(4-fluorophenyl)prop-2-en-1-one (2ad):

The reaction performed according to general procedure 3 afforded $233 \mathrm{mg}(87 \%)$.

Colorless solid (m.p. 129-130 ${ }^{\circ} \mathrm{C}$ ).

${ }^{1} \mathrm{H}$ NMR (400 MHz, 300 K, $\left.\mathrm{CDCl}_{3}\right): \delta 8.50$ (d, J = 15.7 Hz, 1H), 8.19 (ddt, J = 5.2, 1.8, 1.0

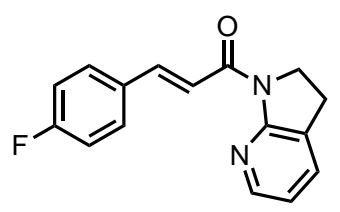

$\mathrm{Hz}, 1 \mathrm{H}), 7.81(\mathrm{~d}, J=15.7 \mathrm{~Hz}, 1 \mathrm{H}), 7.67-7.58(\mathrm{~m}, 2 \mathrm{H}), 7.49(\mathrm{dq}, J=7.3,1.4 \mathrm{~Hz}, 1 \mathrm{H}), 7.14$

$-7.03(\mathrm{~m}, 2 \mathrm{H}), 6.90(\mathrm{dd}, J=7.4,5.1 \mathrm{~Hz}, 1 \mathrm{H}), 4.23(\mathrm{dd}, J=9.1,7.9 \mathrm{~Hz}, 2 \mathrm{H}), 3.10(\mathrm{ddt}, J=$

9.7, 8.6, $1.2 \mathrm{~Hz}, 2 \mathrm{H})$.

${ }^{13} \mathrm{C}$ NMR (101 MHz, 300 K, $\left.\mathrm{CDCl}_{3}\right)$ : $\delta$ 165.5, 163.7 (d, J = 250.0 Hz), 156.4, 146.5, 141.6, 133.7, $132.1(\mathrm{~d}, J=3.3 \mathrm{~Hz}), 130.2(\mathrm{~d}, J=8.3 \mathrm{~Hz}), 126.6,120.7(\mathrm{~d}, J=2.4 \mathrm{~Hz}), 118.2,115.9$ $(\mathrm{d}, J=21.8 \mathrm{~Hz}), 46.2,24.4$.

${ }^{19} \mathrm{~F}$ NMR (376 MHz, $\left.300 \mathrm{~K}, \mathrm{CDCl}_{3}\right): \delta-110.91$.

IR (thin film): $\widetilde{v} 3086,1651,1599,1589,1508,1477,1442,1419,1383,1355,1297,1223$, $1158,1021,981,830,781,745,686 \mathrm{~cm}^{-1}$.

HRMS (ESI): $\mathrm{m} / \mathrm{z}$ calculated for $\mathrm{C}_{16} \mathrm{H}_{13} \mathrm{ON}_{2} \mathrm{FNa}[\mathrm{M}+\mathrm{Na}]^{+}: 291.0904$, found: 291.0907. 
(E)-3-(4-chlorophenyl)-1-(2,3-dihydro-1H-pyrrolo[2,3-b]pyridin-1-yl)prop-2-en-1-one (2ae):

The reaction performed according to general procedure 3 afforded $256 \mathrm{mg}$ (90\%).

Colorless solid (m.p. $107-109^{\circ} \mathrm{C}$ ).

${ }^{1} \mathrm{H}$ NMR (400 MHz, 300 K, $\mathrm{CDCl}_{3}$ ): $\delta 8.53$ (d, J = 15.7 Hz, 1H), 8.18 (ddt, J= 5.1, 1.9, 1.0<smiles>O=C(/C=C/c1ccc(Cl)cc1)N1CCc2cccnc21</smiles>
$\mathrm{Hz}, 1 \mathrm{H}), 7.77(\mathrm{~d}, J=15.7 \mathrm{~Hz}, 1 \mathrm{H}), 7.60-7.54(\mathrm{~m}, 2 \mathrm{H}), 7.48(\mathrm{dq}, J=7.4,1.4 \mathrm{~Hz}, 1 \mathrm{H}), 7.38$ $-7.31(\mathrm{~m}, 2 \mathrm{H}), 6.90(\mathrm{dd}, J=7.4,5.1 \mathrm{~Hz}, 1 \mathrm{H}), 4.21(\mathrm{dd}, J=9.1,7.9 \mathrm{~Hz}, 2 \mathrm{H}), 3.09(\mathrm{ddt}, J=$ 9.7, 8.6, $1.2 \mathrm{~Hz}, 2 \mathrm{H})$.

${ }^{13} \mathrm{C}$ NMR (101 MHz, 300 K, $\left.\mathrm{CDCl}_{3}\right): \delta$ 165.3, 156.3, 146.4, 141.3, 135.5, 134.4, 133.7, 129.6, $129.1,126.5,121.5,118.2,46.1,24.4$.

IR (thin film): $\widetilde{v} 3083,1652,1600,1588,1567,1489,1478,1442,1419,1381,1353,1296$, $1241,1224,1165,1090,1011,984,820,779,701,647,591 \mathrm{~cm}^{-1}$.

HRMS (ESI): $m / z$ calculated for $\mathrm{C}_{16} \mathrm{H}_{14} \mathrm{ON}_{2} \mathrm{Cl}[\mathrm{M}+\mathrm{H}]^{+}:$285.0789, found: 285.0789.

(E)-4-(3-(2,3-dihydro-1H-pyrrolo[2,3-b]pyridin-1-yl)-3-oxoprop-1-en-1-yl)benzonitrile (2af):

The reaction performed according to general procedure 3 afforded $236 \mathrm{mg}$ (86\%).

Yellow solid (m.p. $\left.154-156^{\circ} \mathrm{C}\right)$.

(1)

${ }^{1} \mathrm{H}$ NMR (400 MHz, 300 K, $\left.\mathrm{CDCl}_{3}\right): \delta 8.65$ (d, J = 15.8 Hz, 1H), 8.18 (ddt, J = 5.1, 1.9, 1.0 $\mathrm{Hz}, 1 \mathrm{H}), 7.78(\mathrm{~d}, J=15.8 \mathrm{~Hz}, 1 \mathrm{H}), 7.73-7.64(\mathrm{~m}, 4 \mathrm{H}), 7.50(\mathrm{dq}, J=7.4,1.4 \mathrm{~Hz}, 1 \mathrm{H}), 6.92$ $(\mathrm{dd}, J=7.3,5.1 \mathrm{~Hz}, 1 \mathrm{H}), 4.22(\mathrm{dd}, J=9.1,7.8 \mathrm{~Hz}, 2 \mathrm{H}), 3.15-3.07(\mathrm{~m}, 2 \mathrm{H})$.

${ }^{13} \mathrm{C}$ NMR (101 MHz, 300 K, CDCl $)$ : $\delta$ 164.6, 156.1, 146.5, 140.3, 140.2, 133.9, 132.6, 128.7, 126.6, 124.5, 118.8, 118.5, 112.6, 46.1, 24.4 .

IR (thin film): $\widetilde{v} 3083,2964,2225,1653,1616,1600,1588,1558,1505,1476,1442,1420$, $1383,1353,1299,1241,1226,1165,1020,983,828,784,734,673,592,547 \mathrm{~cm}^{-1}$.

HRMS (ESI): $m / z$ calculated for $\mathrm{C}_{17} \mathrm{H}_{14} \mathrm{ON}_{3}[\mathrm{M}+\mathrm{H}]^{+}: 276.1131$, found: 276.1135 .

\section{Diethyl (2-(2,3-dihydro-1H-pyrrolo[2,3-b]pyridin-1-yl)-2-oxoethyl)phosphonate (9):}

The reaction performed according to general procedure 3 (10 mmol scale, DCM: $100 \mathrm{~mL}$ ) afforded $2.7 \mathrm{~g}(90 \%)$.

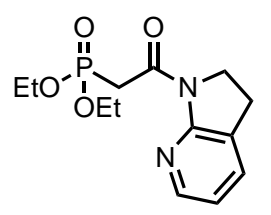

Colorless solid (m.p. $59-61^{\circ} \mathrm{C}$ ).

${ }^{1} \mathrm{H}$ NMR (400 MHz, $\left.300 \mathrm{~K}, \mathrm{CDCl}_{3}\right)$ : $\delta 8.08(\mathrm{ddt}, J=5.1,1.9,1.0 \mathrm{~Hz}, 1 \mathrm{H}), 7.46(\mathrm{dq}, J=7.4$, $1.4 \mathrm{~Hz}, 1 \mathrm{H}), 6.88(\mathrm{dd}, J=7.4,5.1 \mathrm{~Hz}, 1 \mathrm{H}), 4.21(\mathrm{~d}, J=22.0 \mathrm{~Hz}, 2 \mathrm{H}), 4.14(\mathrm{dq}, J=8.1,7.1 \mathrm{~Hz}$, $6 \mathrm{H}), 3.03(\mathrm{ddt}, J=9.8,8.7,1.2 \mathrm{~Hz}, 2 \mathrm{H}), 1.25(\mathrm{t}, J=7.0 \mathrm{~Hz}, 6 \mathrm{H})$.

${ }^{13}$ C NMR (101 MHz, 300 K, $\left.\mathrm{CDCl}_{3}\right): \delta 164.2(\mathrm{~d}, J=6.7 \mathrm{~Hz}), 155.5,146.0,133.8,126.2,118.5$, $62.4(\mathrm{~d}, J=6.1 \mathrm{~Hz}), 46.0,35.0(\mathrm{~d}, J=132.6 \mathrm{~Hz}), 24.1,16.4(\mathrm{~d}, J=6.4 \mathrm{~Hz})$.

${ }^{31} \mathrm{P}$ NMR (162 MHz, $\left.300 \mathrm{~K}, \mathrm{CDCl}_{3}\right)$ : $\delta 22.13$.

IR (thin film): $\widetilde{v} 3474,3054,2985,2931,2908,2869,1734,1660,1590,1480,1444,1423$, $1344,1291,1258,1149,1098,1056,1027,954,856,835,787,729,685,643,607 \mathrm{~cm}^{-1}$.

HRMS (ESI): $m / z$ calculated for $\mathrm{C}_{13} \mathrm{H}_{19} \mathrm{O}_{4} \mathrm{~N}_{2} \mathrm{PNa}[\mathrm{M}+\mathrm{Na}]^{+}: 321.0975$, found: 321.0977 . 

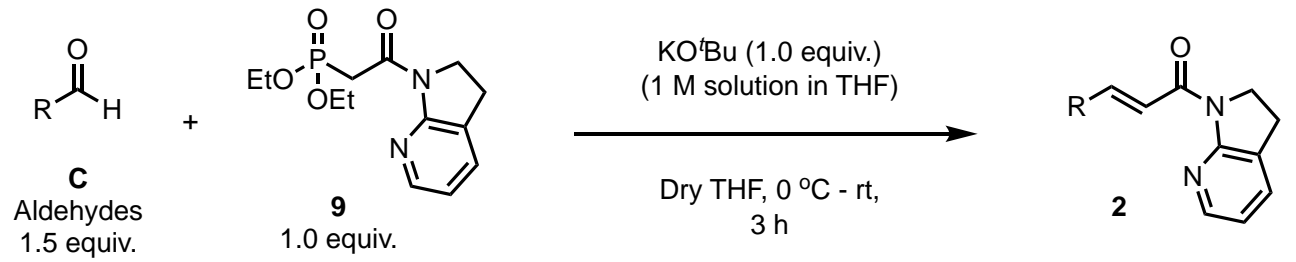

Potassium tert-butoxide solution (1 M solution in THF, $1.0 \mathrm{~mL}, 1.0 \mathrm{mmol}, 1.0$ equiv.) was slowly added to diethyl-(2-(2,3-dihydro-1H-pyrrolo[2,3-b]pyridin-1-yl)-2-oxoethyl)phosphonate 9 (298 mg, $1.0 \mathrm{mmol}, 1.0$ equiv) in anhydrous THF $(15 \mathrm{~mL})$ under a positive argon atmosphere at $0{ }^{\circ} \mathrm{C}$. The resulting mixture allowed stirring for $1 \mathrm{~h}$, followed by slow addition of desired aldehyde $\mathrm{C}$ ( $1.5 \mathrm{mmol}, 1.5$ equiv) in $5 \mathrm{~mL}$ THF over $\sim 2$ $\mathrm{min}$, the resulting mixture stirred under an inert atmosphere for $2 \mathrm{~h}$ at $0{ }^{\circ} \mathrm{C}$. The progress of the reaction monitored by TLC analysis (staining with 2,4-DNP and $\mathrm{KMnO}_{4}$ ). After complete consumption of 9, the reaction was quenched by adding saturated ammonium chloride solution $(10 \mathrm{~mL})$ and extracted with ethyl acetate $(15 \mathrm{~mL} \times 3)$. The combined organic layer was washed with brine $(20 \mathrm{~mL})$, dried over anhydrous $\mathrm{Na}_{2} \mathrm{SO}_{4}$, and concentrated in vacuo. Purification of the crude product by automated flash column chromatography using Hexanes/Ethyl acetate $(\sim 8 / 2)$ solvent system afforded pure $\alpha, \beta$-unsaturated amides 2 . All the solid products were subsequently recrystallized from hot Hexane or $\mathrm{CH}_{2} \mathrm{Cl}_{2} / \mathrm{Hexane}$.

(E)-1-(2,3-dihydro-1H-pyrrolo[2,3-b]pyridin-1-yl)pent-2-en-1-one (2q):

The reaction performed according to general procedure 4 afforded $185 \mathrm{mg}$ (92\%).

Colorless solid (m.p. 29-31 $\left.{ }^{\circ} \mathrm{C}\right)$.

${ }^{1} \mathrm{H}$ NMR (400 MHz, $\left.300 \mathrm{~K}, \mathrm{CDCl}_{3}\right): \delta 8.09$ (d, $\left.J=5.1 \mathrm{~Hz}, 1 \mathrm{H}\right), 7.78(\mathrm{dt}, J=15.4,1.6 \mathrm{~Hz}, 1 \mathrm{H})$,

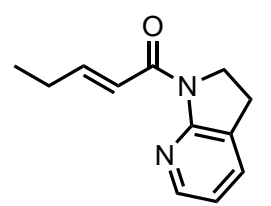
$7.42(\mathrm{dt}, J=7.4,1.5 \mathrm{~Hz}, 1 \mathrm{H}), 7.13(\mathrm{dtd}, J=15.2,6.5,1.3 \mathrm{~Hz}, 1 \mathrm{H}), 6.82(\mathrm{ddd}, J=7.0,5.1,1.3 \mathrm{~Hz}$, $1 \mathrm{H}), 4.16-4.07(\mathrm{~m}, 2 \mathrm{H}), 3.01(\mathrm{t}, J=8.5 \mathrm{~Hz}, 2 \mathrm{H}), 2.30(\mathrm{tt}, J=7.5,5.9 \mathrm{~Hz}, 2 \mathrm{H}), 1.10(\mathrm{td}, J=7.4$, $1.2 \mathrm{~Hz}, 3 \mathrm{H})$.

${ }^{13} \mathrm{C}$ NMR (101 MHz, 300 K, CDCl $\left.{ }_{3}\right): \delta 165.6,156.2,148.9,146.2,133.5,126.4,122.4,117.9,45.9$, 25.8, 24.3, 12.7 .

IR (thin film): $\widetilde{v} 3325,3075,3054,2965,2934,2873,2849,1718,1660,1625,1598,1586,1524$, 1477, 1443, 1418, 1384, 1359, 1322, 1297, 1282, 1241, 1220, 1165, 1092, 1071, 1050, 1003, 982, $860,784,749,691,590 \mathrm{~cm}^{-1}$.

HRMS (ESI): $m / z$ calculated for $\mathrm{C}_{12} \mathrm{H}_{15} \mathrm{ON}_{2}[\mathrm{M}+\mathrm{H}]^{+}:$203.1179, found: 203.1183 .

(E)-1-(2,3-dihydro-1H-pyrrolo[2,3-b]pyridin-1-yl)oct-2-en-1-one (2r):

The reaction performed according to general procedure 4 afforded $229 \mathrm{mg}$ (94\%).

Colorless oil.

${ }^{1} \mathrm{H}$ NMR $\left(400 \mathrm{MHz}, 300 \mathrm{~K}, \mathrm{CDCl}_{3}\right)$ : $\delta 8.12(\mathrm{ddt}, J=5.1,2.0,1.0 \mathrm{~Hz}, 1 \mathrm{H}), 7.80(\mathrm{dt}, J=15.3$, $1.6 \mathrm{~Hz}, 1 \mathrm{H}), 7.45(\mathrm{dq}, J=7.4,1.5 \mathrm{~Hz}, 1 \mathrm{H}), 7.12(\mathrm{dt}, J=15.3,7.0 \mathrm{~Hz}, 1 \mathrm{H}), 6.86(\mathrm{dd}, J=7.4$,

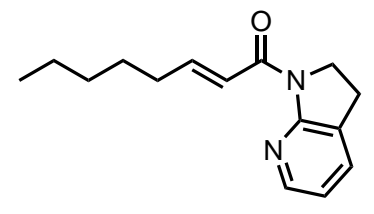
$5.1 \mathrm{~Hz}, 1 \mathrm{H}), 4.16(\mathrm{dd}, J=9.1,7.9 \mathrm{~Hz}, 2 \mathrm{H}), 3.05(\mathrm{tt}, J=8.7,1.2 \mathrm{~Hz}, 2 \mathrm{H}), 2.31(\mathrm{qd}, J=7.1$, $1.6 \mathrm{~Hz}, 2 \mathrm{H}), 1.52(\mathrm{dddd}, J=14.4,9.0,6.0,3.5 \mathrm{~Hz}, 2 \mathrm{H}), 1.40-1.24(\mathrm{~m}, 4 \mathrm{H}), 0.95-0.82(\mathrm{~m}$, $3 \mathrm{H})$.

${ }^{13} \mathrm{C}$ NMR (101 MHz, 300 K, CDCl $): \delta 165.7,156.4,147.9,146.3,133.5,126.5,123.2,118.0$, 46.0, 32.7, 31.6, 28.2, 24.4, 22.6, 14.1 .

IR (thin film): $\widetilde{v} 3075,3051,3010,2956,2928,2857,1660,1627,1601,1587,1479,1443$, $1419,1382,1357,1299,1241,1164,989,781,691,590 \mathrm{~cm}^{-1}$.

HRMS (ESI): $m / z$ calculated for $\mathrm{C}_{15} \mathrm{H}_{21} \mathrm{ON}_{2}[\mathrm{M}+\mathrm{H}]^{+}:$245.1648, found: 245.1651 . 
(E)-1-(2,3-dihydro-1H-pyrrolo[2,3-b]pyridin-1-yl)trideca-2,12-dien-1-one (2s):

The reaction performed according to general procedure 4 afforded $281 \mathrm{mg}$ (90\%).

Colorless oil.

${ }^{1} \mathrm{H}$ NMR (400 MHz, 300 K, $\left.\mathrm{CDCl}_{3}\right): \delta 8.09$ (dd, J = 5.1, $\left.1.8 \mathrm{~Hz}, 1 \mathrm{H}\right), 7.79$ (dd,

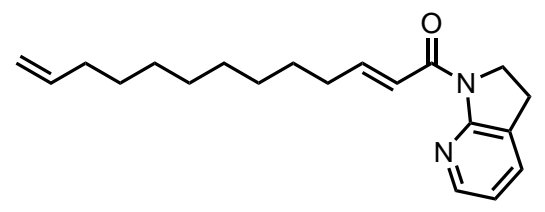
$J=15.4,1.6 \mathrm{~Hz}, 1 \mathrm{H}), 7.42(\mathrm{dq}, J=7.3,1.4 \mathrm{~Hz}, 1 \mathrm{H}), 7.10(\mathrm{dt}, J=15.4,7.0 \mathrm{~Hz}$, $1 \mathrm{H}), 6.83(\mathrm{ddd}, J=7.3,5.1,1.7 \mathrm{~Hz}, 1 \mathrm{H}), 5.78(\mathrm{ddt}, J=16.9,10.2,6.7 \mathrm{~Hz}, 1 \mathrm{H})$, $5.01-4.84(\mathrm{~m}, 2 \mathrm{H}), 4.17-4.07(\mathrm{~m}, 2 \mathrm{H}), 3.08-2.94(\mathrm{~m}, 2 \mathrm{H}), 2.28(\mathrm{qd}, J=7.2$, $1.6 \mathrm{~Hz}, 2 \mathrm{H}), 2.06-1.94(\mathrm{~m}, 2 \mathrm{H}), 1.49(\mathrm{~h}, J=6.9 \mathrm{~Hz}, 2 \mathrm{H}), 1.40-1.20(\mathrm{~m}, 10 \mathrm{H})$. ${ }^{13}$ C NMR (101 MHz, 300 K, CDCl $)$ ): $\delta$ 165.6, 156.3, 147.7, 146.2, 139.2, 133.5, $126.4,123.1,117.9,114.2,45.9,33.8,32.6,29.4,29.3,29.2,29.0,28.5,24.3$.

IR (thin film): $\widetilde{v} 3075,3010,2974,2926,2853,1661,1628,1601,1586,1479$, $1443,1419,1381,1356,1299,1280,1267,1241,1163,1073,990,909,781,691$, $591 \mathrm{~cm}^{-1}$.

HRMS (ESI): $m / z$ calculated for $\mathrm{C}_{20} \mathrm{H}_{29} \mathrm{ON}_{2}[\mathrm{M}+\mathrm{H}]^{+}: 313.2274$, found: 313.2279 .

(E)-1-(2,3-dihydro-1H-pyrrolo[2,3-b]pyridin-1-yl)-5-phenylpent-2-en-1-one (2t):

The reaction performed according to general procedure 4 afforded $233 \mathrm{mg}$ (84\%).

Colorless solid (m.p. $\left.44-46^{\circ} \mathrm{C}\right)$.

${ }^{1} \mathrm{H}$ NMR (400 MHz, 300 K, $\left.\mathrm{CDCl}_{3}\right)$ : $\delta 8.14(\mathrm{ddt}, J=5.1,1.8,1.0 \mathrm{~Hz}, 1 \mathrm{H}), 7.90(\mathrm{dt}, J=15.4$,

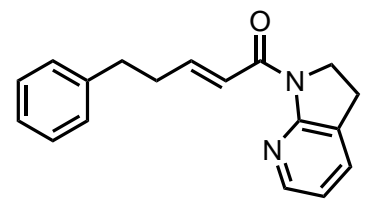
$1.6 \mathrm{~Hz}, 1 \mathrm{H}), 7.46(\mathrm{dq}, J=7.2,1.4 \mathrm{~Hz}, 1 \mathrm{H}), 7.34-7.14(\mathrm{~m}, 6 \mathrm{H}), 6.88(\mathrm{dd}, J=7.3,5.1 \mathrm{~Hz}$, $1 \mathrm{H}), 4.22-4.06(\mathrm{~m}, 2 \mathrm{H}), 3.05(\mathrm{ddd}, J=9.6,7.9,1.2 \mathrm{~Hz}, 2 \mathrm{H}), 2.86(\mathrm{dd}, J=9.0,6.7 \mathrm{~Hz}, 2 \mathrm{H})$, $2.66(\mathrm{dtd}, J=8.6,6.8,1.5 \mathrm{~Hz}, 2 \mathrm{H})$.

${ }^{13} \mathrm{C}$ NMR (101 MHz, 300 K, $\left.\mathrm{CDCl}_{3}\right): \delta 165.4,156.2,146.3,146.2,141.4,133.5,128.5,128.4$, 126.4, 126.0, 123.6, 118.0, 46.0, 34.7, 34.4, 24.3.

IR (thin film): $\widetilde{v} 3024,2924,1659,1625,1600,1587,1495,1477,1454,1442,1419,1382$, $1357,1299,1241,1161,977,784,747,699 \mathrm{~cm}^{-1}$.

HRMS (ESI): $m / z$ calculated for $\mathrm{C}_{18} \mathrm{H}_{19} \mathrm{ON}_{2}[\mathrm{M}+\mathrm{H}]^{+}: 279.1492$, found: 279.1494 .

(E)-1-(2,3-dihydro-1H-pyrrolo[2,3-b]pyridin-1-yl)-3-(thiophen-2-yl)prop-2-en-1-one (2ag):

The reaction performed according to general procedure 4 afforded $256 \mathrm{mg}(88 \%)$.

Colorless solid (m.p. 103-105 $\left.{ }^{\circ} \mathrm{C}\right)$.

${ }^{1} \mathrm{H}$ NMR (400 MHz, 300 K, $\left.\mathbf{C D C l}_{3}\right): \delta 8.37(\mathrm{~d}, J=15.4 \mathrm{~Hz}, 1 \mathrm{H}), 8.17(\mathrm{ddt}, J=5.2,1.8,1.0$

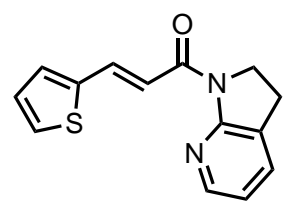
$\mathrm{Hz}, 1 \mathrm{H}), 7.99-7.91(\mathrm{~m}, 1 \mathrm{H}), 7.46(\mathrm{dq}, J=7.3,1.4 \mathrm{~Hz}, 1 \mathrm{H}), 7.34(\mathrm{dt}, J=5.1,1.0 \mathrm{~Hz}, 1 \mathrm{H})$, $7.31-7.27(\mathrm{~m}, 1 \mathrm{H}), 7.04(\mathrm{dd}, J=5.1,3.6 \mathrm{~Hz}, 1 \mathrm{H}), 6.88(\mathrm{dd}, J=7.4,5.1 \mathrm{~Hz}, 1 \mathrm{H}), 4.20(\mathrm{dd}$, $J=9.1,7.8 \mathrm{~Hz}, 2 \mathrm{H}), 3.07(\mathrm{tt}, J=8.6,1.1 \mathrm{~Hz}, 2 \mathrm{H})$.

${ }^{13} \mathrm{C}$ NMR (101 MHz, $\left.300 \mathrm{~K}, \mathrm{CDCl}_{3}\right): \delta 165.2,156.3,146.5,141.2,135.3,133.6,130.3,128.0$, $127.7,126.43,119.8,118.1,46.1,24.4$.

IR (thin film): $\widetilde{v} 3065,1645,1599,1515,1476,1440,1419,1383,1353,1336,1296,1276$, $1241,1213,1164,1043,1019,971,865,827,781,744,702,673,595,585,569 \mathrm{~cm}^{-1}$.

HRMS (ESI): $m / z$ calculated for $\mathrm{C}_{14} \mathrm{H}_{12} \mathrm{ON}_{2} \mathrm{SNa}[\mathrm{M}+\mathrm{Na}]^{+}: 279.0563$, found: 279.0566. 
(E)-1-(2,3-dihydro-1H-pyrrolo[2,3-b]pyridin-1-yl)-3-(furan-2-yl)prop-2-en-1-one (2ah):

The reaction performed according to general procedure 4 afforded $204 \mathrm{mg}$ (85\%).

Colorless solid (m.p. $124-125^{\circ} \mathrm{C}$ ).

${ }^{1} \mathrm{H}$ NMR (400 MHz, $\left.300 \mathrm{~K}, \mathrm{CDCl}_{3}\right)$ : $\delta 8.36$ (d, $\left.J=15.5 \mathrm{~Hz}, 1 \mathrm{H}\right), 8.19$ (ddt, $J=5.1,1.8,1.0$

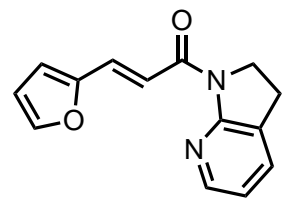
$\mathrm{Hz}, 1 \mathrm{H}), 7.62(\mathrm{~d}, J=15.5 \mathrm{~Hz}, 1 \mathrm{H}), 7.50(\mathrm{~d}, J=1.8 \mathrm{~Hz}, 1 \mathrm{H}), 7.46(\mathrm{dq}, J=7.4,1.4 \mathrm{~Hz}, 1 \mathrm{H}), 6.88$ $(\mathrm{dd}, J=7.3,5.1 \mathrm{~Hz}, 1 \mathrm{H}), 6.62(\mathrm{~d}, J=3.4 \mathrm{~Hz}, 1 \mathrm{H}), 6.46(\mathrm{dd}, J=3.4,1.8 \mathrm{~Hz}, 1 \mathrm{H}), 4.21(\mathrm{dd}, J=$ 9.1, 7.9 Hz, 2H), 3.07 (ddt, $J=9.7,7.5,1.2 \mathrm{~Hz}, 2 \mathrm{H})$.

${ }^{13} \mathrm{C}$ NMR (101 MHz, 300 K, $\left.\mathrm{CDCl}_{3}\right)$ : $\delta$ 165.4, 156.3, 152.3, 146.5, 144.3, 133.6, 129.5, 126.4, $118.4,118.1,114.2,112.2,46.2,24.4$.

IR (thin film): $\widetilde{v} 1650,1599,1588,1556,1478,1442,1419,1380,1348,1260,1240,1227$, $1164,1016,975,883,781,746,717,664,592 \mathrm{~cm}^{-1}$.

HRMS (ESI): $\mathrm{m} / \mathrm{z}$ calculated for $\mathrm{C}_{14} \mathrm{H}_{12} \mathrm{O}_{2} \mathrm{~N}_{2} \mathrm{Na}$ [M+Na] $]^{+}$263.0791, found: 263.0792 . 


\section{Asymmetric Cyclopropanation}

\subsection{Cyclopropanation optimization:}

\section{[A] Initial control experiments}<smiles>CS(C)=CC(=O)c1ccccc1</smiles>

1a<smiles>C/C=C/C(=O)N1CCc2cccnc21</smiles>

2b

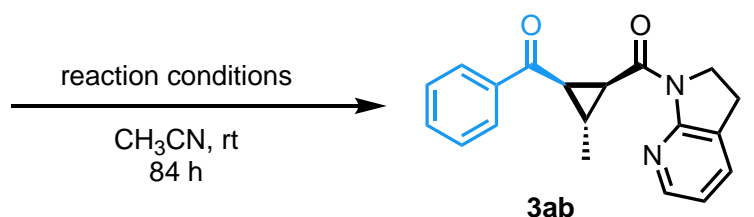

$3 a b$

\begin{tabular}{|c|c|c|c|c|c|}
\hline entry & $\begin{array}{l}\text { metal salt } \\
\text { (10 mol \%) }\end{array}$ & $\begin{array}{l}\text { ligand } \\
\text { (12 mol \%) }\end{array}$ & $\begin{array}{l}\text { yield } \\
(\%)^{b}\end{array}$ & $d r^{c}$ & $e e(\%)^{d}$ \\
\hline 01 & - & - & $<10$ & ND & ND \\
\hline 02 & $\mathrm{Cu}\left(\mathrm{CH}_{3} \mathrm{CN}\right)_{4} \mathrm{PF}_{6}$ & $( \pm)$-MeO-Biphep & 83 & ND & ND \\
\hline 03 & $\mathrm{Cu}\left(\mathrm{CH}_{3} \mathrm{CN}\right)_{4} \mathrm{PF}_{6}$ & (S)-DM-Segphos & 86 & $>99: 1$ & 70 \\
\hline
\end{tabular}

Unless otherwise stated, these are the common conditions maintained for all the optimizations: Table A: ${ }^{a} \mathbf{1 a}: 0.15 \mathrm{mmol}$, $2 \mathbf{b}$ : $0.1 \mathrm{mmol}$, solvent $(0.1 \mathrm{M}), 84 \mathrm{~h}$. ${ }^{b}$ Isolated yield. ${ }^{c}$ The diastereomeric ratio $(d r)$ was determined by ${ }^{1} \mathrm{H}$ NMR analysis of the crude reaction mixture or chiral HPLC analysis. ${ }^{d}$ The enantiomeric excess $(e e)$ was determined by chiral stationary phase HPLC analysis.

[B] Reaction optimization at different temperatures

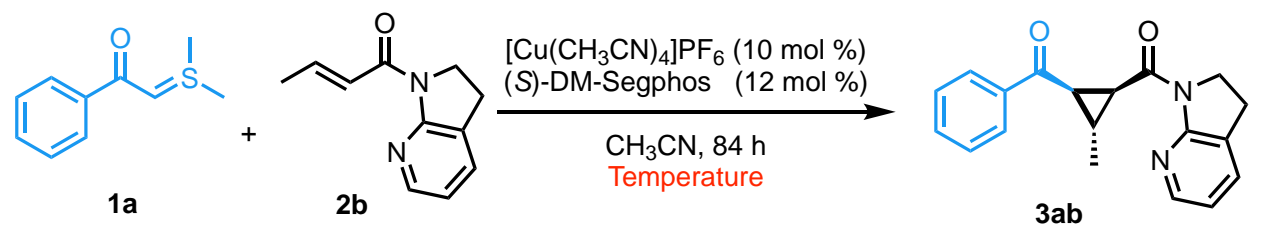

\begin{tabular}{|l|l|l|l|l|}
\hline entry & temperature $\left({ }^{\circ} \mathbf{C}\right)$ & yield (\%) & $d r$ & ee (\%) \\
\hline 01 & $\sim 23(\mathrm{rt})$ & 82 & $>99: 1$ & 71 \\
\hline 02 & 0 & 57 & $>99: 1$ & 73 \\
03 & 32 & $>99: 1$ & 75 \\
\hline 04 & -10 & 11 & $>99: 1$ & 75 \\
\hline
\end{tabular}


[C] Direct use of the sulfonium salt B1 in the presence of an external base

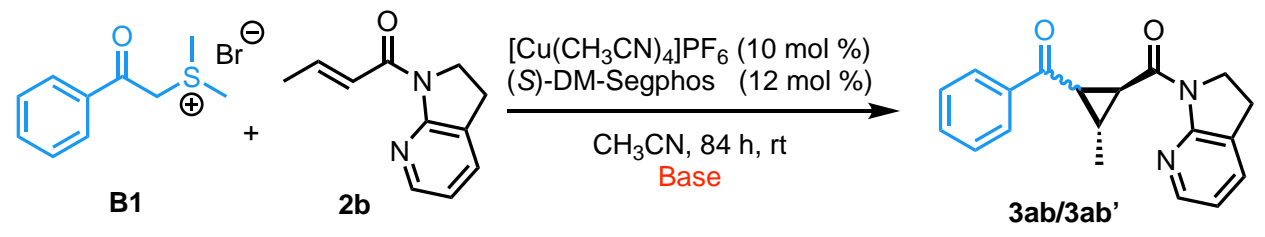

\begin{tabular}{|c|c|c|c|c|}
\hline entry & base (1.5 equiv) & $\begin{array}{c}\text { yield } \\
\left(3 a a+3 a a^{\prime}\right) \\
(\%)\end{array}$ & $\begin{array}{c}d r \\
\left(3 a a / 3 a a^{\prime}\right)\end{array}$ & $\begin{array}{c}e e \\
\left(3 \mathrm{aa} / 3 \mathrm{aa}^{\prime}\right) \\
(\%)\end{array}$ \\
\hline 01 & $\mathrm{~K}_{2} \mathrm{CO}_{3}$ & 65 & $1: 1$ & $71 / 81$ \\
\hline 02 & $\mathrm{Li}_{2} \mathrm{CO}_{3}$ & $<10$ & ND & ND \\
\hline 03 & $\mathrm{Cs}_{2} \mathrm{CO}_{3}$ & 62 & $1: 1$ & $73 / 80$ \\
\hline 04 & $\mathrm{Na}_{2} \mathrm{CO}_{3}$ & $<10$ & ND & ND \\
\hline 05 & $\mathrm{NaHCO}_{3}$ & 0 & ND & ND \\
\hline 06 & $\mathrm{~K}_{3} \mathrm{PO}_{4}$ & 46 & $1: 1$ & $73 / 80$ \\
\hline $07^{*}$ & $\mathrm{NaOH}$ & 0 & ND & ND \\
\hline 08 & - & 0 & ND & ND \\
\hline
\end{tabular}

Observation: When this reaction was carried out in the presence of a sulfonium salt B1 with an external base, the obtained diastereoselectivity was poor ( 1:1). Thus, we continued our further investigations using the corresponding stabilized sulfur ylides 1 in the absence of an external base. 
[D] Ligand variation

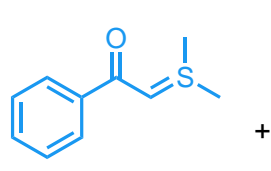

$1 a$<smiles>C/C=C/C(=O)N1CCc2cccnc21</smiles>

$2 b$

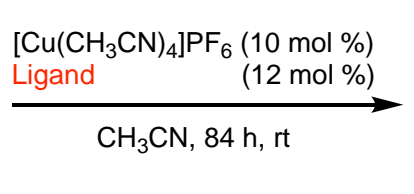

$\mathrm{CH}_{3} \mathrm{CN}, 84 \mathrm{~h}, \mathrm{rt}$

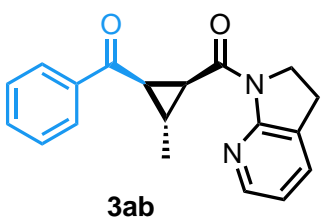

\begin{tabular}{|c|c|c|c|c|}
\hline entry & ligand & yield (\%) & $d r$ & $e e(\%)$ \\
\hline 01 & $(S, S)-i \operatorname{Pr}-\operatorname{Box}(\mathbf{L} 2)$ & $<10$ & ND & ND \\
\hline 02 & $(S, S)-\operatorname{Pr}-\operatorname{PyBox}(\mathbf{L 3})$ & $<10$ & ND & ND \\
\hline 03 & (S)-DTBM-Segphos (L4) & 73 & $>99: 1$ & 87 \\
\hline 04 & (S)-DM-Segphos (L5) & 82 & $>99: 1$ & 71 \\
\hline 05 & (S)-Segphos (L6) & 83 & $>99: 1$ & 49 \\
\hline 06 & $(R)$-DM-BINAP (L7) & 47 & $>20: 1$ & 40 \\
\hline 07 & (R)-MeO-BIPHEP (L8) & 49 & $>99: 1$ & 40 \\
\hline 08 & $(S, S)-B P E($ L9) & 15 & $>99: 1$ & 37 \\
\hline 09 & $(R)$-BINAP (L10) & 49 & $>20: 1$ & 40 \\
\hline 10 & $(R)$-Tol-BINAP (L11) & 45 & $>20: 1$ & 35 \\
\hline 11 & (R)-C3-TUNEPHOS (L12) & 45 & $>99: 1$ & 35 \\
\hline 12 & (R)-SYNPHOS (L13) & 51 & $>99: 1$ & 55 \\
\hline 13 & (R)-Xyl-SDP (L14) & 15 & $>99: 1$ & 13 \\
\hline
\end{tabular}

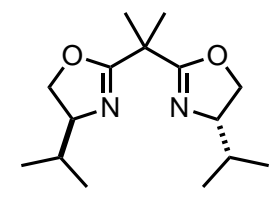

L2: $(S, S)$-iPr-BOX<smiles>CC(C)C1COC(c2cccc(C3=N[C@@H](C(C)C)CO3)n2)=N1</smiles>

L3: $(S, S)-{ }^{-}$Pr-PyBOX<smiles>[R120]c1ccc2c(c1-c1c([P+])ccc3c1OCO3)OCO2</smiles>

L4: (S)-DTBM-Segphos; $\mathrm{Ar}=3,5-\left({ }^{\mathrm{t}} \mathrm{Bu}\right)_{2}-4-\mathrm{MeO}-\mathrm{C}_{6} \mathrm{H}_{2}$ L5: (S)-DM-Segphos; $\mathrm{Ar}=3,5-(\mathrm{Me})_{2}-\mathrm{C}_{6} \mathrm{H}_{3}$ L6: (S)-Segphos; $\mathrm{Ar}=\mathrm{Ph}$<smiles>CCCCc1ccc2ccccc2c1-c1c(C(=O)O)ccc2ccccc12</smiles>

L7: $(R)$-DM-BINAP; $\mathrm{Ar}=3,5-(\mathrm{Me})_{2}-\mathrm{C}_{6} \mathrm{H}_{3}$
L8: (R)-MeO-Biphep

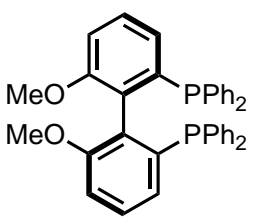<smiles>CC(C)C1CC[C@@H](C)P1CCP1[C@@H](C)CC[C@H]1C</smiles>

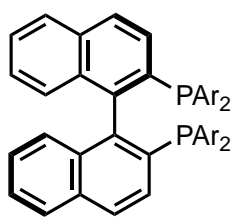

L10: $(R)$-BINAP; $\mathrm{Ar}=\mathrm{Ph}$ L11: $(R)$-Tol-BINAP; $\mathrm{Ar}=4-\mathrm{Me}-\mathrm{C}_{6} \mathrm{H}_{4}$

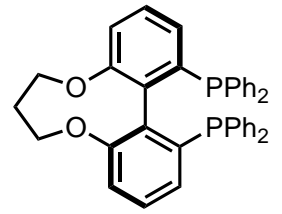

L12: $(R)-\mathrm{C}_{3}$-TunePhos

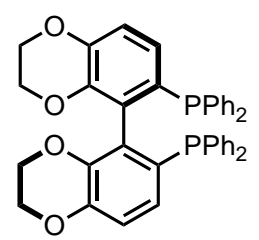

L13: (R)-Synphos

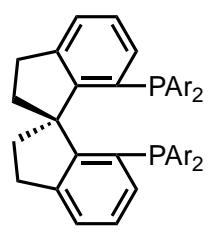

L14: $(R)$-Xyl-SDP; $\mathrm{Ar}=3,5-(\mathrm{Me})_{2}-\mathrm{C}_{6} \mathrm{H}_{3}$ 


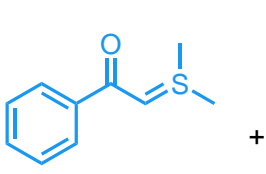

$1 a$<smiles>C/C=C/C(=O)N1CCc2cccnc21</smiles>

$2 b$
$\left[\mathrm{Cu}\left(\mathrm{CH}_{3} \mathrm{CN}\right)_{4}\right] \mathrm{PF}_{6} \quad(10 \mathrm{~mol} \%)$

(S)-DTBM-Segphos (12 mol \%)<smiles>C[C@@H]1C(C(=O)c2ccccc2)[C@H]1C(=O)N1CCc2cccnc21</smiles>

\begin{tabular}{|c|c|c|c|c|}
\hline entry & solvent & yield (\%) & $d r$ & $e e(\%)$ \\
\hline 01 & Toluene & 65 & $>99: 1$ & 42 \\
\hline 02 & THF & 76 & $>99: 1$ & 43 \\
\hline 03 & DME & $<10$ & ND & ND \\
\hline 04 & Acetone & 79 & $>99: 1$ & 60 \\
\hline 05 & $\mathrm{DMF}$ & $<10$ & ND & ND \\
\hline 06 & $\mathrm{MeOH}$ & 0 & ND & ND \\
\hline 07 & $\mathrm{CH}_{2} \mathrm{Cl}_{2}$ & 84 & $>99: 1$ & 93 \\
\hline 08 & $\mathrm{CHCl}_{3}$ & $<10$ & ND & ND \\
\hline 09 & 1,2-DCE & 45 & $>20: 1$ & 55 \\
\hline
\end{tabular}

\section{[E] Concentration}

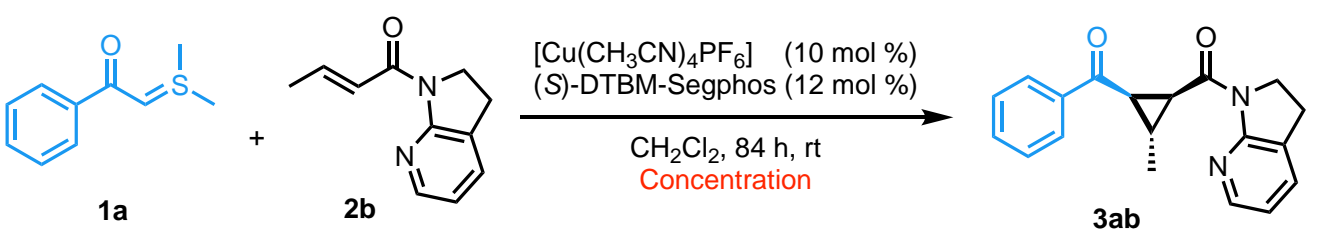

\begin{tabular}{|c|c|c|c|c|}
\hline entry & concentration $(\mathrm{M})$ & yield (\%) & $d r$ & $e e(\%)$ \\
\hline 01 & 0.1 & 83 & $>99: 1$ & 93 \\
\hline 02 & 0.07 & 85 & $>99: 1$ & 95 \\
\hline 03 & 0.05 & 85 & $>99: 1$ & 95 \\
\hline
\end{tabular}


[F] Catalyst loading

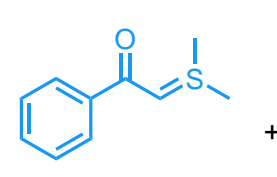

$1 a$<smiles>C/C=C/C(=O)N1CCc2cccnc21</smiles>

$2 b$

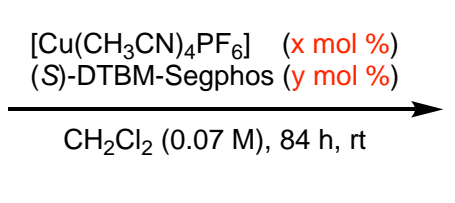

$\mathrm{CH}_{2} \mathrm{Cl}_{2}(0.07 \mathrm{M}), 84 \mathrm{~h}, \mathrm{rt}$

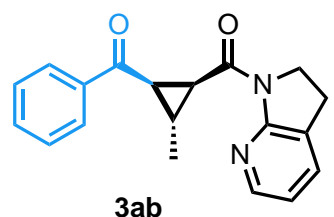

\begin{tabular}{|c|c|c|c|c|}
\hline entry & Cu/L (mol \%) & yield (\%) & $d r$ & ee (\%) \\
\hline 01 & $1 / 1.2$ & 77 & $>99: 1$ & 94 \\
\hline 01 & $2 / 2.5$ & 83 & $>99: 1$ & 95 \\
\hline 02 & $5 / 6$ & 83 & $>99: 1$ & 95 \\
\hline 03 & $10 / 12$ & 85 & $>99: 1$ & 95 \\
\hline
\end{tabular}

\section{[G] Scale}

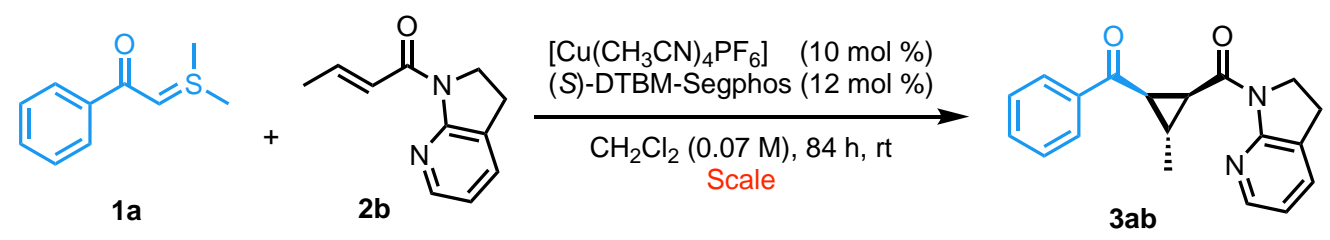

\begin{tabular}{|c|c|c|c|c|}
\hline entry & $\begin{array}{c}\text { scale (mmol) } \\
\mathbf{2 b}\end{array}$ & yield (\%) & $d r$ & $\boldsymbol{e e ~ ( \% )}$ \\
\hline $\mathbf{0 1}$ & $\mathbf{0 . 1}$ & $\mathbf{8 3}$ & $>\mathbf{9 9 : 1}$ & $\mathbf{9 5}$ \\
\hline 02 & 0.2 & 84 & $>99: 1$ & 95 \\
\hline 03 & 0.3 & 84 & $>99: 1$ & 94 \\
\hline
\end{tabular}


An oven-dried $4 \mathrm{~mL}$ vial was charged with 2 (1.0 equiv., $0.1 \mathrm{mmol})$, [ $\left.\mathrm{Cu}\left(\mathrm{CH}_{3} \mathrm{CN}\right)_{4}\right] \mathrm{PF}_{6}(0.75 \mathrm{mg}, 0.02$ equiv., 2 mol \%), (S)-DTBM-Segphos (2.95 mg, 0.025 equiv., $2.5 \mathrm{~mol} \%)$ in the Glovebox. Then, anhydrous $\mathrm{CH}_{2} \mathrm{Cl}_{2}(1.0$ $\mathrm{mL}$ ) was added to the mixture via syringe with a stainless-steel needle at room temperature under positive Argon pressure. The resulting solution was stirred for $30 \mathrm{~min}$ and the pre-dissolved ylide $1 \mathrm{a}(27 \mathrm{mg}$, 1.5 equiv., $0.15 \mathrm{mmol})$ in anhydrous $\mathrm{CH}_{2} \mathrm{Cl}_{2}(0.5 \mathrm{~mL})$ was slowly added to the reaction mixture under the Argon atmosphere. The resulting reaction mixture was then allowed to stir for 24-84 $\mathrm{h}$ at room temperature. After confirming the complete conversion by Thin Layer Chromatography (TLC), the crude residue was directly loaded onto a Preparative Thin Layer Chromatography (PTLC) and eluted using Hexane/Ethyl acetate ( 5/1) solvent system ( $>2 \mathrm{x}$ ). If required, the isolated product(s) was repurified by another PTLC with $\mathrm{CH}_{2} \mathrm{Cl}_{2}$. The $\mathrm{UV}$-visible product band was carefully scratched and filtered through a glass frit funnel using $\mathrm{CH}_{2} \mathrm{Cl}_{2}$ as an eluent to afford a pure cyclopropanation product 3 .

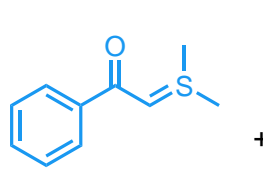

$1 \mathbf{a}$

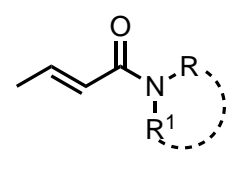

2

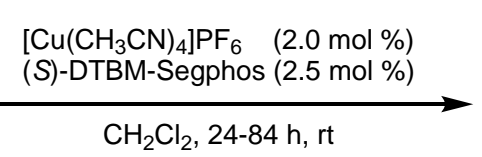

$\mathrm{CH}_{2} \mathrm{Cl}_{2}, 24-84 \mathrm{~h}, \mathrm{rt}$

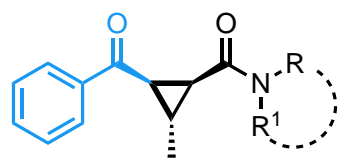

3

3-((1S,2R,3R)-2-benzoyl-3-methylcyclopropane-1-carbonyl)oxazolidin-2-one (3aa):

The reaction performed according to the standard procedure 1 afforded $19 \mathrm{mg}$ (69\%). Colorless oil.

${ }^{1} \mathrm{H}$ NMR (400 MHz, $\left.300 \mathrm{~K}, \mathrm{CDCl}_{3}\right)$ : $\delta 8.01-7.96(\mathrm{~m}, 2 \mathrm{H}), 7.58-7.53(\mathrm{~m}, 1 \mathrm{H}), 7.49-7.43$<smiles>CC1C(C(=O)N2CCOC2=O)[C@H]1C(=O)c1ccccc1</smiles>
$(\mathrm{m}, 2 \mathrm{H}), 4.41-4.28(\mathrm{~m}, 2 \mathrm{H}), 4.04-3.90(\mathrm{~m}, 2 \mathrm{H}), 3.00(\mathrm{dd}, J=9.1,6.3 \mathrm{~Hz}, 1 \mathrm{H}), 2.87(\mathrm{dd}, J$ $=9.0,6.1 \mathrm{~Hz}, 1 \mathrm{H}), 2.35(\mathrm{~h}, J=6.1 \mathrm{~Hz}, 1 \mathrm{H}), 1.35(\mathrm{~d}, J=6.1 \mathrm{~Hz}, 3 \mathrm{H})$.

${ }^{13} \mathrm{C}$ NMR (101 MHz, $\left.300 \mathrm{~K}, \mathrm{CDCl}_{3}\right): \delta$ 196.0, 168.9, 153.9, 137.4, 133.2, 128.7, 128.5, 62.3, $42.8,35.7,32.7,22.7,17.2$.

IR (thin film): $\widetilde{v} 3060,2961,2925,2870,1779,1695,1596,1579,1478,1463,1449,1384$, $1321,1263,1221,1178,1136,1117,1032,1018,978,955,908,852,799,761,724,693 \mathrm{~cm}^{-1}$.

HRMS (ESI): $m / z$ calculated for $\mathrm{C}_{15} \mathrm{H}_{15} \mathrm{O}_{4} \mathrm{NNa}[\mathrm{M}+\mathrm{Na}]^{+}: 296.0893$, found: 296.0893 .

$[\alpha]_{\mathrm{D}}^{23}$ Not determined (because the ee was $\sim 0 \%$ ).

Enantiomeric excess was determined to be $\sim 0 \%$ ee by chiral stationary phase HPLC analysis (CHIRALPAK IF $(\phi 0.46 \mathrm{~cm} \times 25 \mathrm{~cm}), n$-hexane/ $\mathrm{PrOH}=9 / 1$, flow rate 1.0 $\mathrm{mL} / \mathrm{min}$, detection at $254 \mathrm{~nm}, \mathrm{t}_{\mathrm{R}}=62.3 \mathrm{~min}$ and $84.6 \mathrm{~min}$.
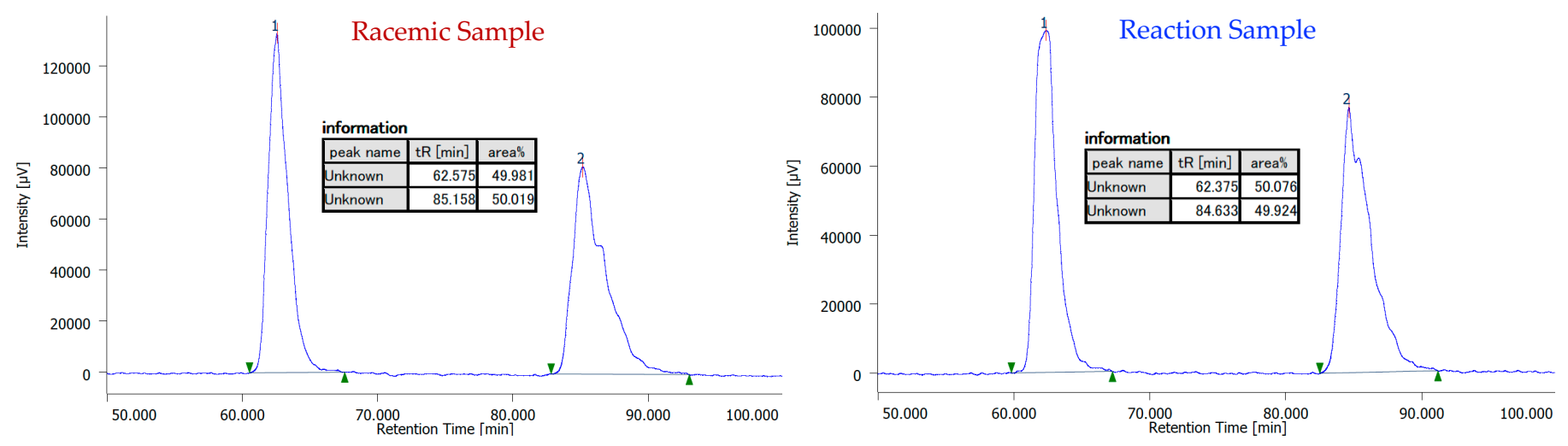
The reaction performed according to the standard procedure 1 afforded $25 \mathrm{mg}(83 \%)$.

Colorless solid (m.p $\left.=91-93^{\circ} \mathrm{C}\right)$.

${ }^{1} \mathrm{H}$ NMR (400 MHz, 300 K, $\left.\mathrm{CDCl}_{3}\right): \delta 8.11(\mathrm{ddt}, J=5.1,1.8,1.0 \mathrm{~Hz}, 1 \mathrm{H}), 7.99-7.94(\mathrm{~m}$,

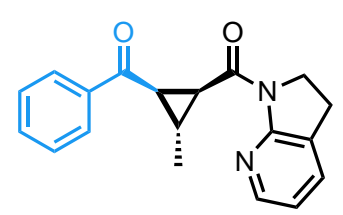
$2 \mathrm{H}), 7.48-7.41(\mathrm{~m}, 1 \mathrm{H}), 7.38(\mathrm{dq}, J=7.3,1.4 \mathrm{~Hz}, 1 \mathrm{H}), 7.35-7.30(\mathrm{~m}, 2 \mathrm{H}), 6.83(\mathrm{dd}, J=$ 7.3, $5.1 \mathrm{~Hz}, 1 \mathrm{H}), 4.08-3.89(\mathrm{~m}, 2 \mathrm{H}), 3.62(\mathrm{dd}, J=9.3,6.3 \mathrm{~Hz}, 1 \mathrm{H}), 3.02-2.85(\mathrm{~m}, 2 \mathrm{H})$, $2.77(\mathrm{dd}, J=9.3,6.1 \mathrm{~Hz}, 1 \mathrm{H}), 2.46(\mathrm{~h}, J=6.1 \mathrm{~Hz}, 1 \mathrm{H}), 1.37(\mathrm{~d}, J=6.1 \mathrm{~Hz}, 3 \mathrm{H})$.

${ }^{13} \mathrm{C}$ NMR (101 MHz, 300 K, CDCl $)$ : $\delta$ 196.3, 168.2, 156.3, 146.0, 137.9, 133.4, 132.6, 128.4, $128.3,126.4,118.0,46.0,35.5,34.8,24.4,22.0,17.5$.

IR (thin film): $\widetilde{v} 3057,2959,2924,2867,2853,1724,1681,1650,1600,1588,1479,1444$, 1420, 1388, 1326, 1294, 1267, 1240, 1219, 1176, 1164, 1134, 1077, 1060, 1038, 1015, 980, 950, $851,782,742,717,694 \mathrm{~cm}^{-1}$.

HRMS (ESI): $m / z$ calculated for $\mathrm{C}_{19} \mathrm{H}_{18} \mathrm{O}_{2} \mathrm{~N}_{2} \mathrm{Na}$ [M+Na] $]^{+}$329.1260, found: 329.1265.

$[\alpha]_{\mathrm{D}}^{23}-172.1$ ( c 1.27, $\mathrm{CHCl}_{3}, 95 \%$ ee sample).

Enantiomeric excess was determined to be $95 \%$ ee by chiral stationary phase HPLC analysis (CHIRALPAK IC3 $(\phi 0.46 \mathrm{~cm} \times 25 \mathrm{~cm}), n$-hexane $/ \mathrm{PrOH}=4 / 1$, flow rate $1.0 \mathrm{~mL} / \mathrm{min}$, detection at $254 \mathrm{~nm}, \mathrm{t}_{\mathrm{R}}=19.4 \mathrm{~min}$ (minor), $33.9 \mathrm{~min}$ (major).
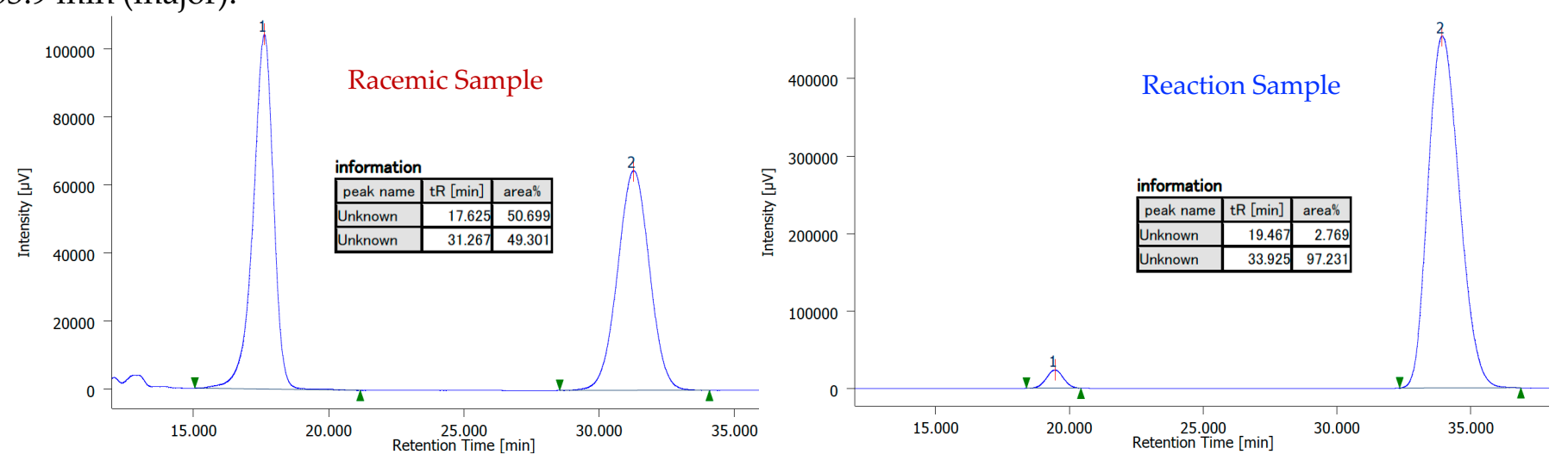

((1S,2R,3R)-2-benzoyl-3-methylcyclopropyl)(1H-pyrrolo[2,3-b]pyridin-1-yl)methanone (3ac):

The reaction performed according to the standard procedure 1 afforded $24 \mathrm{mg}$ (80\%).

Colorless sticky solid.

${ }^{1} \mathrm{H}$ NMR (400 MHz, 300 K, $\left.\mathrm{CDCl}_{3}\right)$ : $\delta 8.38(\mathrm{dd}, J=4.8,1.7 \mathrm{~Hz}, 1 \mathrm{H}), 8.02-7.97(\mathrm{~m}, 2 \mathrm{H})$, $7.89(\mathrm{~d}, J=4.1 \mathrm{~Hz}, 1 \mathrm{H}), 7.84(\mathrm{dd}, J=7.8,1.7 \mathrm{~Hz}, 1 \mathrm{H}), 7.48-7.41(\mathrm{~m}, 1 \mathrm{H}), 7.32(\mathrm{dd}, J=8.3$,

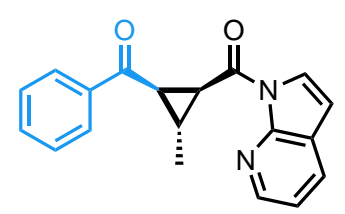

$7.1 \mathrm{~Hz}, 2 \mathrm{H}), 7.18(\mathrm{dd}, J=7.8,4.8 \mathrm{~Hz}, 1 \mathrm{H}), 6.51(\mathrm{~d}, J=4.1 \mathrm{~Hz}, 1 \mathrm{H}), 4.18(\mathrm{dd}, J=9.3,6.1 \mathrm{~Hz}$, $1 \mathrm{H}), 3.01(\mathrm{dd}, J=9.3,6.4 \mathrm{~Hz}, 1 \mathrm{H}), 2.62(\mathrm{~h}, J=6.2 \mathrm{~Hz}, 1 \mathrm{H}), 1.46(\mathrm{~d}, J=6.1 \mathrm{~Hz}, 3 \mathrm{H})$.

${ }^{13}$ C NMR (101 MHz, 300 K, CDCl $): \delta 195.3,167.8,148.0,143.7,137.5,133.0,129.4,128.5$, $128.4,126.1,124.1,118.8,105.6,36.9,34.5,22.9,17.4$.

IR (thin film): $\widetilde{v} 3148,3113,3067,3025,2960,2929,2868,1703,1676,1596,1580,1530$, 1448, 1407, 1381, 1358, 1318, 1288, 1262, 1213, 1177, 1106, 1051, 1013, 955, 889, 846, 8044, $779,754,735,691,656,628,596 \mathrm{~cm}^{-1}$.

HRMS (ESI): $m / z$ calculated for $\mathrm{C}_{19} \mathrm{H}_{16} \mathrm{O}_{2} \mathrm{~N}_{2} \mathrm{Na}$ [M+Na] $]^{+}$327.1104, found: 327.1105. $[\alpha]_{\mathrm{D}}^{23}-56.8\left(c 0.32, \mathrm{CHCl}_{3}, 18 \%\right.$ ee sample).

Enantiomeric excess was determined to be $18 \%$ ee by chiral stationary phase HPLC analysis (CHIRALPAK IG3 $(\phi 0.46 \mathrm{~cm} \times 25 \mathrm{~cm}), n$-hexane $/ \mathrm{PrOH}=4 / 1$, flow rate $1.0 \mathrm{~mL} / \mathrm{min}$, detection at $254 \mathrm{~nm}, \mathrm{t}_{\mathrm{R}}=28.1 \mathrm{~min}$ (minor) and $35.4 \mathrm{~min}$ (major). 

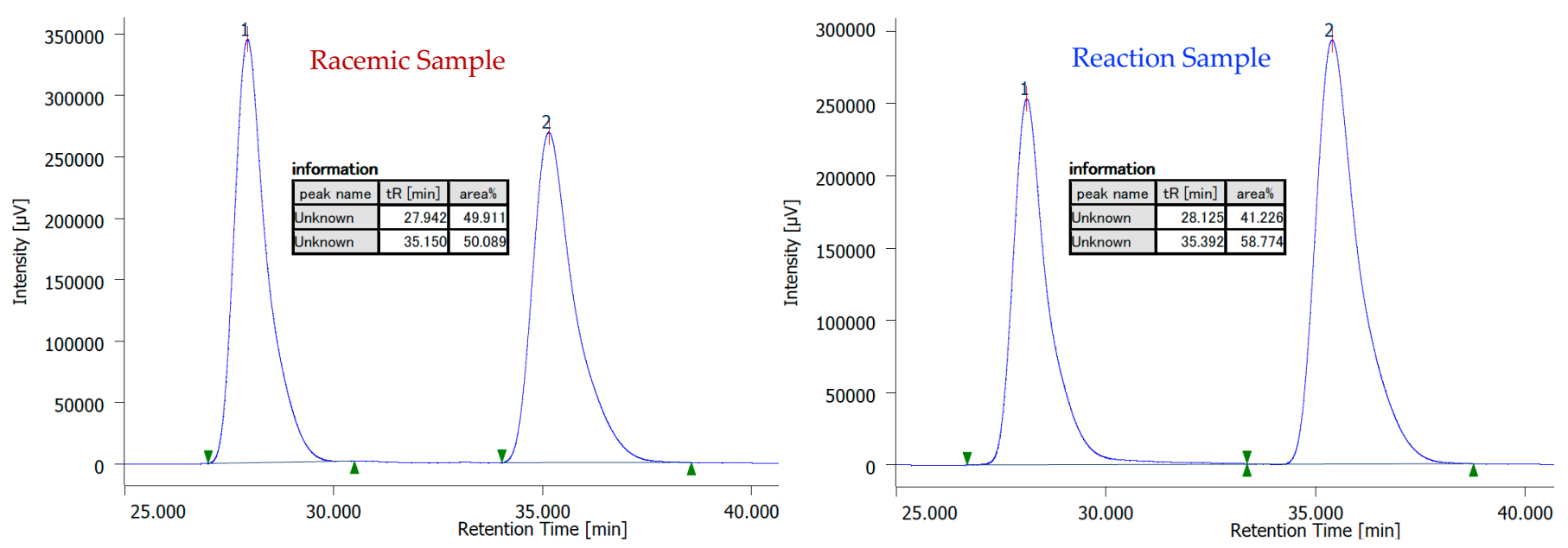

((1S,2R,3R)-2-benzoyl-3-methylcyclopropyl)(6-methyl-2,3-dihydro-1H-pyrrolo[2,3-b]pyridin-1yl)methanone (3ai):

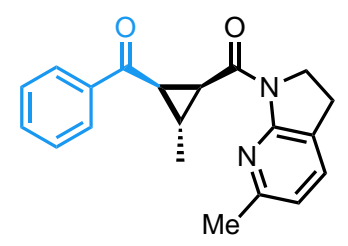

The reaction performed according to the standard procedure 1 afforded $28 \mathrm{mg}(89 \%)$.

Colorless sticky solid.

${ }^{1} \mathrm{H}$ NMR (400 MHz, $\left.300 \mathrm{~K}, \mathrm{CDCl}_{3}\right)$ : $\delta 8.00-7.94(\mathrm{~m}, 2 \mathrm{H}), 7.48-7.42(\mathrm{~m}, 1 \mathrm{H}), 7.37-7.31$

$(\mathrm{m}, 2 \mathrm{H}), 7.28-7.24(\mathrm{~m}, 1 \mathrm{H}), 6.68(\mathrm{~d}, J=7.5 \mathrm{~Hz}, 1 \mathrm{H}), 4.06-3.89(\mathrm{~m}, 2 \mathrm{H}), 3.63(\mathrm{dd}, J=9.3$,

$6.3 \mathrm{~Hz}, 1 \mathrm{H}), 2.98-2.80(\mathrm{~m}, 2 \mathrm{H}), 2.74(\mathrm{dd}, J=9.2,6.0 \mathrm{~Hz}, 1 \mathrm{H}), 2.49-2.40(\mathrm{~m}, 1 \mathrm{H}), 2.46(\mathrm{~s}$, $3 \mathrm{H}), 1.38(\mathrm{~d}, J=6.1 \mathrm{~Hz}, 3 \mathrm{H})$.

${ }^{13}$ C NMR (101 MHz, 300 K, CDCl $)$ : $\delta$ 196.4, 168.1, 155.8, 155.1, 138.1, 133.5, 132.5, 128.4, $128.3,122.9,117.1,46.2,35.4,35.3,24.3,24.1,21.9,17.6$.

IR (thin film): $\widetilde{v} 3065,2957,2923,2867,1681,1648,1593,1478,1449,1417,1390,1363$, $1323,1264,1219,1177,1157,1098,1060,1030,1019,949,921,829,808,765,743,712,693$, $612 \mathrm{~cm}^{-1}$.

HRMS (ESI): $m / z$ calculated for $\mathrm{C}_{20} \mathrm{H}_{20} \mathrm{O}_{2} \mathrm{~N}_{2} \mathrm{Na}$ [M+Na] $]^{+}$343.1417, found: 343.1413 . $[\alpha]_{\mathrm{D}}^{23}-19.9$ ( $c 0.03, \mathrm{CHCl}_{3}, 92 \%$ ee sample).

Enantiomeric excess was determined to be $92 \%$ ee by chiral stationary phase HPLC analysis (CHIRALPAK IG3 $(\phi 0.46 \mathrm{~cm} \times 25 \mathrm{~cm}), n$-hexane $/ \mathrm{PrOH}=4 / 1$, flow rate $1.0 \mathrm{~mL} / \mathrm{min}$, detection at $254 \mathrm{~nm}, \mathrm{t}_{\mathrm{R}}=57.0 \mathrm{~min}$ (major), $66.8 \mathrm{~min}$ (minor).
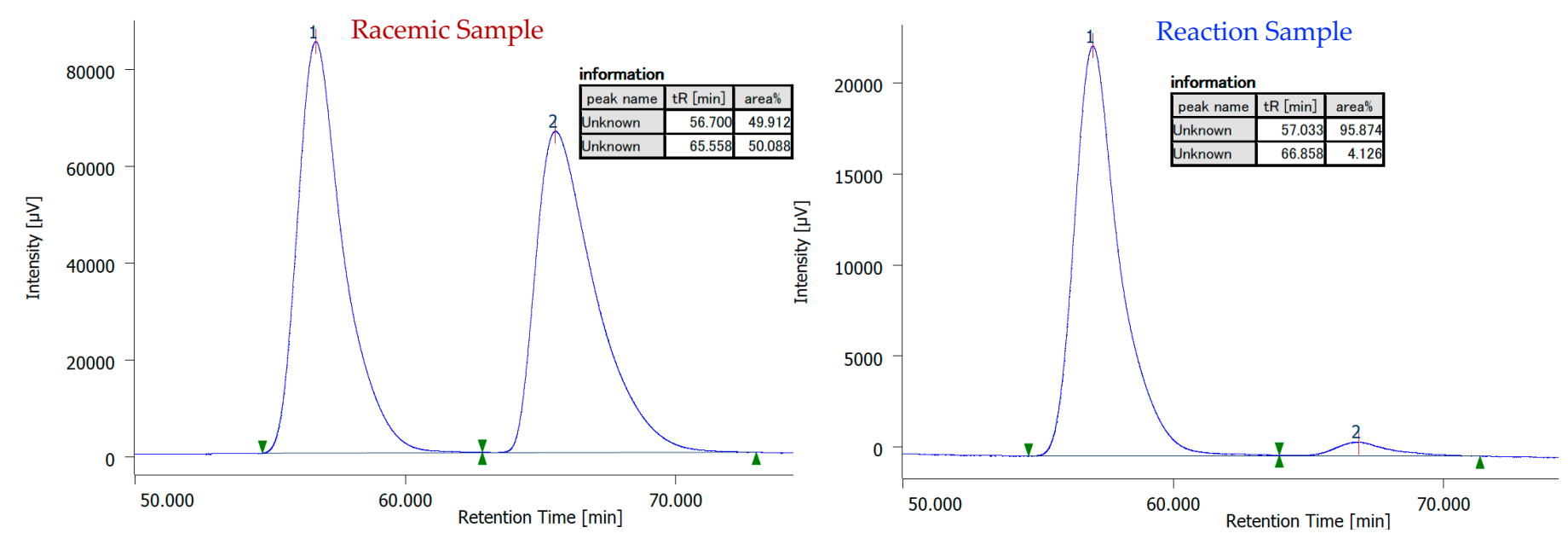
((1S,2R,3R)-2-benzoyl-3-methylcyclopropyl)(6-methoxy-2,3-dihydro-1H-pyrrolo[2,3-b]pyridin-1yl)methanone (3aj):

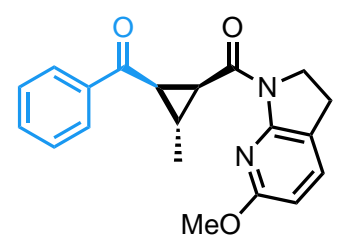

The reaction performed according to the standard procedure 1 afforded $28 \mathrm{mg}(84 \%)$.

Colorless sticky solid.

${ }^{1} \mathrm{H}$ NMR (400 MHz, $\left.300 \mathrm{~K}, \mathrm{CDCl}_{3}\right)$ : $\delta 7.97-7.93(\mathrm{~m}, 2 \mathrm{H}), 7.50-7.44(\mathrm{~m}, 1 \mathrm{H}), 7.38-7.30$ $(\mathrm{m}, 3 \mathrm{H}), 6.33(\mathrm{~d}, J=8.1 \mathrm{~Hz}, 1 \mathrm{H}), 4.06-3.94(\mathrm{~m}, 2 \mathrm{H}), 3.92(\mathrm{~s}, 3 \mathrm{H}), 3.72(\mathrm{dd}, J=9.2,6.1 \mathrm{~Hz}$, $1 \mathrm{H}), 2.97-2.78(\mathrm{~m}, 2 \mathrm{H}), 2.64(\mathrm{dd}, J=9.2,6.2 \mathrm{~Hz}, 1 \mathrm{H}), 2.44(\mathrm{~h}, J=6.1 \mathrm{~Hz}, 1 \mathrm{H}), 1.37(\mathrm{~d}, J=$ $6.1 \mathrm{~Hz}, 3 \mathrm{H})$.

${ }^{13} \mathrm{C}$ NMR (101 MHz, 300 K, CDCl 3 ): $\delta$ 195.8, 167.9, 154.0, 137.8, 136.2, 132.7, 129.4, 128.4, $128.3,117.3,103.5,53.8,46.9,35.7,33.9,23.8,21.5,17.9$.

IR (thin film): $\widetilde{v} 3058,2958,2927,2865,1681,1648,1608,1591,1473,1447,1418,1393$, 1362, 1323, 1313, 1294, 1253, 1220, 1195, 1176, 1159, 1093, 1048, 1018, 952, 925, 891, 809, $768,742,731,712,693,671,535 \mathrm{~cm}^{-1}$.

HRMS (ESI): $m / z$ calculated for $\mathrm{C}_{20} \mathrm{H}_{21} \mathrm{O}_{3} \mathrm{~N}_{2}[\mathrm{M}+\mathrm{H}]^{+}: 337.1547$, found: 337.1546 .

$[\alpha]_{\mathrm{D}}^{23}-420.9$ ( c 0.02, $\mathrm{CHCl}_{3}, 90 \%$ ee sample).

Enantiomeric excess was determined to be $90 \%$ ee by chiral stationary phase HPLC analysis (CHIRALPAK IC3 $(\phi 0.46 \mathrm{~cm} \times 25 \mathrm{~cm}), n$-hexane $/ \mathrm{PrOH}=1 / 1$, flow rate $1.0 \mathrm{~mL} / \mathrm{min}$, detection at $254 \mathrm{~nm}, \mathrm{t}_{\mathrm{R}}=33.9 \mathrm{~min}$ (minor), $57.5 \mathrm{~min}$ (major).
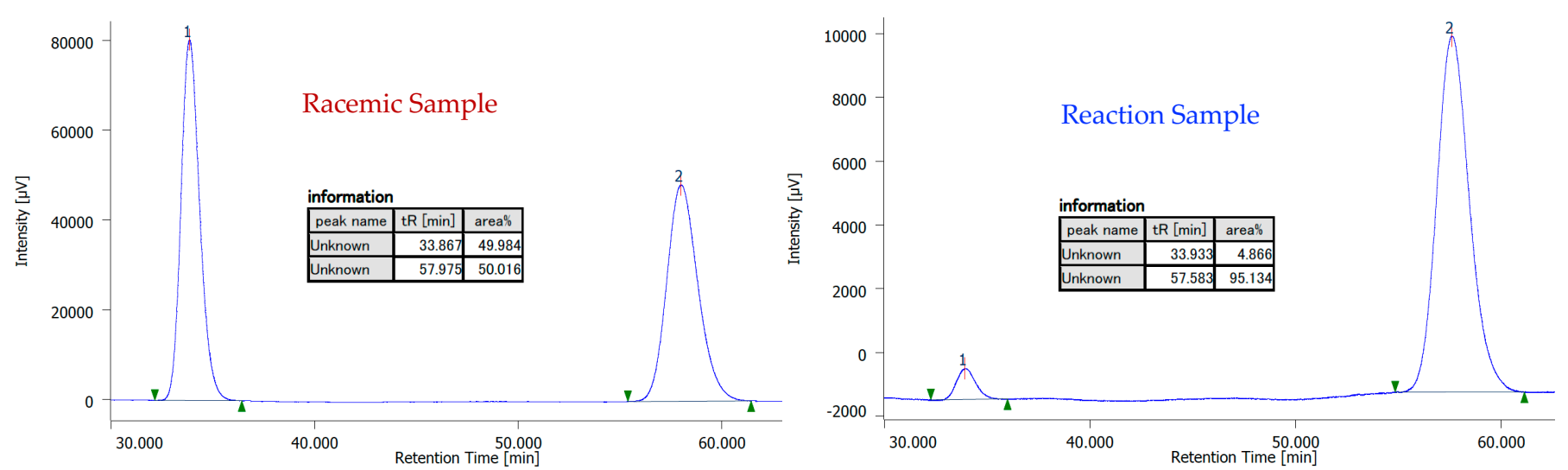

\section{((1S,2R,3R)-2-benzoyl-3-methylcyclopropyl)(6-chloro-2,3-dihydro-1H-pyrrolo[2,3-b]pyridin-1-} yl)methanone (3al):

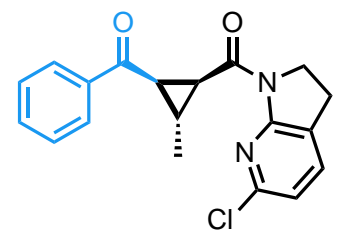

The reaction performed according to the standard procedure 1 afforded $27 \mathrm{mg}$ (79\%). Colorless sticky solid.

${ }^{1} \mathrm{H}$ NMR (400 MHz, $\left.300 \mathrm{~K}, \mathrm{CDCl}_{3}\right)$ : $\delta 8.03-7.98(\mathrm{~m}, 2 \mathrm{H}), 7.50-7.44(\mathrm{~m}, 1 \mathrm{H}), 7.41-7.34$ $(\mathrm{m}, 2 \mathrm{H}), 7.29(\mathrm{dt}, J=7.7,1.3 \mathrm{~Hz}, 1 \mathrm{H}), 6.82(\mathrm{~d}, J=7.7 \mathrm{~Hz}, 1 \mathrm{H}), 4.15-3.97(\mathrm{~m}, 2 \mathrm{H}), 3.21(\mathrm{dd}$, $J=9.2,6.5 \mathrm{~Hz}, 1 \mathrm{H}), 3.02-2.83(\mathrm{~m}, 3 \mathrm{H}), 2.46(\mathrm{~h}, J=6.1 \mathrm{~Hz}, 1 \mathrm{H}), 1.39(\mathrm{~d}, J=6.1 \mathrm{~Hz}, 3 \mathrm{H})$.

${ }^{13}$ C NMR (101 MHz, 300 K, CDCl $)$ : $\delta$ 196.6, 167.7, 155.9, 147.9, 137.9, 135.3, 132.7, 128.5, $128.4,124.7,117.5,46.5,36.2,35.1,23.9,22.8,17.5$.

IR (thin film): $\widetilde{v} 3064,2958,2926,2867,1676,1599,1577,1480,1447,1424,1413,1386$, $1360,1323,1267,1253,1219,1195,1177,1114,1078,1016,950,876,856,825,776,728,699$, $626 \mathrm{~cm}^{-1}$.

HRMS (ESI): $m / z$ calculated for $\mathrm{C}_{19} \mathrm{H}_{17} \mathrm{O}_{2} \mathrm{~N}_{2} \mathrm{ClNa}[\mathrm{M}+\mathrm{Na}]^{+}: 363.0871$, found: 363.0872.

$[\alpha]_{\mathrm{D}}^{23}-74.4\left(c 0.04, \mathrm{CHCl}_{3}, 62 \%\right.$ ee sample).

Enantiomeric excess was determined to be $62 \%$ ee by chiral stationary phase HPLC analysis (CHIRALPAK IE $(\phi 0.46 \mathrm{~cm} \times 25 \mathrm{~cm}), n$-hexane $/ \mathrm{PrOH}=4 / 1$, flow rate $1.0 \mathrm{~mL} / \mathrm{min}$, detection at $254 \mathrm{~nm}, \mathrm{t}_{\mathrm{R}}=51.0 \mathrm{~min}$ (major), $56.6 \mathrm{~min}$ (minor). 

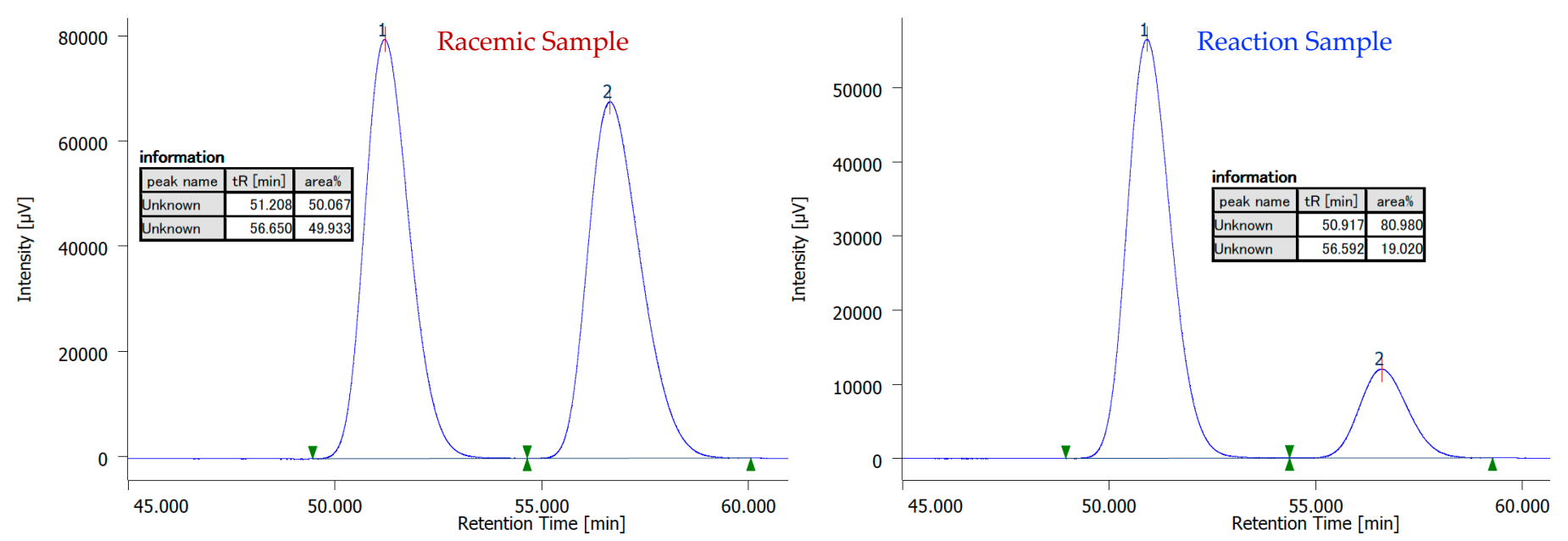

1-((1S,2R,3R)-2-benzoyl-3-methylcyclopropane-1-carbonyl)-2,3-dihydro-1H-pyrrolo[2,3-b]pyridine-6carbonitrile (3am):

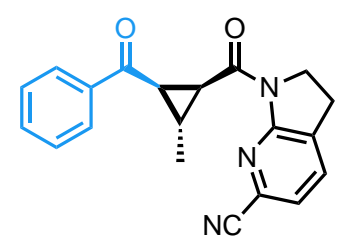

The reaction performed according to the standard procedure 1 afforded $20 \mathrm{mg}(62 \%)$.

Colorless sticky solid.

${ }^{1} \mathrm{H}$ NMR (400 MHz, 300 K, $\left.\mathrm{CDCl}_{3}\right)$ : $\delta 8.01-7.96(\mathrm{~m}, 2 \mathrm{H}), 7.52-7.46(\mathrm{~m}, 1 \mathrm{H}), 7.45-7.37$ $(\mathrm{m}, 3 \mathrm{H}), 7.20(\mathrm{~d}, J=7.4 \mathrm{~Hz}, 1 \mathrm{H}), 4.11(\mathrm{td}, J=8.6,1.3 \mathrm{~Hz}, 2 \mathrm{H}), 3.11(\mathrm{dd}, J=9.2,6.6 \mathrm{~Hz}, 1 \mathrm{H})$, $3.08-3.02(\mathrm{~m}, 2 \mathrm{H}), 2.97(\mathrm{dd}, J=9.1,5.9 \mathrm{~Hz}, 1 \mathrm{H}), 2.45(\mathrm{~h}, J=6.1 \mathrm{~Hz}, 1 \mathrm{H}), 1.41(\mathrm{~d}, J=6.1$ $\mathrm{Hz}, 3 \mathrm{H})$.

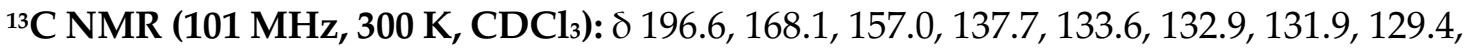
128.6, 128.4, 123.3, 117.7, 46.1, 36.1, 35.1, 24.6, 23.4, 17.5 .

IR (thin film): $\widetilde{v} 3082,2960,2924,2852,2232,1721,1679,1600,1578,1481,1440,1422$, 1384, 1320, 1267, 1218, 1176, 1090, 1060, 1017, 981, 950, 834, 755, 739, 708, 692, $656 \mathrm{~cm}^{-1}$. HRMS (ESI): $m / z$ calculated for $\mathrm{C}_{20} \mathrm{H}_{17} \mathrm{O}_{2} \mathrm{~N}_{3} \mathrm{Na}[\mathrm{M}+\mathrm{Na}]^{+}: 354.1213$, found: 354.1211.

$[\alpha]_{\mathrm{D}}^{23}+59.8$ (c 0.22, $\mathrm{CHCl}_{3}, 33 \%$ ee sample).

Enantiomeric excess was determined to be $33 \%$ ee by chiral stationary phase HPLC analysis (CHIRALPAK IC3 $(\phi 0.46 \mathrm{~cm} \times 25 \mathrm{~cm}), n$-hexane $/ \mathrm{PrOH}=1 / 1$, flow rate $1.0 \mathrm{~mL} / \mathrm{min}$, detection at $254 \mathrm{~nm}, \mathrm{t}=33.0 \mathrm{~min}$ (major), $57.5 \mathrm{~min}$ (minor).
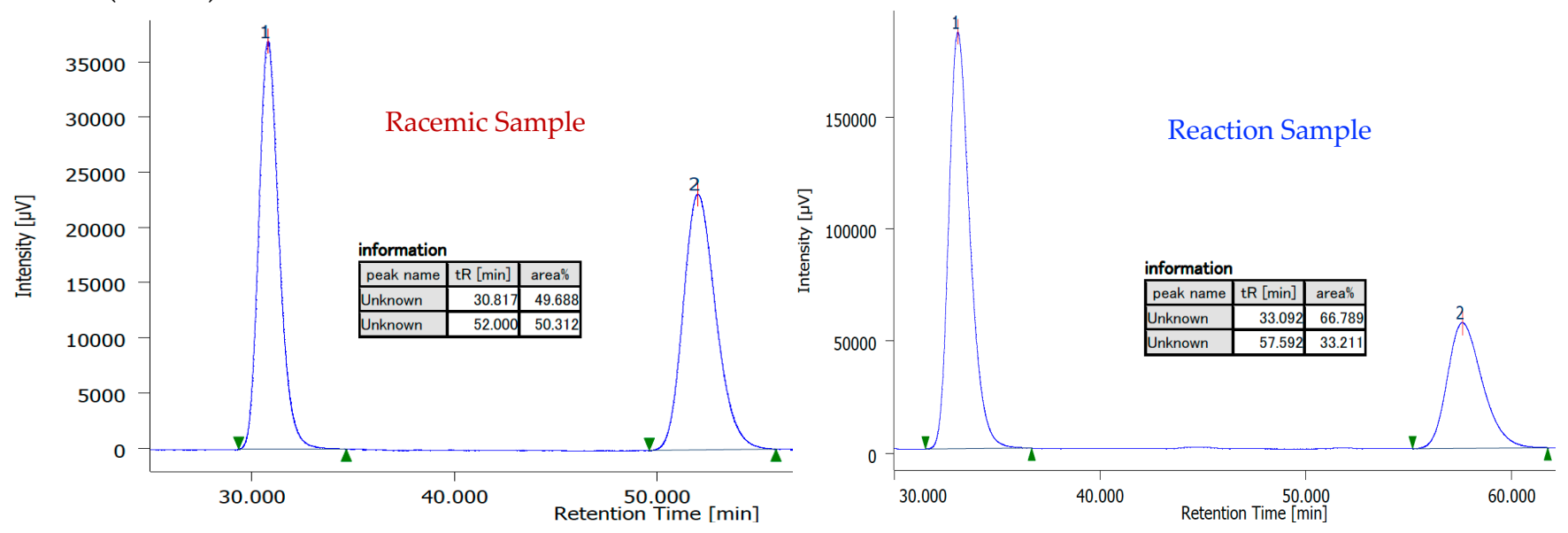
((1S,2R,3R)-2-benzoyl-3-methylcyclopropyl)(4-chloro-2,3-dihydro-1H-pyrrolo[2,3-b]pyridin-1yl)methanone (3an):

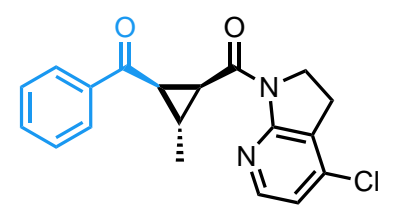

The reaction performed according to the standard procedure 1 afforded $28 \mathrm{mg}(83 \%)$. Colorless solid (m.p $=78-80^{\circ} \mathrm{C}$ ).

${ }^{1} \mathrm{H}$ NMR (400 MHz, 300 K, CDCl $)$ : $\delta 8.00(\mathrm{dd}, J=5.6,1.1 \mathrm{~Hz}, 1 \mathrm{H}), 7.97-7.92(\mathrm{~m}, 2 \mathrm{H})$, $7.50-7.43(\mathrm{~m}, 1 \mathrm{H}), 7.38-7.32(\mathrm{~m}, 2 \mathrm{H}), 6.83(\mathrm{~d}, J=5.6 \mathrm{~Hz}, 1 \mathrm{H}), 4.11-3.94(\mathrm{~m}, 2 \mathrm{H})$, $3.49(\mathrm{dd}, J=9.2,6.3 \mathrm{~Hz}, 1 \mathrm{H}), 3.07-2.88(\mathrm{~m}, 2 \mathrm{H}), 2.79(\mathrm{dd}, J=9.3,6.0 \mathrm{~Hz}, 1 \mathrm{H}), 2.44(\mathrm{~h}$, $J=6.2 \mathrm{~Hz}, 1 \mathrm{H}), 1.37(\mathrm{~d}, J=6.1 \mathrm{~Hz}, 3 \mathrm{H})$.

${ }^{13}$ C NMR (101 MHz, 300 K, CDCl $\left._{3}\right): \delta$ 196.1, 168.3, 157.2, 147.1, 140.7, 137.8, 132.7, $128.4,128.4,125.1,118.4,45.7,35.5,34.8,23.7,22.2,17.4$.

IR (thin film): $\widetilde{v} 3063,2959,2926,2867,1702,1681,1654,1597,1571,1479,1441$, 1410, 1379, 1359, 1323, 1263, 1245, 1220, 1175, 1161, 1092, 1064, 1019, 980, 951, 916, $873,855,804,759,693,630,560 \mathrm{~cm}^{-1}$.

HRMS (ESI): $m / z$ calculated for $\mathrm{C}_{19} \mathrm{H}_{18} \mathrm{O}_{2} \mathrm{~N}_{2} \mathrm{Cl}[\mathrm{M}+\mathrm{H}]^{+}: 341.1051$, found: 341.1055 . $[\alpha]_{\mathrm{D}}^{23}-134.8$ ( c 0.89, $\mathrm{CHCl}_{3}, 94 \%$ ee sample).

Enantiomeric excess was determined to be $94 \%$ ee by chiral stationary phase HPLC analysis (CHIRALPAK IA $(\phi 0.46 \mathrm{~cm} \times 25 \mathrm{~cm}), n$-hexane $/ \mathrm{PrOH}=4 / 1$, flow rate $1.0 \mathrm{~mL} / \mathrm{min}$, detection at $254 \mathrm{~nm}, \mathrm{t}_{\mathrm{R}}=12.0 \mathrm{~min}$ (major), $14.3 \mathrm{~min}$ (minor).
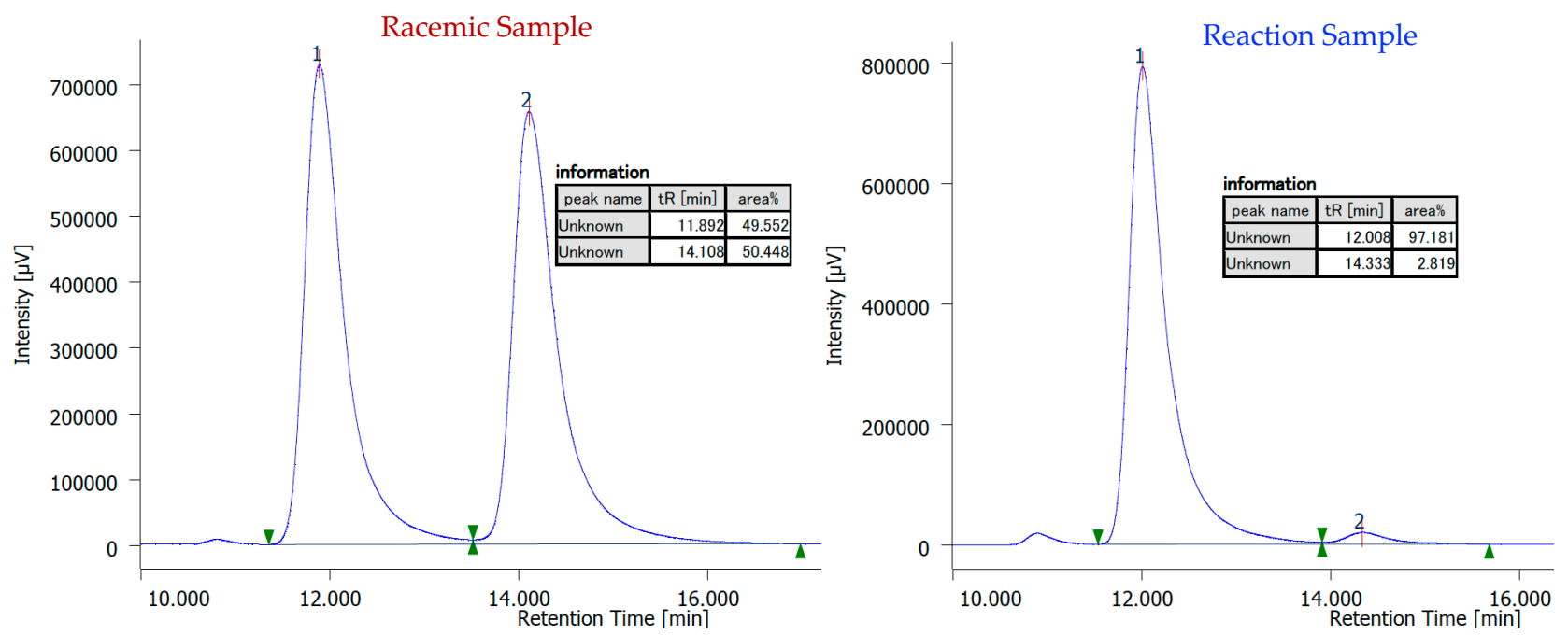

((1S,2R,3R)-2-benzoyl-3-methylcyclopropyl)(3,3-dimethyl-2,3-dihydro-1H-pyrrolo[2,3-b]pyridin-1yl)methanone (3ao):

The reaction performed according to the standard procedure 1 afforded $27 \mathrm{mg}(81 \%)$.

Colorless sticky solid.

${ }^{1} \mathrm{H}$ NMR (400 MHz, $\left.300 \mathrm{~K}, \mathrm{CDCl}_{3}\right)$ : $\delta 8.13(\mathrm{dd}, J=5.1,1.7 \mathrm{~Hz}, 1 \mathrm{H}), 7.99-7.91(\mathrm{~m}, 2 \mathrm{H})$, $7.42(\mathrm{ddt}, J=7.9,6.9,1.3 \mathrm{~Hz}, 1 \mathrm{H}), 7.34-7.27(\mathrm{~m}, 3 \mathrm{H}), 6.87(\mathrm{dd}, J=7.4,5.1 \mathrm{~Hz}, 1 \mathrm{H}), 3.71$

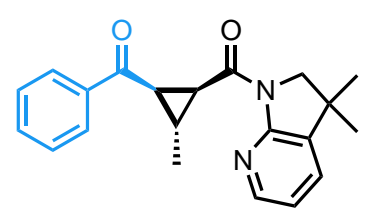

(s, 2H), $3.65(\mathrm{dd}, J=9.4,6.3 \mathrm{~Hz}, 1 \mathrm{H}), 2.83-2.74(\mathrm{~m}, 1 \mathrm{H}), 2.48(\mathrm{~h}, J=6.1 \mathrm{~Hz}, 1 \mathrm{H}), 1.38(\mathrm{~d}$, $J=6.1 \mathrm{~Hz}, 3 \mathrm{H}), 1.27(\mathrm{~s}, 3 \mathrm{H}), 1.09(\mathrm{~s}, 3 \mathrm{H})$.

${ }^{13} \mathrm{C}$ NMR (101 MHz, $\left.300 \mathrm{~K}, \mathrm{CDCl}_{3}\right): \delta 196.0,168.1,154.9,146.2,137.8,135.5,132.6,130.8$, $128.4,128.2,118.4,60.3,36.8,35.3,35.0,28.3,28.2,21.7,17.4$.

IR (thin film): $\widetilde{v} 3063,2959,2927,2869,1786,1677,1655,1597,1474,1467,1438,1421$, $1386,1368,1329,1270,1221,1177,1160,1139,1077,1056,1014,955,798,779,740,716$, $694 \mathrm{~cm}^{-1}$.

HRMS (ESI): $m / z$ calculated for $\mathrm{C}_{21} \mathrm{H}_{23} \mathrm{O}_{2} \mathrm{~N}_{2}[\mathrm{M}+\mathrm{H}]^{+}: 335.1754$, found: 335.1756 . $[\alpha]_{\mathrm{D}}^{23}-153.4$ ( $c$ 0.28, $\mathrm{CHCl}_{3}, 93 \%$ ee sample; major dastereomer). 
Enantiomeric excess was determined to be $93 \%$ ee (major diastereomer) by chiral stationary phase HPLC analysis (CHIRALPAK IG3 $(\phi 0.46 \mathrm{~cm} \times 25 \mathrm{~cm}), n$-hexane/ $\mathrm{PrOH}=4 / 1$, flow rate $1.0 \mathrm{~mL} / \mathrm{min}$, detection at 254 $\mathrm{nm}, \mathrm{t}_{\mathrm{R}}=30.7 \mathrm{~min}$ (minor), $38.2 \mathrm{~min}$ (major).
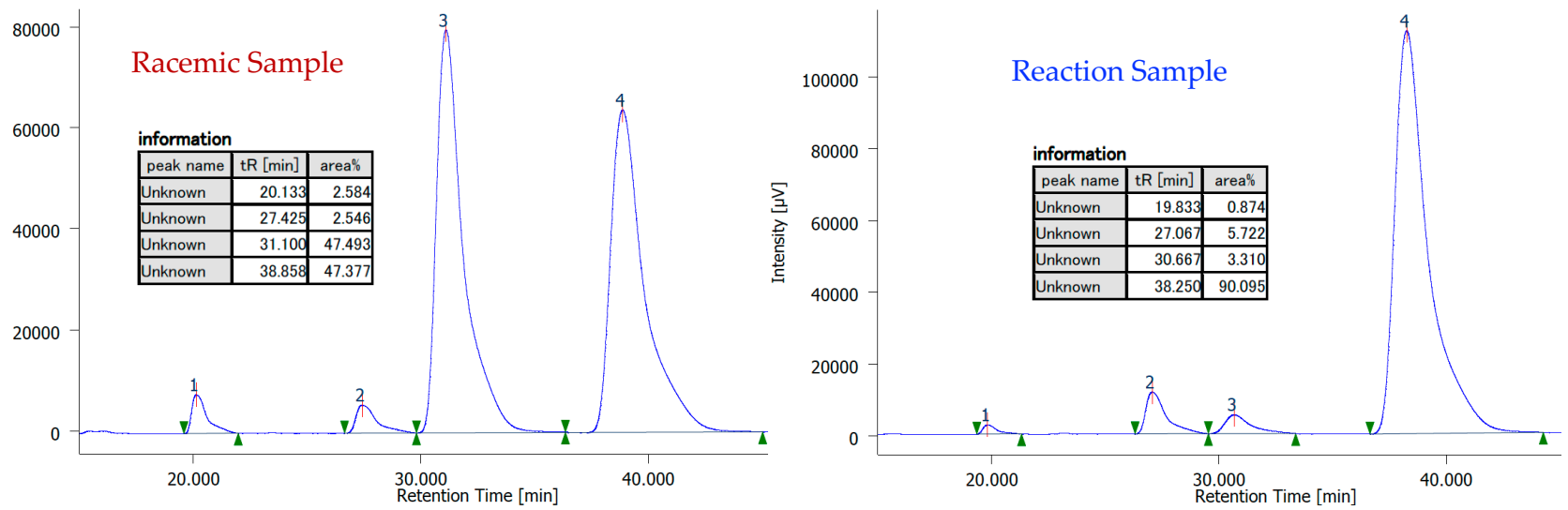
An oven-dried $4 \mathrm{~mL}$ vial was charged with 2p (24 mg, 1.0 equiv., $0.1 \mathrm{mmol})$, [Cu( $\left.\left.\mathrm{CH}_{3} \mathrm{CN}\right)_{4}\right] \mathrm{PF}_{6}(0.75 \mathrm{mg}, 0.02$ equiv., $2 \mathrm{~mol} \%$ ), (S)-DTBM-Segphos (2.95 mg, 0.025 equiv., $2.5 \mathrm{~mol} \%$ ) in the Glovebox. Anhydrous $\mathrm{CH}_{2} \mathrm{Cl}_{2}$ $(1.0 \mathrm{~mL})$ was added to the mixture via syringe with a stainless-steel needle at room temperature under positive Argon pressure. The resulting solution was stirred for $30 \mathrm{~min}$ and the pre-dissolved ylide 1 (1.5 equiv., $0.15 \mathrm{mmol})$ in anhydrous $\mathrm{CH}_{2} \mathrm{Cl}_{2}(0.5 \mathrm{~mL})$ was slowly added to the reaction mixture under the Argon atmosphere. The resulting reaction mixture was then allowed to stir for $24 \mathrm{~h}$ at room temperature. After confirming the complete conversion by Thin Layer Chromatography (TLC), the crude residue was directly loaded onto a Preparative Thin Layer Chromatography (PTLC) and eluted using Hexane/Ethyl acetate ( 5/1) solvent system $(>2 x)$. If required, the isolated product was repurified by another PTLC with $\mathrm{CH}_{2} \mathrm{Cl}_{2}$. The UVvisible product band was carefully scratched and filtered through a glass frit funnel using $\mathrm{CH}_{2} \mathrm{Cl}_{2}$ as an eluent to afford a pure trifluoromethylated cyclopropanation product 4 .
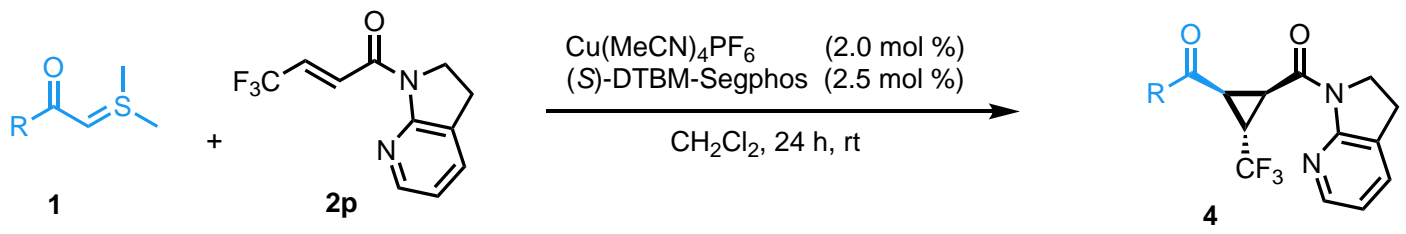

Major diastereomer: ((1R,2S,3R)-2-benzoyl-3-(trifluoromethyl)cyclopropyl)(2,3-dihydro-1H-pyrrolo[2,3b]pyridin-1-yl)methanone (4ap):

The reaction performed according to the standard procedure 2 afforded $34 \mathrm{mg}(95 \%)$.

Colorless solid (m.p $\left.=104-106^{\circ} \mathrm{C}\right)$.

${ }^{1} \mathrm{H}$ NMR (400 MHz, 300 K, $\left.\mathrm{CDCl}_{3}\right)$ : $\delta 8.15$ (ddt, $\left.J=5.1,1.8,1.0 \mathrm{~Hz}, 1 \mathrm{H}\right), 8.02-7.96(\mathrm{~m}$,

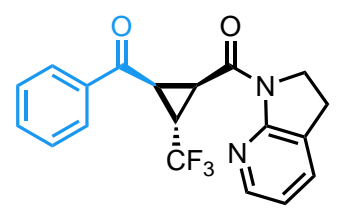
2H), $7.52-7.46(\mathrm{~m}, 1 \mathrm{H}), 7.43(\mathrm{dq}, J=7.4,1.4 \mathrm{~Hz}, 1 \mathrm{H}), 7.38-7.30(\mathrm{~m}, 2 \mathrm{H}), 6.90(\mathrm{dd}, J=$ 7.4, $5.1 \mathrm{~Hz}, 1 \mathrm{H}), 4.34(\mathrm{dd}, J=10.3,6.1 \mathrm{~Hz}, 1 \mathrm{H}), 4.03(\mathrm{ddd}, J=12.3,10.5,6.2 \mathrm{~Hz}, 1 \mathrm{H}), 3.88$ $(\mathrm{ddd}, J=12.3,10.5,6.8 \mathrm{~Hz}, 1 \mathrm{H}), 3.35-3.23(\mathrm{~m}, 2 \mathrm{H}), 3.06-2.85(\mathrm{~m}, 2 \mathrm{H})$.

${ }^{13} \mathrm{C}$ NMR (101 MHz, 300 K, $\left.\mathrm{CDCl}_{3}\right): \delta$ 192.1, 164.6, 155.7, 146.2, 136.6, 133.8, 133.4, 128.7, $128.5,126.2,125.1(q, J=271.7 \mathrm{~Hz}), 118.7,46.0,29.5(q, J=2.5 \mathrm{~Hz}), 28.4(q, J=2.6 \mathrm{~Hz})$, $25.9(\mathrm{q}, J=38.0 \mathrm{~Hz}), 24.3$.

${ }^{19} \mathrm{~F}$ NMR (376 MHz, $\left.300 \mathrm{~K}, \mathrm{CDCl}_{3}\right): \delta-66.14$.

IR (thin film): $\widetilde{v} 3073,1717,1687,1653,1591,1477,1447,1422,1392,1353,1332,1301$, 1277, 1241, 1219, 1137, 1050, 1015, 988, 927, 843, 784, 722, $692 \mathrm{~cm}^{-1}$.

HRMS (ESI): $m / z$ calculated for $\mathrm{C}_{19} \mathrm{H}_{16} \mathrm{O}_{2} \mathrm{~N}_{2} \mathrm{~F}_{3}[\mathrm{M}+\mathrm{H}]^{+}: 361.1158$, found: 361.1155 .

$[\alpha]_{\mathrm{D}}^{23}-219.6$ (c 1.58, $\mathrm{CHCl}_{3}, 96 \%$ ee sample).

Enantiomeric excess was determined to be $96 \%$ ee by chiral stationary phase HPLC analysis (CHIRALPAK IC3 $(\phi 0.46 \mathrm{~cm} \times 25 \mathrm{~cm}), n$-hexane $/ \mathrm{PrOH}=4 / 1$, flow rate $1.0 \mathrm{~mL} / \mathrm{min}$, detection at $254 \mathrm{~nm}, \mathrm{t}=24.4 \mathrm{~min}$ (minor) and $26.9 \mathrm{~min}$ (major).
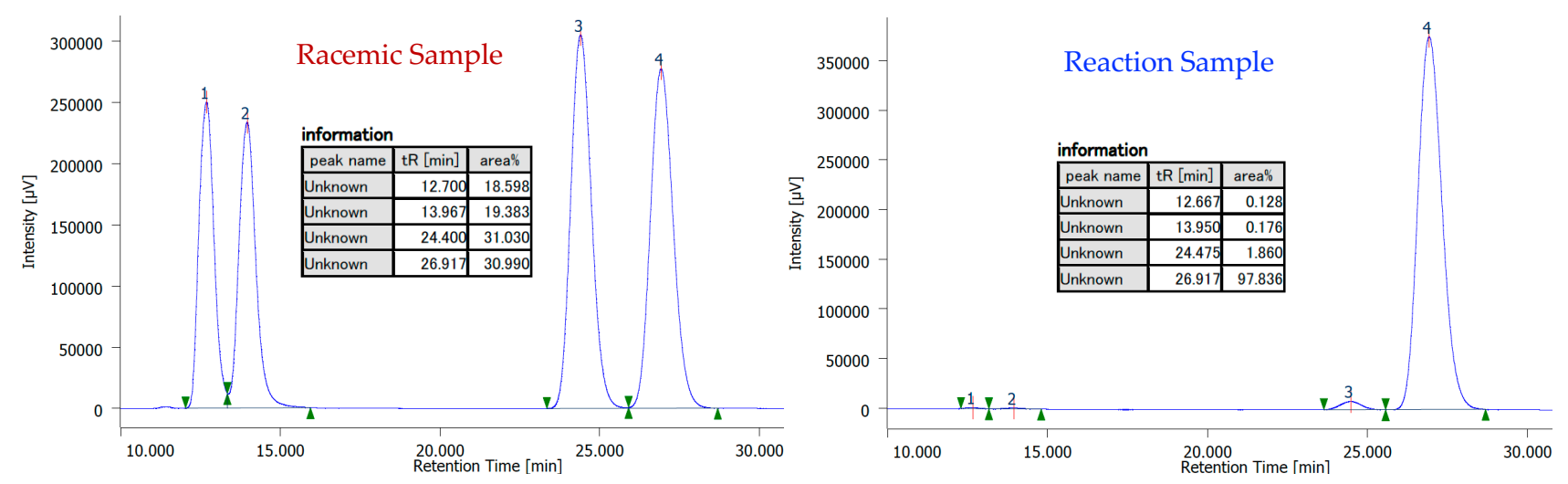
Minor diastereomer: $((1 R, 2 R, 3 R)-2-b e n z o y l-3-(t r i f l u o r o m e t h y l) c y c l o p r o p y l)(2,3-d i h y d r o-1 H-p y r r o l o[2,3-$ b]pyridin-1-yl)methanone (4ap')

This minor diastereomer was separately synthesized by using a ( \pm )-Biphep Ligand (for a diastereomer structure confirmation). In this reaction, the mixture of 4ap and 4ap' was obtained. The desired diastereomer 4ap' was carefully separated and purified by PTLC (16 mg, 44\% yield) as a colorless solid (m.p $\left.=169-171^{\circ} \mathrm{C}\right)$.

${ }^{1} \mathrm{H}$ NMR (400 MHz, $\left.300 \mathrm{~K}, \mathrm{CDCl}_{3}\right): \delta 8.23(\mathrm{ddd}, J=5.0,1.8,0.9 \mathrm{~Hz}, 1 \mathrm{H}), 8.12-8.06(\mathrm{~m}$, $2 \mathrm{H}), 7.63-7.57(\mathrm{~m}, 1 \mathrm{H}), 7.54-7.46(\mathrm{~m}, 3 \mathrm{H}), 6.95(\mathrm{dd}, J=7.4,5.1 \mathrm{~Hz}, 1 \mathrm{H}), 5.44(\mathrm{t}, J=5.9$<smiles></smiles>
$\mathrm{Hz}, 1 \mathrm{H}), 4.19-4.08(\mathrm{~m}, 2 \mathrm{H}), 3.44(\mathrm{dd}, J=10.1,6.1 \mathrm{~Hz}, 1 \mathrm{H}), 3.10(\mathrm{t}, J=8.5 \mathrm{~Hz}, 2 \mathrm{H}), 2.82$ $(\mathrm{dqd}, J=10.2,7.5,5.8 \mathrm{~Hz}, 1 \mathrm{H})$.

${ }^{13} \mathrm{C}$ NMR (101 MHz, 300 K, $\left.\mathrm{CDCl}_{3}\right)$ : $\delta$ 191.7, 168.2, 155.7, 146.7, 136.8, 133.9, 133.7, 128.82, $128.80,126.0,124.6(\mathrm{q}, J=273.7 \mathrm{~Hz}), 119.0,46.3,31.2,30.8(\mathrm{q}, J=38.4 \mathrm{~Hz}), 24.4,22.4(\mathrm{q}, J=$ $2.3 \mathrm{~Hz})$.

${ }^{19}$ F NMR (376 MHz, $\left.300 \mathrm{~K}, \mathrm{CDCl}_{3}\right)$ : $\delta-60.48$.

IR (thin film): $\widetilde{v}$ 3074, 2964, 2918, 2855, 1687, 1649, 1591, 1476, 1447, 1422, 1388, 1353, 1277, 1241, 1215, 1181, 1136, 1103, 1079, 1051, 1020, 989, 970, 934, 867, 786, 752, 722, 689, $642,631,596,533 \mathrm{~cm}^{-1}$.

HRMS (ESI): $\mathrm{m} / \mathrm{z}$ calculated for $\mathrm{C}_{19} \mathrm{H}_{15} \mathrm{O}_{2} \mathrm{~N}_{2} \mathrm{~F}_{3} \mathrm{Na}$ [M+Na] $]^{+}$383.0978, found: 383.0981.

(2,3-dihydro-1H-pyrrolo[2,3-b]pyridin-1-yl)((1R,2S,3R)-2-(4-methylbenzoyl)-3(trifluoromethyl)cyclopropyl)methanone (4bp):

The reaction performed according to the standard procedure 2 afforded $33 \mathrm{mg}(90 \%)$.

Colorless solid (m.p $=88-90^{\circ} \mathrm{C}$ ).

${ }^{1} \mathrm{H}$ NMR (400 MHz, $\left.300 \mathrm{~K}, \mathrm{CDCl}_{3}\right)$ : $\delta 8.15$ (ddt, J= 5.1, 1.8, $\left.1.0 \mathrm{~Hz}, 1 \mathrm{H}\right), 7.92-7.87(\mathrm{~m}$, $2 \mathrm{H}), 7.43(\mathrm{dq}, J=7.4,1.4 \mathrm{~Hz}, 1 \mathrm{H}), 7.16-7.10(\mathrm{~m}, 2 \mathrm{H}), 6.90(\mathrm{dd}, J=7.4,5.1 \mathrm{~Hz}, 1 \mathrm{H}), 4.30$

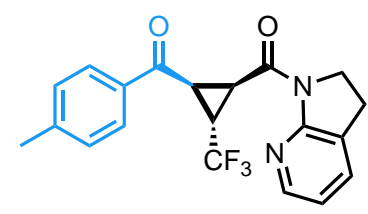
$(\mathrm{dd}, J=10.2,6.2 \mathrm{~Hz}, 1 \mathrm{H}), 3.05-2.87(\mathrm{~m}, 2 \mathrm{H}), 3.34-3.20(\mathrm{~m}, 2 \mathrm{H}), 3.07-2.85(\mathrm{~m}, 2 \mathrm{H})$, $2.35(\mathrm{~s}, 3 \mathrm{H})$.

${ }^{13} \mathrm{C}$ NMR (101 MHz, 300 K, $\left.\mathrm{CDCl}_{3}\right): \delta 191.6,164.7,155.8,146.2,144.3,134.2,133.7,129.2$, 128.8, 126.2, $125.1(\mathrm{q}, J=272.7 \mathrm{~Hz}), 118.6,46.0,29.5(\mathrm{q}, J=2.4 \mathrm{~Hz}), 28.4(\mathrm{q}, J=2.8 \mathrm{~Hz})$, $25.9(\mathrm{q}, J=38.1 \mathrm{~Hz}), 24.4,21.8$.

${ }^{19} \mathrm{~F}$ NMR (376 MHz, $\left.300 \mathrm{~K}, \mathrm{CDCl}_{3}\right)$ : $\delta-66.12$.

IR (thin film): $\widetilde{v} 3073,2922,1684,1654,1603,1590,1478,1444,1423,1391,1351,1332$, $1301,1278,1241,1227,1209,1180,1138,1038,1014,987,844,786,747,700 \mathrm{~cm}^{-1}$.

HRMS (ESI): $m / z$ calculated for $\mathrm{C}_{20} \mathrm{H}_{18} \mathrm{O}_{2} \mathrm{~N}_{2} \mathrm{~F}_{3}[\mathrm{M}+\mathrm{H}]^{+}: 375.1315$, found: 375.1312 .

$[\alpha]_{\mathrm{D}}^{23}-201.5$ ( c 1.44, $\mathrm{CHCl}_{3}, 95 \%$ ee sample; major diastereomer).

Enantiomeric excess was determined to be $95 \%$ ee (major diastereomer) by chiral stationary phase HPLC analysis (CHIRALPAK IG3 $(\phi 0.46 \mathrm{~cm} \times 25 \mathrm{~cm}), n$-hexane/PrOH $=4 / 1$, flow rate $1.0 \mathrm{~mL} / \mathrm{min}$, detection at 254 $\mathrm{nm}, \mathrm{t}_{\mathrm{R}}=32.1 \mathrm{~min}$ (major) and $48.0 \mathrm{~min}$ (minor).
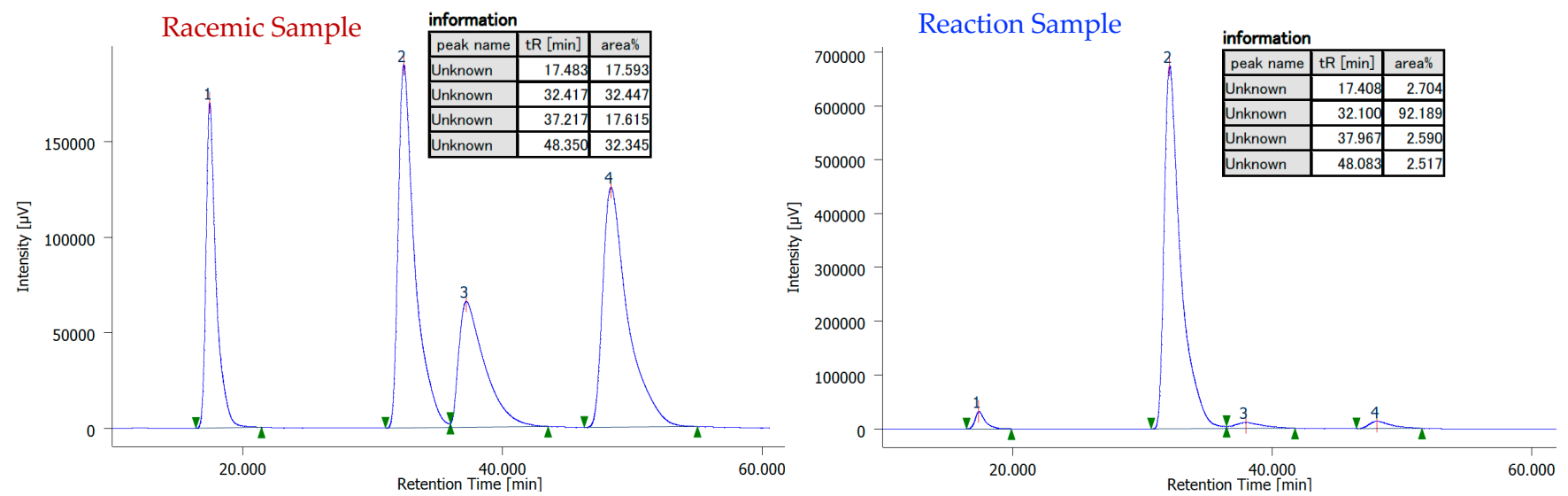
(2,3-dihydro-1H-pyrrolo[2,3- $b$ ]pyridin-1-yl)((1R,2S,3R)-2-(4-methoxybenzoyl)-3-

(trifluoromethyl)cyclopropyl)methanone (4cp):

The reaction performed according to the standard procedure 2 afforded $36 \mathrm{mg}$ (93\%).

Colorless sticky solid.

${ }^{1}$ H NMR (400 MHz, 300 K, CDCl $_{3}$ ): $\delta 8.15$ (ddt, $\left.J=5.2,1.8,1.0 \mathrm{~Hz}, 1 \mathrm{H}\right), 8.01-7.95$ $(\mathrm{m}, 2 \mathrm{H}), 7.43(\mathrm{dq}, J=7.4,1.3 \mathrm{~Hz}, 1 \mathrm{H}), 6.90(\mathrm{dd}, J=7.4,5.1 \mathrm{~Hz}, 1 \mathrm{H}), 6.83-6.77(\mathrm{~m}$, (1) 2H), $4.29(\mathrm{dd}, J=10.1,6.3 \mathrm{~Hz}, 1 \mathrm{H}), 4.04(\mathrm{ddd}, J=12.3,10.5,6.2 \mathrm{~Hz}, 1 \mathrm{H}), 3.95-3.83$ $(\mathrm{m}, 1 \mathrm{H}), 3.82(\mathrm{~s}, 3 \mathrm{H}), 3.31-3.21(\mathrm{~m}, 2 \mathrm{H}), 3.06-2.86(\mathrm{~m}, 2 \mathrm{H})$.

${ }^{13} \mathrm{C}$ NMR (101 MHz, 300 K, CDCl 3 ): $\delta$ 190.4, 164.7, 163.8, 155.8, 146.2, 133.7, 131.0, $129.8,126.2,125.2(\mathrm{q}, J=272.7 \mathrm{~Hz}) 118.6,113.7,55.6,46.0,29.4(\mathrm{q}, J=2.4 \mathrm{~Hz}), 28.2(\mathrm{q}$, $J=2.8 \mathrm{~Hz}), 25.7(\mathrm{q}, J=37.9 \mathrm{~Hz}), 24.4$.

${ }^{19} \mathrm{~F}$ NMR (376 MHz, $\left.300 \mathrm{~K}, \mathrm{CDCl}_{3}\right)$ : $\delta-66.08$.

IR (thin film): $\widetilde{v} 3074,3014,2964,2937,2842,1677,1654,1600,1576,1512,1477$, 1443, 1423, 1391, 1371, 1334, 1302, 1263, 1227, 1170, 1137, 1101, 1020, 988, 933, 840, $786,736,700 \mathrm{~cm}^{-1}$.

HRMS (ESI): $m / z$ calculated for $\mathrm{C}_{20} \mathrm{H}_{18} \mathrm{O}_{3} \mathrm{~N}_{2} \mathrm{~F}_{3}[\mathrm{M}+\mathrm{H}]^{+}: 391.1264$, found: 391.1264 .

$[\alpha]_{\mathrm{D}}^{23}-187.6$ ( $c 1.11, \mathrm{CHCl}_{3}, 94 \%$ ee sample, major diastereomer).

Enantiomeric excess was determined to be $94 \%$ ee (major diastereomer) by chiral stationary phase HPLC analysis (CHIRALPAK IG3 $(\phi 0.46 \mathrm{~cm} \times 25 \mathrm{~cm}), n$-hexane $/ \mathrm{PrOH}=4 / 1$, flow rate $1.0 \mathrm{~mL} / \mathrm{min}$, detection at 254 $\mathrm{nm}, \mathrm{t}_{\mathrm{R}}=53.9 \mathrm{~min}$ (major) and $82.9 \mathrm{~min}$ (minor).
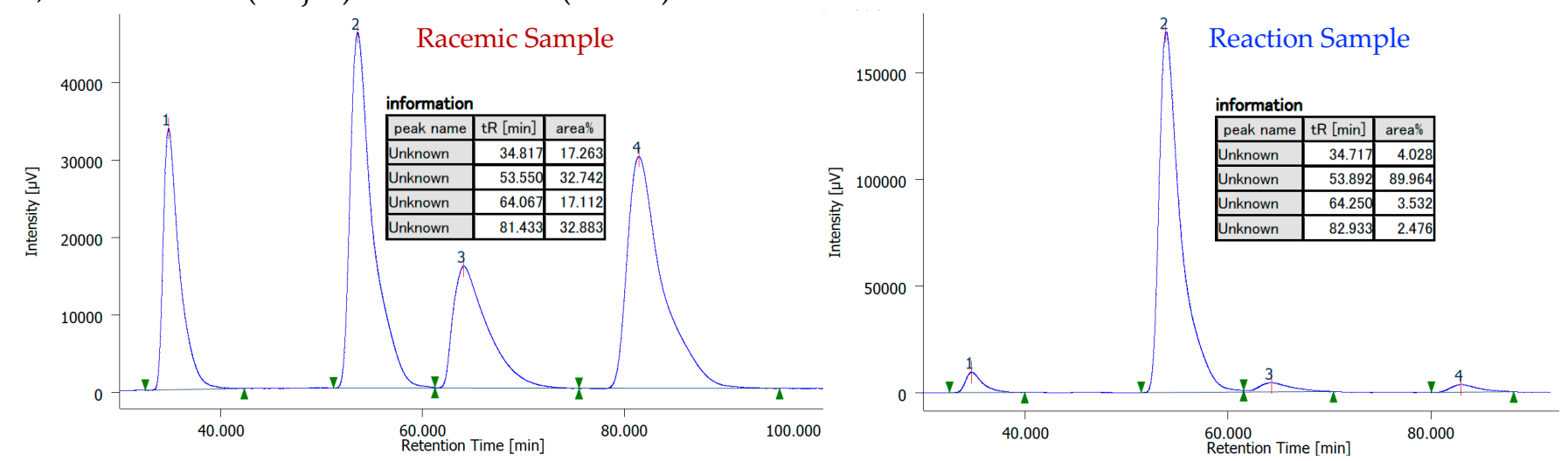

(2,3-dihydro-1H-pyrrolo[2,3-b]pyridin-1-yl)((1R,2S,3R)-2-(4-nitrobenzoyl)-3(trifluoromethyl)cyclopropyl)methanone (4dp):

The reaction performed according to the standard procedure 2 afforded $39 \mathrm{mg}$ (98\%).

Colorless solid (m.p $\left.=153-155^{\circ} \mathrm{C}\right)$.

${ }^{1} \mathrm{H}$ NMR (400 MHz, $\left.300 \mathrm{~K}, \mathrm{CDCl}_{3}\right)$ : $\delta 8.24-8.15(\mathrm{~m}, 5 \mathrm{H}), 7.48(\mathrm{dq}, J=7.4,1.4 \mathrm{~Hz}$,<smiles>O=C(c1ccc([N+](=O)[O-])cc1)C1C(C(=O)N2CCc3cccnc32)C1[C+](F)F</smiles>
$1 \mathrm{H}), 6.96(\mathrm{dd}, J=7.4,5.1 \mathrm{~Hz}, 1 \mathrm{H}), 4.48-4.39(\mathrm{~m}, 1 \mathrm{H}), 4.12-3.79(\mathrm{~m}, 2 \mathrm{H}), 3.33-3.21$ (m, 2H), $3.11-2.88(\mathrm{~m}, 2 \mathrm{H})$.

${ }^{13} \mathrm{C}$ NMR (101 MHz, $\left.300 \mathrm{~K}, \mathrm{CDCl}_{3}\right): \delta$ 190.8, 164.3, 155.5, 150.5, 146.2, 141.01, 134.1, 129.7, 126.3, $124.8(\mathrm{q}, J=272.7 \mathrm{~Hz}), 123.8,119.1,46.1,29.64(\mathrm{q}, J=2.6 \mathrm{~Hz}), 28.7(\mathrm{q}, J$ $=2.8 \mathrm{~Hz}), 26.11(\mathrm{q}, J=38.2 \mathrm{~Hz}), 24.4$.

${ }^{19}$ F NMR (376 MHz, $\left.300 \mathrm{~K}, \mathrm{CDCl}_{3}\right)$ : $\delta$-66.24.

IR (thin film): $\widetilde{v} 3073,2919,1679,1650,1591,1526,1477,1445,1423,1389,1347$, $1300,1276,1241,1211,1139,1011,990,855,786,724 \mathrm{~cm}^{-1}$.

HRMS (ESI): $m / z$ calculated for $\mathrm{C}_{19} \mathrm{H}_{15} \mathrm{O}_{4} \mathrm{~N}_{3} \mathrm{~F}_{3}[\mathrm{M}+\mathrm{H}]^{+}: 406.1009$, found: 406.1010 . $[\alpha]_{\mathrm{D}}^{23}-218.2$ (c 0.84, $\mathrm{CHCl}_{3}, 97 \%$ ee sample; major diastereomer). 
Enantiomeric excess was determined to be $97 \%$ ee (major diastereomer) by chiral stationary phase HPLC analysis (CHIRALPAK IG3 $(\phi 0.46 \mathrm{~cm} \times 25 \mathrm{~cm}), n$-hexane/ $\mathrm{PrOH}=4 / 1$, flow rate $1.0 \mathrm{~mL} / \mathrm{min}$, detection at 254 $\mathrm{nm}, \mathrm{t}_{\mathrm{R}}=39.4 \mathrm{~min}$ (major) and $45.0 \mathrm{~min}$ (minor).
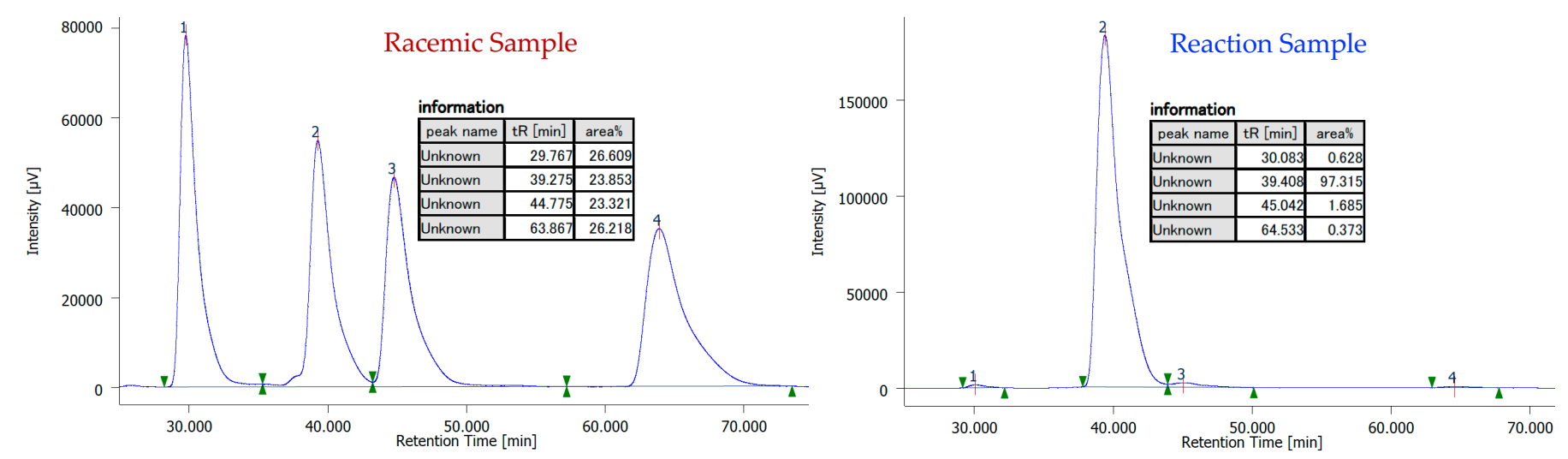

(2,3-dihydro-1H-pyrrolo[2,3-b]pyridin-1-yl)((1R,2S,3R)-2-(2-methoxybenzoyl)-3-

(trifluoromethyl)cyclopropyl)methanone (4ep):

The reaction performed according to the standard procedure 2 afforded $38 \mathrm{mg}$ (97\%).

Colorless sticky solid.

${ }^{1} \mathrm{H}$ NMR (400 MHz, $\left.300 \mathrm{~K}, \mathrm{CDCl}_{3}\right): \delta 8.03(\mathrm{ddt}, J=5.1,1.8,1.0 \mathrm{~Hz}, 1 \mathrm{H}), 7.54(\mathrm{dd}, J=7.7$,<smiles>COc1ccccc1C(=O)C1C(C(=O)N2CCc3cccnc32)[C@@H]1C(F)(F)F</smiles>
$1.8 \mathrm{~Hz}, 1 \mathrm{H}), 7.44-7.38(\mathrm{~m}, 2 \mathrm{H}), 6.92-6.81(\mathrm{~m}, 3 \mathrm{H}), 4.16(\mathrm{dd}, J=10.2,6.1 \mathrm{~Hz}, 1 \mathrm{H}), 4.10$ $-3.98(\mathrm{~m}, 2 \mathrm{H}), 3.79(\mathrm{~s}, 3 \mathrm{H}), 3.48-3.42(\mathrm{~m}, 1 \mathrm{H}), 3.13-3.04(\mathrm{~m}, 1 \mathrm{H}), 3.03-2.94(\mathrm{~m}, 2 \mathrm{H})$. ${ }^{13}$ C NMR (101 MHz, 300 K, CDCl $)$ ): $\delta 194.4,165.6,158.9,155.9,146.1,134.0,133.5,130.9$, 127.6, $125.5(\mathrm{q}, J=272.7 \mathrm{~Hz}), 126.1,120.6,118.4,111.3,55.5,46.0,33.8(\mathrm{q}, J=2.3 \mathrm{~Hz}), 28.3$ $(\mathrm{q}, J=2.6 \mathrm{~Hz}), 27.2(\mathrm{q}, J=37.8 \mathrm{~Hz}), 24.4$.

${ }^{19} \mathrm{~F}$ NMR (376 MHz, $\left.300 \mathrm{~K}, \mathrm{CDCl}_{3}\right)$ : $\delta-66.25$.

IR (thin film): $\widetilde{v} 3077,2964,2922,2843,1673,1596,1486,1422,1395,1335,1301,1260$, $1207,1141,1014,932,864,799,754,700,644,619,599,554,540,525 \mathrm{~cm}^{-1}$.

HRMS (ESI): $m / z$ calculated for $\mathrm{C}_{20} \mathrm{H}_{17} \mathrm{O}_{3} \mathrm{~N}_{2} \mathrm{~F}_{3} \mathrm{Na}$ [M+Na] $: 413.1083$, found: 413.1086 . $[\alpha]_{\mathrm{D}}^{23}-121.4$ ( c $0.12, \mathrm{CHCl}_{3}, 96 \%$ ee sample).

Enantiomeric excess was determined to be $96 \%$ ee by chiral stationary phase HPLC analysis (CHIRALPAK IG3 $(\phi 0.46 \mathrm{~cm} \times 25 \mathrm{~cm}), n$-hexane $/ \mathrm{PrOH}=4 / 1$, flow rate $1.0 \mathrm{~mL} / \mathrm{min}$, detection at $254 \mathrm{~nm}, \mathrm{t}_{\mathrm{R}}=39.0 \mathrm{~min}$ (major) and $55.8 \mathrm{~min}$ (minor).
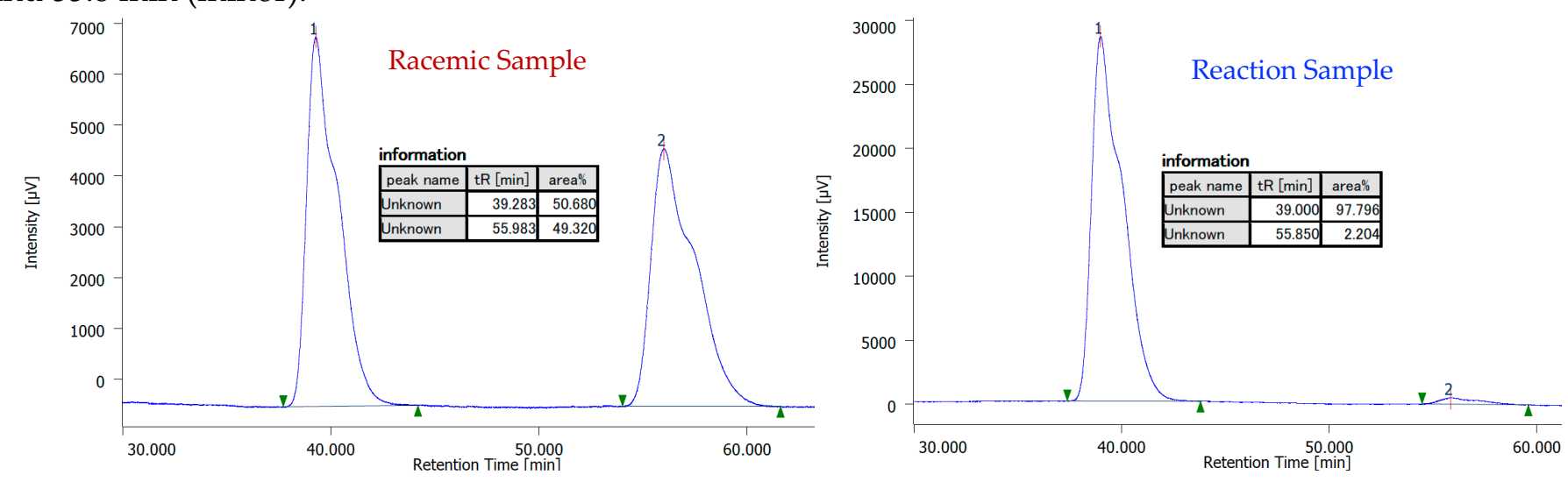
((1R,2S,3R)-2-(2-naphthoyl)-3-(trifluoromethyl)cyclopropyl)(2,3-dihydro-1H-pyrrolo[2,3-b]pyridin-1yl)methanone (4fp):

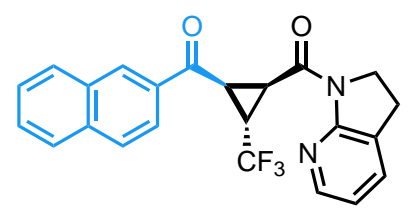

The reaction performed according to the standard procedure 2 afforded $38 \mathrm{mg}$ (93\%).

Colorless solid (m.p $\left.=121-123^{\circ} \mathrm{C}\right)$.

${ }^{1} \mathrm{H}$ NMR (400 MHz, $\left.300 \mathrm{~K}, \mathrm{CDCl}_{3}\right)$ : $\delta 8.59(\mathrm{~d}, J=1.7 \mathrm{~Hz}, 1 \mathrm{H}), 8.29-8.24(\mathrm{~m}, 1 \mathrm{H})$, $8.02(\mathrm{dd}, J=8.7,1.8 \mathrm{~Hz}, 1 \mathrm{H}), 7.80(\mathrm{t}, J=8.5 \mathrm{~Hz}, 2 \mathrm{H}), 7.65(\mathrm{~d}, J=8.2 \mathrm{~Hz}, 1 \mathrm{H}), 7.56$ $(\mathrm{ddd}, J=8.2,6.9,1.3 \mathrm{~Hz}, 1 \mathrm{H}), 7.46(\mathrm{ddd}, J=8.2,6.8,1.2 \mathrm{~Hz}, 1 \mathrm{H}), 7.38(\mathrm{dq}, J=7.5,1.4$ $\mathrm{Hz}, 1 \mathrm{H}), 6.93(\mathrm{dd}, J=7.4,5.1 \mathrm{~Hz}, 1 \mathrm{H}), 4.49(\mathrm{dd}, J=10.5,6.0 \mathrm{~Hz}, 1 \mathrm{H}), 2.94(\mathrm{ddd}, J=$ $16.6,10.7,5.9 \mathrm{~Hz}, 1 \mathrm{H}), 2.75(\mathrm{ddd}, J=16.6,10.7,5.9 \mathrm{~Hz}, 1 \mathrm{H}), 3.46(\mathrm{dd}, J=10.5,6.1 \mathrm{~Hz}$, $1 \mathrm{H}), 3.37(\mathrm{~h}, J=6.4 \mathrm{~Hz}, 1 \mathrm{H}), 3.01-2.68(\mathrm{~m}, 2 \mathrm{H})$.

${ }^{13}$ C NMR (101 MHz, 300 K, CDCl $)$ ): $\delta$ 191.7, 164.6, 155.8, 146.2, 135.8, 133.9, 133.8, 132.4, 131.0, 129.5, 128.7, 128.4, 127.9, 126.8, 126.4, 125.2 (q, J = 272.7 Hz), 124.1, 118.8, 46.0, $29.8(\mathrm{q}, J=2.5 \mathrm{~Hz}), 28.2(\mathrm{q}, J=2.7 \mathrm{~Hz}), 25.6(\mathrm{q}, J=38.1 \mathrm{~Hz}), 24.2$.

${ }^{19} \mathrm{~F}$ NMR (376 MHz, $\left.300 \mathrm{~K}, \mathrm{CDCl}_{3}\right)$ : $\delta$-66.02.

IR (thin film): $\widetilde{v}$ 3073, 1683, 1626, 1590, 1477, 1443, 1422, 1392, 1354, 1327, 1301, $1277,1241,1214,1138,1017,990,946,910,863,824,784,757,738,700,649,624 \mathrm{~cm}^{-1}$. HRMS (ESI): $m / z$ calculated for $\mathrm{C}_{23} \mathrm{H}_{18} \mathrm{O}_{2} \mathrm{~N}_{2} \mathrm{~F}_{3}[\mathrm{M}+\mathrm{H}]^{+}: 411.1315$, found: 411.1312 . $[\alpha]_{\mathrm{D}}^{23}-125.8$ (c 1.77, $\mathrm{CHCl}_{3}, 92 \%$ ee sample; major diastereomer).

Enantiomeric excess was determined to be $92 \%$ ee (major diastereomer) by chiral stationary phase HPLC analysis (CHIRALPAK IC3 $(\phi 0.46 \mathrm{~cm} \times 25 \mathrm{~cm}), n$-hexane/ $\mathrm{PrOH}=4 / 1$, flow rate $1.0 \mathrm{~mL} / \mathrm{min}$, detection at 254 $\mathrm{nm}, \mathrm{t}_{\mathrm{R}}=35.9 \mathrm{~min}$ (minor) and $41.7 \mathrm{~min}$ (major).
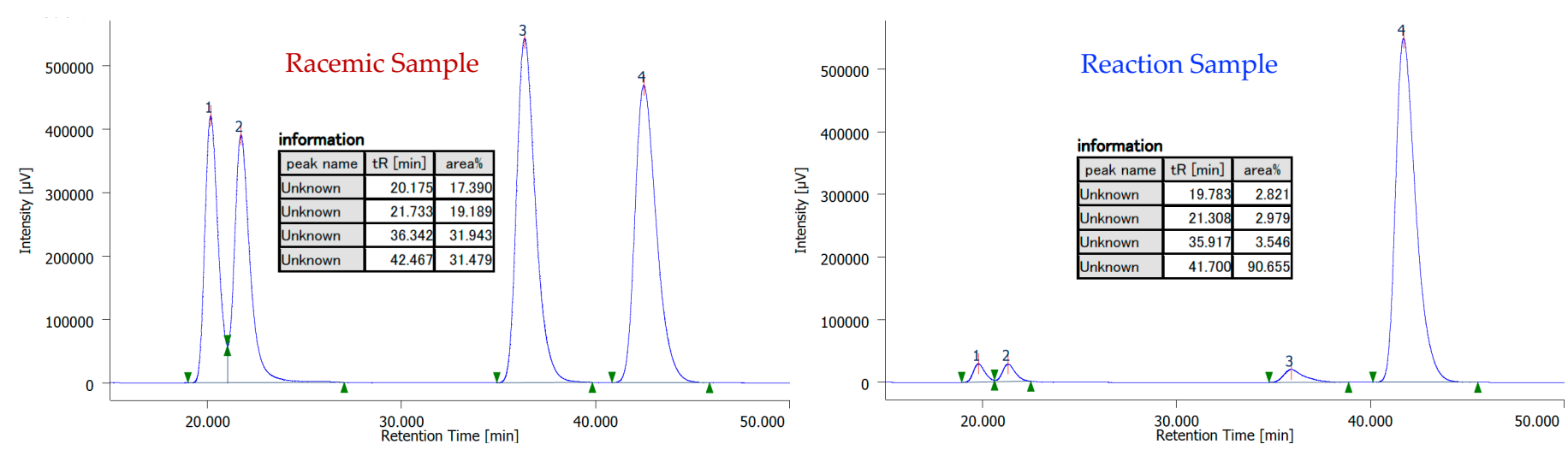
((1R,2S,3R)-2-(benzofuran-2-carbonyl)-3-(trifluoromethyl)cyclopropyl)(2,3-dihydro-1H-pyrrolo[2,3b]pyridin-1-yl)methanone (4gp):

The reaction performed according to the standard procedure 2 afforded $37 \mathrm{mg}(94 \%)$. Colorless sticky solid.

${ }^{1} \mathrm{H}$ NMR (400 MHz, 300 K, $\left.\mathrm{CDCl}_{3}\right): \delta 8.10(\mathrm{ddt}, J=5.0,1.8,0.9 \mathrm{~Hz}, 1 \mathrm{H}), 7.63(\mathrm{dt}, J=$ $7.9,1.1 \mathrm{~Hz}, 1 \mathrm{H}), 7.54(\mathrm{~d}, J=1.0 \mathrm{~Hz}, 1 \mathrm{H}), 7.51-7.41(\mathrm{~m}, 2 \mathrm{H}), 7.38(\mathrm{dq}, J=7.4,1.4 \mathrm{~Hz}$,

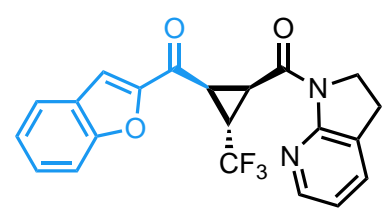

$1 \mathrm{H}), 7.31-7.27(\mathrm{~m}, 1 \mathrm{H}), 6.83(\mathrm{dd}, J=7.4,5.1 \mathrm{~Hz}, 1 \mathrm{H}), 4.21(\mathrm{dd}, J=10.2,6.2 \mathrm{~Hz}, 1 \mathrm{H})$, $4.10-3.93(\mathrm{~m}, 2 \mathrm{H}), 3.40(\mathrm{dd}, J=10.2,6.1 \mathrm{~Hz}, 1 \mathrm{H}), 3.28(\mathrm{~h}, J=6.4 \mathrm{~Hz}, 1 \mathrm{H}), 3.06-2.87$ $(\mathrm{m}, 2 \mathrm{H})$.

${ }^{13} \mathrm{C}$ NMR (101 MHz, 300 K, $\left.\mathrm{CDCl}_{3}\right): \delta$ 183.1, 164.3, 155.9, 155.7, 152.4, 146.2, 133.7, $128.6,127.0,126.2,124.9(\mathrm{q}, J=272.7 \mathrm{~Hz}), 124.1,123.5,118.7,113.9,112.6,46.0,29.1(\mathrm{q}$, $J=2.5 \mathrm{~Hz}), 28.9(\mathrm{q}, J=2.6 \mathrm{~Hz}), 26.5(\mathrm{q}, J=38.1 \mathrm{~Hz}), 24.4$.

${ }^{19}$ F NMR (376 MHz, $\left.300 \mathrm{~K}, \mathrm{CDCl}_{3}\right): \delta-66.30$.

IR (thin film): $\widetilde{v} 3073,3017,2966,2914,1682,1650,1612,1591,1556,1476,1444$, 1423, 1396, 1354, 1336, 1301, 1282, 1258, 1241, 1198, 1139, 1080, 1053, 1021, 995, 939, $887,860,832,786,754,699,666,647,625 \mathrm{~cm}^{-1}$.

HRMS (ESI): $m / z$ calculated for $\mathrm{C}_{21} \mathrm{H}_{16} \mathrm{O}_{3} \mathrm{~N}_{2} \mathrm{~F}_{3}[\mathrm{M}+\mathrm{H}]^{+}: 401.1108$, found: 401.1104 .

$[\alpha]_{\mathrm{D}}^{23}-109.1$ ( $c$ 0.63, $\mathrm{CHCl}_{3}, 97 \%$ ee sample; major diastereomer).

Enantiomeric excess was determined to be $97 \%$ ee (major diastereomer) by chiral stationary phase HPLC analysis (CHIRALPAK IG3 $(\phi 0.46 \mathrm{~cm} \times 25 \mathrm{~cm}), n$-hexane $/ \mathrm{PrOH}=4 / 1$, flow rate $1.0 \mathrm{~mL} / \mathrm{min}$, detection at 254 $\mathrm{nm}, \mathrm{t}_{\mathrm{R}}=39.6 \mathrm{~min}$ (major) and $97.1 \mathrm{~min}$ (minor).
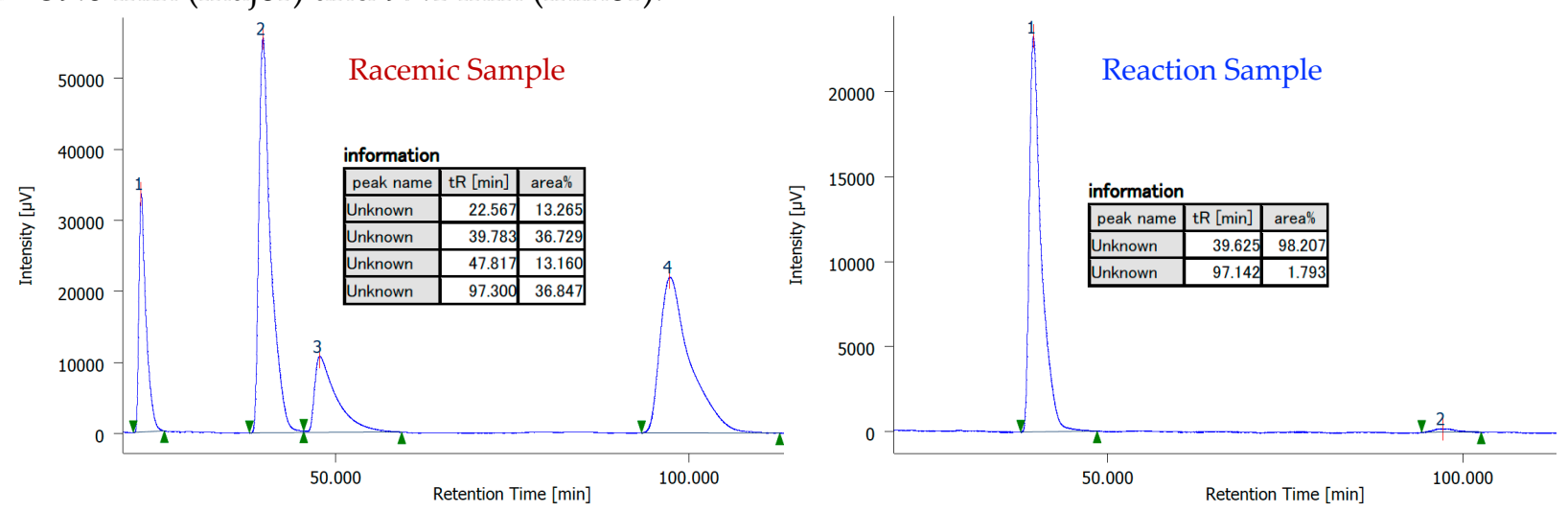

(2,3-dihydro-1H-pyrrolo[2,3-b]pyridin-1-yl)((1R,2S,3R)-2-(thiophene-2-carbonyl)-3(trifluoromethyl)cyclopropyl)methanone (4hp):

The reaction performed according to the standard procedure 2 afforded $33 \mathrm{mg}(91 \%)$.

Colorless solid (m.p $\left.=86-88^{\circ} \mathrm{C}\right)$.

${ }^{1} \mathrm{H}$ NMR (400 MHz, $\left.300 \mathrm{~K}, \mathrm{CDCl}_{3}\right)$ : $\delta 8.11(\mathrm{ddt}, J=5.1,1.8,1.0 \mathrm{~Hz}, 1 \mathrm{H}), 7.78(\mathrm{dd}, J=3.8$, $1.2 \mathrm{~Hz}, 1 \mathrm{H}), 7.58(\mathrm{dd}, J=5.0,1.1 \mathrm{~Hz}, 1 \mathrm{H}), 7.41(\mathrm{dq}, J=7.4,1.4 \mathrm{~Hz}, 1 \mathrm{H}), 7.01(\mathrm{dd}, J=5.0$, $3.8 \mathrm{~Hz}, 1 \mathrm{H}), 6.87(\mathrm{dd}, J=7.4,5.1 \mathrm{~Hz}, 1 \mathrm{H}), 4.18(\mathrm{dd}, J=9.2,7.2 \mathrm{~Hz}, 1 \mathrm{H}), 4.10-3.94(\mathrm{~m}$,

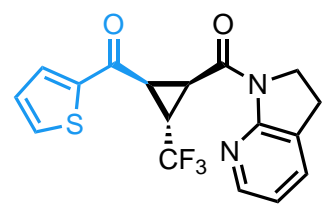
$2 \mathrm{H}), 3.31-3.21(\mathrm{~m}, 2 \mathrm{H}), 3.07-2.90(\mathrm{~m}, 2 \mathrm{H})$.

${ }^{13} \mathrm{C}$ NMR (101 MHz, 300 K, CDCl 3$)$ : $\delta 184.9,164.4,155.7,146.2,143.7,134.3,133.7,133.0$, 128.1, 126.2, $125.0(\mathrm{q}, J=273.71 \mathrm{~Hz}), 118.7,46.1,29.8(\mathrm{q}, J=2.6 \mathrm{~Hz}), 28.5(\mathrm{q}, J=2.7 \mathrm{~Hz})$, $26.2(\mathrm{q}, J=38.1 \mathrm{~Hz}), 24.4$.

${ }^{19} \mathrm{~F}$ NMR (376 MHz, $\left.300 \mathrm{~K}, \mathrm{CDCl}_{3}\right): \delta-66.22$.

IR (thin film): $\widetilde{v} 3075,3018,2965,2913,2855,1669,1590,1518,1477,1444,1423,1391$, $1355,1330,1301,1279,1264,1240,1140,1102,1081,1059,1032,1003,9811,928,856,786$, $729,701,648,616,596,564,542 \mathrm{~cm}^{-1}$.

HRMS (ESI): $m / z$ calculated for $\mathrm{C}_{17} \mathrm{H}_{14} \mathrm{O}_{2} \mathrm{~N}_{2} \mathrm{~F}_{3} \mathrm{~S}$ [M+H] $]^{+}: 367.0723$, found: 367.0720 .

$[\alpha]_{\mathrm{D}}^{23}-210.1$ (c 1.45, $\mathrm{CHCl}_{3}, 96 \%$ ee sample; major diastereomer). 
Enantiomeric excess was determined to be $96 \%$ ee (major diastereomer) by chiral stationary phase HPLC analysis (CHIRALPAK IG3 $(\phi 0.46 \mathrm{~cm} \times 25 \mathrm{~cm}), n$-hexane/ $\mathrm{PrOH}=4 / 1$, flow rate $1.0 \mathrm{~mL} / \mathrm{min}$, detection at 254 $\mathrm{nm}, \mathrm{t}_{\mathrm{R}}=43.1 \mathrm{~min}$ (major) and $65.9 \mathrm{~min}$ (minor).
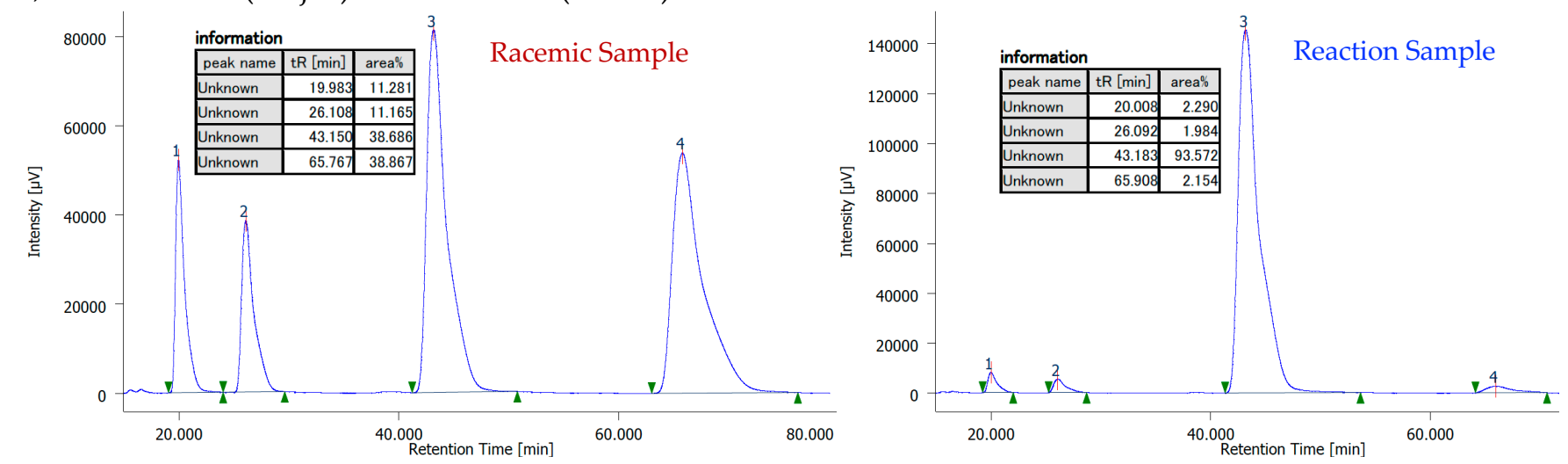

((3S,5S,7S)-adamantan-1-yl)((1S,2R,3R)-2-(2,3-dihydro-1H-pyrrolo[2,3-b]pyridine-1-carbonyl)-3(trifluoromethyl)cyclopropyl)methanone (4ip):

The reaction performed according to the standard procedure 2 afforded $40 \mathrm{mg}$ (96\%).

Colorless sticky solid.

${ }^{1} \mathrm{H}$ NMR (400 MHz, 300 K, $\left.\mathrm{CDCl}_{3}\right)$ : $\delta 8.09$ (dq, $\left.J=5.1,1.2 \mathrm{~Hz}, 1 \mathrm{H}\right), 7.46(\mathrm{dq}, J=7.4,1.4$ $\mathrm{Hz}, 1 \mathrm{H}), 6.88(\mathrm{dd}, J=7.3,5.1 \mathrm{~Hz}, 1 \mathrm{H}), 4.15-4.07(\mathrm{~m}, 2 \mathrm{H}), 3.74(\mathrm{dd}, J=9.6,6.7 \mathrm{~Hz}, 1 \mathrm{H})$, $3.09-3.02(\mathrm{~m}, 2 \mathrm{H}), 3.00-2.89(\mathrm{~m}, 2 \mathrm{H}), 2.00-1.94(\mathrm{~m}, 3 \mathrm{H}), 1.79-1.57(\mathrm{~m}, 12 \mathrm{H})$.

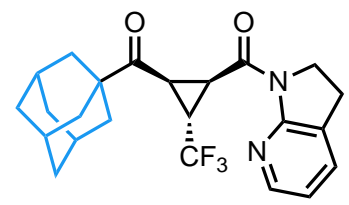

${ }^{13}$ C NMR (101 MHz, 300 K, CDCl CD $_{3}$ : 207.9, 164.7, 155.9, 146.2, 133.6, 126.1, 125.1 (q, J $=272.7 \mathrm{~Hz}), 118.4,46.8,46.0,38.0,36.6,29.4(\mathrm{q}, J=2.8 \mathrm{~Hz}), 27.9,27.3(\mathrm{q}, J=37.9 \mathrm{~Hz}), 26.9$ $(\mathrm{q}, J=2.3 \mathrm{~Hz}), 24.5$.

${ }^{19} \mathrm{~F}$ NMR (376 MHz, $\left.\mathrm{CDCl}_{3}\right)$ : $\delta-66.30$.

IR (thin film): $\widetilde{v} 3074,2907,2851,1702,1653,1590,1478,1445,1422,1387,1350,1314$, $1279,1241,1196,1138,1051,1020,998,973,933,784,754,665,637,596,530 \mathrm{~cm}^{-1}$.

HRMS (ESI): $m / z$ calculated for $\mathrm{C}_{23} \mathrm{H}_{26} \mathrm{O}_{2} \mathrm{~N}_{2} \mathrm{~F}_{3}$ [M+H] $]^{+}: 419.1941$, found: 419.1938 .

$[\alpha]_{\mathrm{D}}^{23}-84.7$ ( $0.44, \mathrm{CHCl}_{3}, 78 \%$ ee sample).

Enantiomeric excess was determined to be $78 \%$ ee by chiral stationary phase HPLC analysis (CHIRALPAK IG3 $(\phi 0.46 \mathrm{~cm} \times 25 \mathrm{~cm}), n$-hexane/PrOH $=4 / 1$, flow rate 1.0 $\mathrm{mL} / \mathrm{min}$, detection at $254 \mathrm{~nm}, \mathrm{t}_{\mathrm{R}}=18.1 \mathrm{~min}$ (major) and $29.8 \mathrm{~min}$ (minor).
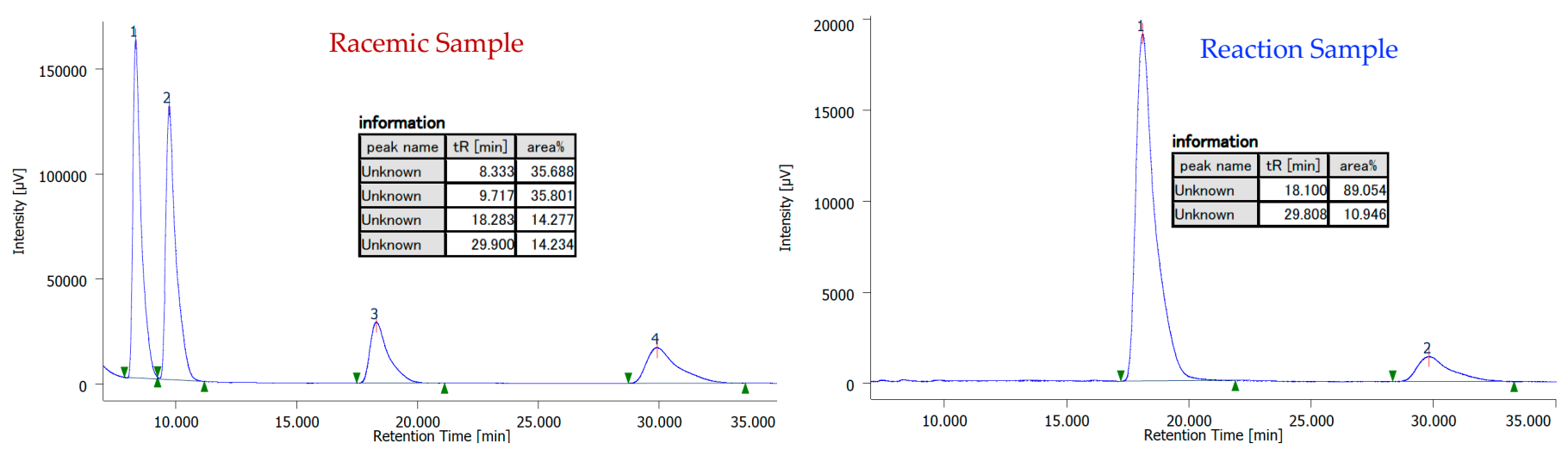
1-((1S,2R,3R)-2-(2,3-dihydro-1H-pyrrolo[2,3-b]pyridine-1-carbonyl)-3-(trifluoromethyl)cyclopropyl)-2,2dimethylpropan-1-one (4jp):

The reaction performed according to the standard procedure 2 afforded $28 \mathrm{mg}(84 \%)$. Colorless solid (m.p $=70-72^{\circ} \mathrm{C}$ ).

${ }^{1} \mathrm{H}$ NMR (400 MHz, $\left.300 \mathrm{~K}, \mathrm{CDCl}_{3}\right)$ : $\delta 8.08$ (ddt, $\left.J=5.1,1.8,1.0 \mathrm{~Hz}, 1 \mathrm{H}\right), 7.45$ (dq, J = 7.4, $1.4 \mathrm{~Hz}, 1 \mathrm{H}), 6.88(\mathrm{dd}, J=7.3,5.1 \mathrm{~Hz}, 1 \mathrm{H}), 4.17-4.05(\mathrm{~m}, 2 \mathrm{H}), 3.76(\mathrm{dd}, J=9.3,7.0 \mathrm{~Hz}, 1 \mathrm{H})$,<smiles>CC(C)(C)C(=O)C1C(C(=O)N2CCc3cccnc32)C1C(C)(F)F</smiles>
$3.10-3.02(\mathrm{~m}, 2 \mathrm{H}), 3.00-2.92(\mathrm{~m}, 2 \mathrm{H}), 1.08(\mathrm{~s}, 9 \mathrm{H})$.

${ }^{13} \mathrm{C}$ NMR (101 MHz, 300 K, $\left.\mathrm{CDCl}_{3}\right)$ : $\delta$ 208.5, 164.5, 155.9, 146.2, 133.6, 126.1, $125.0(\mathrm{q}, J=$ $272.7 \mathrm{~Hz}), 118.5,46.0,44.6,29.6(q, J=2.9 \mathrm{~Hz}), 27.6(q, J=38.4 \mathrm{~Hz}), 27.4(\mathrm{q}, J=3.1 \mathrm{~Hz}), 26.2$, 24.5.

${ }^{19}$ F NMR (376 MHz, 300 K, CDCl $)$ ): $\delta-66.39$.

IR (thin film): $\widetilde{v}$ 3074, 2970, 2873, 1708, 1656, 1590, 1478, 1445, 1423, 1389, 1350, 1333, 1300, 1280, 1241, 1192, 1139, 1080, 1013, 993, 785, 752, 647, 622, 598, $541 \mathrm{~cm}^{-1}$.

HRMS (ESI): $m / z$ calculated for $\mathrm{C}_{17} \mathrm{H}_{20} \mathrm{O}_{2} \mathrm{~N}_{2} \mathrm{~F}_{3}[\mathrm{M}+\mathrm{H}]^{+}: 341.1471$, found: 341.1470 .

$[\alpha]_{\mathrm{D}}^{23}-22.6$ (c $0.19, \mathrm{CHCl}_{3}, 58 \%$ ee sample).

Enantiomeric excess was determined to be $58 \%$ ee by chiral stationary phase HPLC analysis (CHIRALPAK IA $(\phi 0.46 \mathrm{~cm} \times 25 \mathrm{~cm}), n$-hexane $/ \mathrm{PrOH}=9 / 1$, flow rate $1.0 \mathrm{~mL} / \mathrm{min}$, detection at $254 \mathrm{~nm}, \mathrm{tr}=9.1 \mathrm{~min}$ (major) and $13.8 \mathrm{~min}$ (minor).
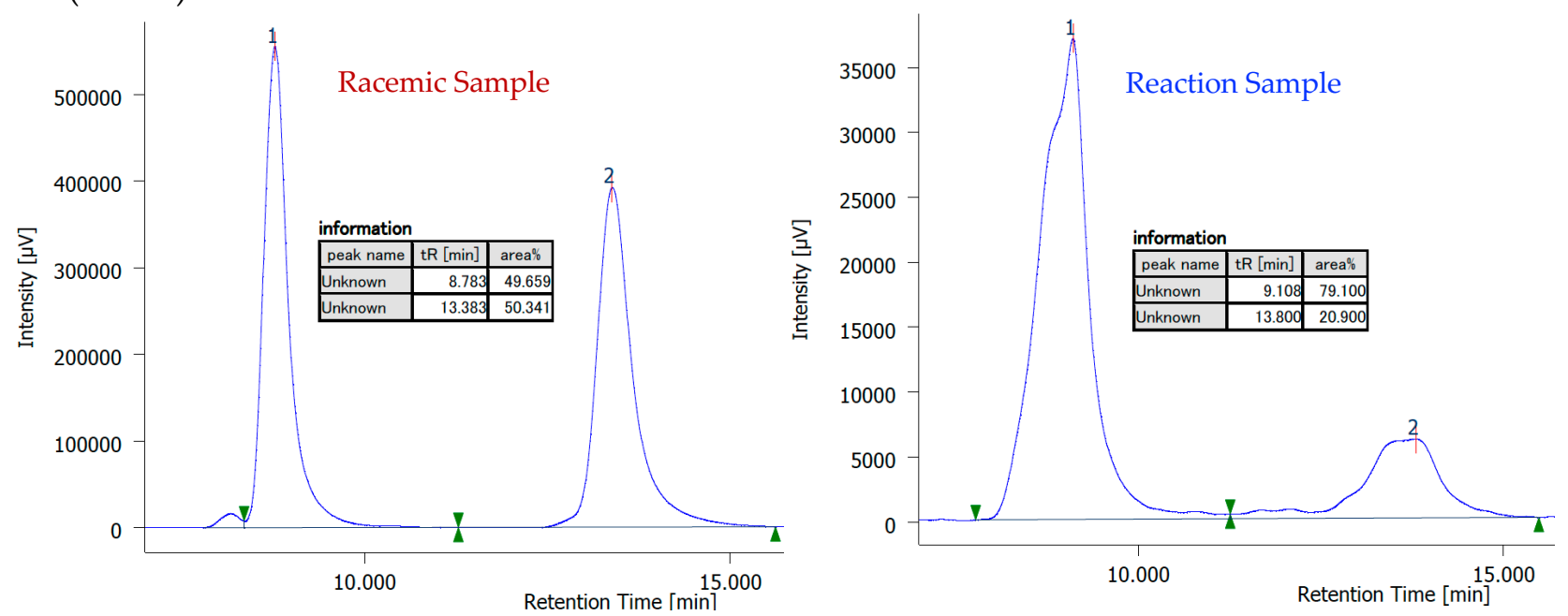
An oven-dried $4 \mathrm{~mL}$ vial was charged with 2 (1.0 equiv., $0.1 \mathrm{mmol})$, [ $\left.\mathrm{Cu}\left(\mathrm{CH}_{3} \mathrm{CN}\right)_{4}\right] \mathrm{PF}_{6}(0.75 \mathrm{mg}, 0.02$ equiv., 2 mol \%), (S)-DTBM-Segphos L4 (2.95 mg, 0.025 equiv., 2.5 mol \%) or (R)-DM-Biphep L15 (1.73 mg, 0.025 equiv., $2.5 \mathrm{~mol} \%)$ in the Glovebox. Then, anhydrous $\mathrm{CH}_{2} \mathrm{Cl}_{2}(1.0 \mathrm{~mL})$ was added to the mixture via syringe with a stainless-steel needle at room temperature under positive Argon pressure. The resulting solution was stirred for $30 \mathrm{~min}$ and the pre-dissolved ylide $1 \mathrm{a}(27 \mathrm{mg}$, 1.5 equiv., $0.15 \mathrm{mmol})$ in anhydrous $\mathrm{CH}_{2} \mathrm{Cl}_{2}(0.5 \mathrm{~mL})$ was slowly added to the reaction mixture. The resulting reaction mixture was then allowed to stir for $24-84 \mathrm{~h}$ at room temperature. After confirming the full conversion by TLC, the crude residue was directly loaded onto a Preparative Thin Layer Chromatography (PTLC) and eluted using Hexane/Ethyl acetate ( 5/1) solvent system $(>2 \mathrm{x})$. If required, the isolated product was repurified by another PTLC with $\mathrm{CH}_{2} \mathrm{Cl}_{2}$. The UV-visible product band was carefully scratched and filtered through a glass frit funnel using $\mathrm{CH}_{2} \mathrm{Cl}_{2}$ as an eluent to afford a pure cyclopropanation product 5/6.

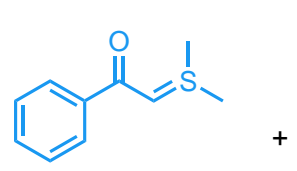

$1 a$

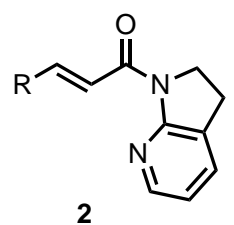

2

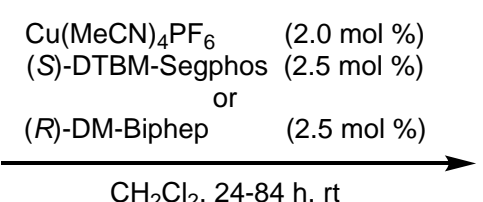

$\mathrm{CH}_{2} \mathrm{Cl}_{2}, 24-84 \mathrm{~h}, \mathrm{rt}$

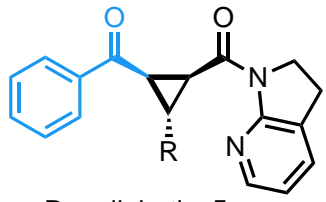

$\mathrm{R}=$ aliphatic: 5

$\mathrm{R}=$ aromatic: $\mathbf{6}$

((1S,2R,3R)-2-benzoyl-3-ethylcyclopropyl)(2,3-dihydro-1H-pyrrolo[2,3-b]pyridin-1-yl)methanone (5aq):

The reaction performed according to the standard procedure 3 afforded $29 \mathrm{mg}(90 \%)$.

Colorless sticky solid.

${ }^{1} \mathrm{H}$ NMR (400 MHz, $\left.300 \mathrm{~K}, \mathrm{CDCl}_{3}\right)$ : $\delta 8.10(\mathrm{ddd}, J=5.1,1.8,0.9 \mathrm{~Hz}, 1 \mathrm{H}), 7.99-7.95(\mathrm{~m}$,

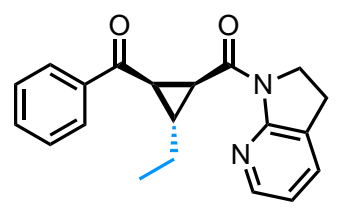
2H), $7.47-7.41(\mathrm{~m}, 1 \mathrm{H}), 7.38(\mathrm{dq}, J=7.3,1.4 \mathrm{~Hz}, 1 \mathrm{H}), 7.35-7.29(\mathrm{~m}, 2 \mathrm{H}), 6.82(\mathrm{dd}, J=$ $7.3,5.1 \mathrm{~Hz}, 1 \mathrm{H}), 4.07-3.90(\mathrm{~m}, 2 \mathrm{H}), 3.72-3.64(\mathrm{~m}, 1 \mathrm{H}), 3.04-2.84(\mathrm{~m}, 2 \mathrm{H}), 2.80(\mathrm{dd}, J$ $=9.4,6.1 \mathrm{~Hz}, 1 \mathrm{H}), 2.45(\mathrm{p}, J=6.6 \mathrm{~Hz}, 1 \mathrm{H}), 1.69-1.59(\mathrm{~m}, 2 \mathrm{H}), 1.08(\mathrm{t}, J=7.4 \mathrm{~Hz}, 3 \mathrm{H})$.

${ }^{13} \mathrm{C}$ NMR (101 MHz, 300 K, $\left.\mathrm{CDCl}_{3}\right): \delta 196.2,168.2,156.4,146.0,137.9,133.3,132.6,128.4$, $128.3,126.4,118.0,46.0,34.2,33.6,29.1,25.7,24.4,13.2$.

IR (thin film): $\widetilde{v} 3061,3010,2960,2926,2871,1681,1649,1599,1588,1479,1446,1420$, $1389,1344,1314,1273,1242,1217,1164,1077,1015,885,862,781,718,693 \mathrm{~cm}^{-1}$.

HRMS (ESI): $m / z$ calculated for $\mathrm{C}_{20} \mathrm{H}_{20} \mathrm{O}_{2} \mathrm{~N}_{2} \mathrm{Na}$ [M+Na] $]^{+}$: 343.1417, found: 343.1422. $[\alpha]_{\mathrm{D}}^{23}-226.0$ ( c 0.21, $\mathrm{CHCl}_{3}, 92 \%$ ee sample).

Enantiomeric excess was determined to be $92 \%$ ee by chiral stationary phase HPLC analysis (CHIRALPAK IC3 $(\phi 0.46 \mathrm{~cm} \times 25 \mathrm{~cm}), n$-hexane $/ \mathrm{PrOH}=4 / 1$, flow rate $1.0 \mathrm{~mL} / \mathrm{min}$, detection at $254 \mathrm{~nm}, \mathrm{t} \mathrm{R}=80.6 \mathrm{~min}$ (minor) and $123.9 \mathrm{~min}$ (major).
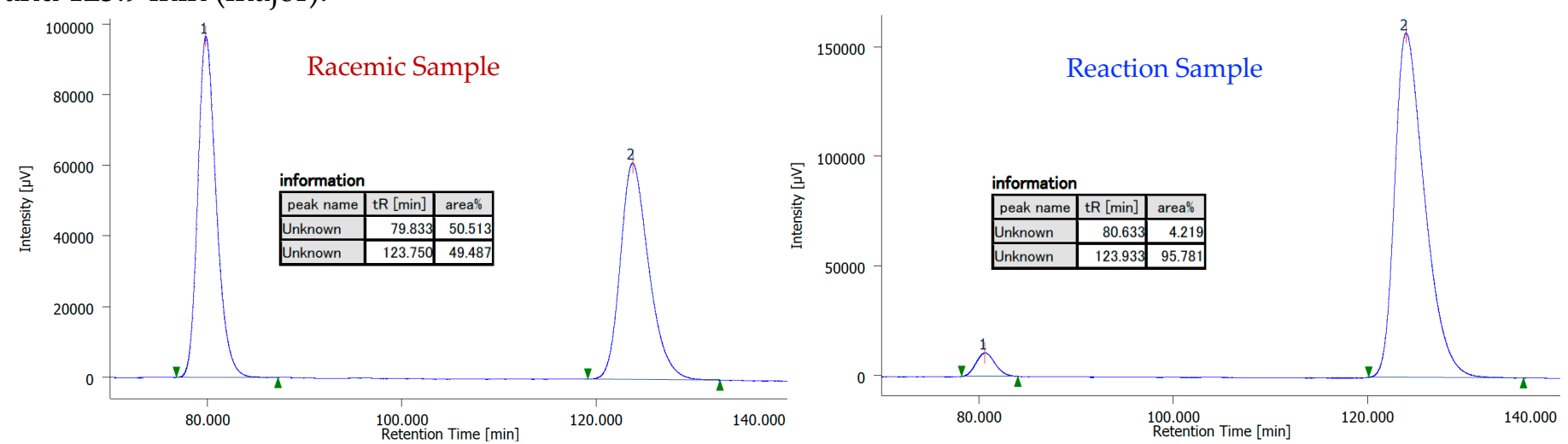
The reaction performed according to the standard procedure 3 afforded $34 \mathrm{mg}$ (94\%).

Colorless sticky solid.

${ }^{1} \mathrm{H}$ NMR (400 MHz, $\left.300 \mathrm{~K}, \mathrm{CDCl}_{3}\right)$ : $\delta 8.10(\mathrm{ddt}, J=5.1,1.8,1.0 \mathrm{~Hz}, 1 \mathrm{H}), 8.00-7.93(\mathrm{~m}$,

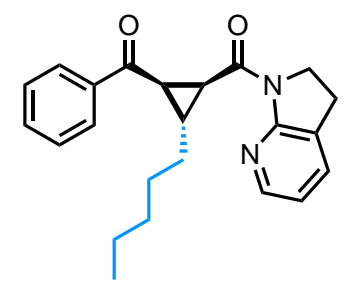
$2 \mathrm{H}), 7.47-7.41(\mathrm{~m}, 1 \mathrm{H}), 7.37(\mathrm{dq}, J=7.3,1.4 \mathrm{~Hz}, 1 \mathrm{H}), 7.35-7.29(\mathrm{~m}, 2 \mathrm{H}), 6.82(\mathrm{dd}, J=$ 7.3, $5.1 \mathrm{~Hz}, 1 \mathrm{H}), 4.08-3.90(\mathrm{~m}, 2 \mathrm{H}), 3.65(\mathrm{dd}, J=9.3,6.3 \mathrm{~Hz}, 1 \mathrm{H}), 3.02-2.84(\mathrm{~m}, 2 \mathrm{H})$, $2.80(\mathrm{dd}, J=9.3,6.1 \mathrm{~Hz}, 1 \mathrm{H}), 2.46(\mathrm{p}, J=6.6 \mathrm{~Hz}, 1 \mathrm{H}), 1.65-1.45(\mathrm{~m}, 4 \mathrm{H}), 1.39-1.25(\mathrm{~m}$, $4 \mathrm{H}), 0.87(\mathrm{t}, J=7.0 \mathrm{~Hz}, 3 \mathrm{H})$.

${ }^{13} \mathrm{C}$ NMR (101 MHz, 300 K, $\left.\mathrm{CDCl}_{3}\right): \delta$ 196.2, 168.2, 156.4, 146.0, 138.0, 133.3, 132.6, 128.4, $128.3,126.4,118.0,46.0,34.5,33.9,32.5,31.6,28.7,27.7,24.4,22.7,14.2$.

IR (thin film): $\widetilde{v} 2955,2925,2855,1682,1650,1600,1588,1479,1446,1420,1388,1322$, $1303,1240,1219,1164,1015,781,717,693 \mathrm{~cm}^{-1}$.

HRMS (ESI): $m / z$ calculated for $\mathrm{C}_{23} \mathrm{H}_{27} \mathrm{O}_{2} \mathrm{~N}_{2}[\mathrm{M}+\mathrm{H}]^{+}: 363.2067$, found: 363.2061 .

$[\alpha]_{\mathrm{D}}^{23}-55.1$ ( $c 0.1, \mathrm{CHCl}_{3}, 90 \%$ ee sample).

Enantiomeric excess was determined to be $90 \%$ ee by chiral stationary phase HPLC analysis (CHIRALPAK IG3 $(\phi 0.46 \mathrm{~cm} \times 25 \mathrm{~cm}), n$-hexane $/ \mathrm{PrOH}=4 / 1$, flow rate $1.0 \mathrm{~mL} / \mathrm{min}$, detection at $254 \mathrm{~nm}, \mathrm{t}=62.2 \mathrm{~min}$ (major) and $101.3 \mathrm{~min}$ (minor).
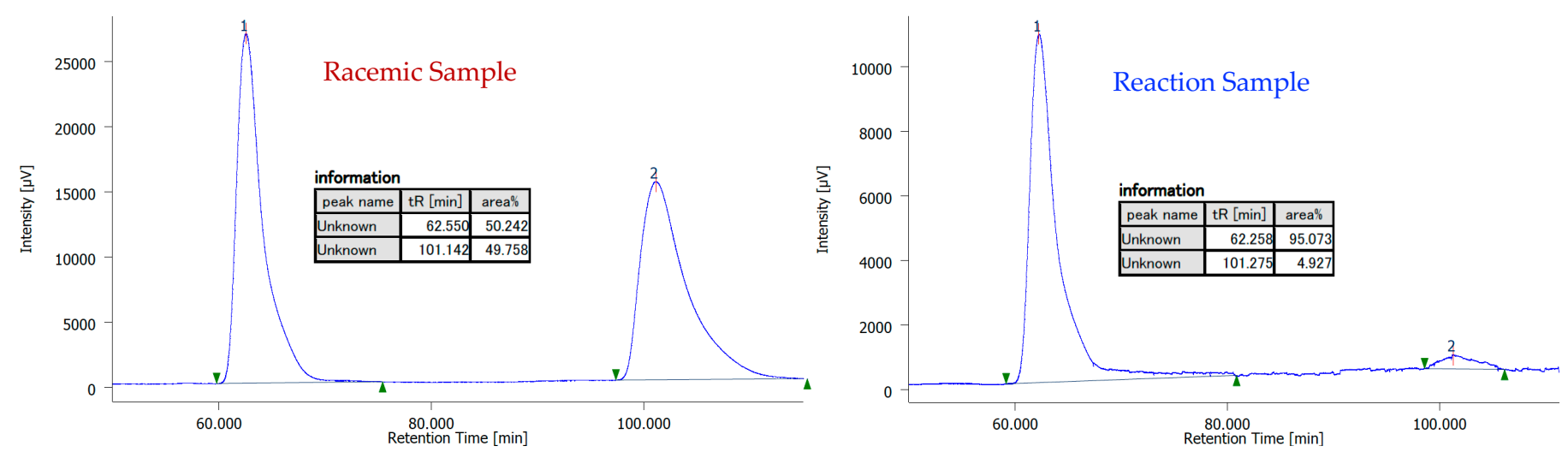

((1S,2R,3R)-2-benzoyl-3-(dec-9-en-1-yl)cyclopropyl)(2,3-dihydro-1H-pyrrolo[2,3-b]pyridin-1yl)methanone (5as):

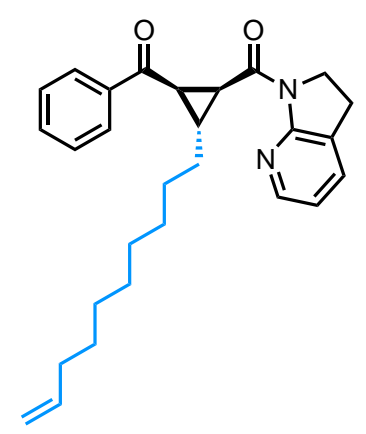

The reaction performed according to the standard procedure 3 afforded $37 \mathrm{mg}(87 \%)$. Colorless sticky solid.

${ }^{1} \mathrm{H}$ NMR (400 MHz, $\left.300 \mathrm{~K}, \mathrm{CDCl}_{3}\right)$ : $\delta 8.10(\mathrm{ddt}, J=5.2,1.8,1.0 \mathrm{~Hz}, 1 \mathrm{H}), 7.99-7.94(\mathrm{~m}$, $2 \mathrm{H}), 7.44(\mathrm{ddt}, J=8.7,6.9,1.3 \mathrm{~Hz}, 1 \mathrm{H}), 7.37(\mathrm{dq}, J=7.3,1.4 \mathrm{~Hz}, 1 \mathrm{H}), 7.35-7.29(\mathrm{~m}, 2 \mathrm{H})$, $6.82(\mathrm{dd}, J=7.3,5.1 \mathrm{~Hz}, 1 \mathrm{H}), 5.80(\mathrm{ddt}, J=17.0,10.2,6.7 \mathrm{~Hz}, 1 \mathrm{H}), 5.02-4.88(\mathrm{~m}, 2 \mathrm{H})$, $4.07-3.89(\mathrm{~m}, 2 \mathrm{H}), 3.65(\mathrm{dd}, J=9.2,6.3 \mathrm{~Hz}, 1 \mathrm{H}), 3.02-2.84(\mathrm{~m}, 2 \mathrm{H}), 2.80(\mathrm{dd}, J=9.3,6.1$ $\mathrm{Hz}, 1 \mathrm{H}), 2.45(\mathrm{p}, J=6.6 \mathrm{~Hz}, 1 \mathrm{H}), 2.07-1.97(\mathrm{~m}, 2 \mathrm{H}), 1.63-1.57(\mathrm{~m}, 2 \mathrm{H}), 1.52-1.43(\mathrm{~m}$, $2 \mathrm{H}), 1.41-1.31(\mathrm{~m}, 4 \mathrm{H}), 1.28-1.20(\mathrm{~m}, 6 \mathrm{H})$.

${ }^{13} \mathrm{C}$ NMR (101 MHz, 300 K, CDCl $)$ ): $\delta$ 196.2, 168.2, 156.4, 146.0, 139.4, 138.0, 133.3, 132.6, 128.4, 128.3, 126.4, 118.0, 114.2, 46.0, 34.5, 33.9, 33.9, 32.6, 29.6, 29.5, 29.4, 29.3, 29.1, 29.0, 27.7, 24.4.

IR (thin film): $\widetilde{v} 3069,2973,2925,2853,1682,1653,1600,1588,1479,1446,1421,1388$, 1322, 1303, 1268, 1241, 1218, 1175, 1164, 1015, 1218, 1175, 1164, 1015, 909, 781, 717, 693 $\mathrm{cm}^{-1}$.

HRMS (ESI): $m / z$ calculated for $\mathrm{C}_{28} \mathrm{H}_{35} \mathrm{O}_{2} \mathrm{~N}_{2}[\mathrm{M}+\mathrm{H}]^{+}: 431.2693$, found: 431.2701 . $[\alpha]_{\mathrm{D}}^{23}-38.3$ ( $c 0.15, \mathrm{CHCl}_{3}, 88 \%$ ee sample).

Enantiomeric excess was determined to be $88 \%$ ee by chiral stationary phase HPLC analysis (CHIRALPAK IC3 $(\phi 0.46 \mathrm{~cm} \times 25 \mathrm{~cm}), n$-hexane/ $\mathrm{PrOH}=4 / 1$, flow rate $1.0 \mathrm{~mL} / \mathrm{min}$, detection at 254 $\mathrm{nm}, \mathrm{t}_{\mathrm{R}}=46.4 \mathrm{~min}$ (minor) and $68.6 \mathrm{~min}$ (major). 

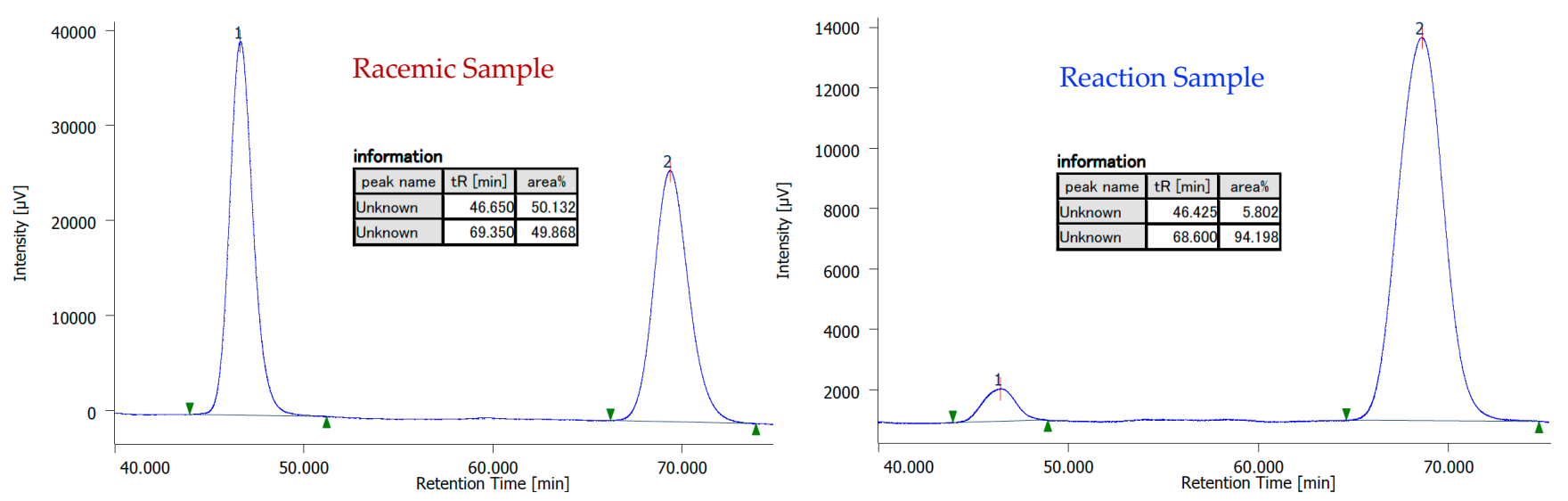

((1S,2R,3R)-2-benzoyl-3-phenethylcyclopropyl)(2,3-dihydro-1H-pyrrolo[2,3-b]pyridin-1-yl)methanone (5at):

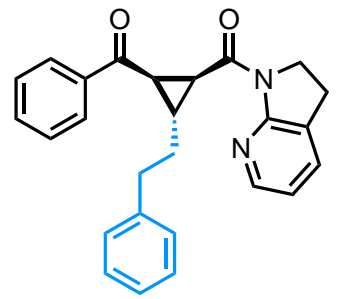

The reaction performed according to the standard procedure 3 afforded $32 \mathrm{mg}(82 \%)$. Colorless sticky solid.

${ }^{1} \mathrm{H}$ NMR $\left(400 \mathrm{MHz}, 300 \mathrm{~K}, \mathrm{CDCl}_{3}\right)$ : $\delta 8.08$ (ddt, J = 5.1, 1.8, $\left.1.0 \mathrm{~Hz}, 1 \mathrm{H}\right), 7.94-7.90(\mathrm{~m}$, $2 \mathrm{H}), 7.47-7.41(\mathrm{~m}, 1 \mathrm{H}), 7.37(\mathrm{dq}, J=7.3,1.4 \mathrm{~Hz}, 1 \mathrm{H}), 7.34-7.28(\mathrm{~m}, 2 \mathrm{H}), 7.24-7.17(\mathrm{~m}$, $4 \mathrm{H}), 7.12-7.07(\mathrm{~m}, 1 \mathrm{H}), 6.82(\mathrm{dd}, J=7.3,5.1 \mathrm{~Hz}, 1 \mathrm{H}), 4.06-3.87(\mathrm{~m}, 2 \mathrm{H}), 3.65(\mathrm{dd}, J=$ 9.4, $6.3 \mathrm{~Hz}, 1 \mathrm{H}), 3.02-2.89(\mathrm{~m}, 2 \mathrm{H}), 2.85-2.80(\mathrm{~m}, 2 \mathrm{H}), 2.76(\mathrm{dd}, J=9.4,6.1 \mathrm{~Hz}, 1 \mathrm{H})$, $2.56-2.47(\mathrm{~m}, 1 \mathrm{H}), 1.99-1.85(\mathrm{~m}, 2 \mathrm{H})$.

${ }^{13} \mathrm{C}$ NMR (101 MHz, 300 K, CDCl $\left.{ }_{3}\right): \delta$ 196.0, 168.0, 156.3, 145.9, 141.9, 137.9, 133.4, 132.6, 128.6, 128.4, 128.4, 128.3, 126.4, 125.9, 118.0, 45.9, 35.5, 34.7, 34.3, 33.7, 27.3, 24.4.

IR (thin film): $\widetilde{v} 3082,3060,3024,2921,2855,1725,1678,1653,1599,1588,1495,1478$, $1448,1421,1390,1351,1324,1304,1268,1240,1220,1175,1078,1050,1038,1014,967,844$, $781,731,719,698 \mathrm{~cm}^{-1}$.

HRMS (ESI): $m / z$ calculated for $\mathrm{C}_{26} \mathrm{H}_{25} \mathrm{O}_{2} \mathrm{~N}_{2}[\mathrm{M}+\mathrm{H}]^{+}: 397.1911$, found: 397.1907. $[\alpha]_{\mathrm{D}}^{23}+105.2$ (c $0.46, \mathrm{CHCl}_{3}, 84 \%$ ee sample).

Enantiomeric excess was determined to be $84 \%$ ee by chiral stationary phase HPLC analysis (CHIRALPAK IA $(\phi 0.46 \mathrm{~cm} \times 25 \mathrm{~cm}), n$-hexane $/ \mathrm{PrOH}=4 / 1$, flow rate $1.0 \mathrm{~mL} / \mathrm{min}$, detection at $254 \mathrm{~nm}, \mathrm{t}_{\mathrm{R}}=20.2 \mathrm{~min}$ (major), $23.2 \mathrm{~min}$ (minor).
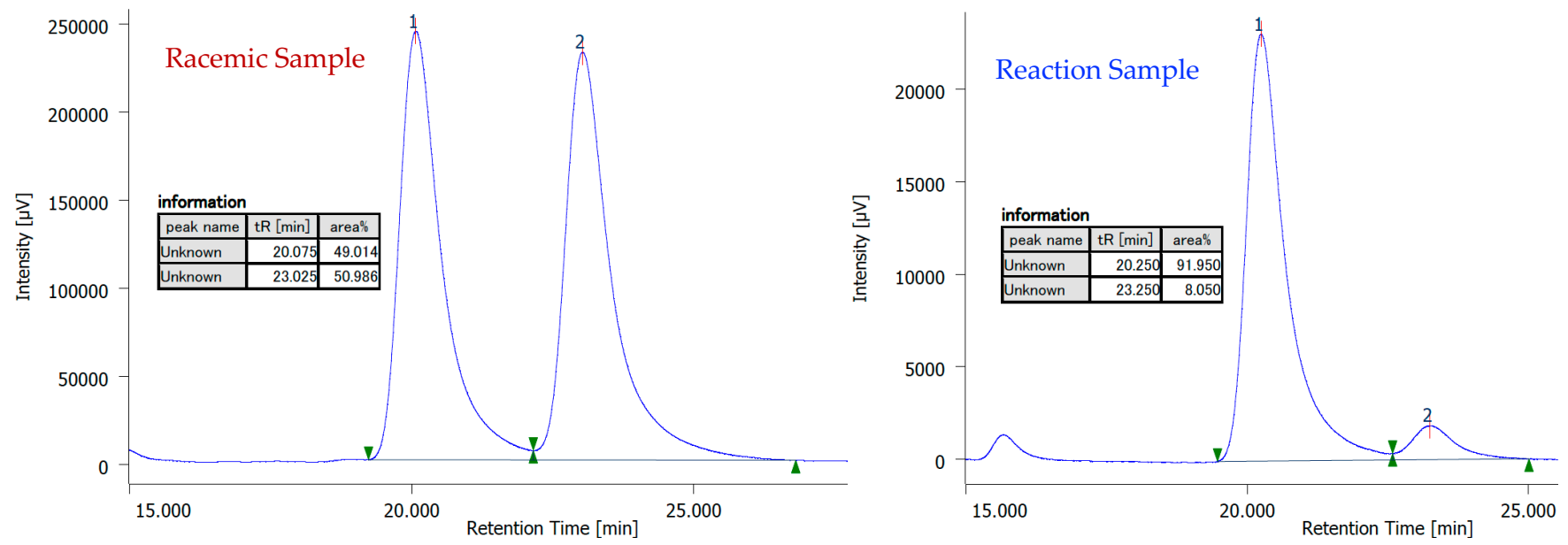
The reaction performed according to the standard procedure 3 afforded $33 \mathrm{mg}(90 \%)$.

Colorless solid (m.p $\left.=135-137^{\circ} \mathrm{C}\right)$.

${ }^{1} \mathrm{H}$ NMR (400 MHz, 300 K, $\left.\mathrm{CDCl}_{3}\right)$ : $\delta 8.08(\mathrm{ddt}, J=5.1,1.8,1.0 \mathrm{~Hz}, 1 \mathrm{H}), 8.02-7.97$ (m,<smiles>O=C(c1ccccc1)C1C(C(=O)N2CCc3cccnc32)[C@H]1c1ccccc1</smiles>
$2 \mathrm{H}), 7.50-7.44(\mathrm{~m}, 1 \mathrm{H}), 7.40(\mathrm{dq}, J=7.3,1.4 \mathrm{~Hz}, 1 \mathrm{H}), 7.37-7.31(\mathrm{~m}, 5 \mathrm{H}), 7.28-7.22(\mathrm{~m}$, $2 \mathrm{H}), 6.83(\mathrm{dd}, J=7.3,5.0 \mathrm{~Hz}, 1 \mathrm{H}), 4.29(\mathrm{dd}, J=9.6,6.4 \mathrm{~Hz}, 1 \mathrm{H}), 4.14-3.97(\mathrm{~m}, 2 \mathrm{H}), 3.62$ $(\mathrm{t}, J=6.3 \mathrm{~Hz}, 1 \mathrm{H}), 3.26(\mathrm{dd}, J=9.5,6.2 \mathrm{~Hz}, 1 \mathrm{H}), 3.08-2.89(\mathrm{~m}, 2 \mathrm{H})$.

${ }^{13} \mathrm{C}$ NMR (101 MHz, 300 K, $\left.\mathrm{CDCl}_{3}\right): \delta$ 195.0, 167.3, 156.2, 146.1, 139.4, 137.6, 133.5, 132.8, $128.6,128.6,128.4,127.1,126.9,126.3,118.2,46.0,36.7,34.6,31.0,24.4$.

IR (thin film): $\widetilde{v} 3059,3028,2959,2916,2850,1680,1650,1599,1589,1478,1459,1440$, $1420,1387,1342,1265,1241,1217,1165,1015,985,784,756,736,696 \mathrm{~cm}^{-1}$.

HRMS (ESI): $m / z$ calculated for $\mathrm{C}_{24} \mathrm{H}_{21} \mathrm{O}_{2} \mathrm{~N}_{2}[\mathrm{M}+\mathrm{H}]^{+}: 369.1598$, found: 369.1590 .

$[\alpha]_{\mathrm{D}}^{23}-46.1$ ( $c 0.03, \mathrm{CHCl}_{3}, 82 \%$ ee sample; major diastereomer).

$[\alpha]_{\mathrm{D}}^{23}+12.6\left(c 0.15, \mathrm{CHCl}_{3}, 76 \%\right.$ ee sample; major diastereomer).

Enantiomeric excess was determined to be $82 \%$ ee (standard procedure 2, using L1) by chiral stationary phase HPLC analysis (CHIRALPAK IG3 $(\phi 0.46 \mathrm{~cm} \times 25 \mathrm{~cm}), n$-hexane $/ \mathrm{PrOH}=7 / 3$, flow rate $1.0 \mathrm{~mL} / \mathrm{min}$, detection at $254 \mathrm{~nm}, \mathrm{t}_{\mathrm{R}}=31.2 \mathrm{~min}$ (major) and $53.4 \mathrm{~min}$ (minor).

Enantiomeric excess was determined to be $76 \%$ ee (standard procedure 2, using L15) by chiral stationary phase HPLC analysis (CHIRALPAK IG3 $(\phi 0.46 \mathrm{~cm} \times 25 \mathrm{~cm}), n$-hexane $/ \mathrm{PrOH}=7 / 3$, flow rate $1.0 \mathrm{~mL} / \mathrm{min}$, detection at $254 \mathrm{~nm}, \mathrm{t}_{\mathrm{R}}=31.4 \mathrm{~min}$ (minor) and $53.1 \mathrm{~min}$ (major).
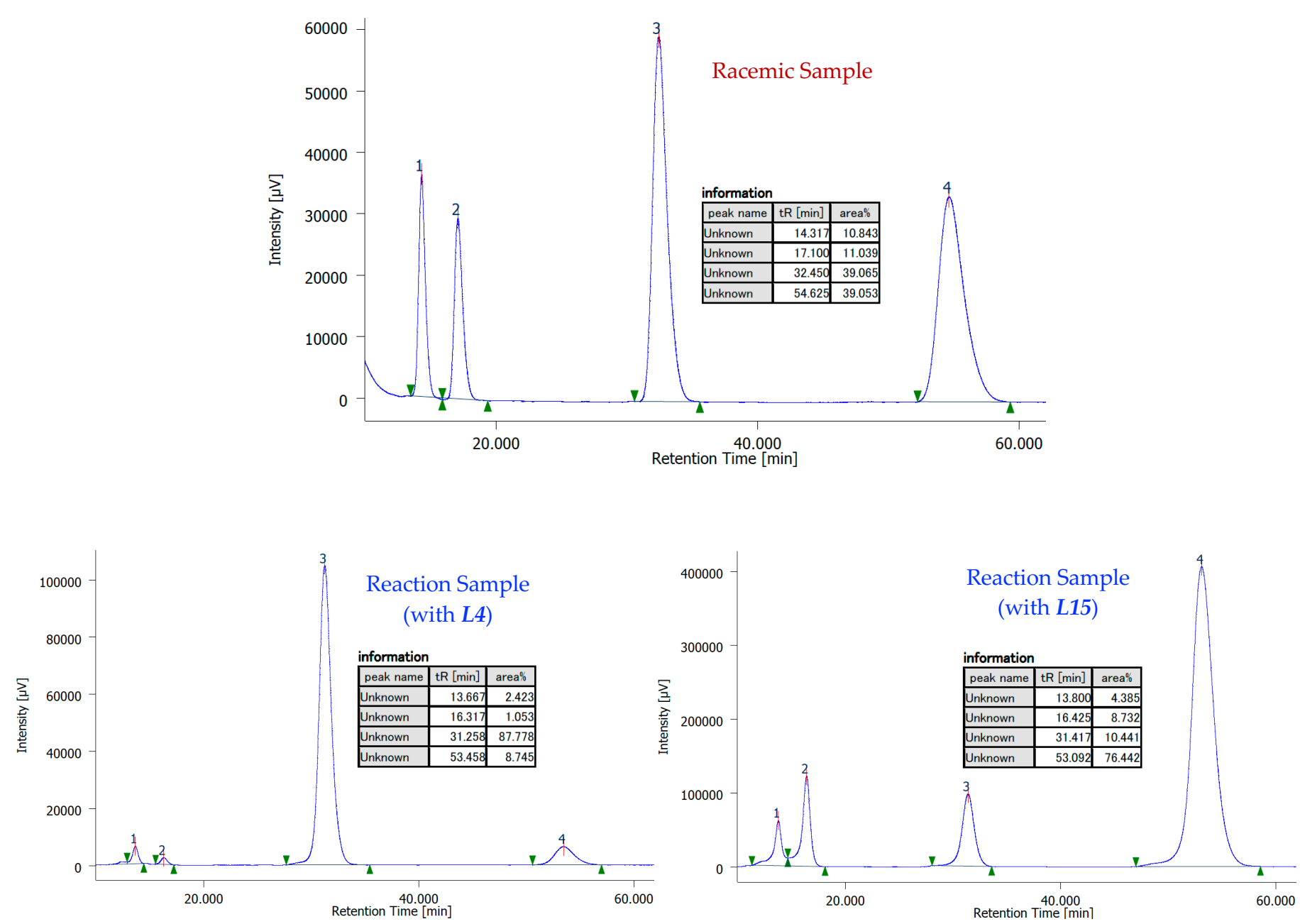
((1S,2R,3R)-2-benzoyl-3-(2-(trifluoromethyl)phenyl)cyclopropyl)(2,3-dihydro-1H-pyrrolo[2,3-b]pyridin-1yl)methanone (6av):

The reaction performed according to the standard procedure 3 afforded $36 \mathrm{mg}$ (83\%).

Colorless solid (m.p $\left.=98-100{ }^{\circ} \mathrm{C}\right)$.

${ }^{1} \mathrm{H}$ NMR (400 MHz, 300 K, $\left.\mathrm{CDCl}_{3}\right)$ : $\delta 8.02(\mathrm{ddt}, J=5.1,1.8,1.0 \mathrm{~Hz}, 1 \mathrm{H}), 7.99$ - $7.94(\mathrm{~m}$, $2 \mathrm{H}), 7.66(\mathrm{ddt}, J=7.9,1.3,0.6 \mathrm{~Hz}, 1 \mathrm{H}), 7.56-7.50(\mathrm{~m}, 1 \mathrm{H}), 7.49-7.42(\mathrm{~m}, 2 \mathrm{H}), 7.40-7.29$

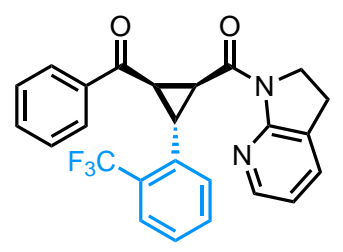

$(\mathrm{m}, 4 \mathrm{H}), 6.80(\mathrm{dd}, J=7.3,5.1 \mathrm{~Hz}, 1 \mathrm{H}), 4.28(\mathrm{dd}, J=9.7,6.7 \mathrm{~Hz}, 1 \mathrm{H}), 4.14-3.98(\mathrm{~m}, 2 \mathrm{H})$, $3.98-3.91(\mathrm{~m}, 1 \mathrm{H}), 3.33(\mathrm{dd}, J=9.7,6.4 \mathrm{~Hz}, 1 \mathrm{H}), 3.07-2.87(\mathrm{~m}, 2 \mathrm{H})$.

${ }^{13} \mathrm{C}$ NMR (101 MHz, $\left.300 \mathrm{~K}, \mathrm{CDCl}_{3}\right): \delta$ 194.5, 166.7, 156.1, 145.9, 137.6, $137.6(\mathrm{q}, J=1.9$ $\mathrm{Hz}), 133.5,132.8,132.0,130.5(\mathrm{q}, J=30.5 \mathrm{~Hz}), 128.6,128.5,128.4,127.1,126.4,126.3(\mathrm{q}, J=$ $5.6 \mathrm{~Hz}), 124.4(\mathrm{q}, J=274.1 \mathrm{~Hz}), 118.2,46.0,35.5,34.2,28.5(\mathrm{q}, J=2.0 \mathrm{~Hz}), 24.4$.

${ }^{19} \mathrm{~F}$ NMR (376 MHz, $\left.300 \mathrm{~K}, \mathrm{CDCl}_{3}\right)$ : $\delta$-60.42.

IR (thin film): $\widetilde{v} 3067,2960,2921,1686,1649,1599,1590,1478,1445,1421,1389,1315$, $1279,1240,1220,1159,1123,1059,1035,1014,842,769,736,718,694,652,597,576 \mathrm{~cm}^{-1}$.

HRMS (ESI): $m / z$ calculated for $\mathrm{C}_{25} \mathrm{H}_{20} \mathrm{O}_{2} \mathrm{~N}_{2} \mathrm{~F}_{3}[\mathrm{M}+\mathrm{H}]^{+}: 437.1471$, found: 437.1472.

$[\alpha]_{\mathrm{D}}^{23}+27.1$ ( $c 1.03, \mathrm{CHCl}_{3}, 76 \%$ ee sample).

Enantiomeric excess was determined to be $76 \%$ ee by chiral stationary phase HPLC analysis (CHIRALPAK IA3 $(\phi 0.46 \mathrm{~cm} \times 25 \mathrm{~cm}), n$-hexane $/ \mathrm{PrOH}=9 / 1$, flow rate $1.0 \mathrm{~mL} / \mathrm{min}$, detection at $254 \mathrm{~nm}, \mathrm{t}_{\mathrm{R}}=44.4 \mathrm{~min}$ (major) and $50.3 \mathrm{~min}$ (minor).
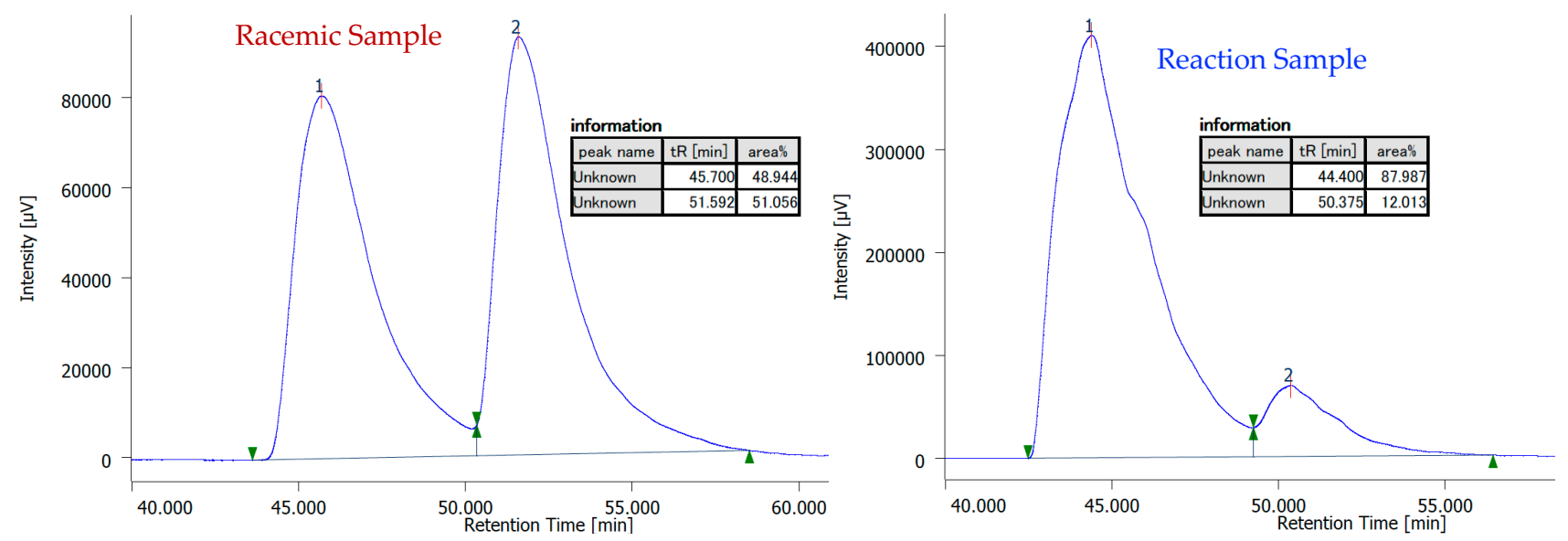

((1S,2R,3R)-2-benzoyl-3-(3-(trifluoromethyl)phenyl)cyclopropyl)(2,3-dihydro-1H-pyrrolo[2,3-b]pyridin-1yl)methanone (6aw):

The reaction performed according to the standard procedure 3 afforded $38 \mathrm{mg}(88 \%)$.

Colorless sticky solid.

${ }^{1} \mathrm{H}$ NMR (400 MHz, 300 K, $\left.\mathrm{CDCl}_{3}\right)$ : $\delta 8.09$ (ddt, $\left.J=5.1,1.8,1.0 \mathrm{~Hz}, 1 \mathrm{H}\right), 8.04-7.99$ (m, $2 \mathrm{H}), 7.64(\mathrm{dq}, J=1.9,1.0 \mathrm{~Hz}, 1 \mathrm{H}), 7.58-7.54(\mathrm{~m}, 1 \mathrm{H}), 7.54-7.40(\mathrm{~m}, 4 \mathrm{H}), 7.39-7.33(\mathrm{~m}$, $2 \mathrm{H}), 6.86(\mathrm{dd}, J=7.4,5.1 \mathrm{~Hz}, 1 \mathrm{H}), 4.37(\mathrm{dd}, J=9.6,6.4 \mathrm{~Hz}, 1 \mathrm{H}), 4.11-4.02(\mathrm{~m}, 1 \mathrm{H}), 4.01-$ $3.92(\mathrm{~m}, 1 \mathrm{H}), 3.67(\mathrm{t}, J=6.3 \mathrm{~Hz}, 1 \mathrm{H}), 3.25(\mathrm{dd}, J=9.7,6.2 \mathrm{~Hz}, 1 \mathrm{H}), 3.07-2.89(\mathrm{~m}, 2 \mathrm{H})$.

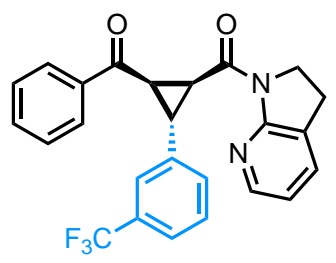

${ }^{13}$ C NMR (101 MHz, 300 K, CDCl $)$ ): $\delta$ 194.5, 166.9, 156.1, 146.1, 140.5, 137.4, 133.6, 133.0, $131.1(\mathrm{q}, J=32.3 \mathrm{~Hz}), 131.0(\mathrm{q}, J=1.5 \mathrm{~Hz}), 129.1,128.6,128.5,128.5(\mathrm{q}, J=11.1 \mathrm{~Hz}), 124.3$ $(q, J=272.7 \mathrm{~Hz}), 124.0(q, J=3.9 \mathrm{~Hz}), 123.7(q, J=3.8 \mathrm{~Hz}), 118.4,46.0,36.4,34.4,30.2,24.4$. ${ }^{19}$ F NMR (376 MHz, $\left.300 \mathrm{~K}, \mathrm{CDCl}_{3}\right)$ : $\delta-62.56$.

IR (thin film): $\widetilde{v} 3058,2958,2924,1681,1651,1599,1590,1478,1444,1422,1388,1332$, $1241,1218,1164,1123,1073,1014,799,783,720,699 \mathrm{~cm}^{-1}$.

HRMS (ESI): $m / z$ calculated for $\mathrm{C}_{25} \mathrm{H}_{19} \mathrm{O}_{2} \mathrm{~N}_{2} \mathrm{~F}_{3} \mathrm{Na}$ [M+Na] $]^{+}: 459.1291$, found: 459.1289 . $[\alpha]_{\mathrm{D}}^{23}-99.2$ ( $0.01, \mathrm{CHCl}_{3}, 60 \%$ ee sample). 
Enantiomeric excess was determined to be $60 \%$ ee by chiral stationary phase HPLC analysis (CHIRALPAK IF $(\phi 0.46 \mathrm{~cm} \times 25 \mathrm{~cm}), n$-hexane $/ \mathrm{PrOH}=4 / 1$, flow rate $1.0 \mathrm{~mL} / \mathrm{min}$, detection at $254 \mathrm{~nm}, \mathrm{t}_{\mathrm{R}}=34.4$ min (major) and $37.3 \mathrm{~min}$ (minor).
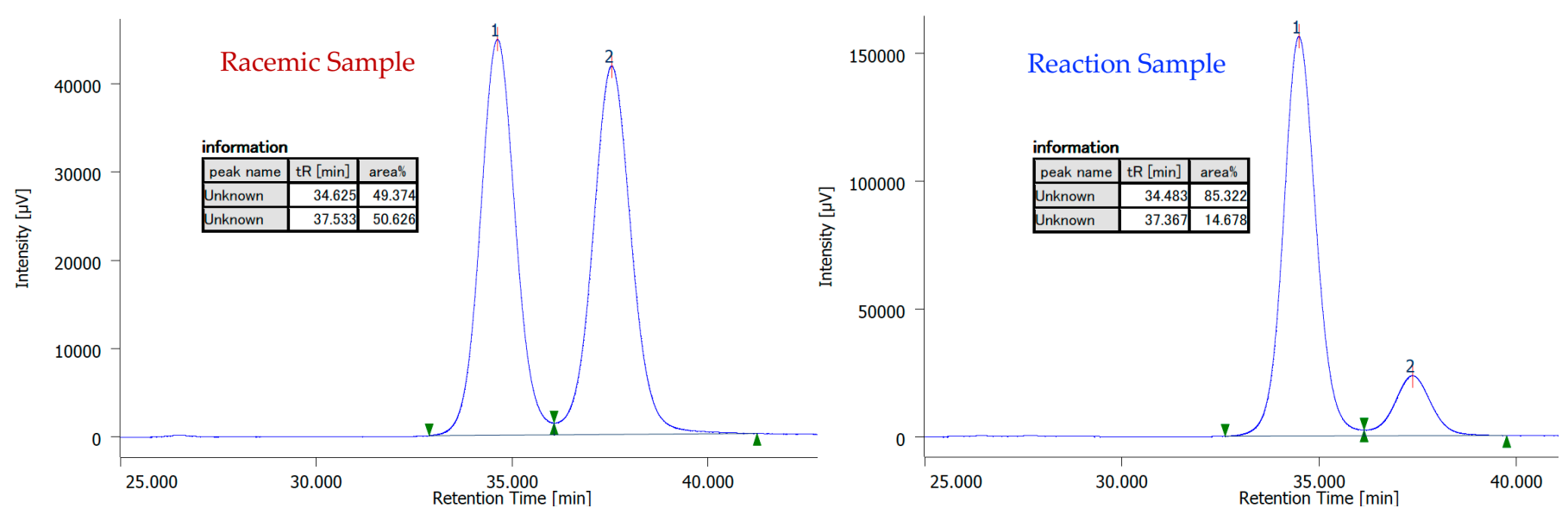

((1S,2R,3R)-2-benzoyl-3-(4-(trifluoromethyl)phenyl)cyclopropyl)(2,3-dihydro-1H-pyrrolo[2,3-b]pyridin-1yl)methanone (6ax):

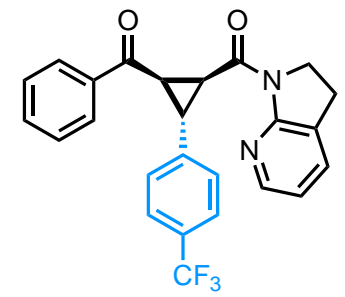

The reaction performed according to the standard procedure 3 afforded $35 \mathrm{mg}(80 \%)$.

Colorless solid (m.p $\left.=118-120^{\circ} \mathrm{C}\right)$.

${ }^{1} \mathrm{H}$ NMR (400 MHz, $\left.300 \mathrm{~K}, \mathrm{CDCl}_{3}\right): \delta 8.07$ (ddt, J = 5.1, 1.8, 1.0 Hz, 1H), $8.02-7.97$ (m, $2 \mathrm{H}), 7.59(\mathrm{~d}, J=8.1 \mathrm{~Hz}, 2 \mathrm{H}), 7.50-7.44(\mathrm{~m}, 3 \mathrm{H}), 7.42(\mathrm{dq}, J=7.4,1.4 \mathrm{~Hz}, 1 \mathrm{H}), 7.35(\mathrm{ddt}, J$ $=7.9,6.8,1.2 \mathrm{~Hz}, 2 \mathrm{H}), 6.86(\mathrm{dd}, J=7.4,5.1 \mathrm{~Hz}, 1 \mathrm{H}), 4.36(\mathrm{dd}, J=9.7,6.4 \mathrm{~Hz}, 1 \mathrm{H}), 4.12-$ $3.94(\mathrm{~m}, 2 \mathrm{H}), 3.66(\mathrm{t}, J=6.3 \mathrm{~Hz}, 1 \mathrm{H}), 3.27(\mathrm{dd}, J=9.7,6.2 \mathrm{~Hz}, 1 \mathrm{H}), 3.05-2.92(\mathrm{~m}, 2 \mathrm{H})$.

${ }^{13} \mathrm{C}$ NMR (101 MHz, 300 K, $\left.\mathrm{CDCl}_{3}\right)$ : $\delta$ 194.4, 166.8, 156.1, 146.1, 143.6, 137.4, 133.6, 133.1, $129.8,128.6,128.5,128.4,127.5,126.4,(q, J=272.7 \mathrm{~Hz}), 125.6$ (q, $J=3.8 \mathrm{~Hz}), 118.4,46.1$, $36.7,34.7,30.3,24.4$.

${ }^{19}$ F NMR (376 MHz, $\left.\mathrm{CDCl}_{3}\right): \delta-62.40$.

IR (thin film): $\widetilde{v} 3066,2962,2918,2854,1683,1649,1618,1599,1590,1479,1446,1421$, $1386,1325,1288,1267,1241,1218,1164,1114,1068,1050,1016,985,954,828,783,733,715$, $692,639,617,604,592 \mathrm{~cm}^{-1}$.

HRMS (ESI): $m / z$ calculated for $\mathrm{C}_{25} \mathrm{H}_{20} \mathrm{O}_{2} \mathrm{~N}_{2} \mathrm{~F}_{3}[\mathrm{M}+\mathrm{H}]^{+}: 437.1471$, found: 437.1474 .

$[\alpha]_{\mathrm{D}}^{23}-16.2$ (c $0.11, \mathrm{CHCl}_{3}, 84 \%$ ee sample).

Enantiomeric excess was determined to be $84 \%$ ee by chiral stationary phase HPLC analysis (CHIRALPAK IC3 $(\phi 0.46 \mathrm{~cm} \times 25 \mathrm{~cm}), n$-hexane $/ \mathrm{PrOH}=4 / 1$, flow rate $1.0 \mathrm{~mL} / \mathrm{min}$, detection at $254 \mathrm{~nm}, \mathrm{t}_{\mathrm{R}}=59.1 \mathrm{~min}$ (minor) and $89.4 \mathrm{~min}$ (major).
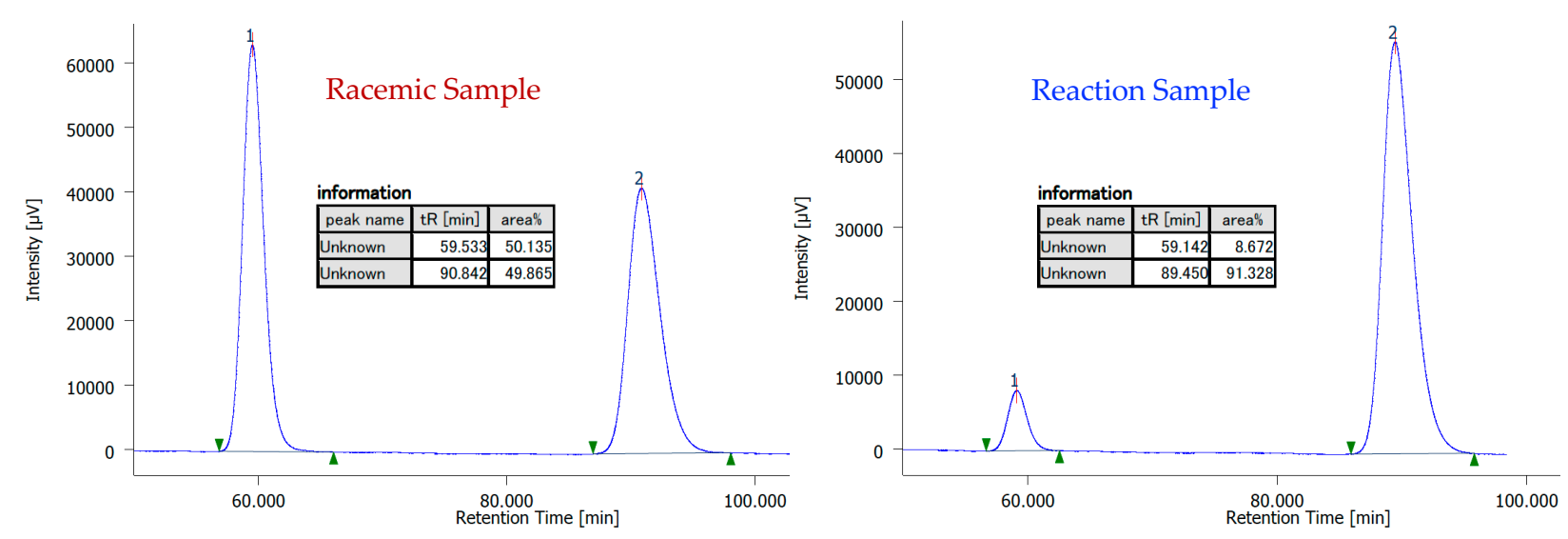
((1S,2R,3R)-2-benzoyl-3-(2-methoxyphenyl)cyclopropyl)(2,3-dihydro-1H-pyrrolo[2,3-b]pyridin-1yl)methanone (6ay):

The reaction performed according to the standard procedure 3 afforded $35 \mathrm{mg}(88 \%)$.

Colorless solid (m.p $=153-155^{\circ} \mathrm{C}$ ).

${ }^{1} \mathrm{H}$ NMR (400 MHz, $\left.300 \mathrm{~K}, \mathrm{CDCl}_{3}\right)$ : $\delta 8.04(\mathrm{ddd}, J=5.1,1.8,0.9 \mathrm{~Hz}, 1 \mathrm{H}), 8.01-7.97$ (m, $2 \mathrm{H}), 7.49-7.43(\mathrm{~m}, 1 \mathrm{H}), 7.38(\mathrm{dq}, J=7.3,1.4 \mathrm{~Hz}, 1 \mathrm{H}), 7.36-7.31(\mathrm{~m}, 2 \mathrm{H}), 7.26-7.20(\mathrm{~m}$,

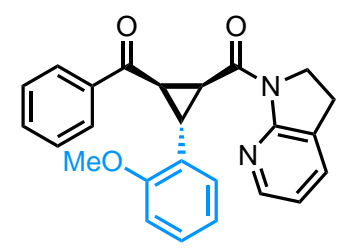

2H), $6.94(\mathrm{td}, J=7.4,1.1 \mathrm{~Hz}, 1 \mathrm{H}), 6.87(\mathrm{dd}, J=8.6,1.1 \mathrm{~Hz}, 1 \mathrm{H}), 6.80(\mathrm{dd}, J=7.3,5.1 \mathrm{~Hz}$, $1 \mathrm{H}), 4.24(\mathrm{dd}, J=9.4,6.7 \mathrm{~Hz}, 1 \mathrm{H}), 4.15-4.01(\mathrm{~m}, 2 \mathrm{H}), 3.79(\mathrm{~s}, 3 \mathrm{H}), 3.75(\mathrm{t}, J=6.6 \mathrm{~Hz}, 1 \mathrm{H})$, $3.24(\mathrm{dd}, J=9.4,6.4 \mathrm{~Hz}, 1 \mathrm{H}), 3.06-2.90(\mathrm{~m}, 2 \mathrm{H})$.

${ }^{13} \mathrm{C}$ NMR (101 MHz, 300 K, $\left.\mathrm{CDCl}_{3}\right): \delta$ 195.7, 167.8, 158.7, 156.4, 146.0, 137.9, 133.3, 132.6, $128.5,128.3,128.0,127.8,127.4,126.3,120.5,118.0,110.6,55.6,46.0,35.6,33.6,27.2,24.5$.

IR (thin film): $\widetilde{v} 3060,3004,2958,2928,2835,1680,1651,1599,1589,1498,1478,1463$, 1444, 1420, 1386, 1329, 1245, 1217, 1176, 1163, 1113, 1049, 1024, 1015, 875, 784, 754, 731, $718,694,590 \mathrm{~cm}^{-1}$.

HRMS (ESI): $m / z$ calculated for $\mathrm{C}_{25} \mathrm{H}_{22} \mathrm{O}_{3} \mathrm{~N}_{2} \mathrm{Na}$ [M+Na] $]^{+}$421.1523, found: 421.1520 . $[\alpha]_{\mathrm{D}}^{23}+15.8\left(c 0.67, \mathrm{CHCl}_{3}, 82 \%\right.$ ee sample).

Enantiomeric excess was determined to be $82 \%$ ee by chiral stationary phase HPLC analysis (CHIRALPAK IF $(\phi 0.46 \mathrm{~cm} \times 25 \mathrm{~cm}), n$-hexane $/ \mathrm{PrOH}=4 / 1$, flow rate $1.0 \mathrm{~mL} / \mathrm{min}$, detection at $254 \mathrm{~nm}, \mathrm{t}_{\mathrm{R}}=54.5 \mathrm{~min}($ minor) and $58.7 \mathrm{~min}$ (major).
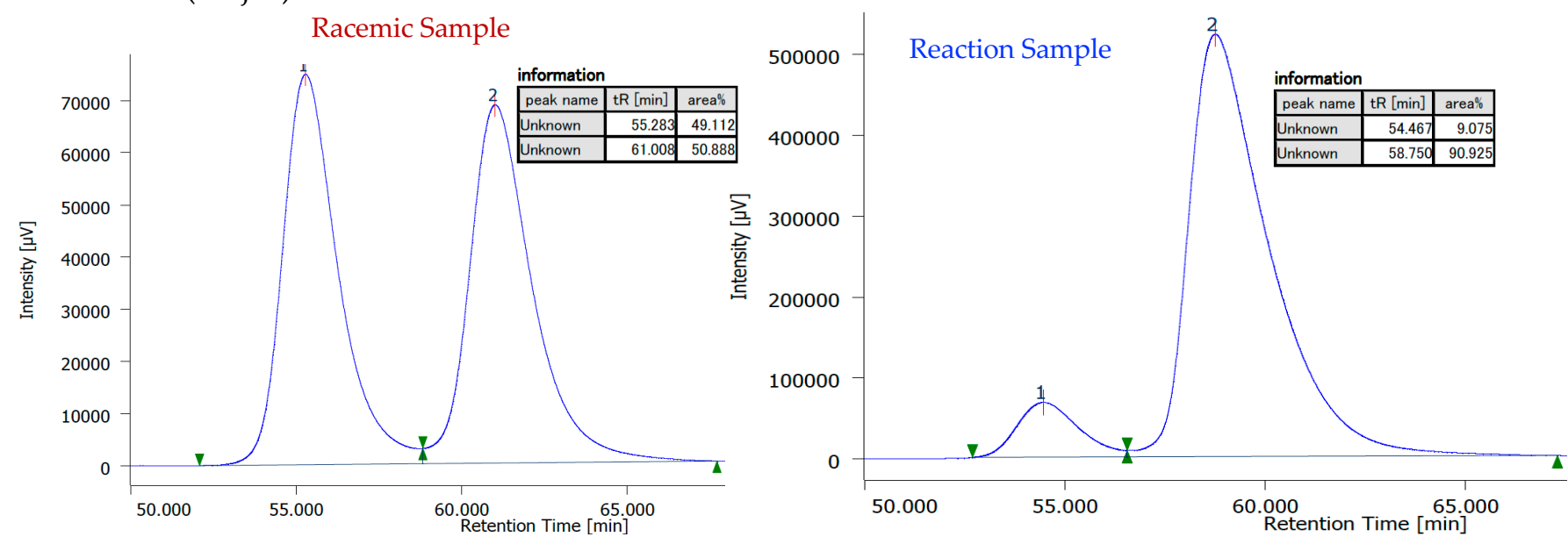

((1S,2R,3R)-2-benzoyl-3-(3-methoxyphenyl)cyclopropyl)(2,3-dihydro-1H-pyrrolo[2,3-b]pyridin-1yl)methanone (6az):

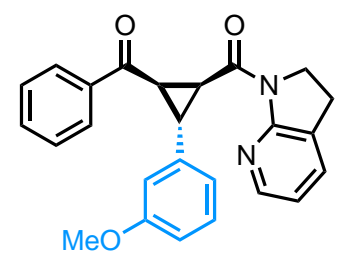

The reaction performed according to the standard procedure 3 afforded $32 \mathrm{mg}(80 \%)$.

Colorless sticky solid.

${ }^{1} \mathrm{H}$ NMR (400 MHz, $\left.300 \mathrm{~K}, \mathrm{CDCl}_{3}\right)$ : $\delta 8.09(\mathrm{dd}, J=5.1,1.6 \mathrm{~Hz}, 1 \mathrm{H}), 8.02-7.97(\mathrm{~m}, 2 \mathrm{H})$, $7.54-7.43(\mathrm{~m}, 1 \mathrm{H}), 7.40(\mathrm{dq}, J=7.4,1.4 \mathrm{~Hz}, 1 \mathrm{H}), 7.37-7.31(\mathrm{~m}, 2 \mathrm{H}), 7.28-7.22(\mathrm{~m}, 1 \mathrm{H})$, $6.97-6.76(\mathrm{~m}, 4 \mathrm{H}), 4.29(\mathrm{dd}, J=9.7,6.4 \mathrm{~Hz}, 1 \mathrm{H}), 4.12-3.96(\mathrm{~m}, 2 \mathrm{H}), 3.82(\mathrm{~s}, 3 \mathrm{H}), 3.60(\mathrm{t}$, $J=6.3 \mathrm{~Hz}, 1 \mathrm{H}), 3.25(\mathrm{dd}, J=9.6,6.2 \mathrm{~Hz}, 1 \mathrm{H}), 3.07-2.88(\mathrm{~m}, 2 \mathrm{H})$.

${ }^{13}$ C NMR (101 MHz, 300 K, CDCl $\left._{3}\right): \delta$ 195.0, 167.3, 159.9, 156.2, 146.1, 141.1, 137.6, 133.5, 132.9, 129.7, 128.6, 128.4, 126.3, 119.4, 118.2, 113.1, 112.3, 55.4, 46.0, 36.7, 34.6, 31.0, 24.4 . IR (thin film): $\widetilde{v} 3057,2958,2936,2911,2834,1681,1648,1600,1589,1493,1477,1442$, 1420, 1386, 1343, 1294, 1267, 1242, 1217, 1176, 1161, 1153, 1044, 1015, 988, 849, 782, 731, $720,693,589,539 \mathrm{~cm}^{-1}$.

HRMS (ESI): $m / z$ calculated for $\mathrm{C}_{25} \mathrm{H}_{23} \mathrm{O}_{3} \mathrm{~N}_{2}[\mathrm{M}+\mathrm{H}]^{+}: 399.1703$, found: 399.1701 . $[\alpha]_{\mathrm{D}}^{23}-54.1$ ( c 0.01, $\mathrm{CHCl}_{3}, 75 \%$ ee sample). 
Enantiomeric excess was determined to be $75 \%$ ee by chiral stationary phase HPLC analysis (CHIRALPAK IC3 $(\phi 0.46 \mathrm{~cm} \times 25 \mathrm{~cm}), n$-hexane $/ \mathrm{PrOH}=1 / 1$, flow rate $1.0 \mathrm{~mL} / \mathrm{min}$, detection at $254 \mathrm{~nm}, \mathrm{t}=59.9$ min (major) and $64.9 \mathrm{~min}$ (minor).
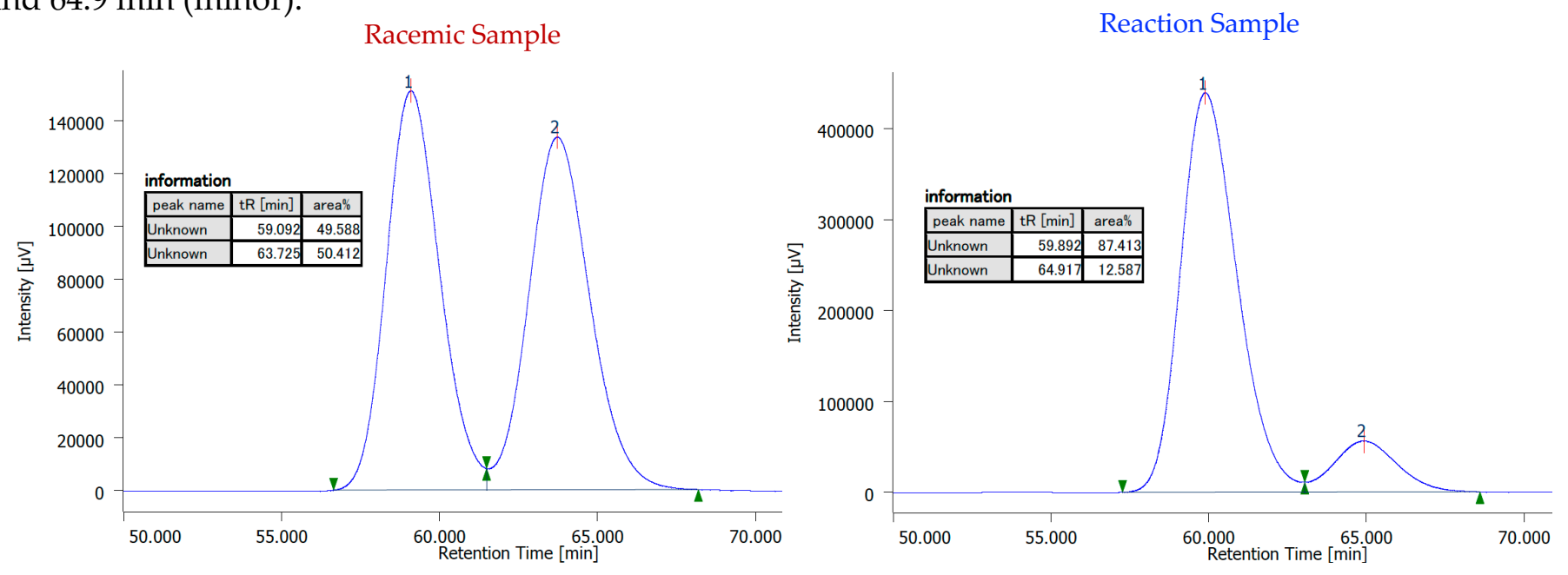

((1S,2R,3R)-2-benzoyl-3-(4-methoxyphenyl)cyclopropyl)(2,3-dihydro-1H-pyrrolo[2,3-b]pyridin-1yl)methanone (6aaa):

The reaction performed according to the standard procedure 3 afforded $31 \mathrm{mg}(77 \%)$.

Colorless sticky solid.

${ }^{1} \mathrm{H}$ NMR (400 MHz, $\left.300 \mathrm{~K}, \mathrm{CDCl}_{3}\right): \delta 8.08(\mathrm{ddt}, J=5.1,1.9,1.0 \mathrm{~Hz}, 1 \mathrm{H}), 8.01-7.97$ (m, $2 \mathrm{H}), 7.46(\mathrm{ddt}, J=8.1,6.9,1.3 \mathrm{~Hz}, 1 \mathrm{H}), 7.39(\mathrm{dq}, J=7.4,1.4 \mathrm{~Hz}, 1 \mathrm{H}), 7.37-7.31(\mathrm{~m}, 2 \mathrm{H})$,<smiles>COc1ccc(C2C(C(=O)c3ccccc3)C2C(=O)N2CCc3cccnc32)cc1</smiles>
$7.30-7.26(\mathrm{~m}, 2 \mathrm{H}), 6.90-6.85(\mathrm{~m}, 2 \mathrm{H}), 6.83(\mathrm{dd}, J=7.3,5.1 \mathrm{~Hz}, 1 \mathrm{H}), 4.23(\mathrm{dd}, J=9.5,6.4$ $\mathrm{Hz}, 1 \mathrm{H}), 4.12-3.97(\mathrm{~m}, 2 \mathrm{H}), 3.81(\mathrm{~s}, 3 \mathrm{H}), 3.57(\mathrm{t}, J=6.3 \mathrm{~Hz}, 1 \mathrm{H}), 3.20(\mathrm{dd}, J=9.5,6.3 \mathrm{~Hz}$, $1 \mathrm{H}), 3.06-2.89(\mathrm{~m}, 2 \mathrm{H})$.

${ }^{13}$ C NMR (101 MHz, 300 K, CDCl $)$ ): $\delta$ 195.2, 167.5, 158.7, 156.3, 146.1, 137.7, 133.4, 132.8, $131.4,128.5,128.4,128.3,126.3,118.2,114.1,55.5,46.0,36.6,34.5,30.6,24.4$.

IR (thin film): $\widetilde{v} 3061,3003,2956,2924,2852,2837,1682,1648,1599,1589,1517,1478$, $1447,1420,1386,1331,1305,1245,1217,1176,1111,1032,1014,984,824,781,768,720,693$, $550,535,525 \mathrm{~cm}^{-1}$.

HRMS (ESI): $m / z$ calculated for $\mathrm{C}_{25} \mathrm{H}_{22} \mathrm{O}_{3} \mathrm{~N}_{2} \mathrm{Na}[\mathrm{M}+\mathrm{Na}]^{+}: 421.1523$, found: 421.1522 . $[\alpha]_{\mathrm{D}}^{23}-8.9\left(c 0.12, \mathrm{CHCl}_{3}, 72 \%\right.$ ee sample).

Enantiomeric excess was determined to be $72 \%$ ee by chiral stationary phase HPLC analysis (CHIRALPAK IC3 $(\phi 0.46 \mathrm{~cm} \times 25 \mathrm{~cm}), n$-hexane/PrOH $=1 / 1$, flow rate $1.0 \mathrm{~mL} / \mathrm{min}$, detection at 254 $\mathrm{nm}, \mathrm{t}_{\mathrm{R}}=70.3 \mathrm{~min}$ (minor) and $81.0 \mathrm{~min}$ (major).
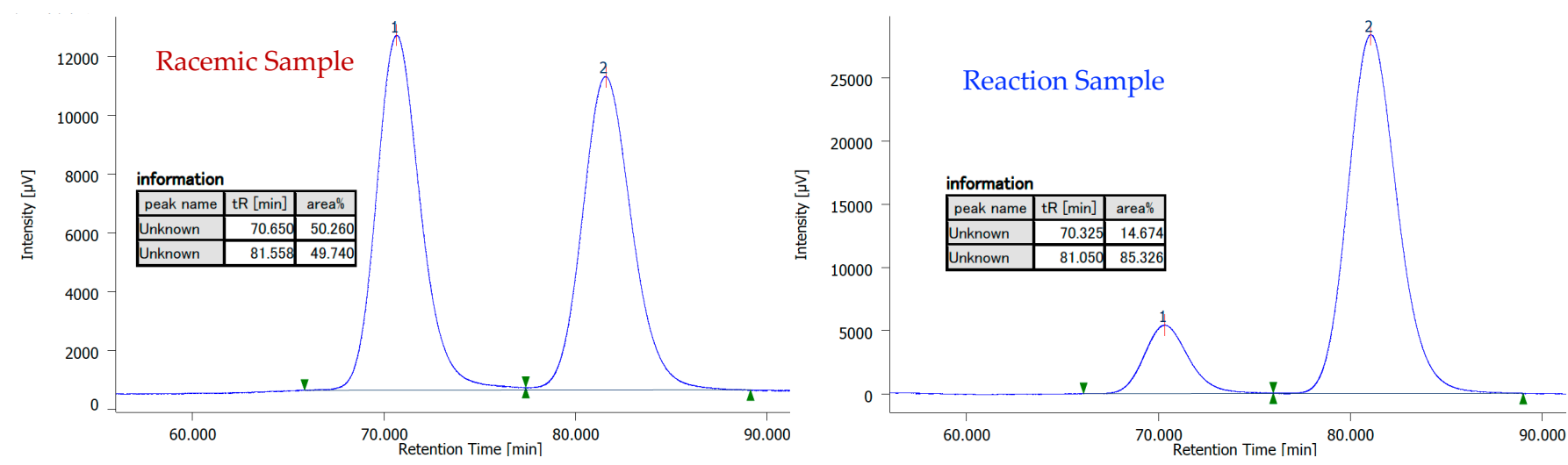

(6aab):

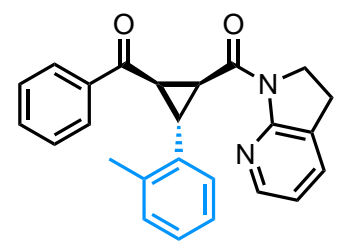

The reaction performed according to the standard procedure 3 afforded $34 \mathrm{mg}$ (89\%). Colorless solid (m.p $=147-148^{\circ} \mathrm{C}$ ).

${ }^{1} \mathrm{H}$ NMR (400 MHz, $\left.300 \mathrm{~K}, \mathrm{CDCl}_{3}\right): \delta 8.08$ (ddt, J = 5.1, 1.8, $\left.1.0 \mathrm{~Hz}, 1 \mathrm{H}\right), 8.03-7.98$ (m, $2 \mathrm{H}), 7.49-7.44(\mathrm{~m}, 1 \mathrm{H}), 7.39(\mathrm{dq}, J=7.3,1.4 \mathrm{~Hz}, 1 \mathrm{H}), 7.37-7.31(\mathrm{~m}, 2 \mathrm{H}), 7.29(\mathrm{dd}, J=6.3$, $3.2 \mathrm{~Hz}, 1 \mathrm{H}), 7.18(\mathrm{~d}, J=2.9 \mathrm{~Hz}, 3 \mathrm{H}), 6.83(\mathrm{dd}, J=7.4,5.1 \mathrm{~Hz}, 1 \mathrm{H}), 4.27(\mathrm{dd}, J=9.5,6.7 \mathrm{~Hz}$, $1 \mathrm{H}), 4.13-3.95(\mathrm{~m}, 2 \mathrm{H}), 3.66(\mathrm{t}, J=6.6 \mathrm{~Hz}, 1 \mathrm{H}), 3.22(\mathrm{dd}, J=9.6,6.5 \mathrm{~Hz}, 1 \mathrm{H}), 3.06-2.88$ $(\mathrm{m}, 2 \mathrm{H}), 2.43(\mathrm{~s}, 3 \mathrm{H})$.

${ }^{13} \mathrm{C}$ NMR (101 MHz, 300 K, $\left.\mathrm{CDCl}_{3}\right): \delta$ 195.3, 167.5, 156.2, 146.0, 138.7, 137.7, 137.4, 133.5, 132.8, 130.1, 128.5, 128.4, 127.1, 126.7, 126.3, 125.9, 118.2, 46.0, 35.2, 33.8, 29.7, 24.4, 19.9.

IR (thin film): $\widetilde{v} 3060,3016,2967,2914,1680,1650,1599,1590,1494,1478,1442,1420$, $1386,1326,1285,1265,1241,1217,1164,1111,1099,1037,1016,986,951,894,872,784$, 756, 734, 717, 694, 641, $591 \mathrm{~cm}^{-1}$.

HRMS (ESI): $m / z$ calculated for $\mathrm{C}_{25} \mathrm{H}_{23} \mathrm{O}_{2} \mathrm{~N}_{2}[\mathrm{M}+\mathrm{H}]^{+}: 383.1754$, found: 383.1753 .

$[\alpha]_{\mathrm{D}}^{23}+103.9$ ( c $0.04, \mathrm{CHCl}_{3}, 82 \%$ ee sample, major diastereomer).

Enantiomeric excess of the major diastereomer was determined to be $82 \%$ ee by chiral stationary phase HPLC analysis (CHIRALPAK IF $(\phi 0.46 \mathrm{~cm} \times 25 \mathrm{~cm}), n$-hexane $/ \mathrm{PrOH}=9 / 1$, flow rate $1.0 \mathrm{~mL} / \mathrm{min}$, detection at 254 $\mathrm{nm}, \mathrm{t}_{\mathrm{R}}=38.5 \mathrm{~min}$ (minor), $59.8 \mathrm{~min}$ (major) and $\mathrm{t}_{\mathrm{R}}=98.6 \mathrm{~min}$ (minor), and $106.1 \mathrm{~min}$ (major).
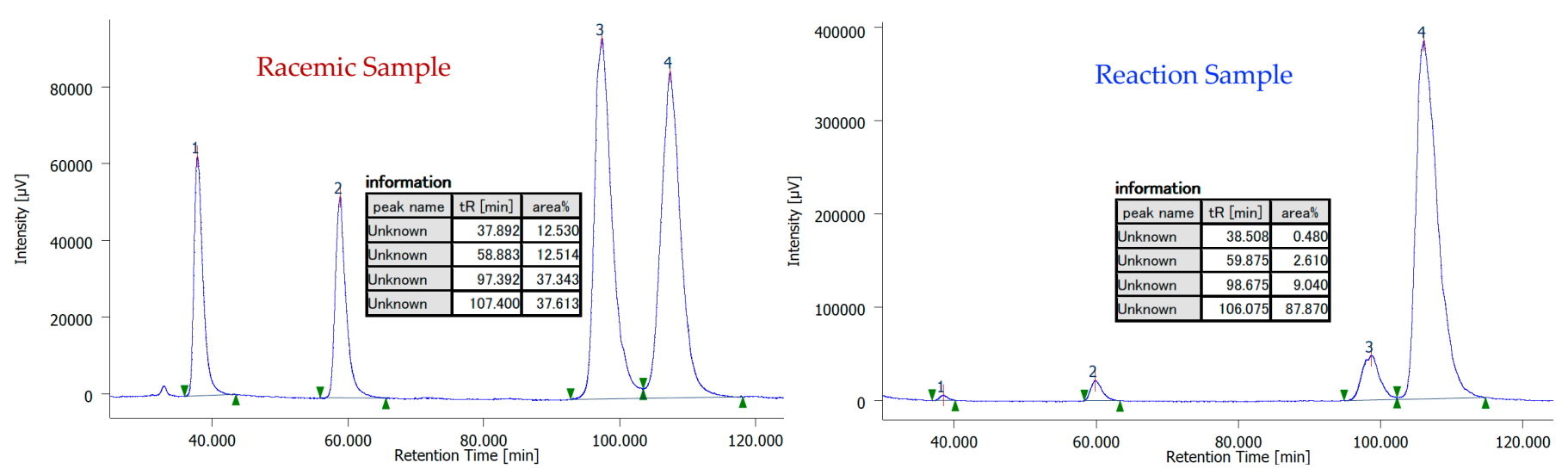

((1S,2R,3R)-2-benzoyl-3-( $p$-tolyl)cyclopropyl)(2,3-dihydro-1H-pyrrolo[2,3-b]pyridin-1-yl)methanone (6aac):

The reaction performed according to the standard procedure 3 afforded $34 \mathrm{mg}$ (91\%).

Colorless sticky solid.

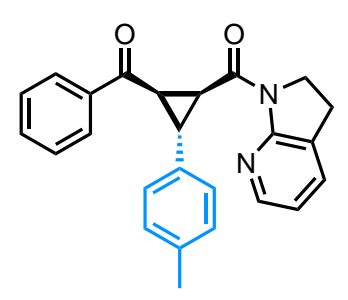

${ }^{1} \mathrm{H}$ NMR (400 MHz, 300 K, $\left.\mathrm{CDCl}_{3}\right)$ : $\delta 8.08$ (ddt, $\left.J=5.1,1.8,1.0 \mathrm{~Hz}, 1 \mathrm{H}\right), 8.01-7.96(\mathrm{~m}$, $2 \mathrm{H}), 7.49-7.43(\mathrm{~m}, 1 \mathrm{H}), 7.39(\mathrm{dq}, J=7.4,1.4 \mathrm{~Hz}, 1 \mathrm{H}), 7.37-7.31(\mathrm{~m}, 2 \mathrm{H}), 7.25-7.12(\mathrm{~m}$, $4 \mathrm{H}), 6.83(\mathrm{dd}, J=7.3,5.1 \mathrm{~Hz}, 1 \mathrm{H}), 4.25(\mathrm{dd}, J=9.5,6.4 \mathrm{~Hz}, 1 \mathrm{H}), 4.13-3.97(\mathrm{~m}, 2 \mathrm{H}), 3.58$ $(\mathrm{t}, J=6.3 \mathrm{~Hz}, 1 \mathrm{H}), 3.22(\mathrm{dd}, J=9.5,6.3 \mathrm{~Hz}, 1 \mathrm{H}), 3.08-2.88(\mathrm{~m}, 2 \mathrm{H}), 2.34(\mathrm{~s}, 3 \mathrm{H})$.

${ }^{13}$ C NMR (101 MHz, 300 K, CDCl $)$ : $\delta$ 195.2, 167.5, 156.3, 146.1, 137.7, 136.5, 136.3, 133.4, $132.8,129.3,128.6,128.4,127.1,126.3,118.1,46.0,36.7,34.5,30.9,24.4,21.2$.

IR (thin film): $\widetilde{v} 3053,3018,2964,2918,1682,1649,1599,1589,1518,1478,1444,1420$, $1385,1332,1310,1264,1241,1217,1164,1049,1015,985,808,782,768,721,692 \mathrm{~cm}^{-1}$.

HRMS (ESI): $m / z$ calculated for $\mathrm{C}_{25} \mathrm{H}_{23} \mathrm{O}_{2} \mathrm{~N}_{2}[\mathrm{M}+\mathrm{H}]^{+}: 383.1754$, found: 383.1749 .

$[\alpha]_{\mathrm{D}}^{23}-24.6$ ( $0.02, \mathrm{CHCl}_{3}, 77 \%$ ee sample).

Enantiomeric excess was determined to be $77 \%$ ee by chiral stationary phase HPLC analysis (CHIRALPAK IG3 $(\phi 0.46 \mathrm{~cm} \times 25 \mathrm{~cm}), n$-hexane $/ \mathrm{PrOH}=4 / 1$, flow rate $1.0 \mathrm{~mL} / \mathrm{min}$, detection at $254 \mathrm{~nm}, \mathrm{t}=154.6 \mathrm{~min}($ minor) and $307.8 \mathrm{~min}$ (major). 

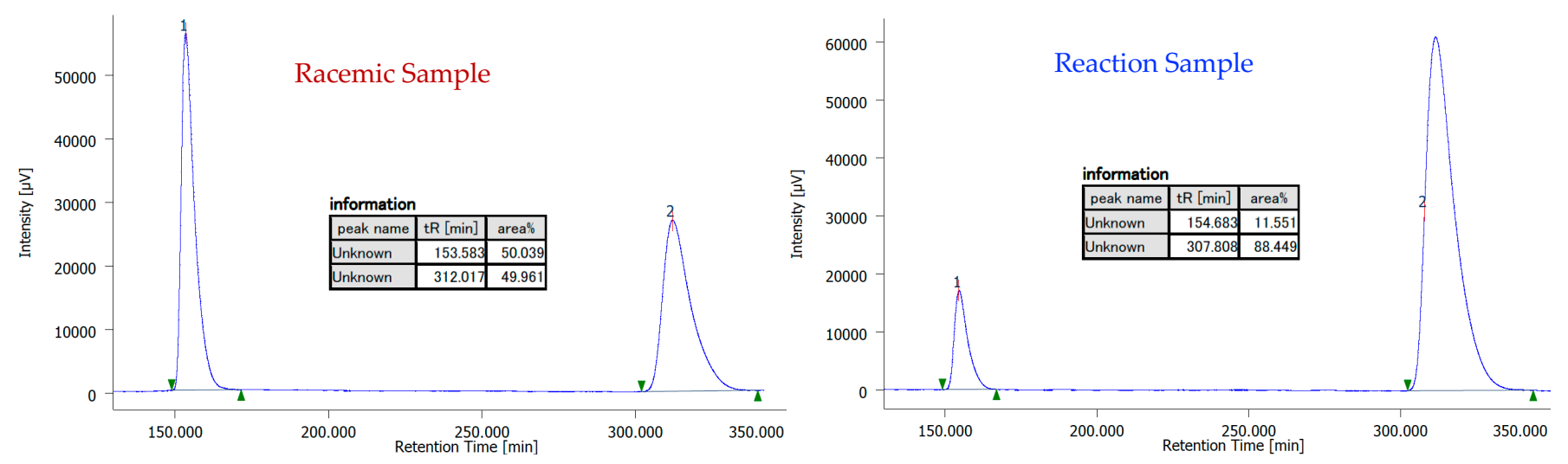

((1S,2R,3R)-2-benzoyl-3-(4-fluorophenyl)cyclopropyl)(2,3-dihydro-1H-pyrrolo[2,3- $b$ ]pyridin-1yl)methanone (6aad):

The reaction performed according to the standard procedure 3 afforded $34 \mathrm{mg}(89 \%)$.

Colorless sticky solid.

${ }^{1} \mathrm{H}$ NMR (400 MHz, 300 K, $\left.\mathbf{C D C l}_{3}\right)$ : $\delta 8.08$ (ddt, J = 5.1, 1.9, $\left.1.0 \mathrm{~Hz}, 1 \mathrm{H}\right), 8.01-7.97$ (m, $2 \mathrm{H}), 7.51-7.44(\mathrm{~m}, 1 \mathrm{H}), 7.40(\mathrm{dq}, J=7.4,1.4 \mathrm{~Hz}, 1 \mathrm{H}), 7.38-7.29(\mathrm{~m}, 4 \mathrm{H}), 7.06-6.98(\mathrm{~m}$,<smiles>O=C(c1ccccc1)C1C(C(=O)N2CCc3cccnc32)[C@H]1c1ccc(F)cc1</smiles>

$2 \mathrm{H}), 6.84(\mathrm{dd}, J=7.4,5.1 \mathrm{~Hz}, 1 \mathrm{H}), 4.26(\mathrm{dd}, J=9.6,6.4 \mathrm{~Hz}, 1 \mathrm{H}), 4.12-3.94(\mathrm{~m}, 2 \mathrm{H}), 3.60$ $(\mathrm{t}, J=6.3 \mathrm{~Hz}, 1 \mathrm{H}), 3.21(\mathrm{dd}, J=9.6,6.2 \mathrm{~Hz}, 1 \mathrm{H}), 3.07-2.89(\mathrm{~m}, 2 \mathrm{H})$.

${ }^{13} \mathrm{C}$ NMR (101 MHz, 300 K, CDCl 3 ): $\delta$ 194.7, 167.0, 161.8 (d, J = $\left.244.9 \mathrm{~Hz}\right), 156.0,145.9$, 137.4, $134.9(\mathrm{~d}, J=3.2 \mathrm{~Hz}), 133.4,132.8,128.6(\mathrm{~d}, J=8.1 \mathrm{~Hz}), 128.3(\mathrm{~d}, J=9.2 \mathrm{~Hz}), 126.2$, $118.1,115.4,115.2,45.9,36.4,34.5,30.0,24.3$.

${ }^{19}$ F NMR (376 MHz, $\left.300 \mathrm{~K}, \mathrm{CDCl}_{3}\right)$ : $\delta$-116.07.

IR (thin film): $\widetilde{v}$ 3066, 3014, 2965, 2913, 1682, 1649, 1599, 1590, 1590, 1513, 1478, 1446, 1421, 1386, 1343, 1330, 1304, 1266, 1240, 1219, 1159, 1100, 1049, 1015, 985, 875, 827, 785, $772,734,723,692,638,589,545 \mathrm{~cm}^{-1}$.

HRMS (ESI): $m / z$ calculated for $\mathrm{C}_{24} \mathrm{H}_{20} \mathrm{O}_{2} \mathrm{~N}_{2} \mathrm{~F}[\mathrm{M}+\mathrm{H}]^{+}: 387.1503$, found: 387.1497 .

$[\alpha]_{\mathrm{D}}^{23}-28.9$ ( $c 0.01, \mathrm{CHCl}_{3}, 82 \%$ ee sample; major diastereomer).

Enantiomeric excess was determined to be $82 \%$ ee (standard procedure 2, using $\mathbf{L 1}$ ) by chiral stationary phase HPLC analysis (CHIRALPAK IC3 $(\phi 0.46 \mathrm{~cm} \times 25 \mathrm{~cm}), n$-hexane $/ \mathrm{PrOH}=1 / 1$, flow rate $1.0 \mathrm{~mL} / \mathrm{min}$, detection at $254 \mathrm{~nm}, \mathrm{t}_{\mathrm{R}}=29.6 \mathrm{~min}$ (minor) and $37.5 \mathrm{~min}$ (major).
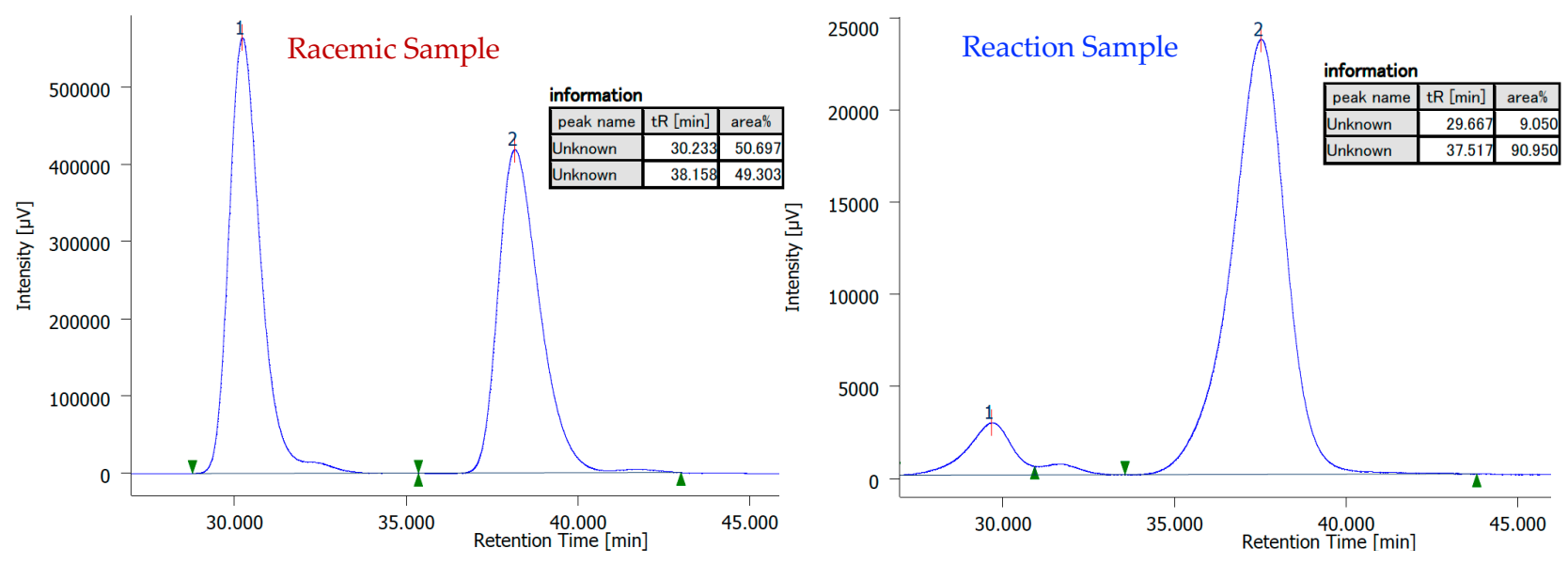
((1S,2R,3R)-2-benzoyl-3-(4-chlorophenyl)cyclopropyl)(2,3-dihydro-1H-pyrrolo[2,3-b]pyridin-1yl)methanone (6aae):

The reaction performed according to the standard procedure 3 afforded $36 \mathrm{mg}(90 \%)$.

Colorless sticky solid.

${ }^{1} \mathrm{H}$ NMR (400 MHz, $\left.300 \mathrm{~K}, \mathrm{CDCl}_{3}\right): \delta 8.07$ (ddt, $\left.J=5.1,1.9,1.0 \mathrm{~Hz}, 1 \mathrm{H}\right), 8.01-7.96(\mathrm{~m}$,

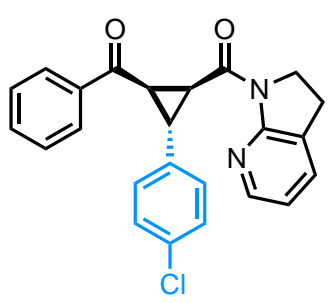

$2 \mathrm{H}), 7.50-7.44(\mathrm{~m}, 1 \mathrm{H}), 7.41(\mathrm{dq}, J=7.4,1.4 \mathrm{~Hz}, 1 \mathrm{H}), 7.38-7.31(\mathrm{~m}, 2 \mathrm{H}), 7.31-7.26(\mathrm{~m}$, $4 \mathrm{H}), 6.85(\mathrm{dd}, J=7.4,5.1 \mathrm{~Hz}, 1 \mathrm{H}), 4.27(\mathrm{dd}, J=9.6,6.4 \mathrm{~Hz}, 1 \mathrm{H}), 4.12-3.95(\mathrm{~m}, 2 \mathrm{H}), 3.58$ $(\mathrm{t}, J=6.3 \mathrm{~Hz}, 1 \mathrm{H}), 3.21(\mathrm{dd}, J=9.6,6.2 \mathrm{~Hz}, 1 \mathrm{H}), 3.07-2.89(\mathrm{~m}, 2 \mathrm{H})$.

${ }^{13} \mathrm{C}$ NMR (101 MHz, 300 K, $\left.\mathrm{CDCl}_{3}\right): \delta 194.6,166.9,156.0,145.9,137.8,137.3,133.4,132.8$, $132.5,128.6,128.41,128.40,128.3,126.2,118.2,45.9,36.5,34.5,30.1,24.3$.

IR (thin film): $\widetilde{v} 3060,2964,2912,1684,1647,1599,1590,1496,1477,1446,1421,1402$, $1385,1330,1305,1276,1264,1241,1217,1165,1092,1049,1014,985,817,776,735,694,590$ $\mathrm{cm}^{-1}$.

HRMS (ESI): $m / z$ calculated for $\mathrm{C}_{24} \mathrm{H}_{20} \mathrm{O}_{2} \mathrm{~N}_{2} \mathrm{Cl}[\mathrm{M}+\mathrm{H}]^{+}: 403.1208$, found: 403.1208 .

$[\alpha]_{\mathrm{D}}^{23}+0.52\left(\right.$ c $0.12, \mathrm{CHCl}_{3}, 76 \%$ ee sample).

Enantiomeric excess was determined to be $76 \%$ ee by chiral stationary phase HPLC analysis (CHIRALPAK IG3 $(\phi 0.46 \mathrm{~cm} \times 25 \mathrm{~cm}), n$-hexane $/ \mathrm{PrOH}=4 / 1$, flow rate $1.0 \mathrm{~mL} / \mathrm{min}$, detection at $254 \mathrm{~nm}, \mathrm{t}_{\mathrm{R}}=138.6 \mathrm{~min}$ (minor) and $252.6 \mathrm{~min}$ (major).
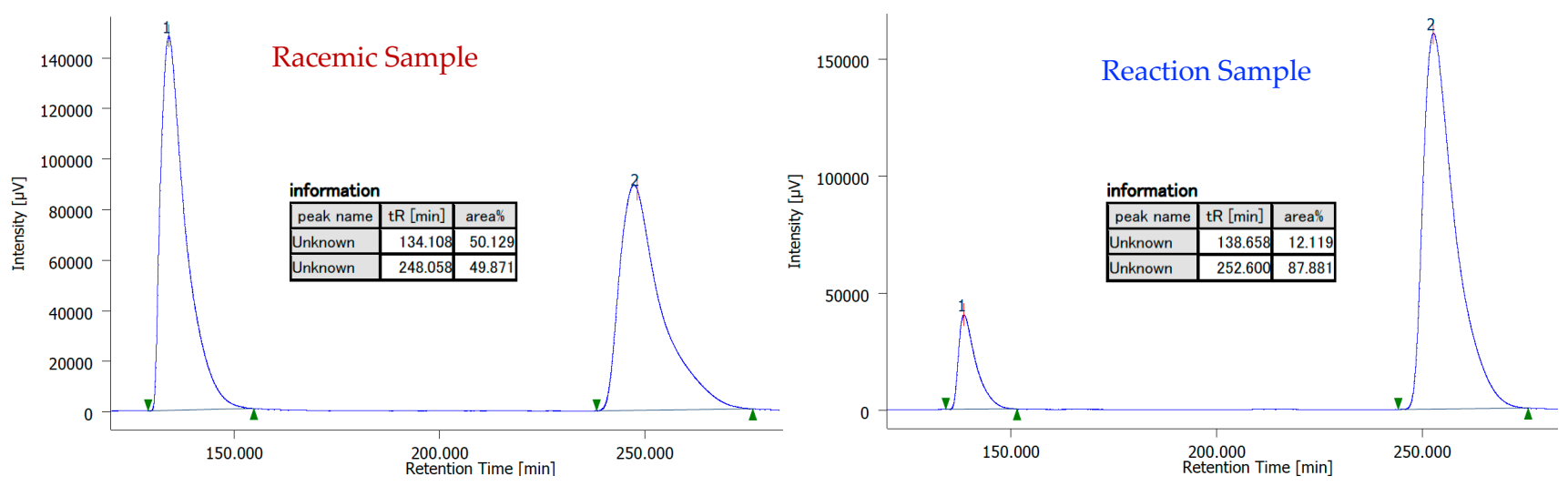

4-((1R,2R,3S)-2-benzoyl-3-(2,3-dihydro-1H-pyrrolo[2,3-b]pyridine-1-carbonyl)cyclopropyl)benzonitrile (6aaf):

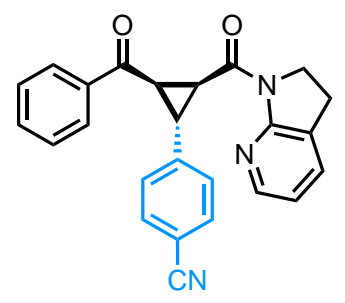

The reaction performed according to the standard procedure 3 afforded $29 \mathrm{mg}(75 \%)$. Colorless solid (m.p $=157-159^{\circ} \mathrm{C}$ ).

${ }^{1} \mathrm{H}$ NMR (400 MHz, $\left.300 \mathrm{~K}, \mathrm{CDCl}_{3}\right): \delta 8.06(\mathrm{ddt}, J=5.1,1.8,1.0 \mathrm{~Hz}, 1 \mathrm{H}), 8.02-7.97(\mathrm{~m}$, $2 \mathrm{H}), 7.66-7.61(\mathrm{~m}, 2 \mathrm{H}), 7.55-7.40(\mathrm{~m}, 4 \mathrm{H}), 7.39-7.32(\mathrm{~m}, 2 \mathrm{H}), 6.86(\mathrm{dd}, J=7.4,5.1 \mathrm{~Hz}$, $1 \mathrm{H}), 4.36(\mathrm{dd}, J=9.7,6.4 \mathrm{~Hz}, 1 \mathrm{H}), 4.02(\mathrm{dddd}, J=34.2,12.2,10.2,6.8 \mathrm{~Hz}, 2 \mathrm{H}), 3.64(\mathrm{t}, J=$ $6.2 \mathrm{~Hz}, 1 \mathrm{H}), 3.27(\mathrm{dd}, J=9.7,6.1 \mathrm{~Hz}, 1 \mathrm{H}), 3.06-2.87(\mathrm{~m}, 2 \mathrm{H})$.

${ }^{13}$ C NMR (101 MHz, 300 K, CDCl CD): $\delta_{194.1}$ 166.4, 156.0, 146.1, 145.2, 137.2, 133.7, 133.1, $132.5,128.5,128.5,127.8,126.4,119.0,118.5,110.6,46.0,36.9,35.0,30.3,24.4$.

IR (thin film): $\widetilde{v} 3065,2912,2226,1684,1677,1654,1647,1598,1589,1506,1477,1447$, $1421,1387,1369,1333,1310,1267,1240,1219,1178,1165,1078,1049,1014,984,964,825$, $783,734,714,693,610,589,558 \mathrm{~cm}^{-1}$.

HRMS (ESI): $m / z$ calculated for $\mathrm{C}_{25} \mathrm{H}_{20} \mathrm{O}_{2} \mathrm{~N}_{3}[\mathrm{M}+\mathrm{H}]^{+}: 394.1550$, found: 394.1549 .

$[\alpha]_{\mathrm{D}}^{23}-3.8$ ( c 1.0, $\mathrm{CHCl}_{3}, 74 \%$ ee sample).

Enantiomeric excess was determined to be $74 \%$ ee by chiral stationary phase HPLC analysis (CHIRALPAK IA $(\phi 0.46 \mathrm{~cm} \times 25 \mathrm{~cm}), n$-hexane $/ \mathrm{PrOH}=4 / 1$, flow rate $1.0 \mathrm{~mL} / \mathrm{min}$, detection at $254 \mathrm{~nm}, \mathrm{t}_{\mathrm{R}}=68.8 \mathrm{~min}($ minor) and $89.3 \mathrm{~min}$ (major). 

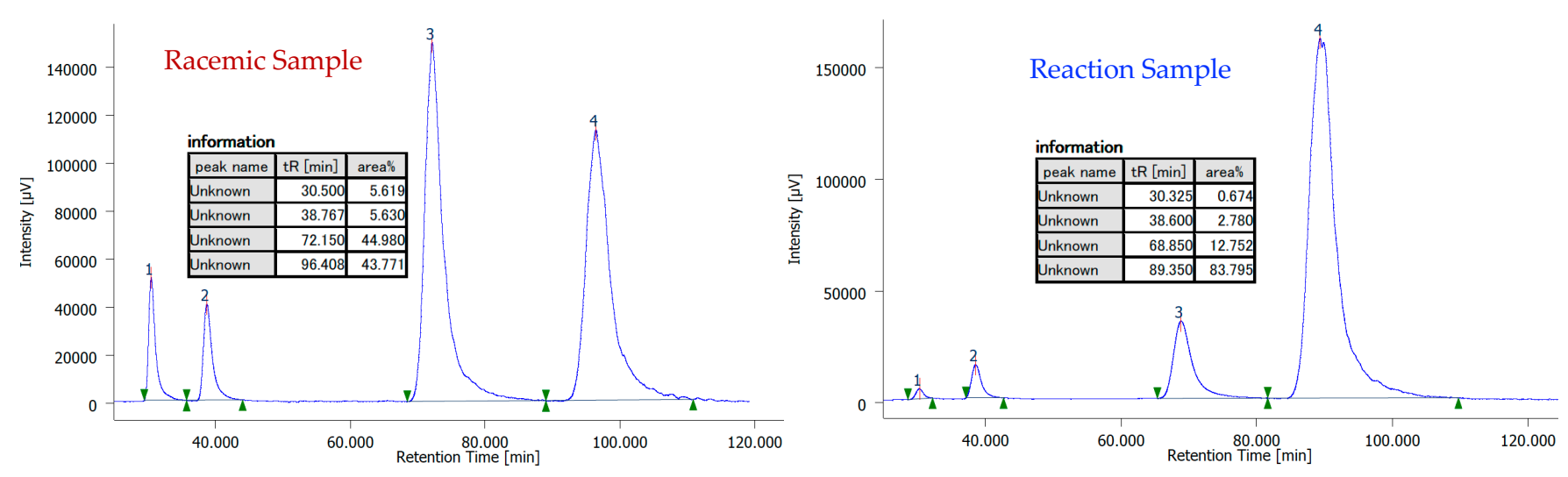

((1R,2S,3R)-2-benzoyl-3-(thiophen-2-yl)cyclopropyl)(2,3-dihydro-1H-pyrrolo[2,3-b]pyridin-1yl)methanone (6aag):

The reaction performed according to the standard procedure 3 afforded $33 \mathrm{mg}(88 \%)$.<smiles>O=C(c1ccccc1)C1C(C(=O)N2CCc3cccnc32)[C@H]1c1cccs1</smiles>

Colorless sticky solid.

${ }^{1} \mathrm{H}$ NMR (400 MHz, 300 K, $\left.\mathrm{CDCl}_{3}\right)$ : $\delta 8.10(\mathrm{dd}, J=5.1,1.5 \mathrm{~Hz}, 1 \mathrm{H}), 8.03-7.99(\mathrm{~m}, 2 \mathrm{H})$, $7.53-7.45(\mathrm{~m}, 1 \mathrm{H}), 7.40(\mathrm{dq}, J=7.4,1.4 \mathrm{~Hz}, 1 \mathrm{H}), 7.36(\mathrm{dd}, J=8.4,7.0 \mathrm{~Hz}, 2 \mathrm{H}), 7.16(\mathrm{dd}, J$ $=5.1,1.2 \mathrm{~Hz}, 1 \mathrm{H}), 7.03(\mathrm{dt}, J=3.5,1.1 \mathrm{~Hz}, 1 \mathrm{H}), 6.96(\mathrm{dd}, J=5.1,3.5 \mathrm{~Hz}, 1 \mathrm{H}), 6.84(\mathrm{dd}, J=$ $7.4,5.1 \mathrm{~Hz}, 1 \mathrm{H}), 4.28(\mathrm{dd}, J=9.6,6.2 \mathrm{~Hz}, 1 \mathrm{H}), 4.13-3.95(\mathrm{~m}, 2 \mathrm{H}), 3.76(\mathrm{td}, J=6.2,0.8 \mathrm{~Hz}$, $1 \mathrm{H}), 3.29(\mathrm{dd}, J=9.6,6.1 \mathrm{~Hz}, 1 \mathrm{H}), 3.07-2.88(\mathrm{~m}, 2 \mathrm{H})$.

${ }^{13} \mathrm{C}$ NMR (101 MHz, 300 K, $\left.\mathrm{CDCl}_{3}\right): \delta$ 194.5, 166.8, 156.1, 146.1, 143.4, 137.4, 133.5, 133.0, $128.6,128.5,127.1,126.3,124.9,123.7,118.3,46.0,37.5,35.7,26.2,24.4$.

IR (thin film): $\widetilde{v} 3068,2960,2924,2852,1732,1681,1650,1599,1589,1477,1443,1420$, 1387, 1351, 1316, 1266, 1240, 1219, 1165, 1056, 1013, 850, 784, 715, 693, 615, $591 \mathrm{~cm}^{-1}$.

HRMS (ESI): $m / z$ calculated for $\mathrm{C}_{22} \mathrm{H}_{19} \mathrm{O}_{2} \mathrm{~N}_{2} \mathrm{~S}[\mathrm{M}+\mathrm{H}]^{+}: 375.1162$, found: 375.1160 .

$[\alpha]_{\mathrm{D}}^{23}+2.4$ ( $0.09, \mathrm{CHCl}_{3}, 75 \%$ ee sample; major diastereomer).

Enantiomeric excess was determined to be $75 \%$ ee (major diastereomer) by chiral stationary phase HPLC analysis (CHIRALPAK IF $(\phi 0.46 \mathrm{~cm} \times 25 \mathrm{~cm}), n$-hexane/PrOH $=4 / 1$, flow rate $1.0 \mathrm{~mL} / \mathrm{min}$, detection at $254 \mathrm{~nm}, \mathrm{t}_{\mathrm{R}}=64.5 \mathrm{~min}$ (minor) and $86.0 \mathrm{~min}$ (major).
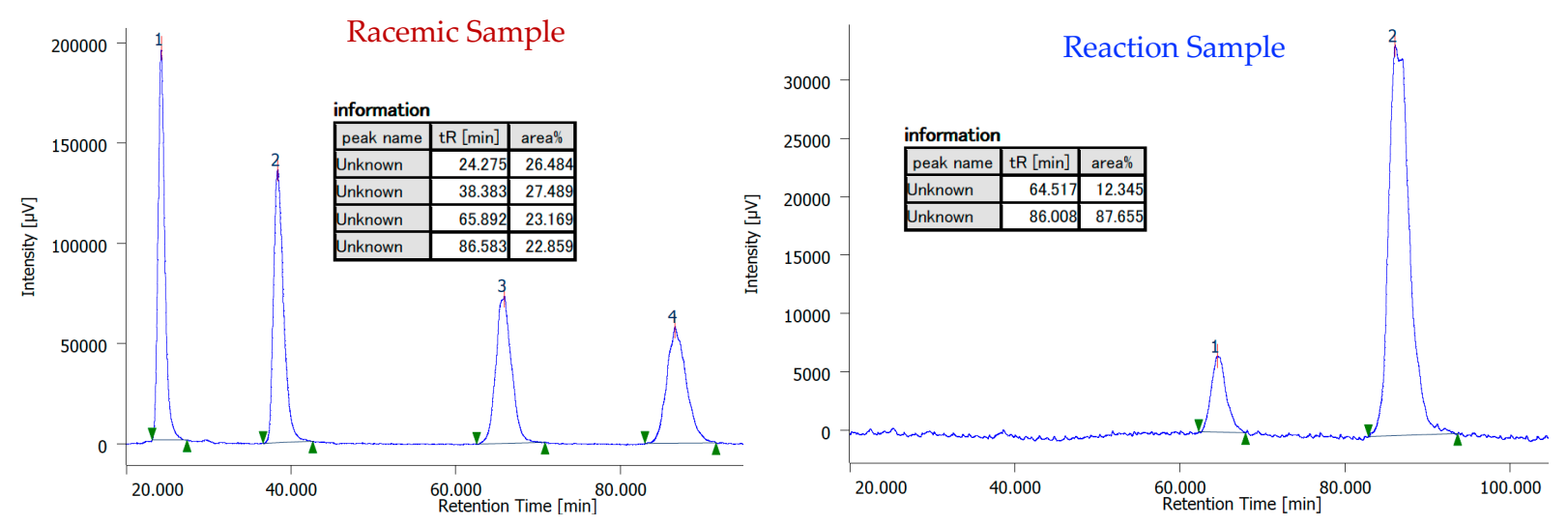
((1S,2R,3S)-2-benzoyl-3-(furan-2-yl)cyclopropyl)(2,3-dihydro-1H-pyrrolo[2,3-b]pyridin-1-yl)methanone (6aah):

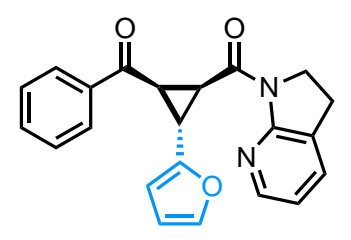

The reaction performed according to the standard procedure 3 afforded $30 \mathrm{mg}$ (85\%).

Colorless sticky solid.

${ }^{1} \mathrm{H}$ NMR (400 MHz, 300 K, $\left.\mathrm{CDCl}_{3}\right)$ : $\delta 8.10(\mathrm{ddt}, J=5.1,1.8,1.0 \mathrm{~Hz}, 1 \mathrm{H}), 8.03-7.98(\mathrm{~m}$, $2 \mathrm{H}), 7.50-7.43(\mathrm{~m}, 1 \mathrm{H}), 7.39(\mathrm{dq}, J=7.3,1.4 \mathrm{~Hz}, 1 \mathrm{H}), 7.37-7.31(\mathrm{~m}, 3 \mathrm{H}), 6.84(\mathrm{dd}, J=7.3$, $5.1 \mathrm{~Hz}, 1 \mathrm{H}), 6.34(\mathrm{dd}, J=3.3,1.8 \mathrm{~Hz}, 1 \mathrm{H}), 6.27(\mathrm{dt}, J=3.2,0.7 \mathrm{~Hz}, 1 \mathrm{H}), 4.30-4.20(\mathrm{~m}, 1 \mathrm{H})$, $4.12-3.95(\mathrm{~m}, 2 \mathrm{H}), 3.59(\mathrm{t}, J=6.2 \mathrm{~Hz}, 1 \mathrm{H}), 3.42(\mathrm{dd}, J=9.6,6.1 \mathrm{~Hz}, 1 \mathrm{H}), 3.07-2.88(\mathrm{~m}$, $2 \mathrm{H})$.

${ }^{13}$ C NMR (101 MHz, 300 K, CDCl $\left.{ }_{3}\right): \delta 194.6,166.7,156.1,152.7,146.2,141.3,137.4,133.5$, 132.9, 128.6, 128.4, 126.2, 118.2, 110.8, 106.5, 46.0, 34.5, 33.2, 24.5, 24.4.

IR (thin film): $\widetilde{v} 3057,2919,1682,1648,1598,1589,1477,1442,1420,1388,1356,1321$, $1265,1240,1218,1175,1146,1076,1013,987,785,717,693,591 \mathrm{~cm}^{-1}$.

HRMS (ESI): $m / z$ calculated for $\mathrm{C}_{22} \mathrm{H}_{19} \mathrm{O}_{3} \mathrm{~N}_{2}[\mathrm{M}+\mathrm{H}]^{+}:$359.1390, found: 359.1391 .

$[\alpha]_{\mathrm{D}}^{23}+8.6\left(\right.$ c $0.13, \mathrm{CHCl}_{3}, 71 \%$ ee sample).

Enantiomeric excess was determined to be $71 \%$ ee by chiral stationary phase HPLC analysis (CHIRALPAK IF $(\phi 0.46 \mathrm{~cm} \times 25 \mathrm{~cm}$ ), $n$-hexane/ $\mathrm{PrOH}=4 / 1$, flow rate 1.0 $\mathrm{mL} / \mathrm{min}$, detection at $254 \mathrm{~nm}, \mathrm{t}_{\mathrm{R}}=61.7 \mathrm{~min}$ (major) and $88.4 \mathrm{~min}$ (minor).
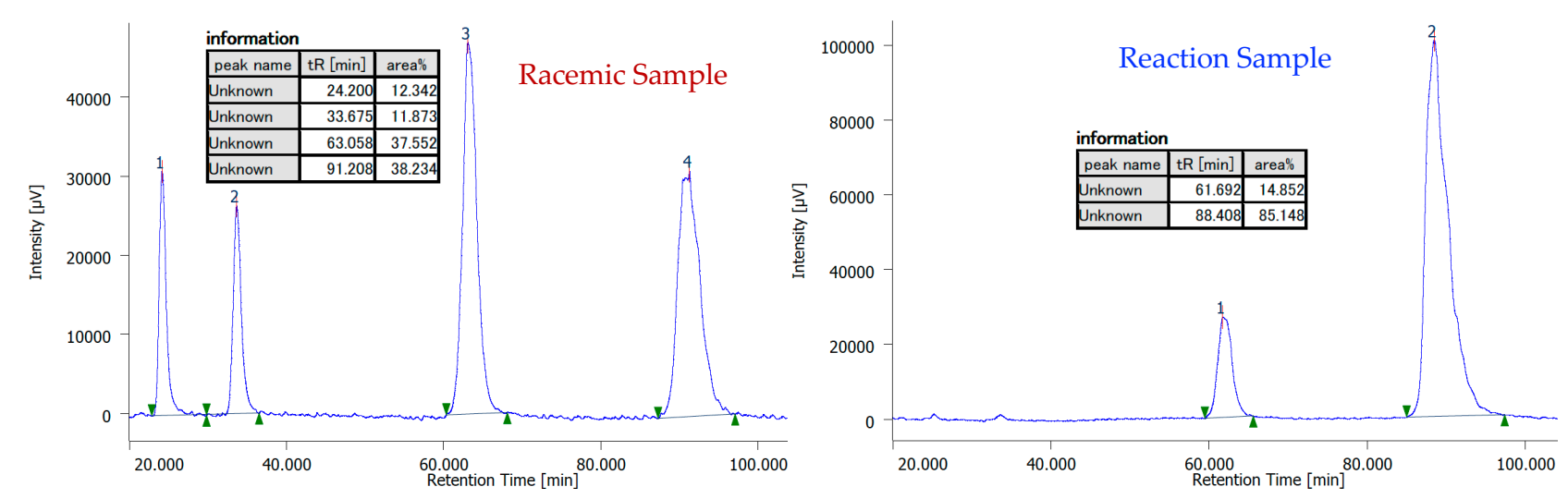


\section{Gram-Scale Reactions}

\section{Gram scale synthesis of 4ap:}

An oven-dried $50 \mathrm{~mL}$ round-bottomed flask equipped with a magnetic stirrer bar was charged with $2 \mathbf{p}(1.21$ g, $5.0 \mathrm{mmol}, 1.0$ equiv.), [Cu( $\left.\left.\mathrm{CH}_{3} \mathrm{CN}\right)_{4}\right] \mathrm{PF}_{6}(18.7 \mathrm{mg}, 0.05 \mathrm{mmol}, 0.01$ equiv.), L4 (S)-DTBM-Segphos (71 mg, $0.06 \mathrm{mmol}, 0.012$ equiv.) under inert atmosphere. To the mixture was added anhydrous $\mathrm{CH}_{2} \mathrm{Cl}_{2}(15 \mathrm{~mL})$ via syringe with a stainless-steel needle at room temperature under positive Argon pressure. The readily prepared ylide $1 \mathrm{a}$ (1.35 g, $7.5 \mathrm{mmol}, 1.5$ equiv.), was dissolved in $\mathrm{CH}_{2} \mathrm{Cl}_{2}$ (15 mL) and the resulting solution was slowly added to $50 \mathrm{~mL}$ round bottomned flask containing $\mathbf{2} \mathbf{p},\left[\mathrm{Cu}\left(\mathrm{CH}_{3} \mathrm{CN}\right)_{4}\right] \mathrm{PF}_{6}$, and (S)-DTBM-Segphos. The resulting reaction mixture was then allowed to stir for an additional $24 \mathrm{~h}$ at room temperature. After confirming the complete conversion by TLC (see the picture), purification of the crude product was done by automated flash column chromatography using Hexanes/Ethyl acetate $(\sim 8 / 2)$ solvent system to afford pure 4ap as a colorless solid (1.80 g, 99\% yield).

Enantiomeric excess of the major diastereomer was determined to be $95 \%$ ee by chiral stationary phase HPLC analysis (CHIRALPAK IC3 $(\phi 0.46 \mathrm{~cm} \times 25 \mathrm{~cm}), n$-hexane/ $\mathrm{PrOH}=8 / 2$, flow rate $1.0 \mathrm{~mL} / \mathrm{min}$, detection at 254 $\mathrm{nm}, \mathrm{t}_{\mathrm{R}}=12.7$ ( $1^{\text {st }}$ diastereomer, major), $14.0 \mathrm{~min}$ ( $1^{\text {st }}$ diastereomer, minor), 24.2 ( $2^{\text {nd }}$ diastereomer, minor $)$, and $26.5 \mathrm{~min}\left(2^{\text {nd }}\right.$ diastereomer, major $)$.
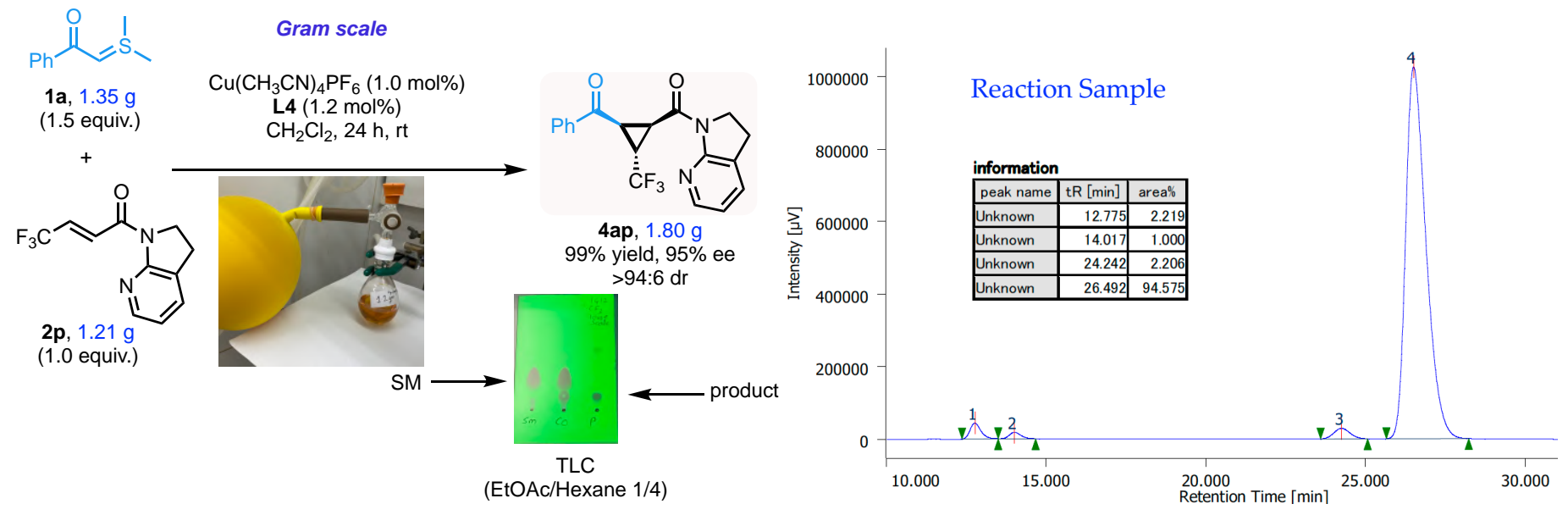

\section{Gram scale synthesis of 4ep:}

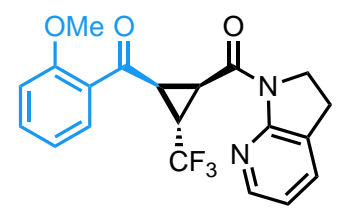

Similar to the gram scale synthesis of 4ap, the $4 \mathrm{ep}$ was synthesized in $1.51 \mathrm{~g}[99 \%$ yield, $96 \% e e,>97: 3 d r$ (before recrystalliazation) $\rightarrow 97 \%$ ee, $>99: 1 d r$ (after recrystallization)] by using 1 e $(1.51 \mathrm{~g}$, $7.19 \mathrm{mmol}, 1.5$ equiv) and $2 \mathrm{p}(1.16 \mathrm{~g}, 4.79 \mathrm{mmol}, 1.0$ equiv) in the presence of a $\left[\mathrm{Cu}\left(\mathrm{CH}_{3} \mathrm{CN}\right)_{4}\right] \mathrm{PF}_{6}(17.9$ $\mathrm{mg}, 0.05 \mathrm{mmol}, 0.01$ equiv.) and L4 (S)-DTBMSegphos (68 mg, $0.06 \mathrm{mmol}, 0.012$ equiv.) under argon atmosphere at room temperature conditions. Enantiomeric excess of the major diastereomer was

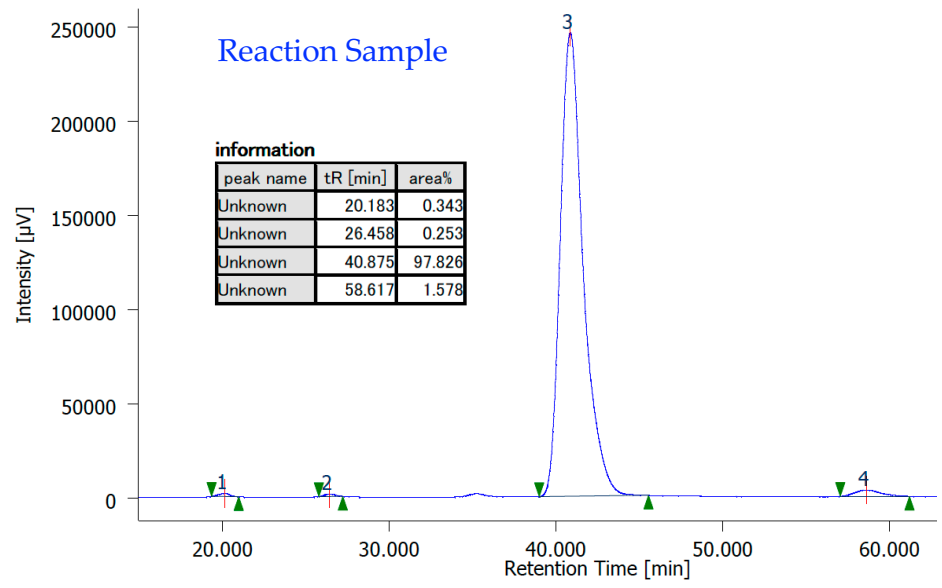
determined to be $97 \%$ ee by chiral stationary phase HPLC analysis (CHIRALPAK IG3 $(\phi 0.46 \mathrm{~cm} \times 25 \mathrm{~cm}), n$ hexane $/ \mathrm{PrOH}=4 / 1$, flow rate $1.0 \mathrm{~mL} / \mathrm{min}$, detection at $254 \mathrm{~nm}, \mathrm{t}_{\mathrm{R}}=20.1 \mathrm{~min}$ ( $1^{\text {st }}$ diastereomer, major), 26.4 $\min \left(1^{\text {st }}\right.$ diastereomer, minor), $40.8 \mathrm{~min}$ ( $2^{\text {nd }}$ diastereomer, major $)$, and $58.6 \mathrm{~min}\left(2^{\text {nd }}\right.$ diastereomer, minor). 


\section{Auxiliary Removal}

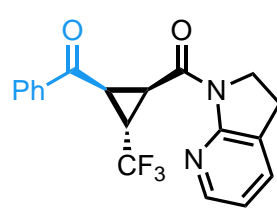

4ap, $1.80 \mathrm{~g}$
Auxiliary removal

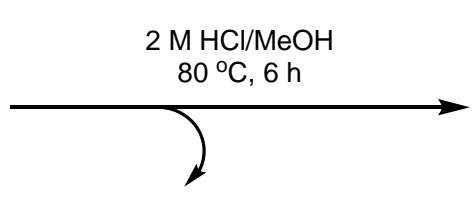<smiles>COC(=O)C1C(C(=O)c2ccccc2)C1C(F)(F)F</smiles>

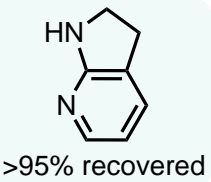
$7 \mathrm{a}, 1.22 \mathrm{~g}$ $90 \%$ yield $95 \%$ ee, $>94: 6 \mathrm{dr}$ $>99 \%$ ee, $>99: 1 \mathrm{dr}$ (after recryst.) $91 \%$ recrystallization yield

A solution of the amide (4ap) $(1.80 \mathrm{~g}, 5 \mathrm{mmol})$ in $2 \mathrm{M} \mathrm{MeOH} / \mathrm{HCl}(10 \mathrm{~mL})$ was heated in a sealed pressure tube to $80^{\circ} \mathrm{C}$ for $6 \mathrm{~h}$. After cooling to room temperature the volatiles was removed under reduced pressure and the residue was basified using saturated $\mathrm{NaHCO}_{3}(30 \mathrm{~mL})$ and extracted by ethyl acetate $(3 \times 30 \mathrm{~mL})$. The combined organic layers were dried over $\mathrm{Na}_{2} \mathrm{SO}_{4}$ and purified by flash chromatography eluting with Hexane/Ethyl acetate ( 9/1) to afford $7 \mathbf{a}$ (1.22 g, 90\% yield). The obtained product 7a was subsequently recrystallized from hot hexane.

methyl (1R,2S,3R)-2-benzoyl-3-(trifluoromethyl)cyclopropane-1-carboxylate (7a):

Colorless solid (m.p $=73-75^{\circ} \mathrm{C}$ ).

${ }^{1} \mathrm{H}$ NMR (400 MHz, 300 K, $\left.\mathbf{C D C l}_{3}\right)$ : $\delta 8.02-7.98(\mathrm{~m}, 2 \mathrm{H}), 7.64-7.58(\mathrm{~m}, 1 \mathrm{H}), 7.53-7.46(\mathrm{~m}$, $2 \mathrm{H}), 3.60(\mathrm{~s}, 3 \mathrm{H}), 3.15(\mathrm{dd}, J=10.1,6.2 \mathrm{~Hz}, 1 \mathrm{H}), 3.01(\mathrm{~h}, J=6.2 \mathrm{~Hz}, 1 \mathrm{H}), 2.66(\mathrm{dd}, J=10.1,5.8$ $\mathrm{Hz}, 1 \mathrm{H})$.

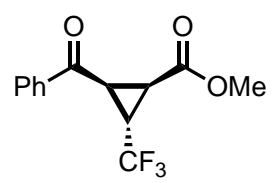

${ }^{13} \mathrm{C}$ NMR (101 MHz, $\left.300 \mathrm{~K}, \mathrm{CDCl}_{3}\right): \delta$ 191.1, 167.5, 136.1, 134.0, 129.0, 128.6, $124.4(\mathrm{q}, J=271.9$ $\mathrm{Hz}), 52.71,28.18(\mathrm{q}, J=2.4 \mathrm{~Hz}), 25.5(\mathrm{q}, J=38.4 \mathrm{~Hz}), 25.4(\mathrm{q}, J=2.4 \mathrm{~Hz})$.

${ }^{19}$ F NMR (376 MHz, $\left.300 \mathrm{~K}, \mathrm{CDCl}_{3}\right)$ : $\delta-66.86$.

IR (thin film): $\widetilde{v} 3068,3028,3009,2965,1730,1682,1596,1579,1490,1448,1435,1404,1375$, 1322, 1309, 1274, 1231, 1144, 1098, 1077, 1051, 1022, 999, 989, 934, 882, 860, 815, 760, 696, 662, $619,579,540 \mathrm{~cm}^{-1}$.

HRMS (ESI): $m / z$ calculated for $\mathrm{C}_{13} \mathrm{H}_{11} \mathrm{O}_{3} \mathrm{~F}_{3} \mathrm{Na}[\mathrm{M}+\mathrm{Na}]^{+}: 295.0553$, found: 295.0558 .

$[\alpha]_{\mathrm{D}}^{23}+59.0$ (c 1.39, $\mathrm{CHCl}_{3}, 99 \%$ ee sample).

Enantiomeric excess was determined to be $99 \%$ ee by chiral stationary phase HPLC analysis (CHIRALPAK IG3 $(\phi 0.46 \mathrm{~cm} \times 25 \mathrm{~cm}), n$-hexane $/ \mathrm{PrOH}=95 / 5$, flow rate $1.0 \mathrm{~mL} / \mathrm{min}$, detection at $254 \mathrm{~nm}, \mathrm{t}_{\mathrm{R}}=11.0 \mathrm{~min}$ (major) and $12.0 \mathrm{~min}$ (minor).
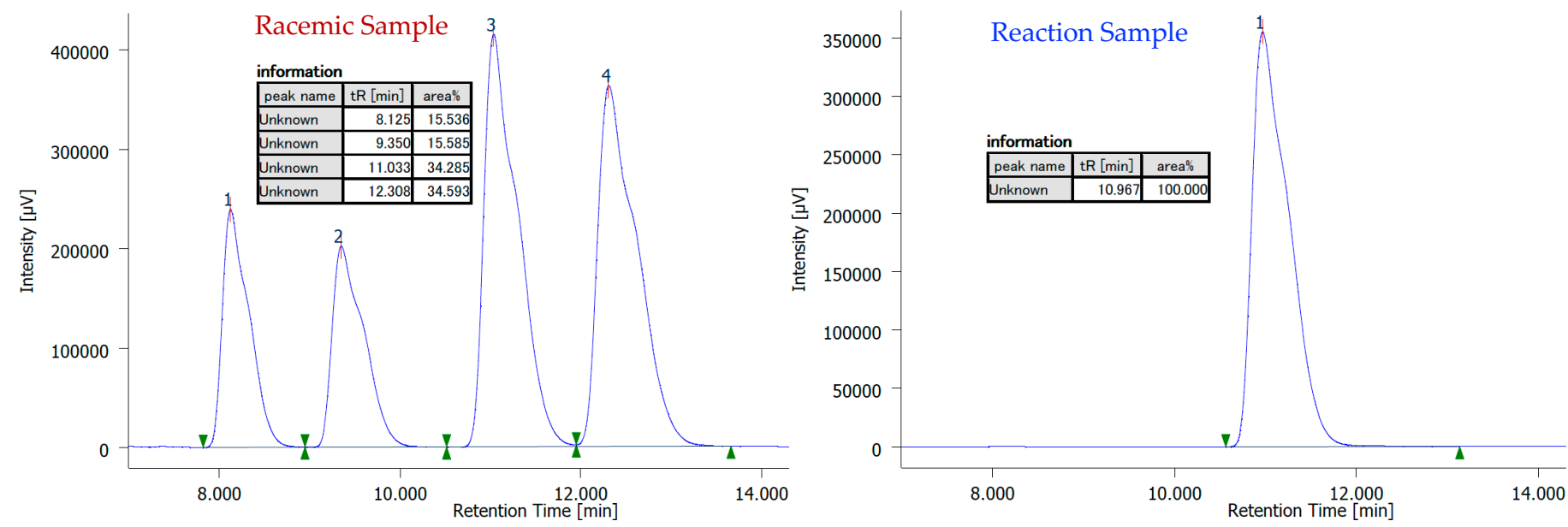
methyl (1R,2S,3R)-2-(2-methoxybenzoyl)-3-(trifluoromethyl)cyclopropane-1-carboxylate (7b):

A solution of the amide $(4 \mathrm{ep}, 1.00 \mathrm{~g}, 2.56 \mathrm{mmol})$ in $2 \mathrm{M} \mathrm{MeOH} / \mathrm{HCl}(10 \mathrm{~mL})$ was heated in a sealed pressure tube to $80^{\circ} \mathrm{C}$ for $6 \mathrm{~h}$. After cooling to room temperature the volatiles was removed under reduced pressure and the residue was basified using saturated $\mathrm{NaHCO}_{3}(30 \mathrm{~mL})$ and extracted by ethyl acetate $(3 \times 30 \mathrm{~mL})$. The combined organic layers were dried over $\mathrm{Na}_{2} \mathrm{SO}_{4}$ and purified by flash chromatography eluting with Hexane/Ethyl acetate $(\sim 9 / 1)$ to afford $7 \mathbf{b}(0.720 \mathrm{~g}, 93 \%$ yield $)$ as a colorless solid (m.p $\left.=35-37^{\circ} \mathrm{C}\right)$.

${ }^{1} \mathrm{H}$ NMR $\left(400 \mathrm{MHz}, 300 \mathrm{~K}, \mathrm{CDCl}_{3}\right): \delta 7.70(\mathrm{dd}, J=7.7,1.8 \mathrm{~Hz}, 1 \mathrm{H}), 7.50(\mathrm{ddd}, J=8.3,7.3$, $1.9 \mathrm{~Hz}, 1 \mathrm{H}), 7.03-6.96(\mathrm{~m}, 2 \mathrm{H}), 3.92(\mathrm{~s}, 3 \mathrm{H}), 3.65(\mathrm{~s}, 3 \mathrm{H}), 3.31(\mathrm{dd}, J=10.2,6.2 \mathrm{~Hz}, 1 \mathrm{H})$, $2.93(\mathrm{~h}, J=6.4 \mathrm{~Hz}, 1 \mathrm{H}), 2.51(\mathrm{dd}, J=10.2,5.9 \mathrm{~Hz}, 1 \mathrm{H})$.

$\underbrace{\mathrm{OMMe}}_{\mathrm{C}_{\mathrm{F}_{3}}}$

${ }^{13} \mathrm{C}$ NMR (101 MHz, $\left.300 \mathrm{~K}, \mathrm{CDCl}_{3}\right): \delta 192.9,168.0,159.2,134.8,131.0,126.9,124.5(\mathrm{q}, J=$ $271.6 \mathrm{~Hz}), 120.9,111.7,55.7,52.5,32.5(\mathrm{q}, J=2.2 \mathrm{~Hz}), 26.4(\mathrm{q}, J=38.3 \mathrm{~Hz}), 25.7(\mathrm{q}, J=2.8$ $\mathrm{Hz})$.

${ }^{19} \mathrm{~F}$ NMR (376 MHz, $\left.300 \mathrm{~K}, \mathrm{CDCl}_{3}\right): \delta-66.93(\mathrm{~d}, J=6.9 \mathrm{~Hz})$.

IR (thin film): $\widetilde{v} 3067,3011,2956,2843,1769,1741,1673,1598,1502,1486,1465,1437$, $1368,1320,1280,1259,1217,1199,1143,1109,1041,1024,937,751,621 \mathrm{~cm}^{-1}$.

HRMS (ESI): $m / z$ calculated for $\mathrm{C}_{14} \mathrm{H}_{14} \mathrm{O}_{4} \mathrm{~F}_{3}[\mathrm{M}+\mathrm{H}]^{+}: 303.0839$, found: 303.0841 .

$[\alpha]_{\mathrm{D}}^{23}+80.1$ ( c 2.4, $\mathrm{CHCl}_{3}, 96 \%$ ee sample).

Enantiomeric excess was determined to be $96 \%$ ee by chiral stationary phase HPLC analysis (CHIRALPAK IG3 $(\phi 0.46 \mathrm{~cm} \times 25 \mathrm{~cm}), n$-hexane/ $\mathrm{PrOH}=95 / 5$, flow rate $1.0 \mathrm{~mL} / \mathrm{min}$, detection at 254 $\mathrm{nm}, \mathrm{t}_{\mathrm{R}}=11.7 \mathrm{~min}\left(1^{\text {st }}\right.$ diastereomer, major $), 12.6 \mathrm{~min}$ ( $1^{\text {st }}$ diastereomer, minor $), 19.5 \mathrm{~min}$ ( $2^{\text {nd }}$ diastereomer, major), and $22.8 \mathrm{~min}$ ( $2^{\text {nd }}$ diastereomer, minor).
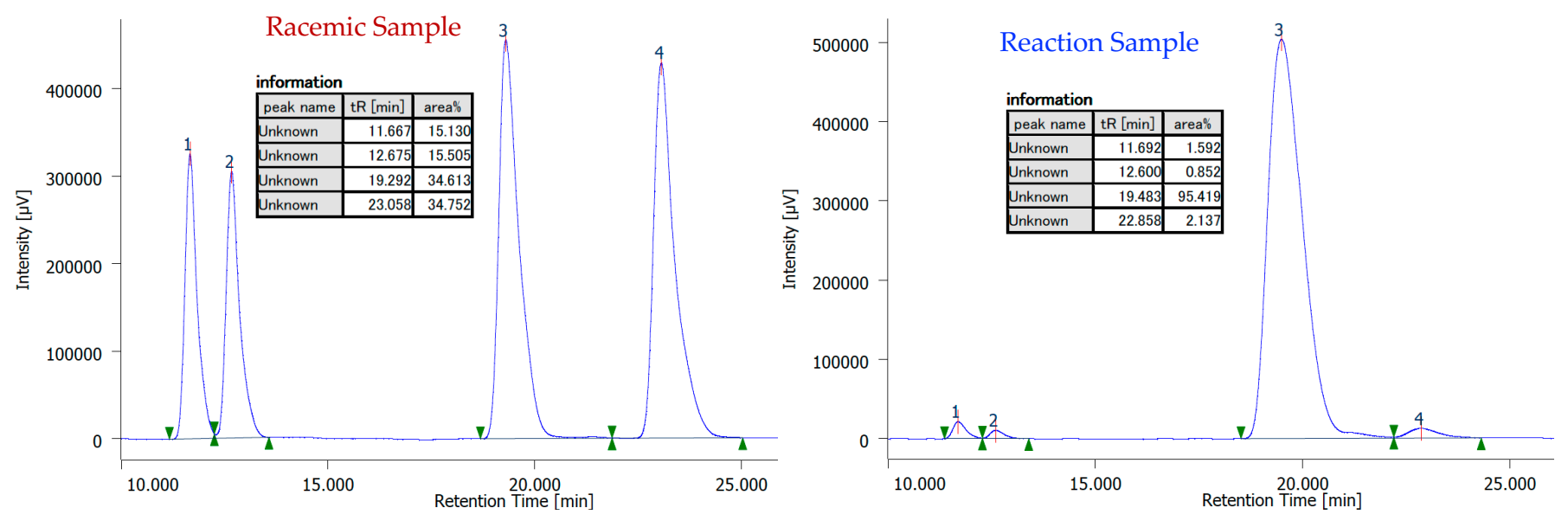


\section{Schmidt Reaction}

This procedure was adapted from the literature: ${ }^{3} \mathrm{TMSN}_{3}(0.250 \mathrm{~mL}, 1.87 \mathrm{mmol})$ was slowly added to the solution of $7 \mathbf{a}(0.338 \mathrm{~g}, 1.25 \mathrm{mmol})$ and $\mathrm{FeCl}_{3}(0.202 \mathrm{~g}, 1.25 \mathrm{mmol})$ in 1,2 DCE $(10 \mathrm{~mL})$ under Argon atmosphere at room temperature. The resulting mixture was stirred for $24 \mathrm{~h}$ at room temperature. After the complete conversion of $\mathbf{7 b}$ (judged by TLC), the reaction mixture was filtered through Celite and washed with DCM $(5 \times 10 \mathrm{~mL})$. The filtrate was concentrated under reduced pressure and the residue was purified by flash chromatography eluting with Hexane/Ethyl acetate ( 8/2) to afford $8 \mathbf{a}(0.322 \mathrm{~g}, 90 \%$ yield) as a colorless solid (m.p $\left.=100-102^{\circ} \mathrm{C}\right)$.<smiles>COC(=O)C1C(C(=O)c2ccccc2)C1C(F)(F)F</smiles>

$7 a$

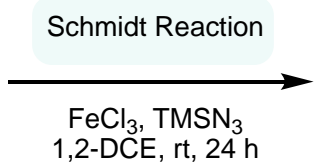

1,2-DCE, rt, $24 \mathrm{~h}$

methyl (1S,2S,3S)-2-benzamido-3-(trifluoromethyl)cyclopropane-1-carboxylate (8a):

${ }^{1} \mathrm{H}$ NMR (400 MHz, 300 K, $\left.\mathrm{CDCl}_{3}\right): \delta 7.63-7.52(\mathrm{~m}, 3 \mathrm{H}), 7.50-7.46(\mathrm{~m}, 2 \mathrm{H}), 5.39$ (d, $J=7.5 \mathrm{~Hz}, 1 \mathrm{H}, \mathrm{NH}), 4.04(\mathrm{td}, J=7.7,5.1 \mathrm{~Hz}, 1 \mathrm{H}), 3.71(\mathrm{~s}, 3 \mathrm{H}), 2.49(\mathrm{dd}, J=7.9,5.6 \mathrm{~Hz}$,<smiles>COC(=O)C1C(NC(=O)c2ccccc2)C1C(F)(F)F</smiles>
$1 \mathrm{H}), 2.46-2.37(\mathrm{~m}, 1 \mathrm{H})$.

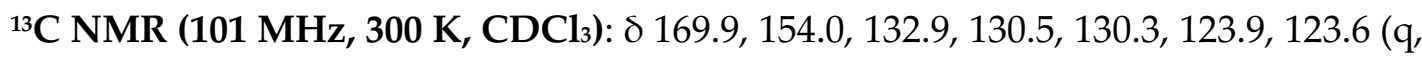
$J=272.7 \mathrm{~Hz}), 53.1,35.6(\mathrm{q}, J=3.6 \mathrm{~Hz}), 28.6(\mathrm{q}, J=38.6 \mathrm{~Hz}), 22.5(\mathrm{q}, J=2.6 \mathrm{~Hz})$.

${ }^{19}$ F NMR (376 MHz, $\left.300 \mathrm{~K}, \mathrm{CDCl}_{3}\right)$ : $\delta-66.47(\mathrm{~d}, J=6.6 \mathrm{~Hz})$.

IR (thin film): $\widetilde{v} 3248,3058,2956,1733,1603,1585,1499,1458,1438,1411,1373,1321$, 1263, 1210, 1164, 1130, 1113, 1090, 1070, 1018, 992, 945, 922, 869, 762, 735, 688, 654, 616 $\mathrm{cm}^{-1}$.

$[\alpha]_{\mathrm{D}}^{23}+14.1$ ( c 0.26, $\mathrm{CHCl}_{3},>99 \%$ ee sample).

HRMS (ESI): This compound was decomposing under HRMS conditions.

Enantiomeric excess was determined to be $>99 \%$ ee by chiral stationary phase HPLC analysis (CHIRALPAK IE $(\phi 0.46 \mathrm{~cm} \times 25 \mathrm{~cm}), n$-hexane $/ \mathrm{PrOH}=95 / 5$, flow rate $1.0 \mathrm{~mL} / \mathrm{min}$, detection at $254 \mathrm{~nm}, \mathrm{t}_{\mathrm{R}}=59.7 \mathrm{~min}$ (major) and $67.9 \mathrm{~min}$ (minor).
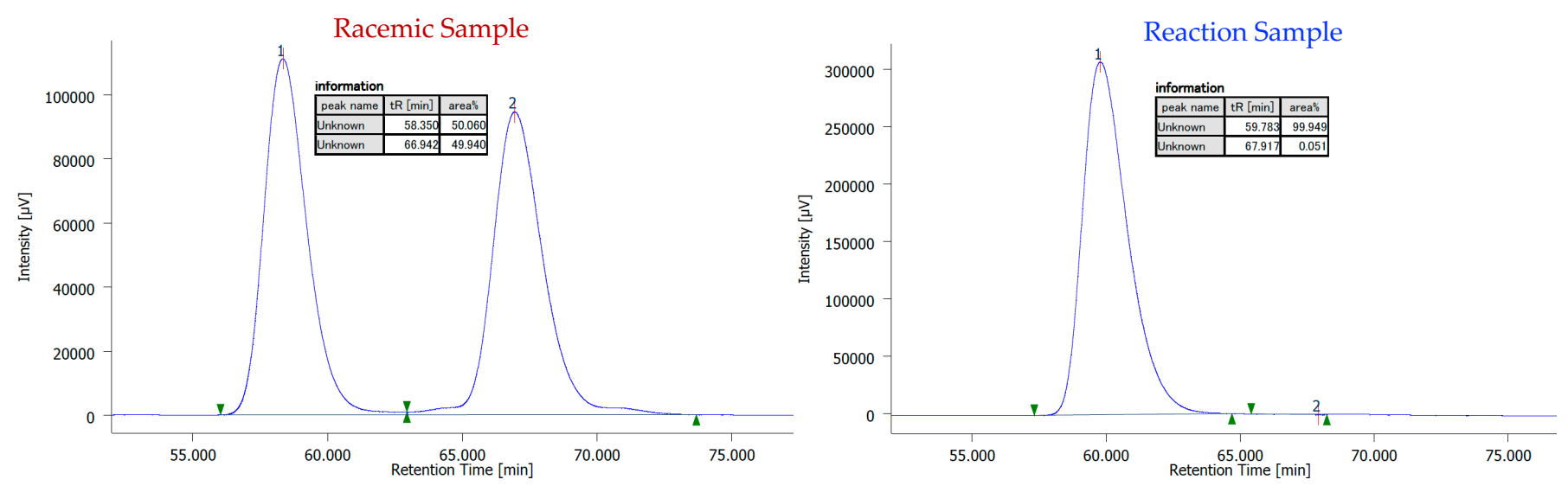


\section{Crystal Structures:}

\subsection{Solid-state structure of $2 p$}

Single crystals of $2 \mathbf{p}$ were obtained by vapor diffusion from $\mathrm{CH}_{2} \mathrm{Cl}_{2} / \mathrm{Hexane}$ at room temperature. A suitable crystal was selected and the sample was measured on a Rigaku R-AXIS RAPIS II diffractometer using multilayer mirror monochromated $\mathrm{Cu}-\mathrm{K} \alpha$ radiation $(\lambda=1.54184)$. The data were collected at $93.15 \mathrm{~K}$. Refined structure and crystallographic parameters are summarized in Table S1 and Fig. S1. CCDC 2079329 contains the supplementary crystallographic data for $\mathbf{2 p}$.

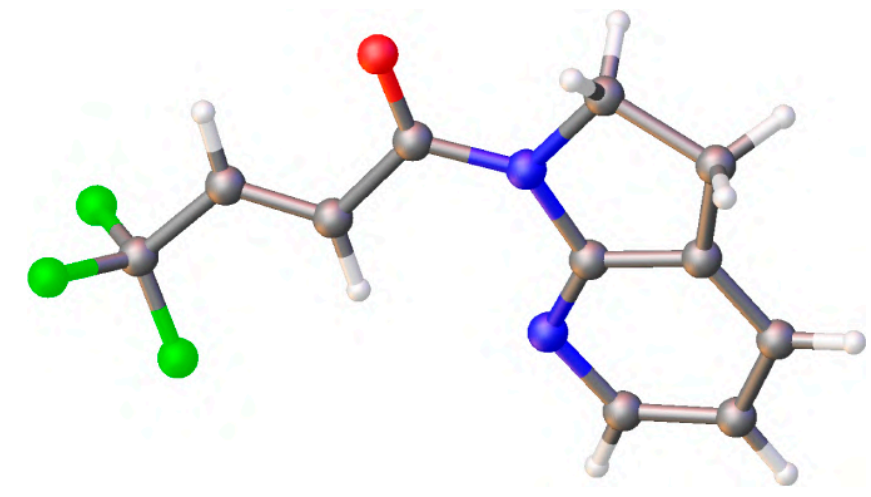

Figure S1. Structure of $2 p$ in the solid-state. Color code: red: oxygen; blue: nitrogen; gray: carbon; white: hydrogen; green: fluorine.
Table S1. Selected crystal data of $2 p$.

Empirical Formula $\quad \mathrm{C}_{11} \mathrm{H}_{9} \mathrm{~F}_{3} \mathrm{~N}_{2} \mathrm{O}$

Formula Weight

242.20

Crystal Color, Habit white, needles

Crystal Dimensions

Crystal System $0.200 \times 0.200 \times 0.100 \mathrm{~mm}$ Space group monoclinic

Lattice Parameters

$\begin{array}{lll} & \mathrm{a} & 12.90270(10) \AA \\ & \mathrm{b} & 8.76560(10) \AA \\ & \mathrm{c} & 19.0963(2) \AA \\ & \mathrm{V} & 2098.61(4) \AA^{3} \\ \mathrm{Z} \text { value } & & 8 \\ \mathrm{R}_{1} & & 0.0385 \\ \mathrm{wR}_{2} & & 0.1001 \\ \mathrm{D}_{\text {calc }} & & 1.533 \mathrm{~g} / \mathrm{cm}^{3} \\ \text { Fooo } & & 992.00\end{array}$




\subsection{Solid-state structure of 2 af}

Single crystals of 2 af were obtained by vapor diffusion from $\mathrm{CH}_{2} \mathrm{Cl}_{2} / \mathrm{Hexane}$ at room temperature. A suitable crystal was selected and the sample was measured on a Rigaku R-AXIS RAPIS II diffractometer using multilayer mirror monochromated $\mathrm{Cu}-\mathrm{K} \alpha$ radiation $(\lambda=1.54184)$. The data were collected at $93.15 \mathrm{~K}$. Refined structure and crystallographic parameters are summarized in Table S2 and Fig. S2. CCDC 2079331 contains the supplementary crystallographic data for 2 af.

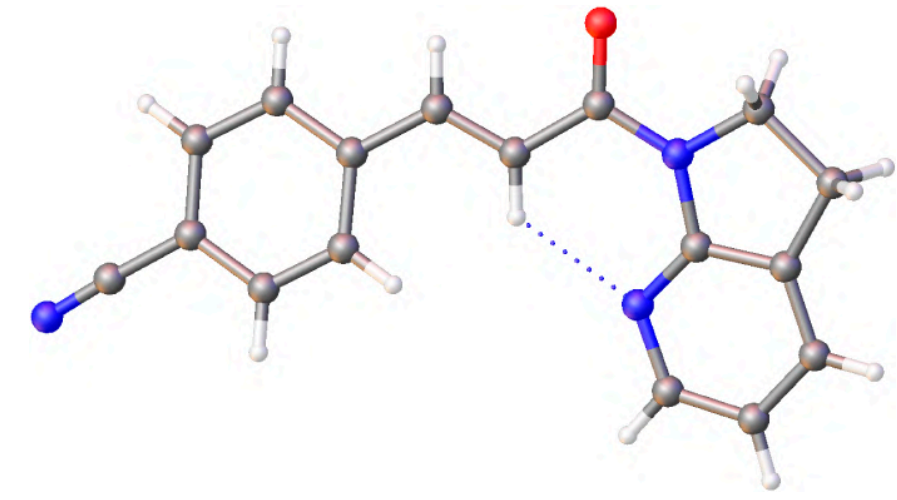

Figure S2. Structure of 2af in the solid-state. Color code: red: oxygen; blue: nitrogen; gray: carbon; white: hydrogen.
Table S2. Selected crystal data of 2af.

Empirical Formula $\quad \mathrm{C}_{17} \mathrm{H}_{13} \mathrm{~N}_{3} \mathrm{O}$

Formula Weight

275.30

Crystal Color, Habit yellow, needles

Crystal Dimensions $\quad 0.300 \times 0.300 \times 0.100 \mathrm{~mm}$

Crystal System

orthorhombic

Space group

Pbcn

Lattice Parameters

$\begin{array}{lll} & \mathrm{a} & 16.2773(2) \AA \\ & \mathrm{b} & 15.51970(10) \AA \\ & \mathrm{c} & 10.68650(10) \AA \\ & \mathrm{V} & 2699.61(5) \AA^{3} \\ \text { Z value } & & 8 \\ \mathrm{R}_{1} & & 0.0391 \\ \mathrm{wR}_{2} & & 0.1014 \\ \mathrm{D}_{\text {calc }} & & 1.355 \mathrm{~g} / \mathrm{cm}^{3} \\ \text { Fo00 } & & 1152.00\end{array}$


Single crystals of $4 a p$ were obtained by vapor diffusion from $\mathrm{CH}_{2} \mathrm{Cl}_{2} / \mathrm{Hexane}$ at room temperature. A suitable crystal was selected and the sample was measured on a Rigaku R-AXIS RAPIS II diffractometer using multilayer mirror monochromated $\mathrm{Cu}-\mathrm{K} \alpha$ radiation $(\lambda=1.54184)$. The data were collected at $93.15 \mathrm{~K}$. Refined structure and crystallographic parameters are summarized in Table S3 and Fig. S3. CCDC 2079328 contains the supplementary crystallographic data for 4ap. The absolute configuration of the cyclopropane ring is $1 R, 2 R, 3 S$.

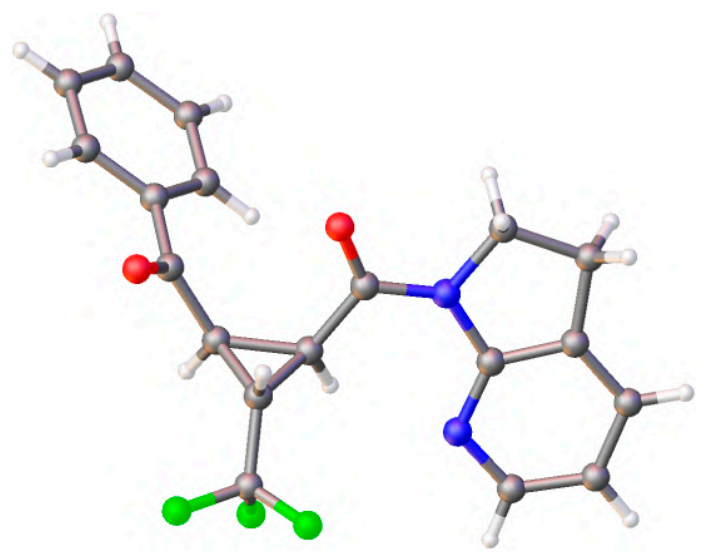

Figure S3. Structure of $4 a p$ in the solid-state. Color code: red: oxygen; blue: nitrogen; gray: carbon; white: hydrogen; green: fluorine.
Table S3. Selected crystal data of 4ap.

Empirical Formula

$\mathrm{C}_{19} \mathrm{H}_{15} \mathrm{~F}_{3} \mathrm{~N}_{2} \mathrm{O}_{2}$

Formula Weight 360.33

Crystal Color, Habit colorless, needles Crystal Dimensions Crystal System $0.300 \times 0.050 \times 0.050 \mathrm{~mm}$ orthorhombic

Space group P212121

Lattice Parameters

$\begin{array}{lll} & \mathrm{a} & 4.89770(10) \AA \\ & \mathrm{b} & 9.72600(10) \AA \\ & \mathrm{c} & 34.0348(4) \AA \\ & \mathrm{V} & 1621.25(4) \AA^{3} \\ \mathrm{Z} \text { value } & & 4 \\ \mathrm{R}_{1} & & 0.0312 \\ \mathrm{wR}_{2} & & 0.0717 \\ \mathrm{D}_{\text {calc }} & & 1.476 \mathrm{~g} / \mathrm{cm}^{3} \\ \text { Fooo } & 744.00 \\ \text { Flack parameter } & -0.02(6)\end{array}$


Single crystals of 4ap' were obtained by vapor diffusion from $\mathrm{CH}_{2} \mathrm{Cl}_{2} / \mathrm{Hexane}$ at room temperature. A suitable crystal was selected and the sample was measured on a Rigaku R-AXIS RAPIS II diffractometer using multilayer mirror monochromated $\mathrm{Cu}-\mathrm{K} \alpha$ radiation $(\lambda=1.54184)$. The data were collected at $93.15 \mathrm{~K}$. Refined structure and crystallographic parameters are summarized in Table S4 and Fig. S4. CCDC 2079332 contains the supplementary crystallographic data for $4 \mathbf{a p}^{\prime}$.

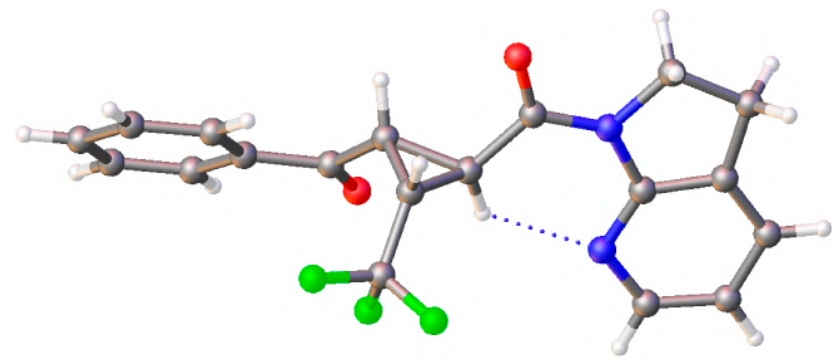

Figure S4. Structure of 4ap' in the solid-state Color code: red: oxygen; blue: nitrogen; gray: carbon; white: hydrogen; green: fluorine.
Table S4. Selected crystal data of 4ap'.

Empirical Formula $\quad \mathrm{C}_{19} \mathrm{H}_{15} \mathrm{~F}_{3} \mathrm{~N}_{2} \mathrm{O}_{2}$

Formula Weight $\quad 360.33$

Crystal Color, Habit $\quad$ colorless, needles

Crystal Dimensions $\quad 0.250 \times 0.100 \times 0.080 \mathrm{~mm}$

Crystal System triclinic

Space group $\quad$ P-1

Lattice Parameters

$\begin{array}{lll} & \mathrm{a} & 7.47030(10) \AA \\ & \mathrm{b} & 8.30920(10) \AA \\ & \mathrm{c} & 14.2834(2) \AA \\ & \mathrm{V} & 797.389(19) \AA^{3} \\ \mathrm{Z} \text { value } & & 2 \\ \mathrm{R}_{1} & & 0.0421 \\ \mathrm{wR}_{2} & & 0.1058 \\ \mathrm{D}_{\text {calc }} & & 1.501 \mathrm{~g} / \mathrm{cm}^{3} \\ \mathrm{~F}_{000} & & 372.00\end{array}$


Single crystals of 7a were obtained by vapor diffusion from $\mathrm{CH}_{2} \mathrm{Cl}_{2} / \mathrm{Hexane}$ at room temperature. A suitable crystal was selected and the sample was measured on a Rigaku R-AXIS RAPIS II diffractometer using multilayer mirror monochromated $\mathrm{Cu}-\mathrm{K} \alpha$ radiation $(\lambda=1.54184)$. The data were collected at $93.15 \mathrm{~K}$. Refined structure and crystallographic parameters are summarized in Table S5 and Fig. S5. CCDC 2079330 contains the supplementary crystallographic data for $7 \mathbf{a}$. The absolute configuration of the cyclopropane ring is $1 R, 2 R, 3 S$.

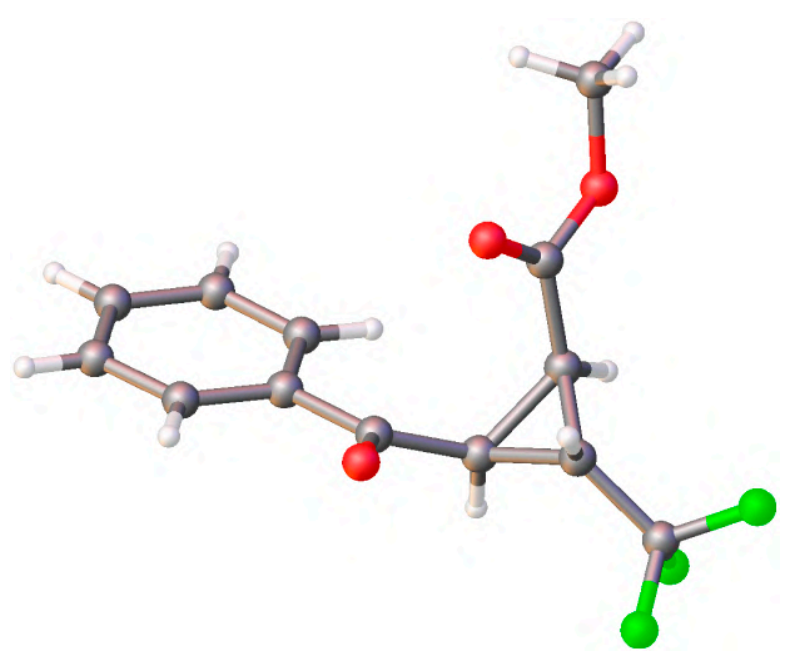

Figure S5. Structure of $7 \mathrm{a}$ in the solid-state.

Color code: red: oxygen; gray: carbon; white: hydrogen; green: fluorine.
Table S5. Selected crystal data of $7 \mathbf{a}$

Empirical Formula $\quad \mathrm{C}_{13} \mathrm{H}_{11} \mathrm{~F}_{3} \mathrm{O}_{3}$

Formula Weight $\quad 272.22$

Crystal Color, Habit colorless, needles

Crystal Dimensions $0.300 \times 0.200 \times 0.080 \mathrm{~mm}$

Crystal System monoclinic

Space group $\quad \mathrm{P} 21$

Lattice Parameters

$\begin{array}{ll}\mathrm{a} & 5.14490(10) \AA \\ \mathrm{b} & 8.4717(2) \AA \\ \mathrm{c} & 13.8636(2) \AA \\ \mathrm{V} & 604.13(2) \AA^{3}\end{array}$

$\mathrm{Z}$ value 2

$\mathrm{R}_{1} \quad 0.0387$

$\mathrm{wR}_{2} \quad 0.1042$

D calc $\quad 1.496 \mathrm{~g} / \mathrm{cm}^{3}$

Foo0 280.00

Flack parameter $\quad 0.02(9)$ 


\section{References}

1. The representative references for the synthesis of ylides, see:

(a) Chen, W.; Luo, F.; Wu, Y.; Cen, J.; Shao J.; Yu, Y., Efficient access to fluorescent benzofuro[3,2$b$ ]carbazoles via TFA-promoted cascade annulations of sulfur ylides, 2-hydroxy- $\beta$-nitrostyrenes and indoles. Org. Chem. Front., 2020, 7, 873-878.

(b) Yu, P. Ushakov; Khatuntseva, E. A.; Nelyubina, Y. V.; Tabolin, A. A.; Ioffe, Sukhorukov, A. Y., Synthesis of Isoxazolines from Nitroalkanes via a [4+1]-Annulation Strategy. Adv. Synth. Catal. 2019, 361, 5322-5327.

(c) Dong, J.; Du, H.; Xu, J., Synthesis of Functionalized Thietanes via Electrophilic Carbenoid-Induced Ring Expansion of Thiiranes with Sulfonium Acylmethylides as Carbene Precursors. J. Org. Chem. 2019, 84, 10724-10739.

(d) Nambu, H.; Onuki, Y.; Ono, N.; Tsuge, K.; Yakura, T., Ring-opening cyclization of spirocyclopropanes with stabilized sulfonium ylides for the construction of a chromane skeleton. Chem. Commun., 2019, 55, 6539-6542.

(e) Wang, J.; Liu, X.; Dong, S.; Lin, L. Feng, X., Asymmetric Organocatalytic Cyclopropanation of Cinnamone Derivatives with Stabilized Sulfonium Ylides. J. Org. Chem. 2013, 78, 6322-6327.

2. The representative references for the synthesis of 7-azaindoline and related derivatives, see:

(a) Pagire, S. K.; Kumagai, N.; Shibasaki, M., Introduction of a 7-aza-6-MeO-indoline auxiliary in Lewisacid/photoredox cooperative catalysis: highly enantioselective aminomethylation of $\alpha, \beta$-unsaturated amides. Chem. Sci. 2020, 11, 5168-5174.

(b) Casado-Sánchez, A.; Domingo-Legarda, P.; Cabrera, S.; Alemán, J., Visible light photocatalytic asymmetric synthesis of pyrrolo[1,2-a]indoles via intermolecular [3+2] cycloaddition. Chem. Commun., 2019, 55, 1130311306

(c) Brewitz, L.; Noda, H.; Kumagai, N.; Shibasaki, M., Structural and Computational Investigation of Intramolecular N...H Interactions in $\alpha$ - and $\beta$-Fluorinated 7-Azaindoline Amides. Eur. J. Org. Chem. 2018, 714722.

(d) Zhang, M.; Kumagai, N.; Shibasaki, M., $\alpha, \beta$-Unsaturated Amides as Dipolarophiles: Catalytic Asymmetric exo-Selective 1,3-Dipolar Cycloaddition with Nitrones. Chem. Eur. J. 2017, 23, 12450-12455.

(e) Zhang, M.; Kumagai, N.; Shibasaki, M., Electrophilic Activation of $\alpha, \beta$-Unsaturated Amides: Catalytic Asymmetric Vinylogous Conjugate Addition of Unsaturated $\gamma$-Butyrolactones. Chem. Eur. J. 2016, 22, 55255529.

3. For Schmidt reaction procedure, see: Yadav, J. S.; Subba Reddy, B. V.; Subba Reddy, U. V.; Praneeth, K., Azido-Schmidt Reaction for the Formation of Amides, Imides and Lactams from Ketones in the Presence of $\mathrm{FeCl}_{3}$. Tetrahedron Lett. 2008, 49, 4742-4745. 
11. Representative 7-azaindolines and their copper complexes in $\mathrm{CD}_{2} \mathrm{Cl}_{2}$ :

[A] Before complexation with 7-azaindolines:

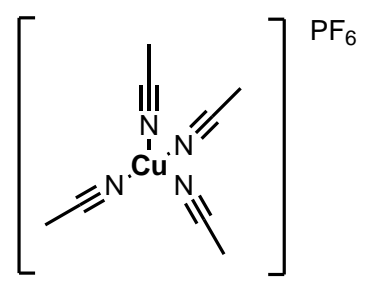

Tetrakis(acetonitrile)copper(I) hexafluorophosphate $\left[\mathrm{Cu}\left(\mathrm{CH}_{3} \mathrm{CN}\right)_{4}\right] \mathrm{PF} 6$ :

Colorless solid.

${ }^{1} \mathrm{H}$ NMR (400 MHz, $\left.300 \mathrm{~K}, \mathrm{CD}_{2} \mathrm{Cl}_{2}\right)$ : $\delta 2.18$ (s, 12H).

${ }^{13} \mathrm{C}$ NMR (101 MHz, $\left.300 \mathrm{~K}, \mathrm{CD}_{2} \mathrm{Cl}_{2}\right): \delta 117.4,2.7$.

${ }^{19} \mathrm{~F}$ NMR (376 MHz, $\left.300 \mathrm{~K}, \mathrm{CD}_{2} \mathrm{Cl}_{2}\right)$ : $\delta$-70.54, -72.43.

31P NMR (162 MHz, 300 K, CD2 $\mathbf{C l}_{2}$ ): $\delta$-144.55 (hept, $J=710.1 \mathrm{~Hz}$ ).

(S)-(+)-5,5'-Bis[di(3,5-di-tert-butyl-4-methoxyphenyl)phosphino]-4,4'-bi-1,3-benzodioxole

[(S)-DTBMSegphos]:
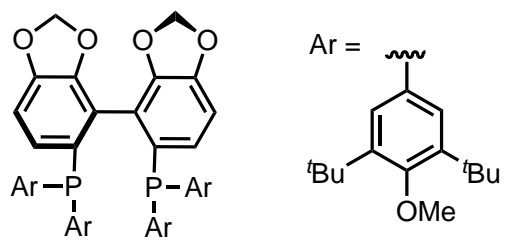

Colorless solid.

${ }^{1} \mathrm{H}$ NMR (400 MHz, $\left.300 \mathrm{~K}, \mathrm{CD}_{2} \mathrm{Cl}_{2}\right): \delta 7.29-7.26(\mathrm{~m}, 4 \mathrm{H}), 7.13-7.09(\mathrm{~m}$, $4 \mathrm{H}), 6.75(\mathrm{~s}, 1 \mathrm{H}), 6.73(\mathrm{~s}, 1 \mathrm{H}), 6.62-6.60(\mathrm{~m}, 1 \mathrm{H}), 6.60-6.58(\mathrm{~m}, 1 \mathrm{H}), 5.69(\mathrm{~d}$, $J=1.6 \mathrm{~Hz}, 2 \mathrm{H}), 4.94(\mathrm{~d}, J=1.6 \mathrm{~Hz}, 2 \mathrm{H}), 3.68(\mathrm{~s}, 6 \mathrm{H}), 3.64(\mathrm{~s}, 6 \mathrm{H}), 1.32(\mathrm{~s}, 36 \mathrm{H})$, $1.32(\mathrm{~s}, 36 \mathrm{H})$.

${ }^{13} \mathrm{C}$ NMR (101 MHz, 300 K, CD $\left.\mathrm{Cl}_{2}\right)$ : $\delta$ 160.9, 160.2, 148.1, 147.2, 147.1, 147.1, 143.7, 143.6, 143.6, 143.4, 143.4, 143.3, 133.4, 133.4, 133.3, 133.3, 133.2, 132.8, 132.7, 132.6, 132.6, 132.5, 132.5, 132.0, 131.9, 131.9, 128.1, 122.3, 122.1, 121.9, 108.5, 101.3, 64.8, 64.7, 36.3, 36.2, 32.5, 32.3.

${ }^{31}$ P NMR (162 MHz, $\left.300 \mathrm{~K}, \mathrm{CD}_{2} \mathrm{Cl}_{2}\right)$ : $\delta$-13.66.

$\left[\mathrm{Cu}\left(\mathrm{CH}_{3} \mathrm{CN}\right)_{2}(S)-D T B M-S e g p h o s\right] \mathrm{PF}_{6}+2 \mathrm{CH}_{3} \mathrm{CN}$ :

${ }^{1} \mathrm{H}$ NMR (400 MHz, 300 K, $\left.\mathrm{CD}_{2} \mathrm{Cl}_{2}\right): \delta 7.62(\mathrm{t}, J=5.6 \mathrm{~Hz}, 4 \mathrm{H}), 7.29(\mathrm{t}$, $J=5.7 \mathrm{~Hz}, 4 \mathrm{H}), 6.56(\mathrm{q}, J=8.1,6.2 \mathrm{~Hz}, 4 \mathrm{H}), 5.59(\mathrm{~s}, 2 \mathrm{H}), 5.50(\mathrm{~s}, 2 \mathrm{H})$,

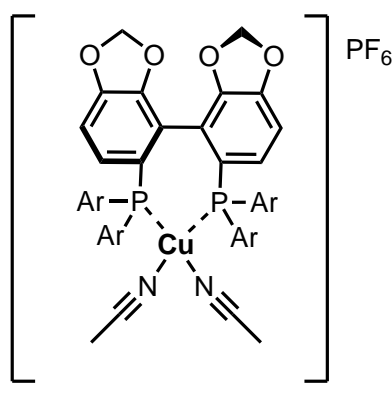

$\mathrm{Ar}=$ $3.76(\mathrm{~s}, 6 \mathrm{H}), 3.66(\mathrm{~s}, 6 \mathrm{H}), 2.12(\mathrm{~s}, 12 \mathrm{H}), 1.45(\mathrm{~s}, 36 \mathrm{H}), 1.31(\mathrm{~s}, 36 \mathrm{H})$. ${ }^{13} \mathrm{C}$ NMR (101 MHz, $\left.300 \mathrm{~K}, \mathrm{CD}_{2} \mathrm{Cl}_{2}\right)$ : $\delta$ 162.5, 162.4, 148.4, 147.8, $147.7,147.7,145.3,145.3,145.2,144.3,144.3,144.2,133.9,133.8,133.7$, $132.8,132.8,132.7,128.1,127.9,127.7,126.9,126.7,126.6,126.0,125.8$, $125.6,125.3,125.2,125.2,118.7,118.7,118.6,118.4,108.5,108.5,108.5$, 101.2, 65.0, 64.7, 36.6, 36.2, 32.4, 32.2.

${ }^{19} \mathrm{~F}$ NMR (376 MHz, $300 \mathrm{~K}, \mathrm{CD}_{2} \mathrm{Cl}_{2}$ ): $\delta$-72.36, -74.25.

${ }^{31}$ P NMR (162 MHz, $300 \mathrm{~K}, \mathrm{CD}_{2} \mathrm{Cl}_{2}$ ): $\delta-2.41,-144.50$ (hept, $J=710.1$ $\mathrm{Hz}$ ). 
[B] Pure 7-azaindolines:

(E)-1-(2,3-dihydro-1H-pyrrolo[2,3-b]pyridin-1-yl)but-2-en-1-one (2b):

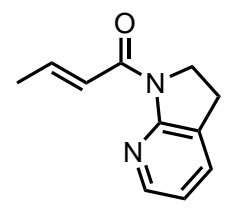

Colorless solid.

${ }^{1} \mathrm{H}$ NMR (400 MHz, 300 K, CD $\left.\mathbf{C l}_{2}\right)$ : $\delta 8.10(\mathrm{ddt}, J=5.1,1.8,1.0 \mathrm{~Hz}, 1 \mathrm{H}), 7.82(\mathrm{dq}, J=15.2$, $1.7 \mathrm{~Hz}, 1 \mathrm{H}), 7.47(\mathrm{dq}, J=7.3,1.4 \mathrm{~Hz}, 1 \mathrm{H}), 7.04(\mathrm{dq}, J=15.2,6.9 \mathrm{~Hz}, 1 \mathrm{H}), 6.87(\mathrm{dd}, J=7.3,5.1$ $\mathrm{Hz}, 1 \mathrm{H}), 4.12-4.05(\mathrm{~m}, 2 \mathrm{H}), 3.04(\mathrm{~m}, 2 \mathrm{H}), 1.96(\mathrm{dd}, J=6.9,1.7 \mathrm{~Hz}, 3 \mathrm{H})$.

${ }^{13} \mathrm{C}$ NMR (101 MHz, 300 K, $\mathrm{CD}_{2} \mathrm{Cl}_{2}$ ): $\delta$ 165.5, 156.9, 146.5, 142.4, 134.0, 127.1, 125.4, 118.3, $46.4,24.8,18.6$.

(E)-1-(6-phenyl-2,3-dihydro-1H-pyrrolo[2,3-b]pyridin-1-yl)but-2-en-1-one (2k):

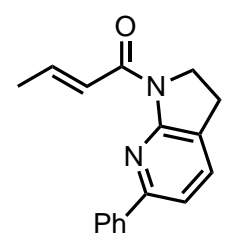

Colorless solid.

${ }^{1} \mathrm{H}$ NMR (400 MHz, $\left.300 \mathrm{~K}, \mathrm{CD}_{2} \mathrm{Cl}_{2}\right): \delta 8.09-7.99(\mathrm{~m}, 3 \mathrm{H}), 7.54(\mathrm{dt}, J=7.7,1.3 \mathrm{~Hz}, 1 \mathrm{H}), 7.51$ $7.45(\mathrm{~m}, 2 \mathrm{H}), 7.44-7.36(\mathrm{~m}, 2 \mathrm{H}), 7.06(\mathrm{dq}, J=15.3,6.9 \mathrm{~Hz}, 1 \mathrm{H}), 4.14(\mathrm{dd}, J=9.2,7.7 \mathrm{~Hz}, 2 \mathrm{H})$, $3.07(\mathrm{ddd}, J=9.6,7.5,1.3 \mathrm{~Hz}, 2 \mathrm{H}), 2.02(\mathrm{dd}, J=6.9,1.7 \mathrm{~Hz}, 3 \mathrm{H})$.

${ }^{13} \mathrm{C}$ NMR (101 MHz, $\left.300 \mathrm{~K}, \mathrm{CD}_{2} \mathrm{Cl}_{2}\right)$ : $\delta$ 165.6, 156.8, 154.6, 141.9, 139.6, 134.6, 129.3, 129.3, 129.2, 127.0, 126.0, 125.8, 114.7, 46.6, 24.6, 18.8 . 
[Cu(2b)(S)-DTBM-Segphos]PF $6+4 \mathrm{CH}_{3} \mathrm{CN}$ :

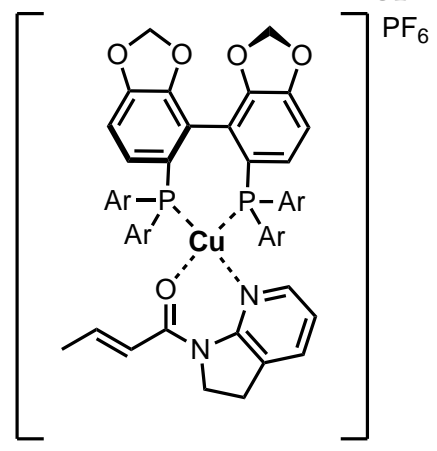

${ }_{\mathrm{OMe}}^{\mathrm{Ar}}=$
${ }^{1} \mathrm{H}$ NMR (400 MHz, $\left.300 \mathrm{~K}, \mathrm{CD}_{2} \mathrm{Cl}_{2}\right): \delta 7.80-7.38(\mathrm{~m}, 5 \mathrm{H}), 7.27(\mathrm{q}, J=$ 7.6, 7.2 Hz, 2H), 7.05-6.86 (m, 3H), $6.68(\mathrm{dd}, J=23.5,8.0 \mathrm{~Hz}, 2 \mathrm{H}), 6.55$ $-6.30(\mathrm{~m}, 2 \mathrm{H}), 5.84-5.57(\mathrm{~m}, 2 \mathrm{H}), 5.42(\mathrm{~s}, 1 \mathrm{H}), 4.32(\mathrm{dd}, J=44.9,9.3$ $\mathrm{Hz}, 2 \mathrm{H}), 3.69(\mathrm{~s}, 6 \mathrm{H}), 3.65(\mathrm{~s}, 6 \mathrm{H}), 3.29(\mathrm{~d}, J=9.4 \mathrm{~Hz}, 2 \mathrm{H}), 1.99(\mathrm{~s}, 12 \mathrm{H})$, $1.36(\mathrm{~s}, 18 \mathrm{H}), 1.31(\mathrm{~s}, 18 \mathrm{H}), 1.24(\mathrm{~s}, 18 \mathrm{H}), 1.22(\mathrm{~s}, 18 \mathrm{H})$.

${ }^{13} \mathrm{C}$ NMR (101 MHz, $\left.300 \mathrm{~K}, \mathrm{CD}_{2} \mathrm{Cl}_{2}\right)$ : $\delta 167.7,162.3,162.2,156.4,149.2$, 148.7, 148.4, 147.6, 145.0, 144.8, 144.3, 136.2, 133.8, 133.7, 133.5, 132.5, $129.7,128.4,128.3,127.6,127.4,126.7,126.2,124.9,123.0,121.6,119.2$, 118.4, 117.3, 108.6, 101.2, 64.9, 64.6, 48.9, 36.3, 36.2, 32.3, 32.1, 25.2, 18.7 . ${ }^{19} \mathrm{~F}$ NMR (376 MHz, $\left.300 \mathrm{~K}, \mathrm{CD}_{2} \mathrm{Cl}_{2}\right)$ : $\delta-72.43,-74.32$.

${ }^{31}$ P NMR (162 MHz, $300 \mathrm{~K}, \mathrm{CD}_{2} \mathrm{Cl}_{2}$ ): $\delta-4.23,-144.45$ (hept, $J=710.2$ $\mathrm{Hz})$.

$[\mathrm{Cu}(2 \mathrm{k})(S)-\mathrm{DTBM}-\mathrm{Segphos}] \mathrm{PF}_{6}+4 \mathrm{CH}_{3} \mathrm{CN}$ :

${ }^{1} \mathrm{H}$ NMR $\left(400 \mathrm{MHz}, 300 \mathrm{~K}, \mathrm{CD}_{2} \mathrm{Cl}_{2}\right): \delta 8.02(\mathrm{~d}, J=7.7 \mathrm{~Hz}, 3 \mathrm{H}), 7.64(\mathrm{t}$,

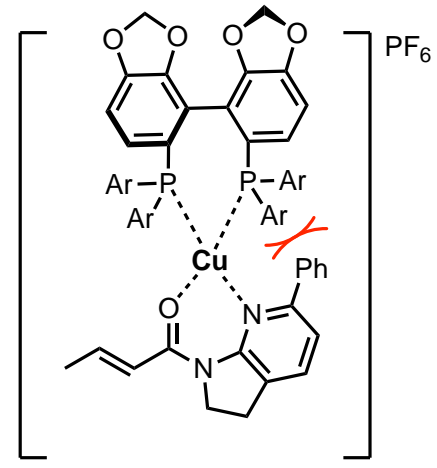
$J=5.6 \mathrm{~Hz}, 4 \mathrm{H}), 7.57(\mathrm{~d}, J=7.7 \mathrm{~Hz}, 1 \mathrm{H}), 7.48(\mathrm{t}, J=7.5 \mathrm{~Hz}, 2 \mathrm{H}), 7.44-$ $7.38(\mathrm{~m}, 2 \mathrm{H}), 7.31(\mathrm{t}, J=5.7 \mathrm{~Hz}, 4 \mathrm{H}), 7.06(\mathrm{dq}, J=13.9,6.9 \mathrm{~Hz}, 1 \mathrm{H}), 6.64$ $-6.51(\mathrm{~m}, 4 \mathrm{H}), 5.65-5.55(\mathrm{~m}, 2 \mathrm{H}), 5.51(\mathrm{~s}, 2 \mathrm{H}), 4.14(\mathrm{t}, J=8.4 \mathrm{~Hz}, 2 \mathrm{H})$, $3.77(\mathrm{~s}, 6 \mathrm{H}), 3.67(\mathrm{~s}, 6 \mathrm{H}), 3.08(\mathrm{t}, J=8.4 \mathrm{~Hz}, 2 \mathrm{H}), 2.12(\mathrm{~s}, 12 \mathrm{H}), 2.02(\mathrm{dd}$,<smiles>COc1c(Br)cc(C[Mg])cc1Br</smiles>
$J=6.9,1.7 \mathrm{~Hz}, 3 \mathrm{H}), 1.47(\mathrm{~s}, 36 \mathrm{H}), 1.47(\mathrm{~s}, 36 \mathrm{H})$.

${ }^{13} \mathrm{C}$ NMR (101 MHz, 300 K, CD $\left.\mathbf{C l}_{2}\right)$ : $\delta 165.6,162.5,162.3,156.7,154.6$, 148.4, 147.8, 147.7, 145.3, 145.2, 144.3, 144.2, 141.8, 139.5, 134.7, 133.9, $133.8,133.7,132.9,132.8,132.7,129.3,129.2,128.1,128.0,127.8,127.0$, $126.9,126.8,126.6,126.0,125.9,125.8,125.6,125.2,118.8,118.7,118.6$, $118.5,114.9,108.5,108.4,101.2,65.0,64.7,46.6,36.6,36.2,32.4,32.2$, 24.6, 18.8 .

${ }^{19}$ F NMR (376 MHz, $\left.300 \mathrm{~K}, \mathrm{CD}_{2} \mathrm{Cl}_{2}\right)$ : $\delta$-72.27, -74.16. ${ }^{31 P}$ NMR (162 MHz, 300 K, $\mathrm{CD}_{2} \mathbf{C l}_{2}$ ): $\delta-2.55,-144.46$ (hept, $J=710.2$ $\mathrm{Hz})$. 


\section{NMR Spectra:}

[Note: unless otherwise stated, all the NMR spectra were recorded in $\mathrm{CDCl}_{3}$ ]. ${ }^{1} \mathrm{H}$ NMR: $\mathbf{C u}\left(\mathbf{C H}_{3} \mathbf{C N}\right)_{4} \mathbf{P F}_{6}\left(\mathrm{CD}_{2} \mathrm{Cl}_{2}\right)$

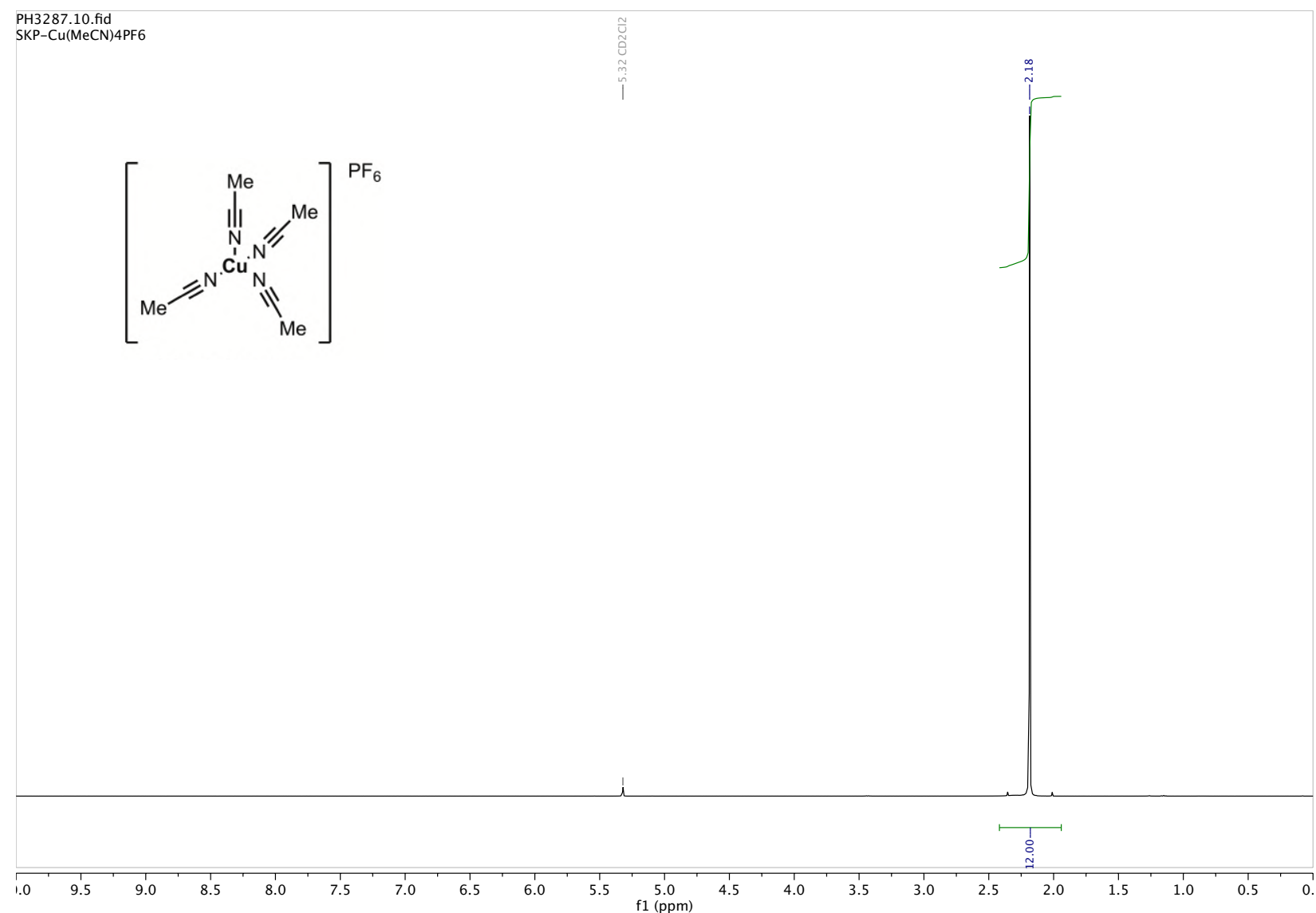

${ }^{13} \mathrm{C}$ NMR: $\mathrm{Cu}\left(\mathrm{CH}_{3} \mathrm{CN}\right)_{4} \mathrm{PF}_{6}\left(\mathrm{CD}_{2} \mathrm{Cl}_{2}\right)$

PH3287.12.fid

SKP-Cu(MeCN)4PF6

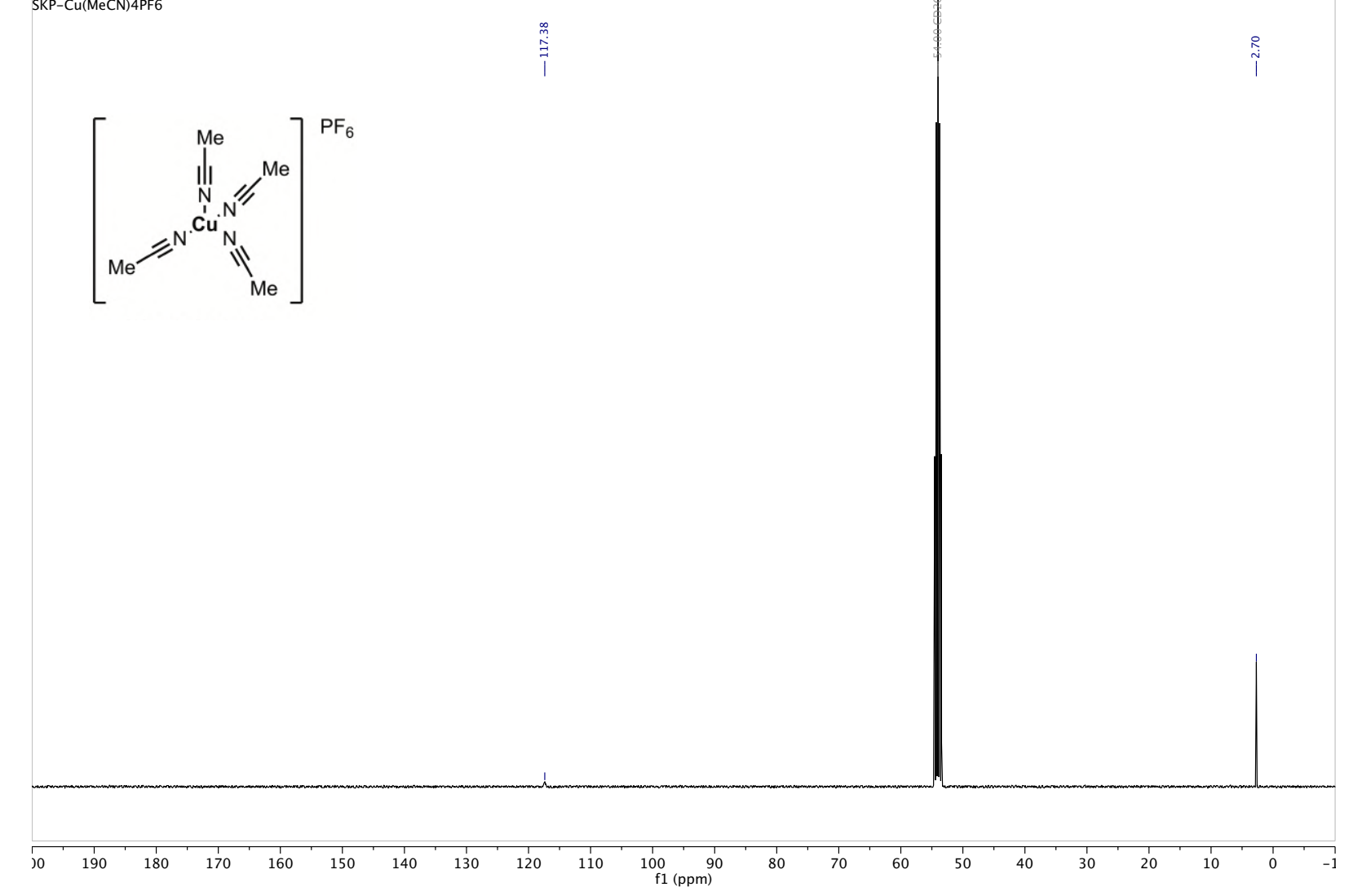


${ }^{19} \mathrm{~F}$ NMR: $\mathrm{Cu}\left(\mathrm{CH}_{3} \mathrm{CN}\right)_{4} \mathrm{PF}_{6}\left(\mathrm{CD}_{2} \mathrm{Cl}_{2}\right)$

PH3287.11.fid

PH3287.11.fid
SKP-Cu(MeCN)4PF6

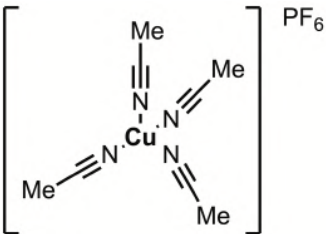

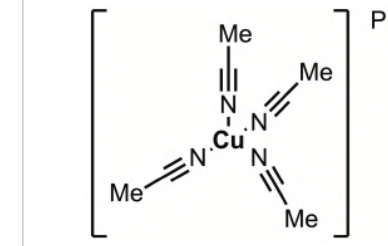

1)

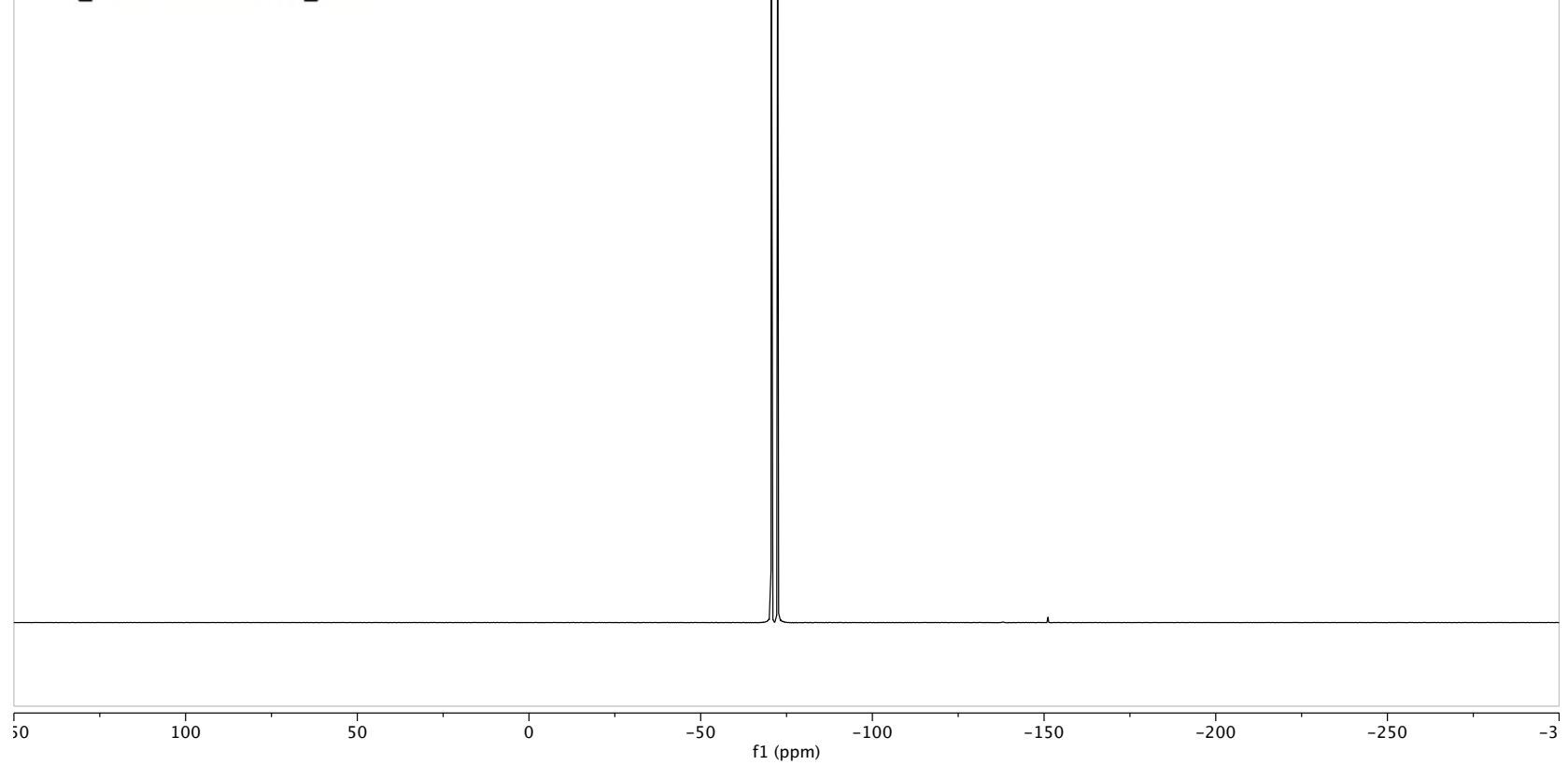

${ }^{31} P$ NMR: $\mathrm{Cu}\left(\mathrm{CH}_{3} \mathrm{CN}\right)_{4} \mathrm{PF}_{6}\left(\mathrm{CD}_{2} \mathrm{Cl}_{2}\right)$

PH3410.11.fid
Cu(MeCN)4PF6
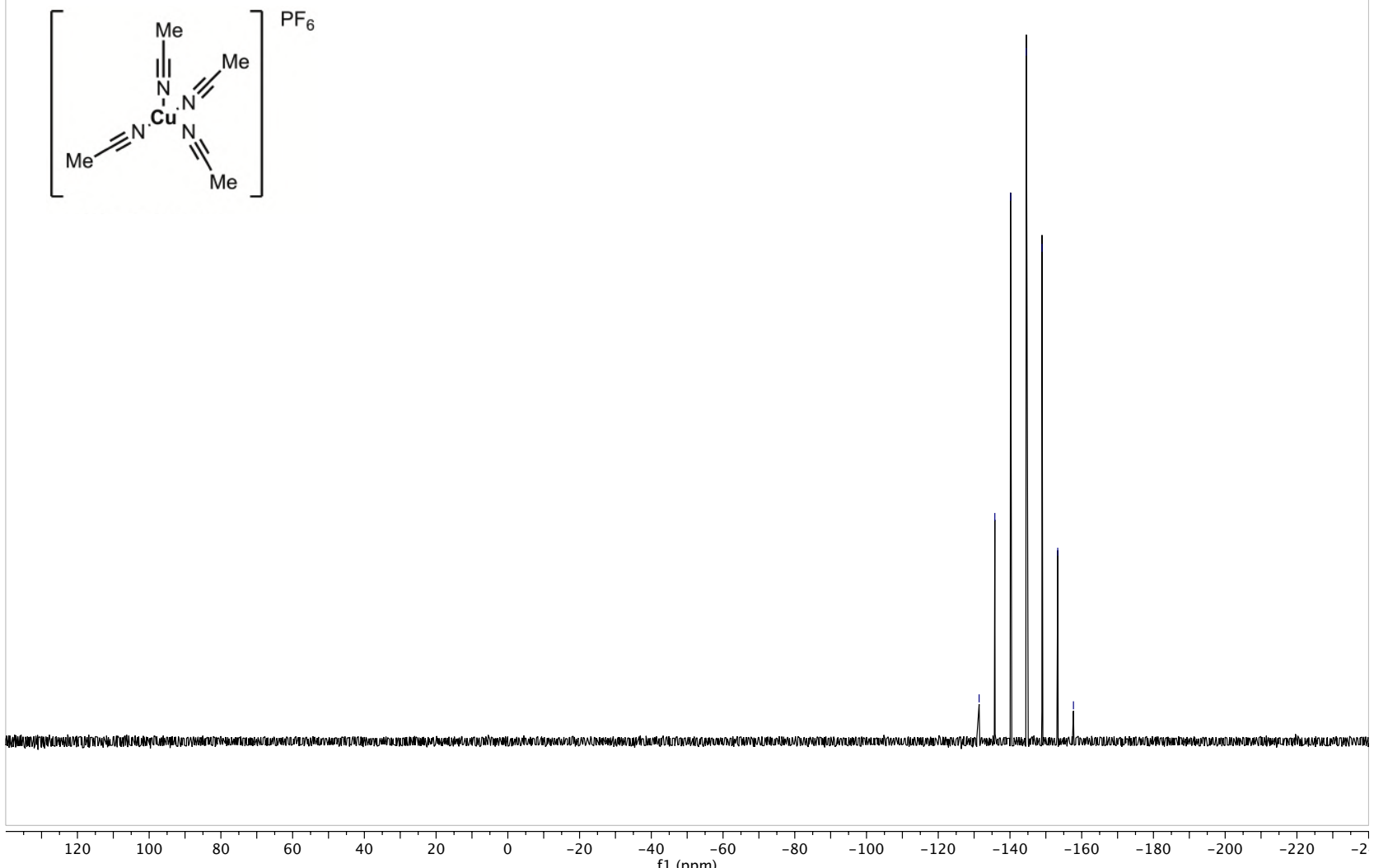
${ }^{1} \mathrm{H}$ NMR: (S)-DTBM-Segphos $\left(\mathrm{CD}_{2} \mathrm{Cl}_{2}\right)$

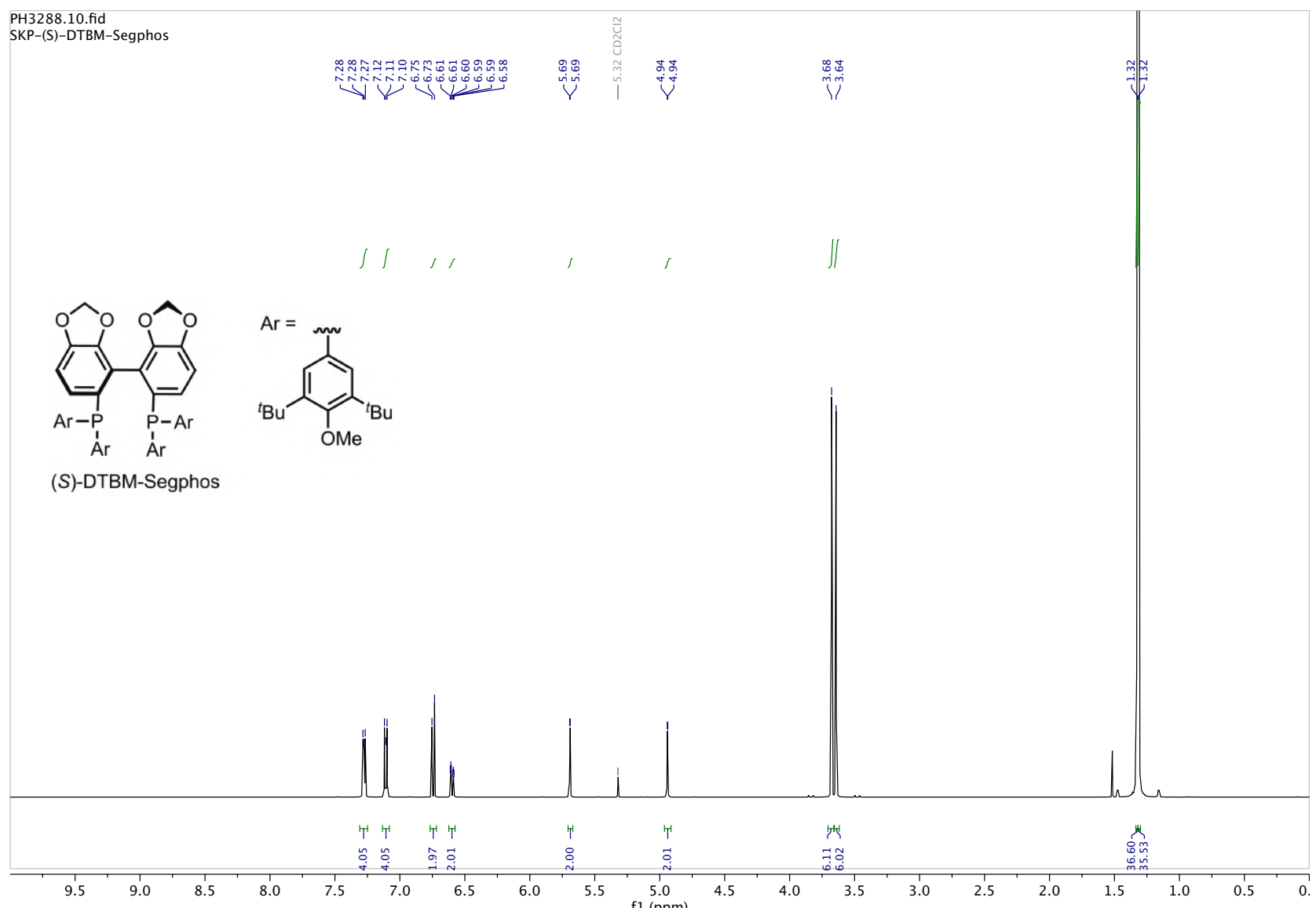

${ }^{13}$ C NMR: (S)-DTBM-Segphos $\left(\mathrm{CD}_{2} \mathrm{Cl}_{2}\right)$

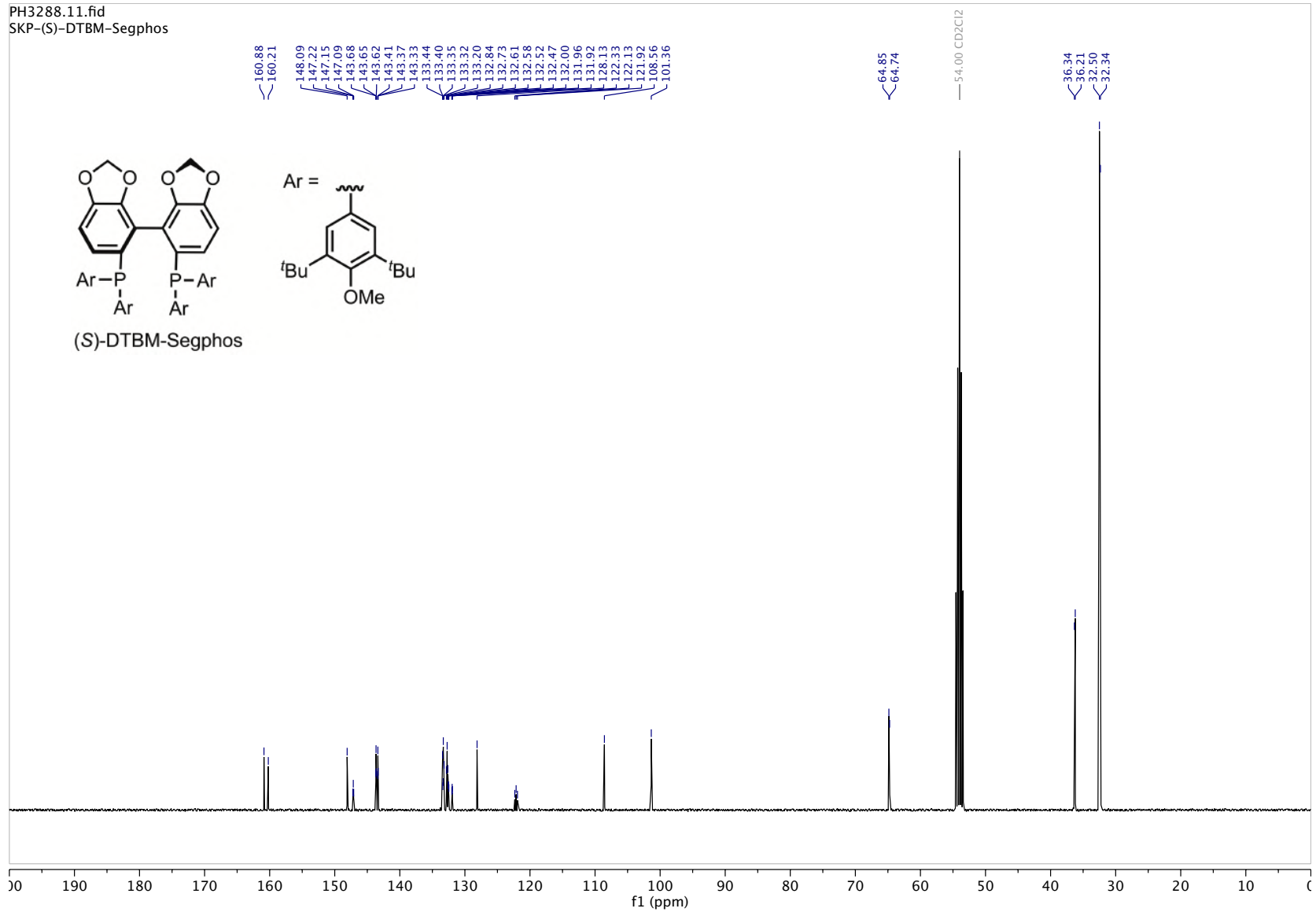


${ }^{31}$ P NMR: (S)-DTBM-Segphos $\left(\mathrm{CD}_{2} \mathrm{Cl}_{2}\right)$

PH3397.11.fi

DTBM-Segphos in DCM
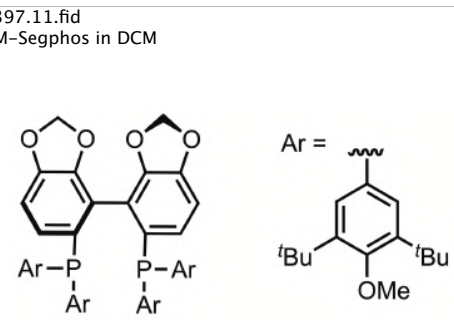

(S)-DTBM-Segphos

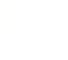

$+2$

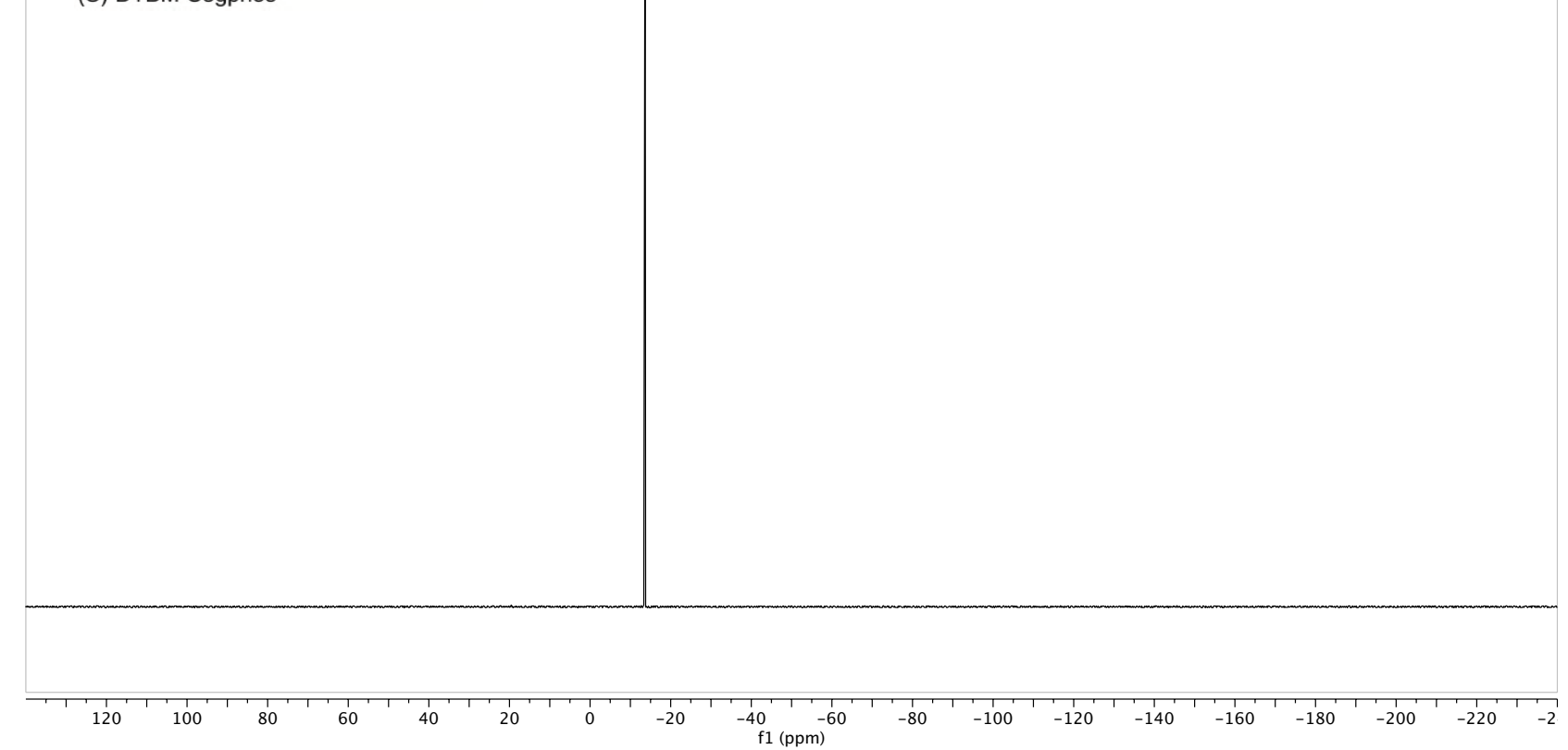

${ }^{1} \mathrm{H}$ NMR: From top: 3: $\left[\mathrm{Cu}\left(\mathrm{CH}_{3} \mathrm{CN}\right)_{2}(S)-\mathrm{DTBM}-S e g p h o s\right] \mathrm{PF}_{6}+2 \mathrm{CH}_{3} \mathrm{CN}$; 2: (S)-DTBM-Segphos; 1: $\mathrm{Cu}\left(\mathrm{CH}_{3} \mathrm{CN}_{4} \mathrm{PF}_{6}\left(\mathrm{CD}_{2} \mathrm{Cl}_{2}\right)\right.$

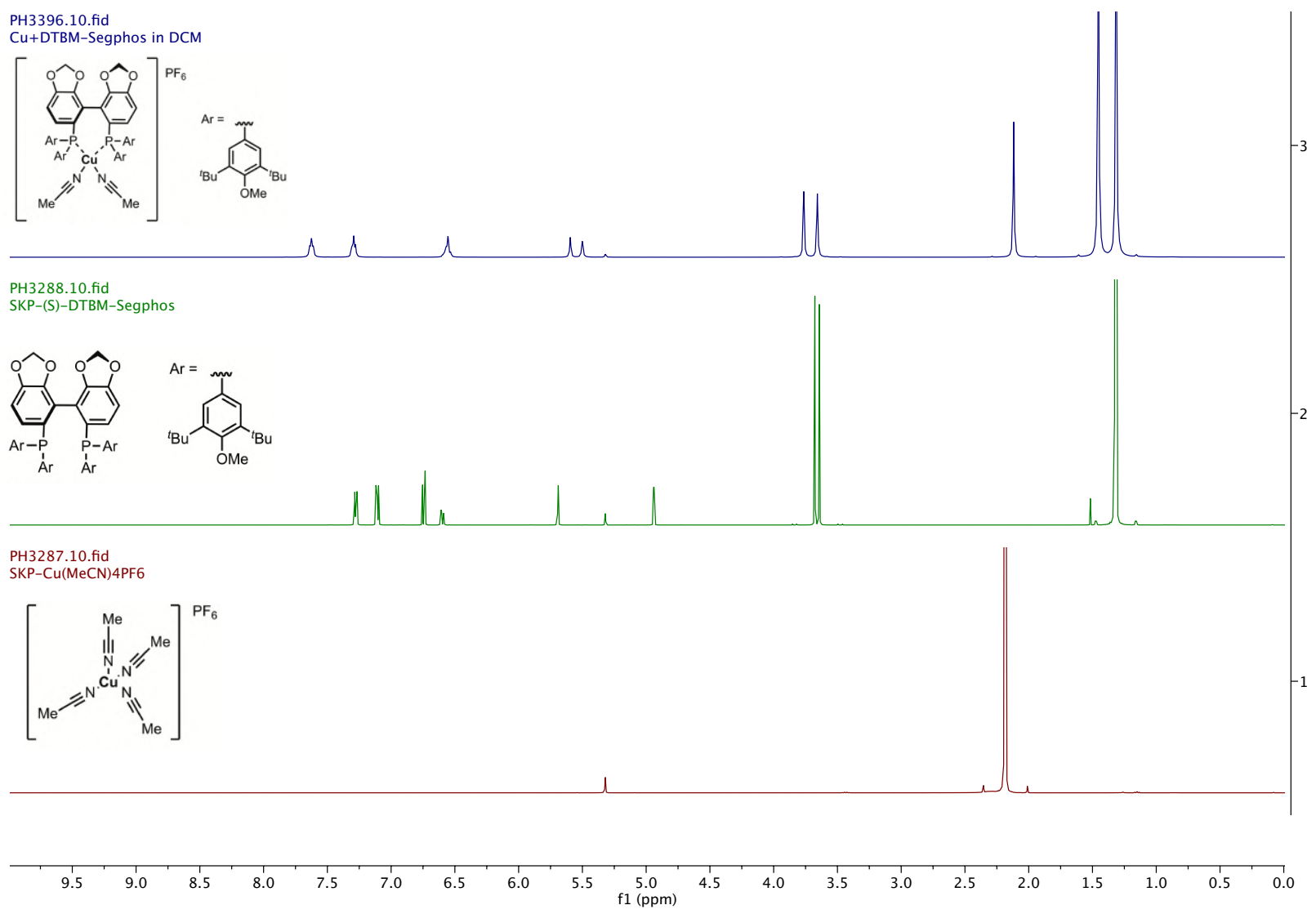


${ }^{1} \mathrm{H}$ NMR: $\left[\mathrm{Cu}\left(\mathrm{CH}_{3} \mathrm{CN}\right)_{2}(S)-\mathrm{DTBM}-S e g p h o s\right] \mathrm{PF}_{6}+2 \mathrm{CH}_{3} \mathrm{CN}\left(\mathrm{CD}_{2} \mathrm{Cl}_{2}\right)$

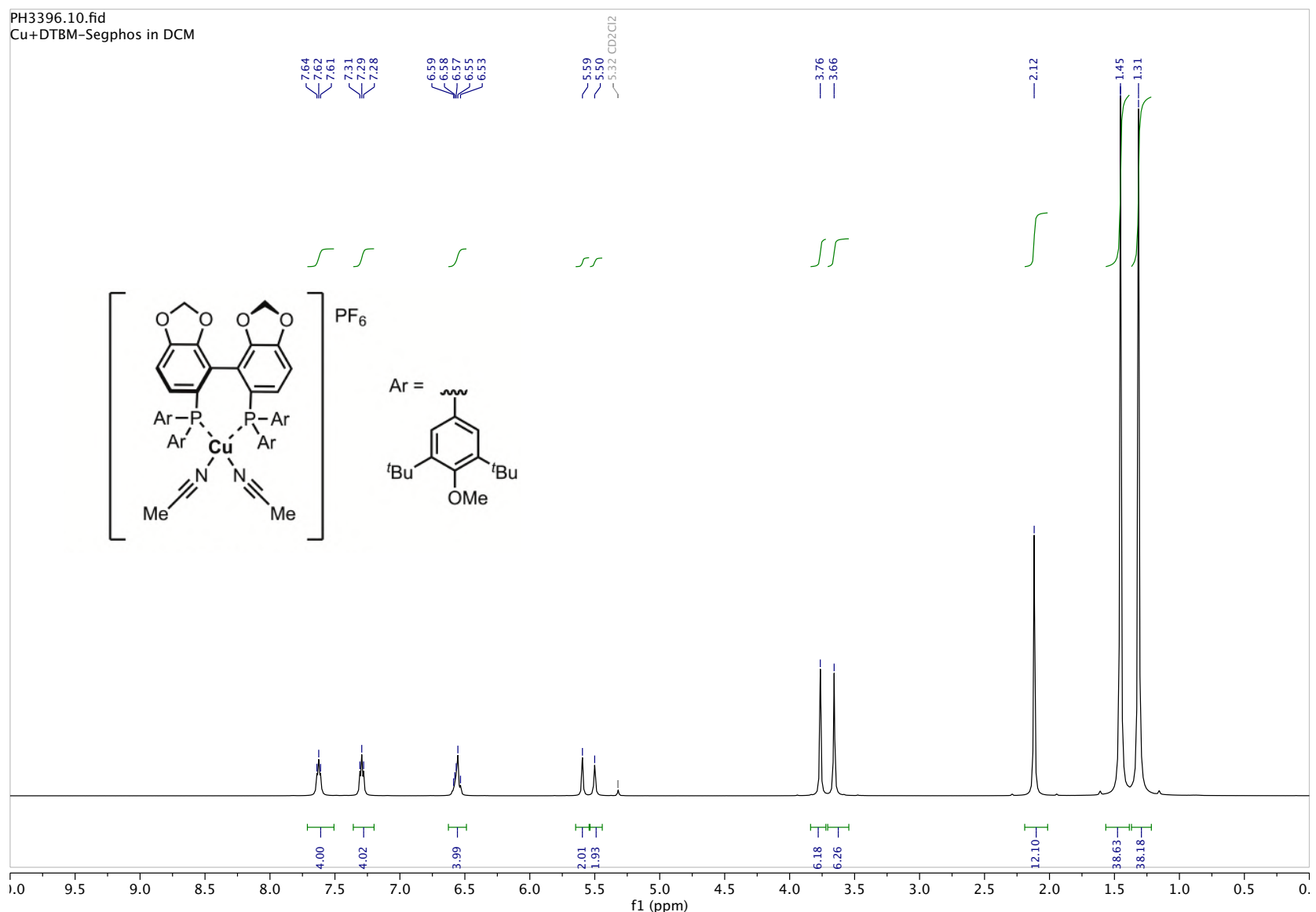

${ }^{13} \mathrm{C}$ NMR: $\left[\mathrm{Cu}\left(\mathrm{CH}_{3} \mathrm{CN}\right)_{2}(S)-\mathrm{DTBM}-S e g p h o s\right] \mathrm{PF}_{6}+2 \mathrm{CH}_{3} \mathrm{CN}\left(\mathrm{CD}_{2} \mathrm{Cl}_{2}\right)$

PH3396.14. fid

Cu+DTBM-Segphos in DCM
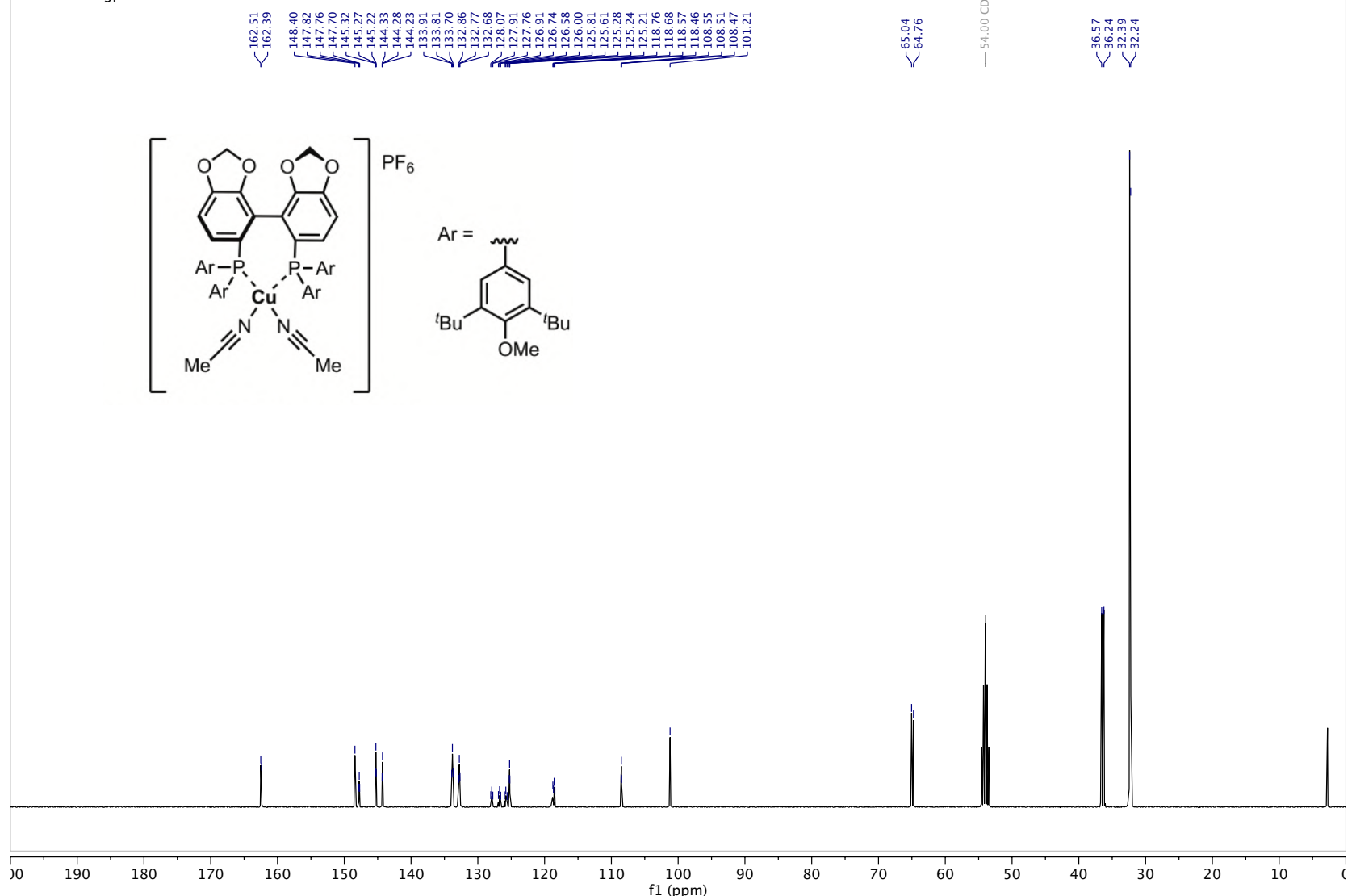
${ }^{19} \mathrm{~F}$ NMR: $\left[\mathrm{Cu}\left(\mathrm{CH}_{3} \mathrm{CN}\right)_{2}(S)-\mathrm{DTBM}-S e g p h o s\right] \mathrm{PF}_{6}+2 \mathrm{CH}_{3} \mathrm{CN}\left(\mathrm{CD}_{2} \mathrm{Cl}_{2}\right)$

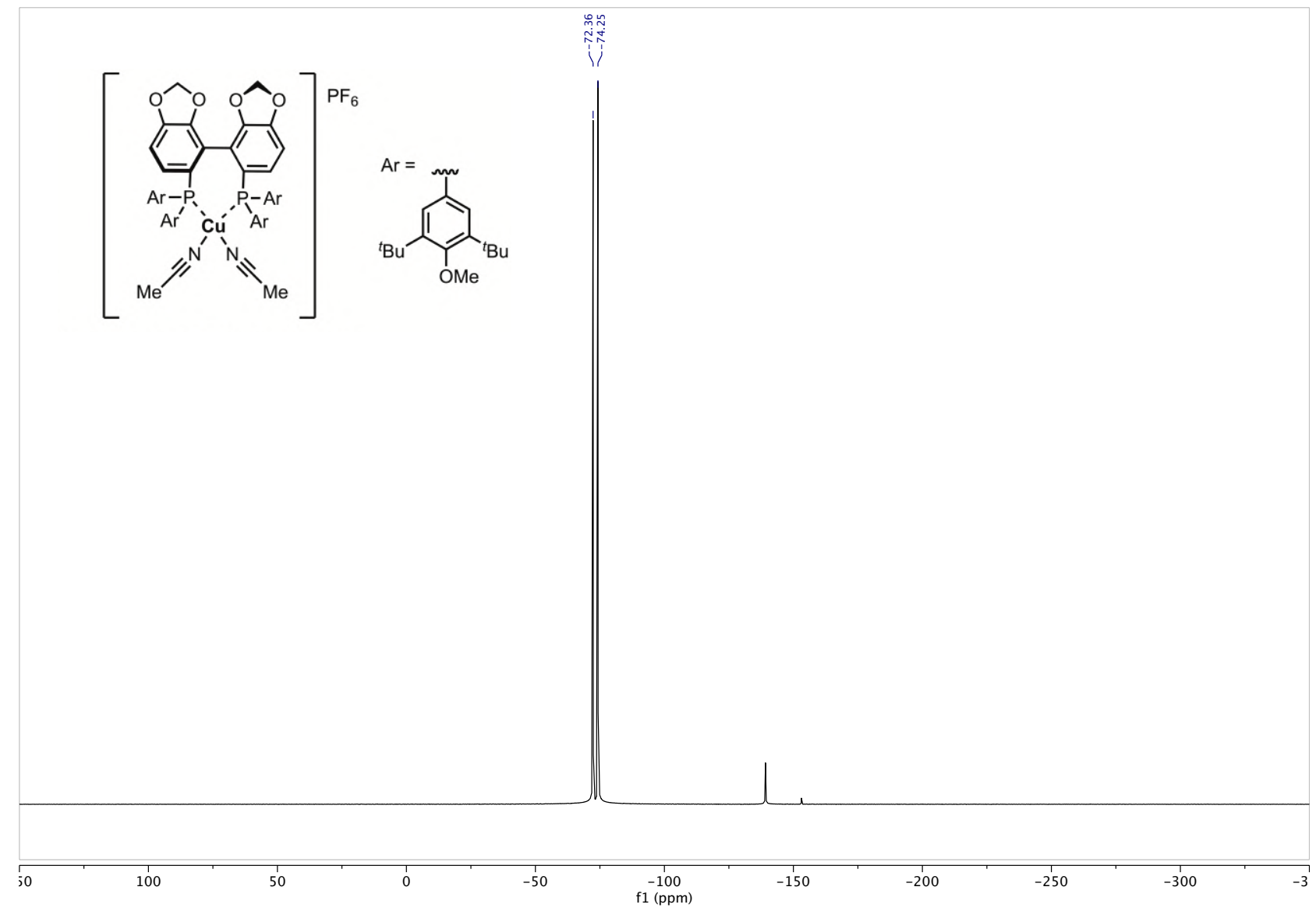

31P NMR: $\left[\mathrm{Cu}\left(\mathrm{CH}_{3} \mathrm{CN}\right)_{2}(S)-\mathrm{DTBM}-\mathrm{Segphos}\right] \mathrm{PF}{ }_{6}+2 \mathrm{CH}_{3} \mathrm{CN}\left(\mathrm{CD}_{2} \mathrm{Cl}_{2}\right)$

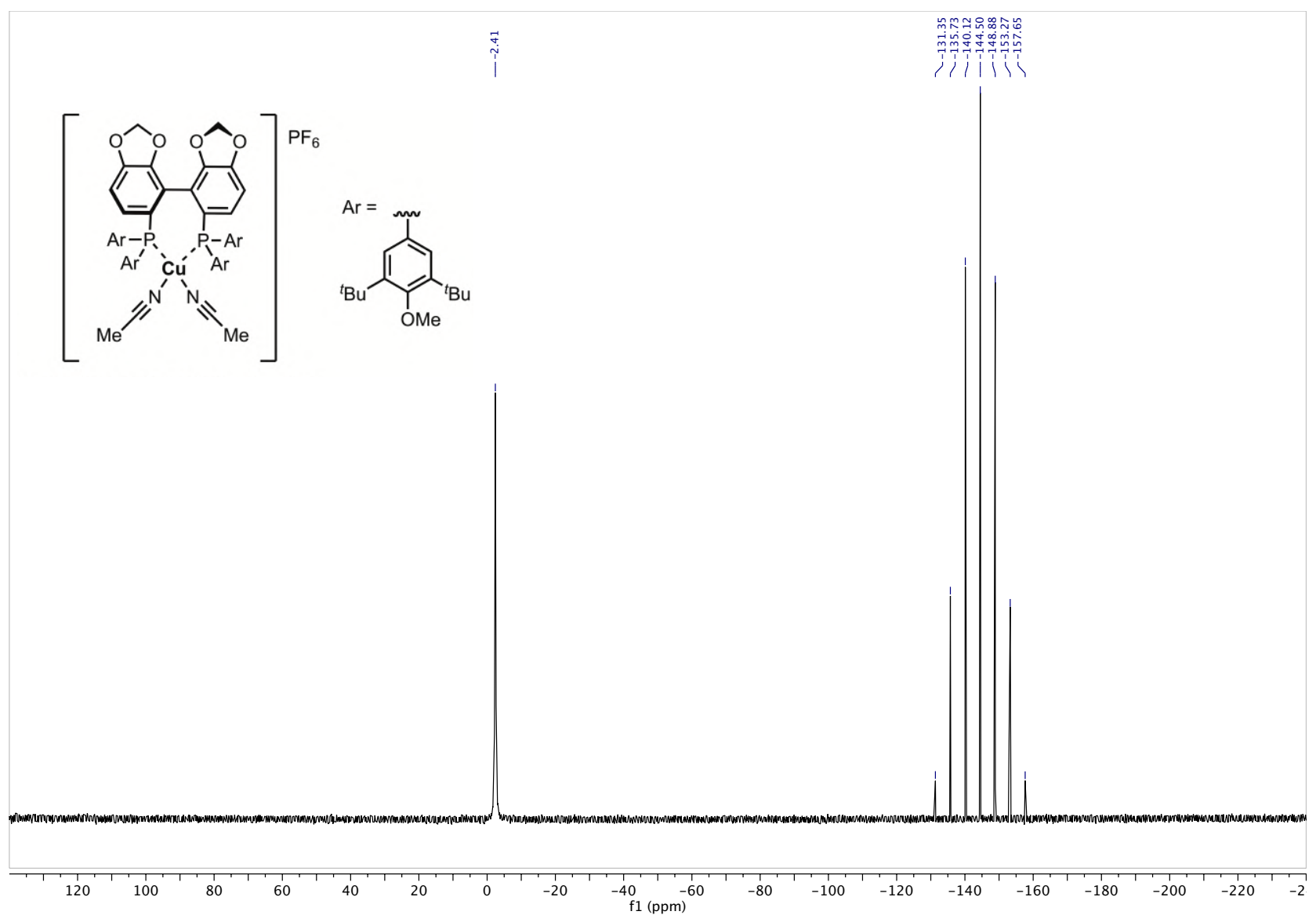


Supporting Information

${ }^{1} \mathrm{H}$ NMR: $\mathbf{2 b}\left(\mathrm{CD}_{2} \mathrm{Cl}_{2}\right)$

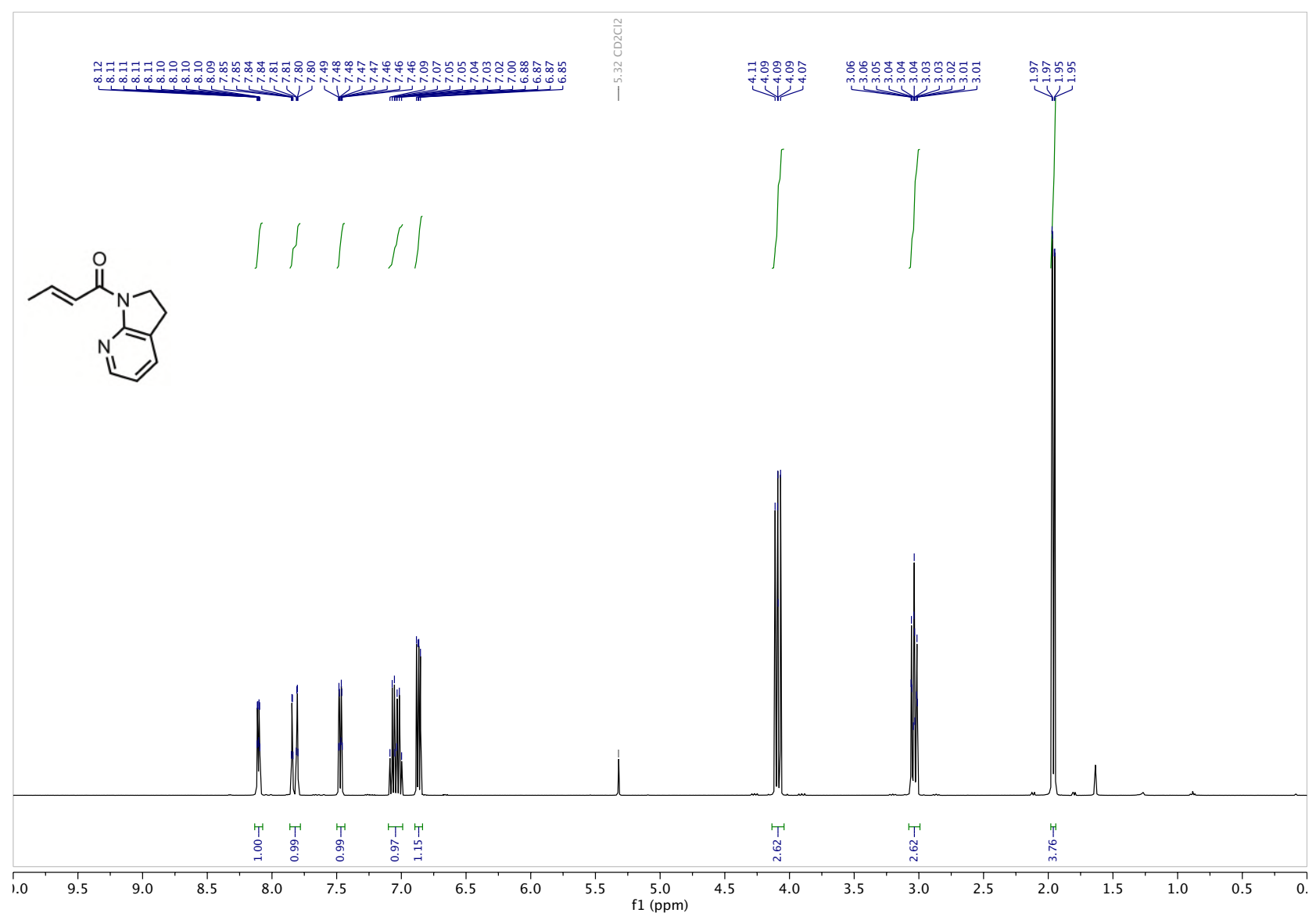

${ }^{13} \mathrm{C}$ NMR: $\mathbf{2 b}\left(\mathrm{CD}_{2} \mathrm{Cl}_{2}\right)$

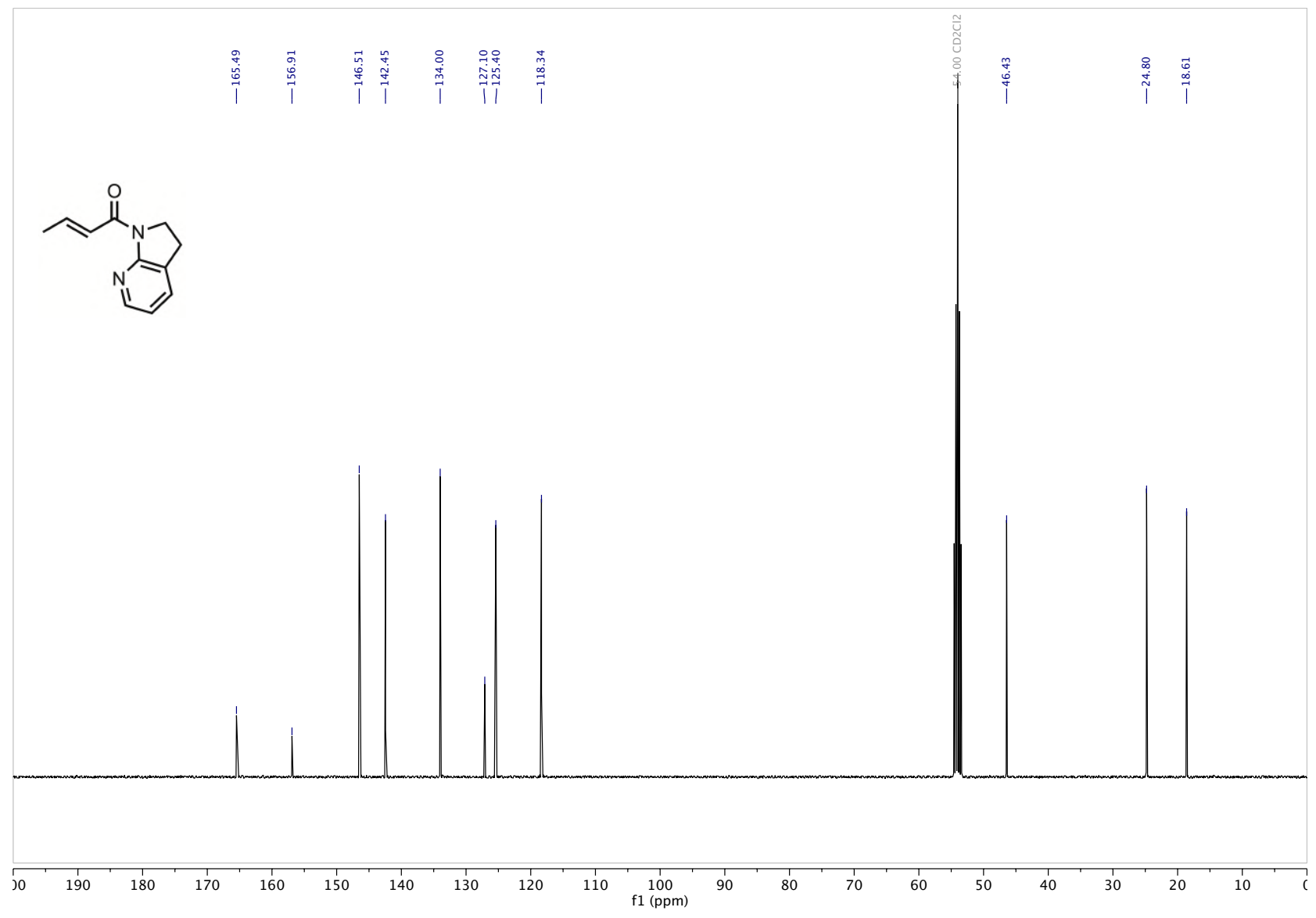

S71 
${ }^{1} \mathrm{H}$ NMR: $2 \mathrm{~b}+\left[\mathrm{Cu}\left(\mathrm{CH}_{3} \mathrm{CN}\right)_{2}(S)-\mathrm{DTBM}-S\right.$ egphos $] \mathrm{PF}_{6}+2 \mathrm{CH}_{3} \mathrm{CN}\left(\mathrm{CD}_{2} \mathrm{Cl}_{2}\right)$

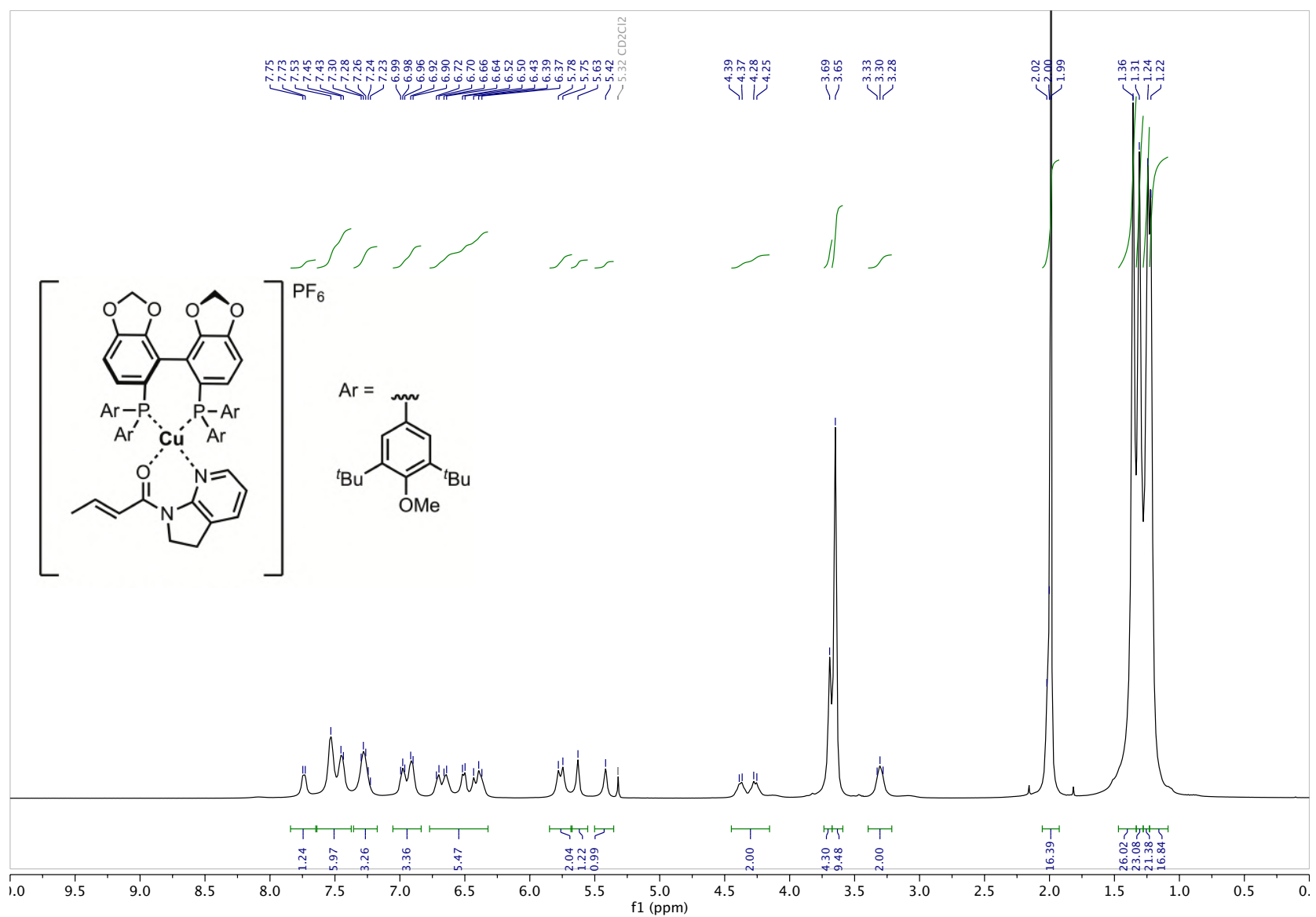

${ }^{13} \mathrm{C} \mathrm{NMR:} \mathbf{2 b}+\left[\mathrm{Cu}\left(\mathrm{CH}_{3} \mathrm{CN}\right)_{2}(S)-\mathrm{DTBM}-S\right.$ egphos $] \mathrm{PF}_{6}+2 \mathrm{CH}_{3} \mathrm{CN}\left(\mathrm{CD}_{2} \mathrm{Cl}_{2}\right)$

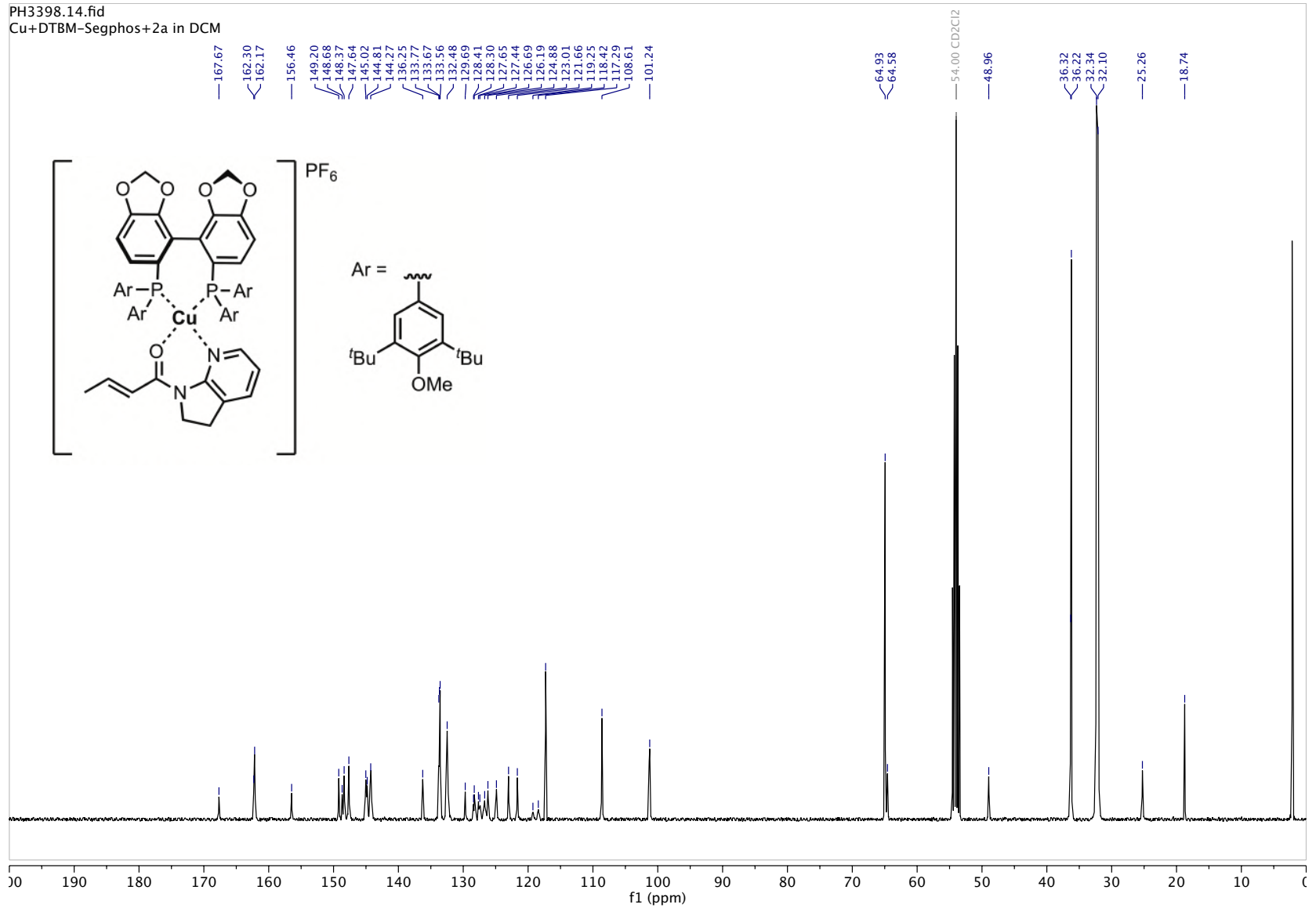


${ }^{19} \mathrm{~F}$ NMR: $2 \mathrm{~b}+\left[\mathrm{Cu}\left(\mathrm{CH}_{3} \mathrm{CN}\right)_{2}(S)-\mathrm{DTBM}-\mathrm{Segphos}\right] \mathrm{PF}_{6}+2 \mathrm{CH}_{3} \mathrm{CN}\left(\mathrm{CD}_{2} \mathrm{Cl}_{2}\right)$

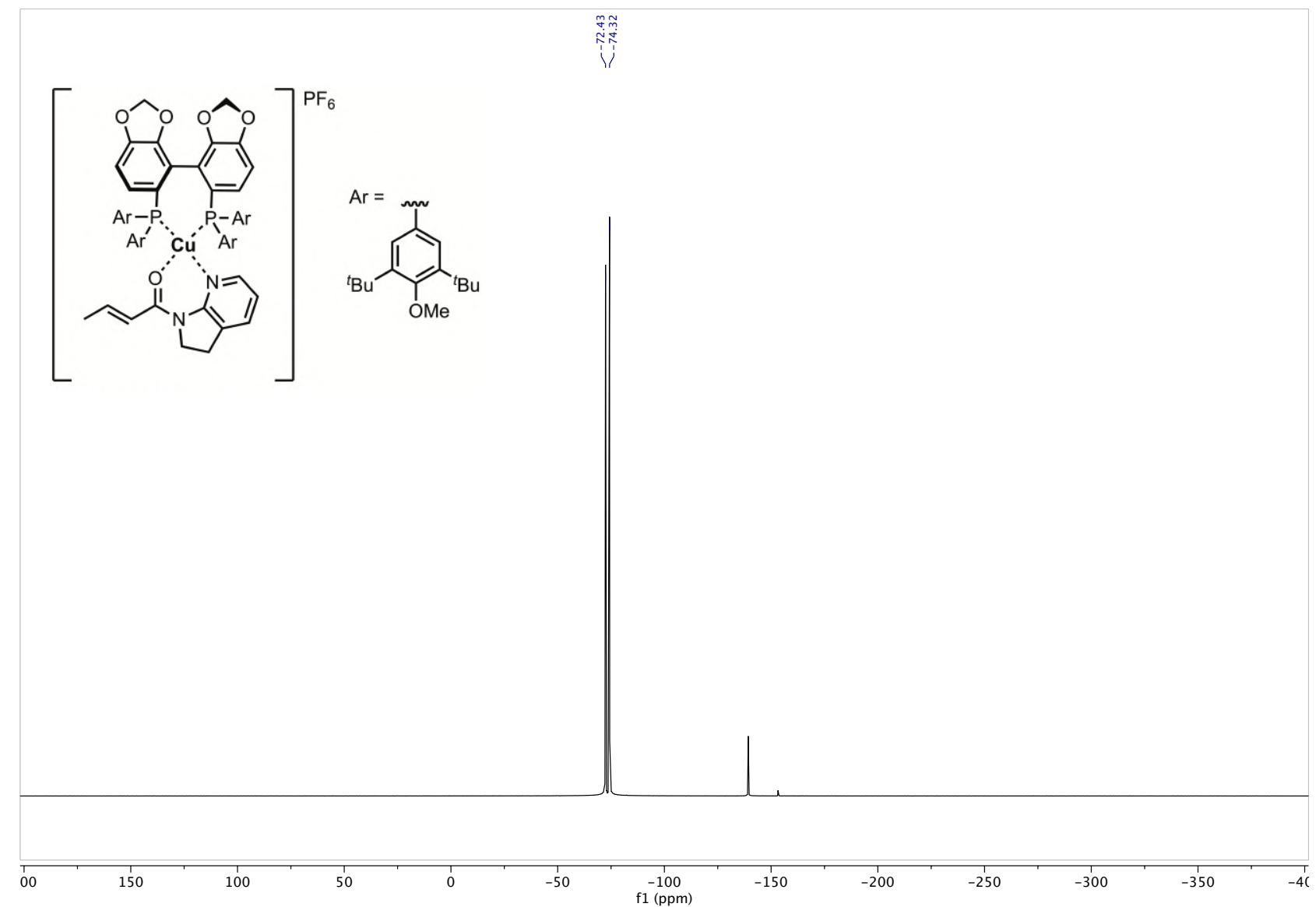

${ }^{31}$ P NMR: $2 b+\left[\mathrm{Cu}\left(\mathrm{CH}_{3} \mathrm{CN}\right)_{2}(S)-\mathrm{DTBM}-\right.$ Segphos $] \mathrm{PF}_{6}+2 \mathrm{CH}_{3} \mathrm{CN}\left(\mathrm{CD}_{2} \mathrm{Cl}_{2}\right)$

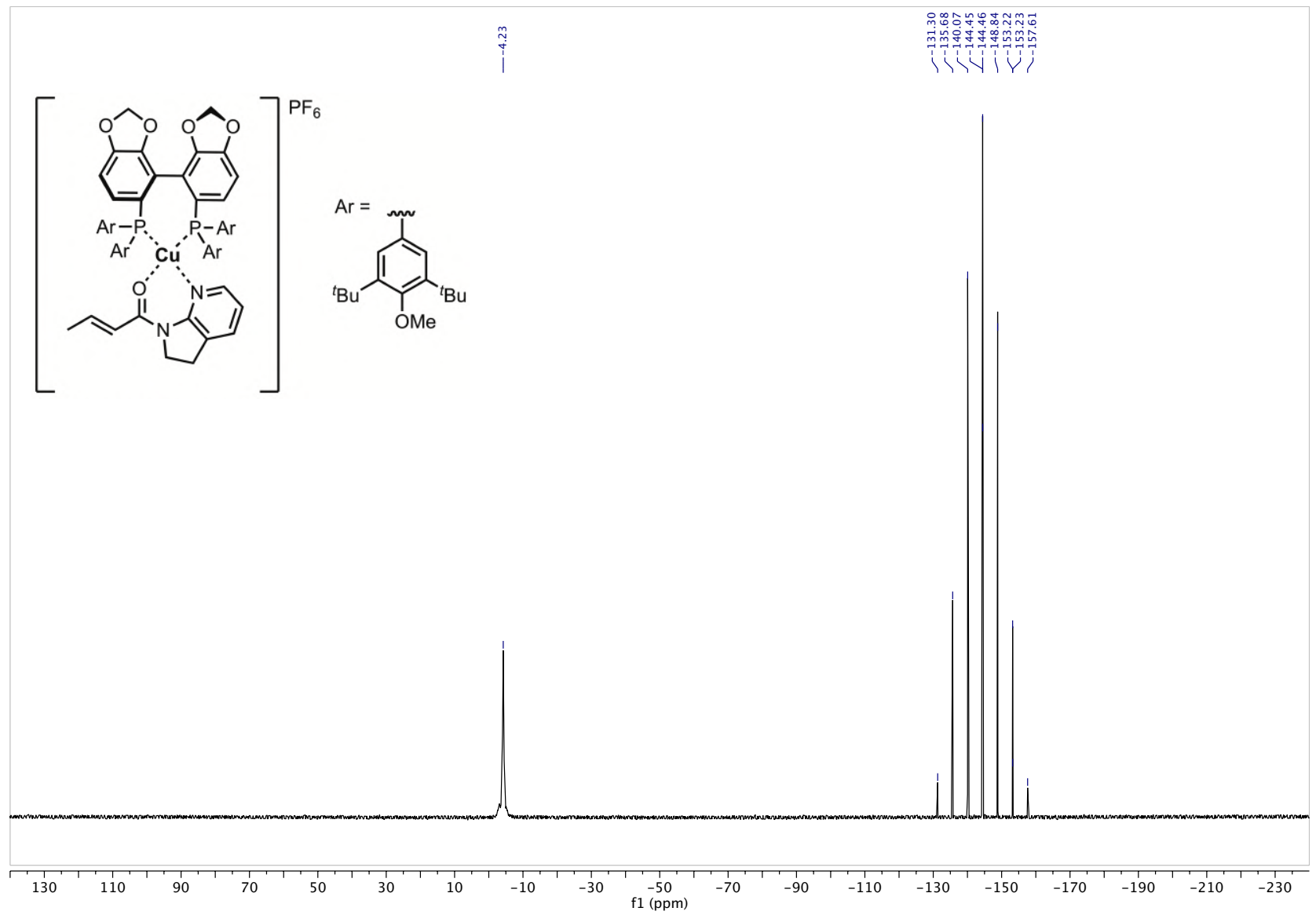


${ }^{1} \mathrm{H}$ NMR: 2k $\left(\mathrm{CD}_{2} \mathrm{Cl}_{2}\right)$

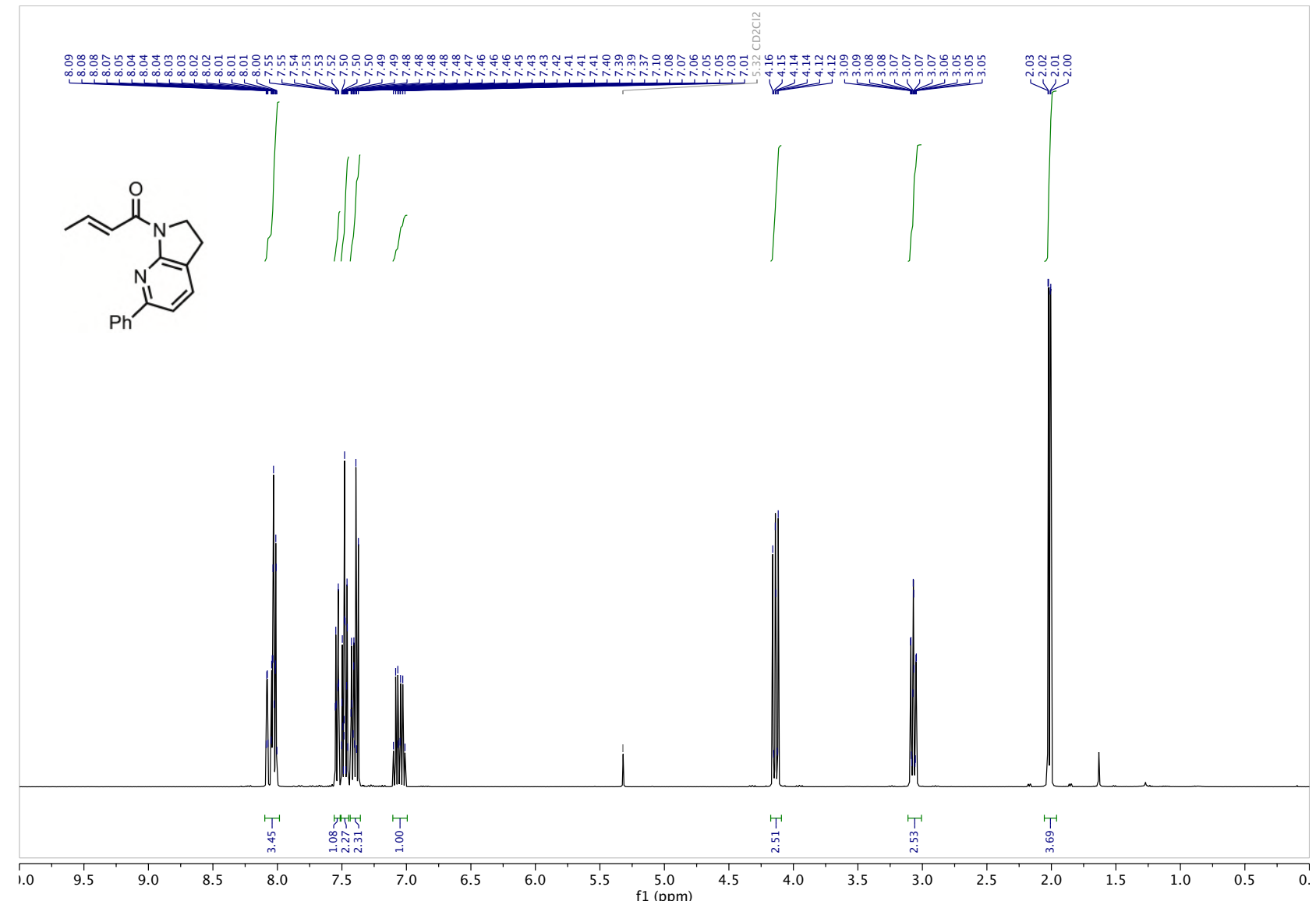

${ }^{13} \mathrm{C}$ NMR: 2k $\left(\mathrm{CD}_{2} \mathrm{Cl}_{2}\right)$

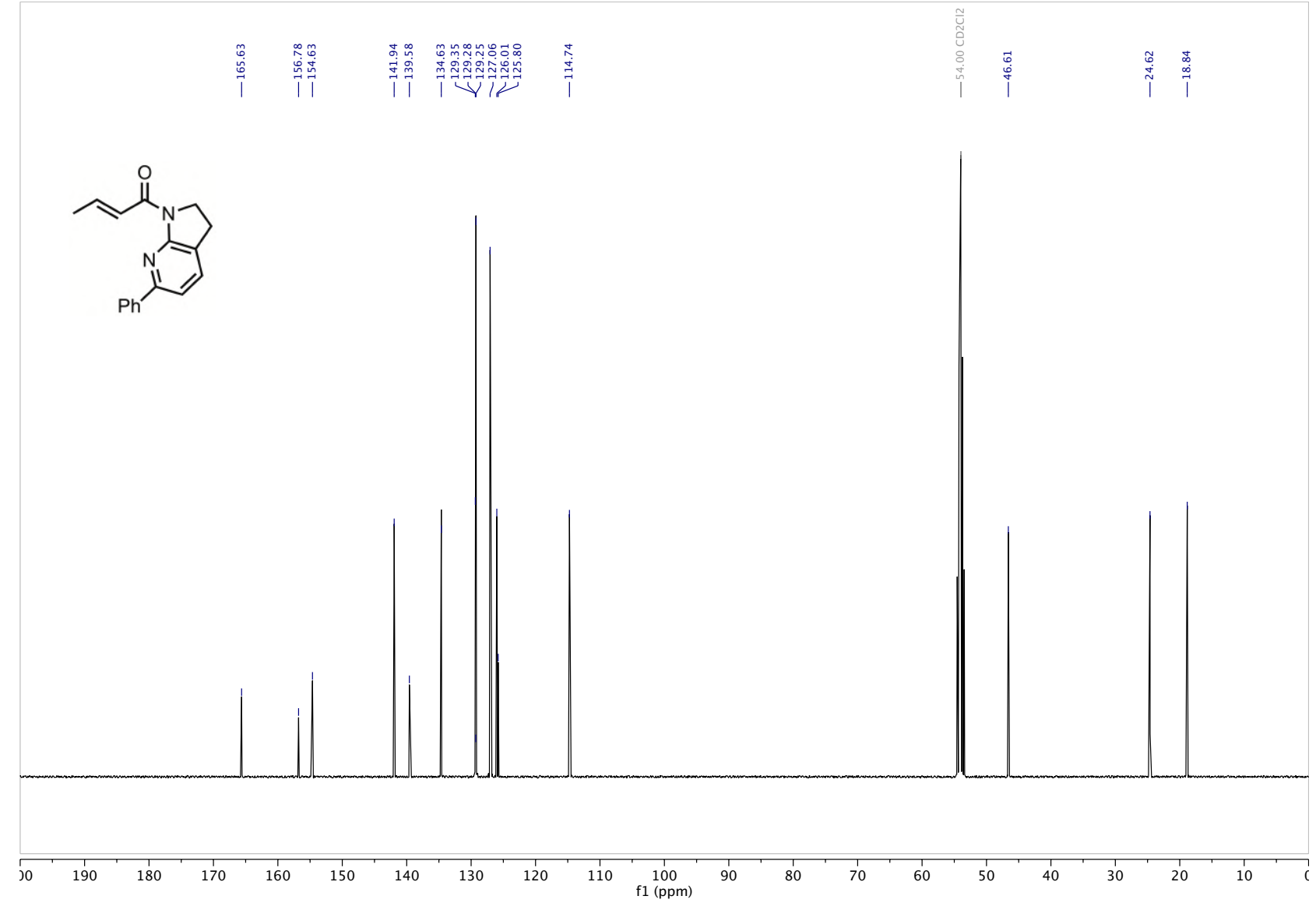


${ }^{1} \mathrm{H}$ NMR: $2 \mathrm{k}+\left[\mathrm{Cu}\left(\mathrm{CH}_{3} \mathrm{CN}\right)_{2}(S)-\mathrm{DTBM}-S\right.$ egphos $] \mathrm{PF}_{6}+2 \mathrm{CH}_{3} \mathrm{CN}\left(\mathrm{CD}_{2} \mathrm{Cl}_{2}\right)$

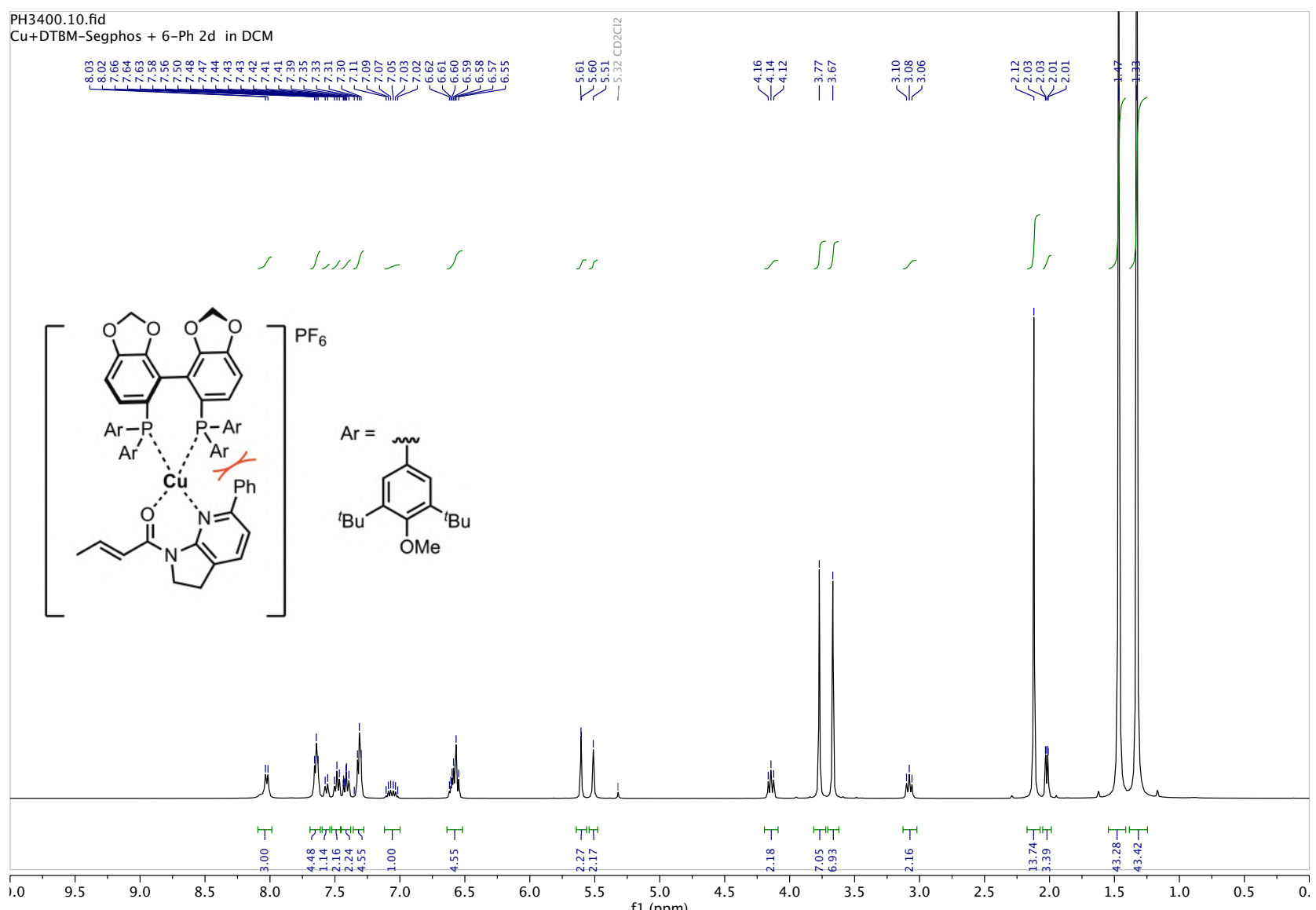

${ }^{13} \mathrm{C}$ NMR: $2 \mathbf{k}+\left[\mathrm{Cu}\left(\mathrm{CH}_{3} \mathrm{CN}\right)_{2}(S)-\mathrm{DTBM}-\mathrm{Segphos}\right] \mathrm{PF}_{6}+2 \mathrm{CH}_{3} \mathrm{CN}\left(\mathrm{CD}_{2} \mathrm{Cl}_{2}\right)$

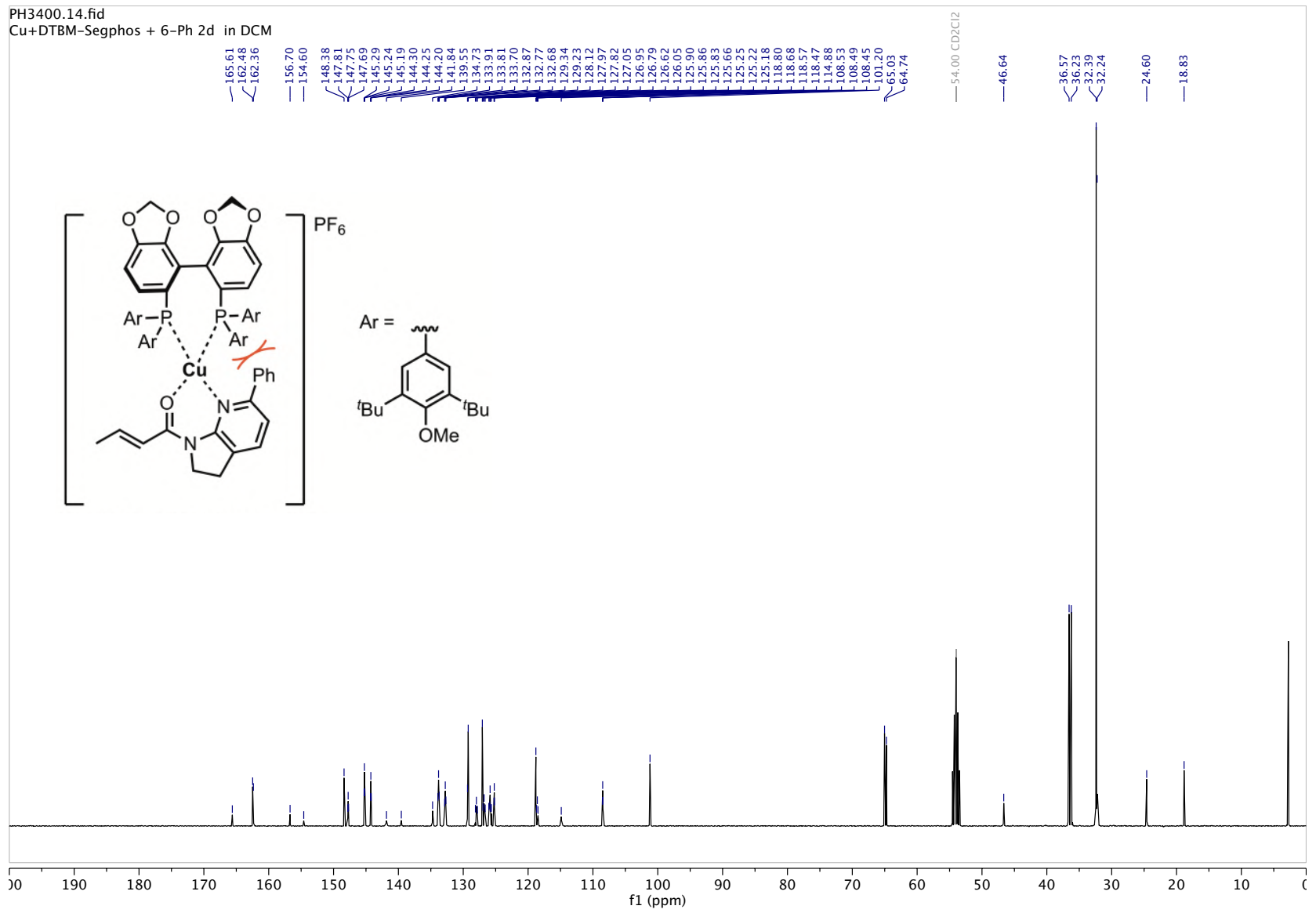


${ }^{19} \mathrm{~F}$ NMR: $2 \mathrm{k}+\left[\mathrm{Cu}\left(\mathrm{CH}_{3} \mathrm{CN}\right)_{2}(S)-\mathrm{DTBM}-\mathrm{Segphos}\right] \mathrm{PF}_{6}+2 \mathrm{CH}_{3} \mathrm{CN}\left(\mathrm{CD}_{2} \mathrm{Cl}_{2}\right)$
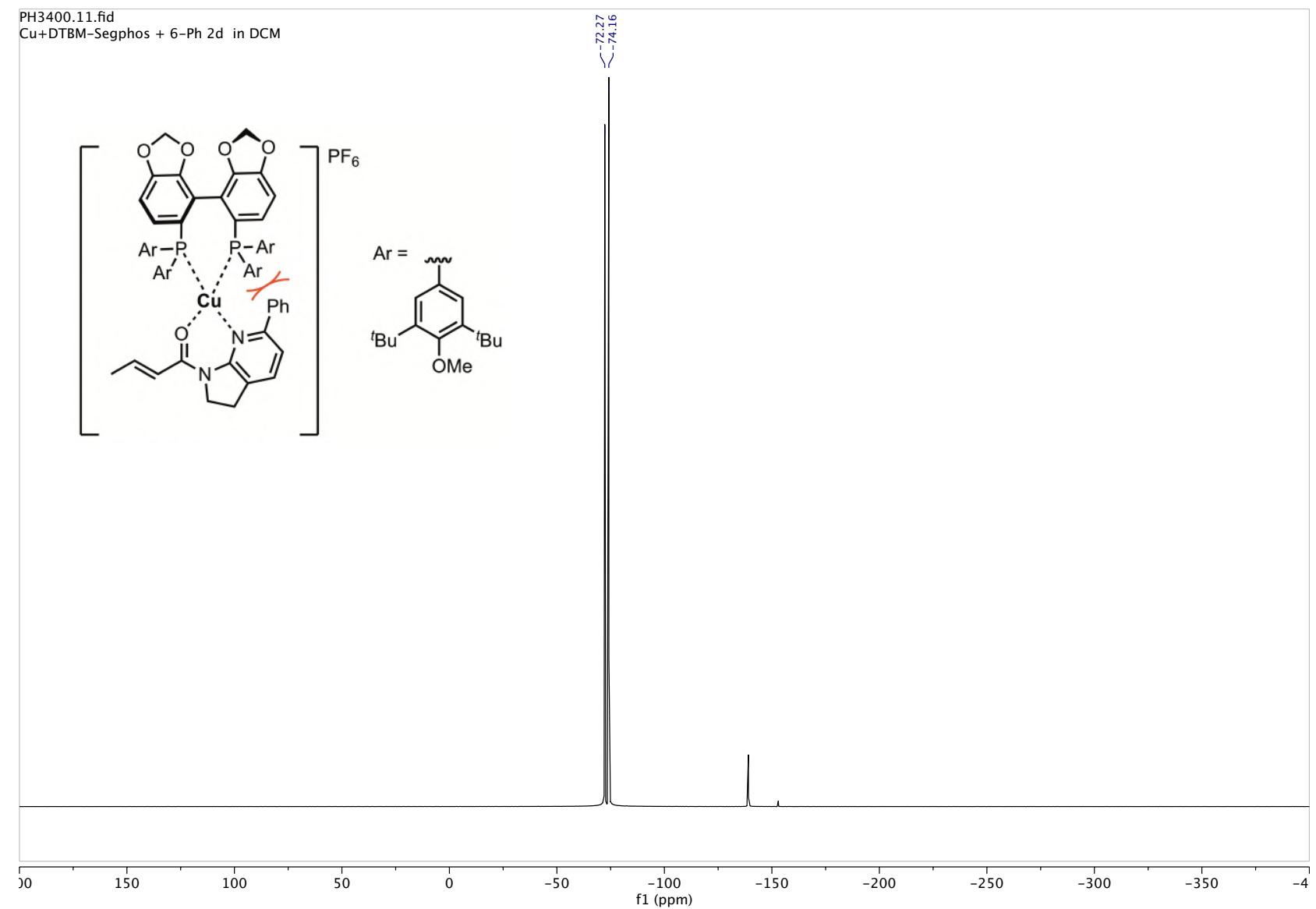

${ }^{31}$ P NMR: $2 \mathbf{k}+\left[\mathrm{Cu}\left(\mathrm{CH}_{3} \mathrm{CN}\right)_{2}(S)-\mathrm{DTBM}-\mathrm{Segphos}\right] \mathrm{PF}$ + $2 \mathrm{CH}_{3} \mathrm{CN}\left(\mathrm{CD}_{2} \mathrm{Cl}_{2}\right)$

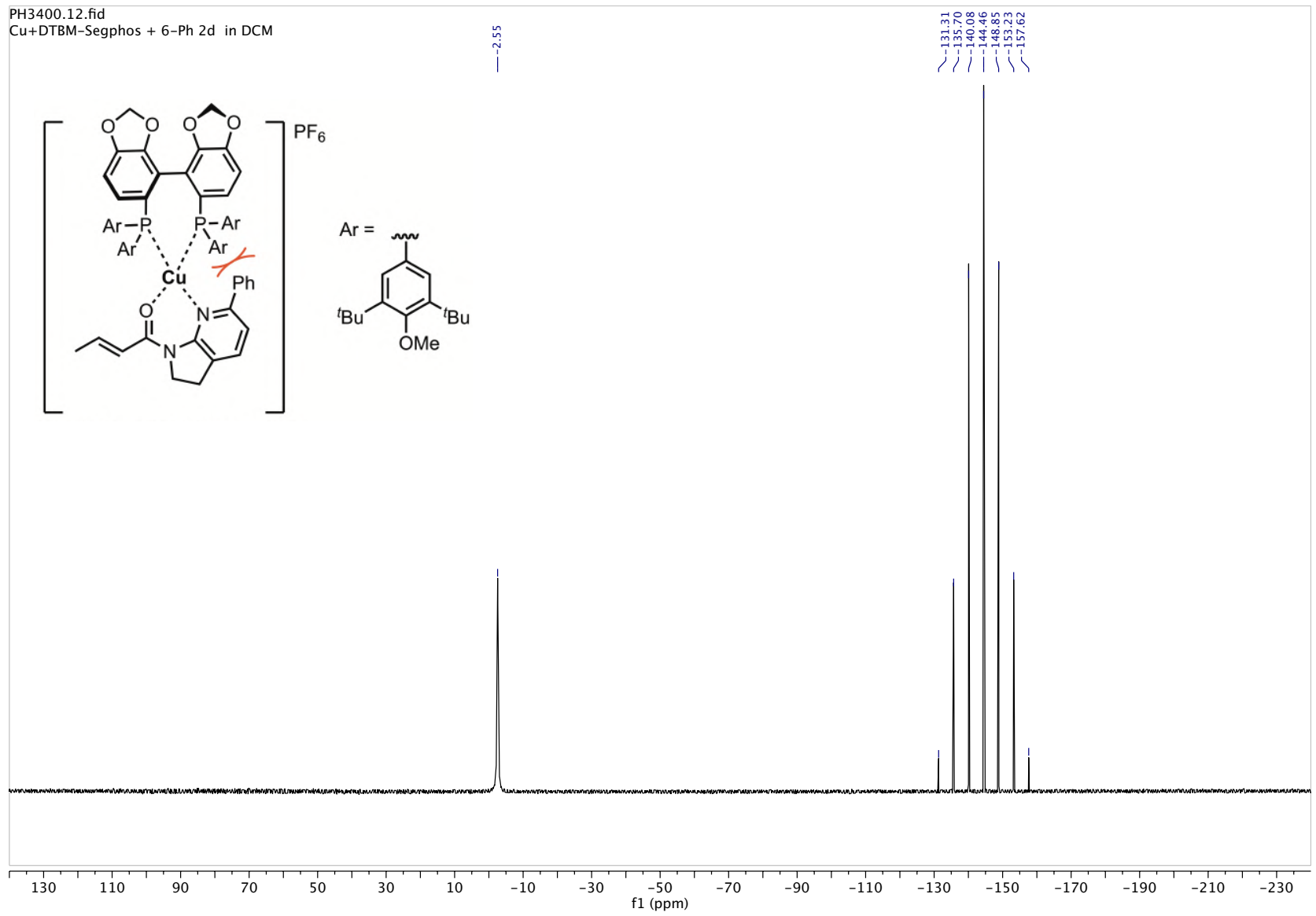


${ }^{1} \mathrm{H}$ NMR: B1 (DMSO-d $\left.d_{6}\right)$

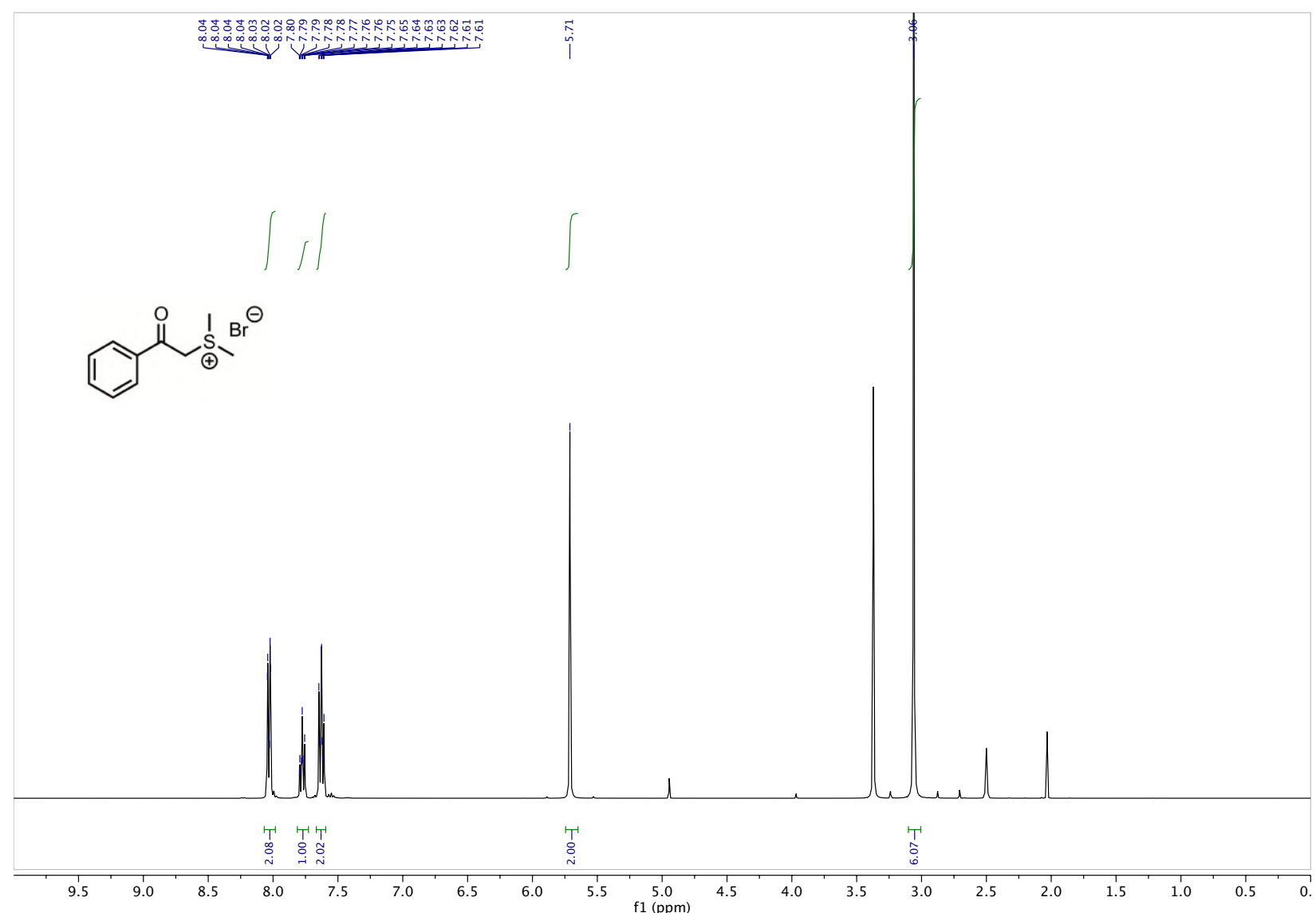

${ }^{13}$ C NMR: B1 (DMSO- $\left.d_{6}\right)$

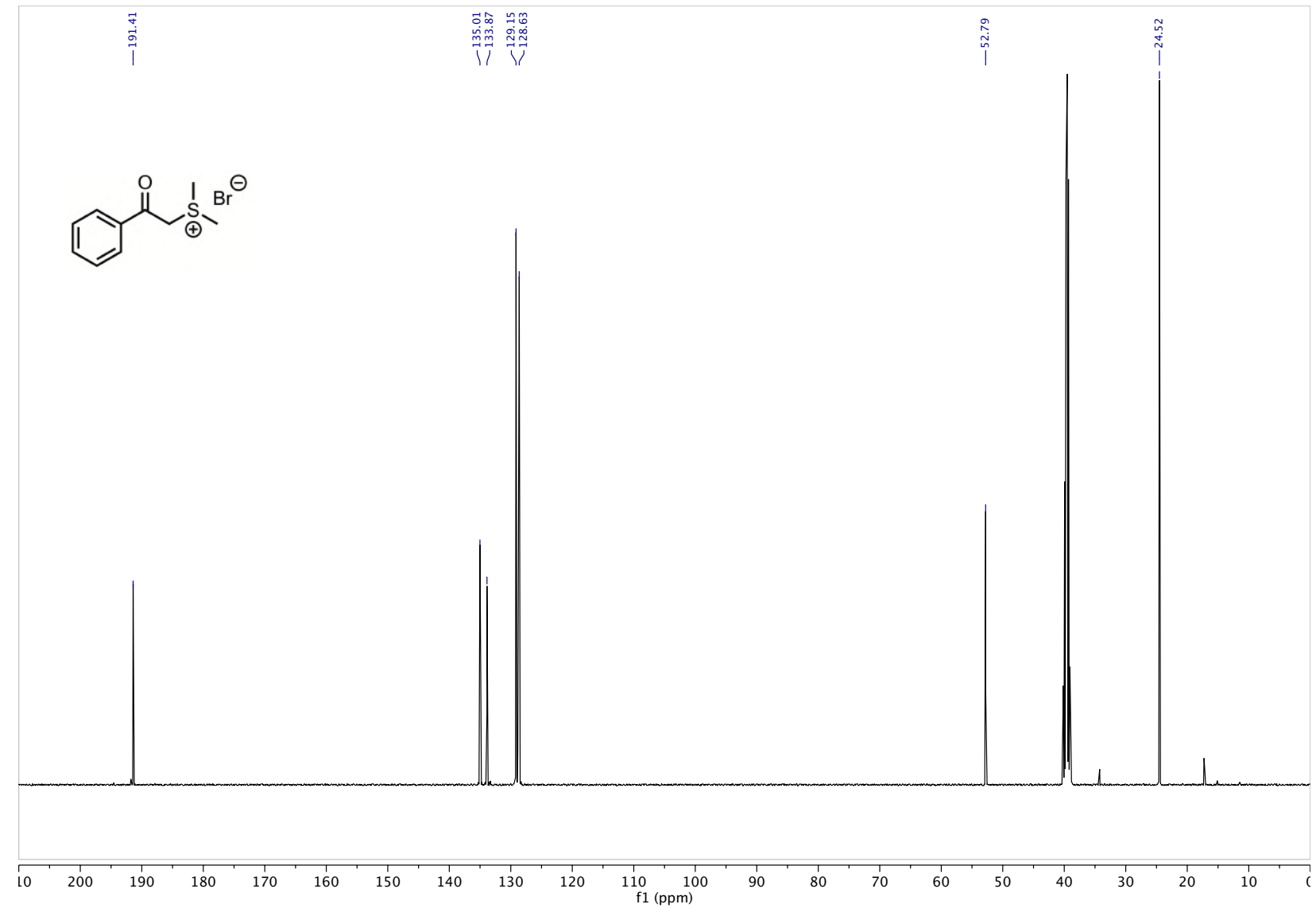


Supporting Information

${ }^{1} \mathrm{H}$ NMR: $1 \mathrm{a}$

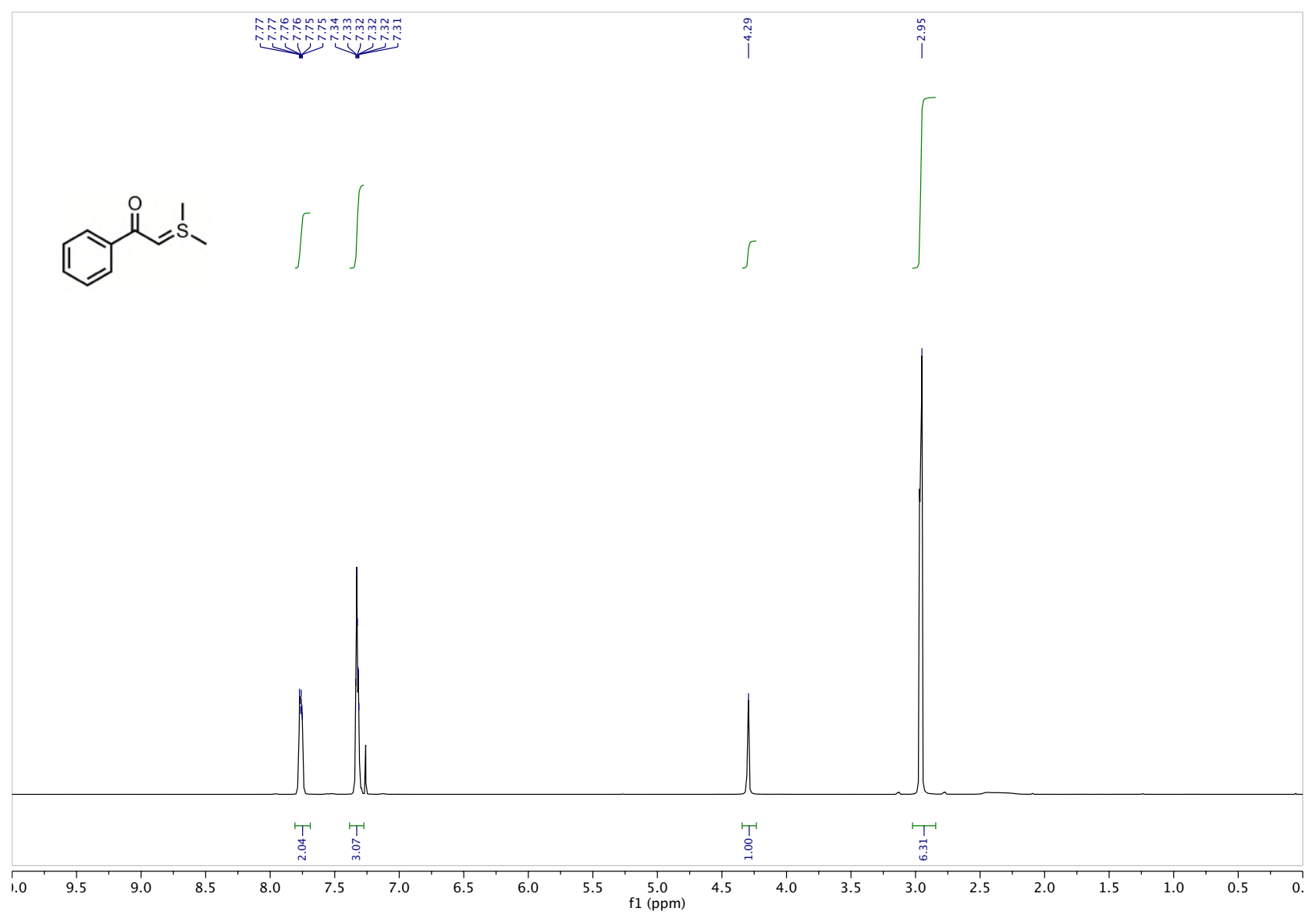

${ }^{13}$ C NMR: Ia

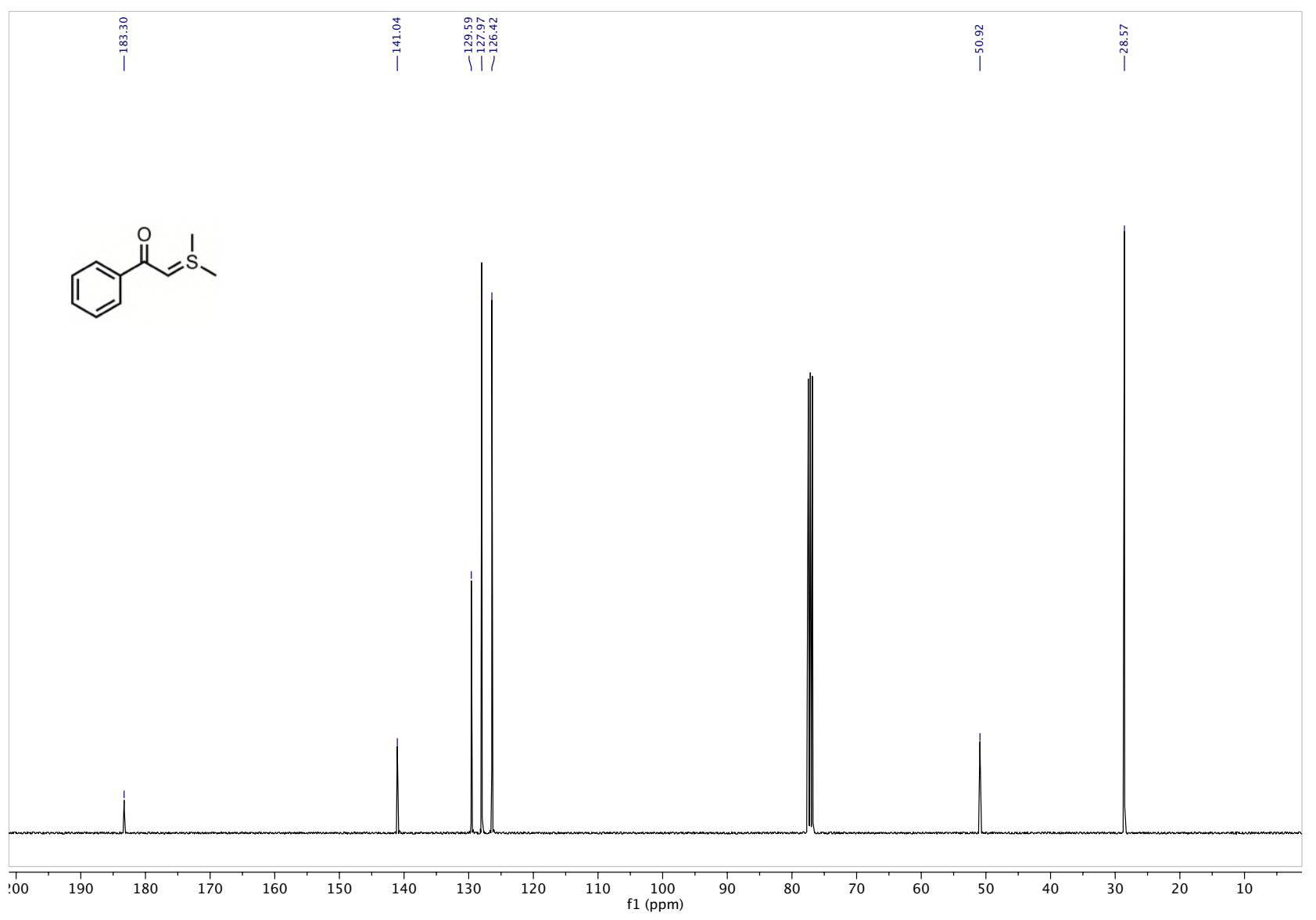

S78 
Supporting Information

${ }^{1} \mathrm{H}$ NMR: $\mathbf{1 b}$

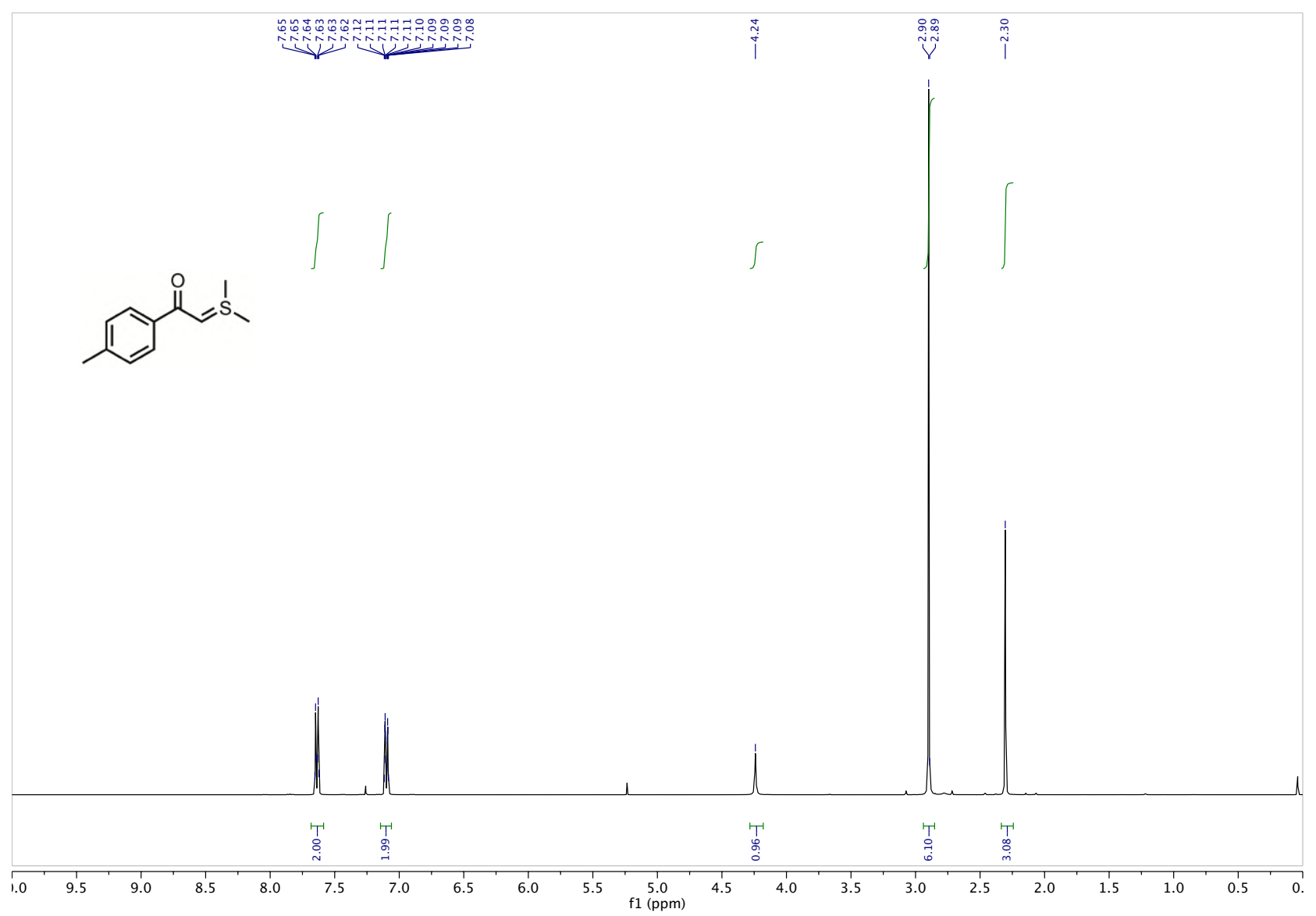

${ }^{13}$ C NMR: Ib

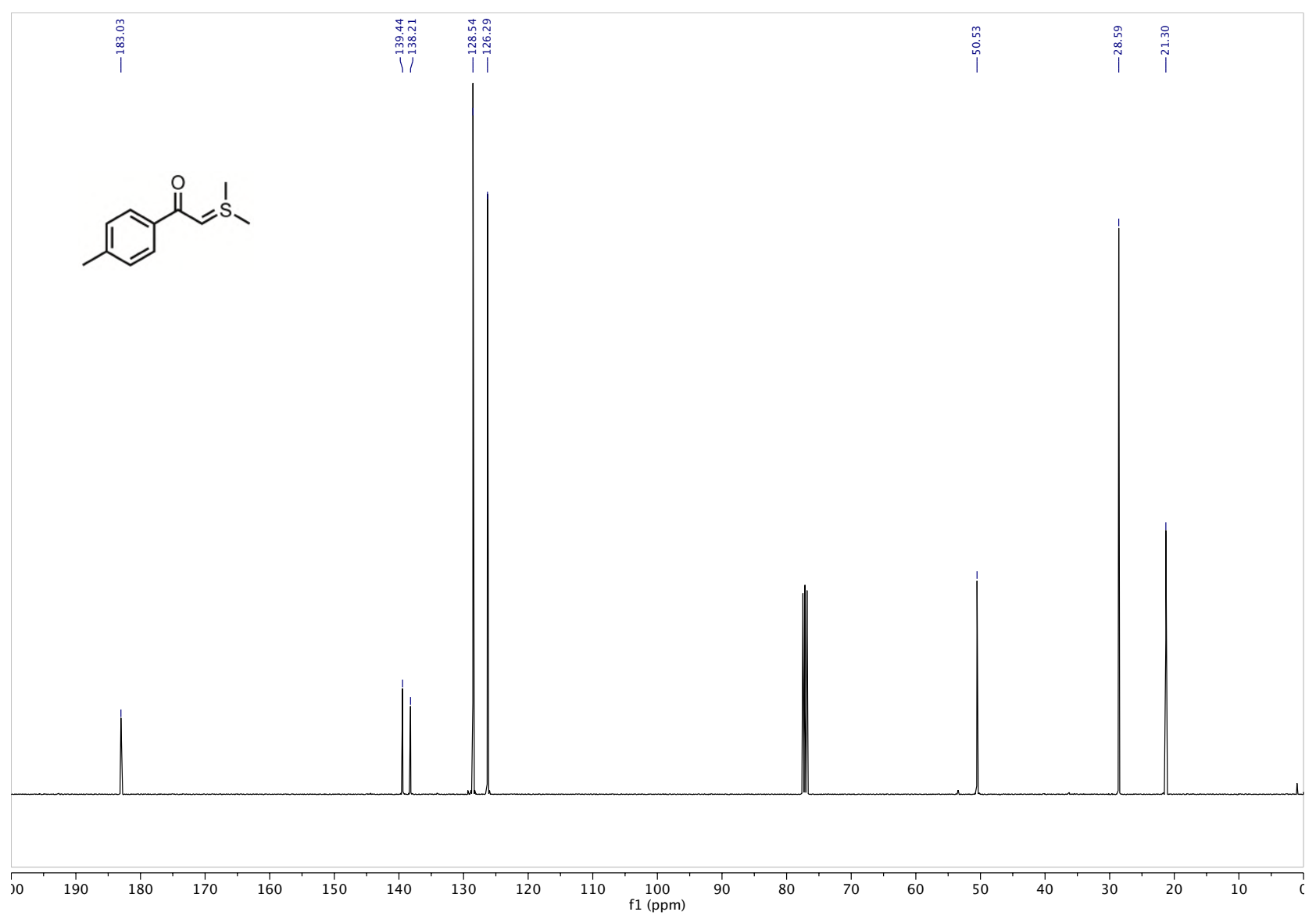

S79 
Supporting Information

${ }^{1} \mathrm{H}$ NMR: Ic

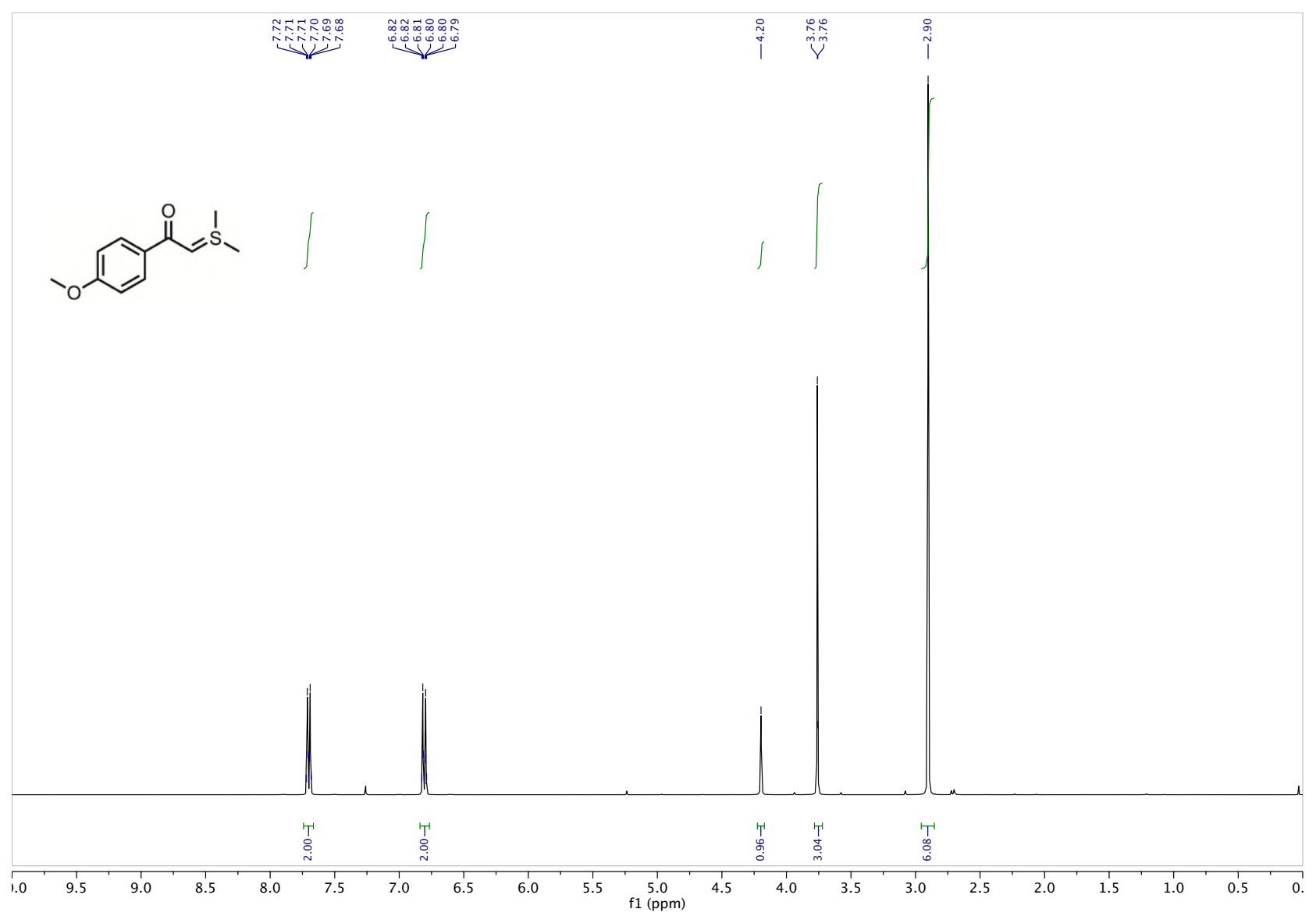

${ }^{13}$ C NMR: Ic

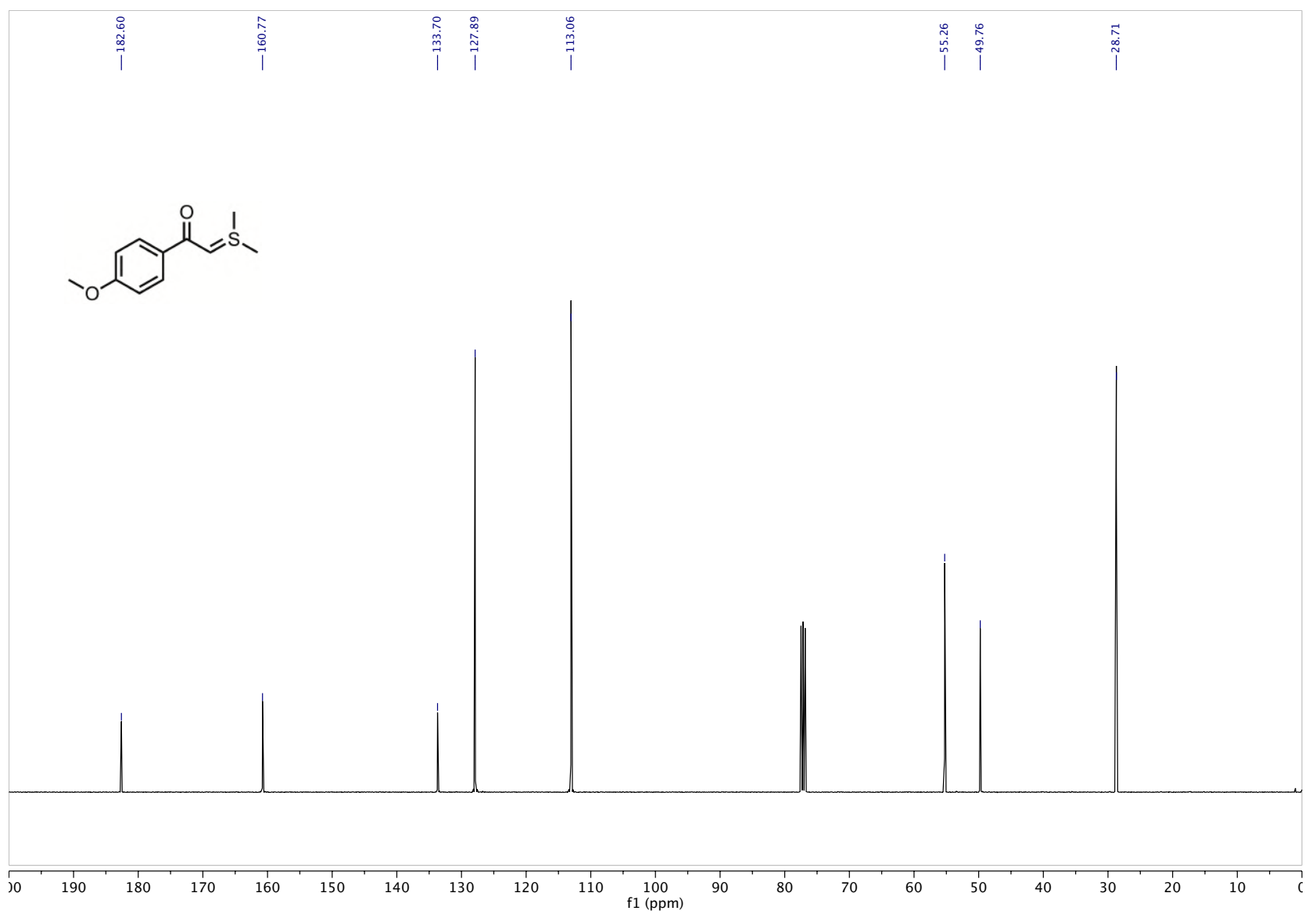

S80 
Supporting Information

${ }^{1} \mathrm{H}$ NMR: Id

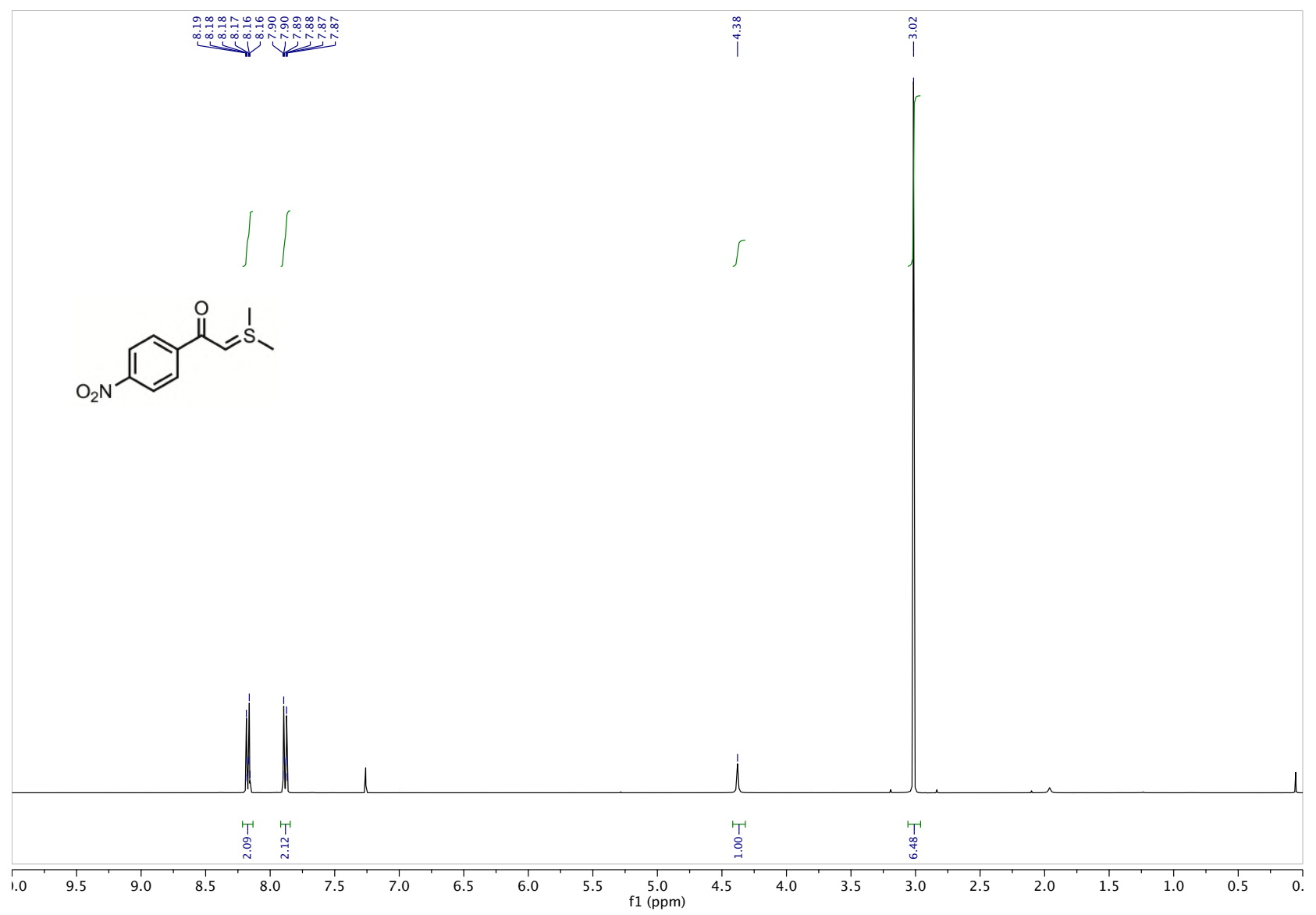

${ }^{13} \mathrm{C}$ NMR: Id

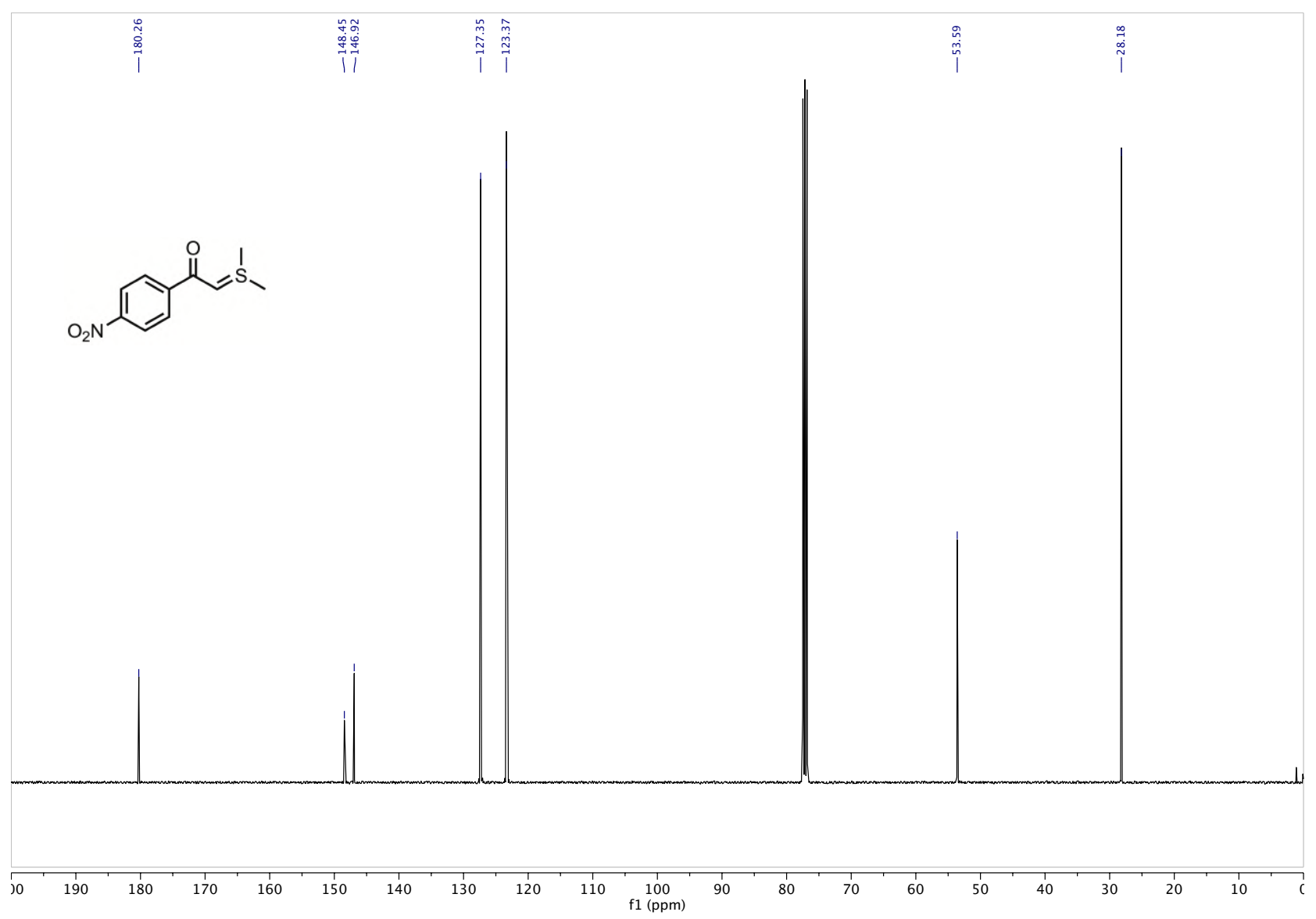

S81 
Supporting Information

${ }^{1} \mathrm{H}$ NMR: Ie

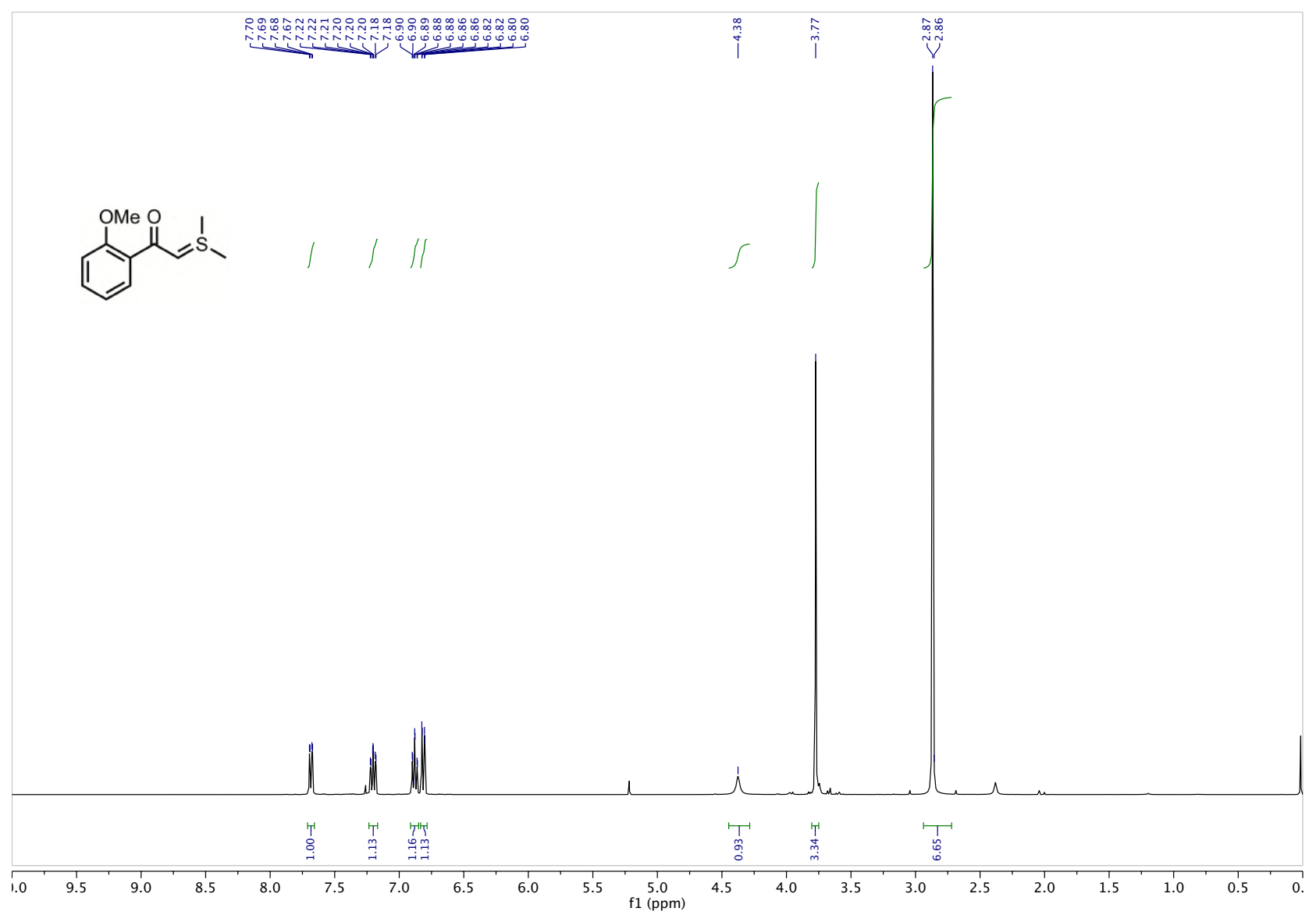

${ }^{13}$ C NMR: Ie

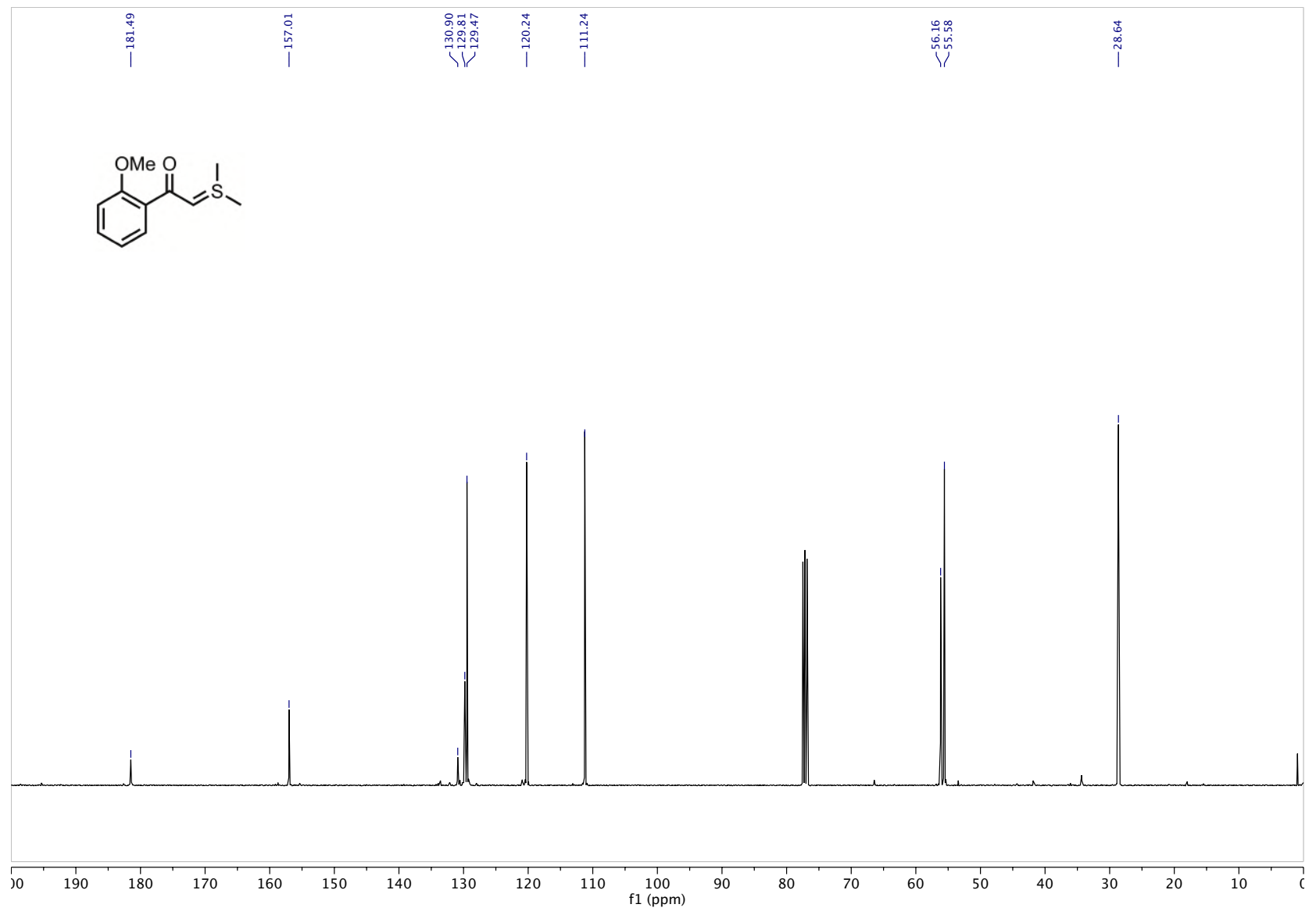

S82 
Supporting Information

${ }^{1} \mathrm{H}$ NMR: $1 f$

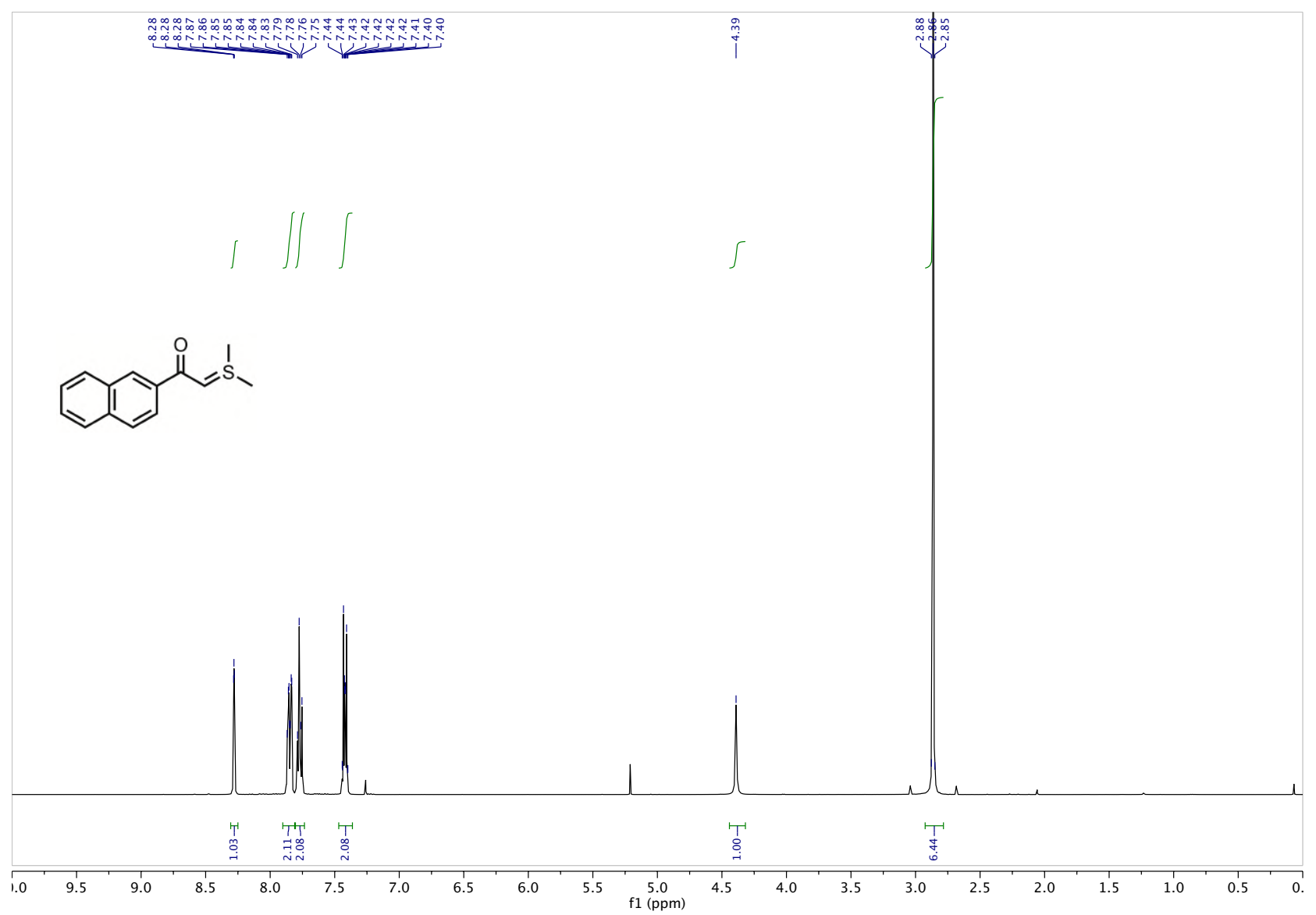

${ }^{13}$ C NMR: If

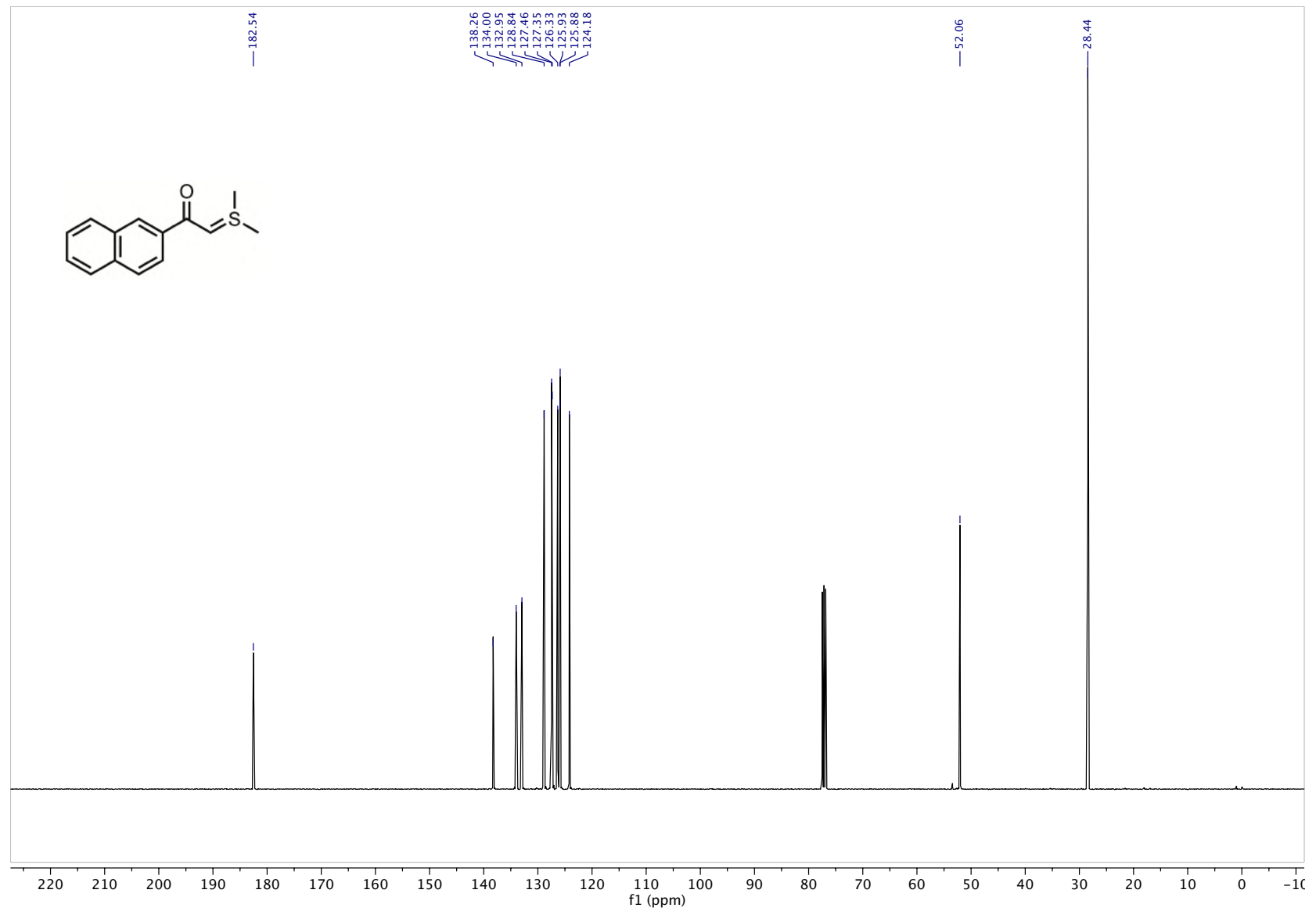

S83 
${ }^{1} \mathrm{H}$ NMR: 19

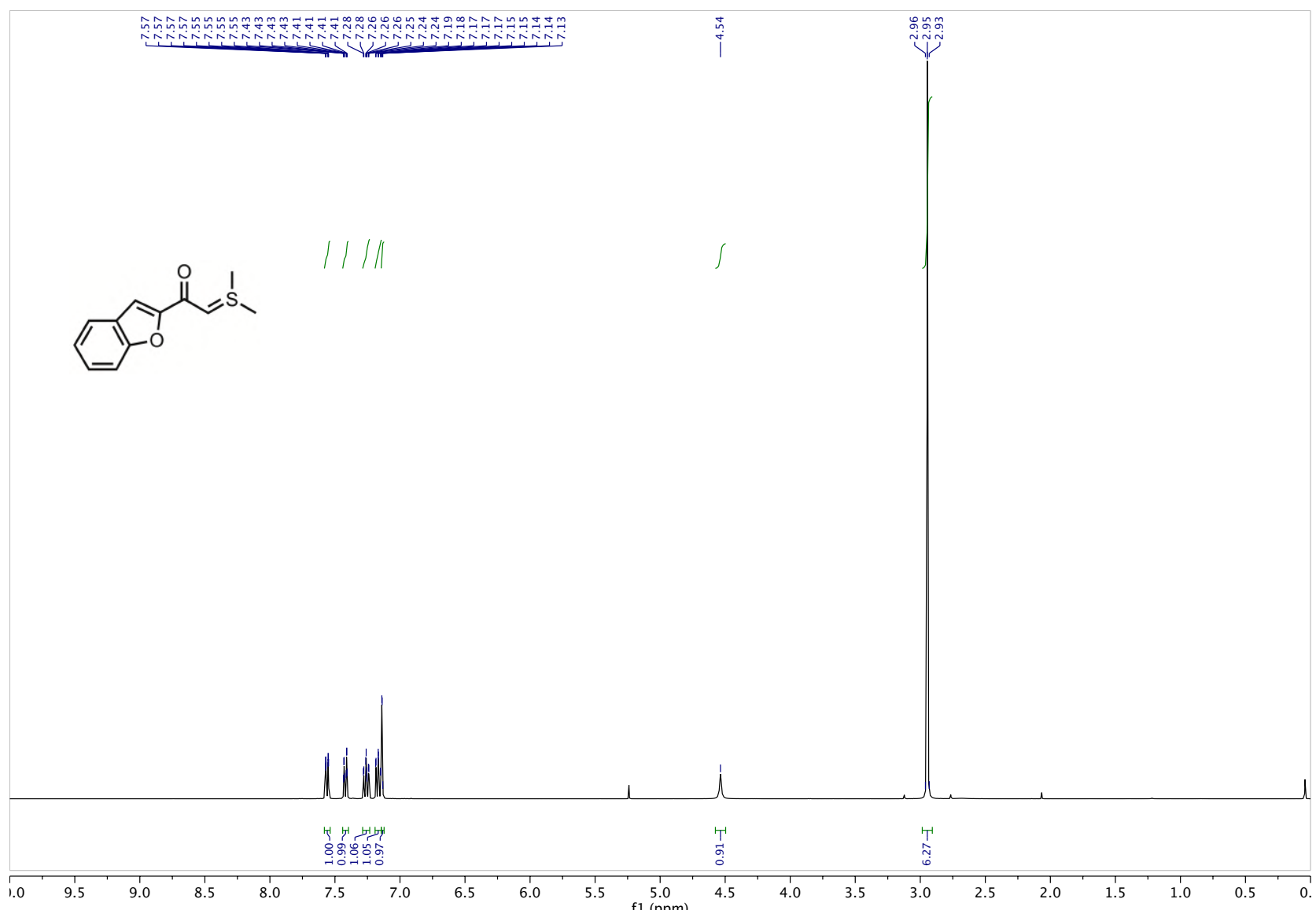

${ }^{13} \mathrm{C}$ NMR: 19

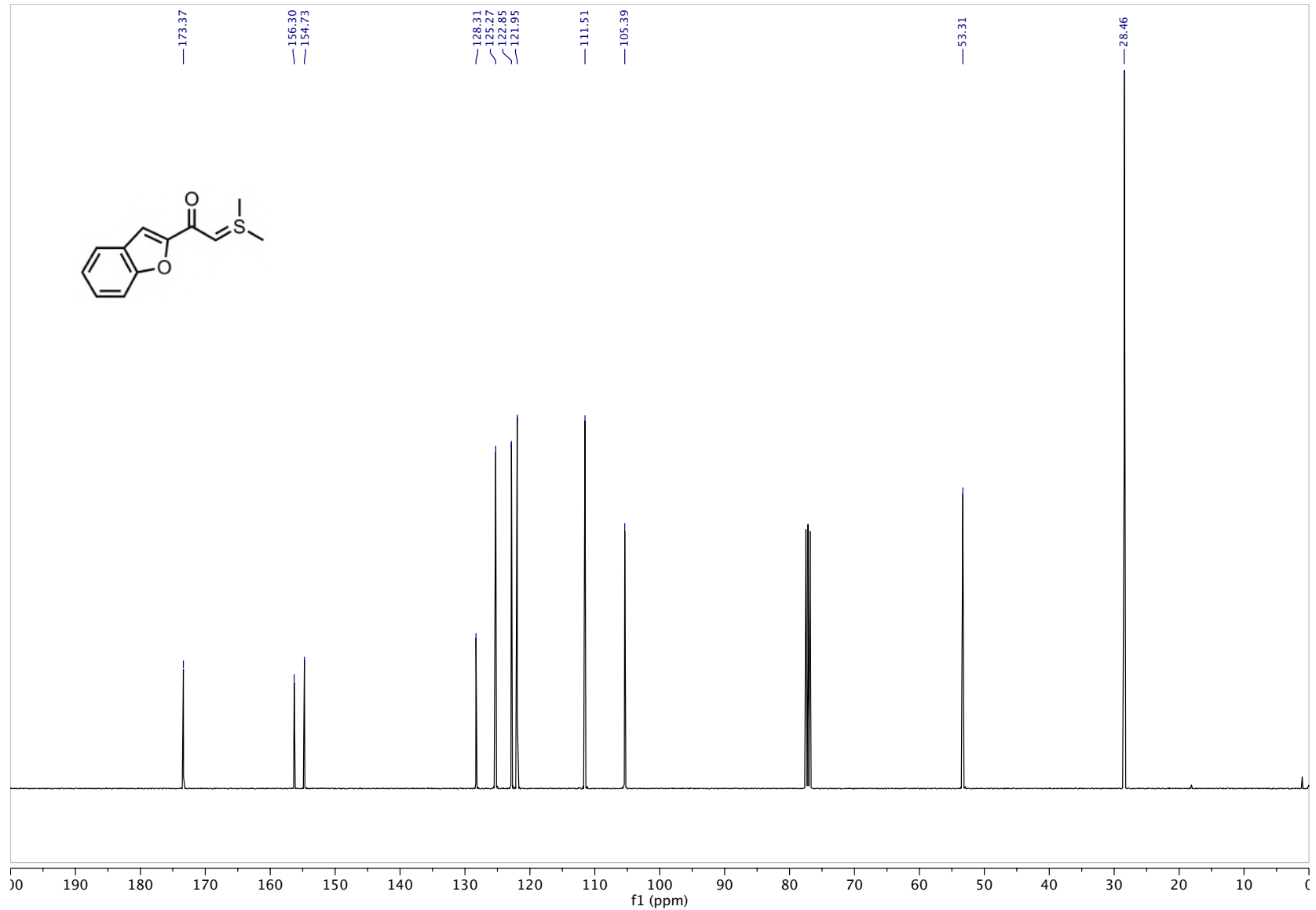


Supporting Information

${ }^{1} \mathrm{H}$ NMR: $\mathbf{1 h}$

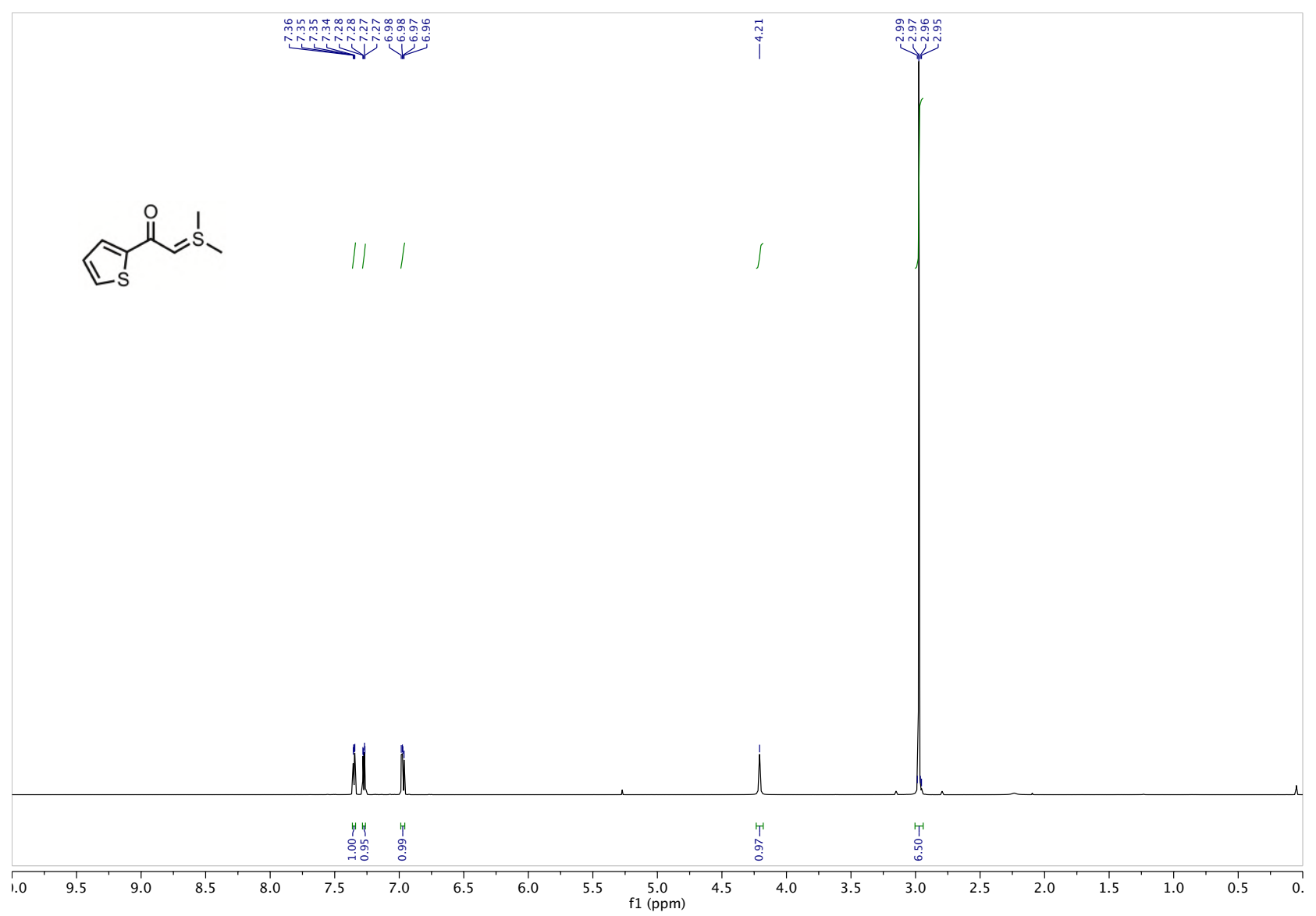

${ }^{13}$ C NMR: $\mathbf{1 h}$

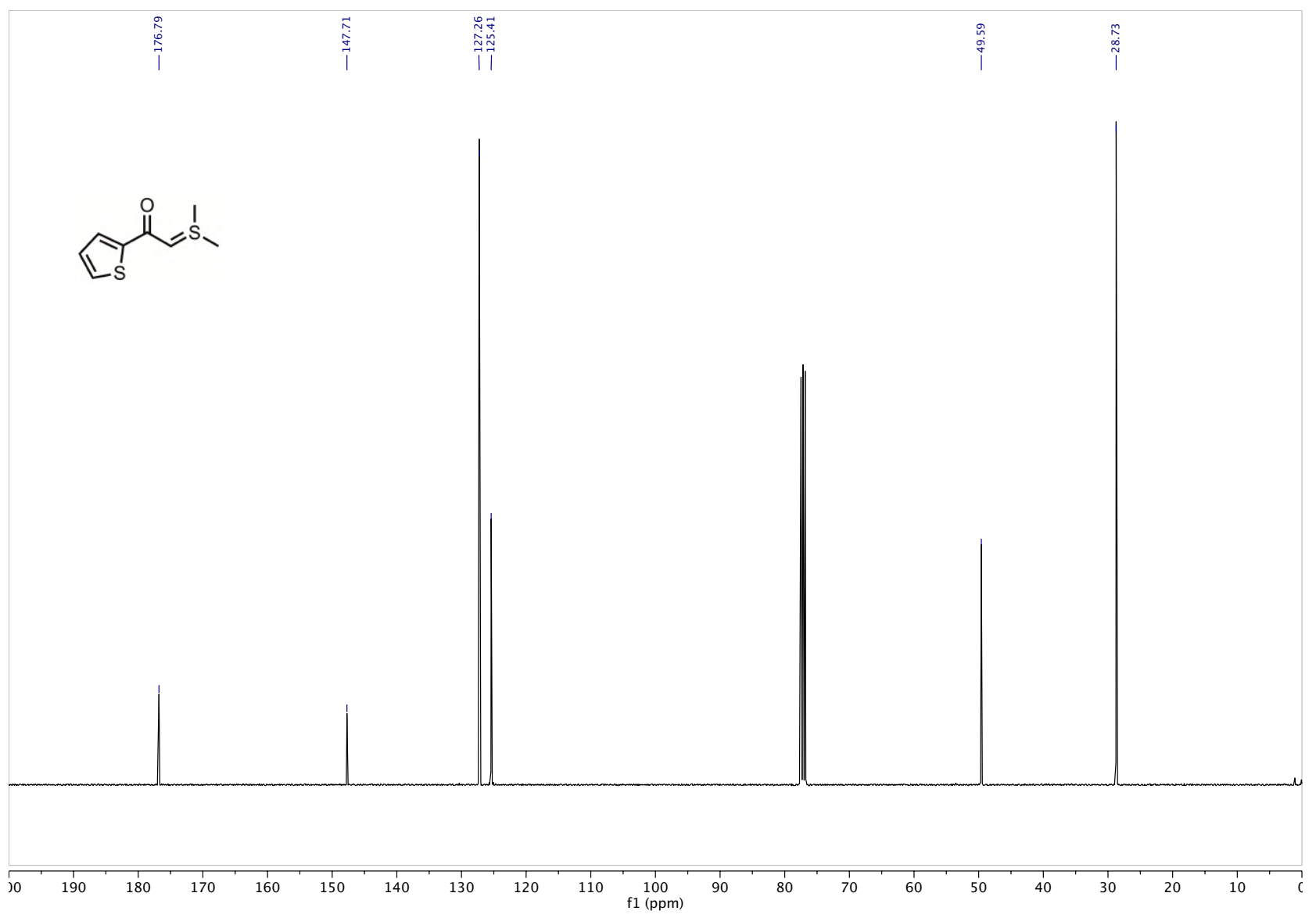

S85 
Supporting Information

${ }^{1} \mathrm{H}$ NMR: $1 \mathbf{i}$

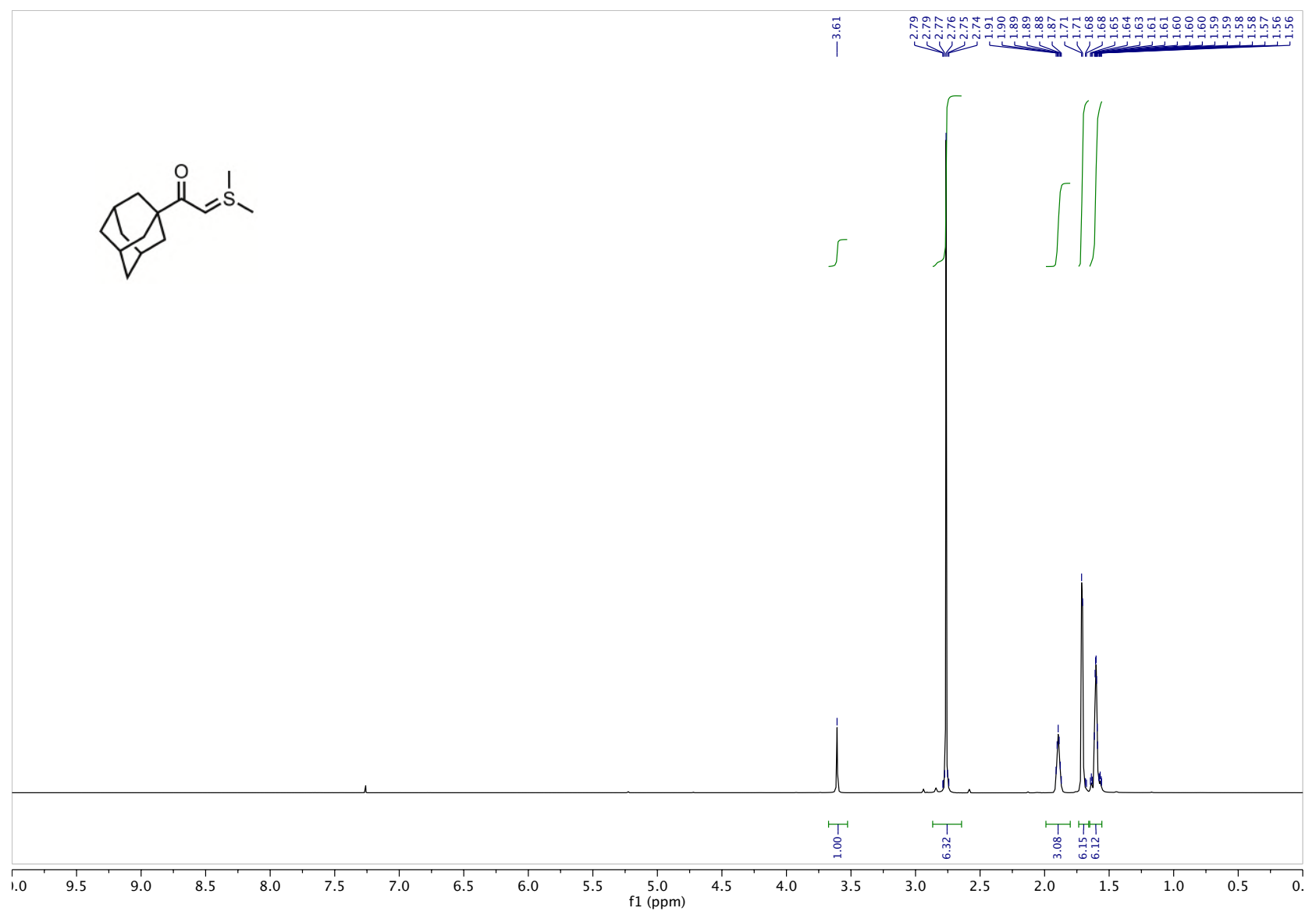

${ }^{13}$ C NMR: $1 \mathbf{i}$

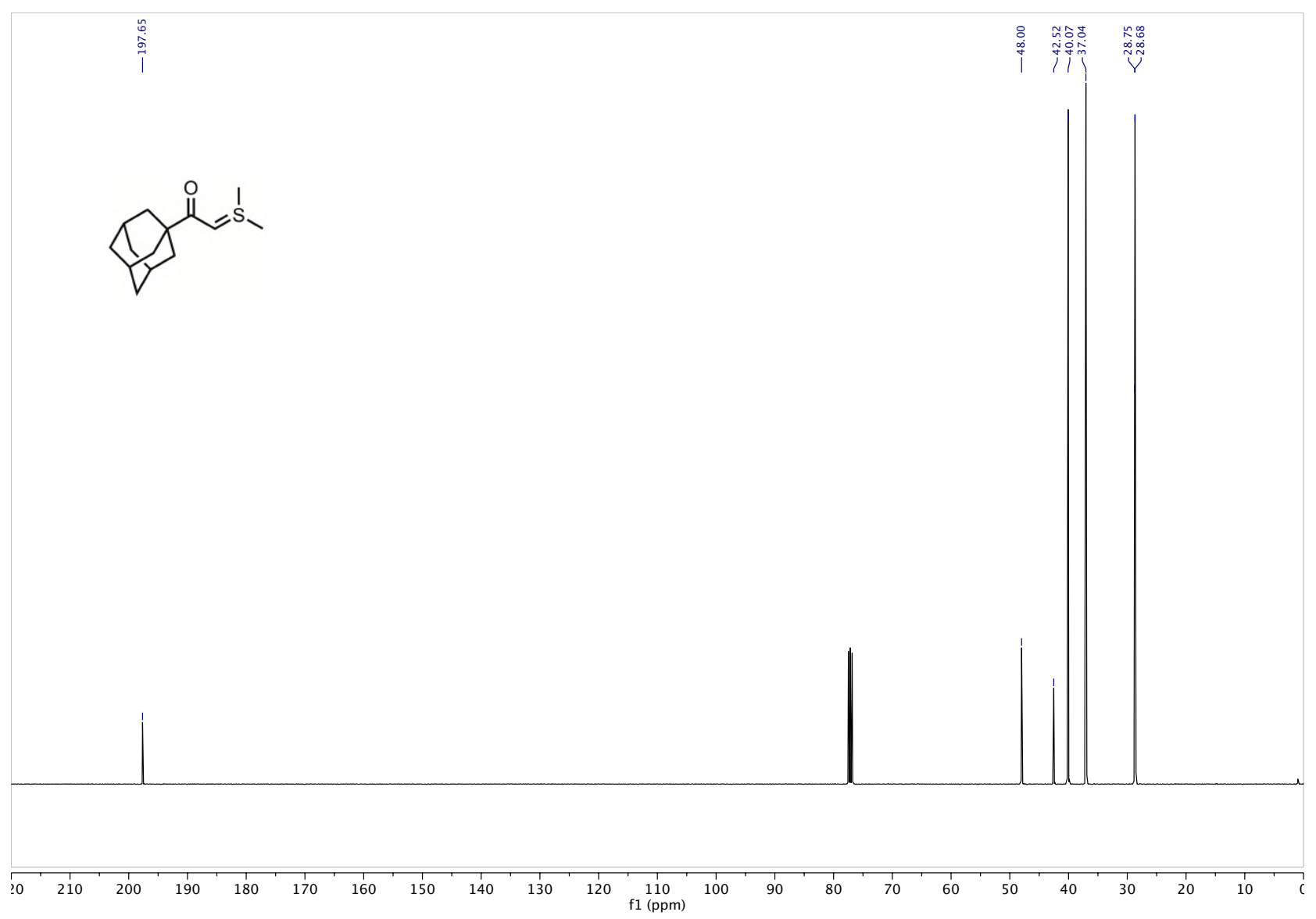

S86 
Supporting Information

${ }^{1} \mathrm{H}$ NMR: $\mathbf{1 j}$

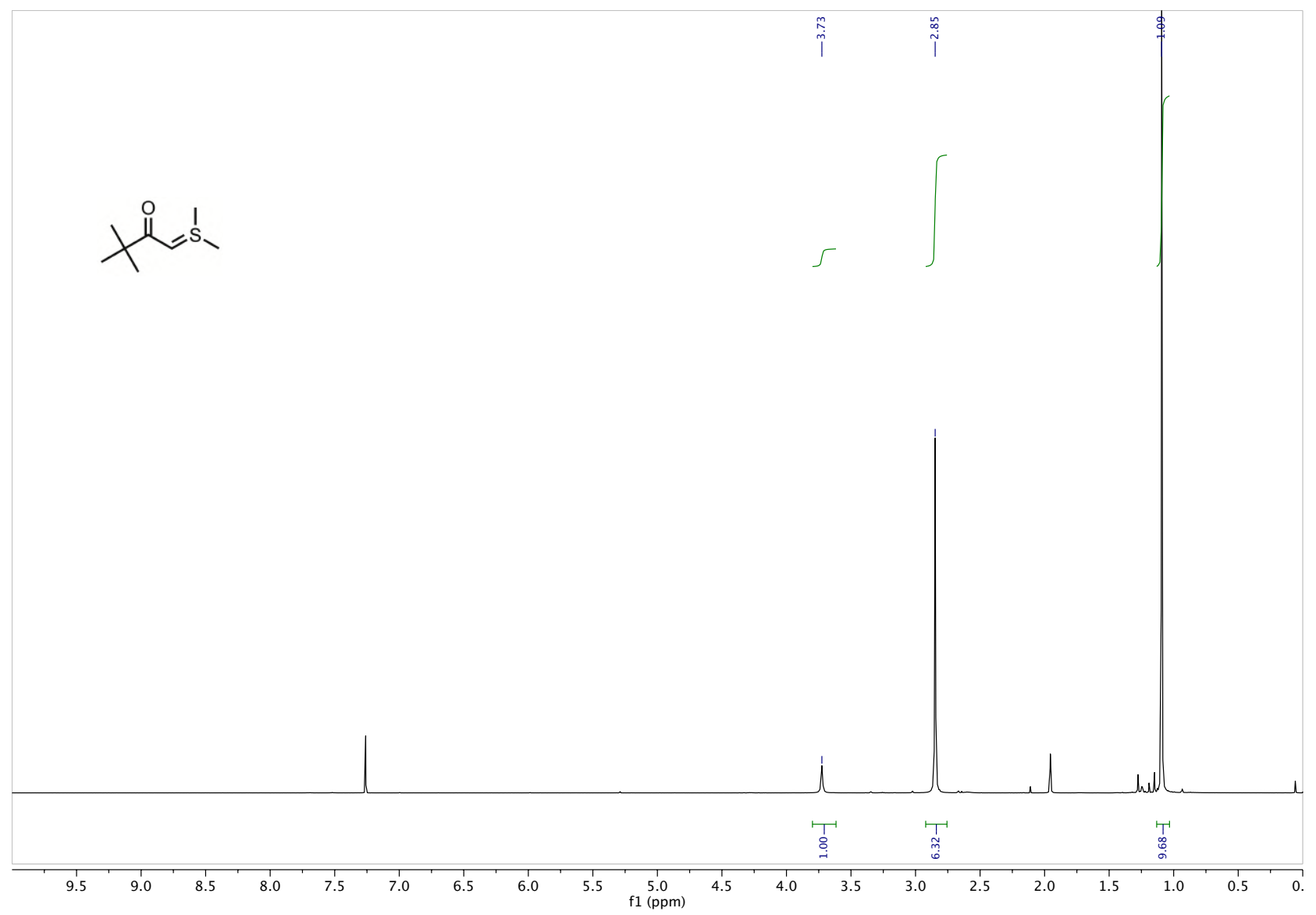

${ }^{13} \mathrm{C}$ NMR: $\mathbf{1 j}$

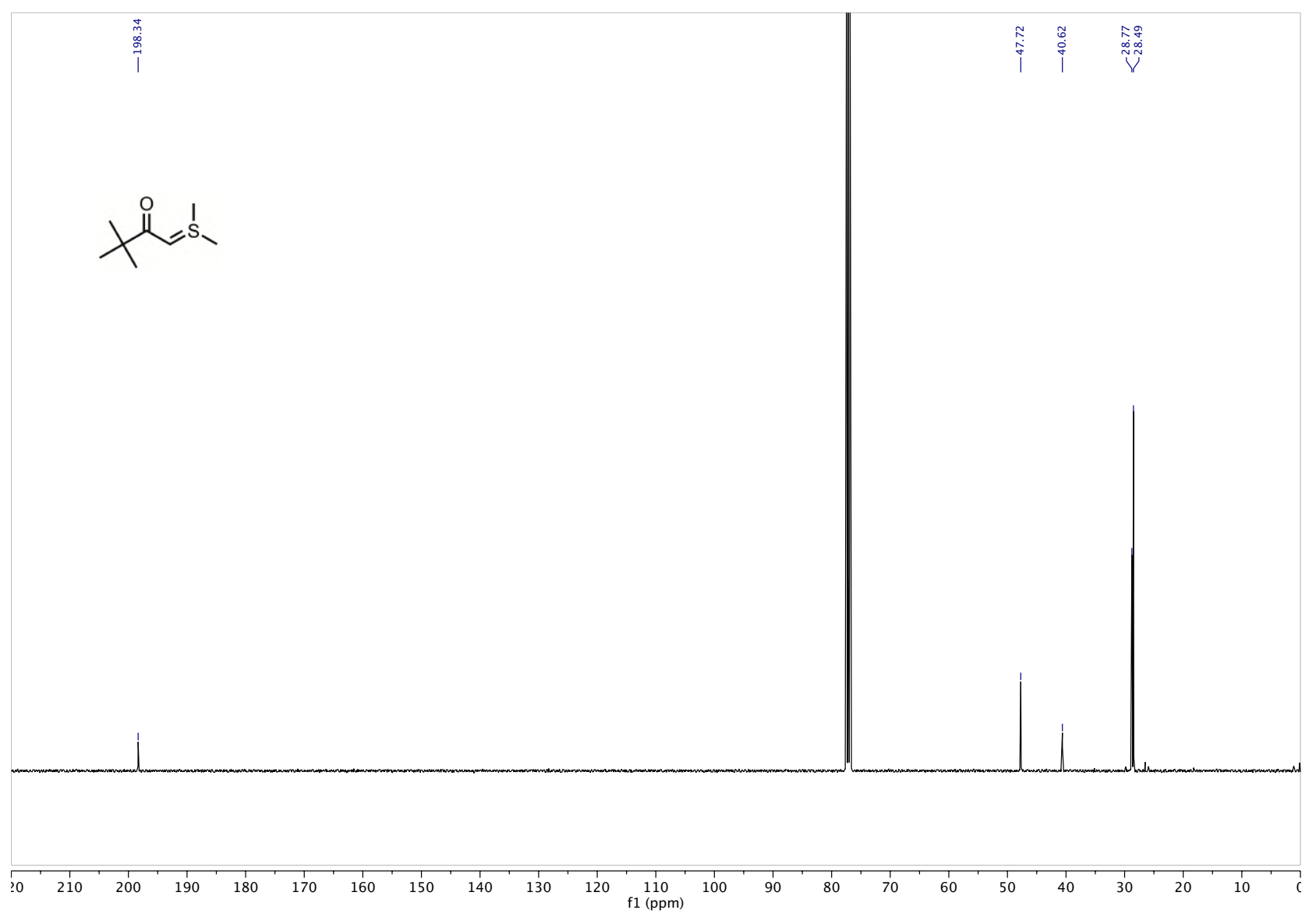

S87 
Supporting Information

${ }^{1} \mathrm{H}$ NMR: 2a

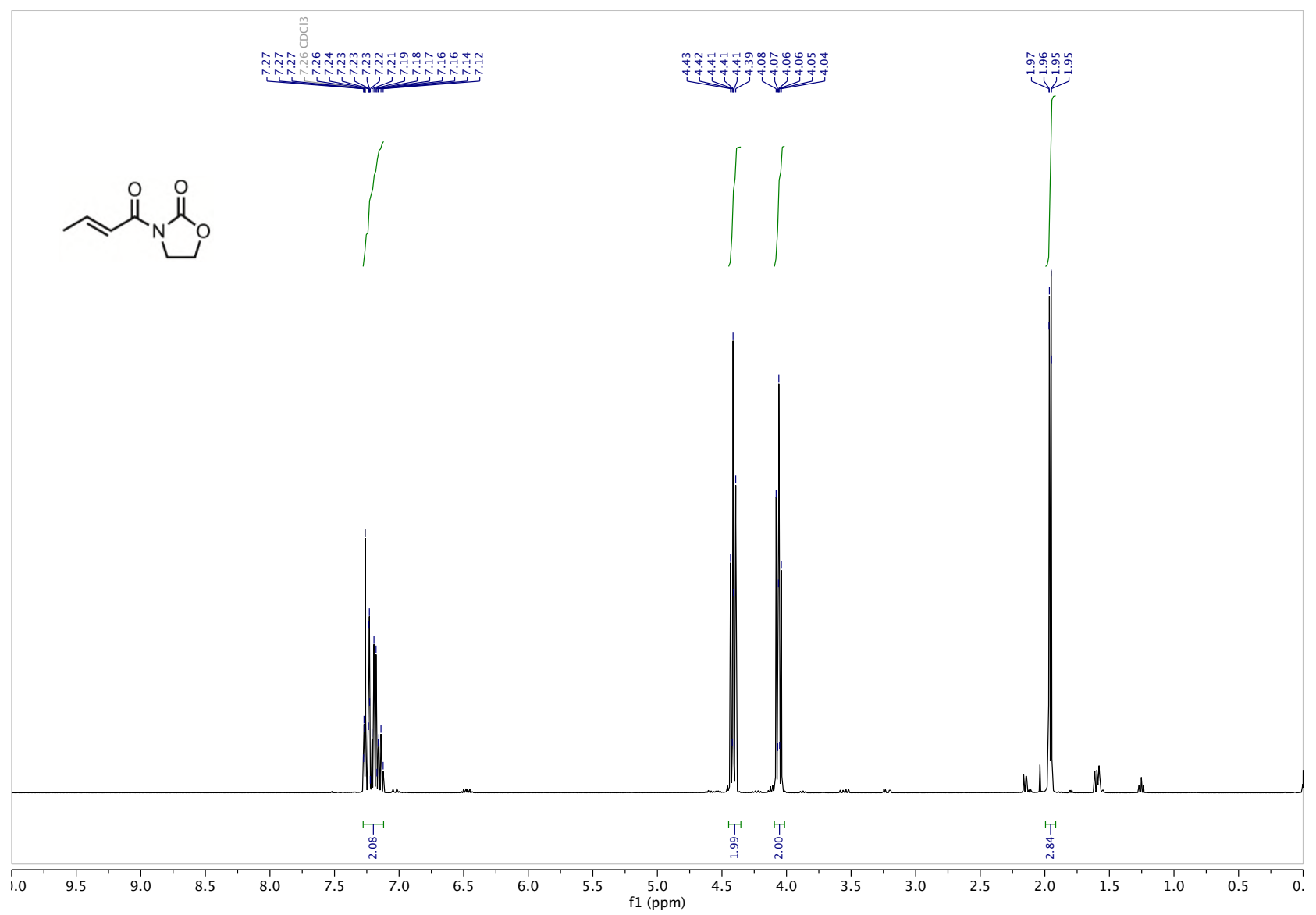

${ }^{13} \mathrm{C}$ NMR: 2a

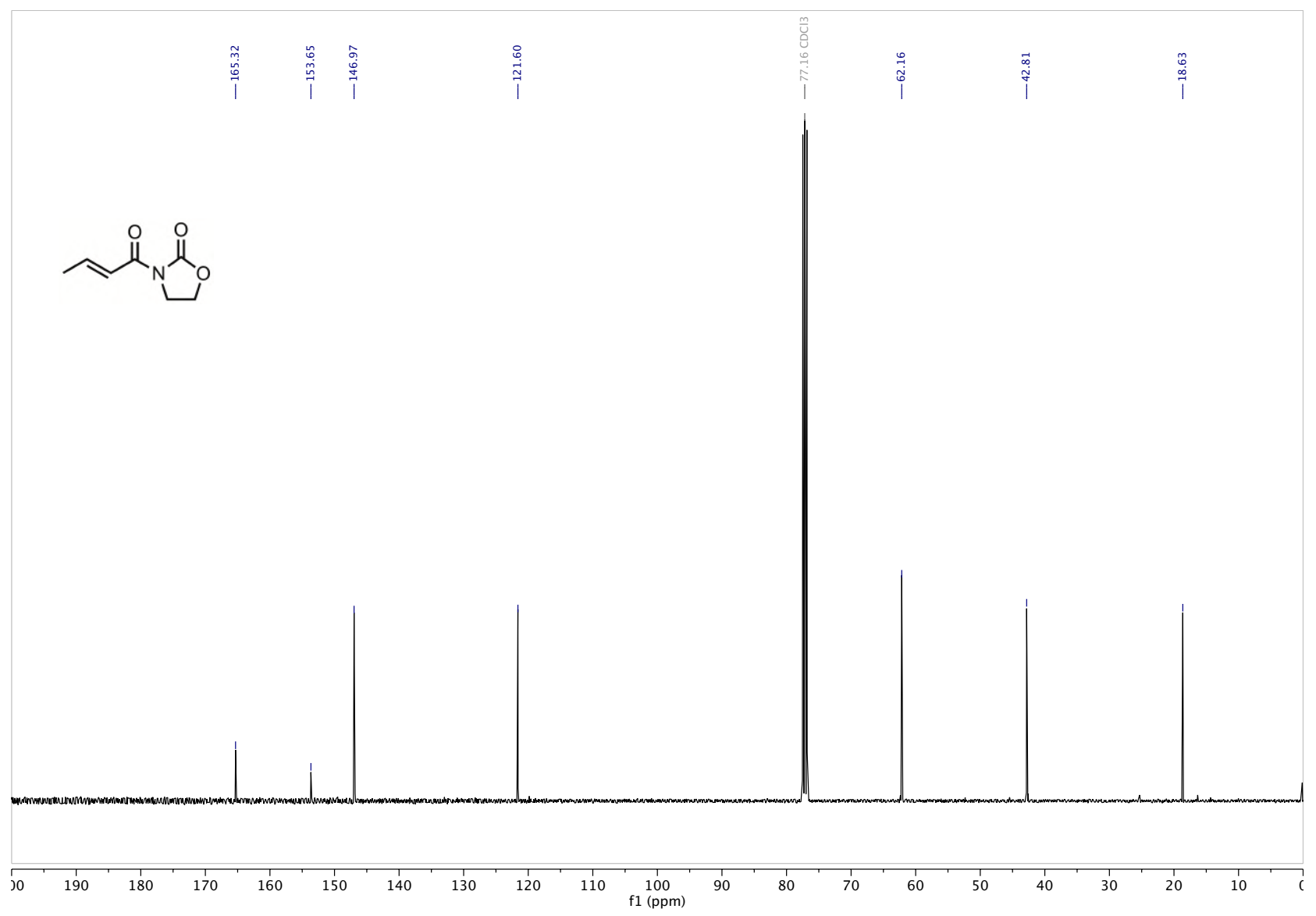

S88 
${ }^{1} \mathrm{H}$ NMR: 2b

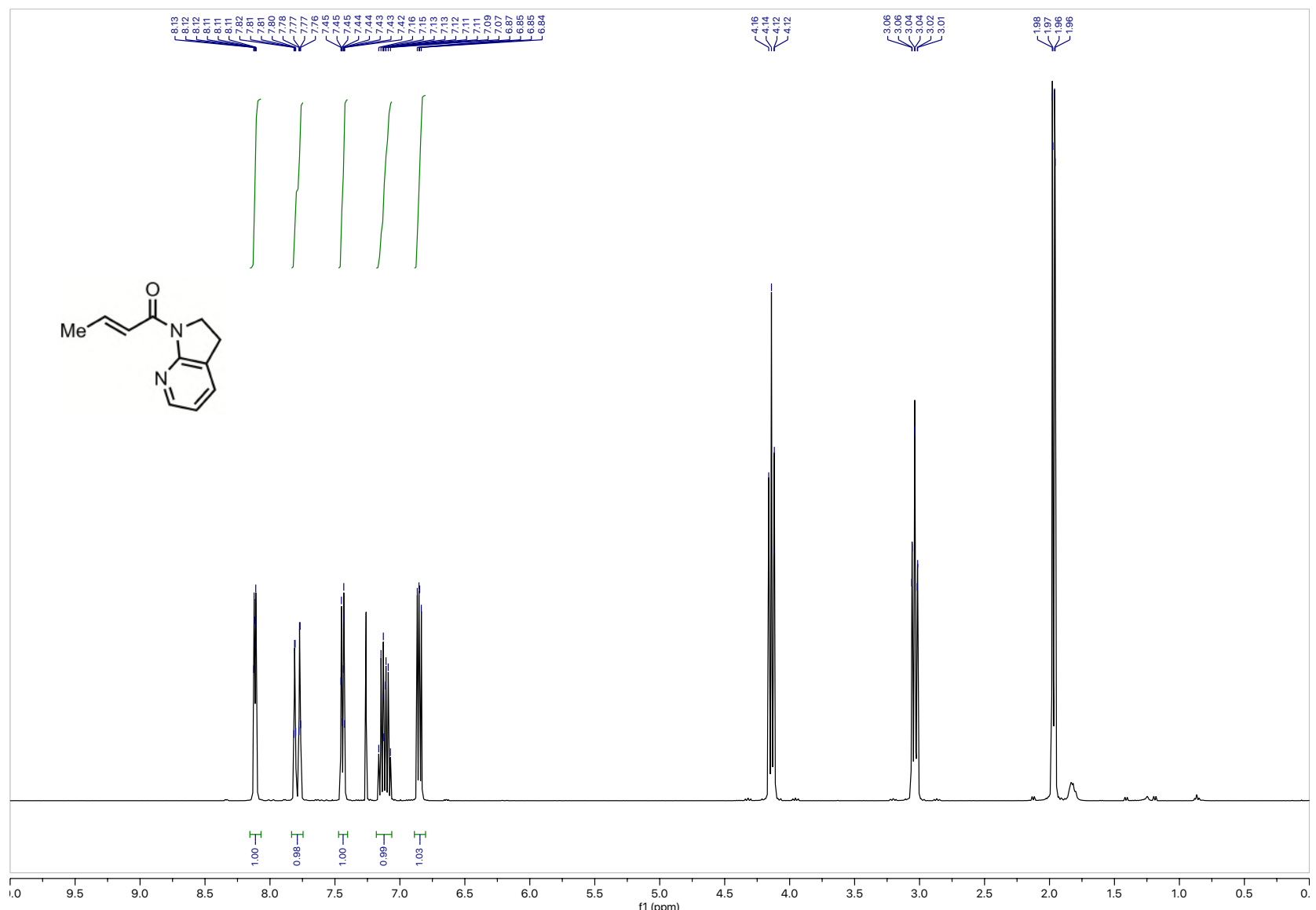

${ }^{13} \mathrm{C}$ NMR: 2b
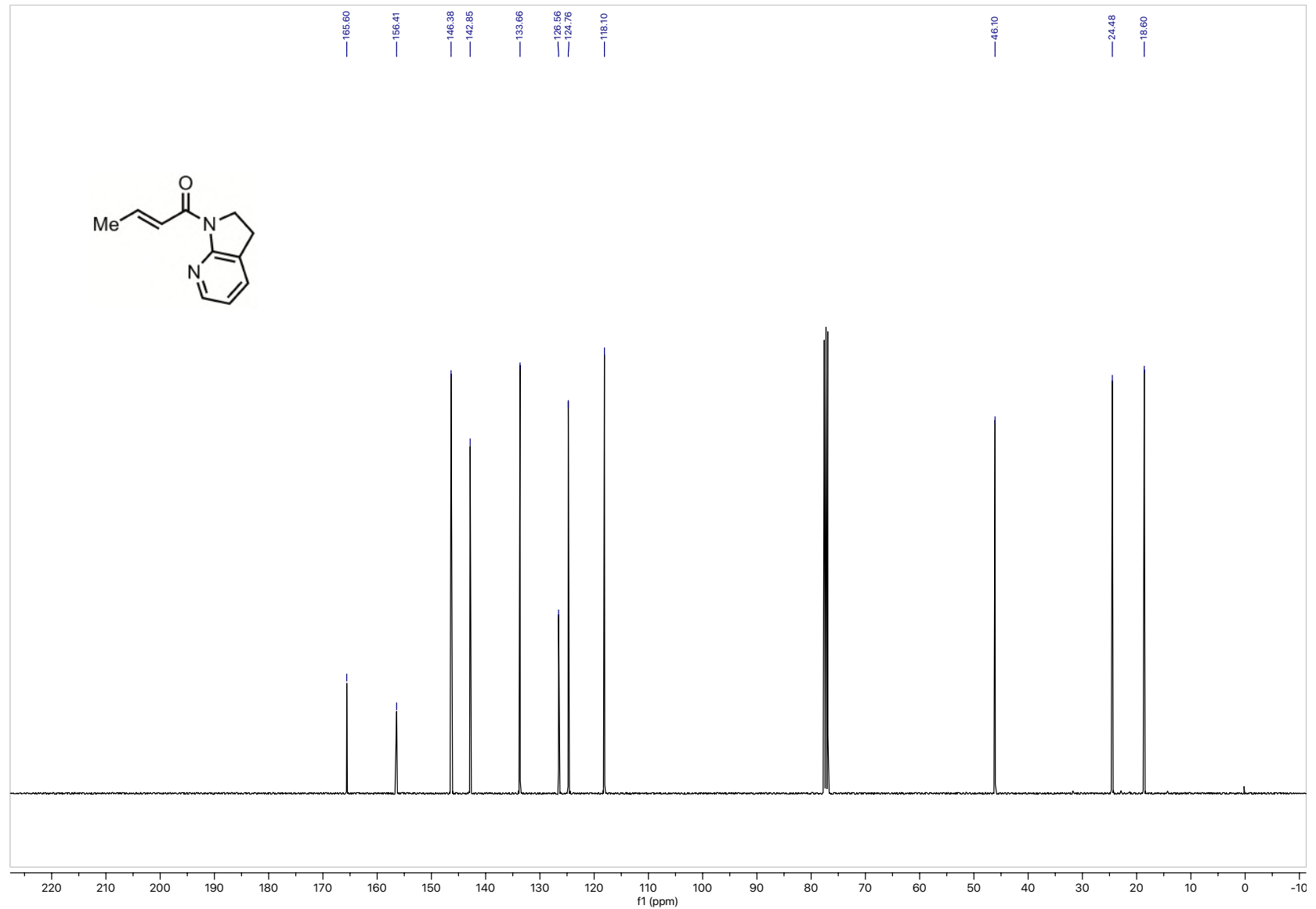
Supporting Information

${ }^{1} \mathrm{H}$ NMR: Qc

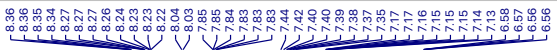

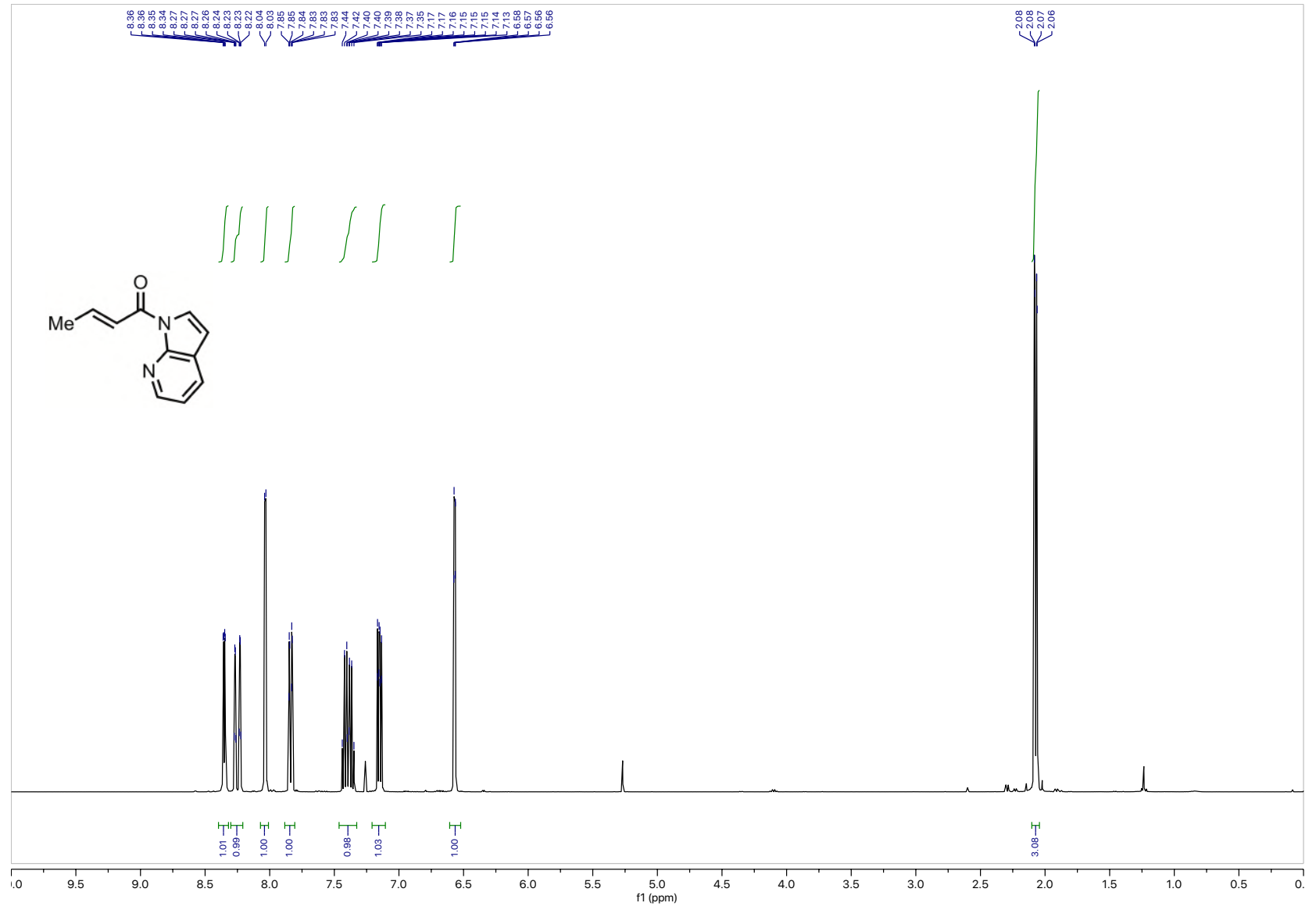

${ }^{13} \mathrm{C}$ NMR: ic

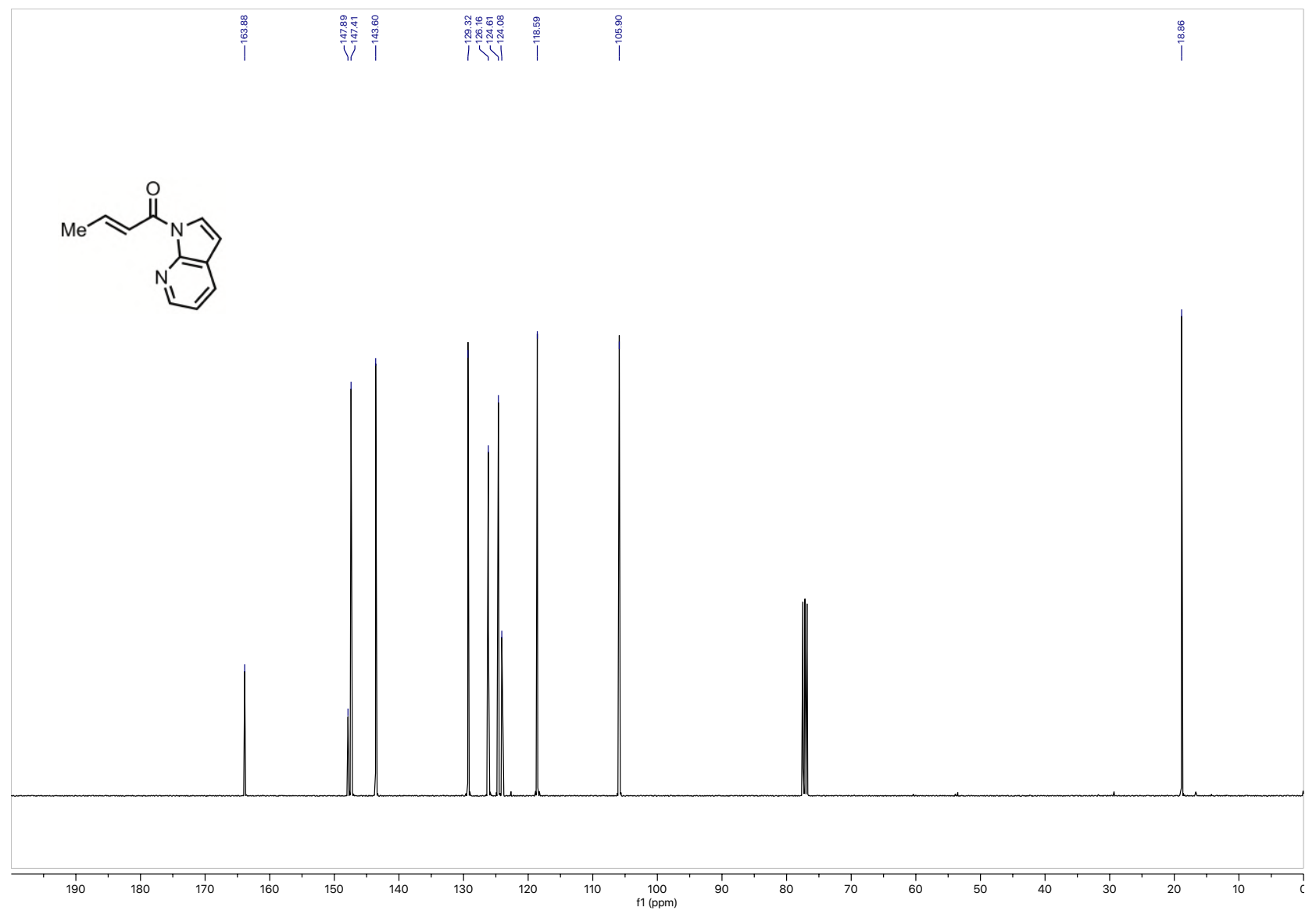

S90 
${ }^{1} \mathrm{H}$ NMR: 2d (DMSO-d6)

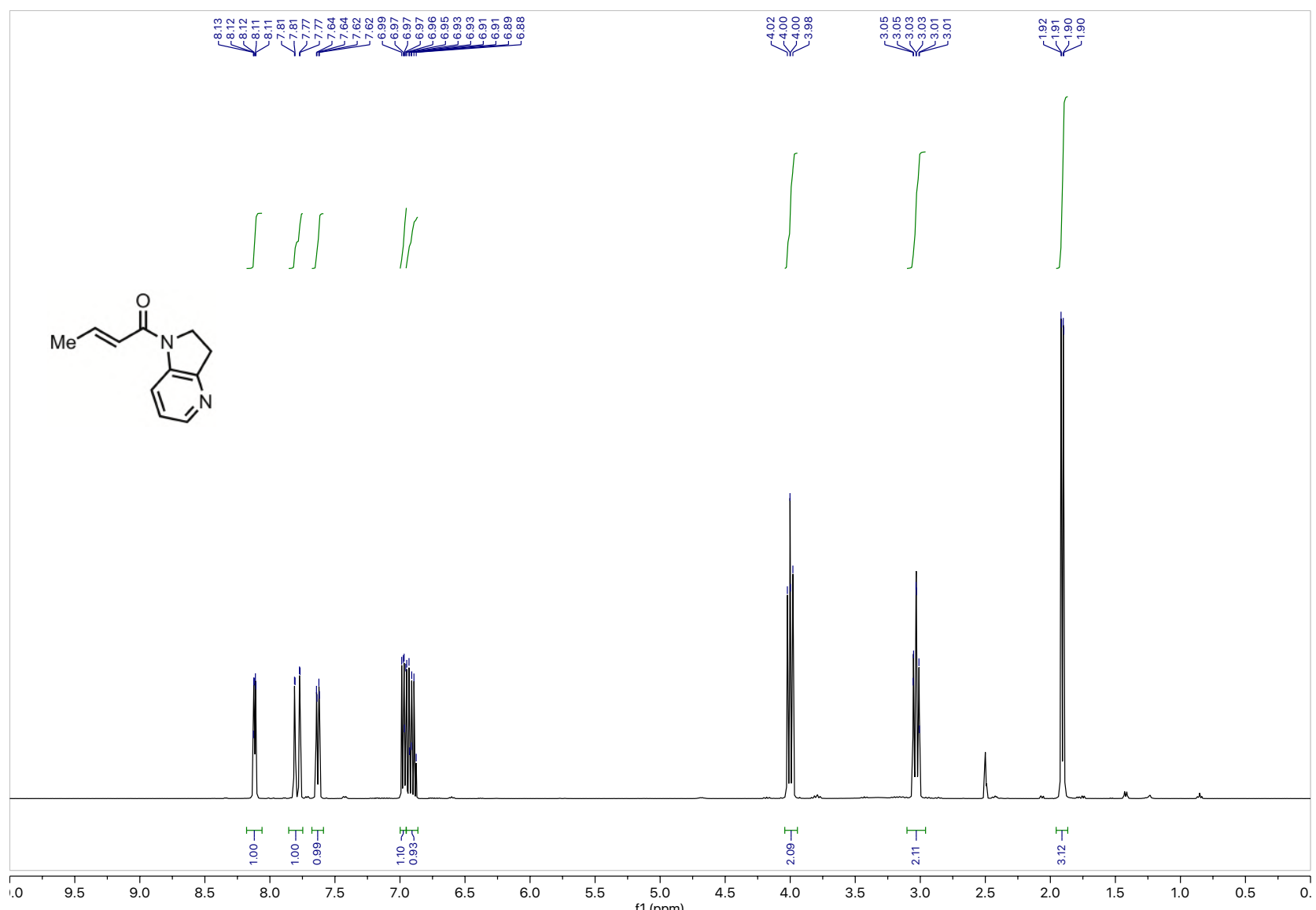

${ }^{13}$ C NMR: 2d (DMSO- $d_{6}$ )

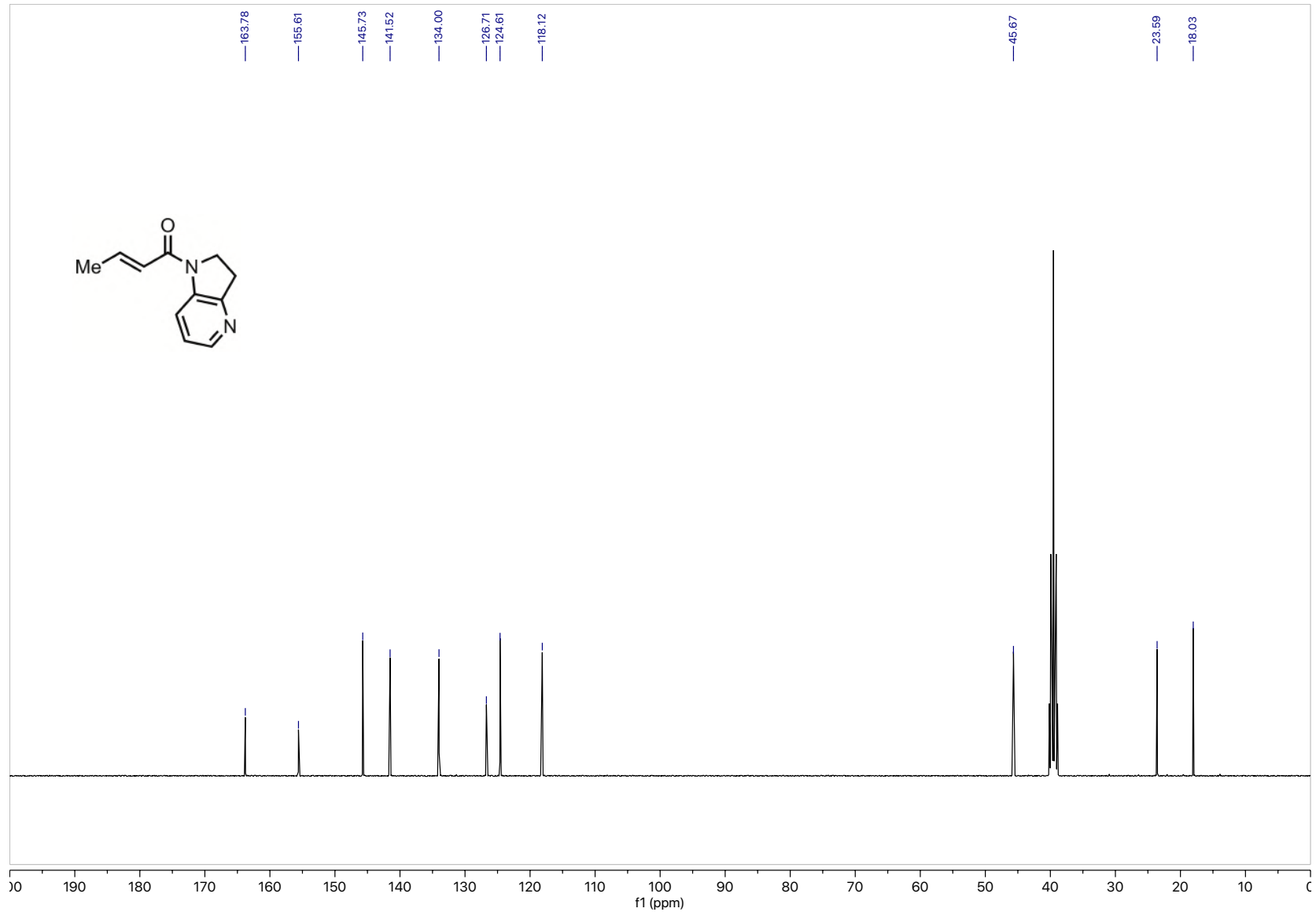


${ }^{1} \mathrm{H}$ NMR: $2 \mathbf{e}$

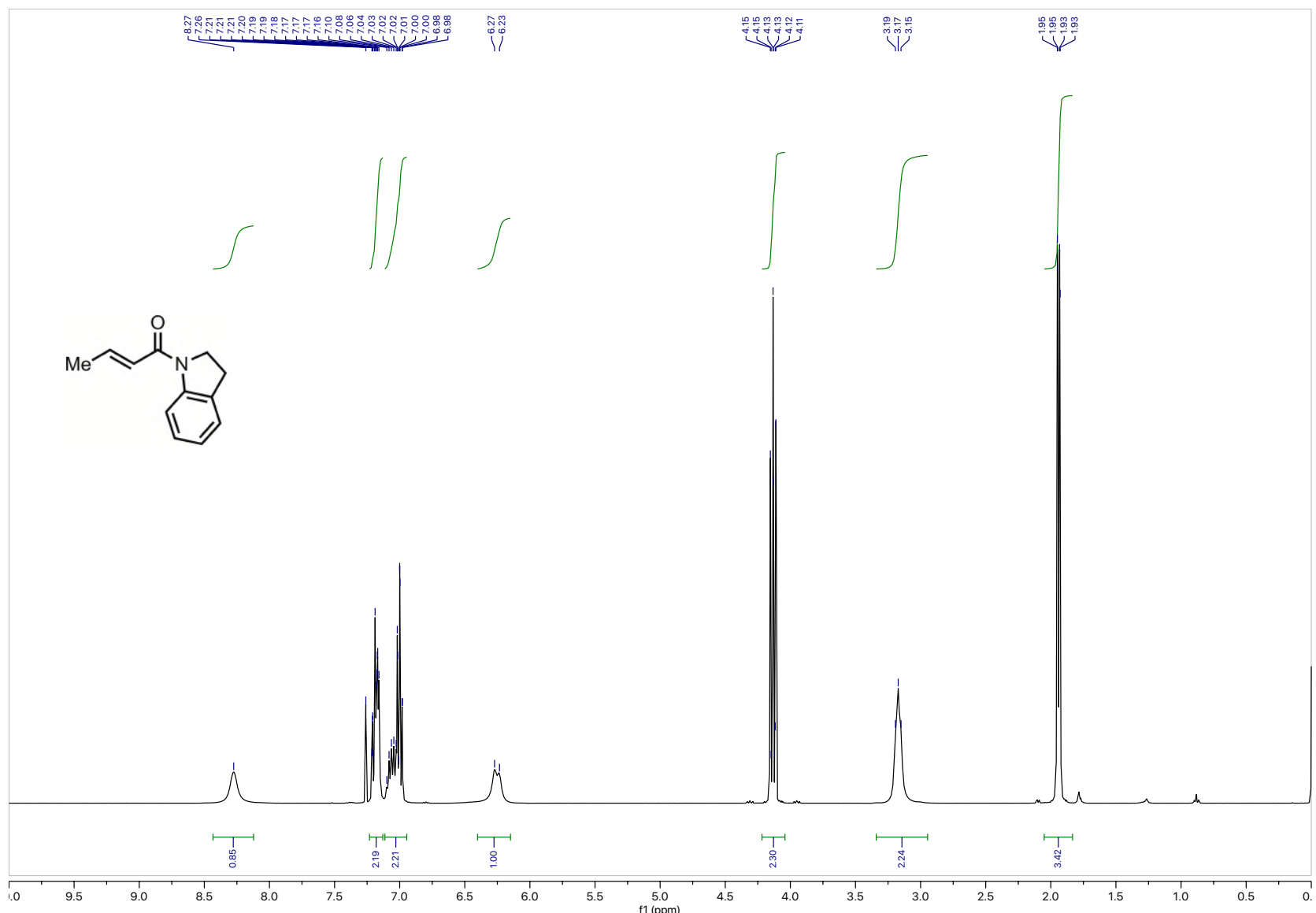

${ }^{13} \mathrm{C}$ NMR: $2 \mathrm{e}$
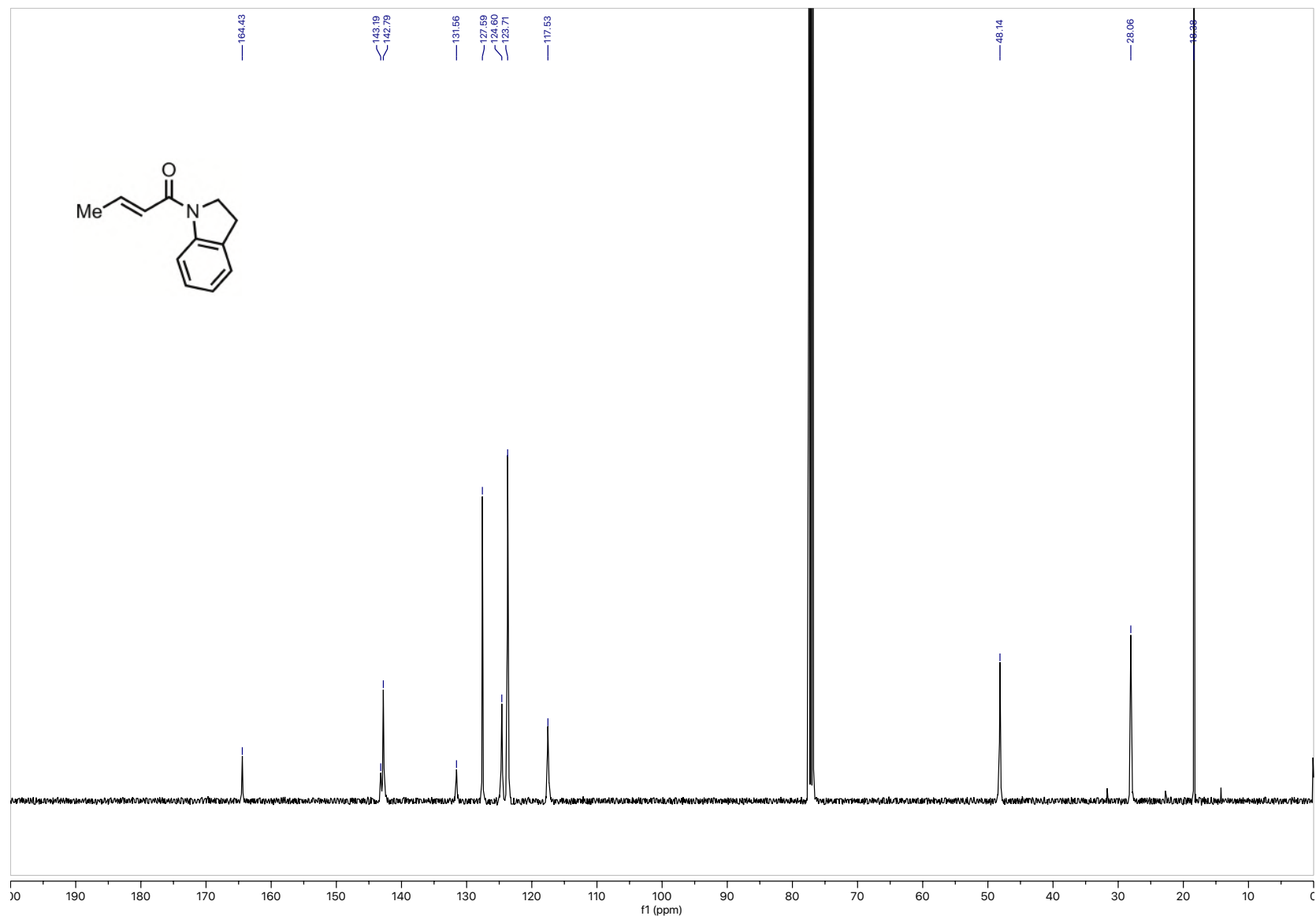
Supporting Information

${ }^{1} \mathrm{H}$ NMR: $2 \mathrm{f}$

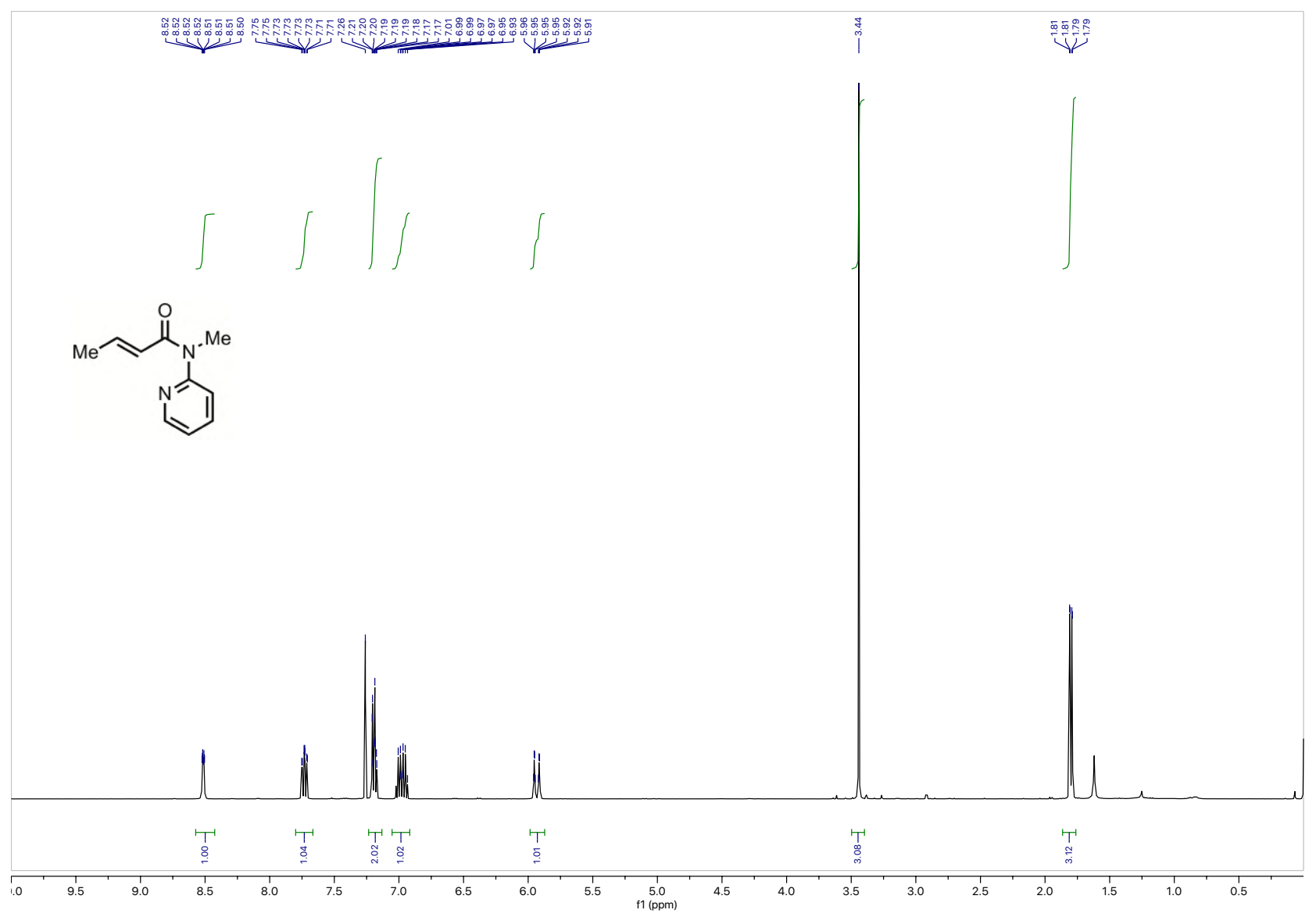

${ }^{13}$ C NMR: $2 f$

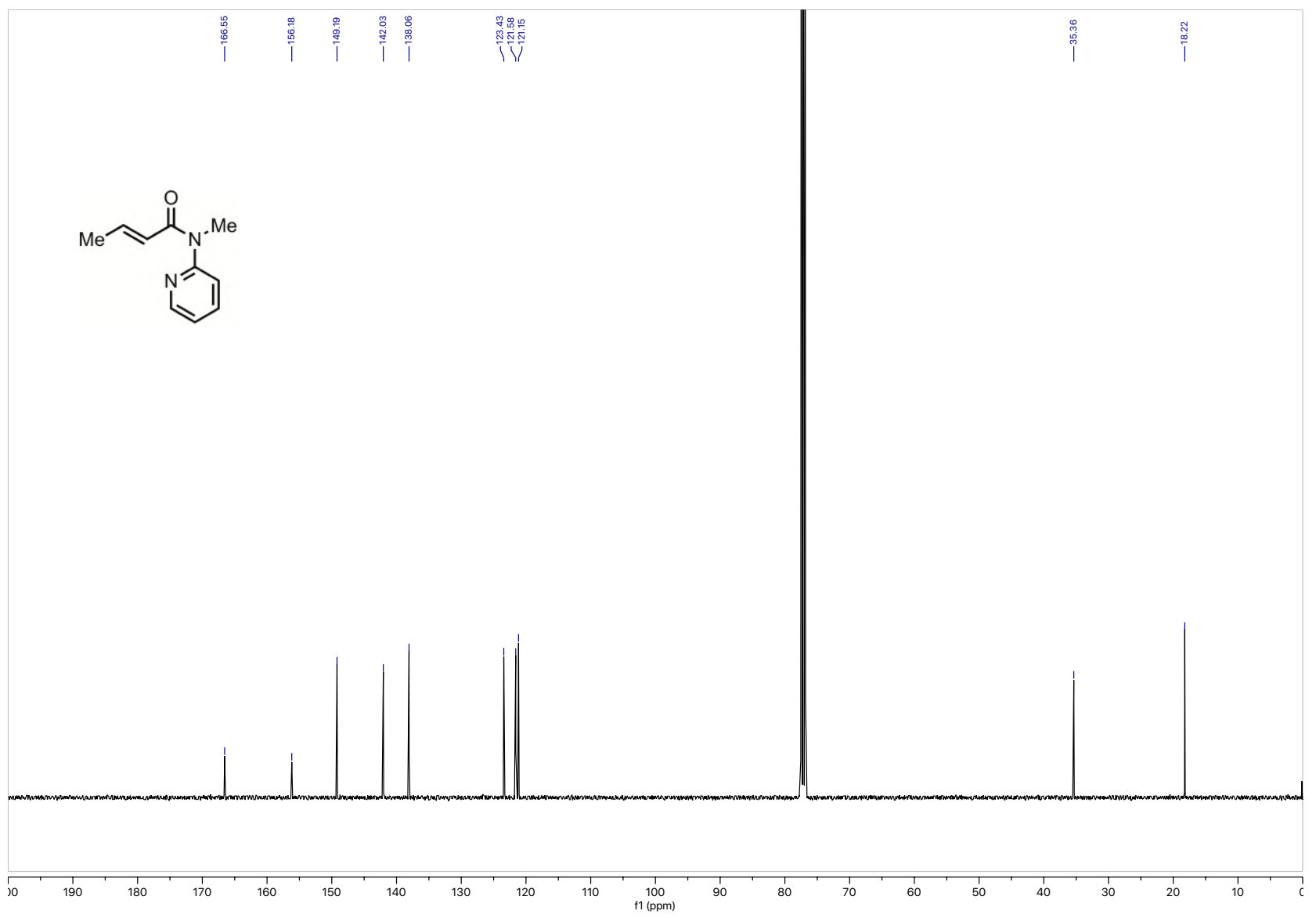

S93 
${ }^{1} \mathrm{H}$ NMR: $2 \mathrm{~g}$

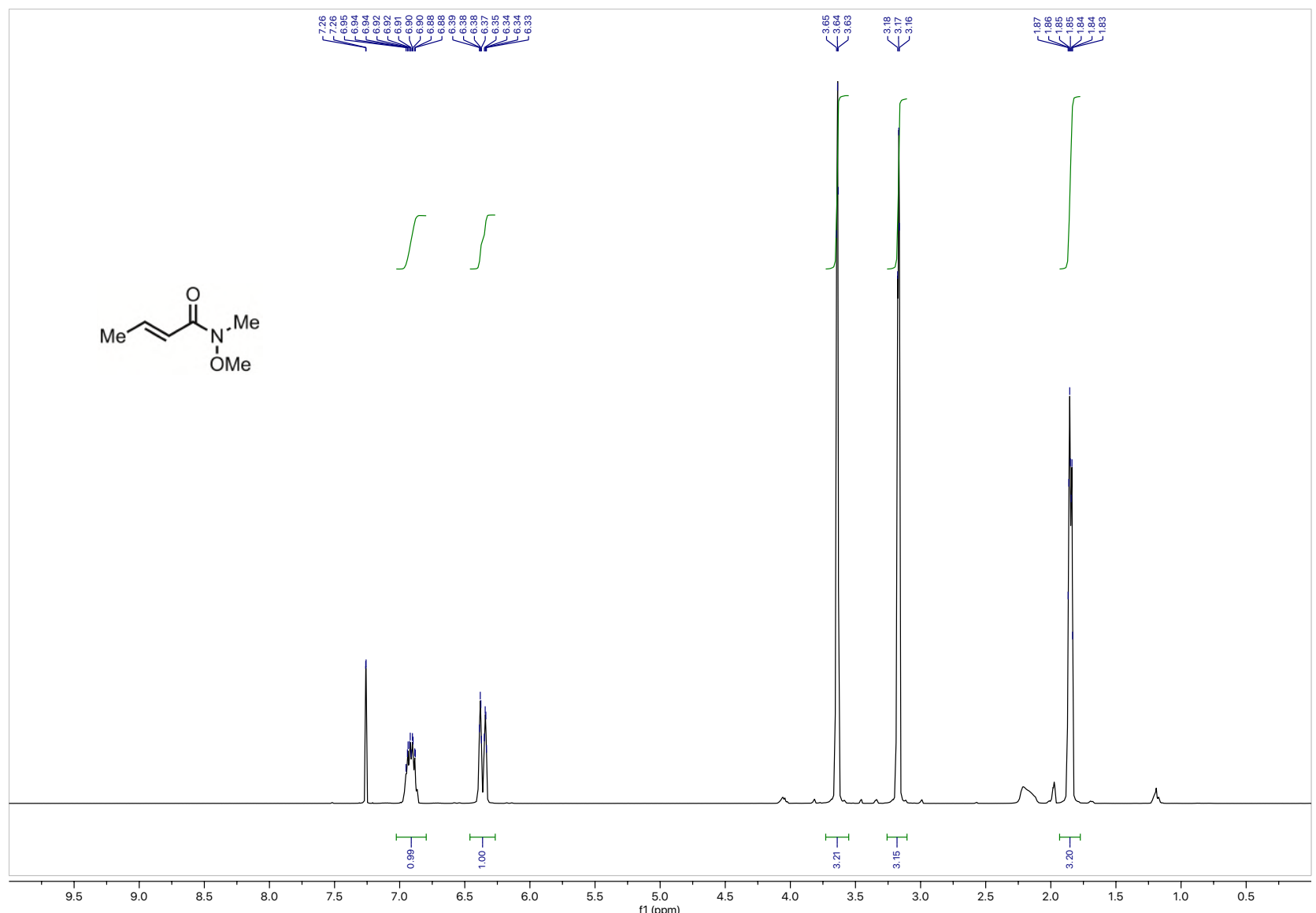

${ }^{13} \mathrm{C}$ NMR: $2 \mathrm{~g}$

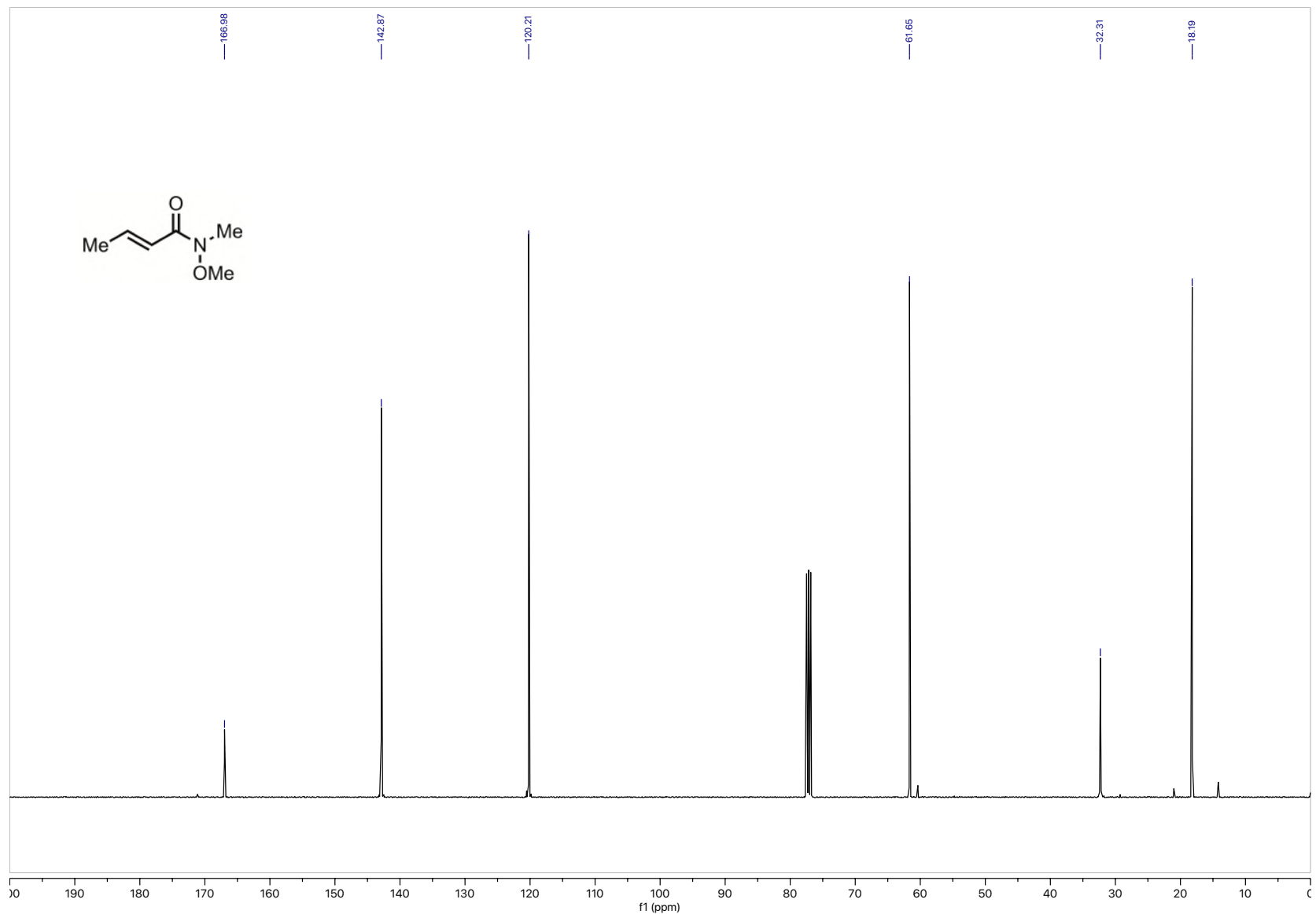


Supporting Information

${ }^{1} \mathrm{H}$ NMR: $\mathbf{2 h}$

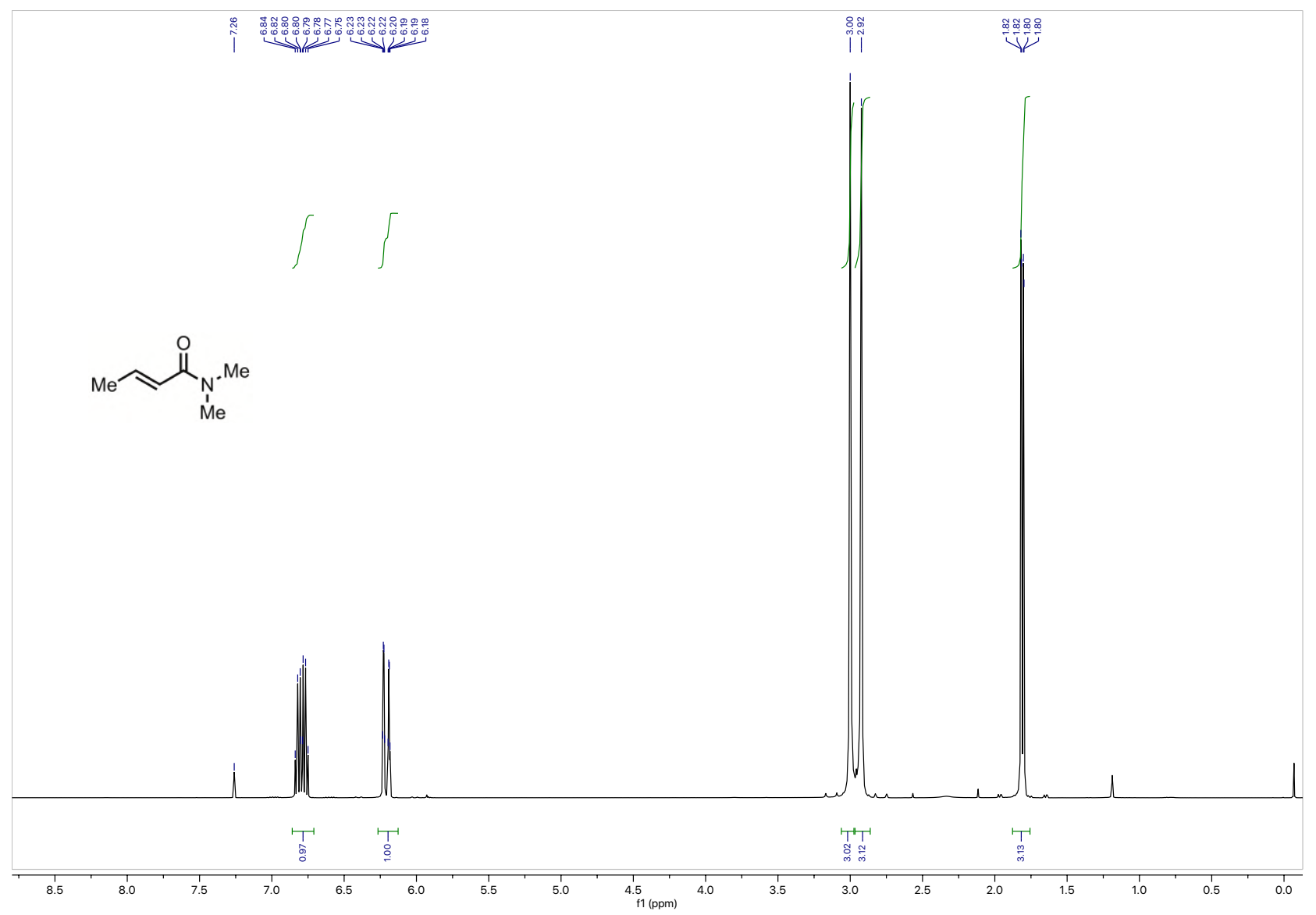

${ }^{13}$ C NMR: $\mathbf{2 h}$

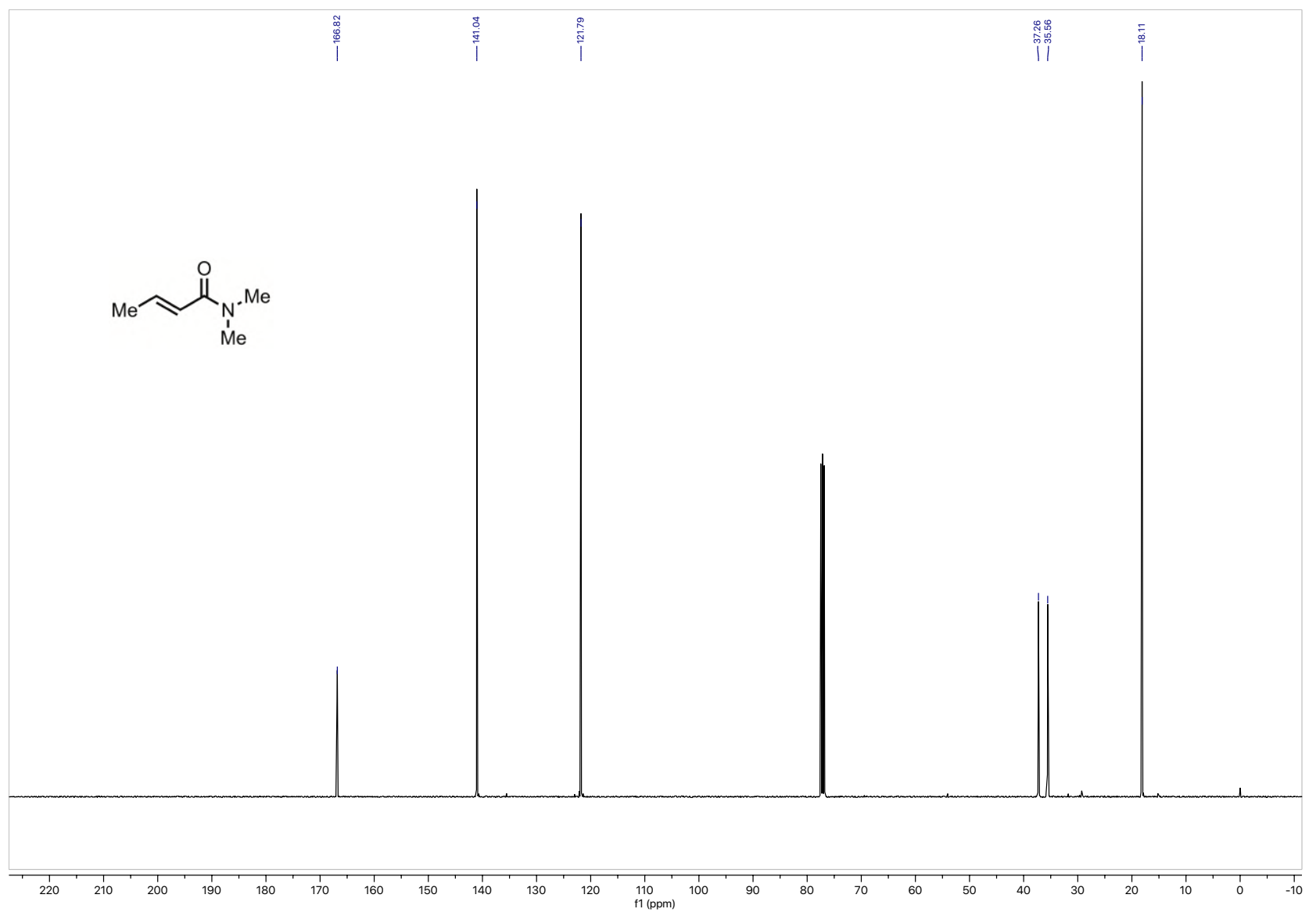

S95 
${ }^{1} \mathrm{H}$ NMR: 2i

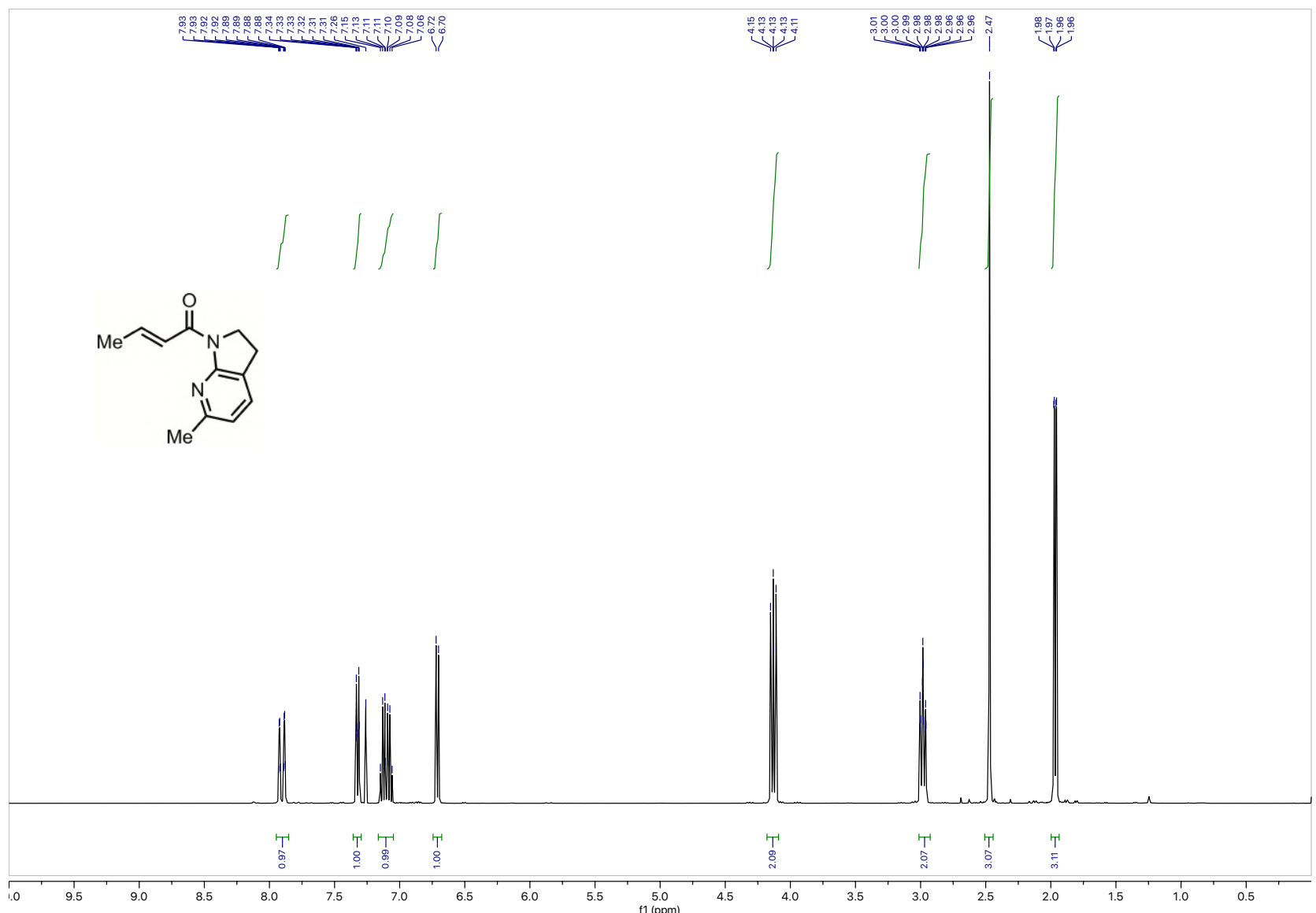

${ }^{13}$ C NMR: $2 \mathbf{i}$

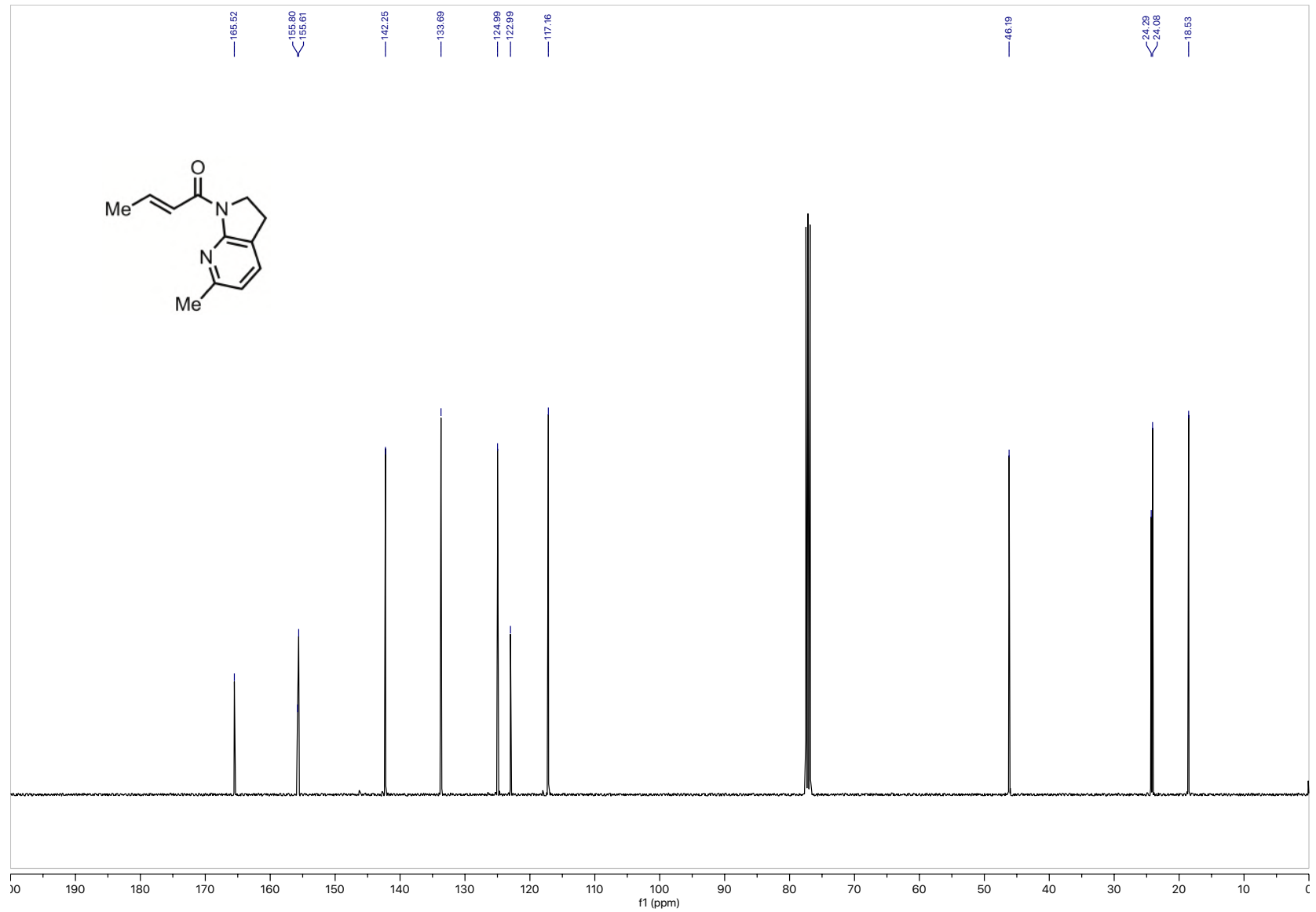


${ }^{1} \mathrm{H}$ NMR: $\mathbf{2 j}$

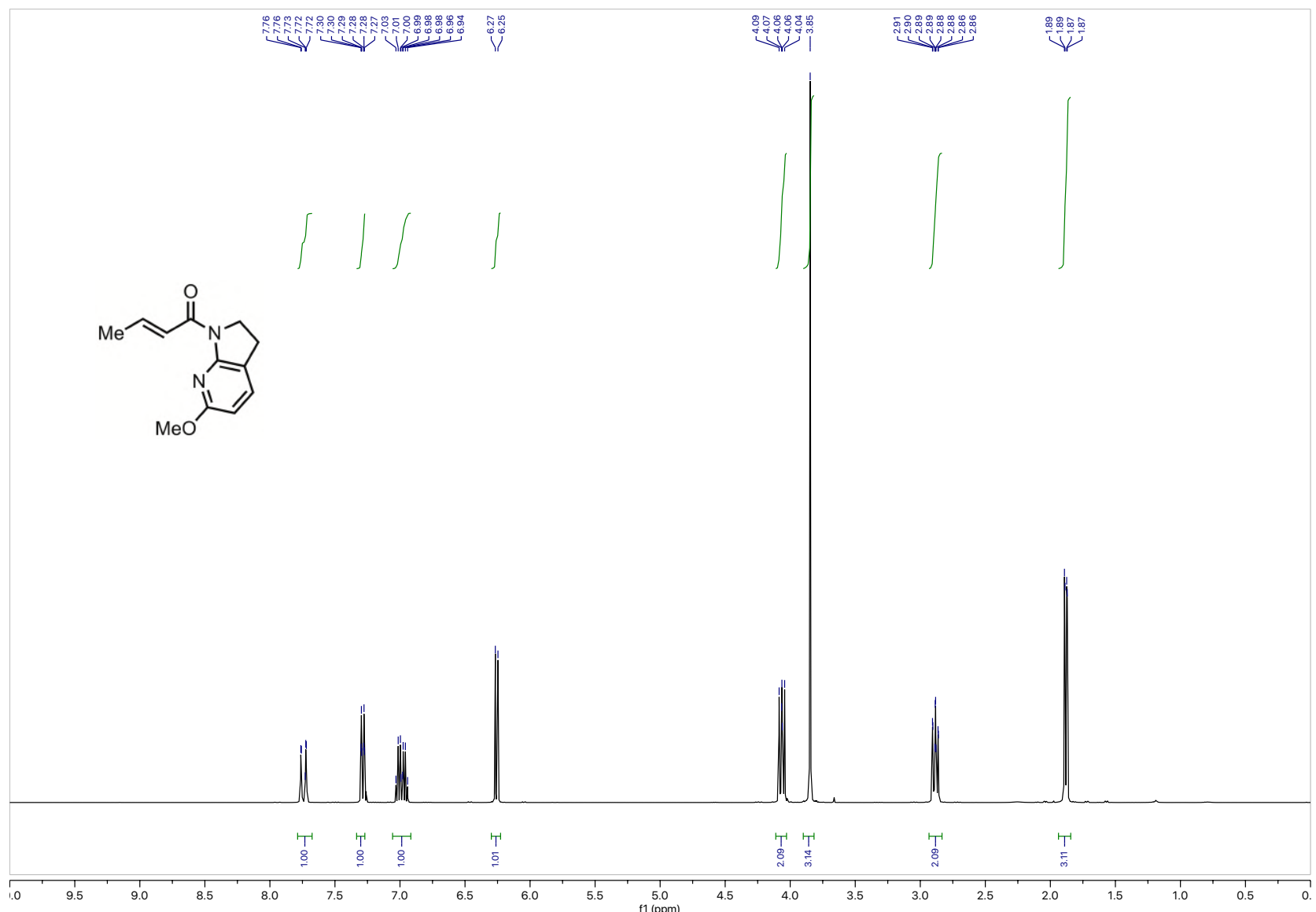

${ }^{13}$ C NMR: 2j

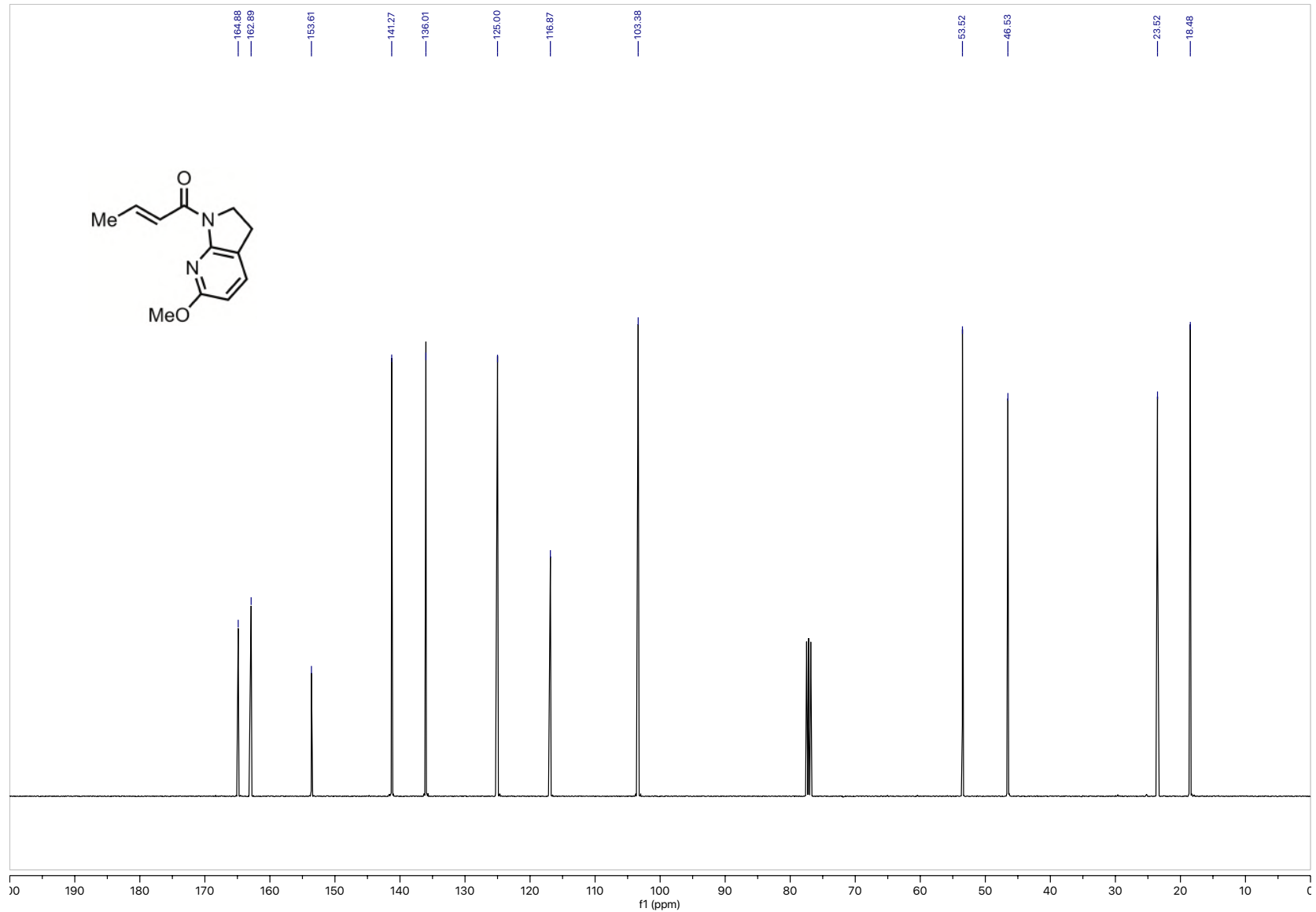


Supporting Information

${ }^{1} \mathrm{H}$ NMR: 2k

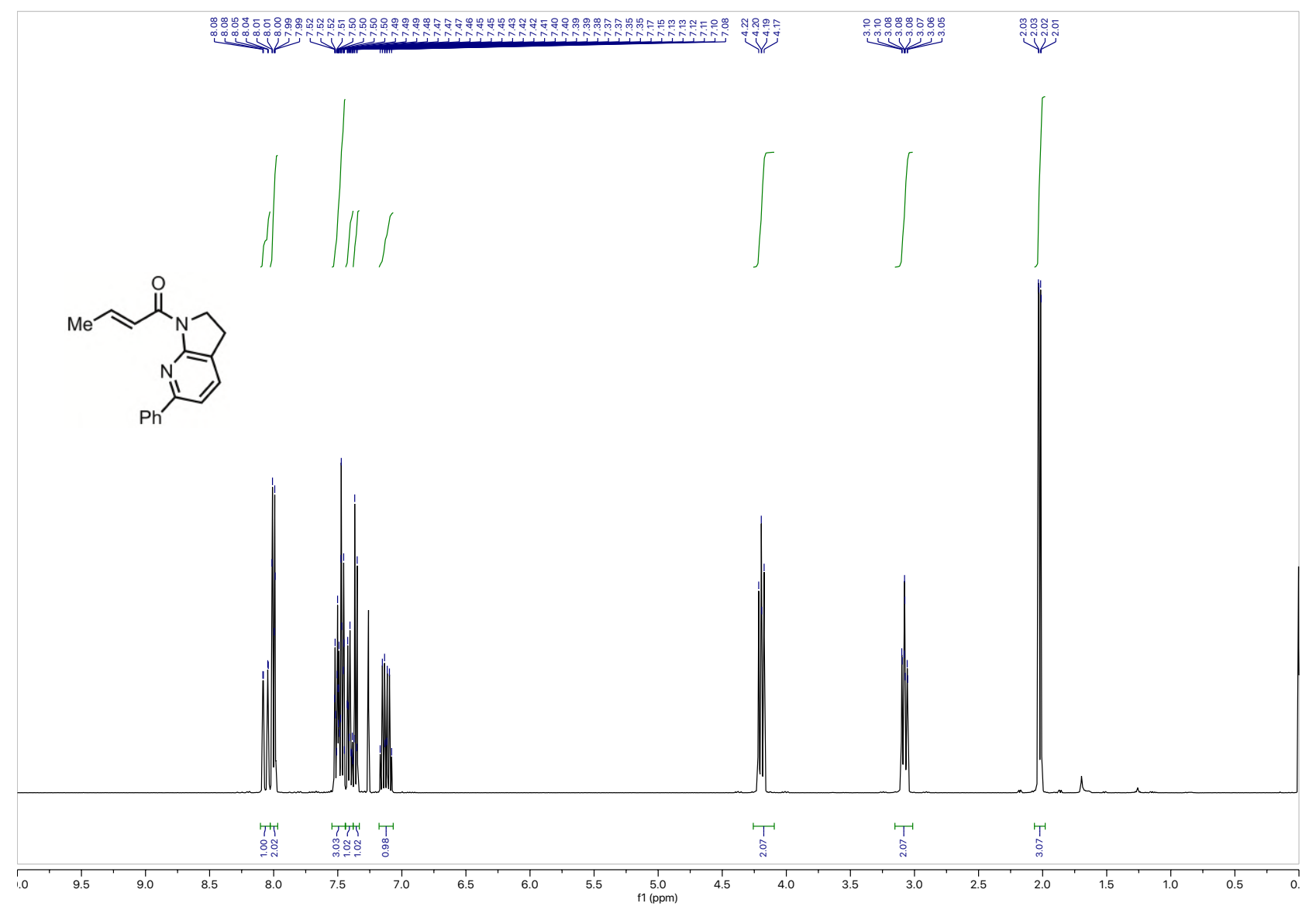

${ }^{13}$ C NMR: 2k

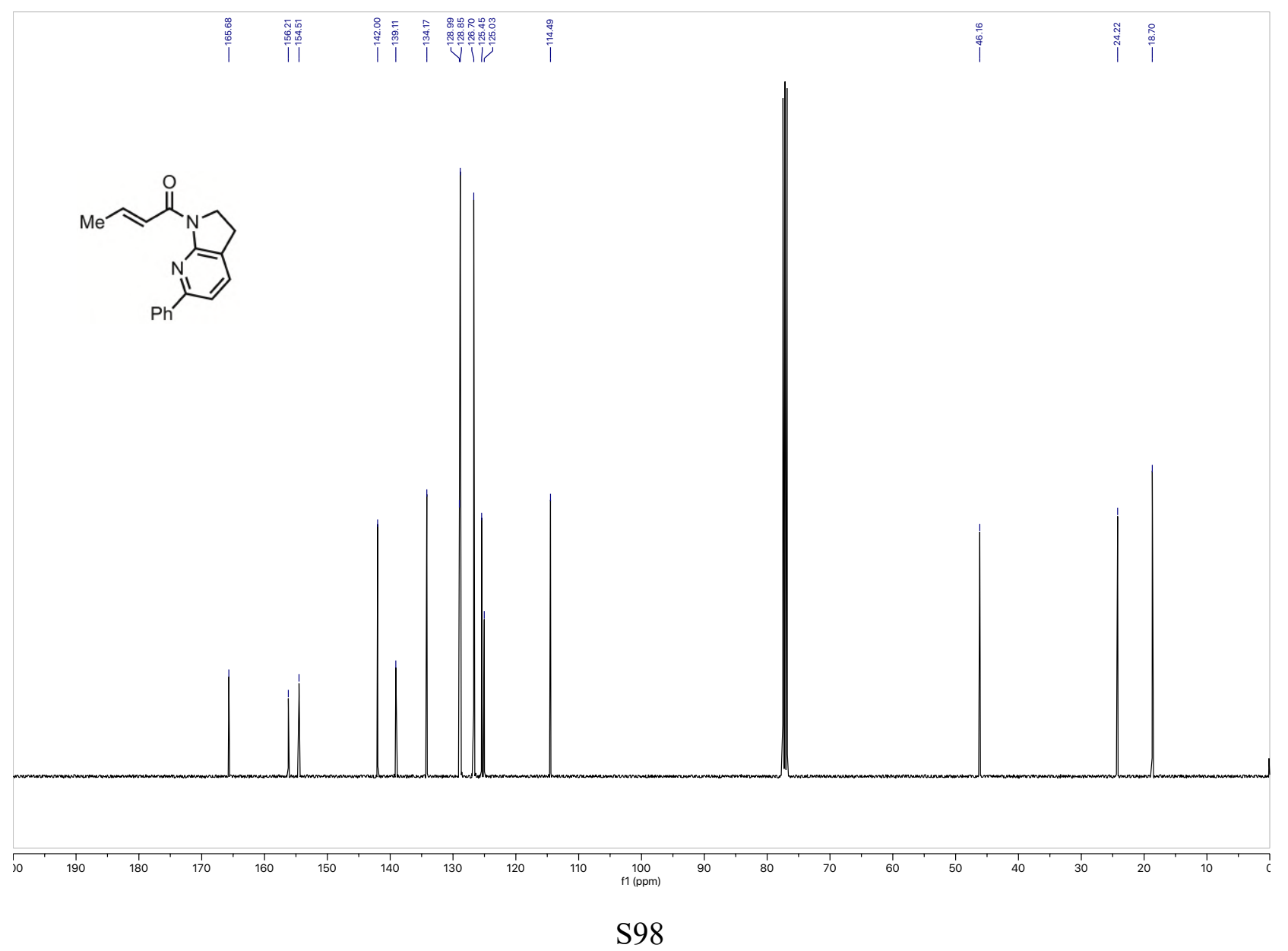


${ }^{1} \mathrm{H}$ NMR: 21

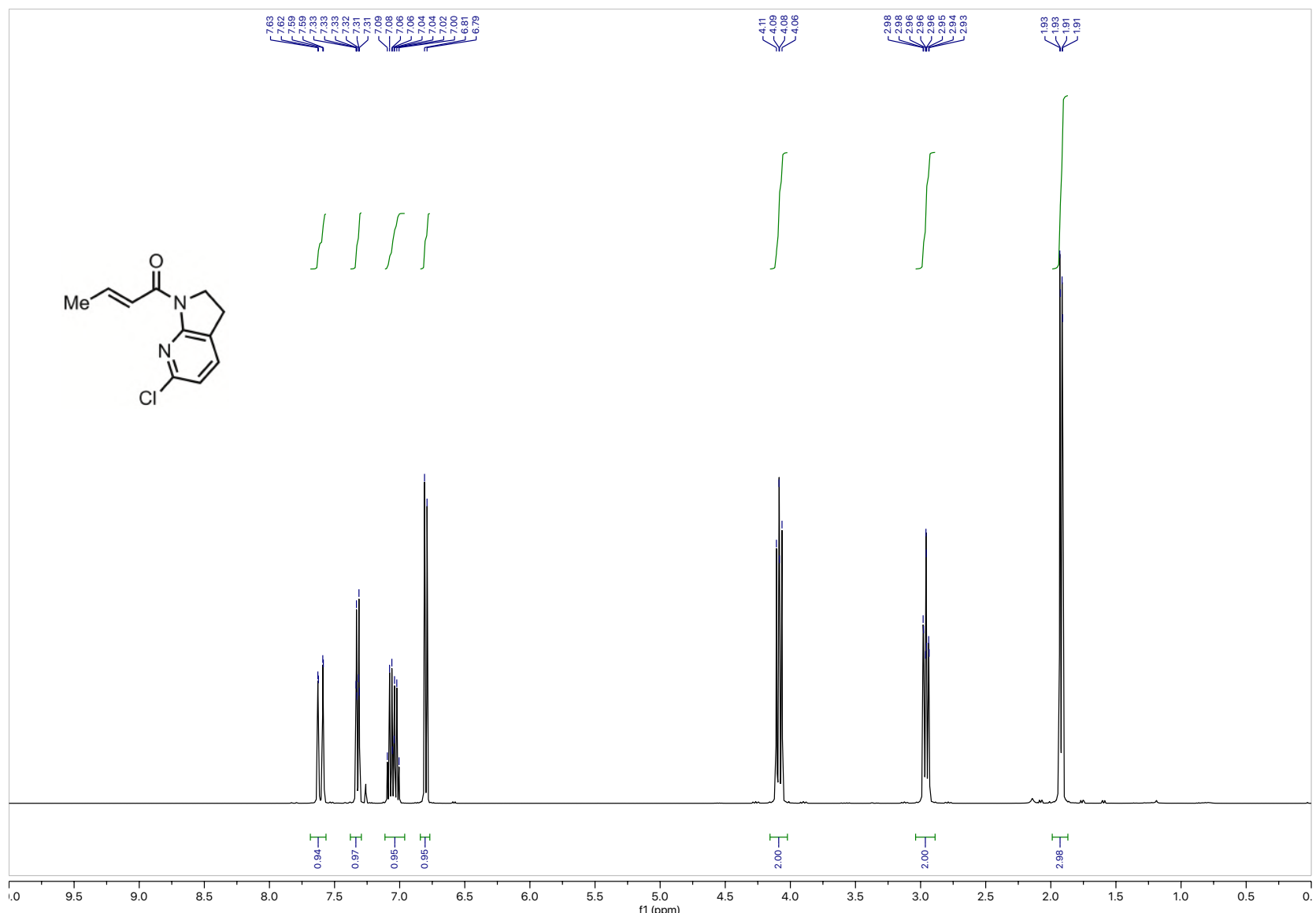

${ }^{13}$ C NMR: 21

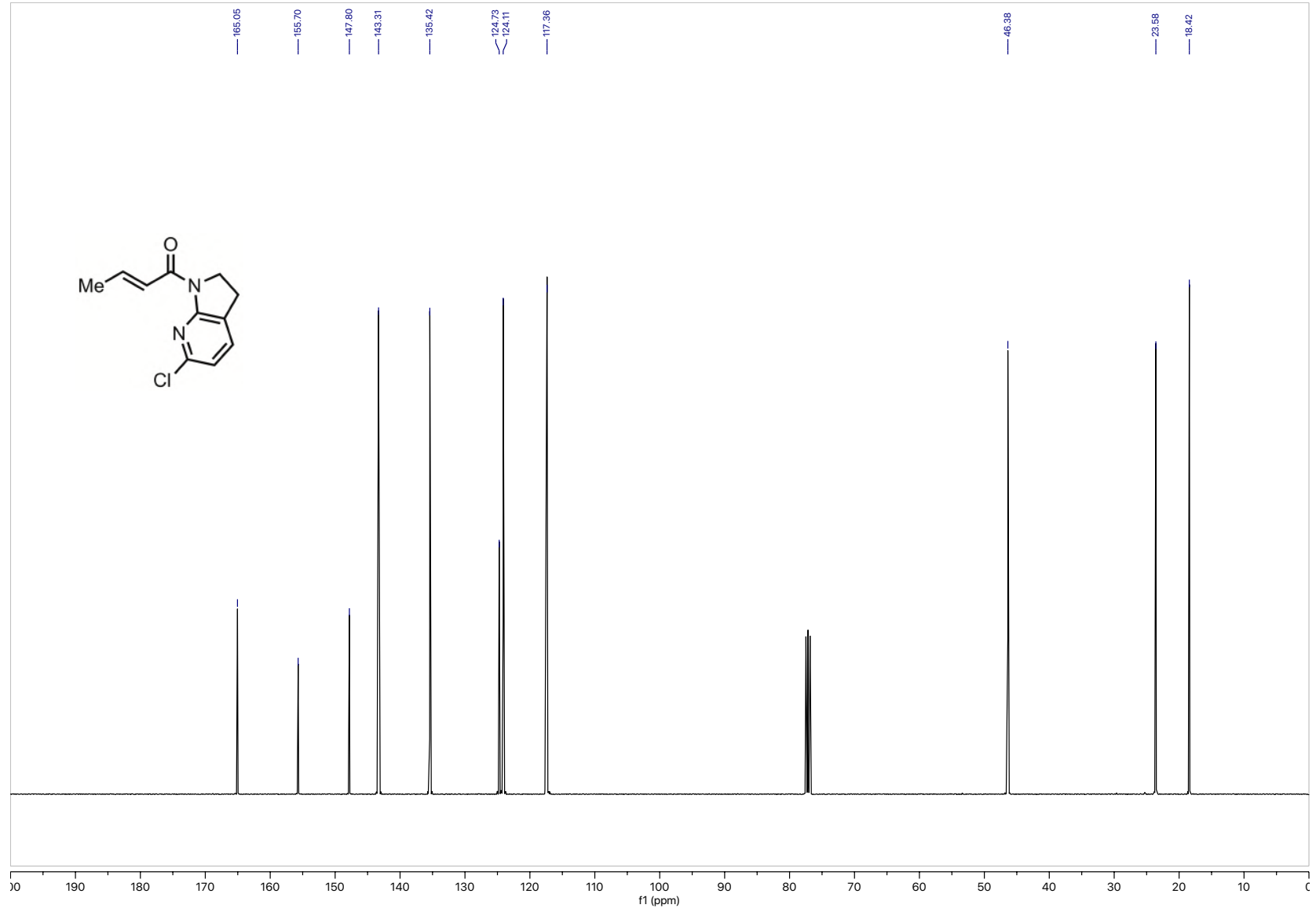


${ }^{1} \mathrm{H}$ NMR: $2 \mathrm{~m}$

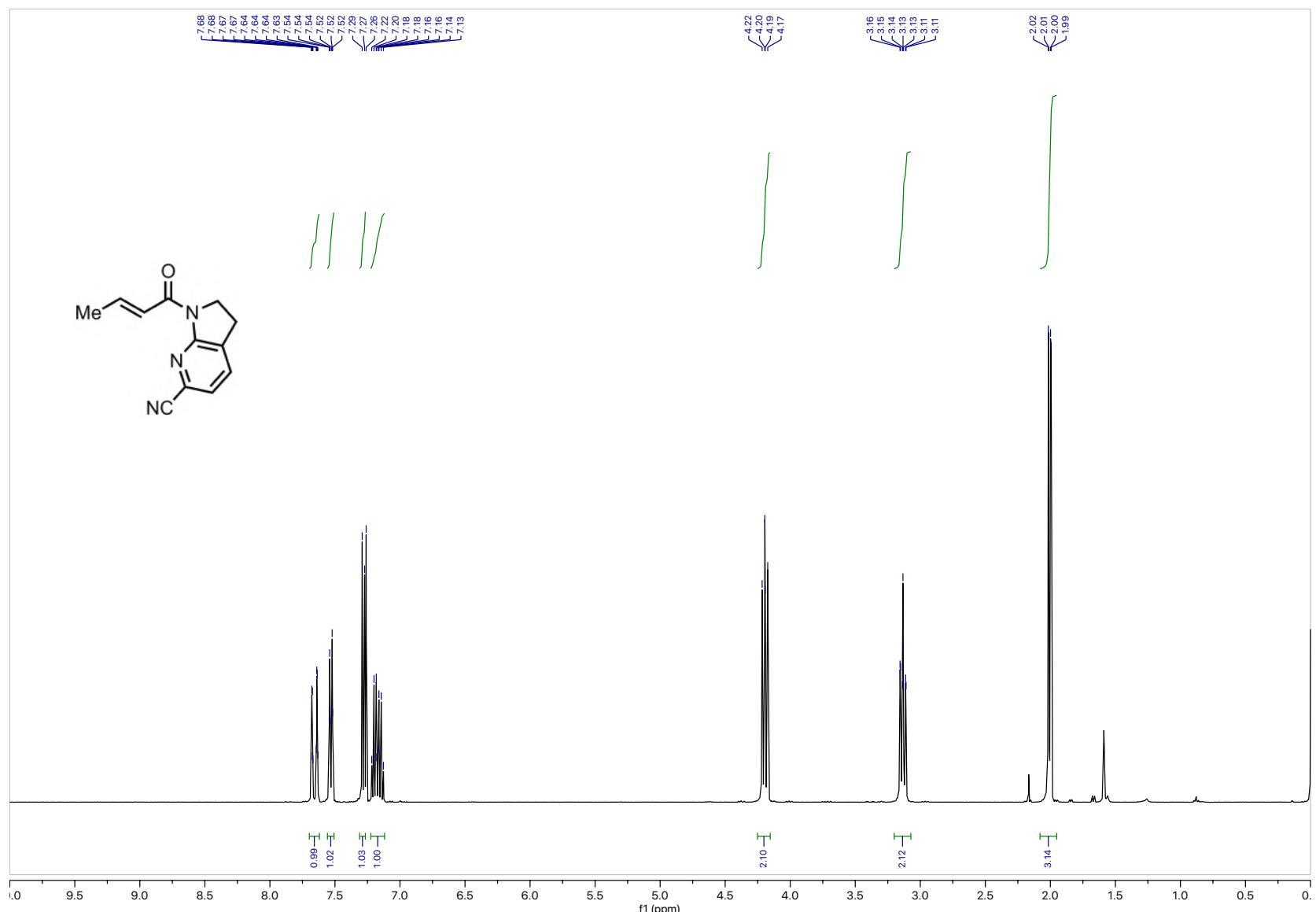

${ }^{13}$ C NMR: 2m
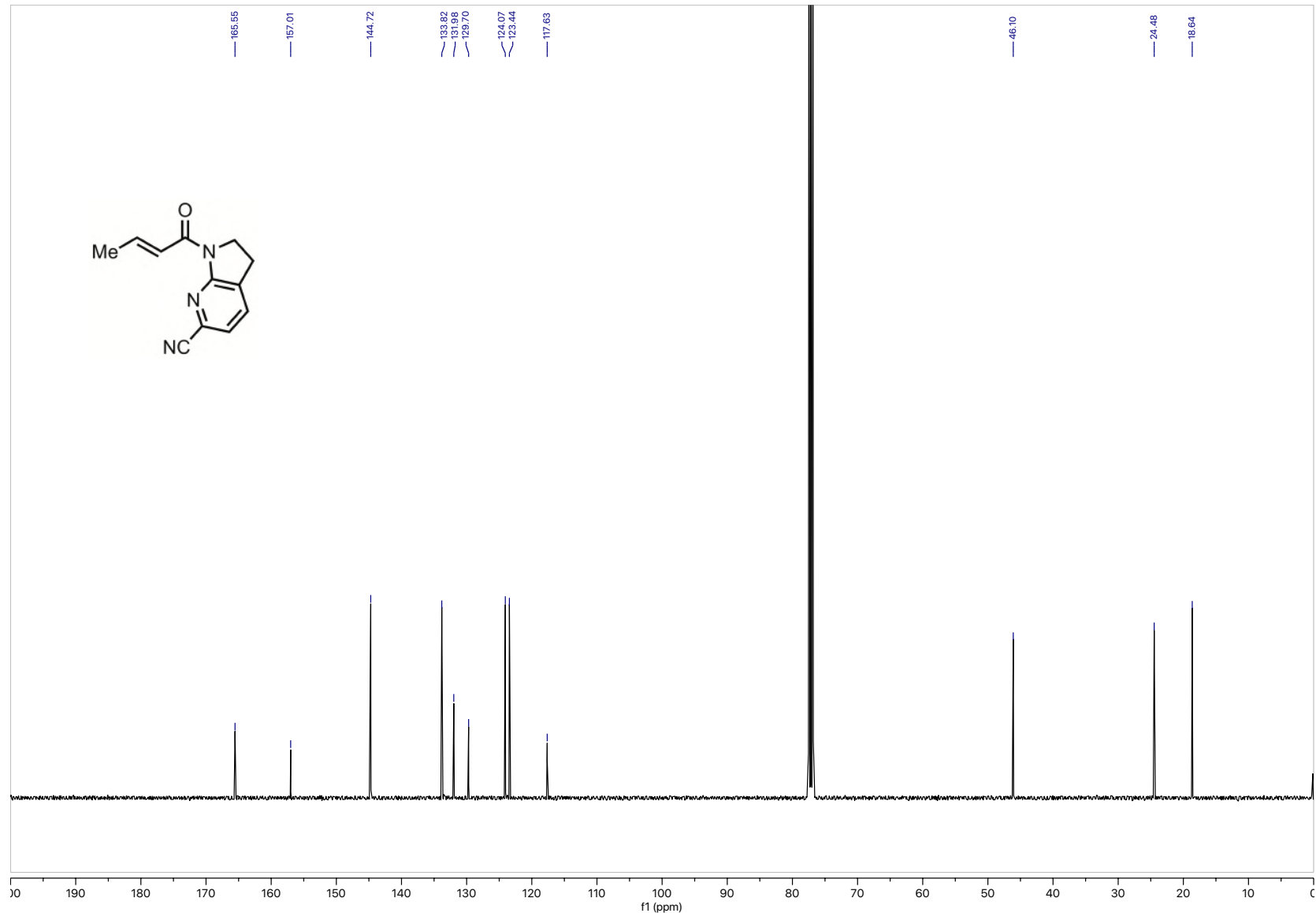
Supporting Information

${ }^{1} \mathrm{H}$ NMR: 2n

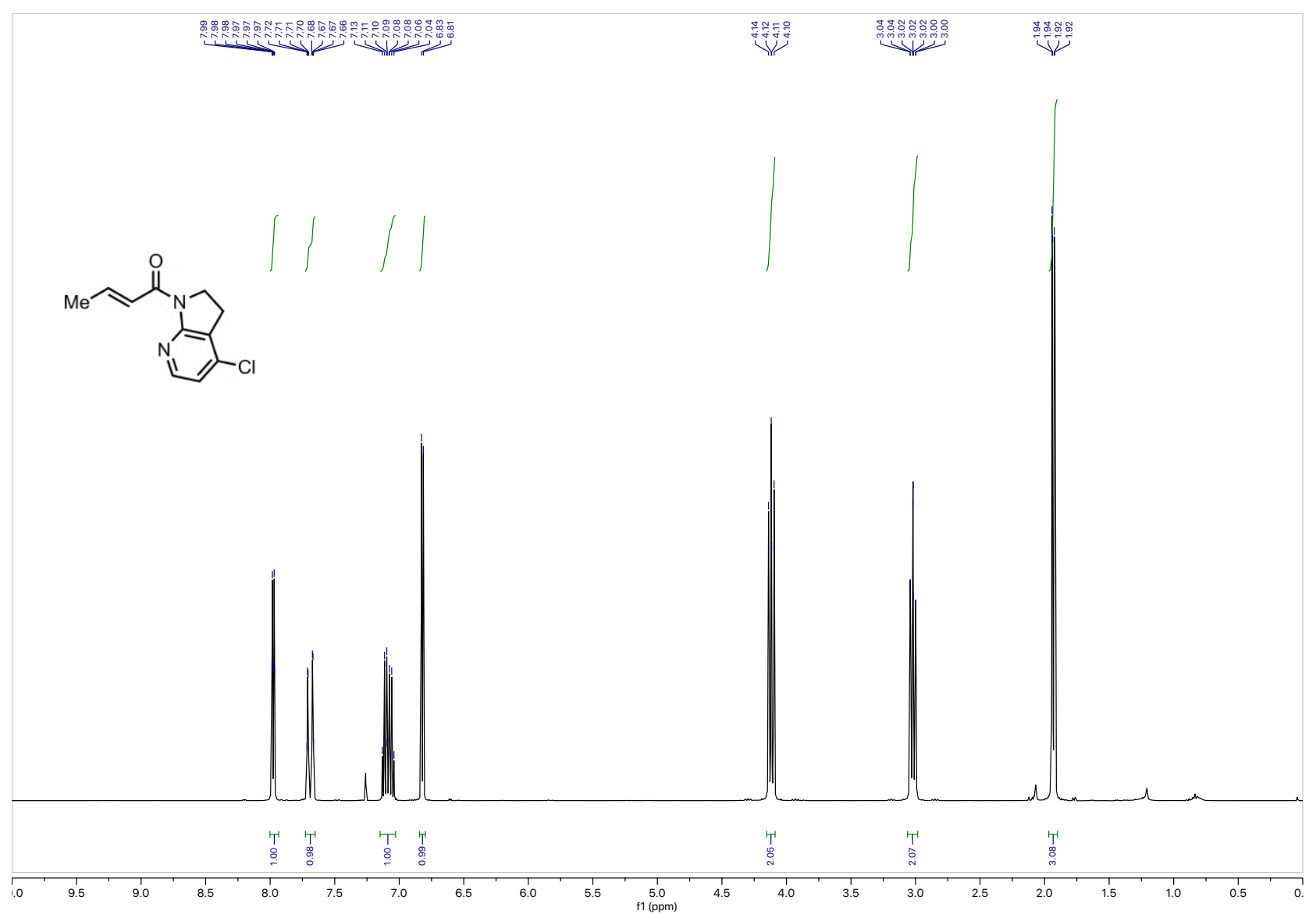

${ }^{13} \mathrm{C}$ NMR: $2 \mathbf{n}$

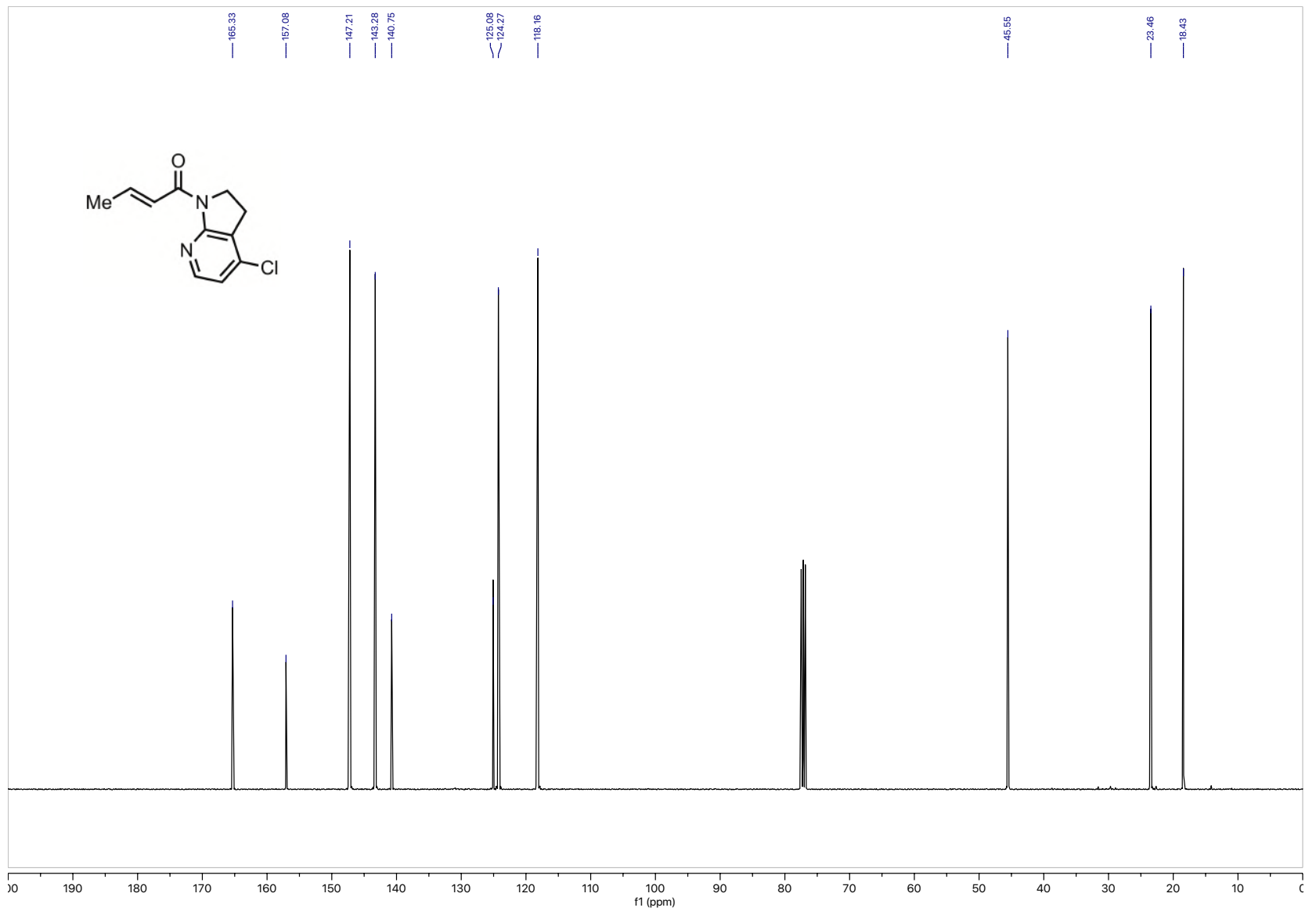

S101 
Supporting Information

${ }^{1} \mathrm{H}$ NMR: 20

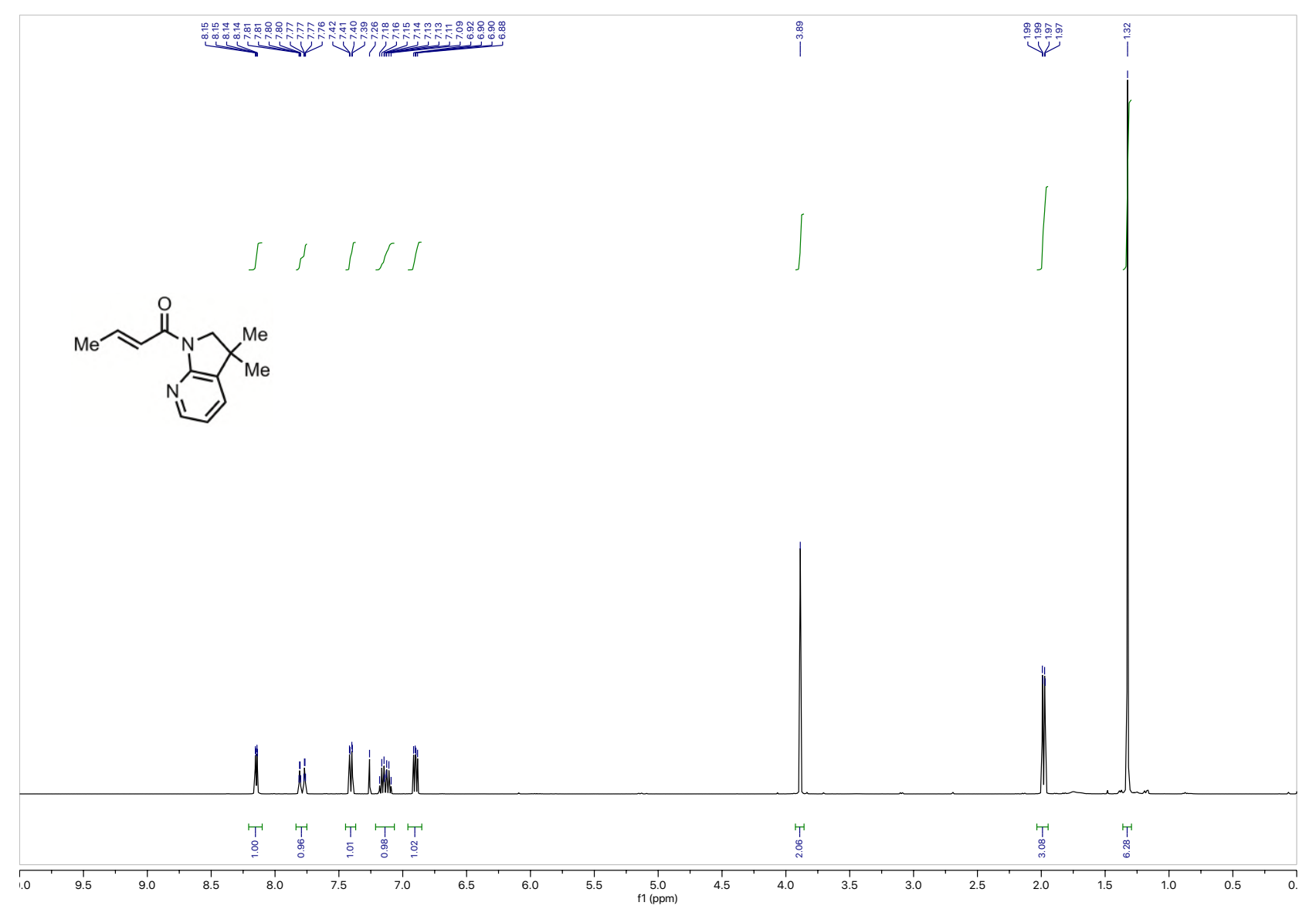

${ }^{13} \mathrm{C}$ NMR: 20

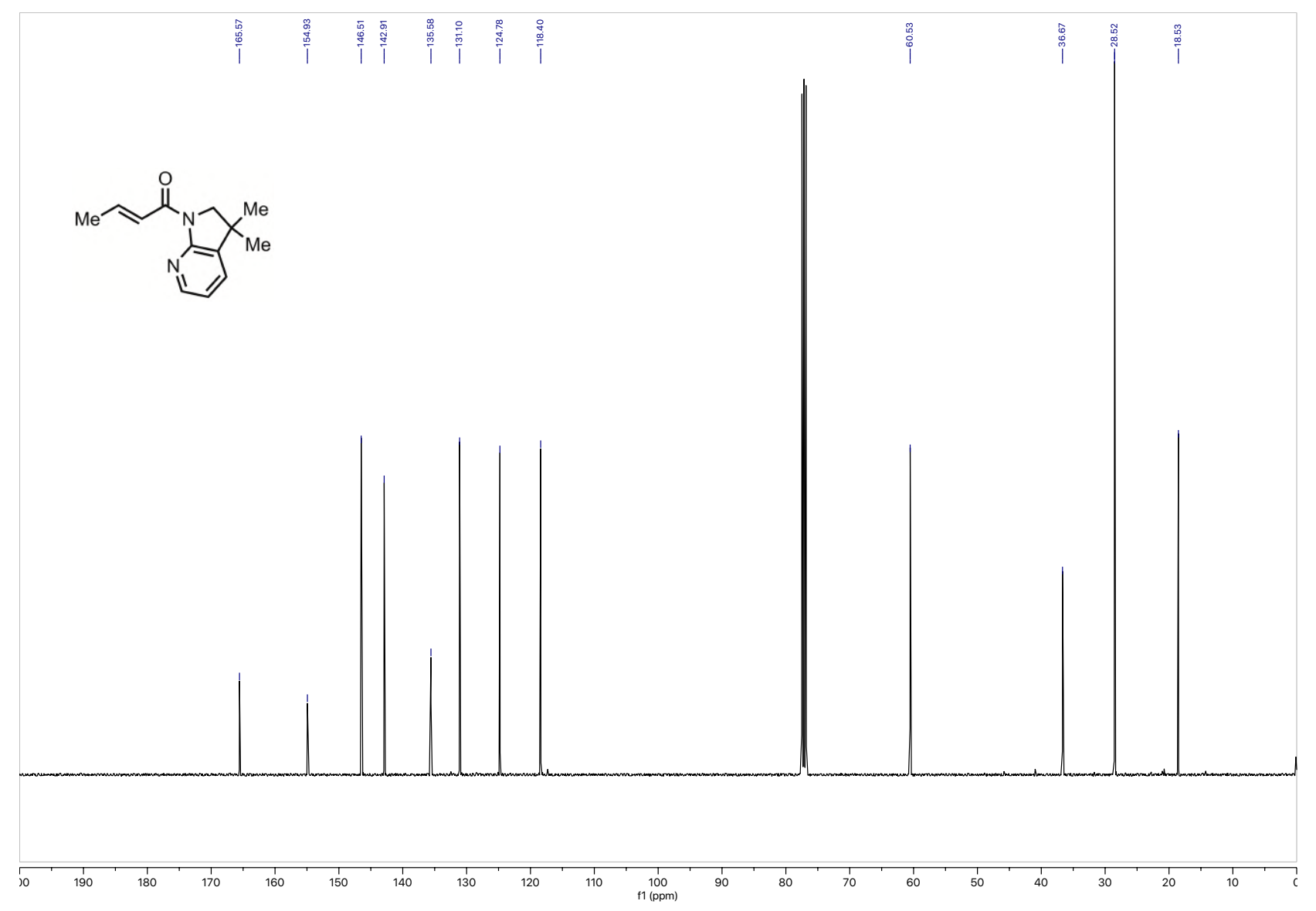

S102 
${ }^{1} \mathrm{H}$ NMR: 2p

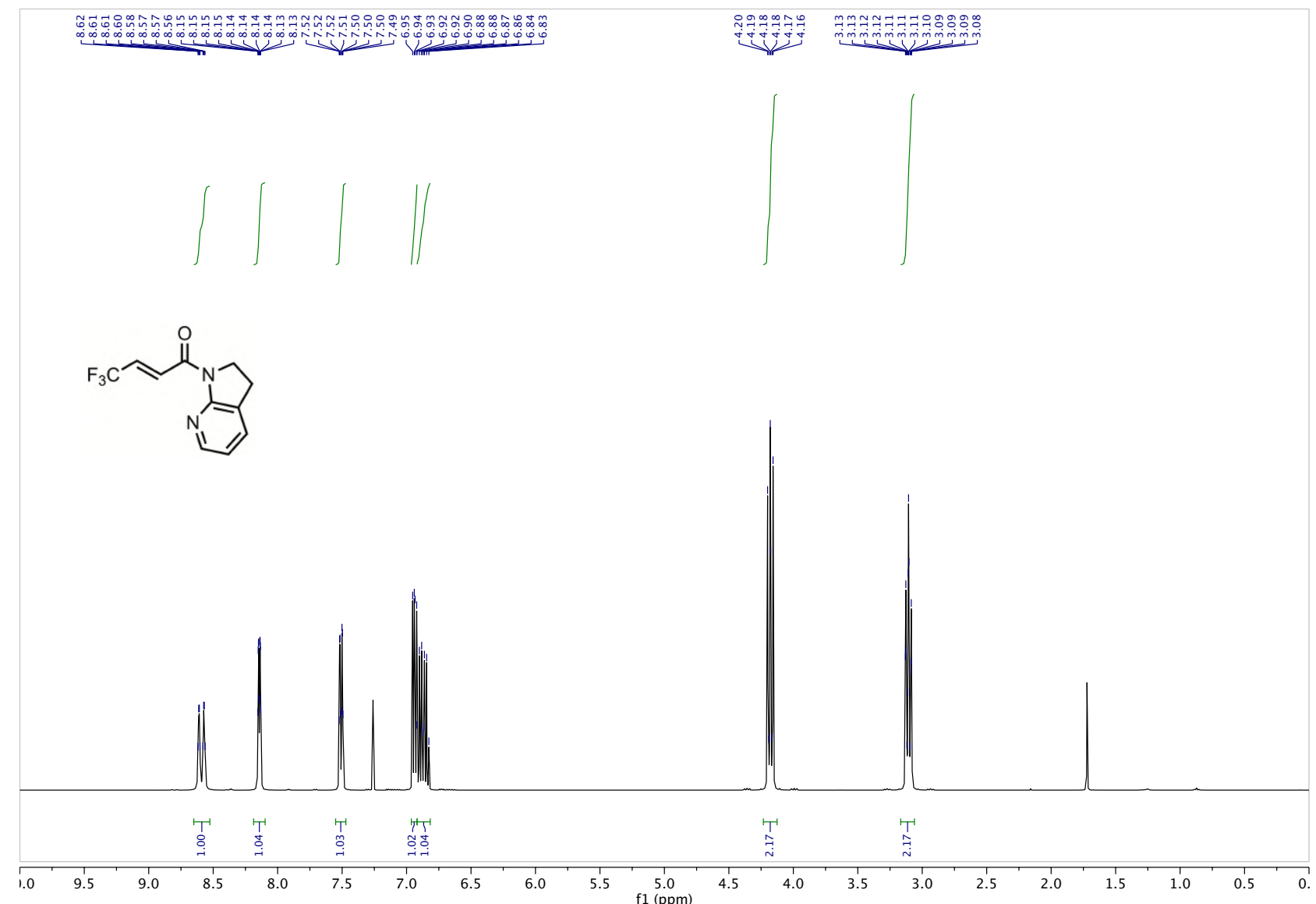

${ }^{13}$ C NMR: 2p

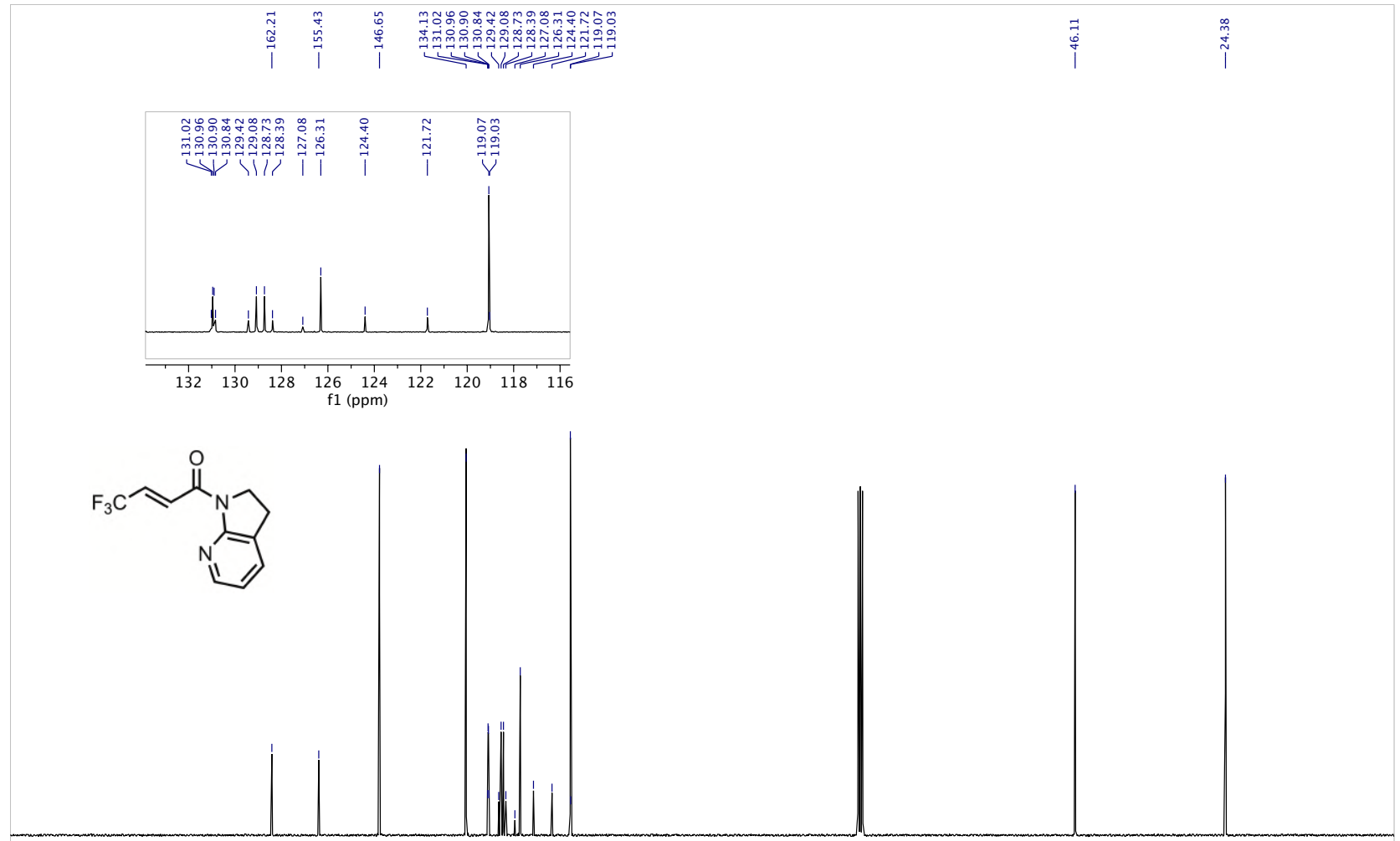

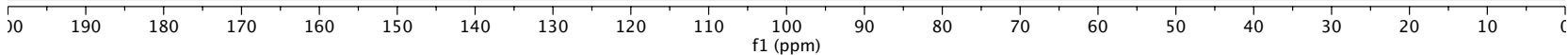


${ }^{19} \mathrm{~F}$ NMR: 2p

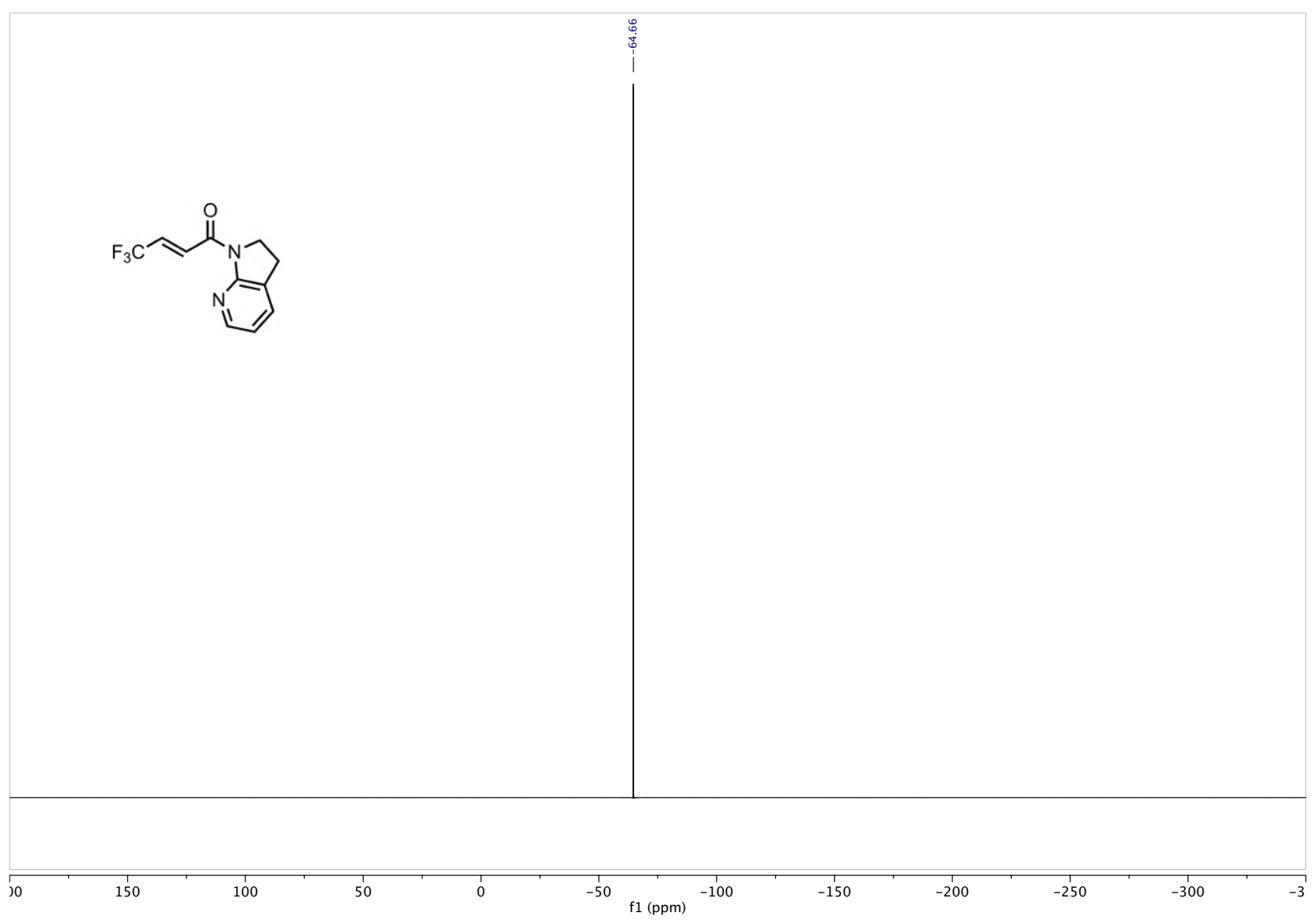


${ }^{1} \mathrm{H}$ NMR: $2 \mathrm{q}$

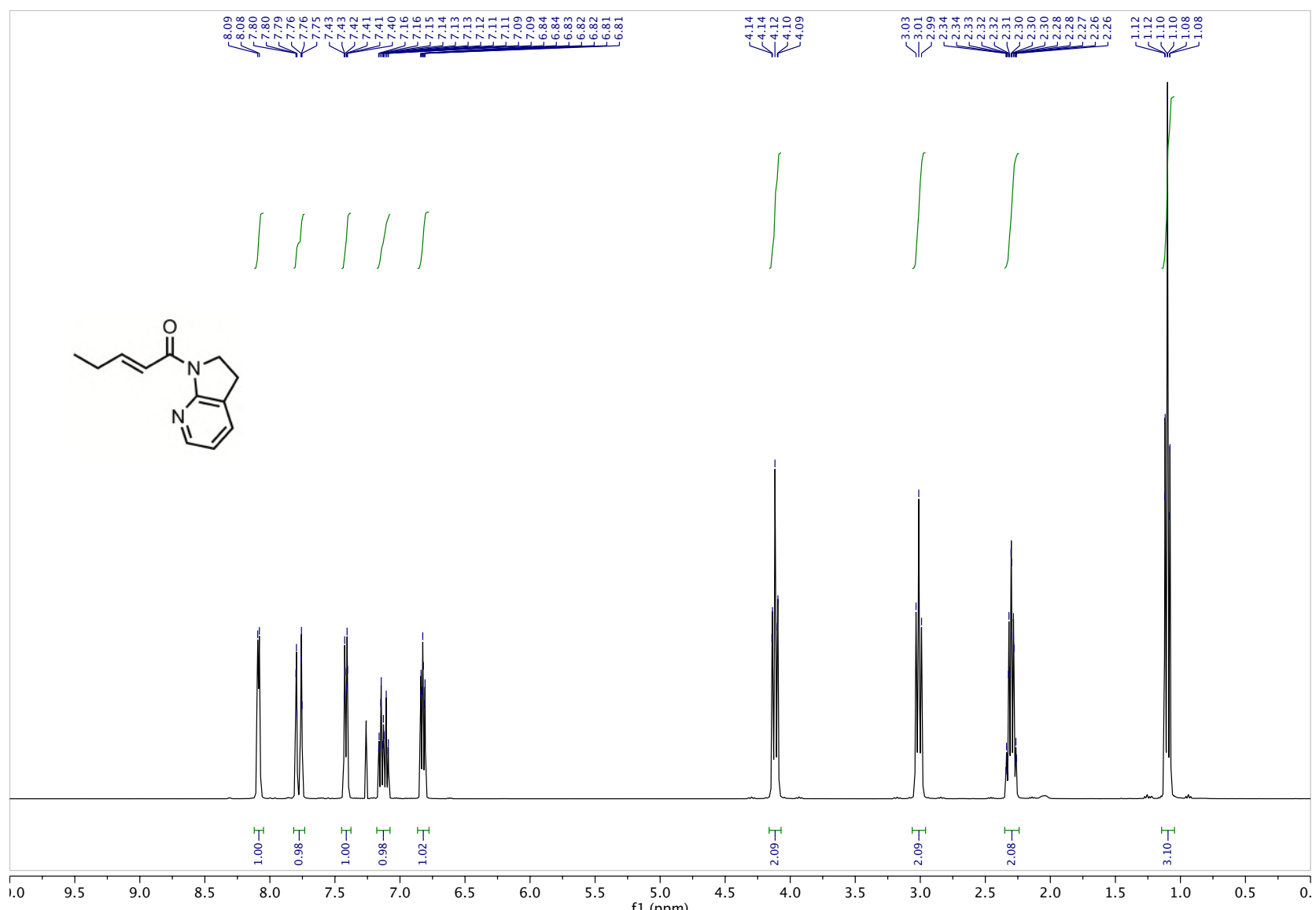

${ }^{13}$ C NMR: 2q

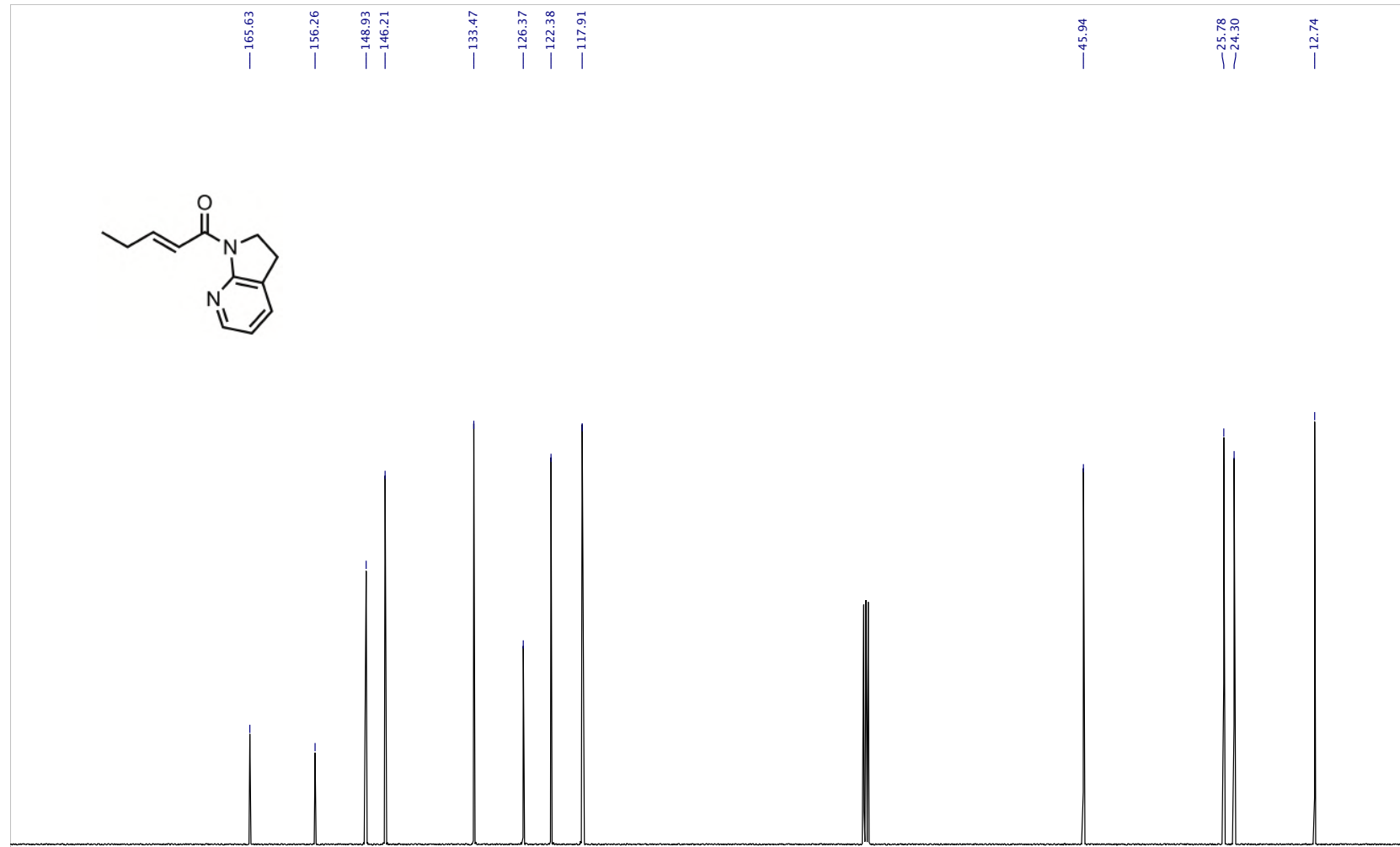

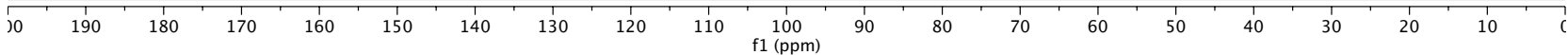


${ }^{1} \mathrm{H}$ NMR: 2r

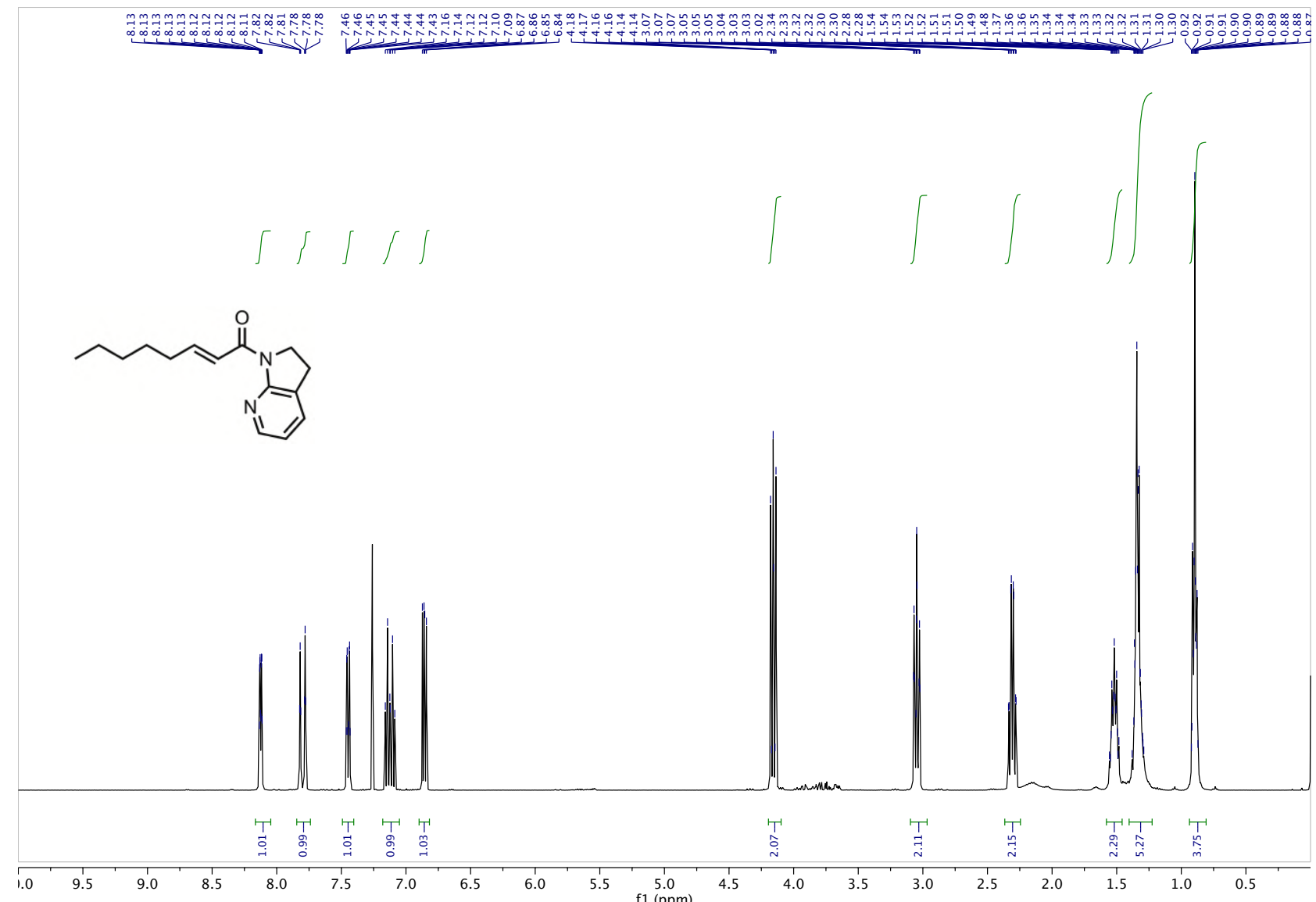

${ }^{13} \mathrm{C}$ NMR: 2r

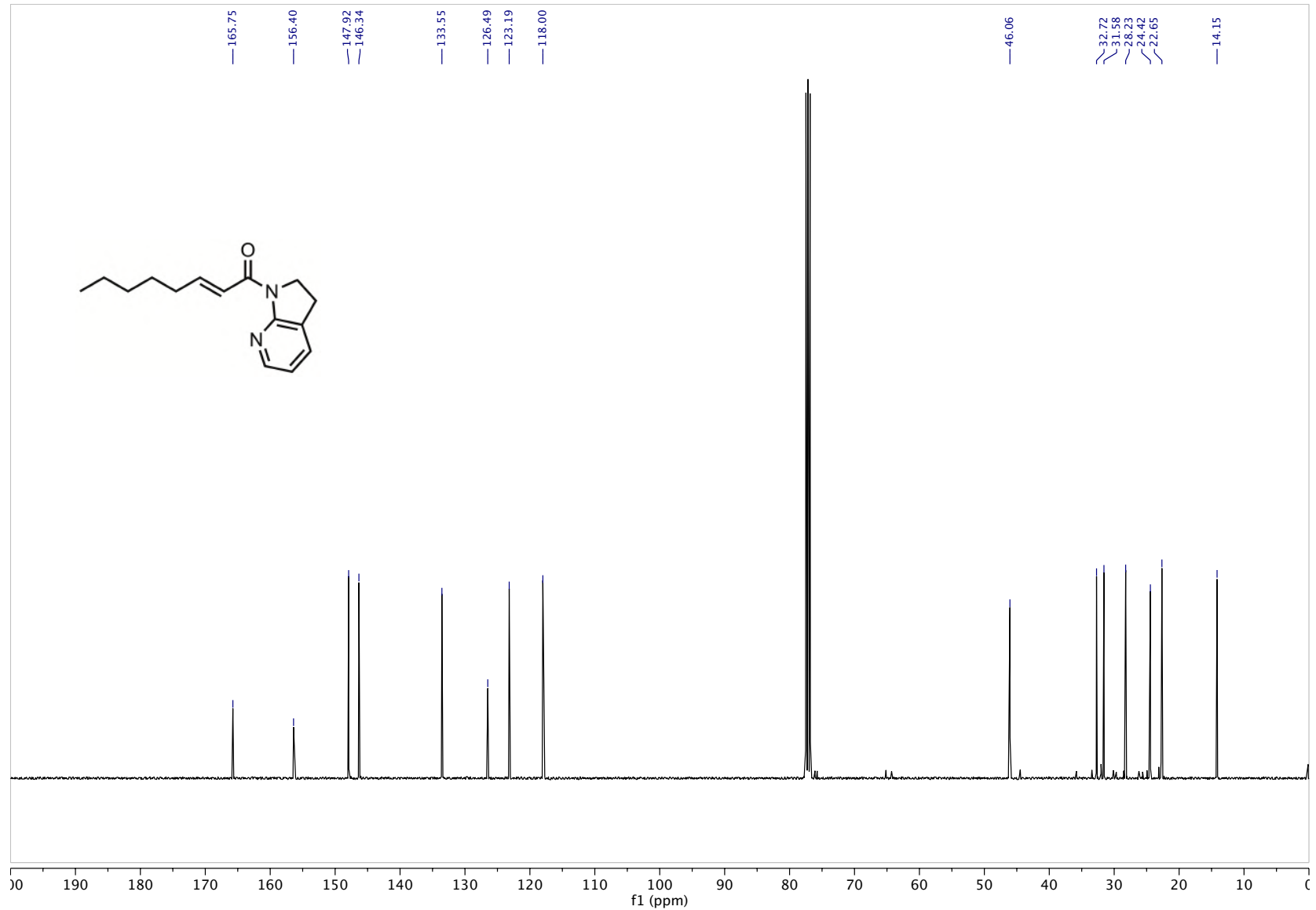


${ }^{1} \mathrm{H}$ NMR: 2s

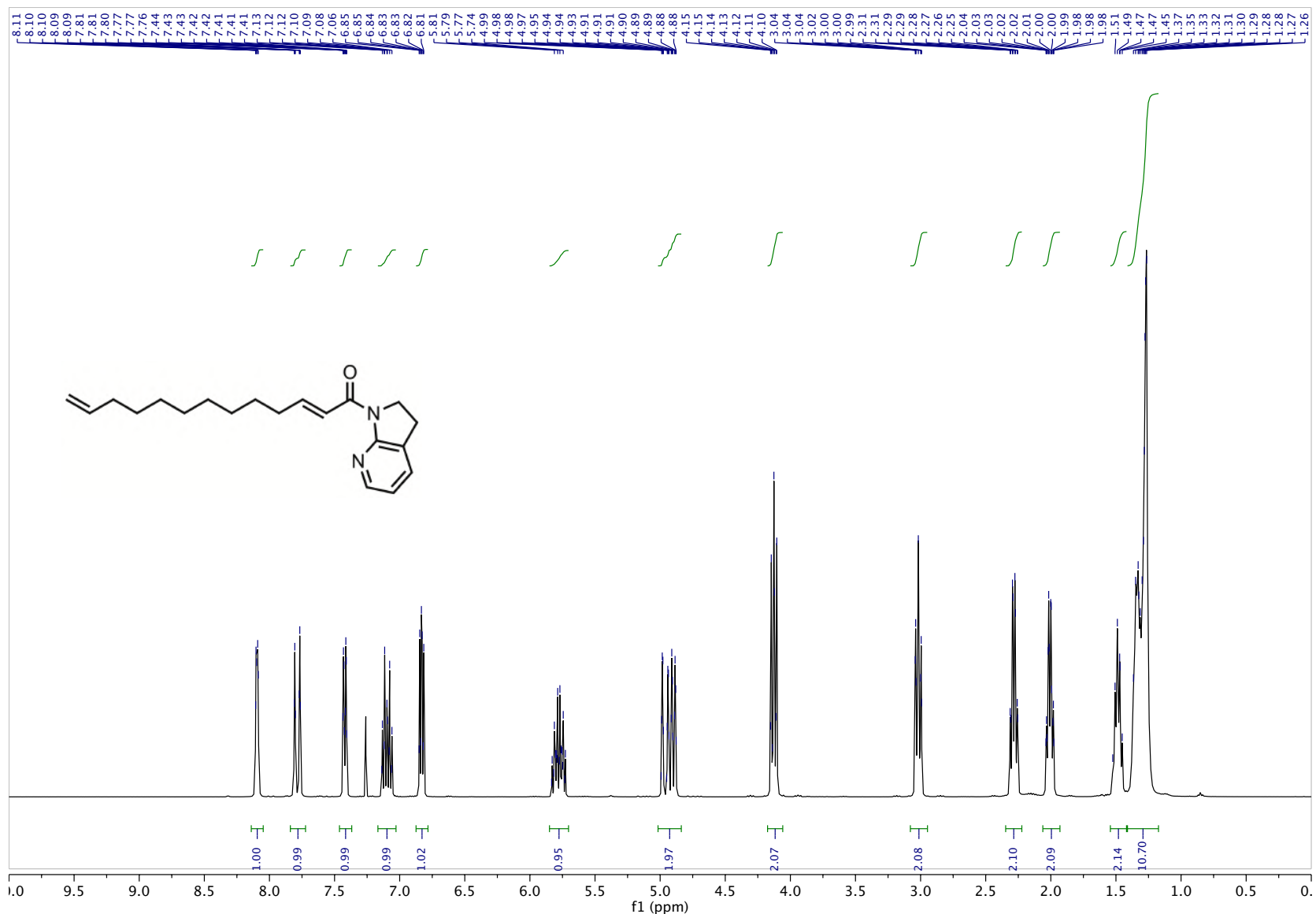

${ }^{13} \mathrm{C}$ NMR: 2s

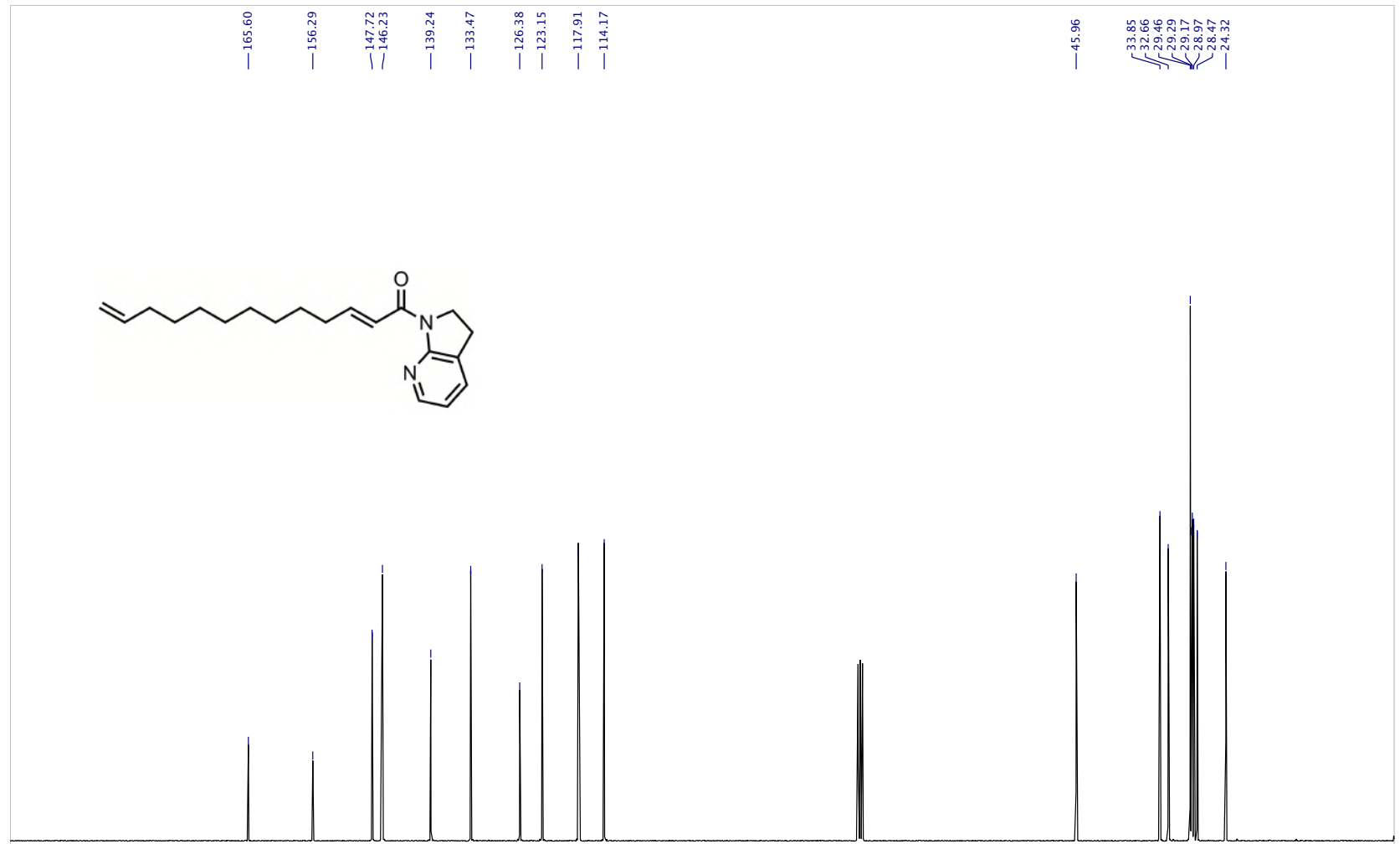

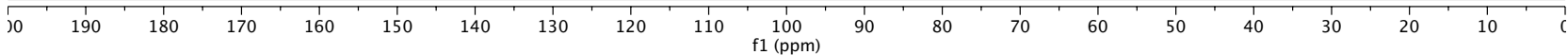


Supporting Information

${ }^{1} \mathrm{H}$ NMR: $2 \mathbf{t}$

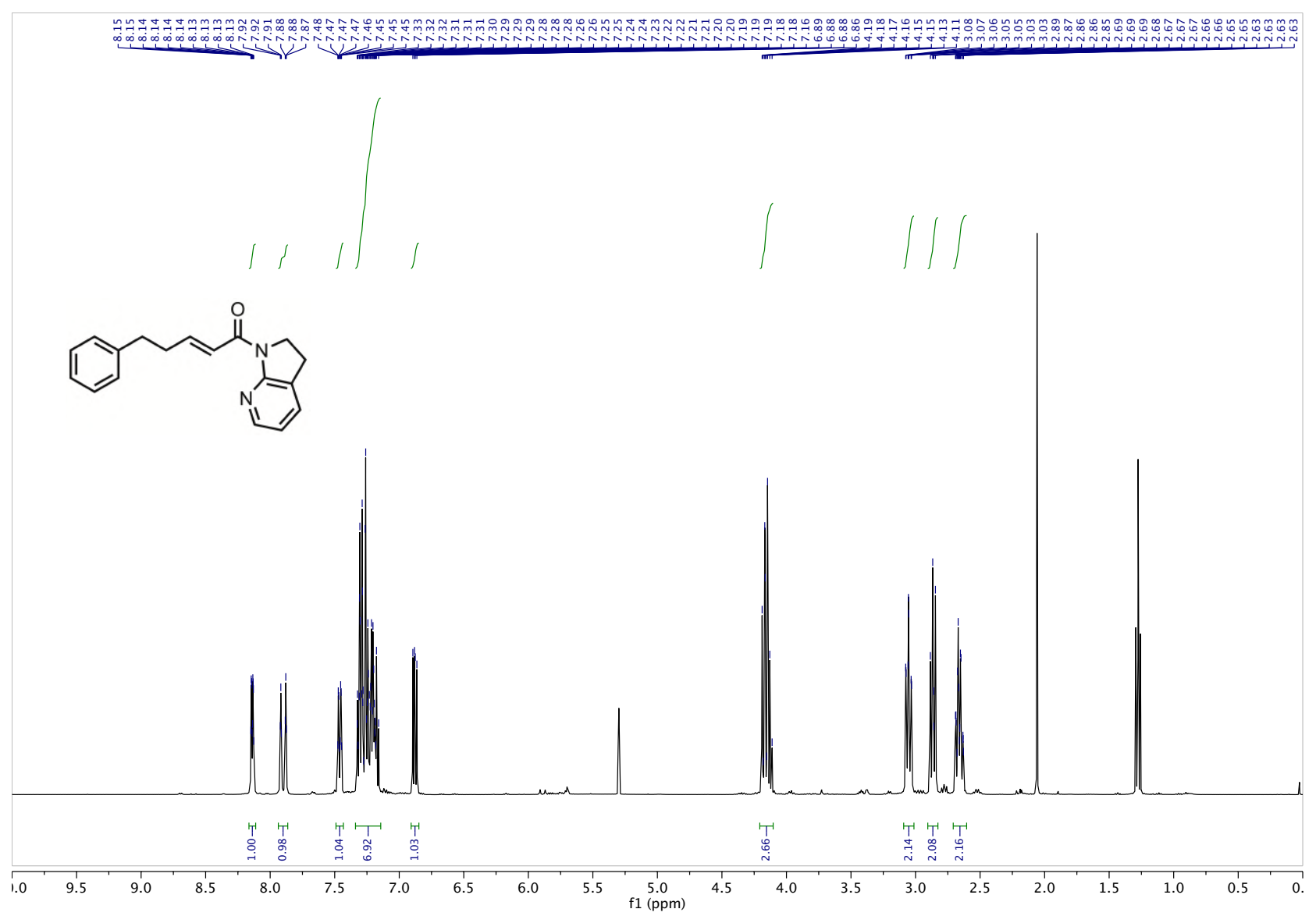

${ }^{13} \mathrm{C}$ NMR: $2 \mathrm{t}$

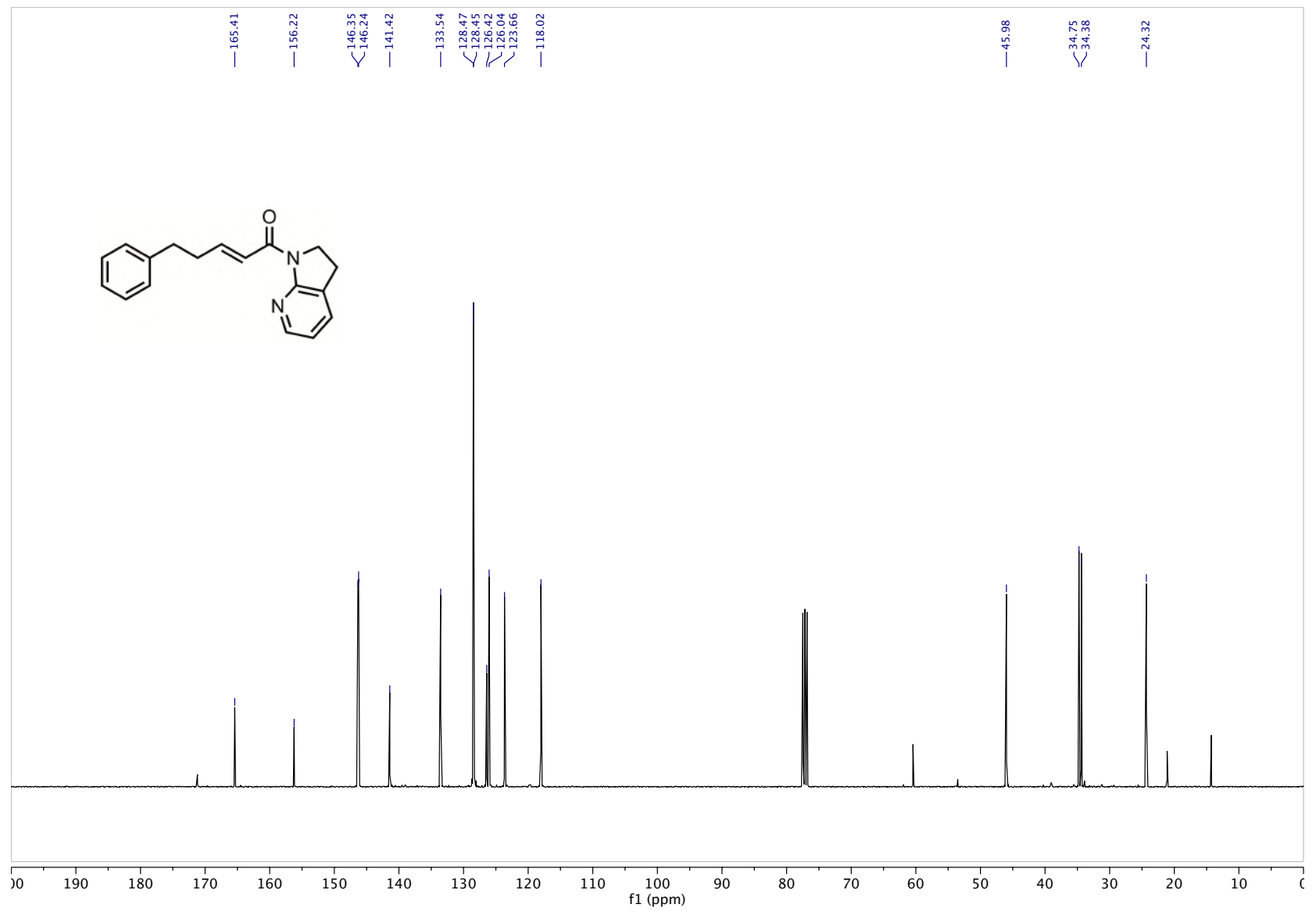

S108 
${ }^{1} \mathrm{H}$ NMR: $\mathbf{2 u}$

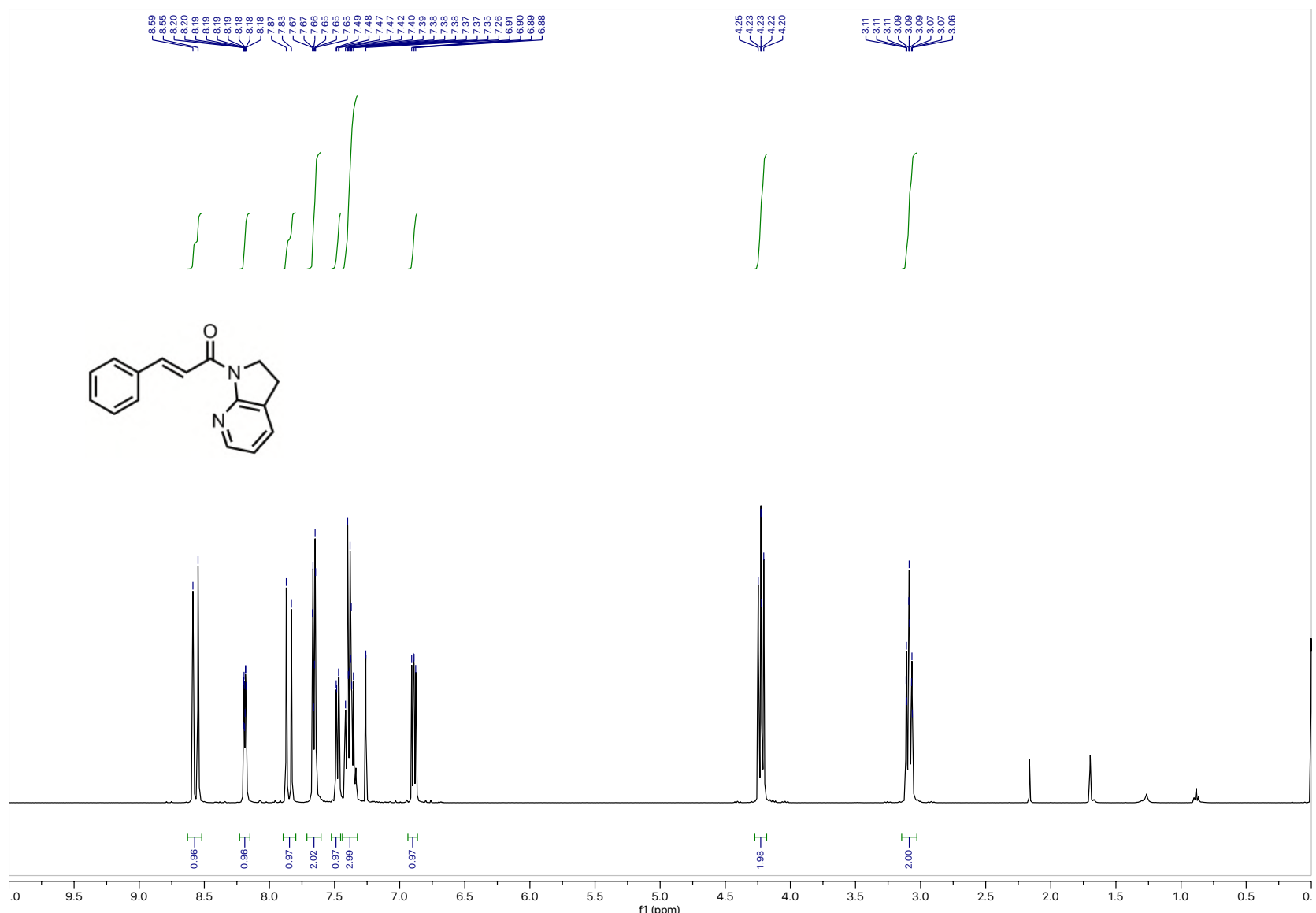

${ }^{13} \mathrm{C}$ NMR: $\mathbf{2 u}$
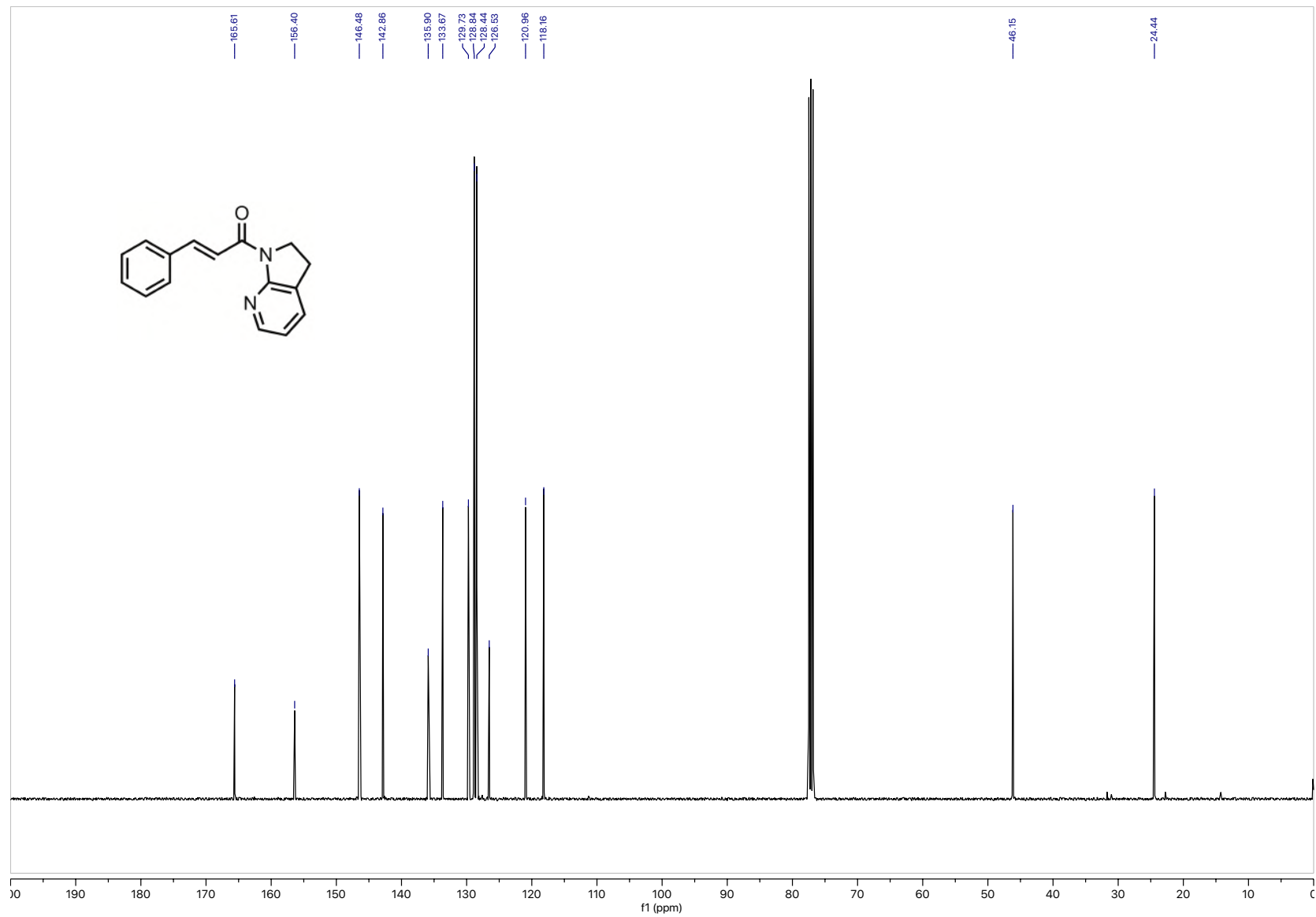
${ }^{1} \mathrm{H}$ NMR: 2v

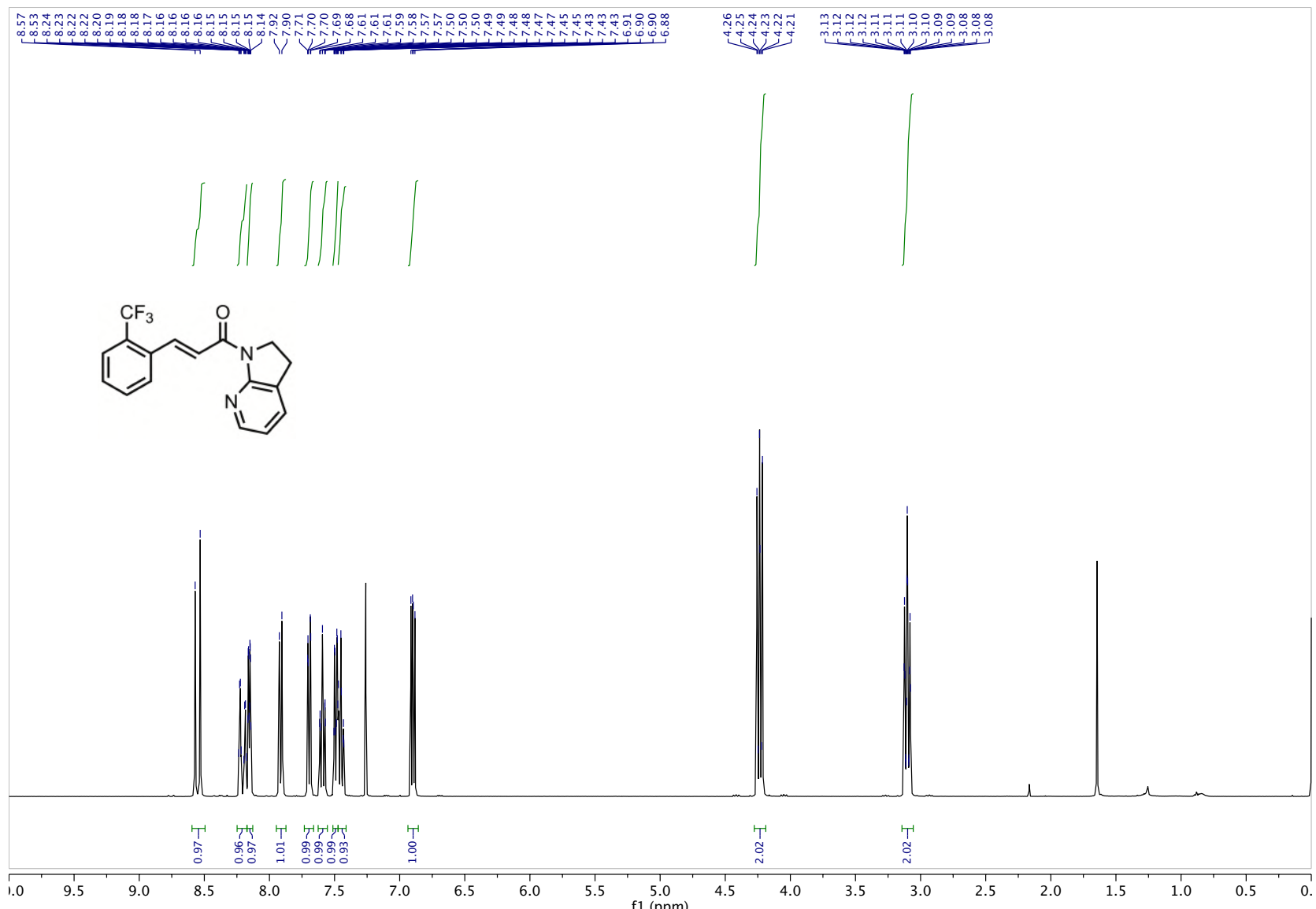

${ }^{13} \mathrm{C}$ NMR: 2v

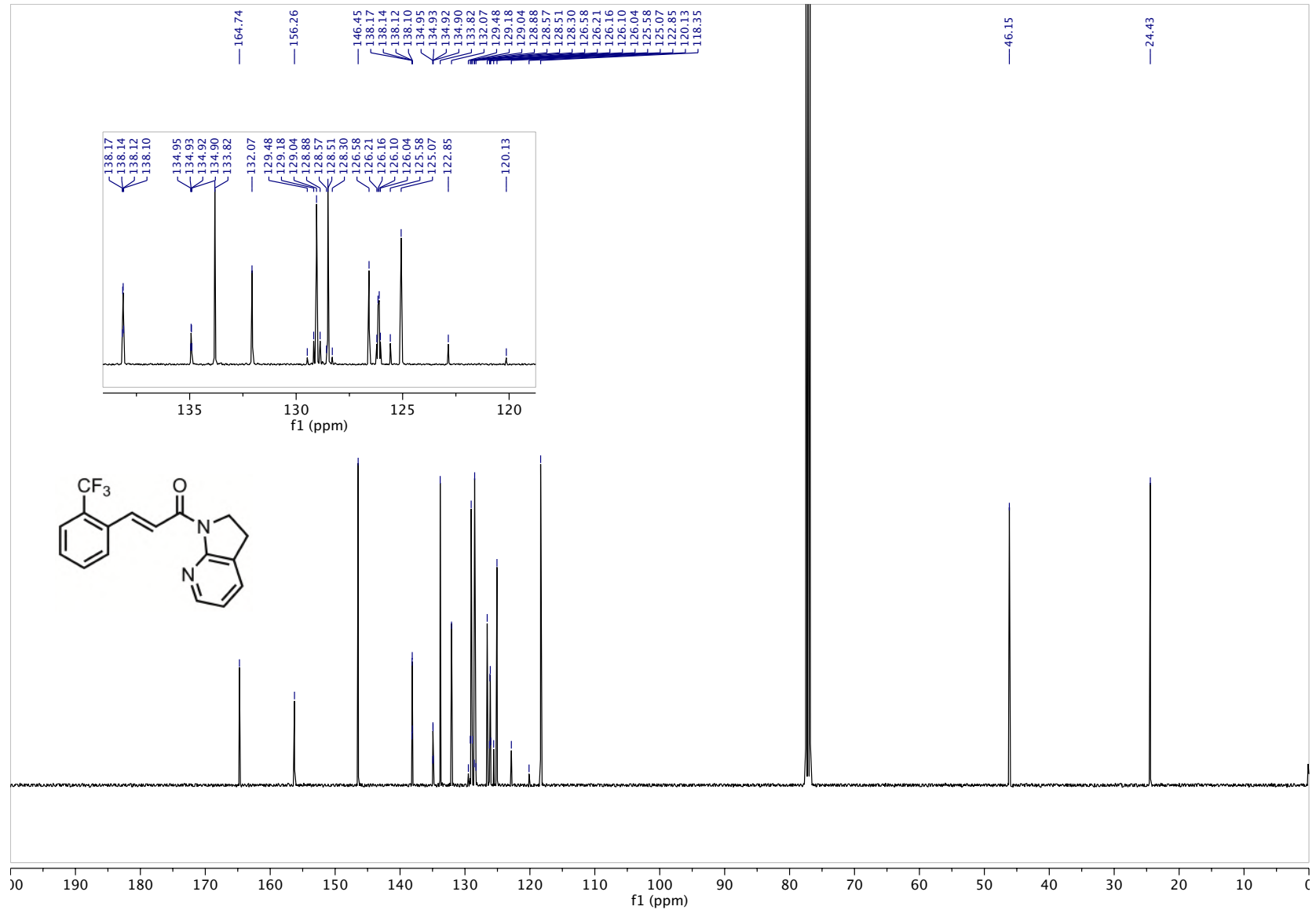


${ }^{19}$ F NMR: 2v

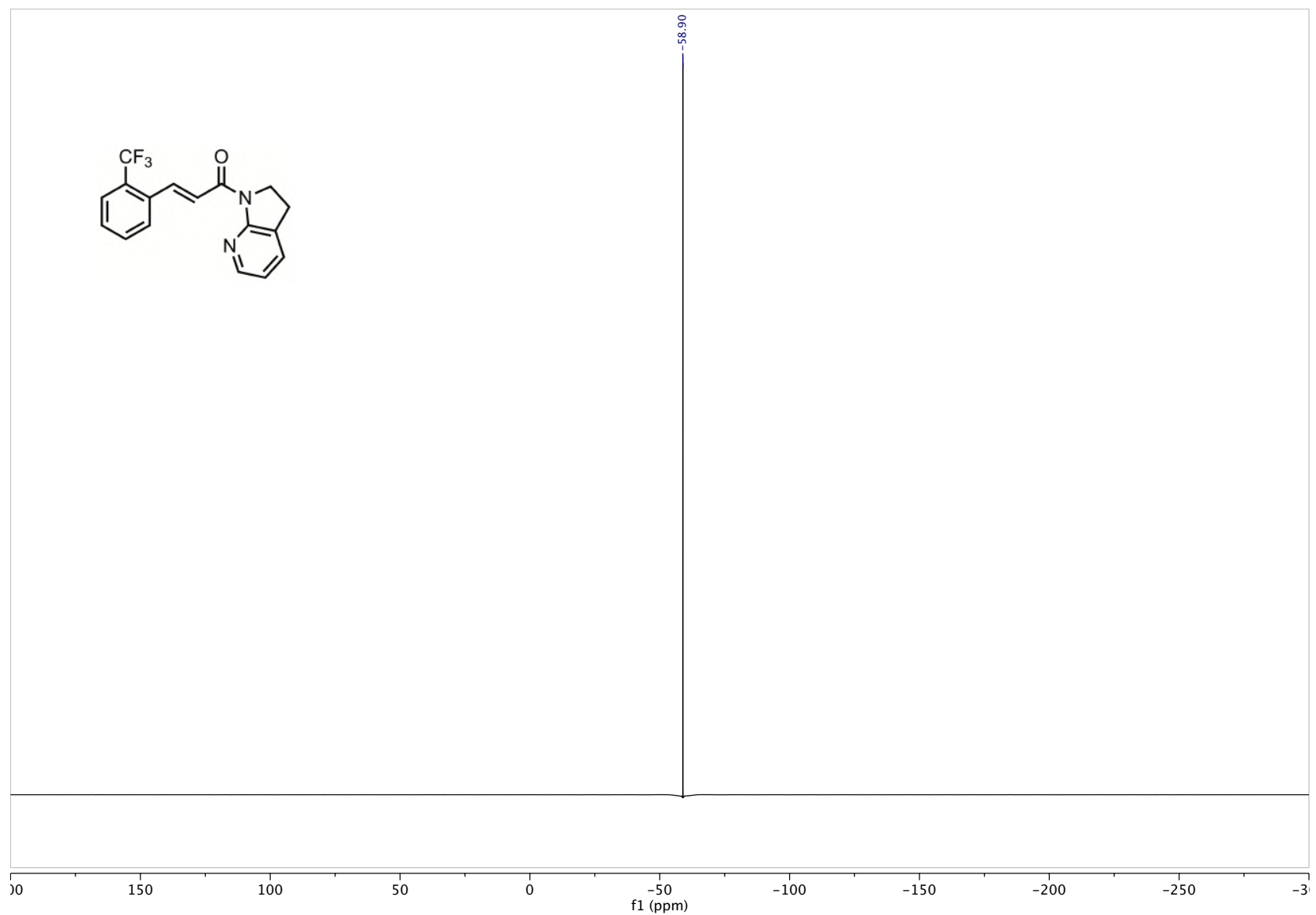


${ }^{1} \mathrm{H}$ NMR: 2w

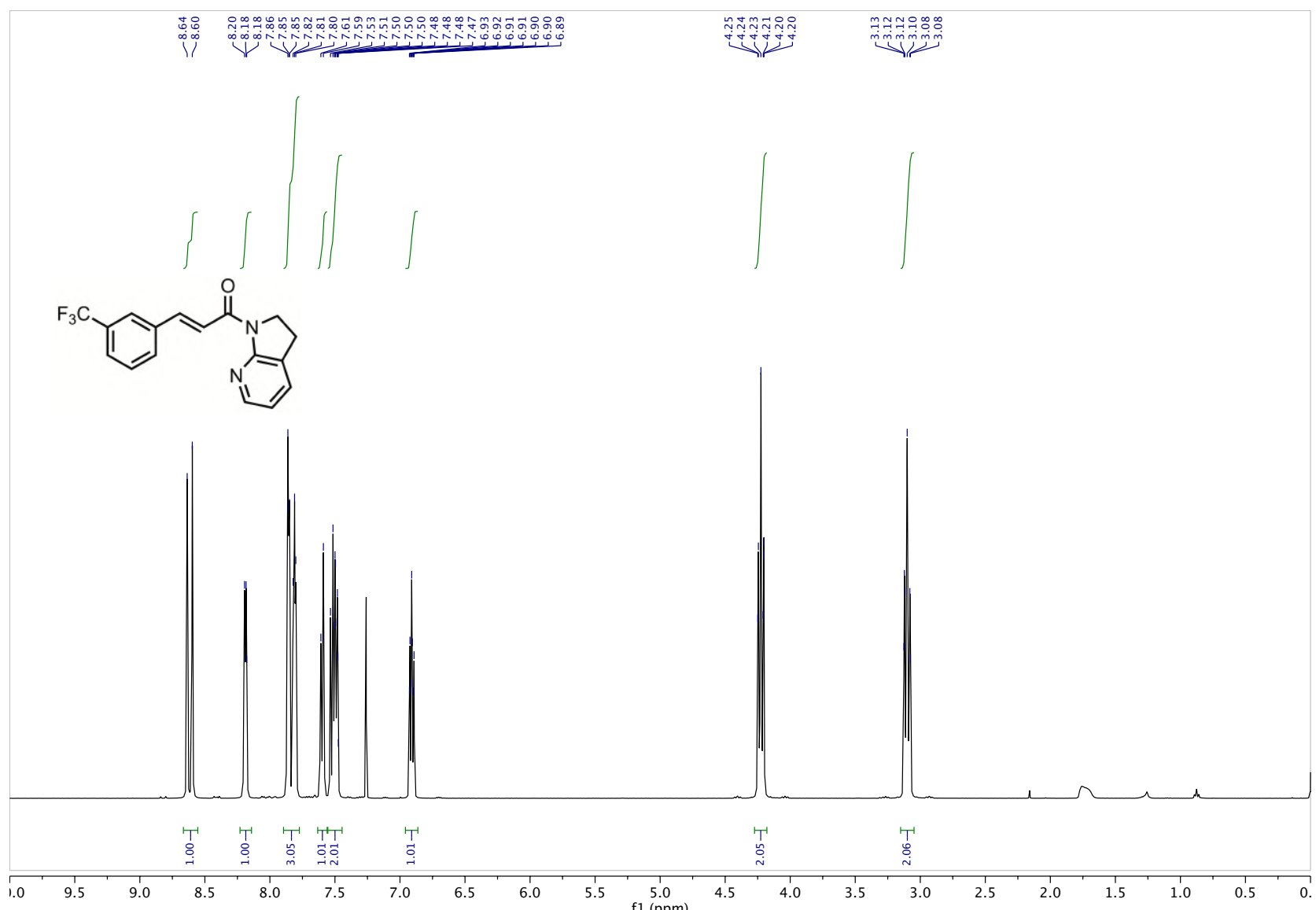

${ }^{13}$ C NMR: 2w

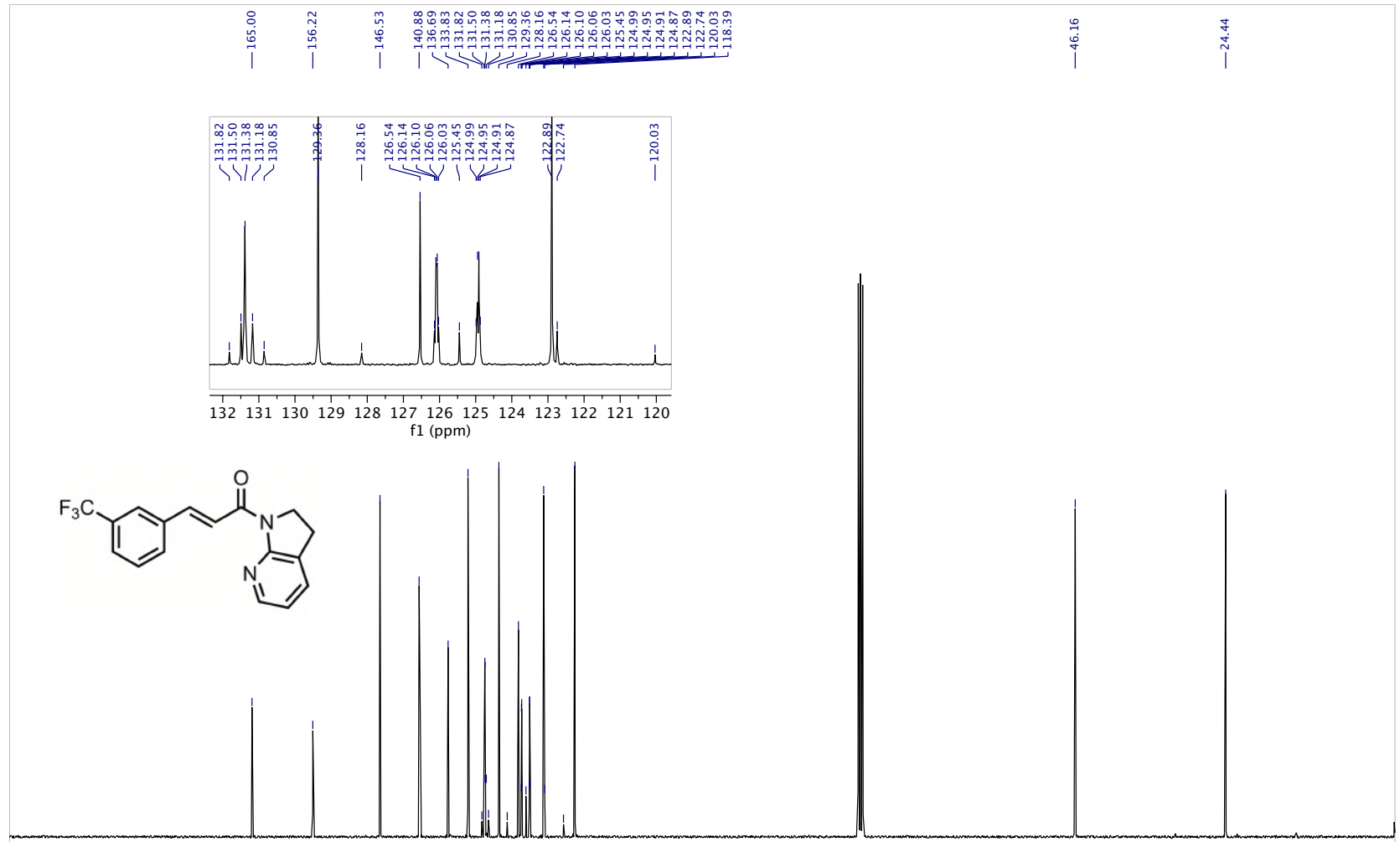

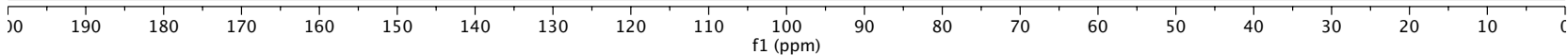


${ }^{19}$ F NMR: 2w

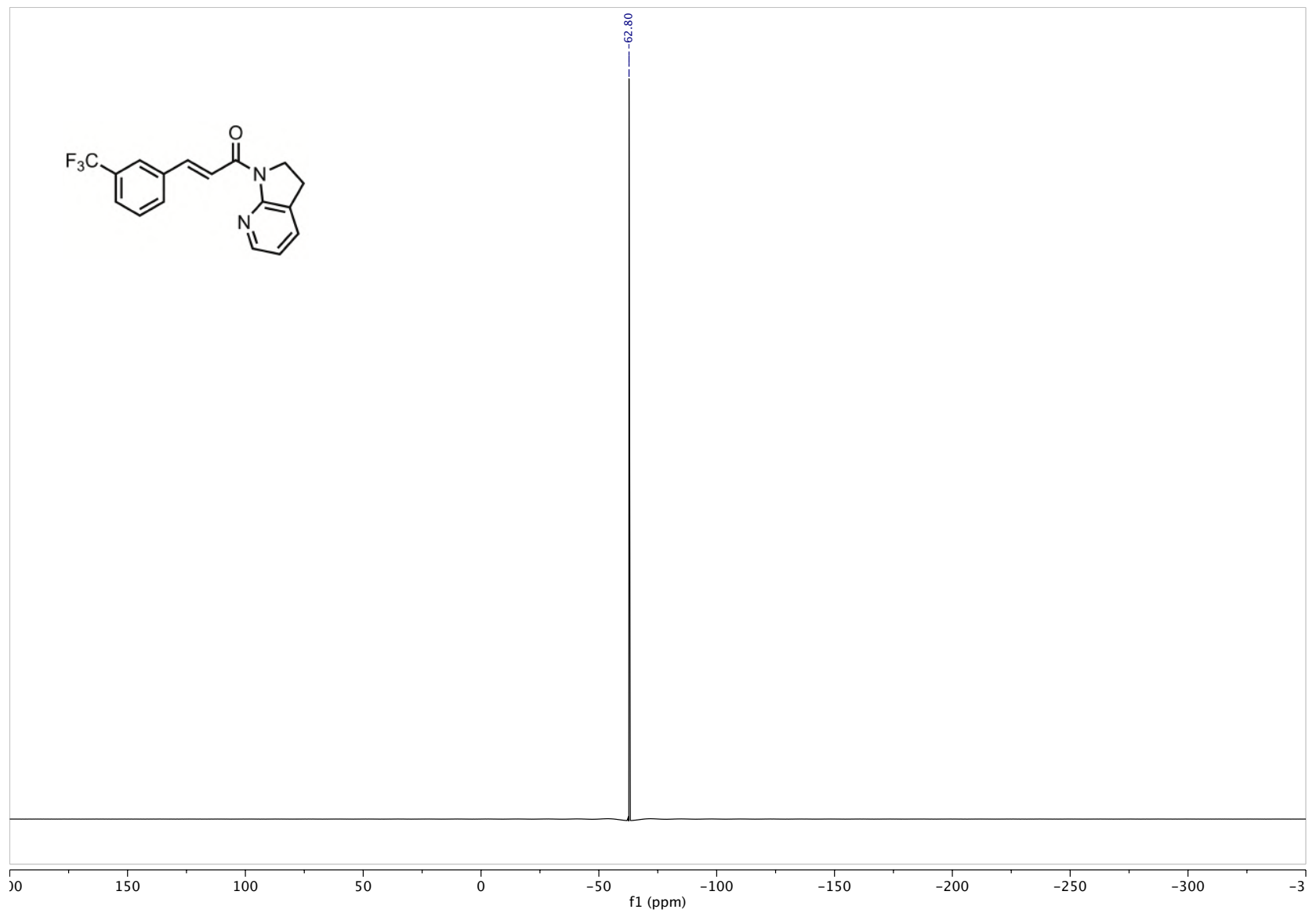


${ }^{1} \mathrm{H}$ NMR: 2x

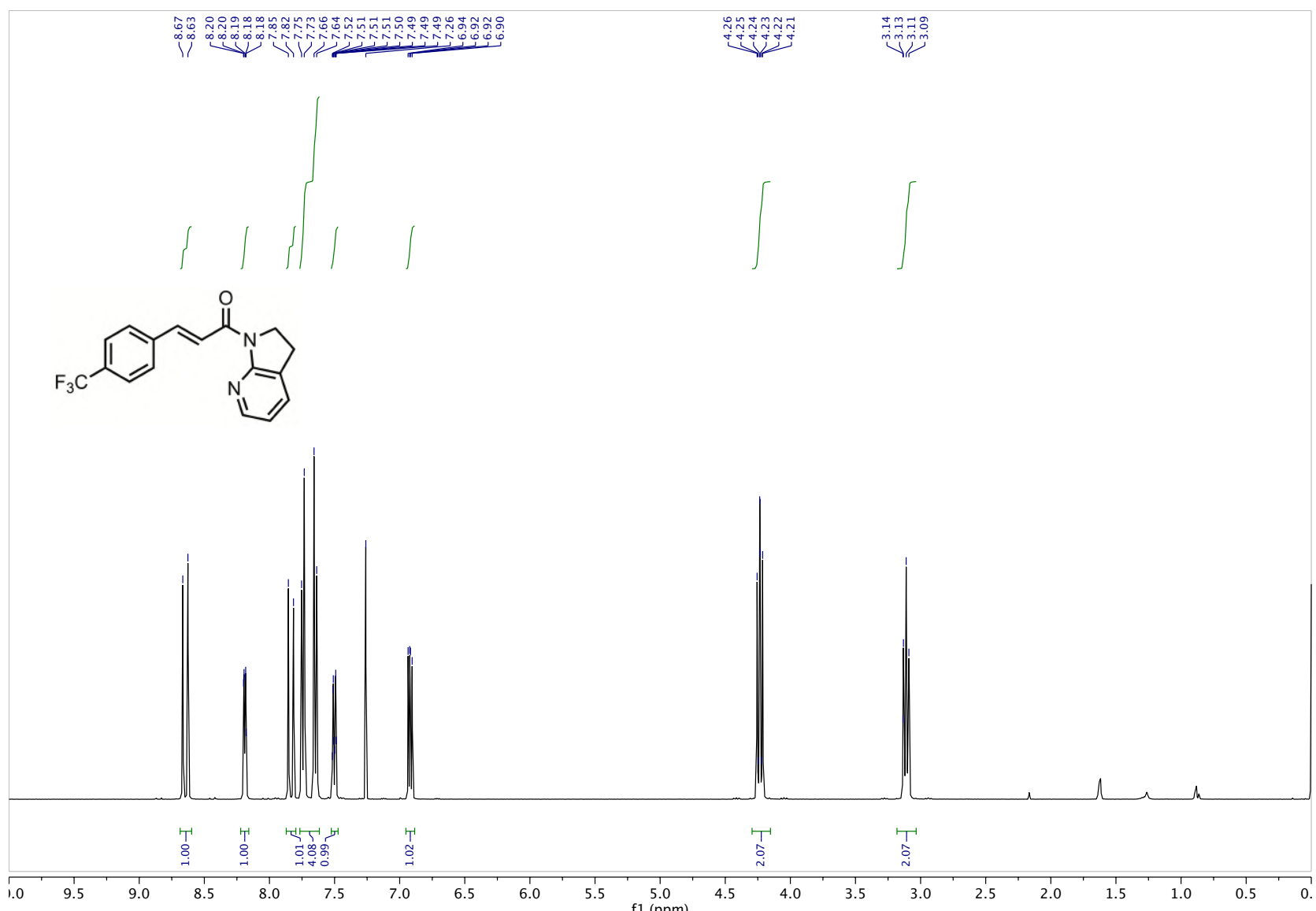

${ }^{1} \mathrm{H}$ NMR: 2x

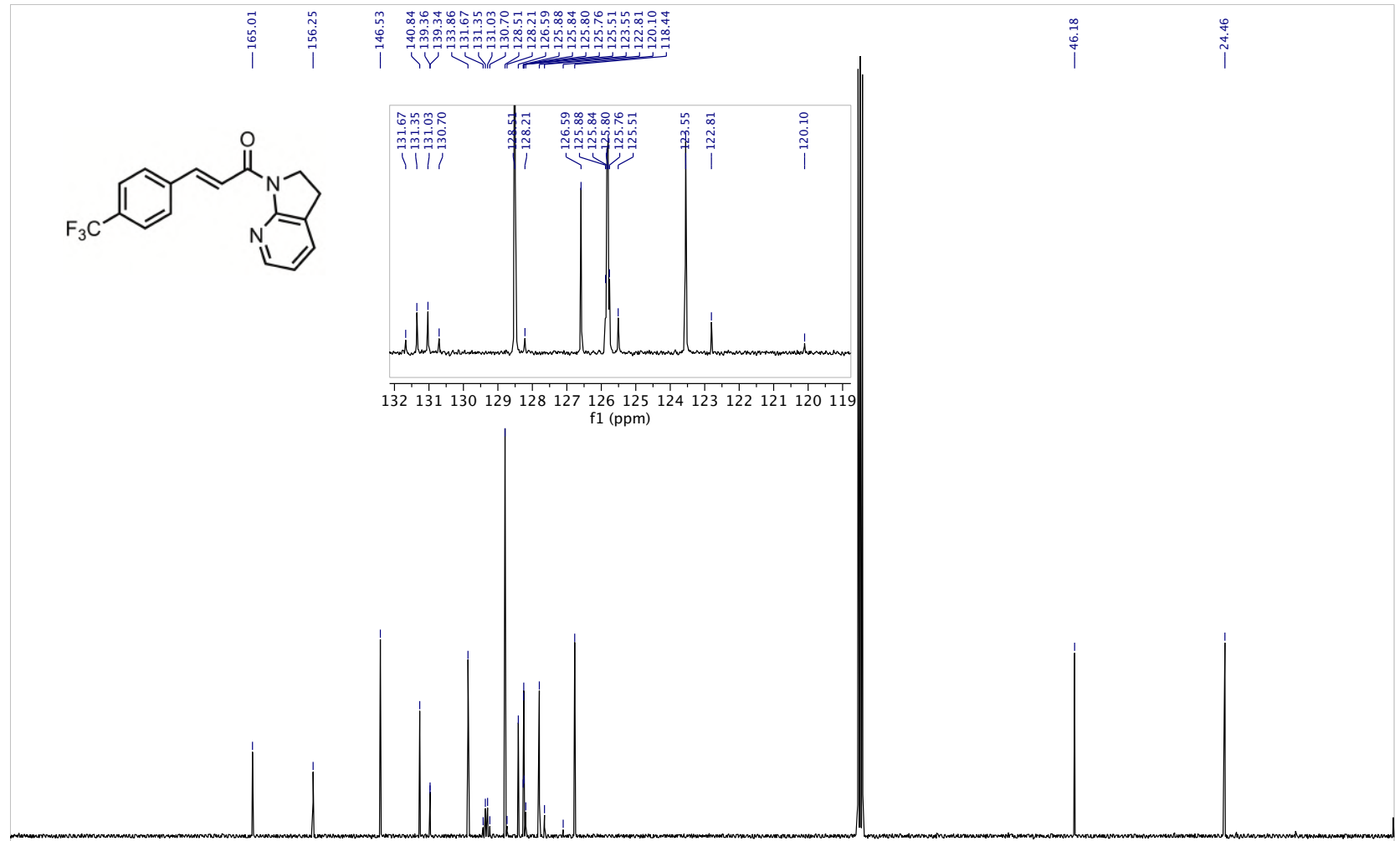

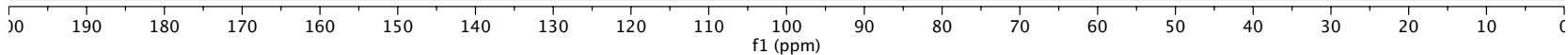


${ }^{19}$ F NMR: 2x

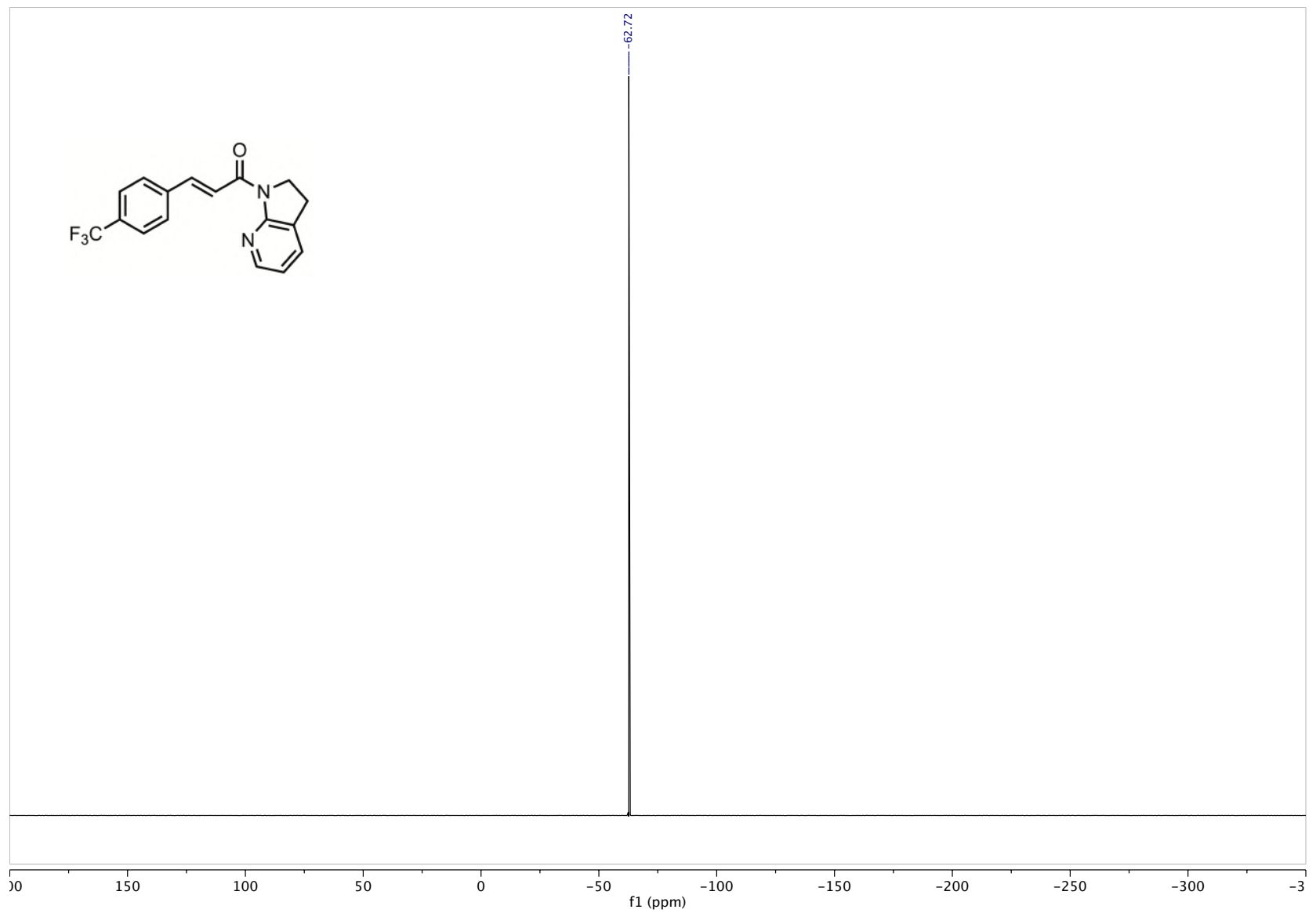


Supporting Information

${ }^{1} \mathrm{H}$ NMR: 2y

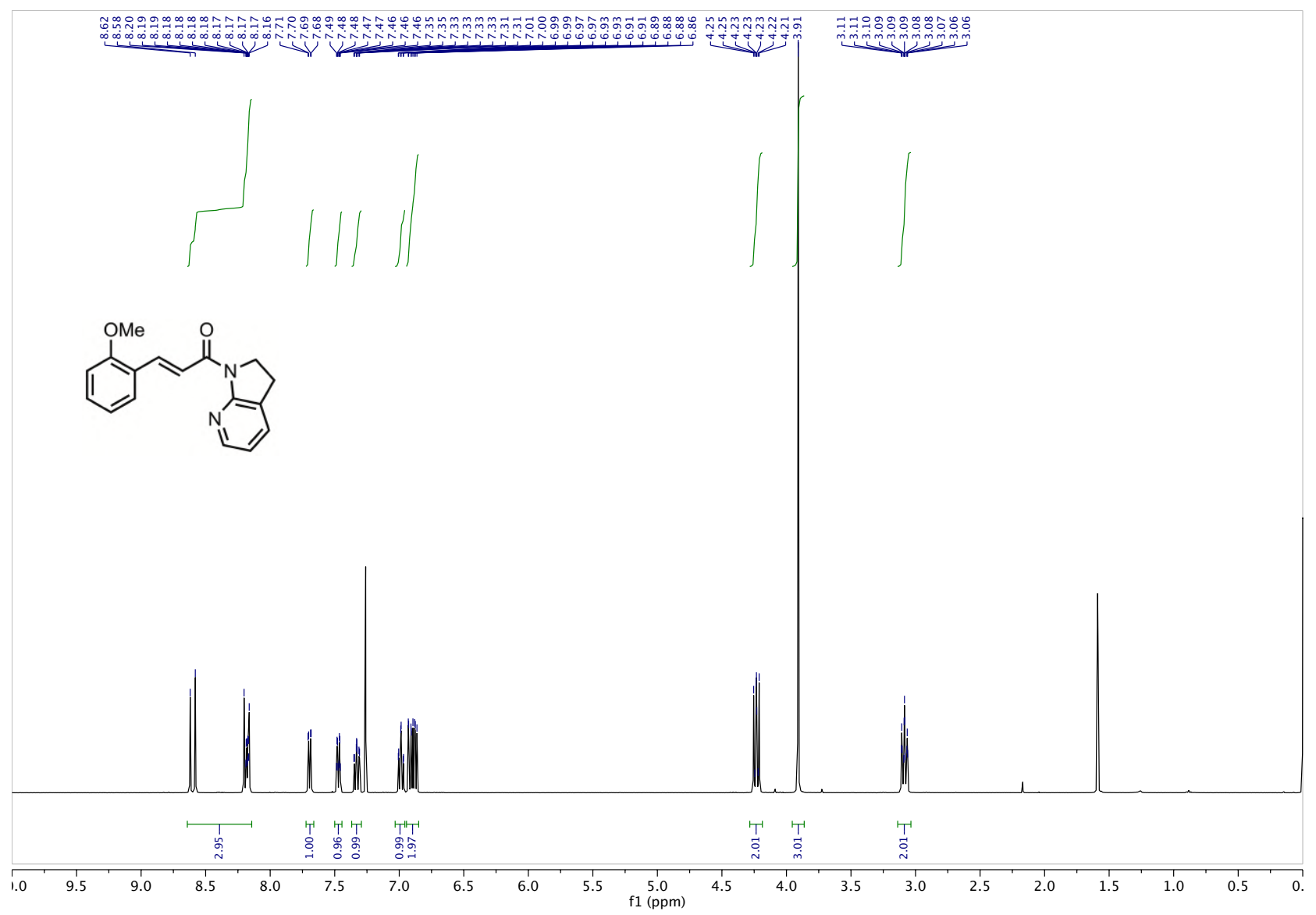

${ }^{13} \mathrm{C}$ NMR: $\mathbf{2 y}$

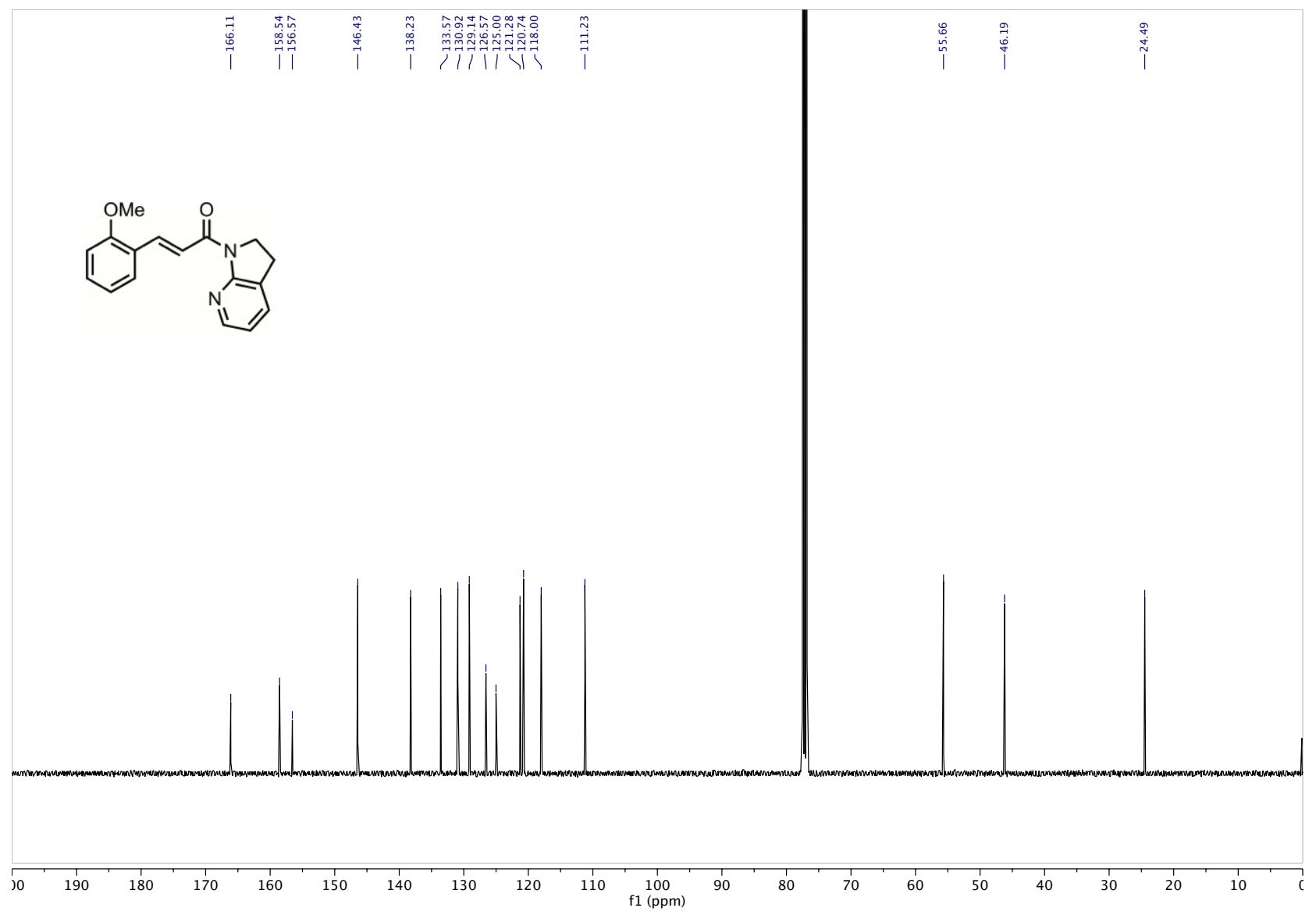

S116 
Supporting Information

${ }^{1}$ H NMR: $2 z$

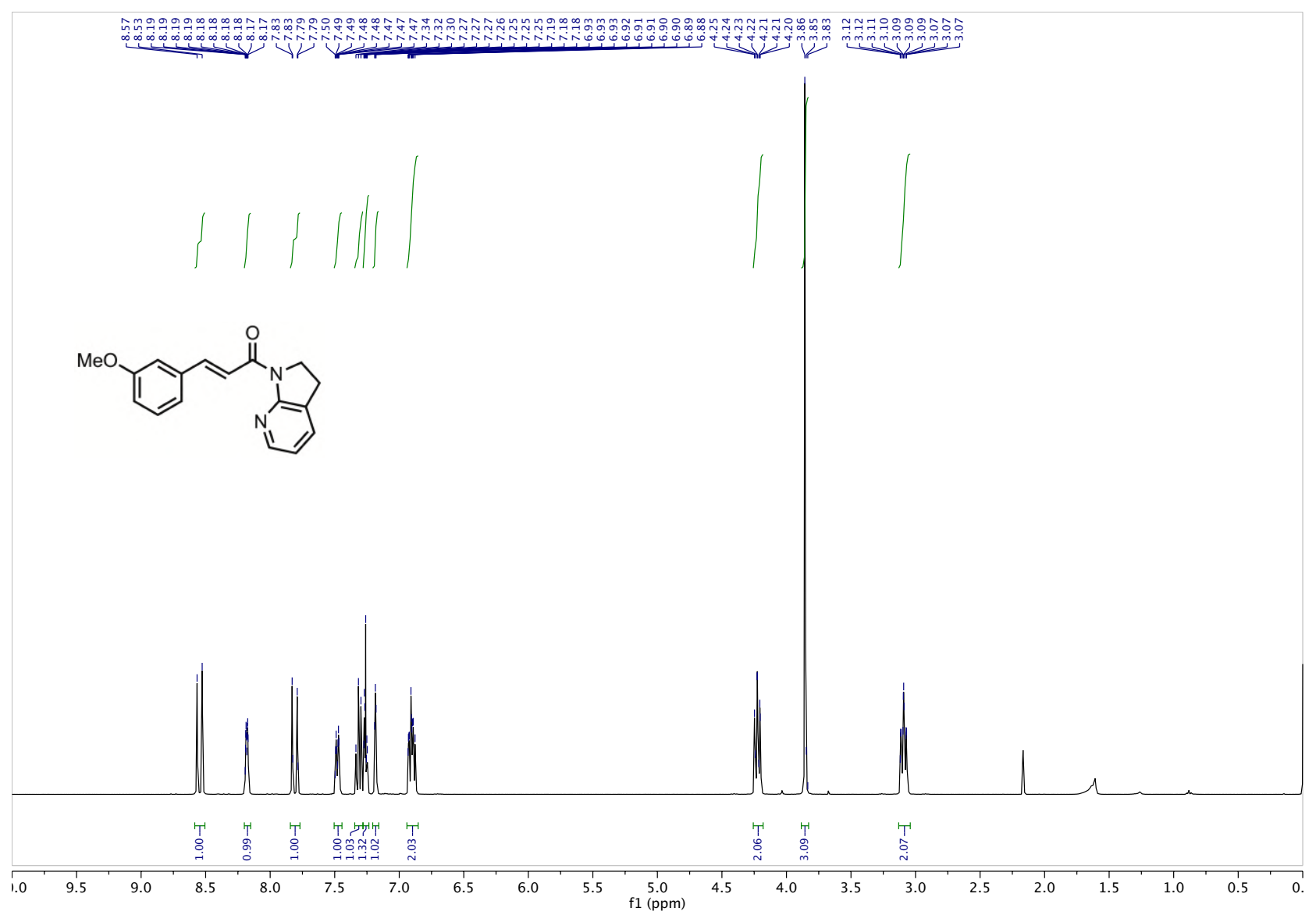

${ }^{13}$ C NMR: $2 z$

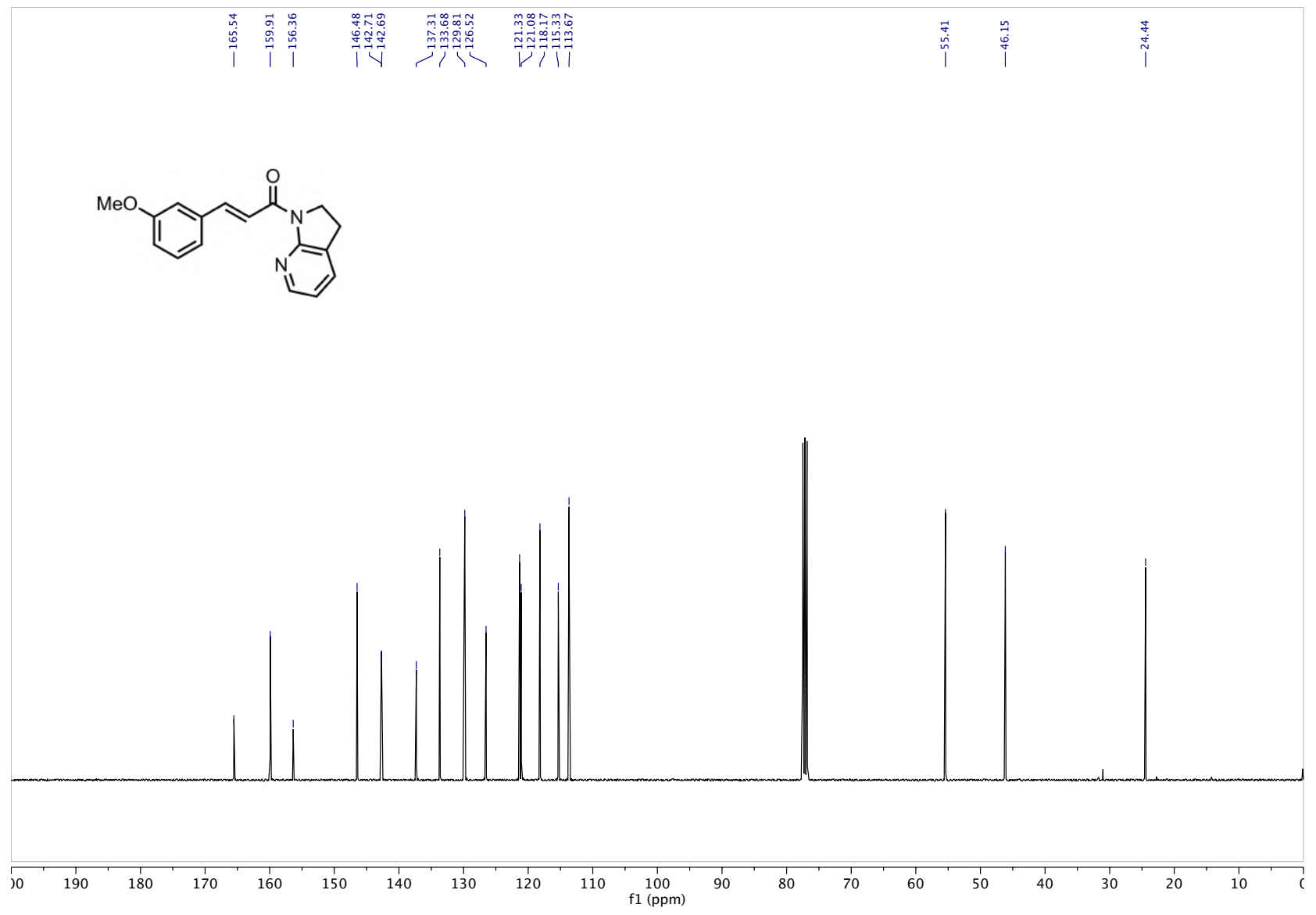

S117 
${ }^{1} \mathrm{H}$ NMR: 2aa

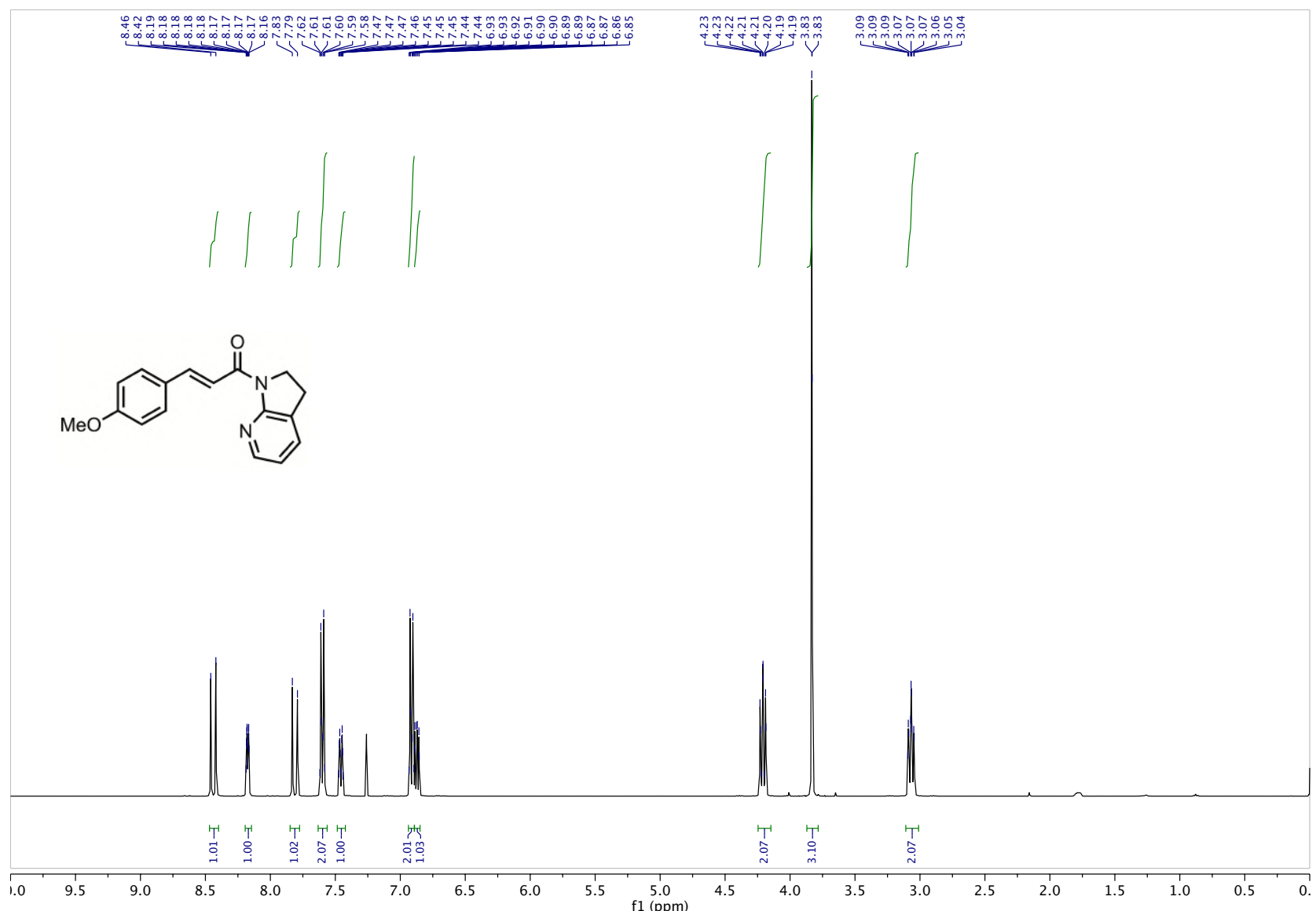

${ }^{13}$ C NMR: 2aa

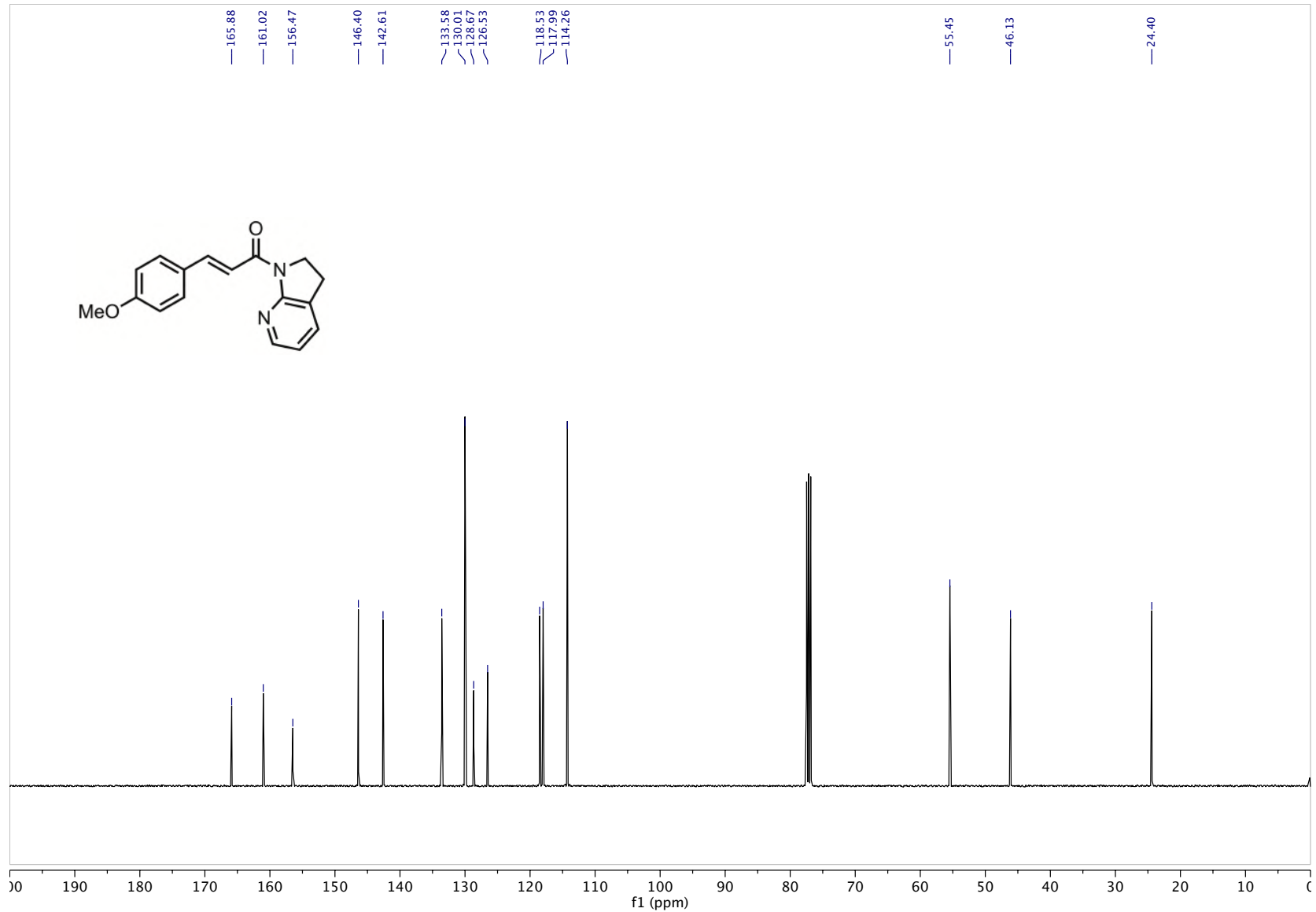


${ }^{1} \mathrm{H}$ NMR: 2ab

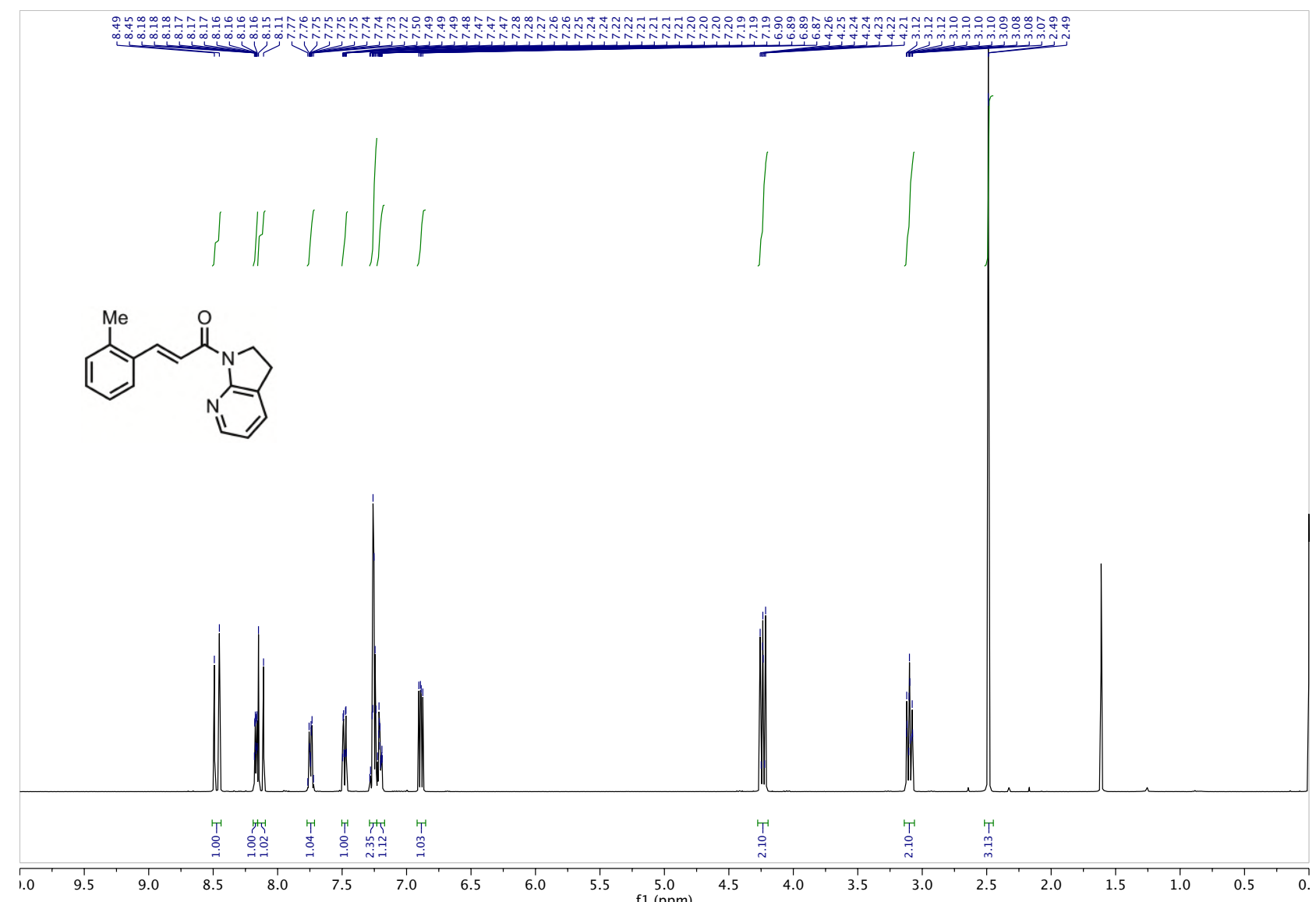

${ }^{13} \mathrm{C}$ NMR: 2ab

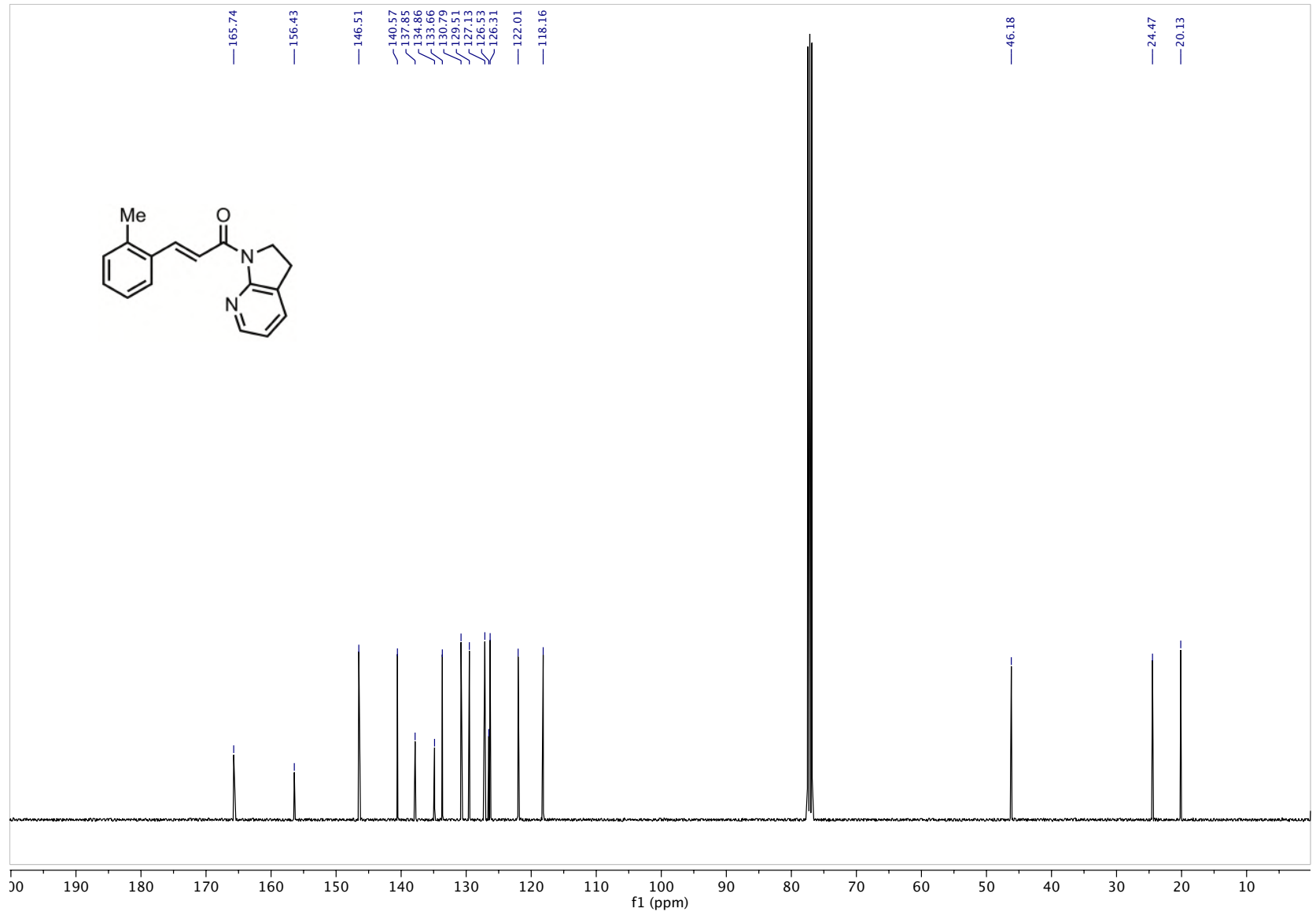


${ }^{1} \mathrm{H}$ NMR: 2ac

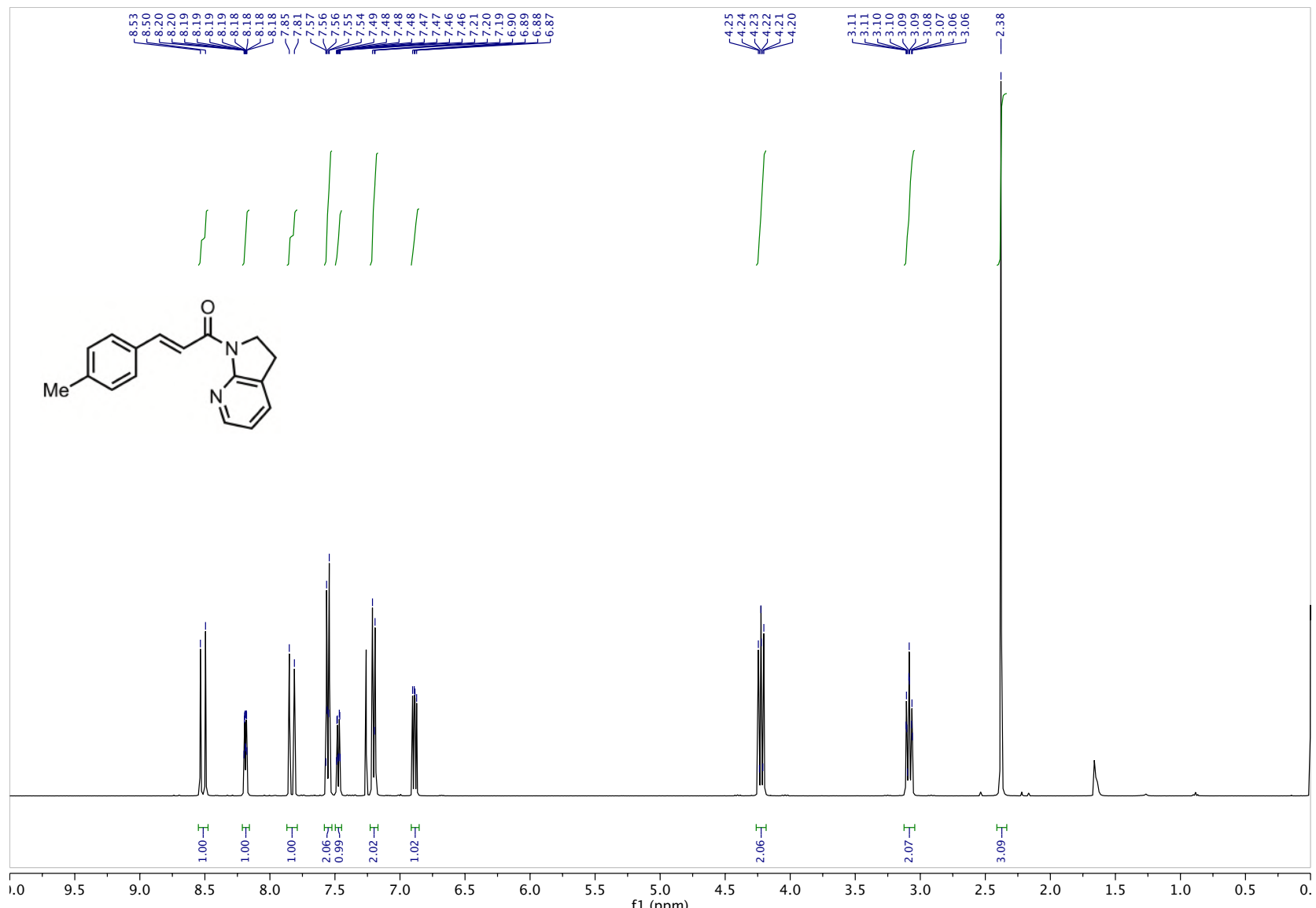

${ }^{13} \mathrm{C}$ NMR: 2ac

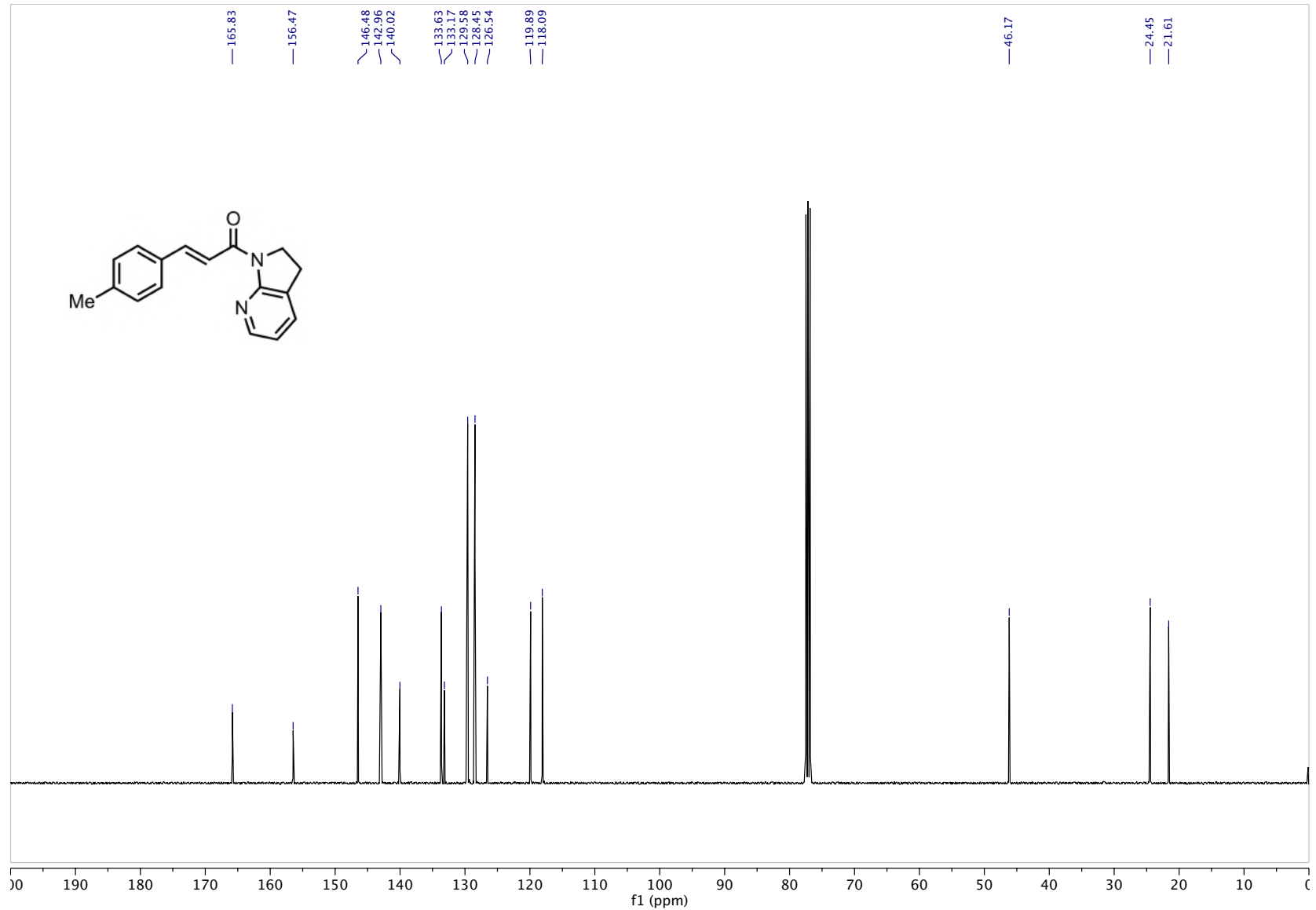


${ }^{1} \mathrm{H}$ NMR: 2ad

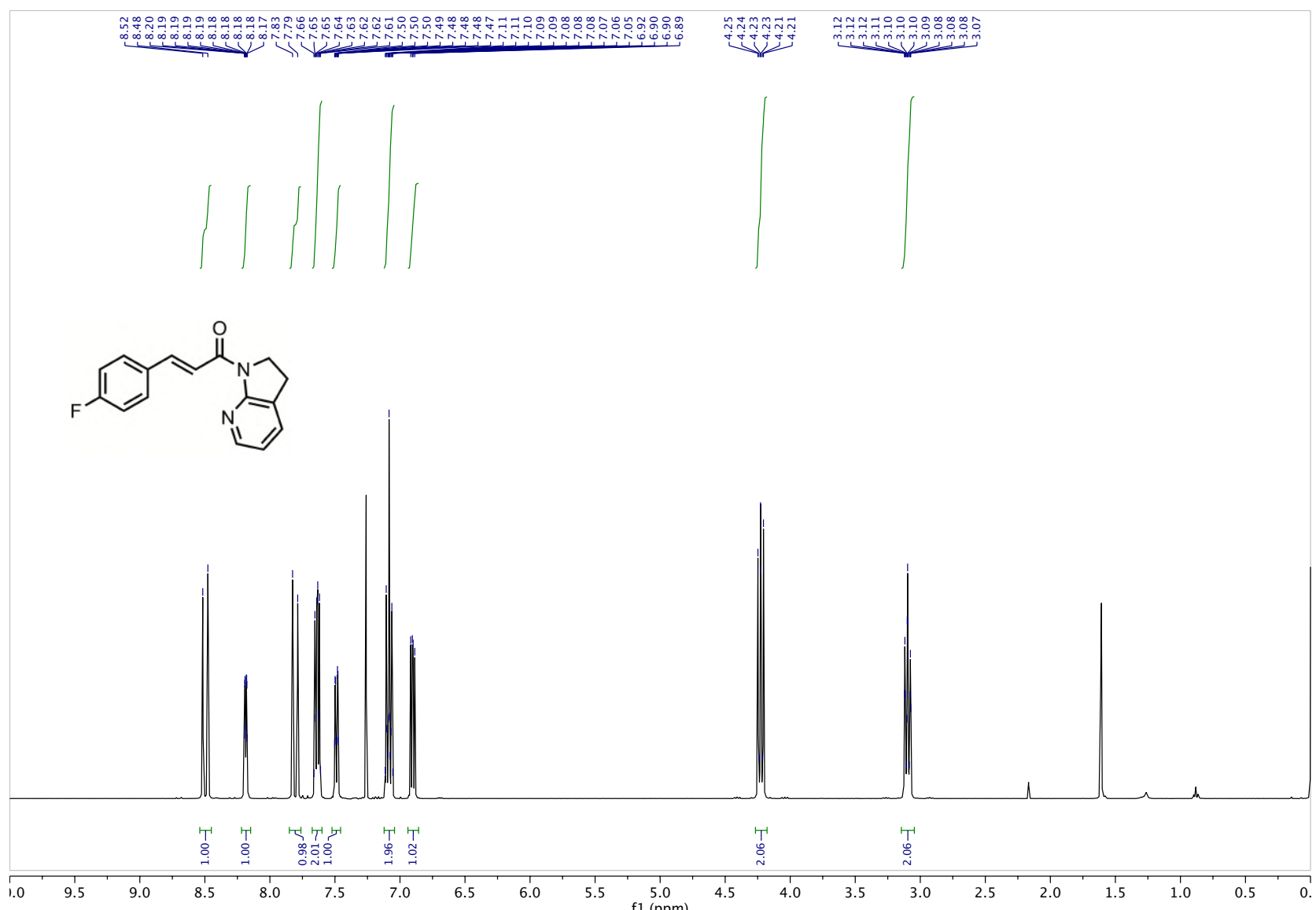

${ }^{13}$ C NMR: 2ad

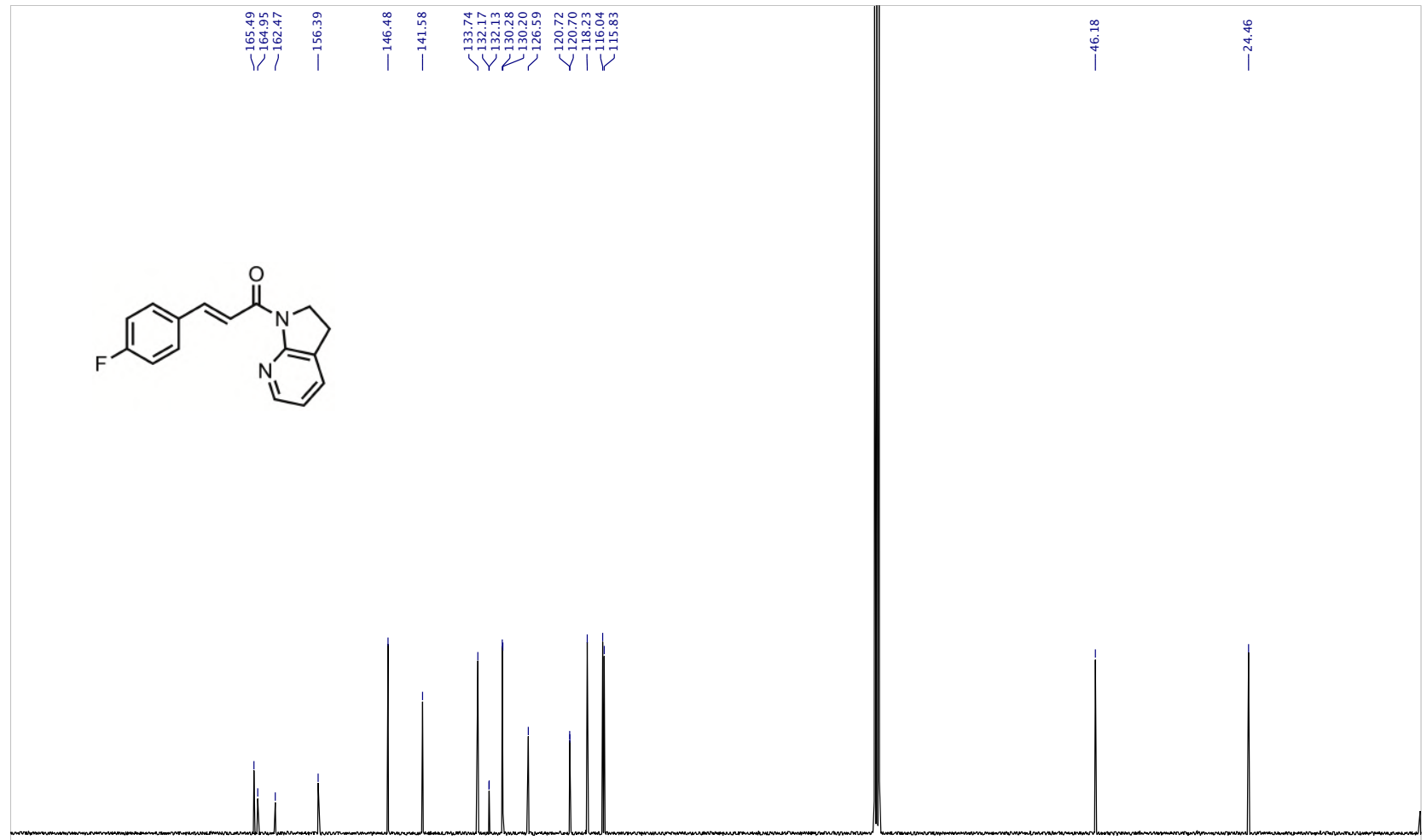

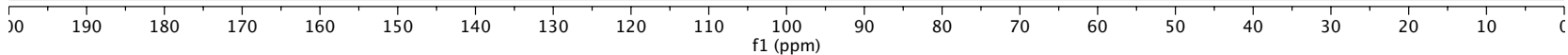


${ }^{19}$ F NMR: 2ad

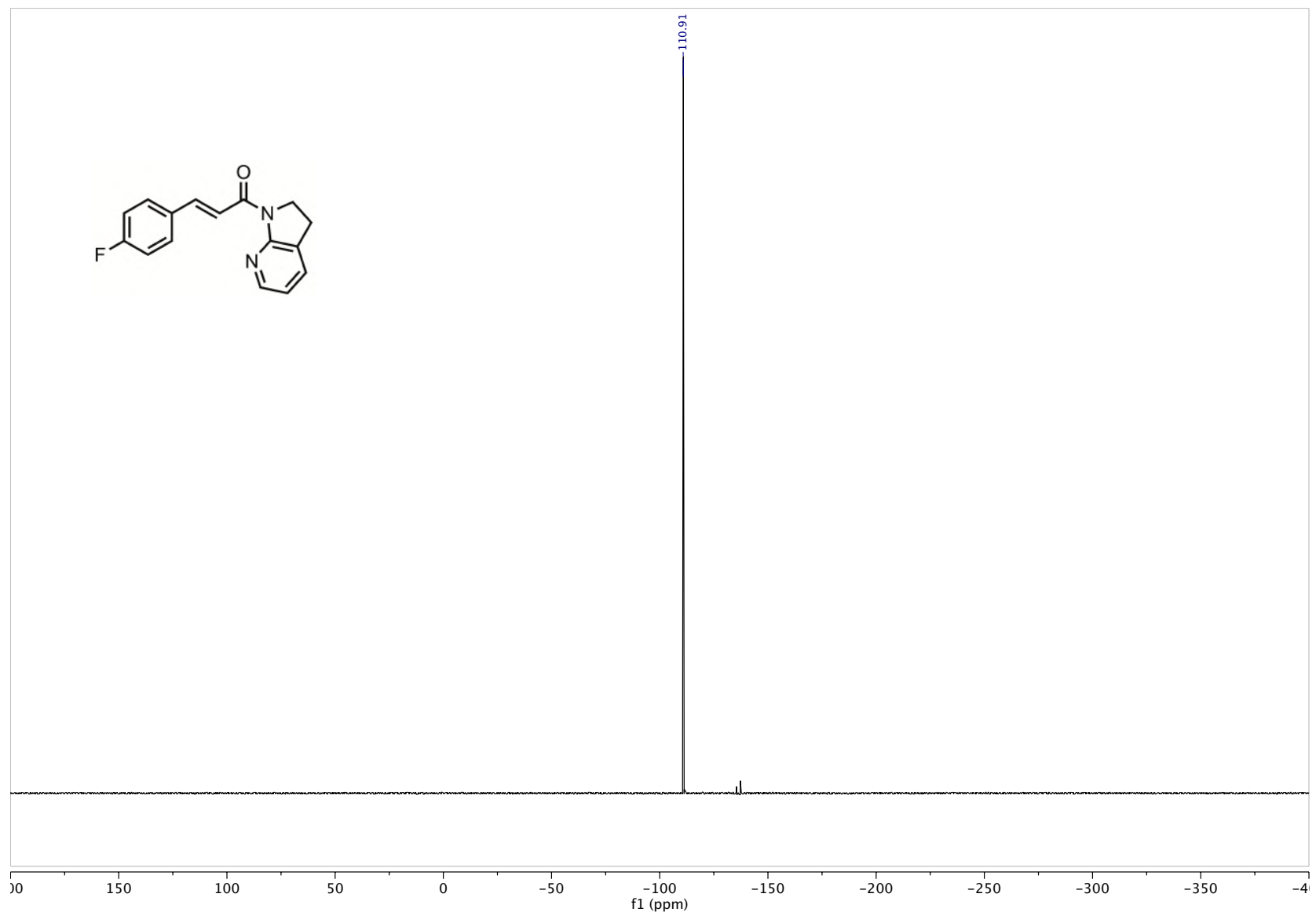


Supporting Information

${ }^{1} \mathrm{H}$ NMR: 2ae

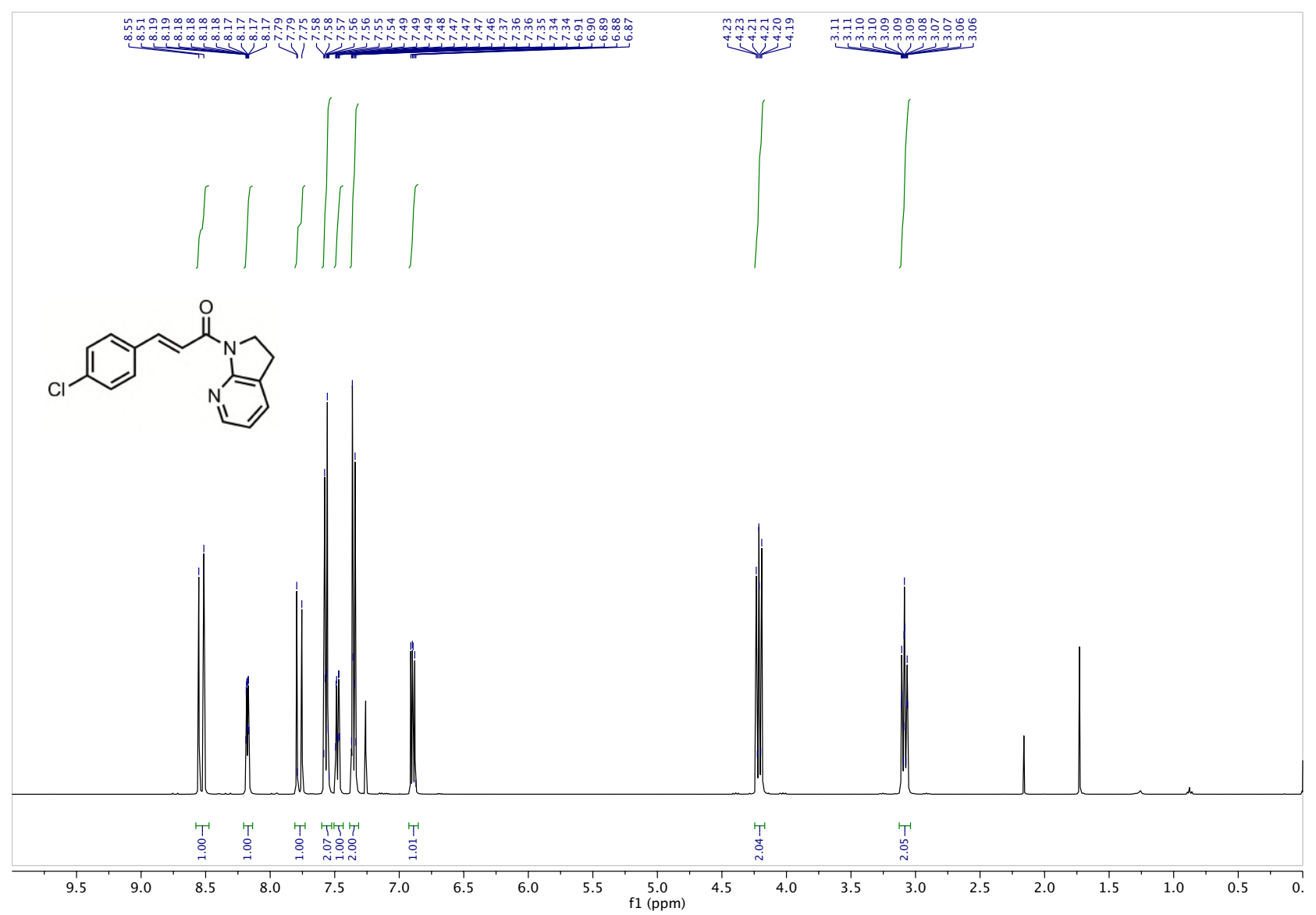

${ }^{13} \mathrm{C}$ NMR: ae

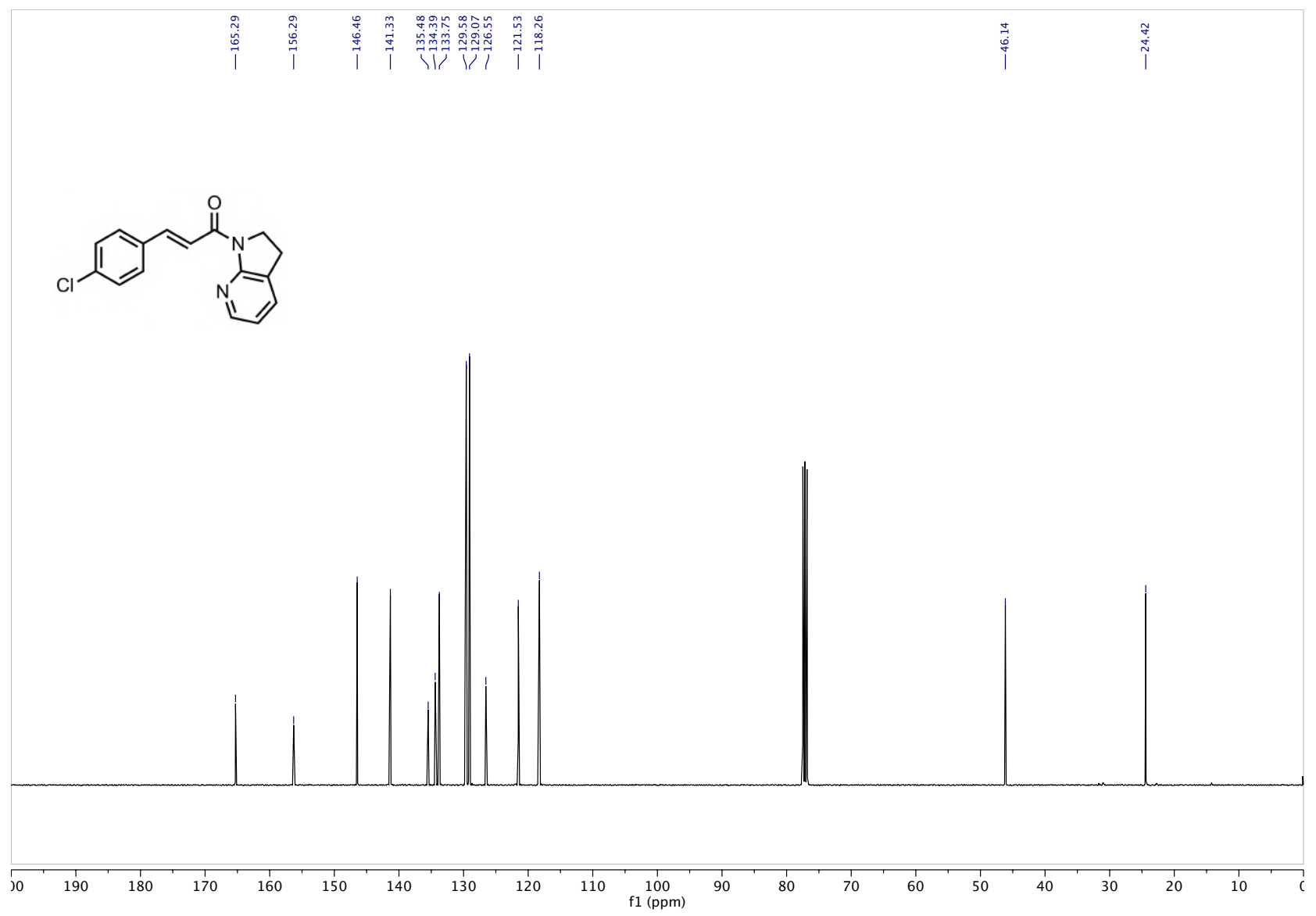

S123 
${ }^{1} \mathrm{H}$ NMR: 2af

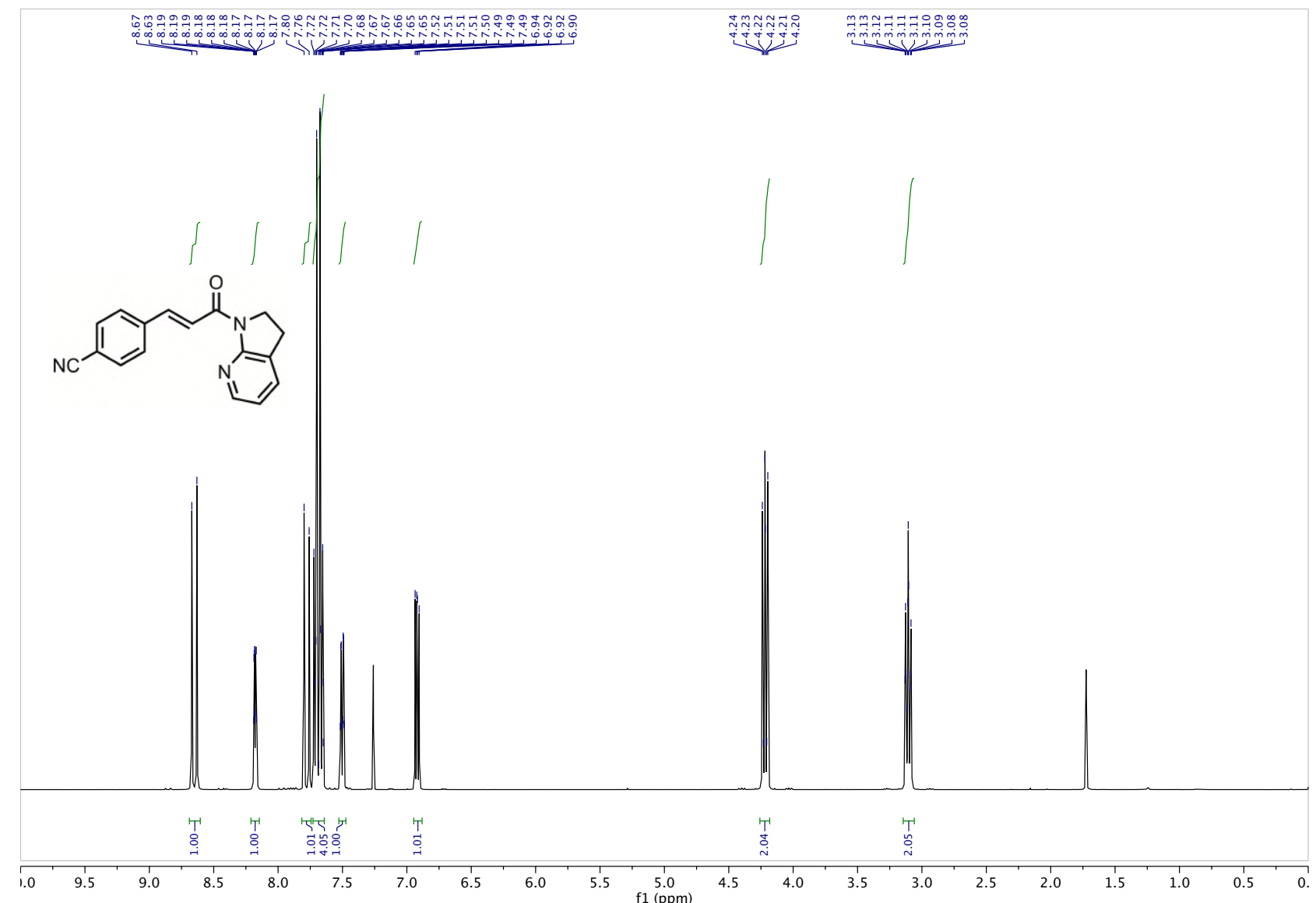

${ }^{13}$ C NMR: 2af

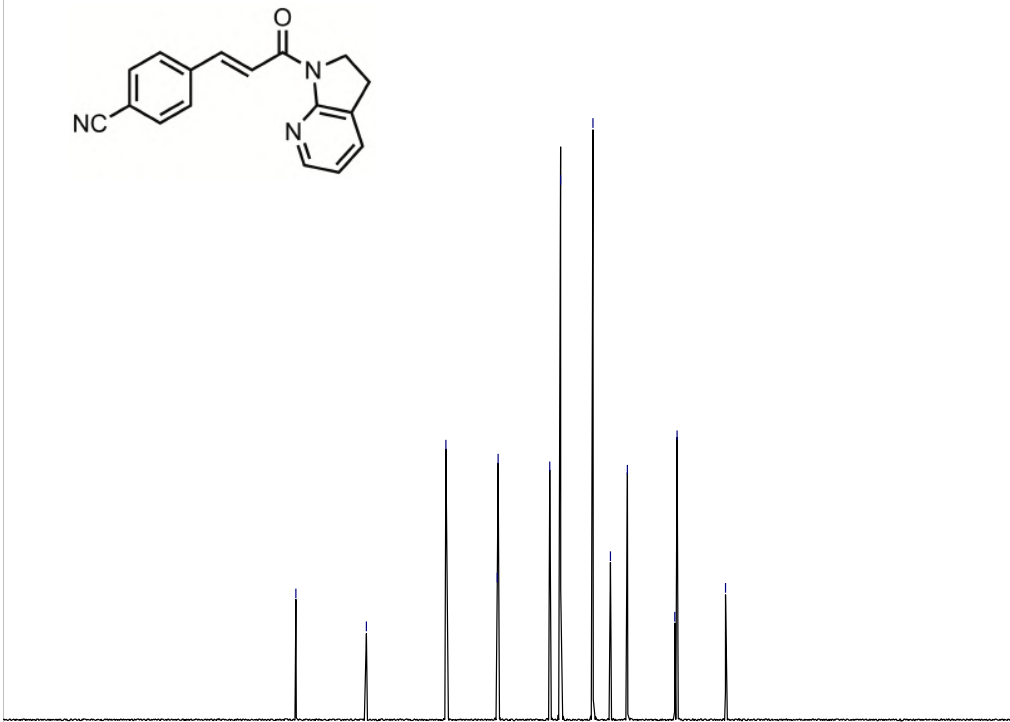

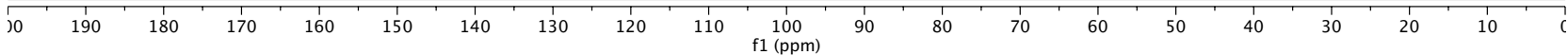


${ }^{1} \mathrm{H}$ NMR: 2ag

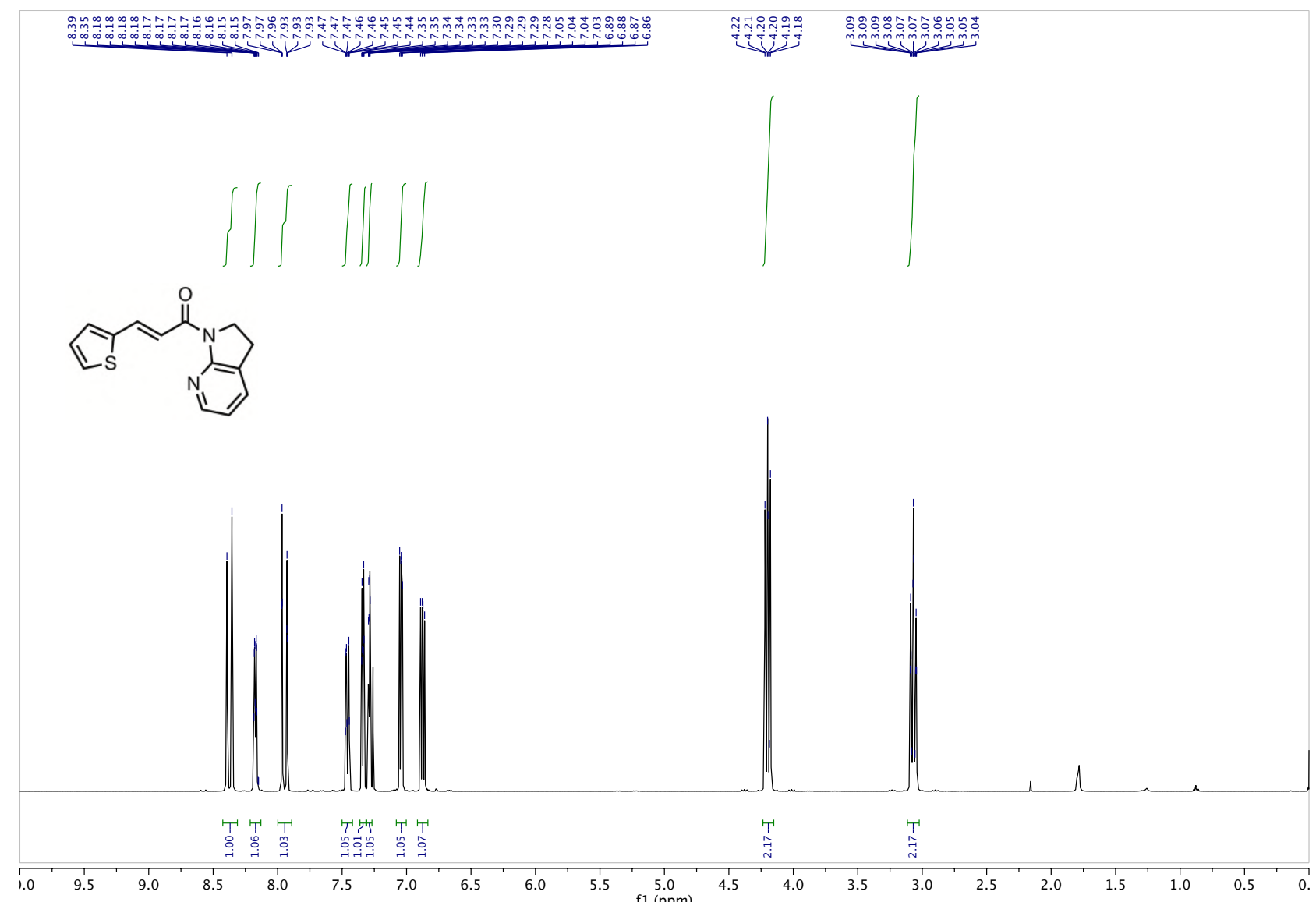

${ }^{13} \mathrm{C}$ NMR: 2 ag

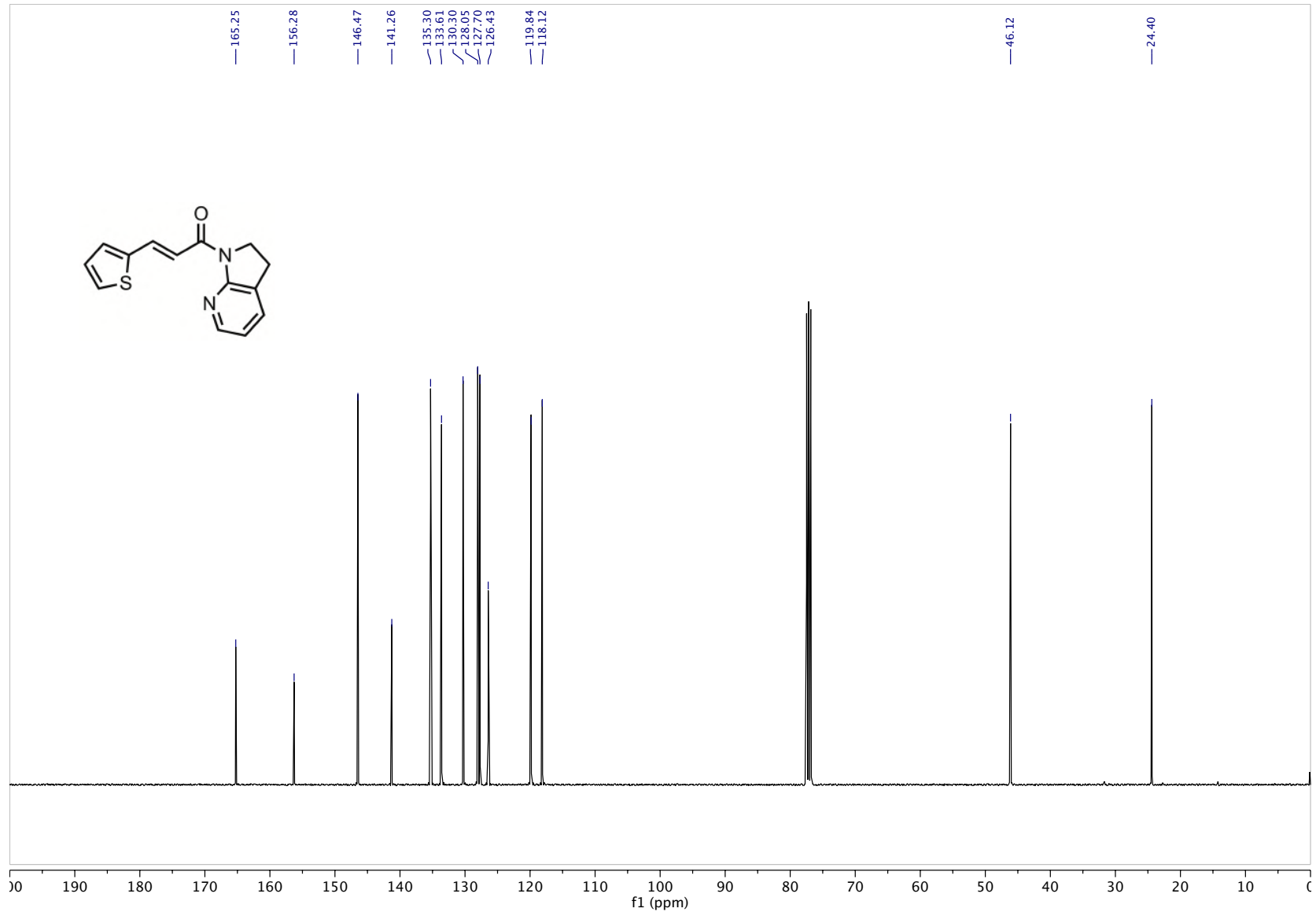


${ }^{1} \mathrm{H}$ NMR: 2ah

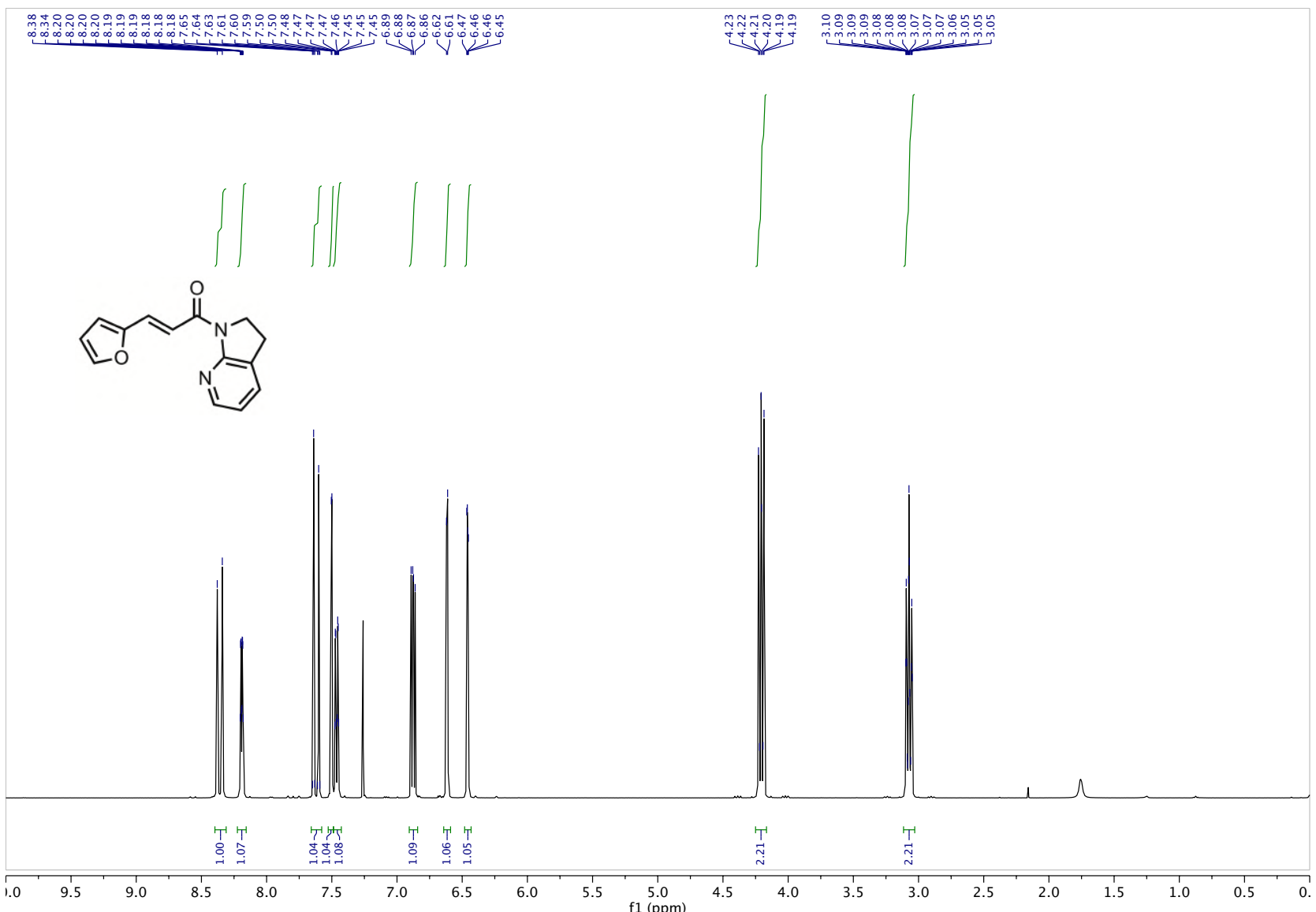

${ }^{13}$ C NMR: 2ah

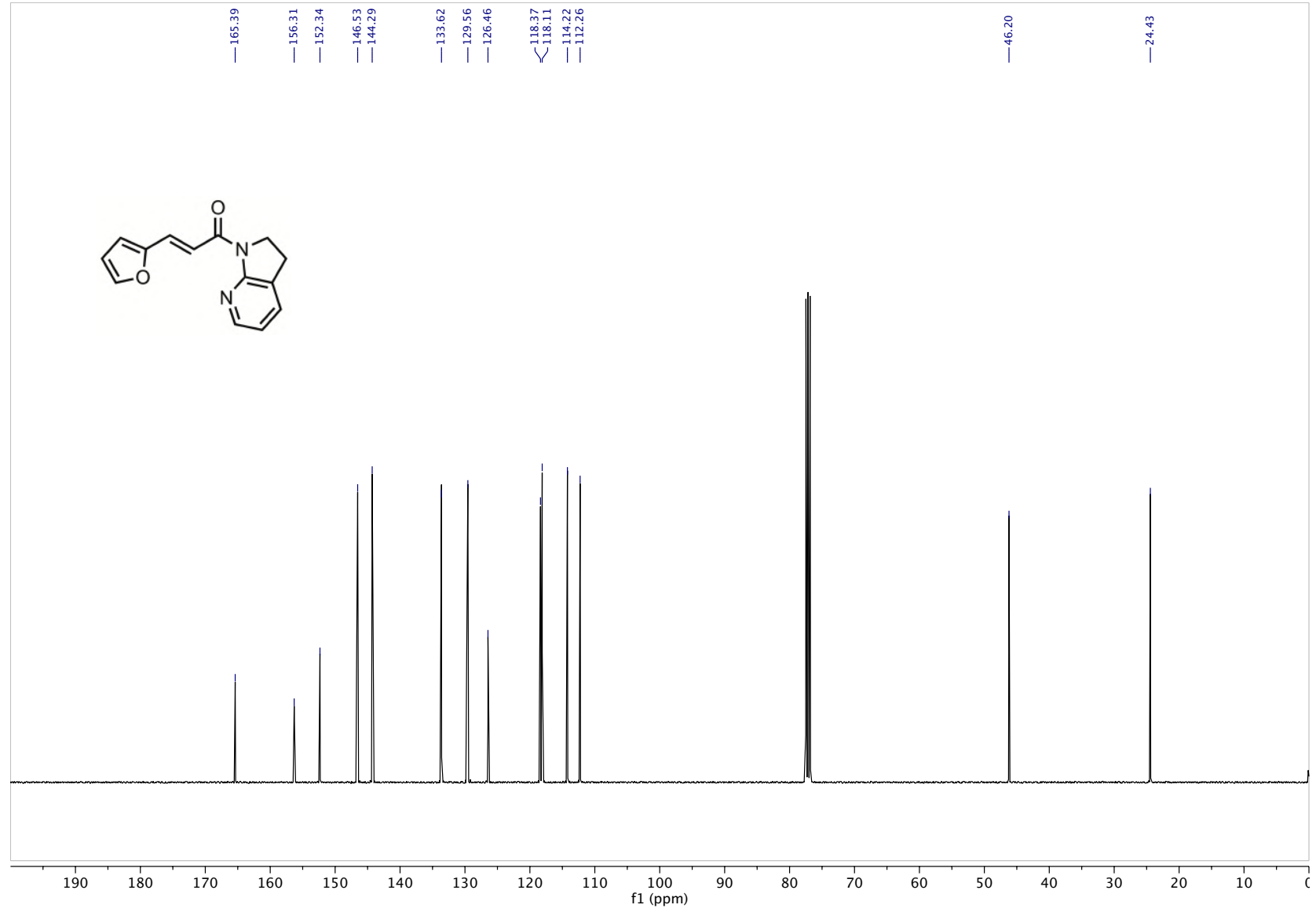


${ }^{1} \mathrm{H}$ NMR: 3aa

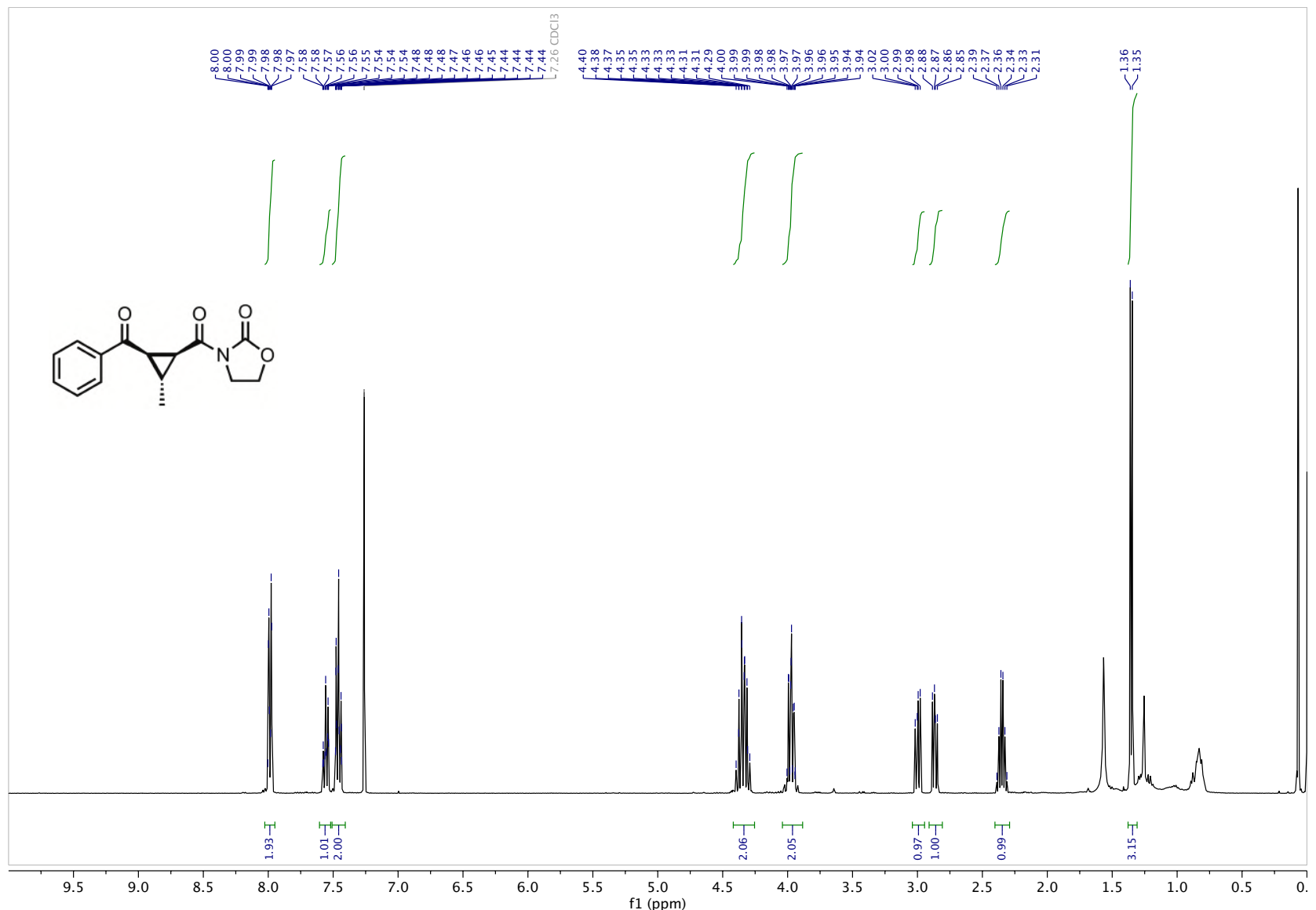

${ }^{13} \mathrm{C}$ NMR: 3aa

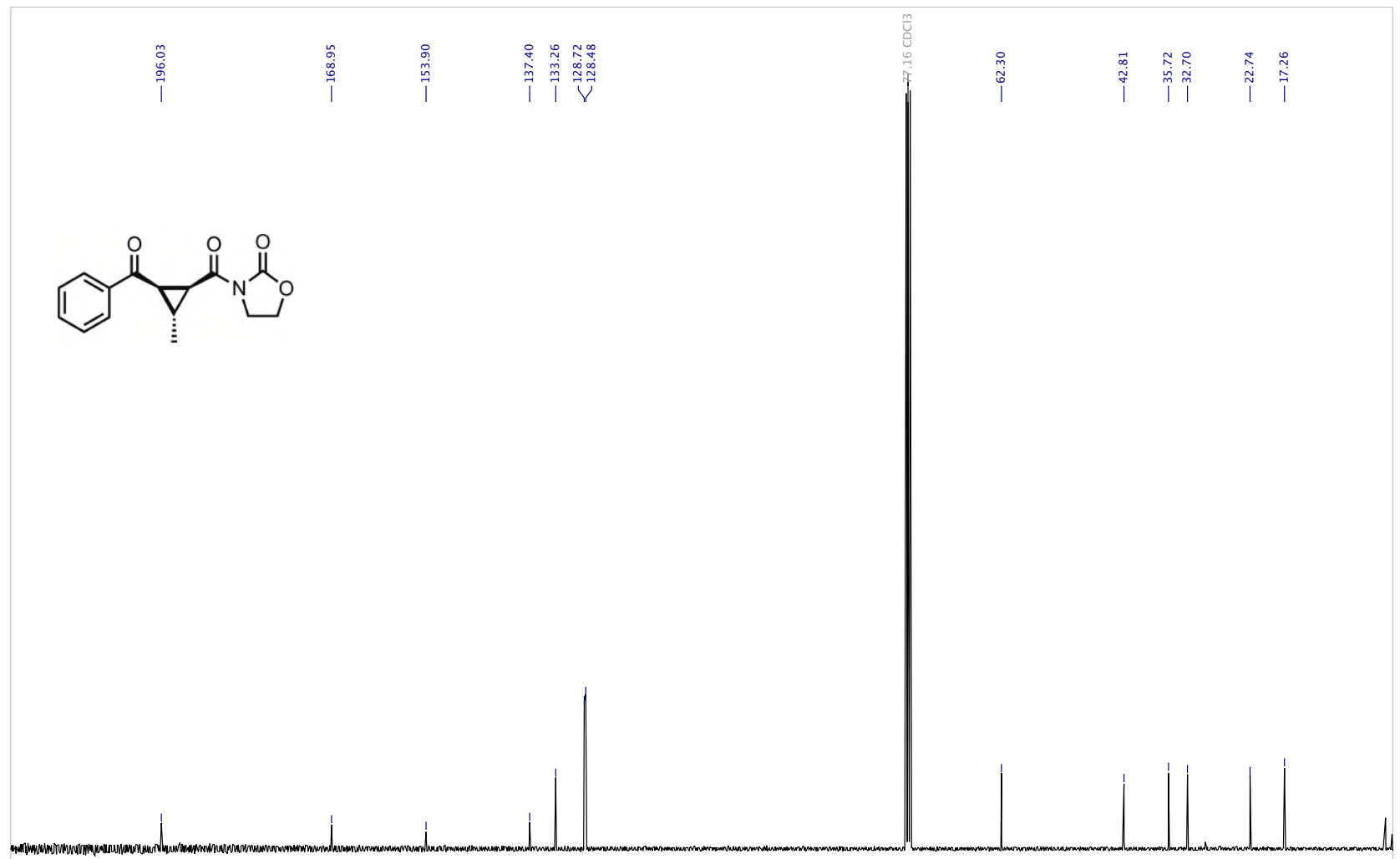

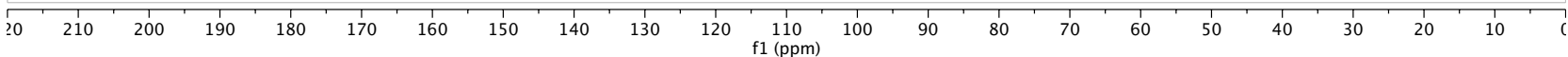


${ }^{1} \mathrm{H}$ NMR: 3ab

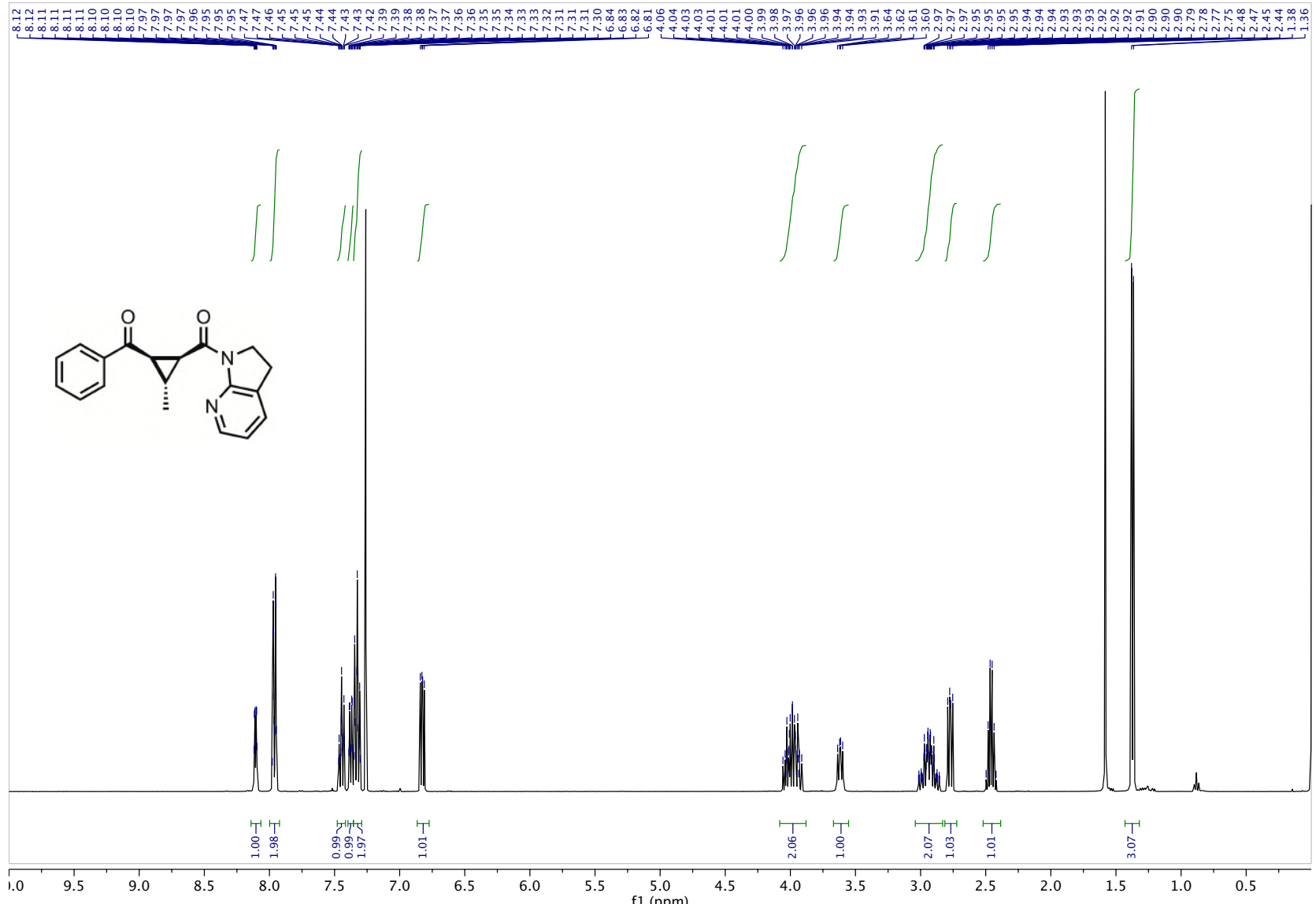

${ }^{13} \mathrm{C}$ NMR: 3ab

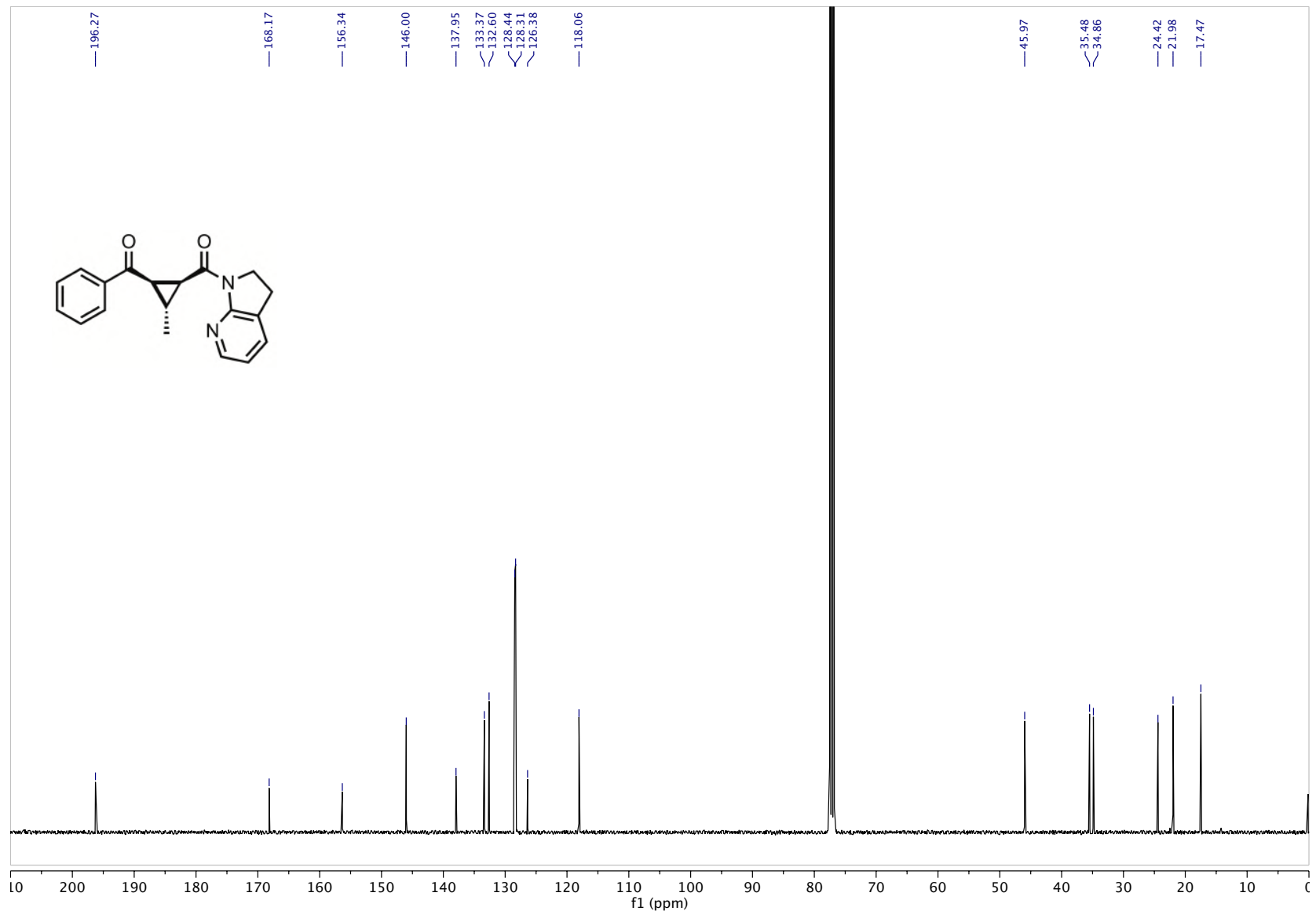


${ }^{1} \mathrm{H}$ NMR: 3ab (NOESY)

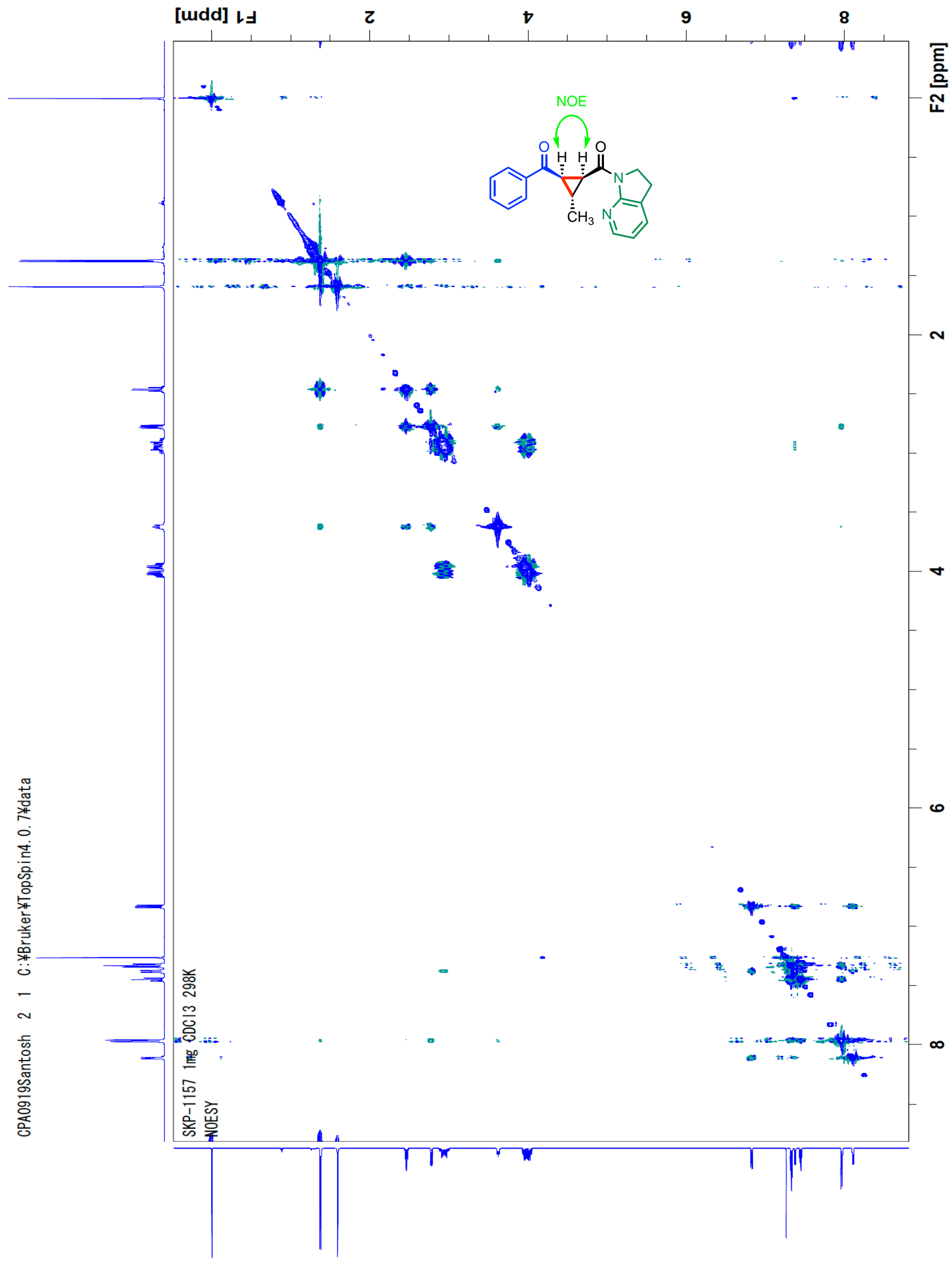


${ }^{1} \mathrm{H}$ NMR: 3ab (COSY)

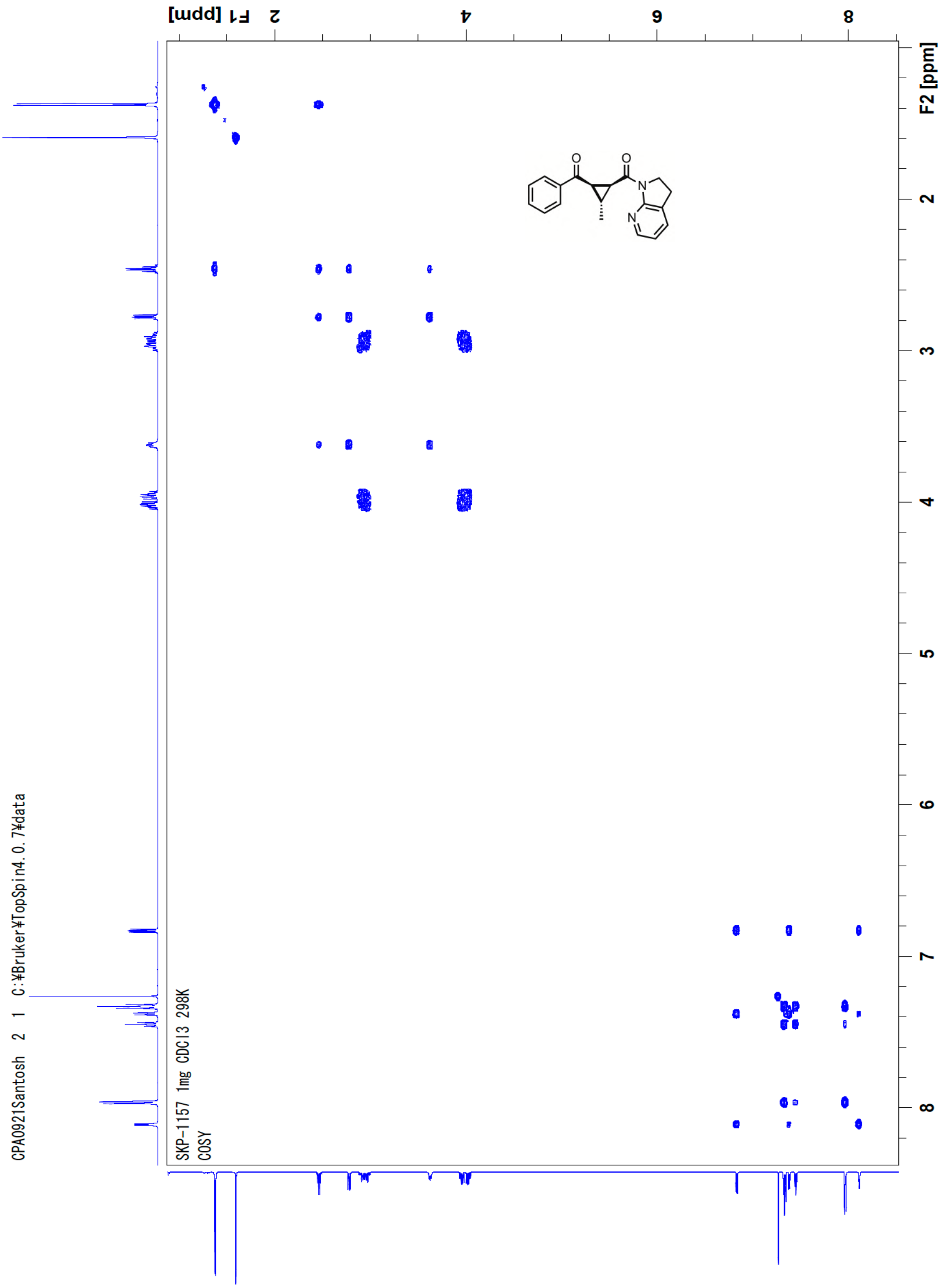


${ }^{1} \mathrm{H}$ NMR: 3ab (HMQC)

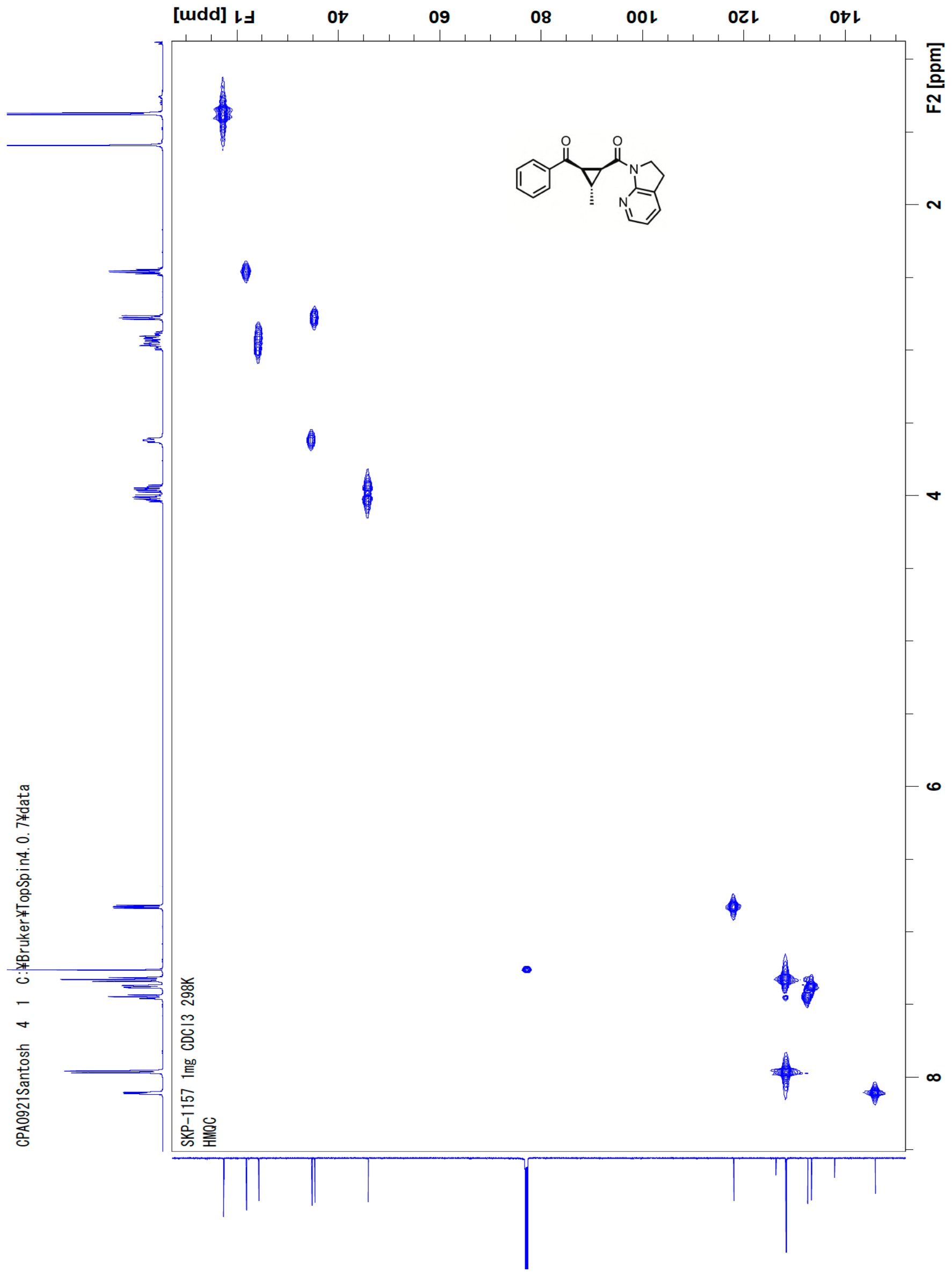


${ }^{1} \mathrm{H}$ NMR: 3ac

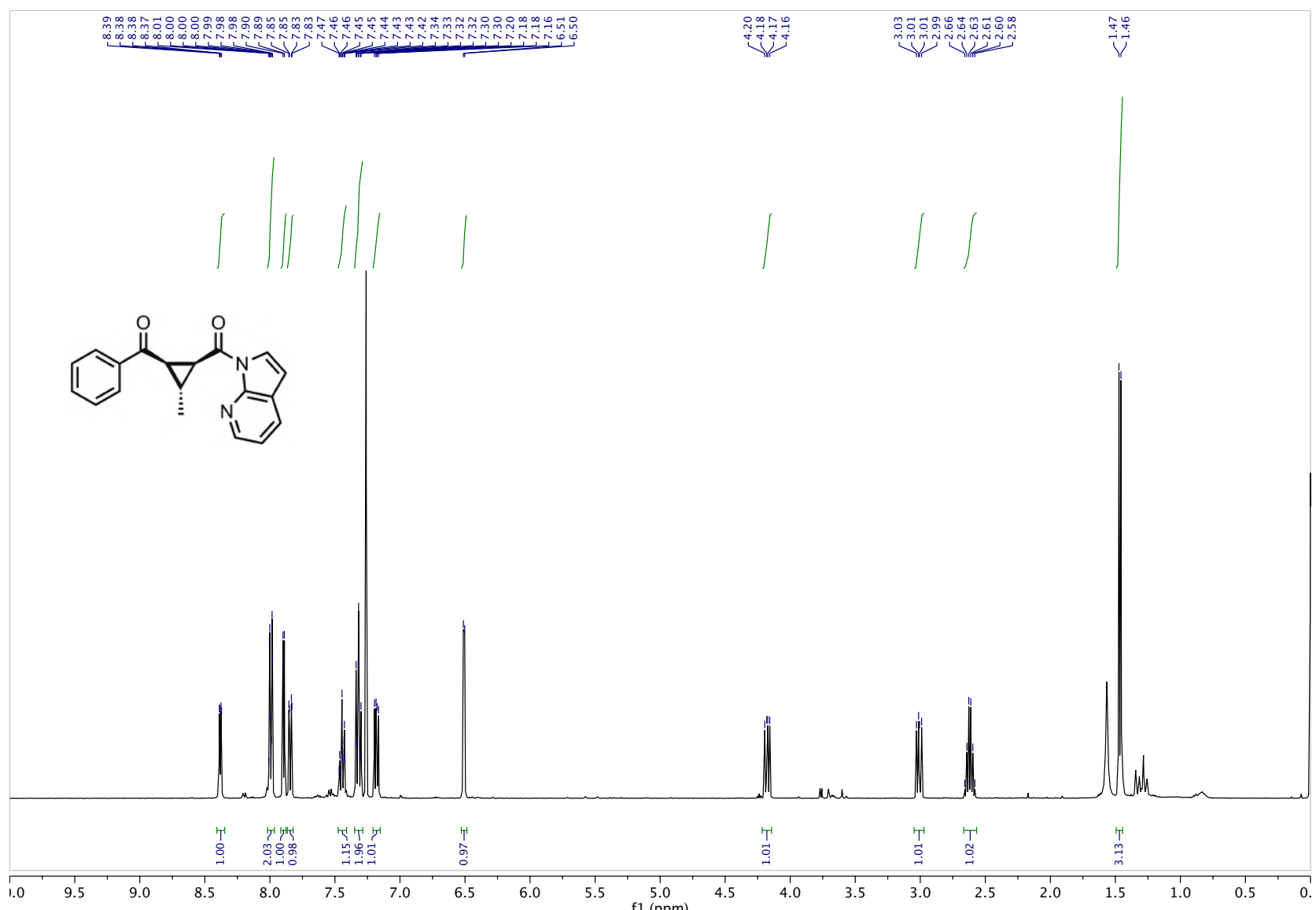

${ }^{13}$ C NMR: 3ac

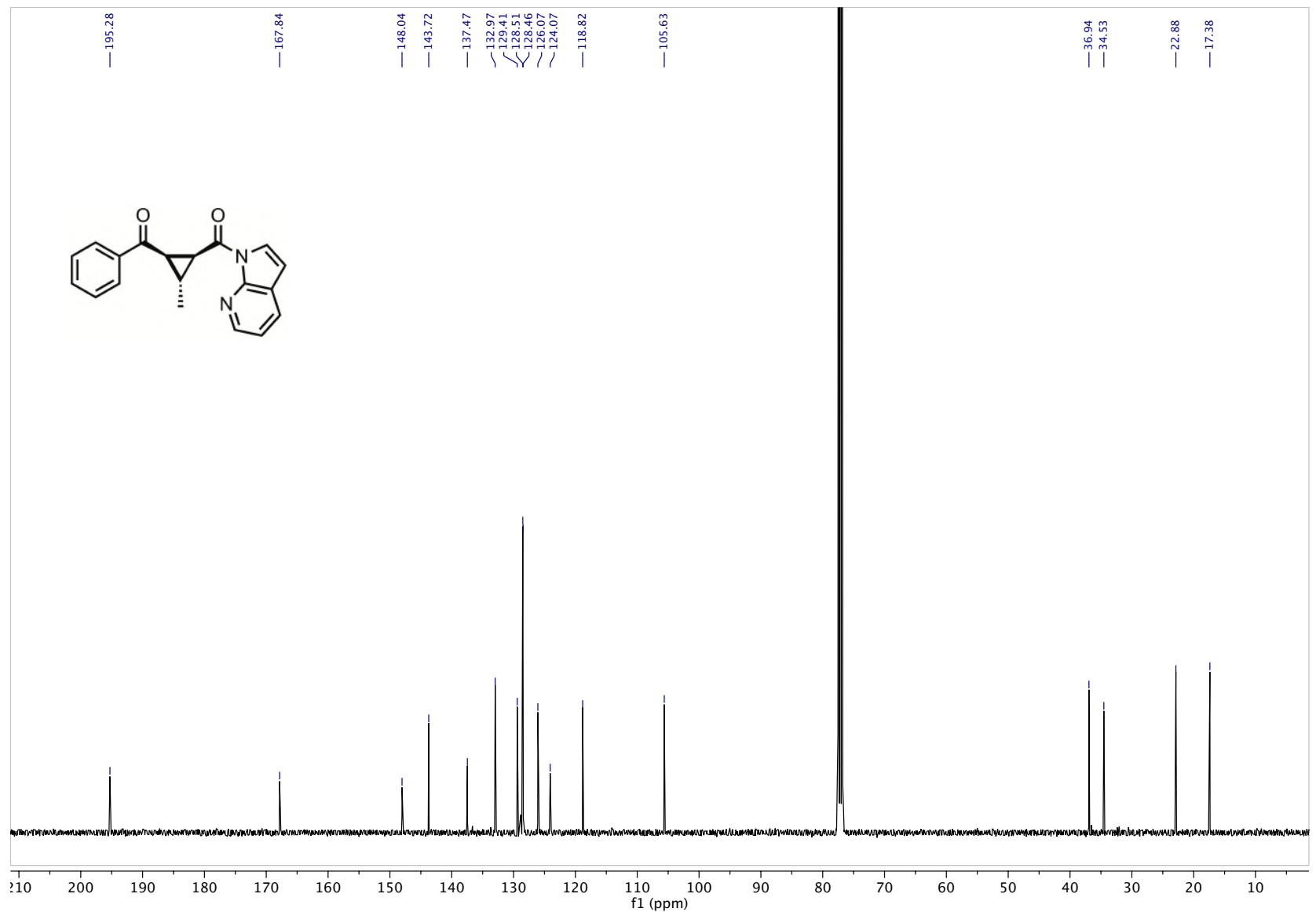




\section{${ }^{1} \mathrm{H}$ NMR: 3 ai}

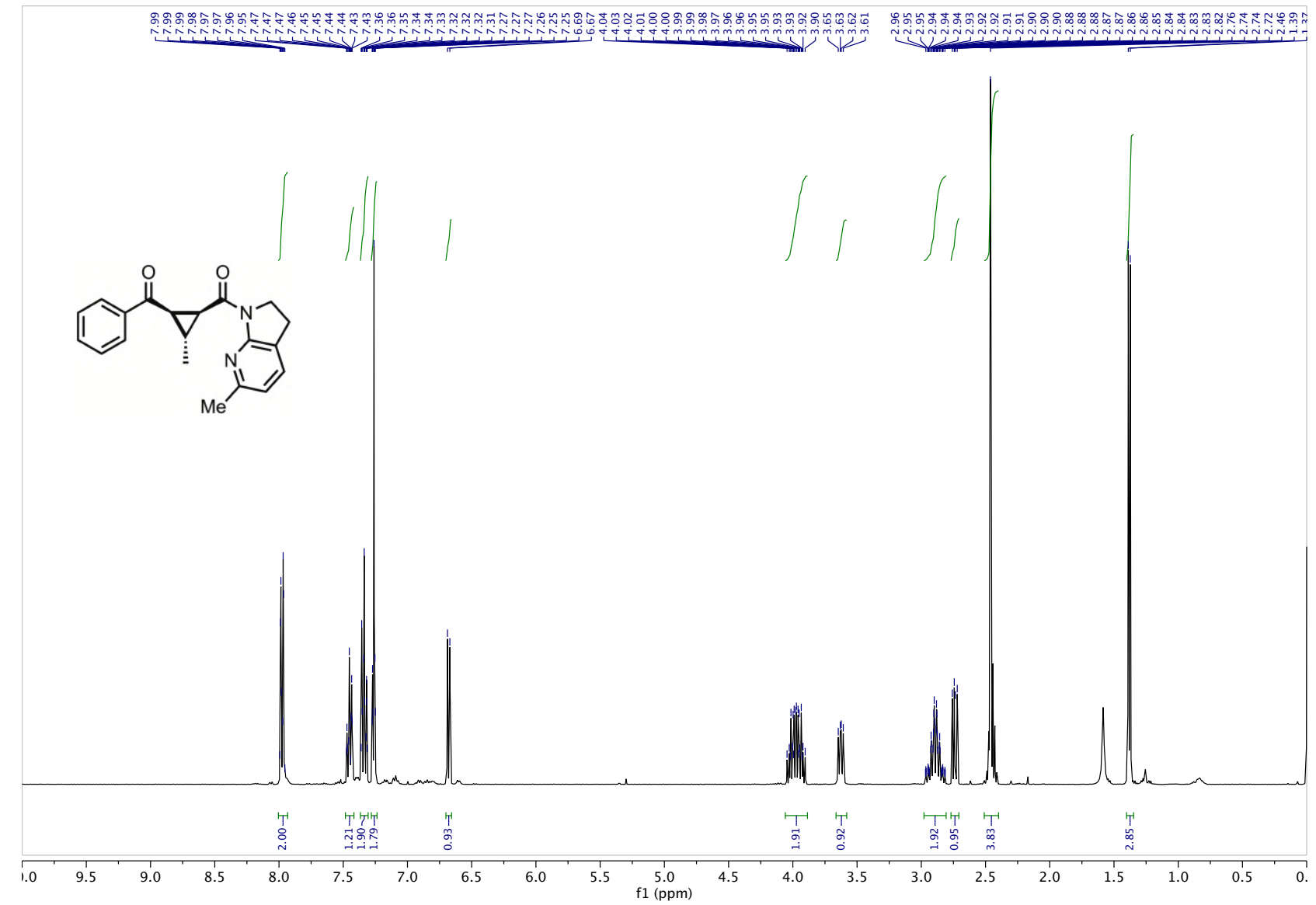

${ }^{13} \mathrm{C}$ NMR: 3ai

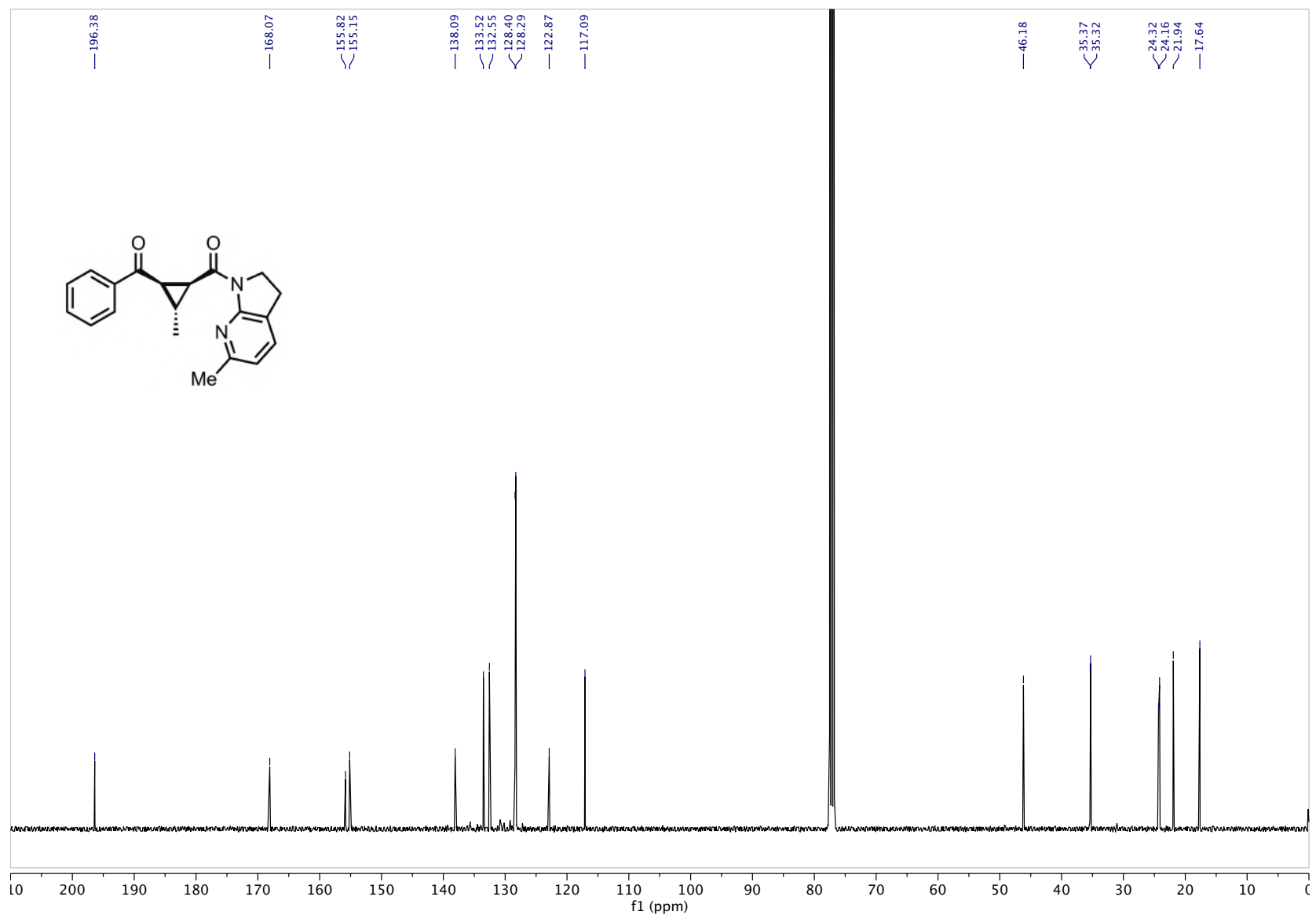


${ }^{1} \mathrm{H}$ NMR: 3aj

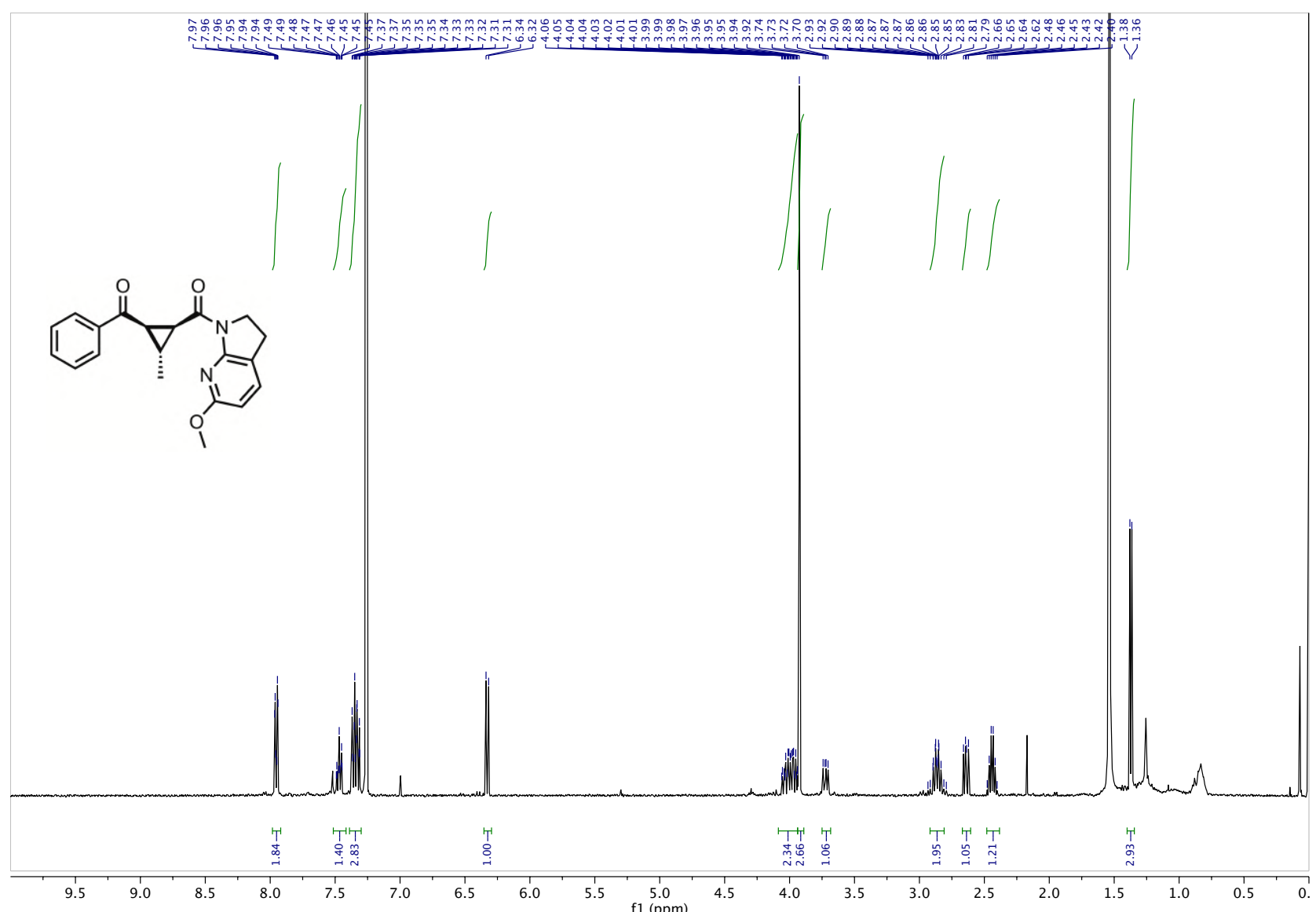

${ }^{13} \mathrm{C}$ NMR: 3aj

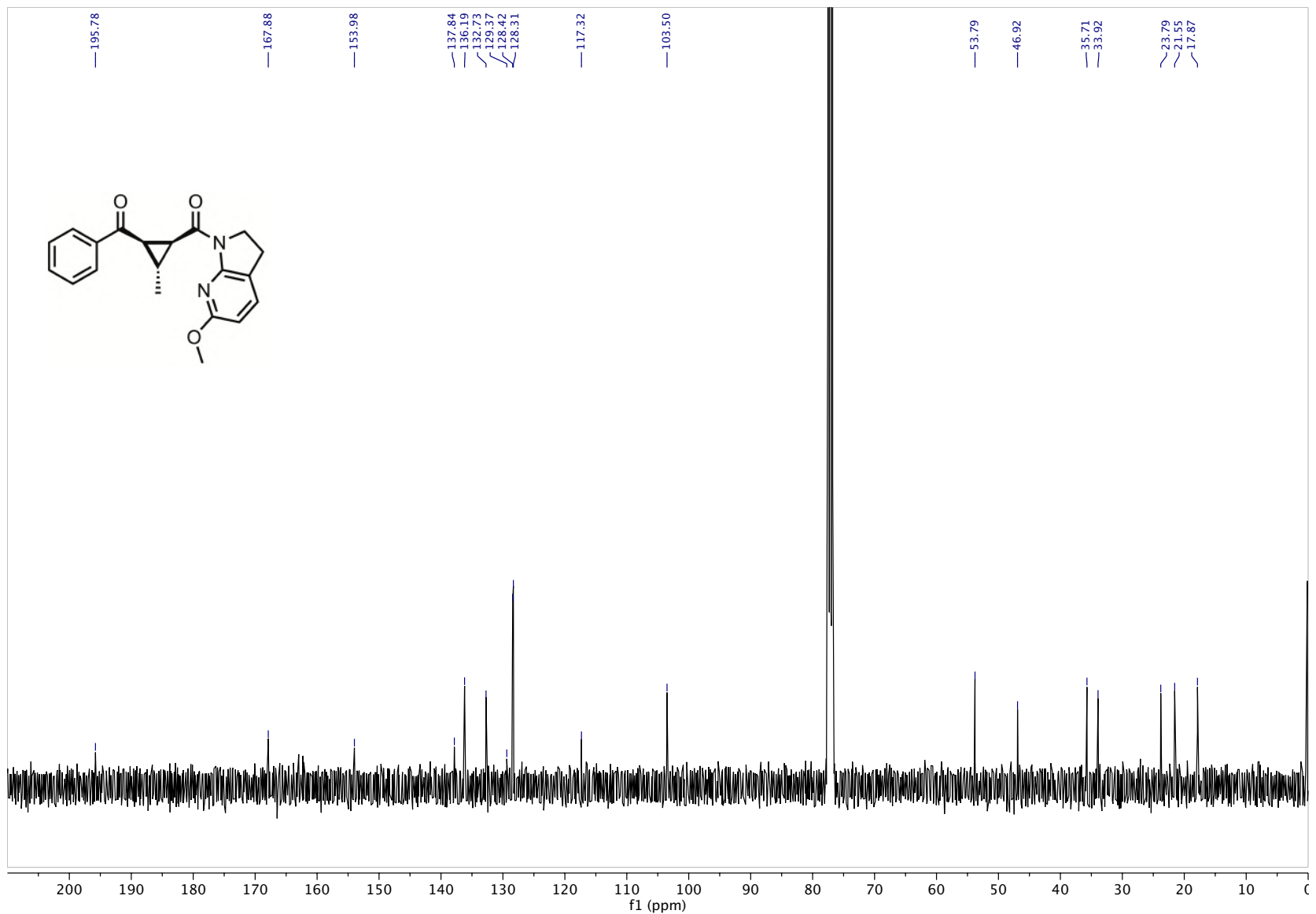


${ }^{1} \mathrm{H}$ NMR: 3al

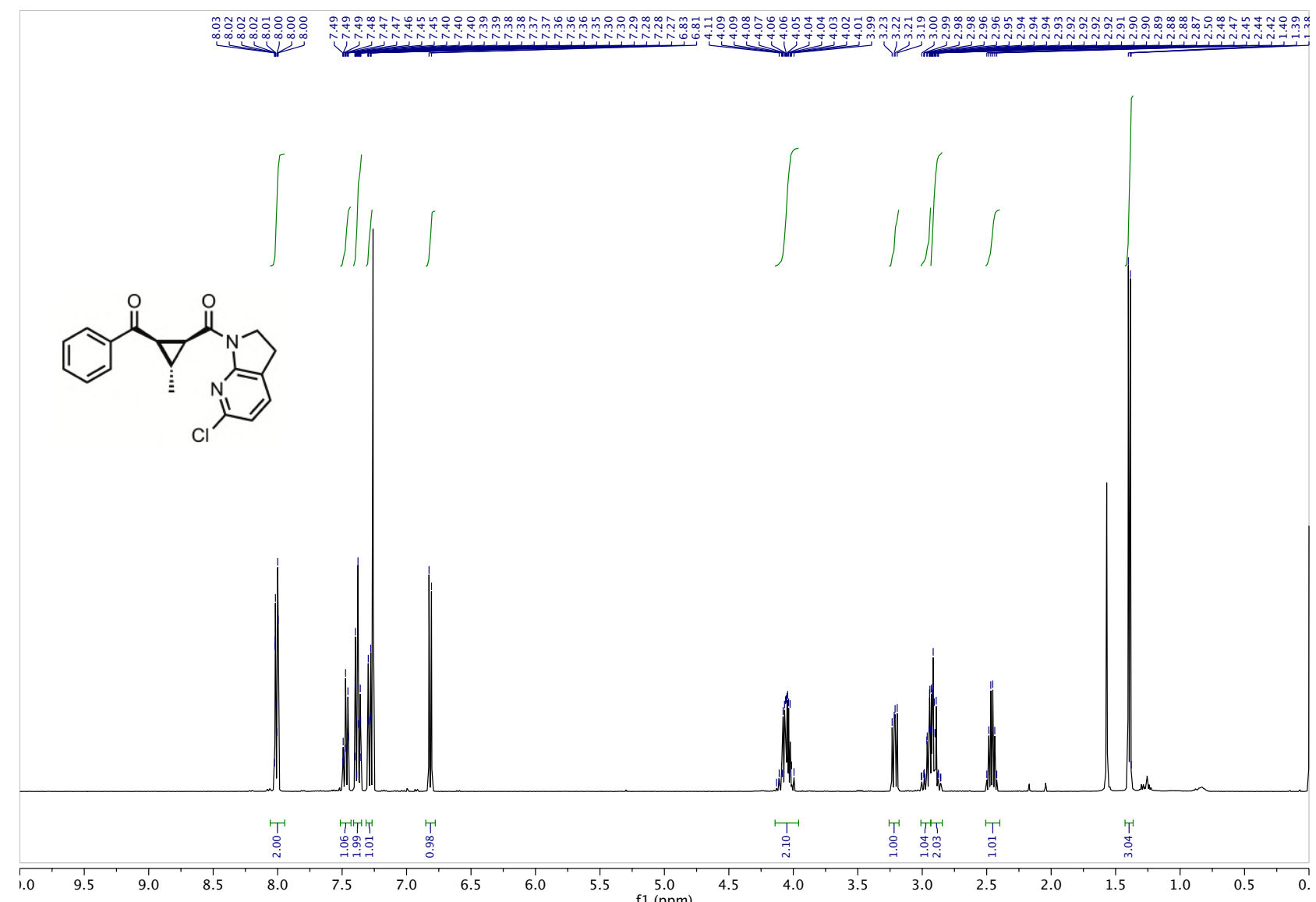

${ }^{13}$ C NMR: 3al

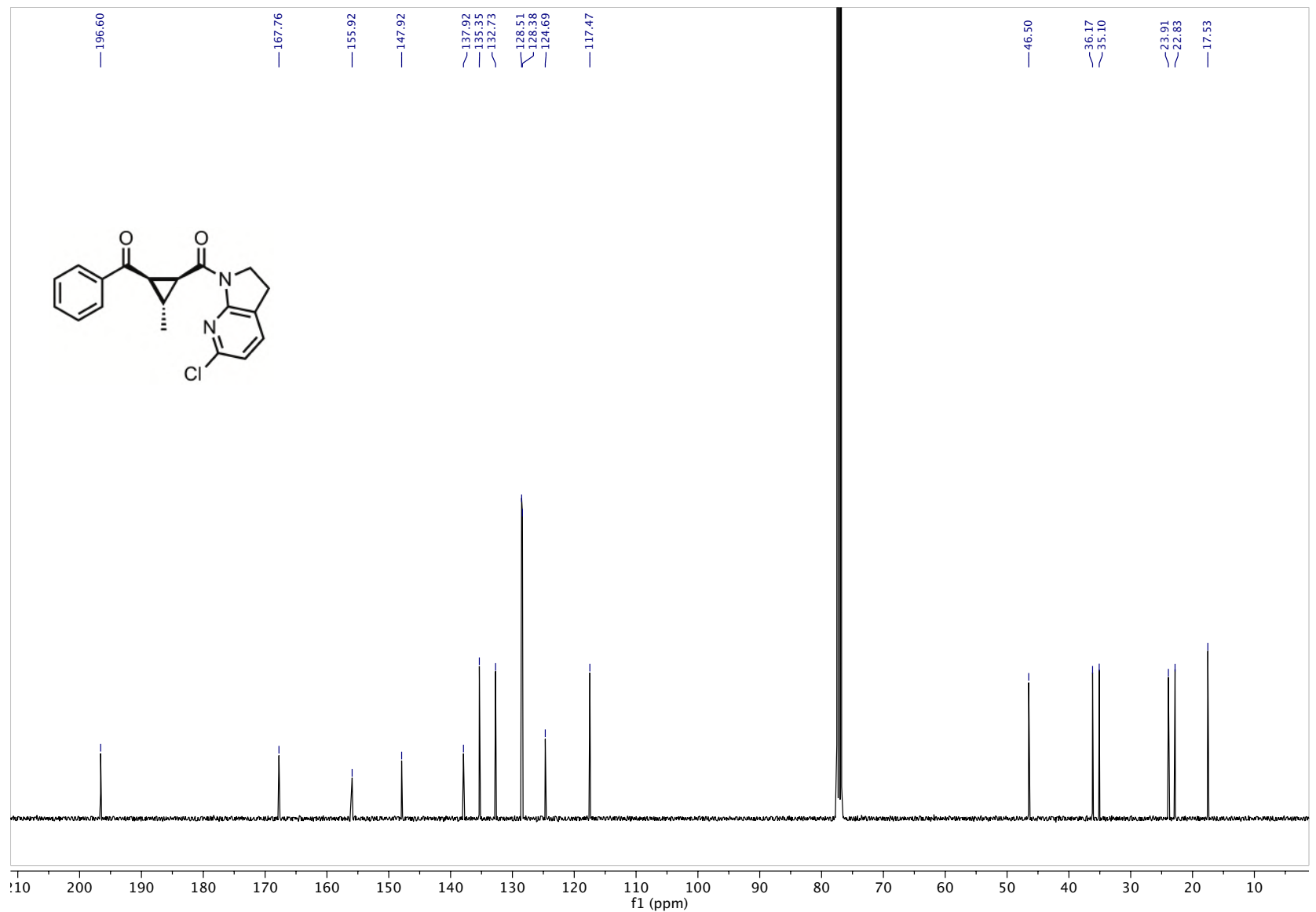


${ }^{1} \mathrm{H}$ NMR: 3am

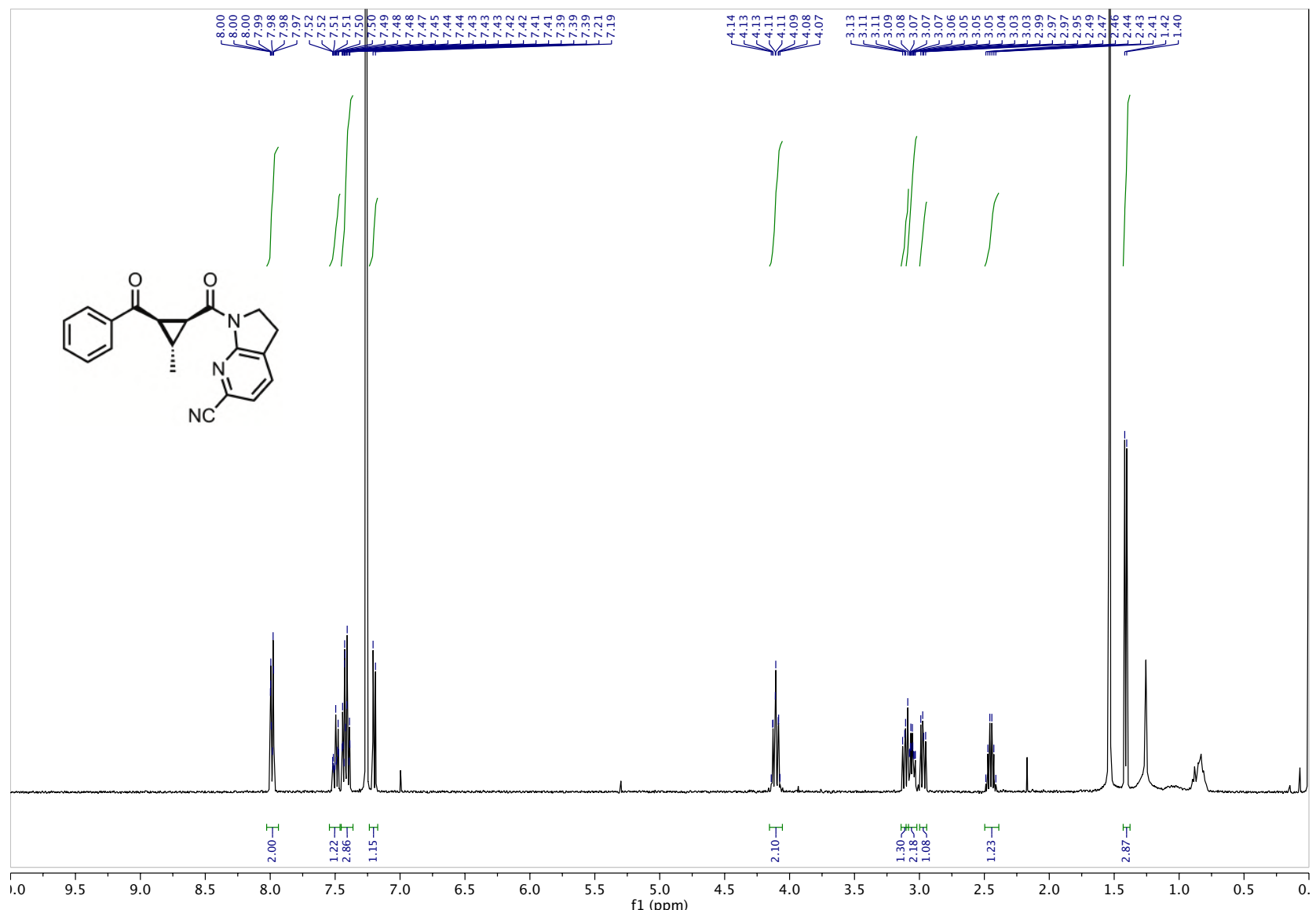

${ }^{13}$ C NMR: 3am

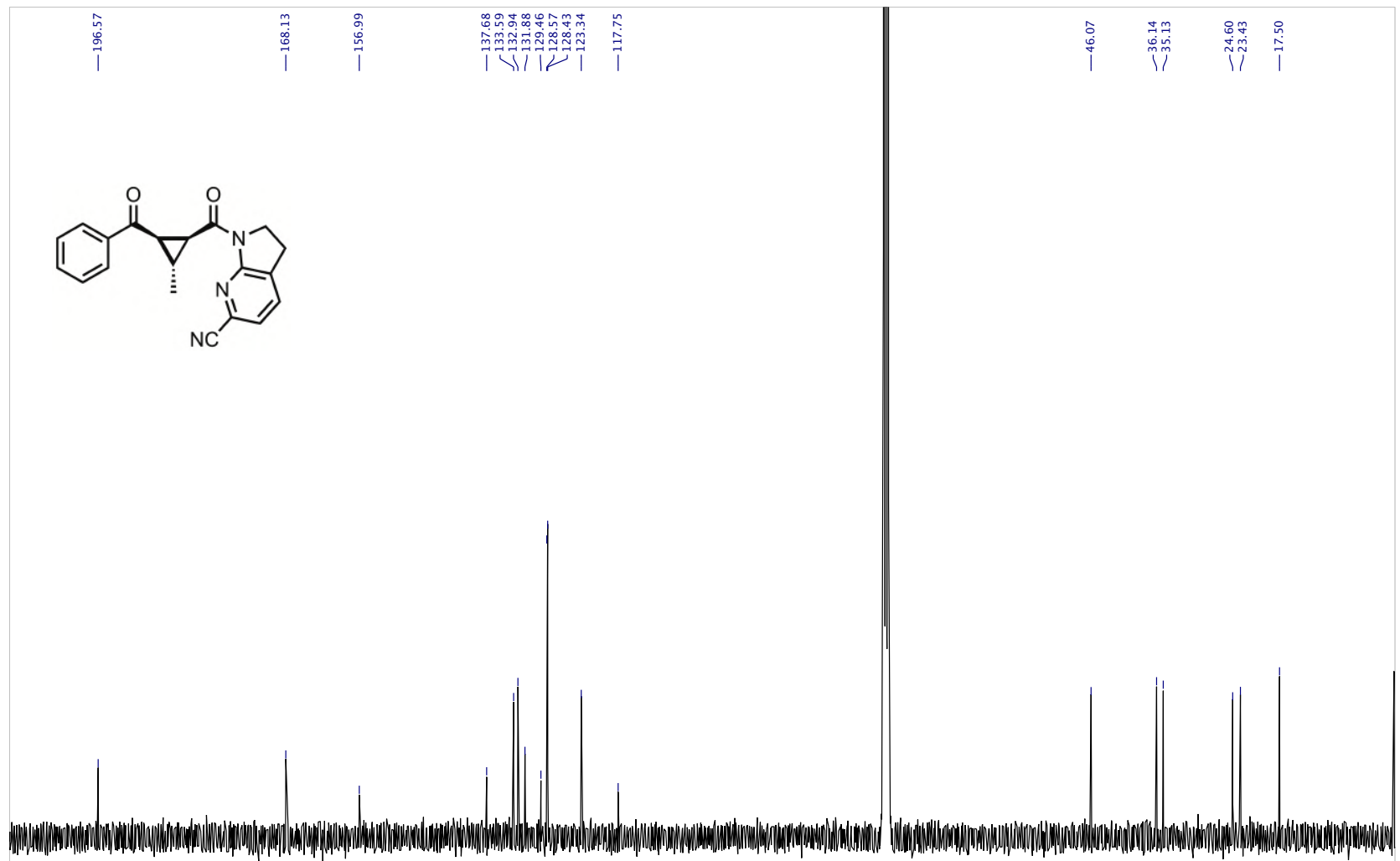

$\begin{array}{lllllllllll}10 & 200 & 190 & 180 & 170 & 160 & 150 & 140 & 130 & 120 & \underset{\mathrm{f} 1(\mathrm{ppm})}{1100}\end{array}$ 
${ }^{1} \mathrm{H}$ NMR: 3an

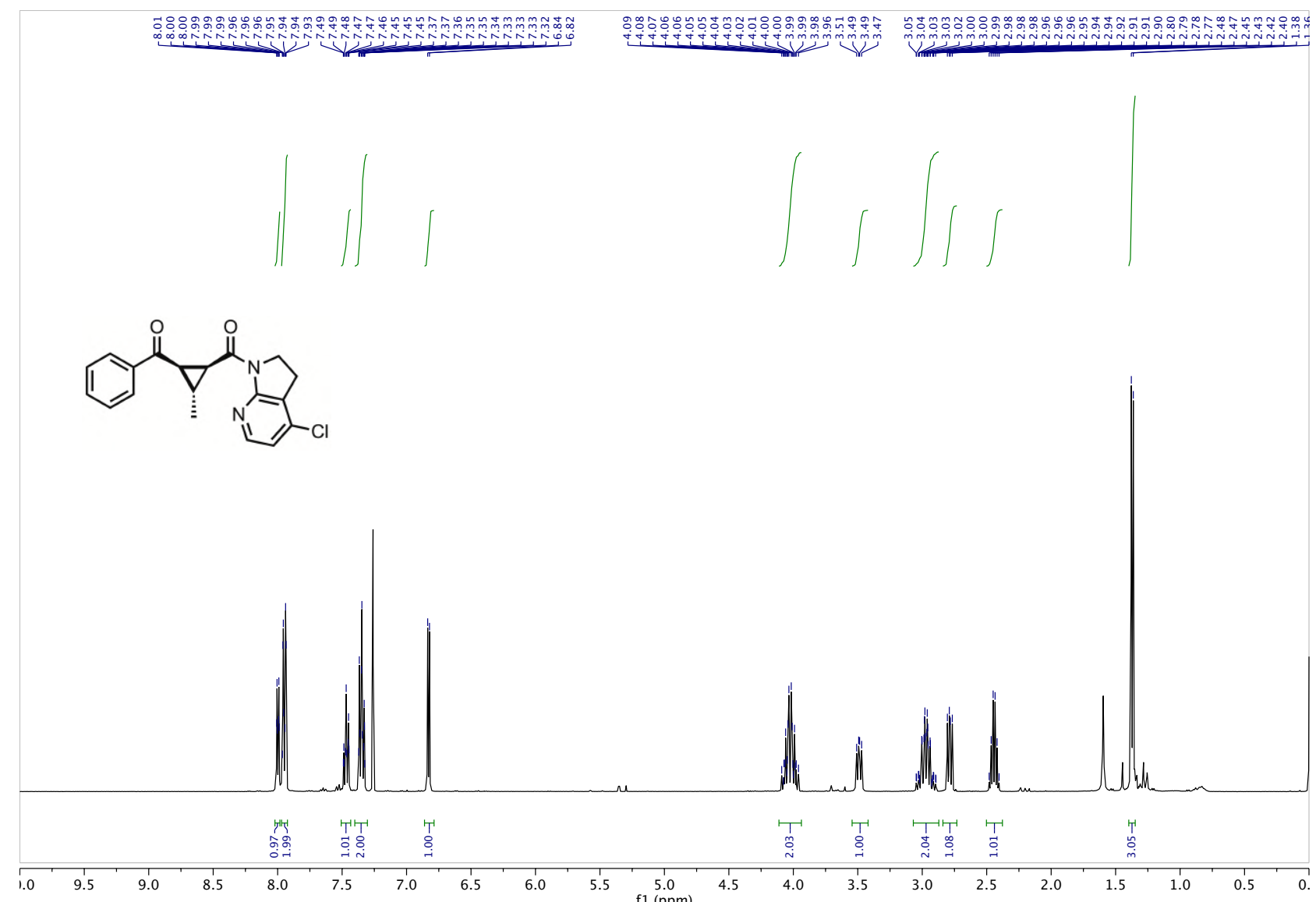

${ }^{13} \mathrm{C}$ NMR: 3an

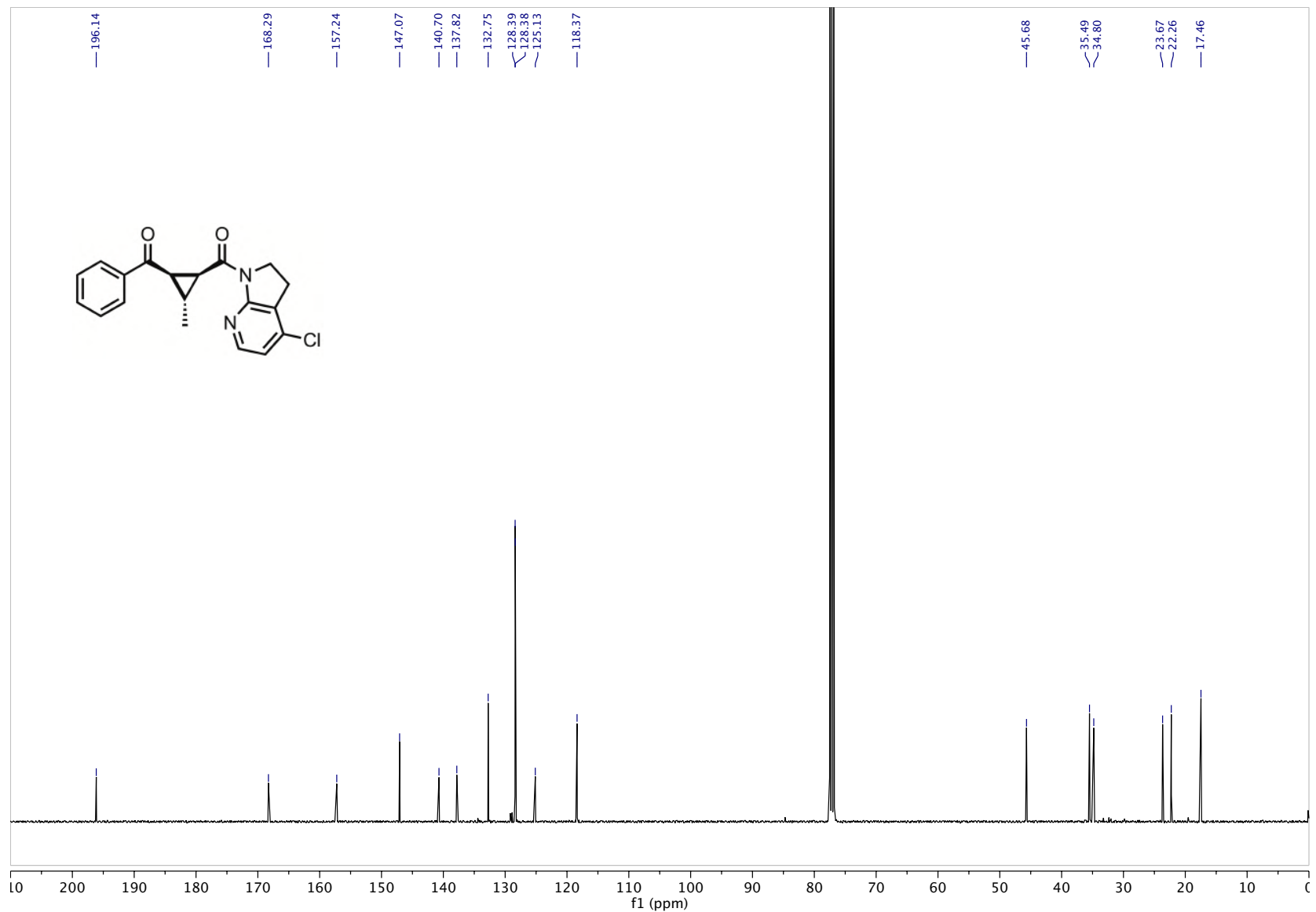


Supporting Information

${ }^{1} \mathrm{H}$ NMR: 3 oo

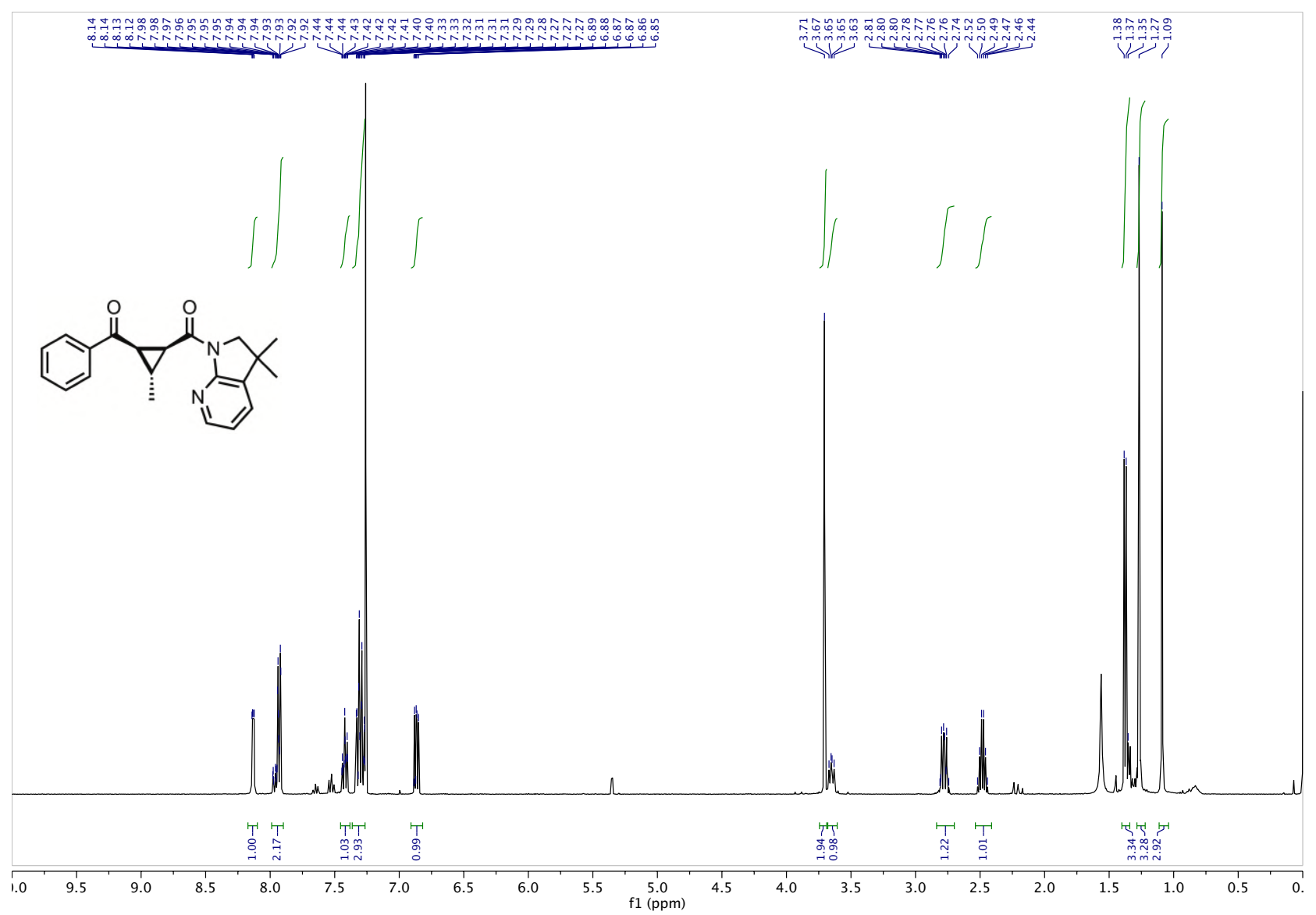

${ }^{13} \mathrm{C}$ NMR: 3 as

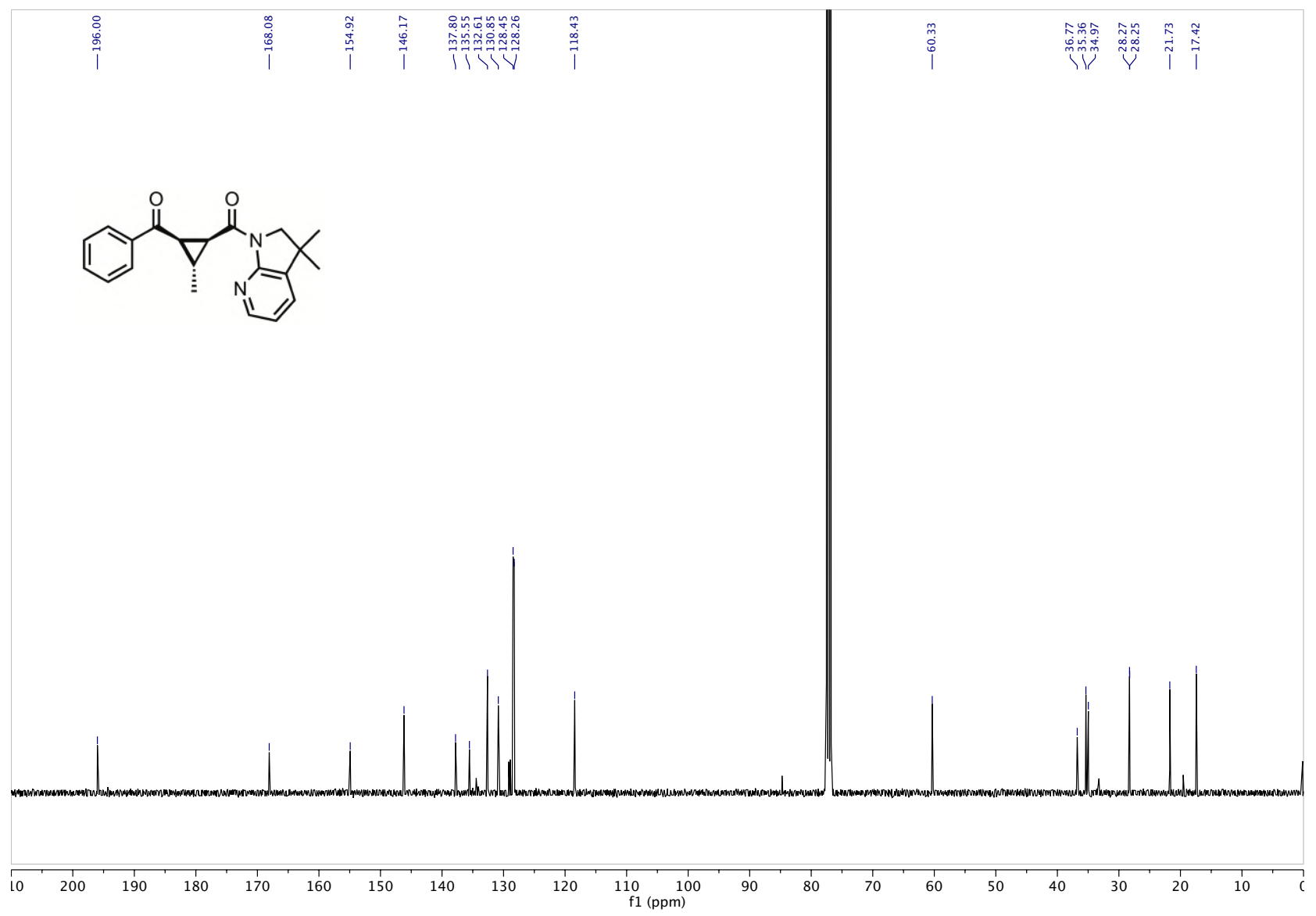

S138 
${ }^{1} \mathrm{H}$ NMR: 4ap

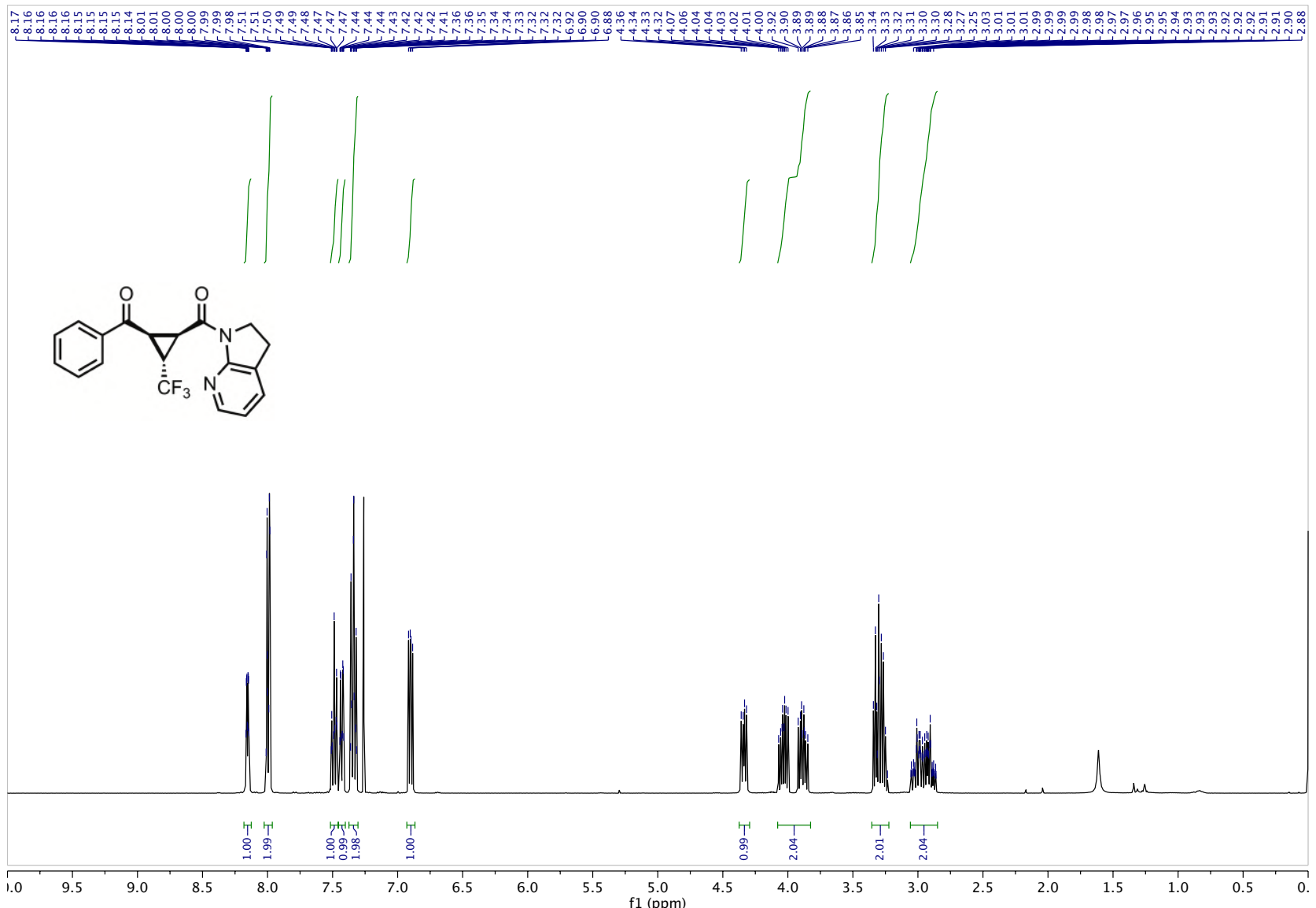

${ }^{13} \mathrm{C}$ NMR: 4ap

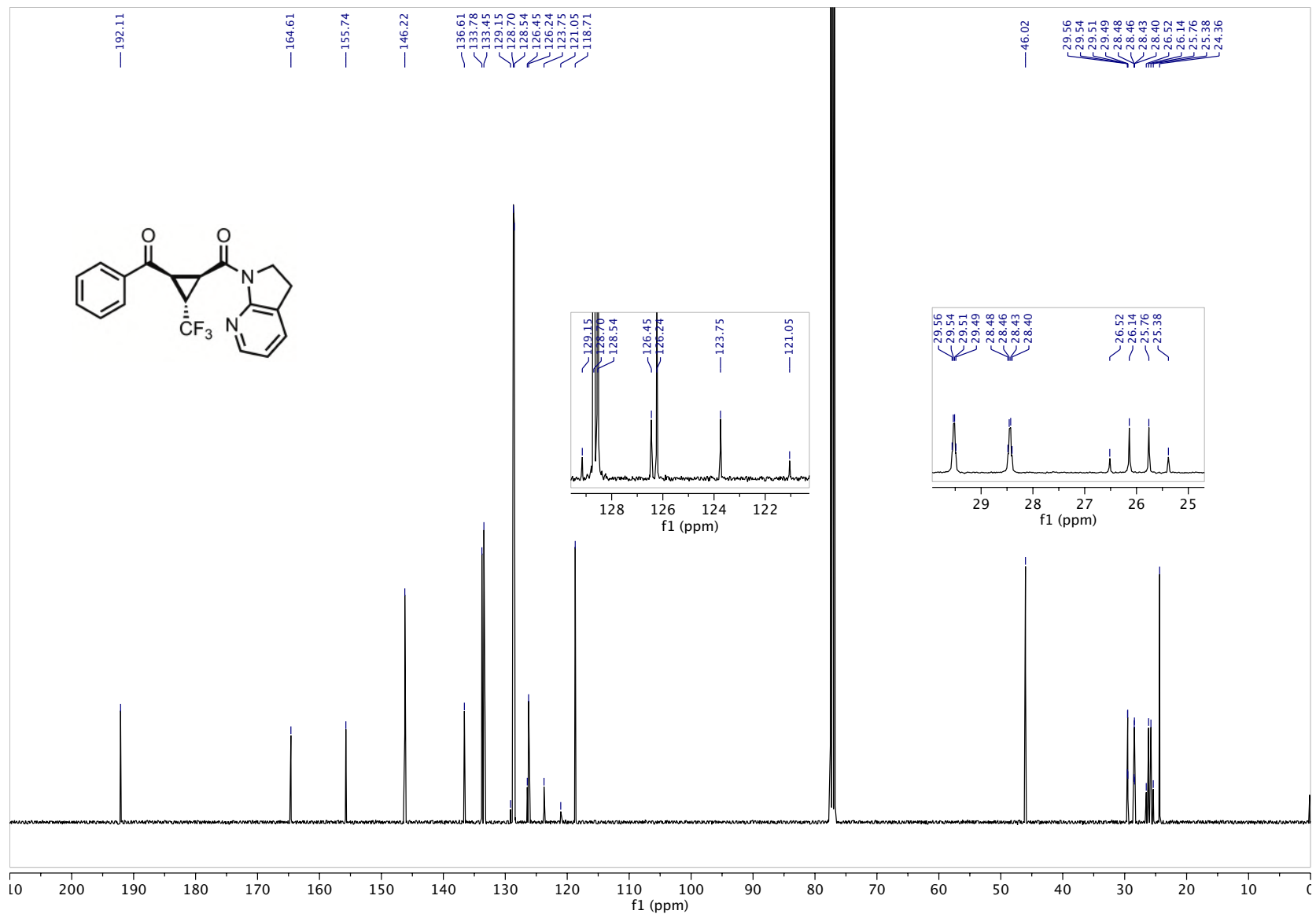


${ }^{19}$ F NMR: 4ap

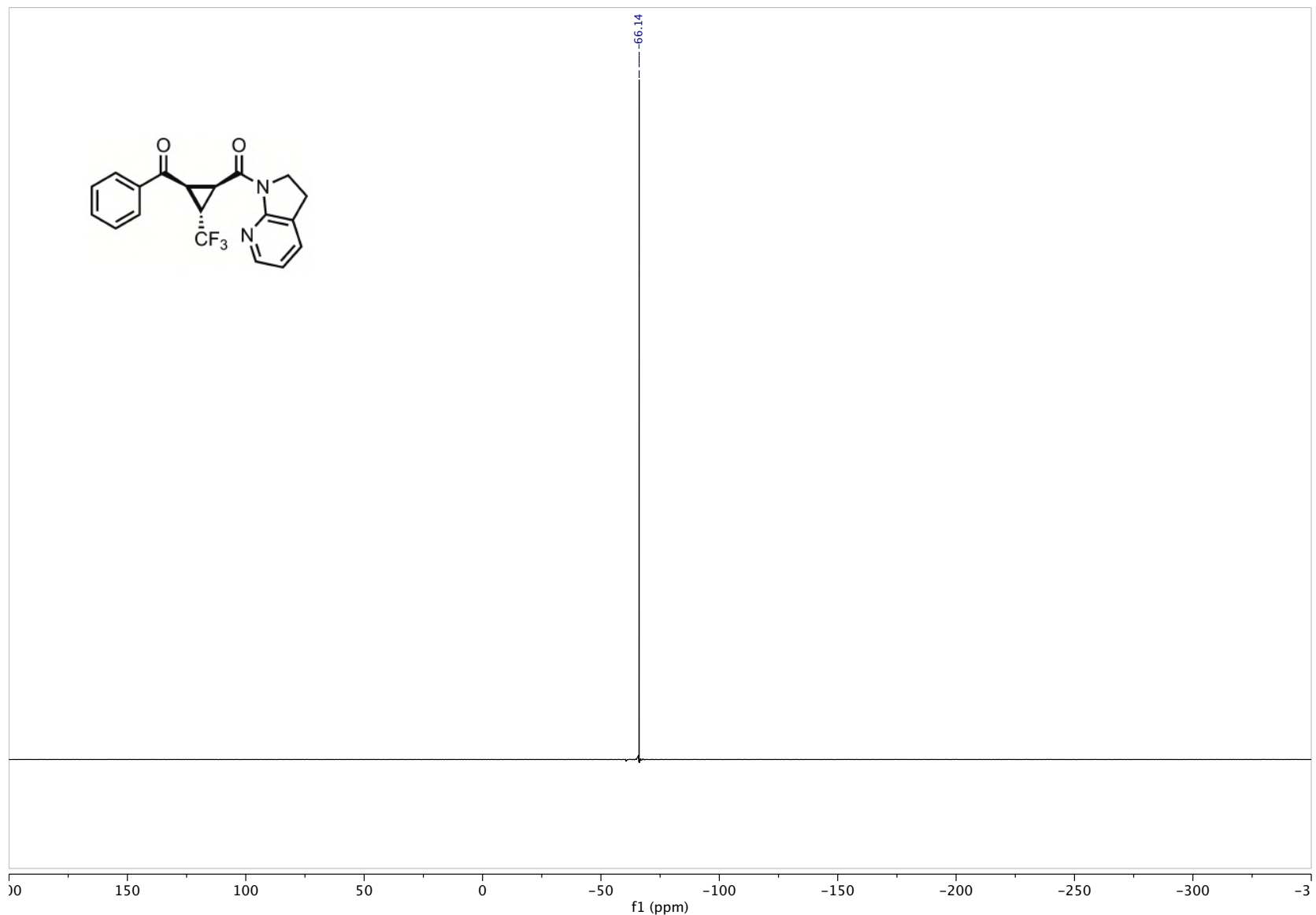


${ }^{1} \mathrm{H}$ NMR: 4ap' (Minor diastereomer)

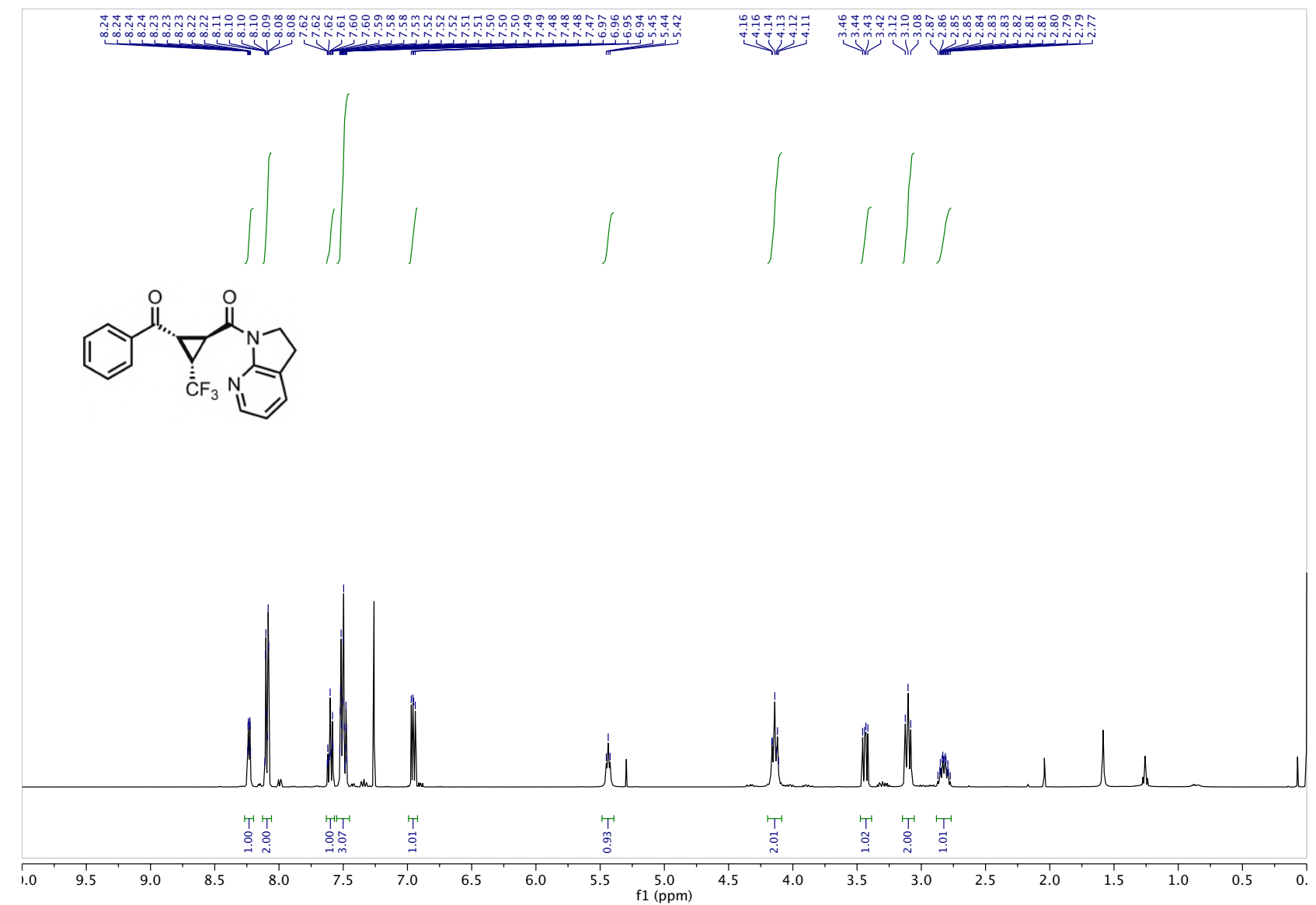

${ }^{13} \mathrm{C}$ NMR: 4ap' (Minor diastereomer)

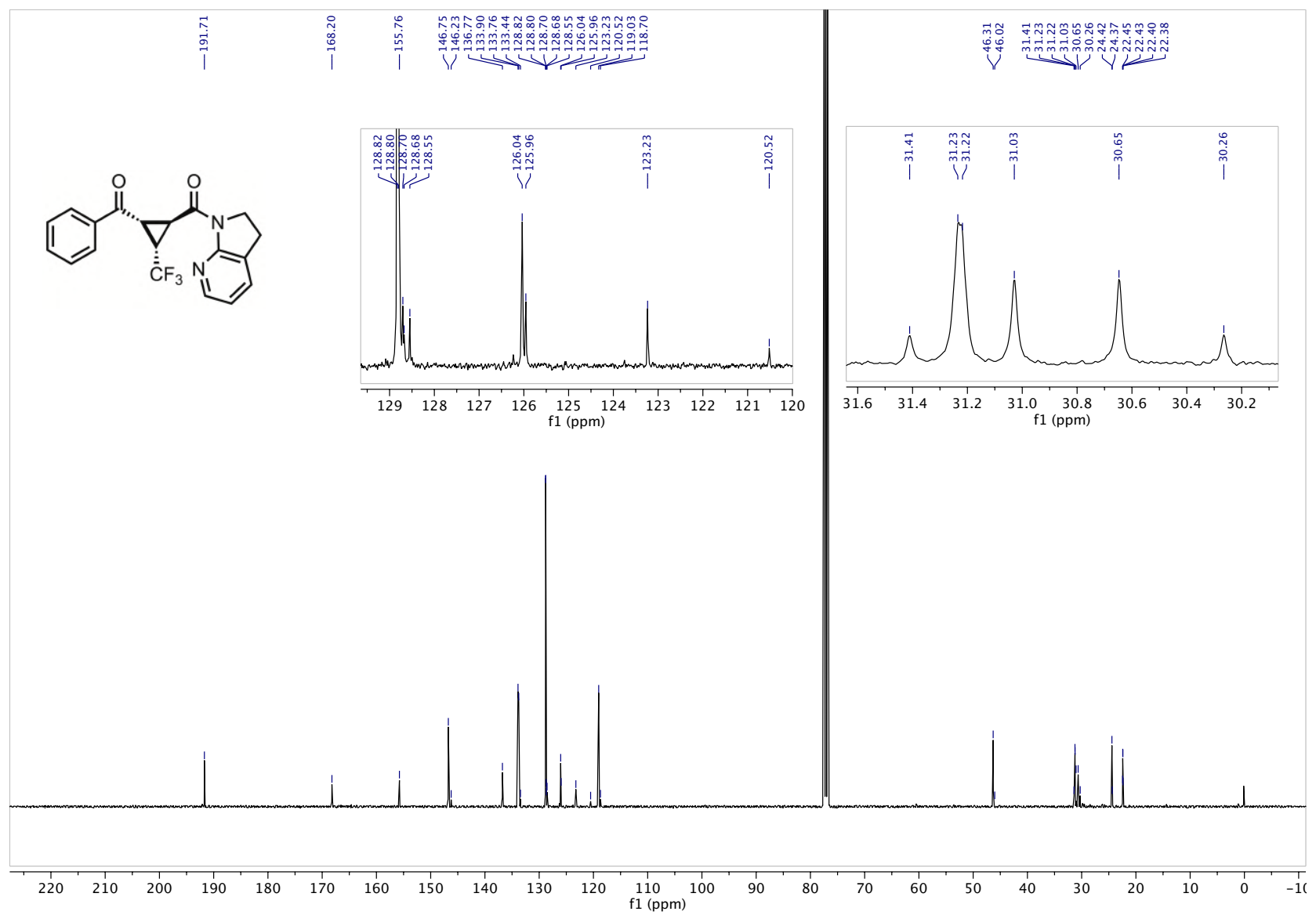


${ }^{19}$ F NMR: 4ap' (Minor diastereomer)

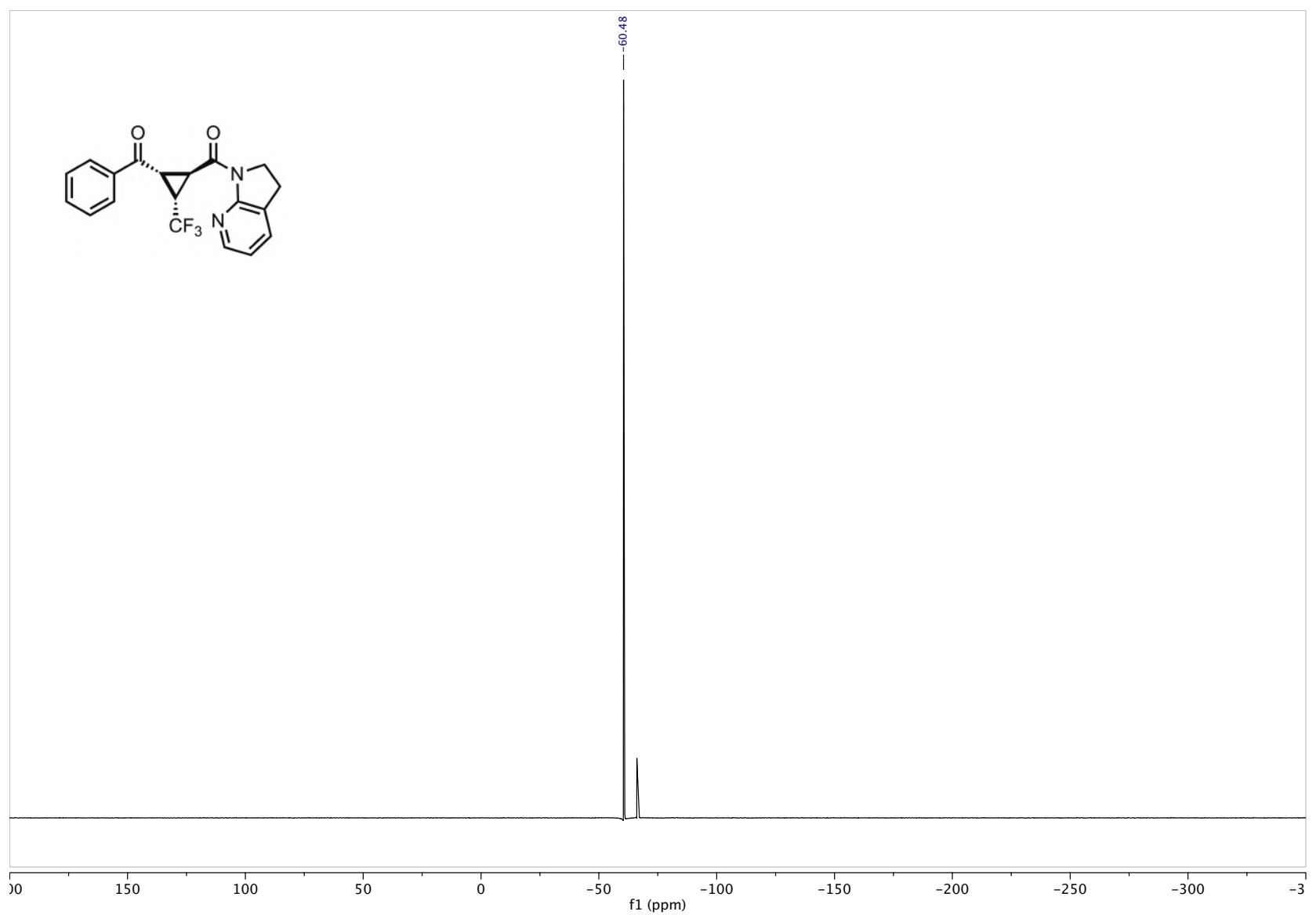


${ }^{1} \mathrm{H}$ NMR: $4 \mathrm{bp}$

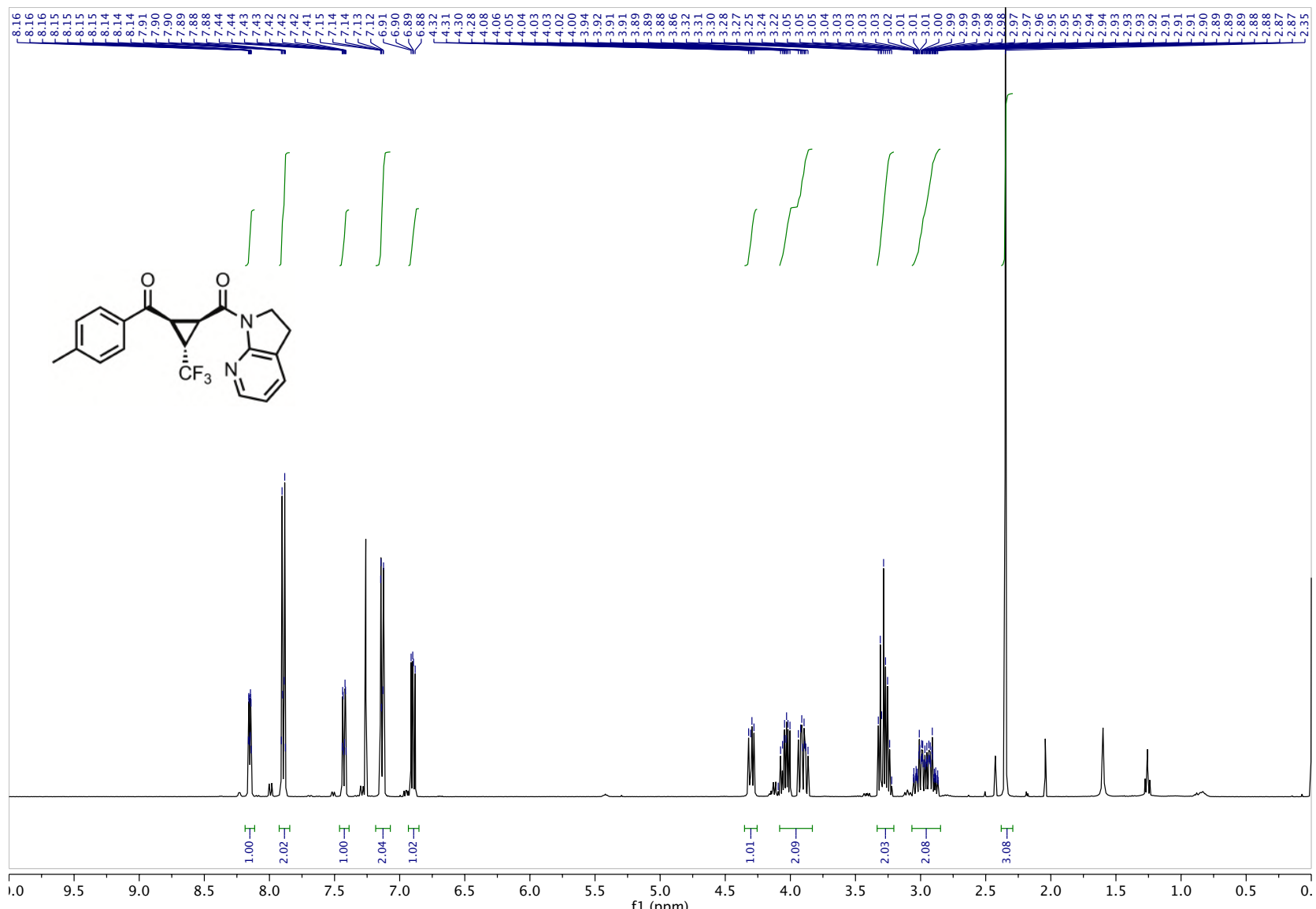

${ }^{13} \mathrm{C}$ NMR: $4 \mathrm{bp}$

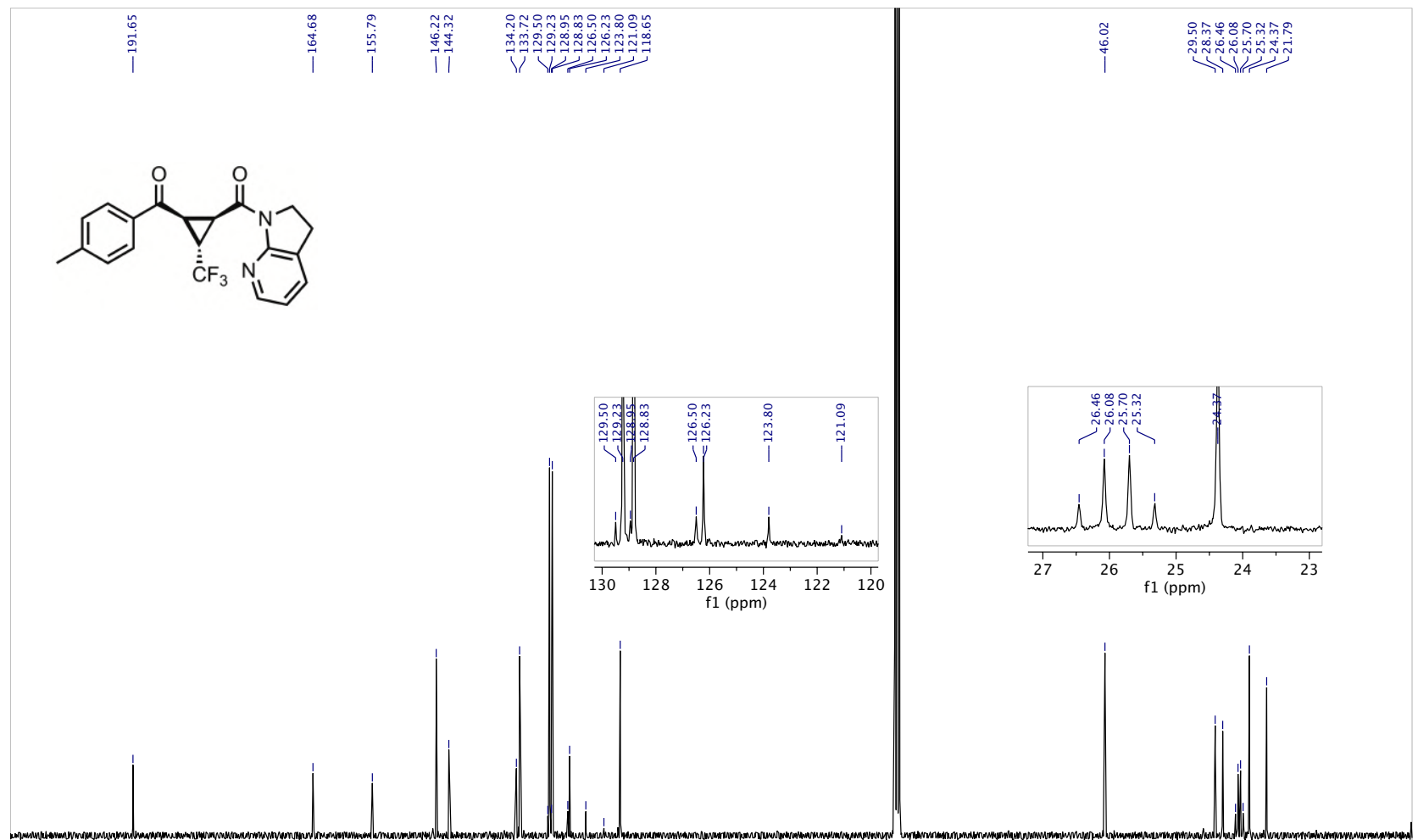

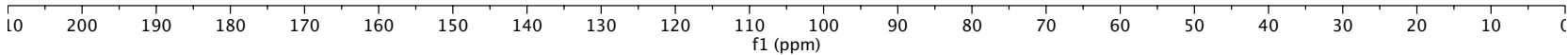


${ }^{19}$ F NMR: 4bp

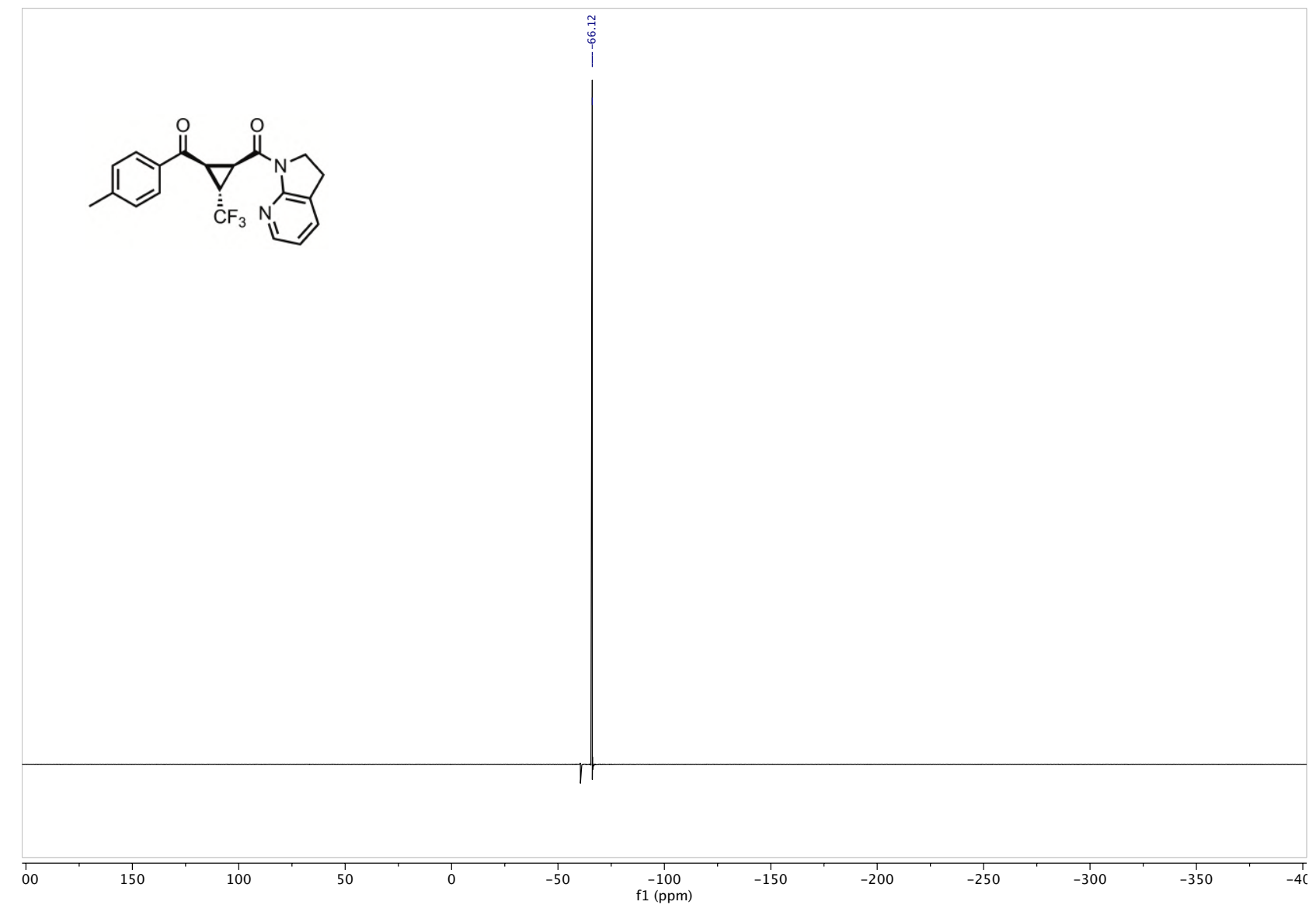


${ }^{1} \mathrm{H}$ NMR: 4cp

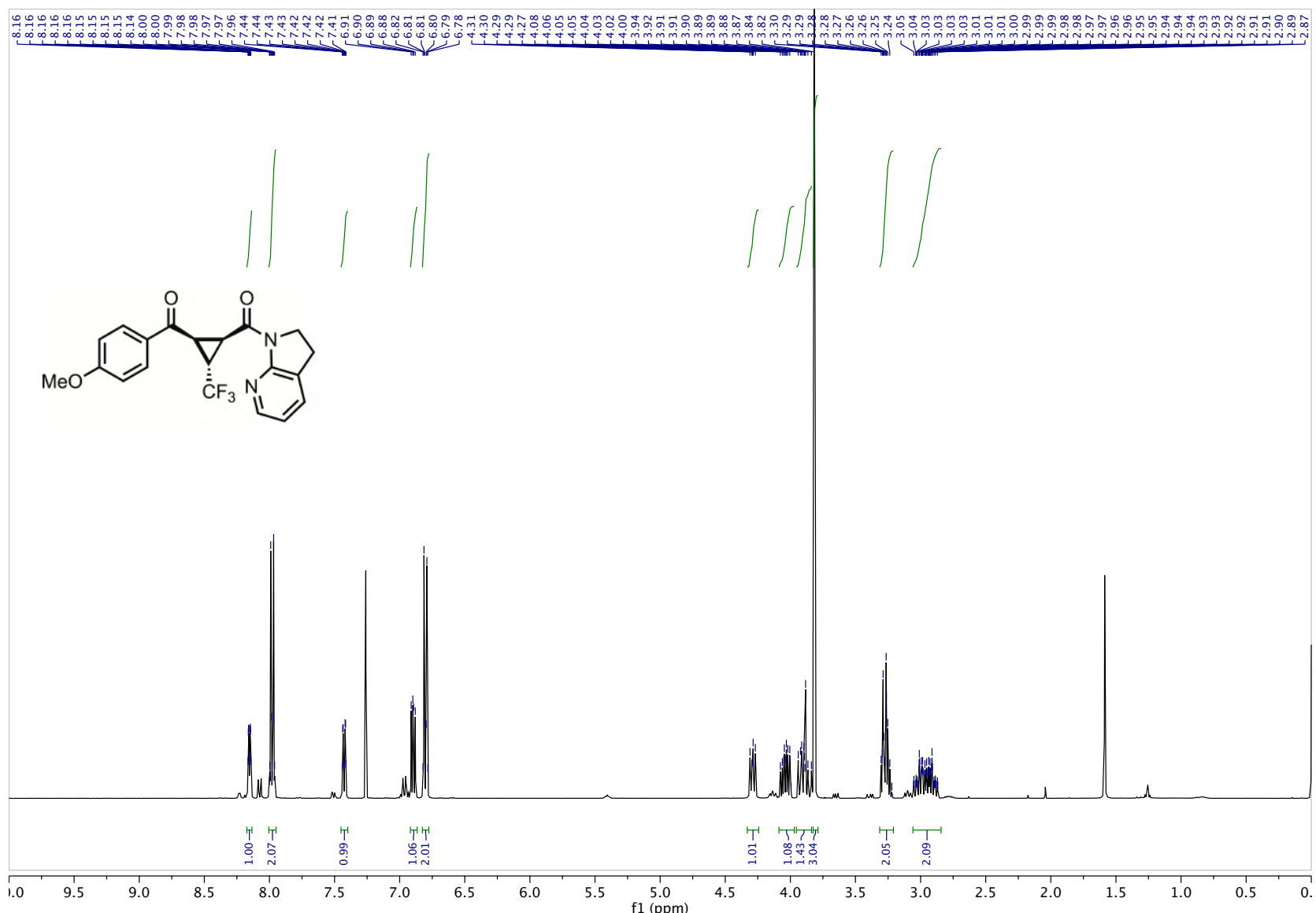

${ }^{13} \mathrm{C}$ NMR: $4 \mathrm{cp}$

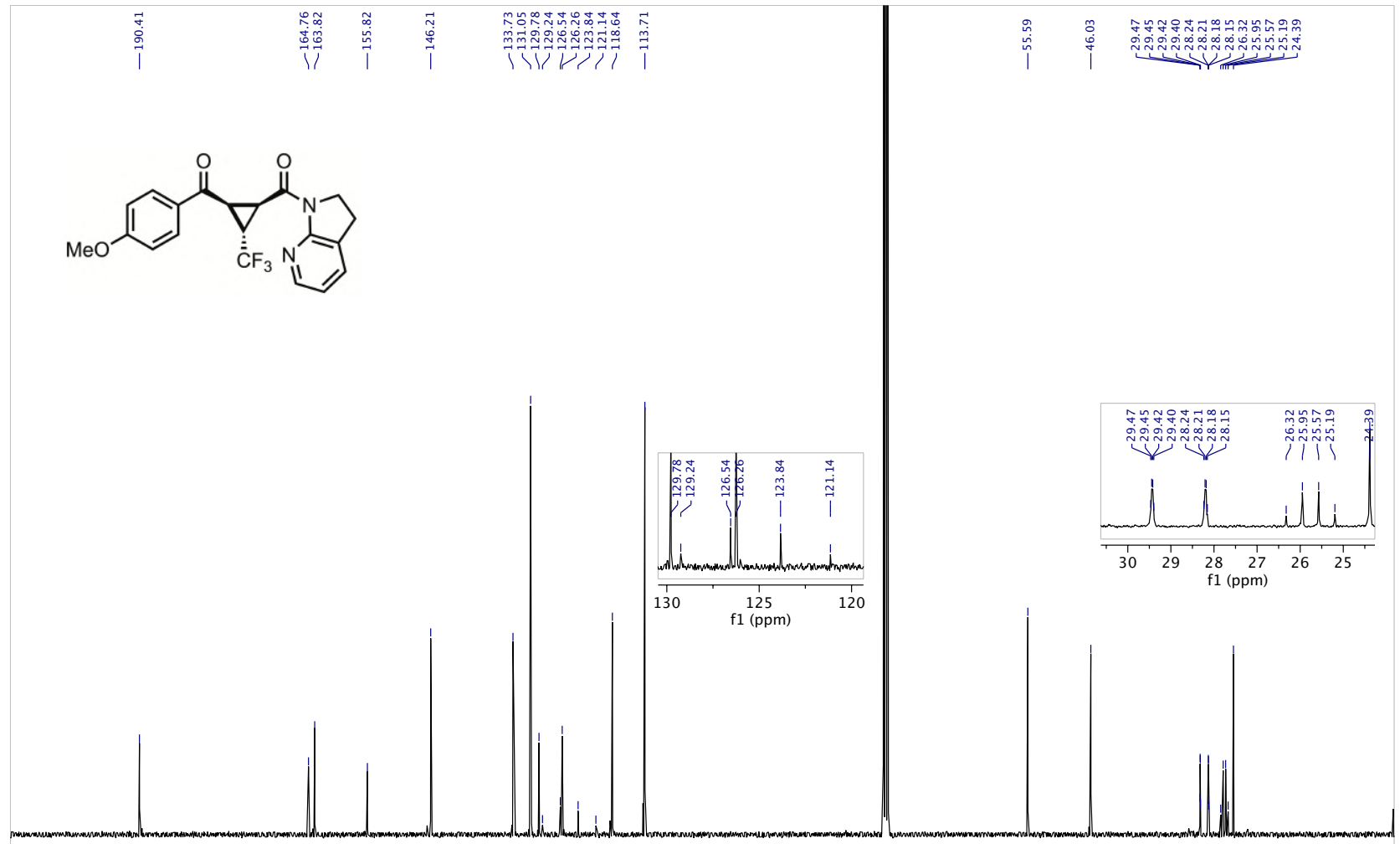

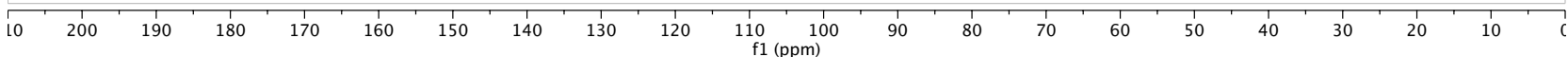


${ }^{19}$ F NMR: 4cp

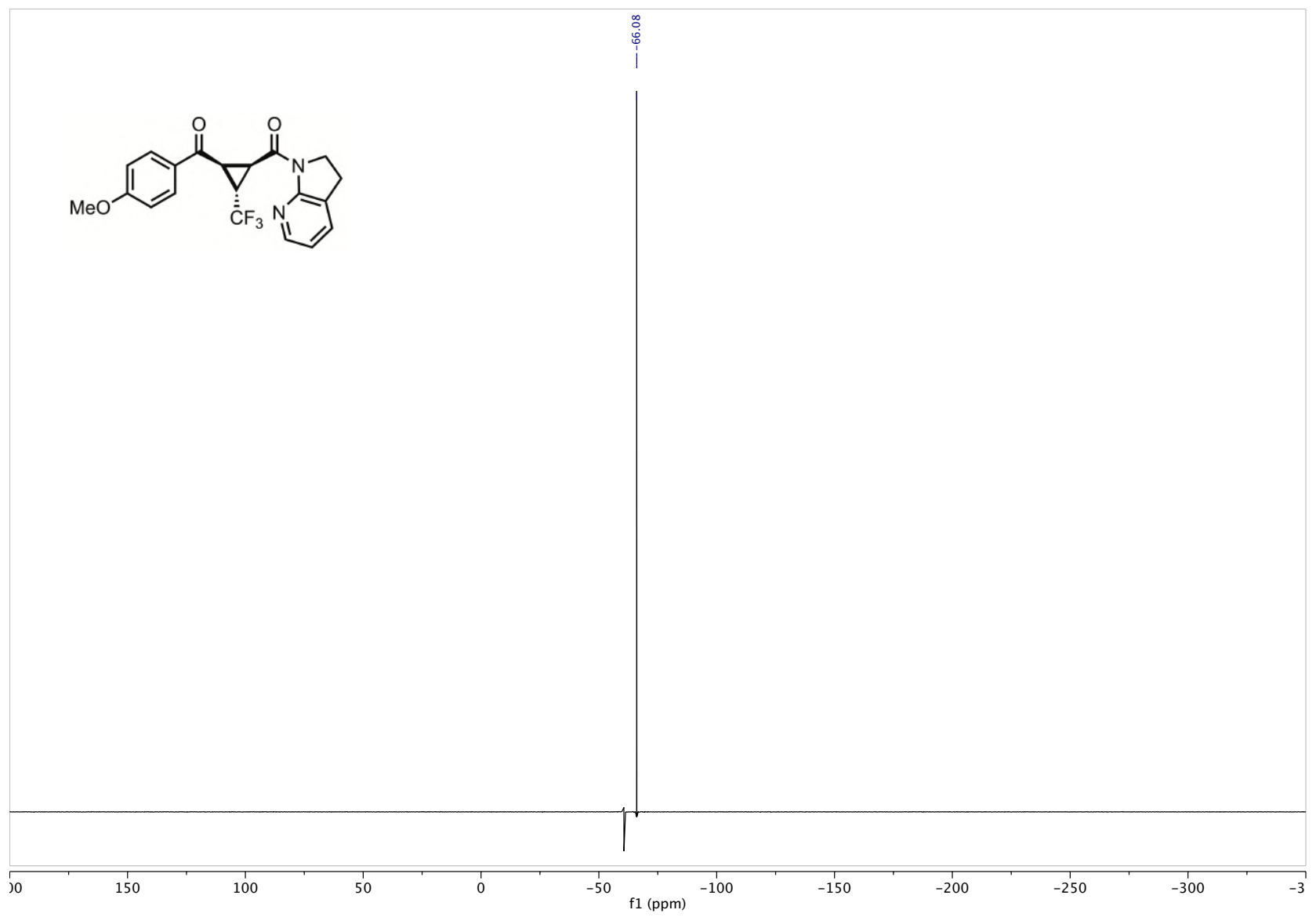


${ }^{1} \mathrm{H}$ NMR: 4dp

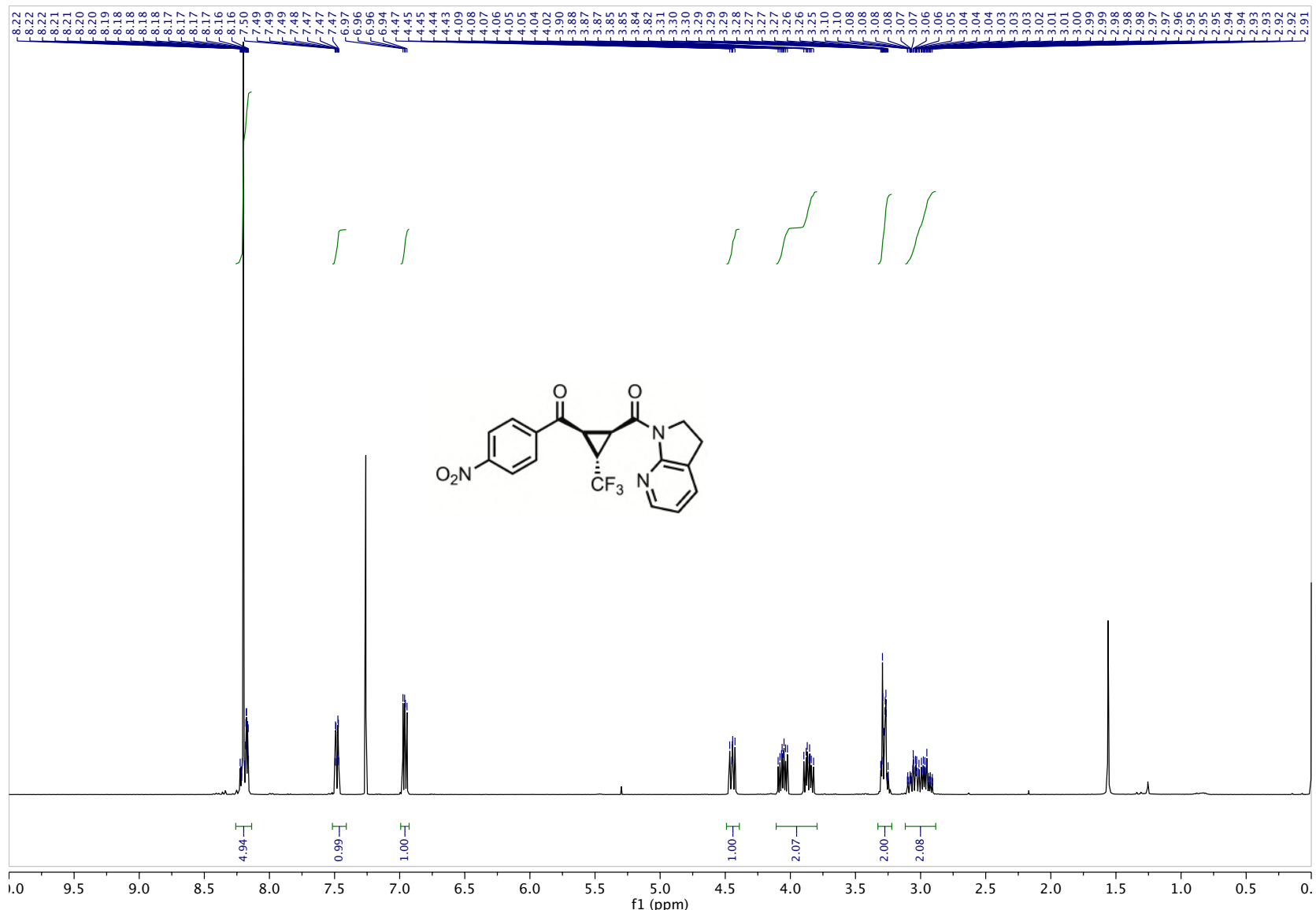

${ }^{13}$ C NMR: 4dp

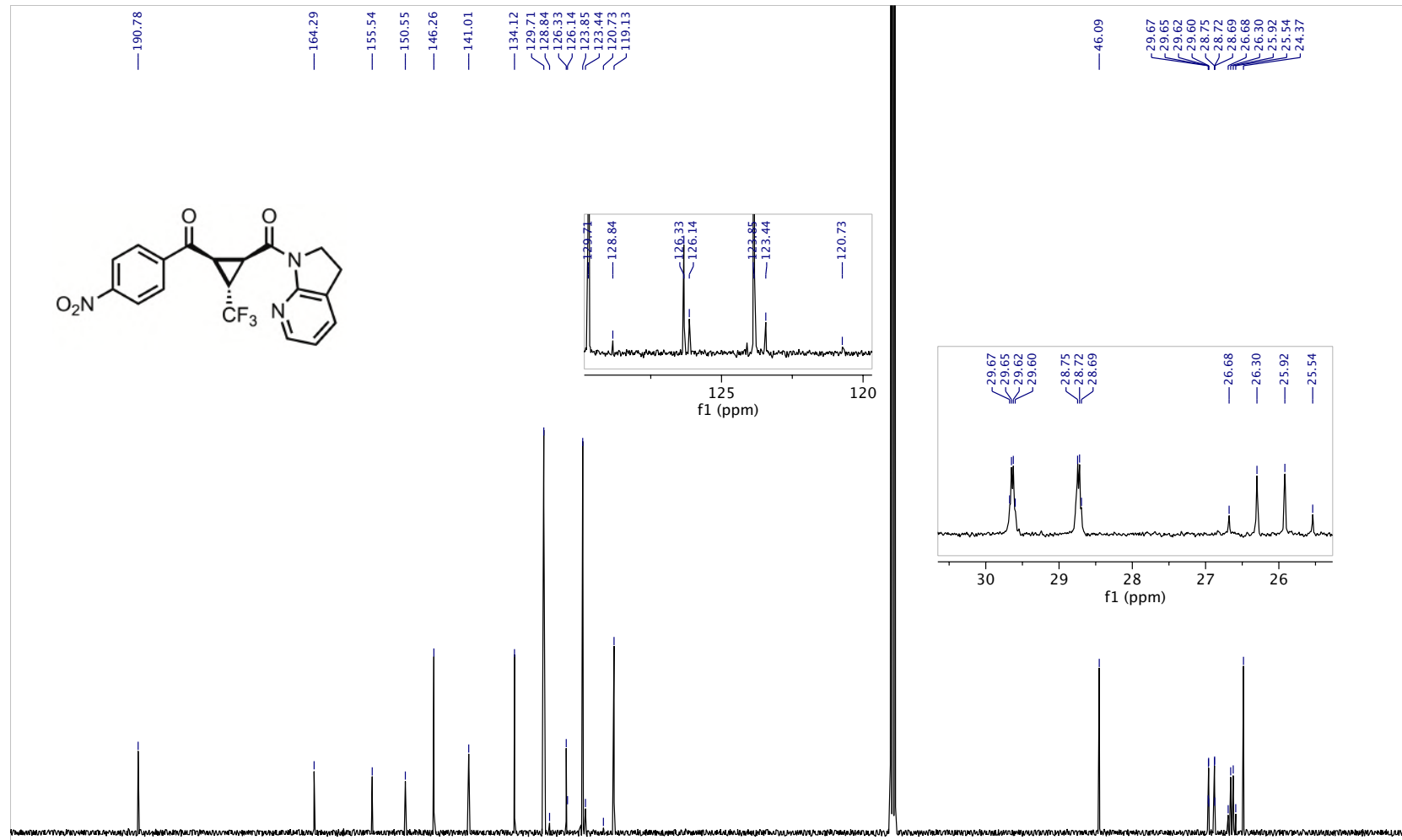

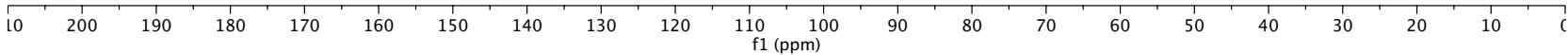




\section{${ }^{19} \mathrm{~F}$ NMR: 4dp}

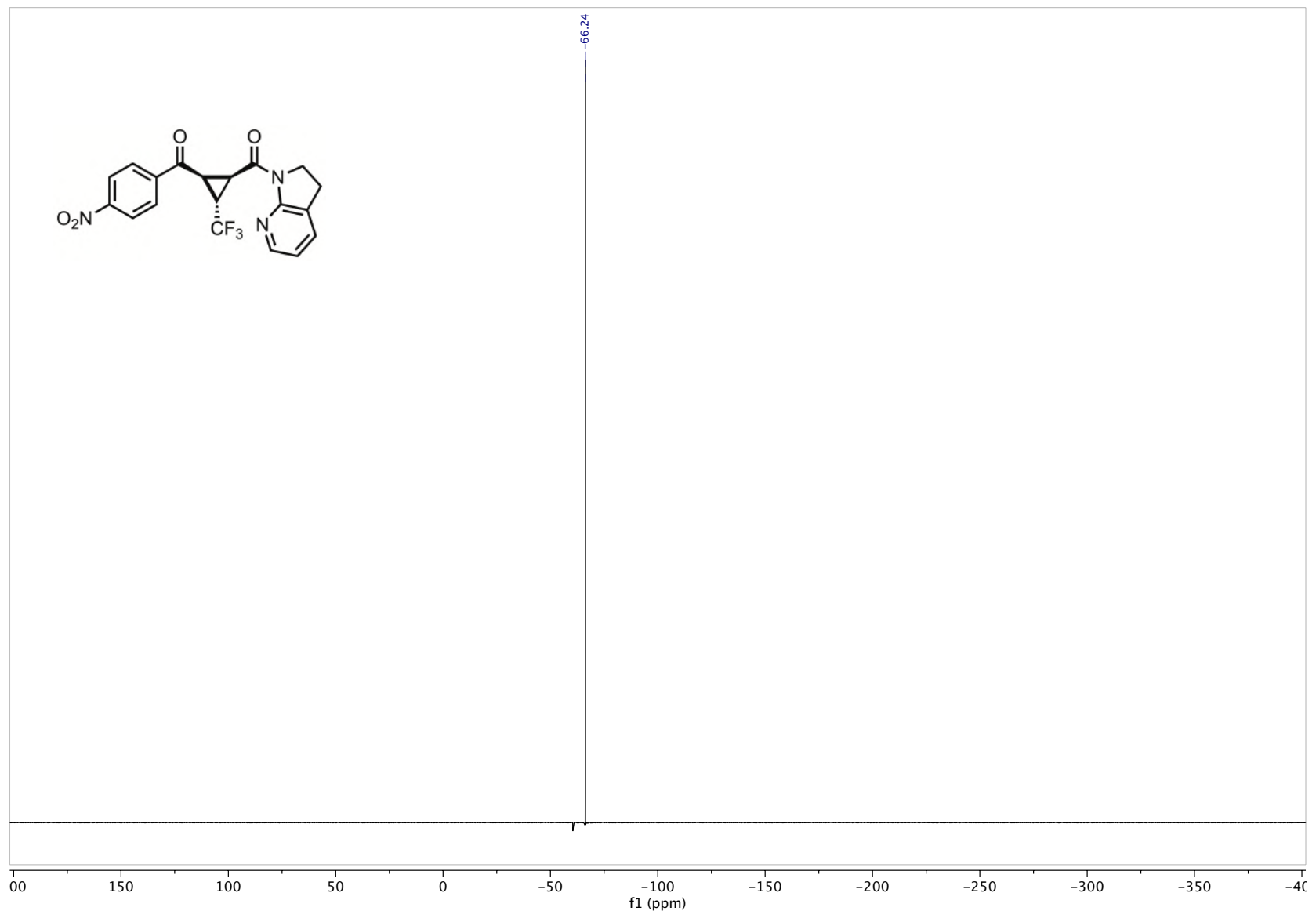


${ }^{1} \mathrm{H}$ NMR: 4ep

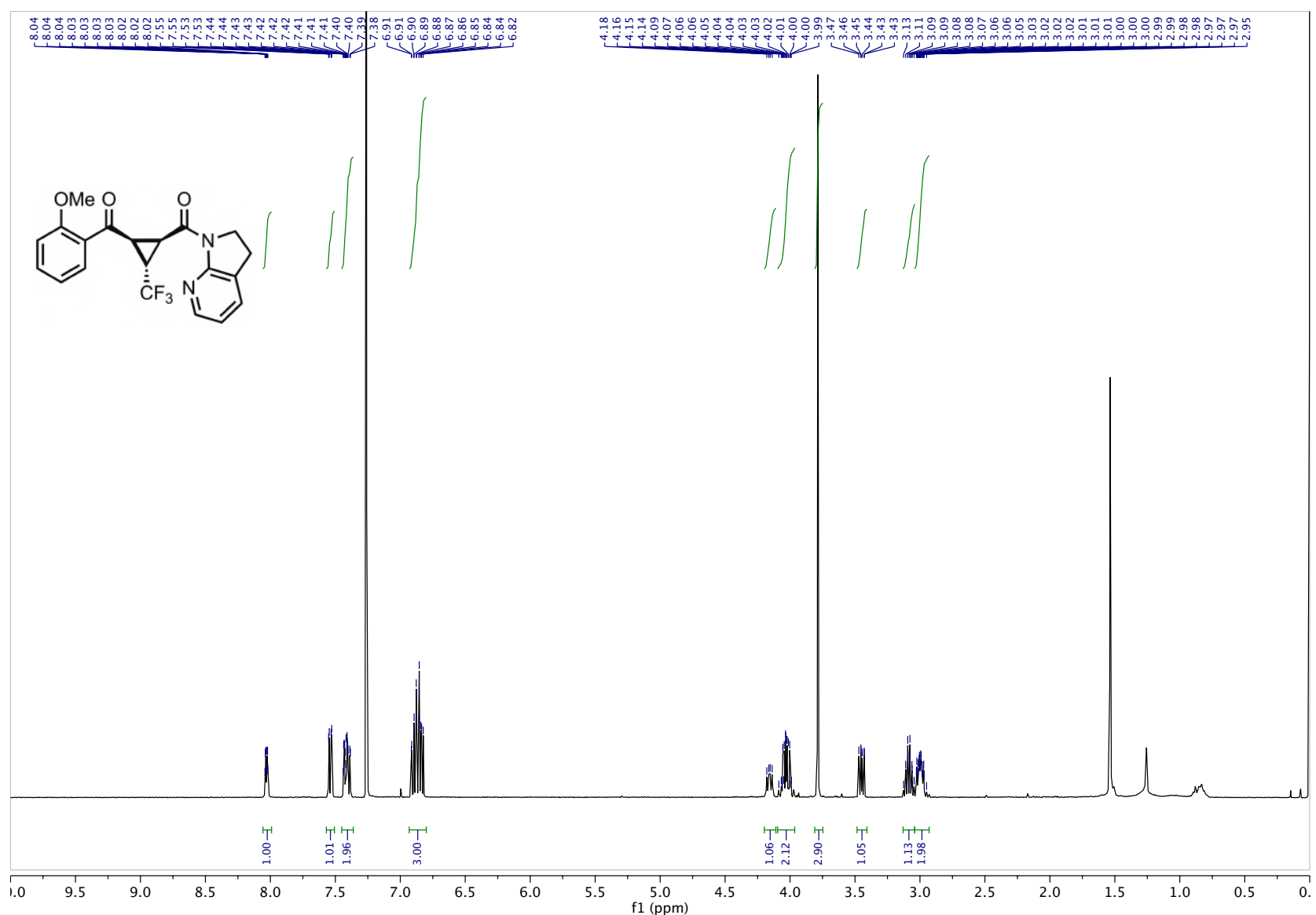

${ }^{13}$ C NMR: 4ep

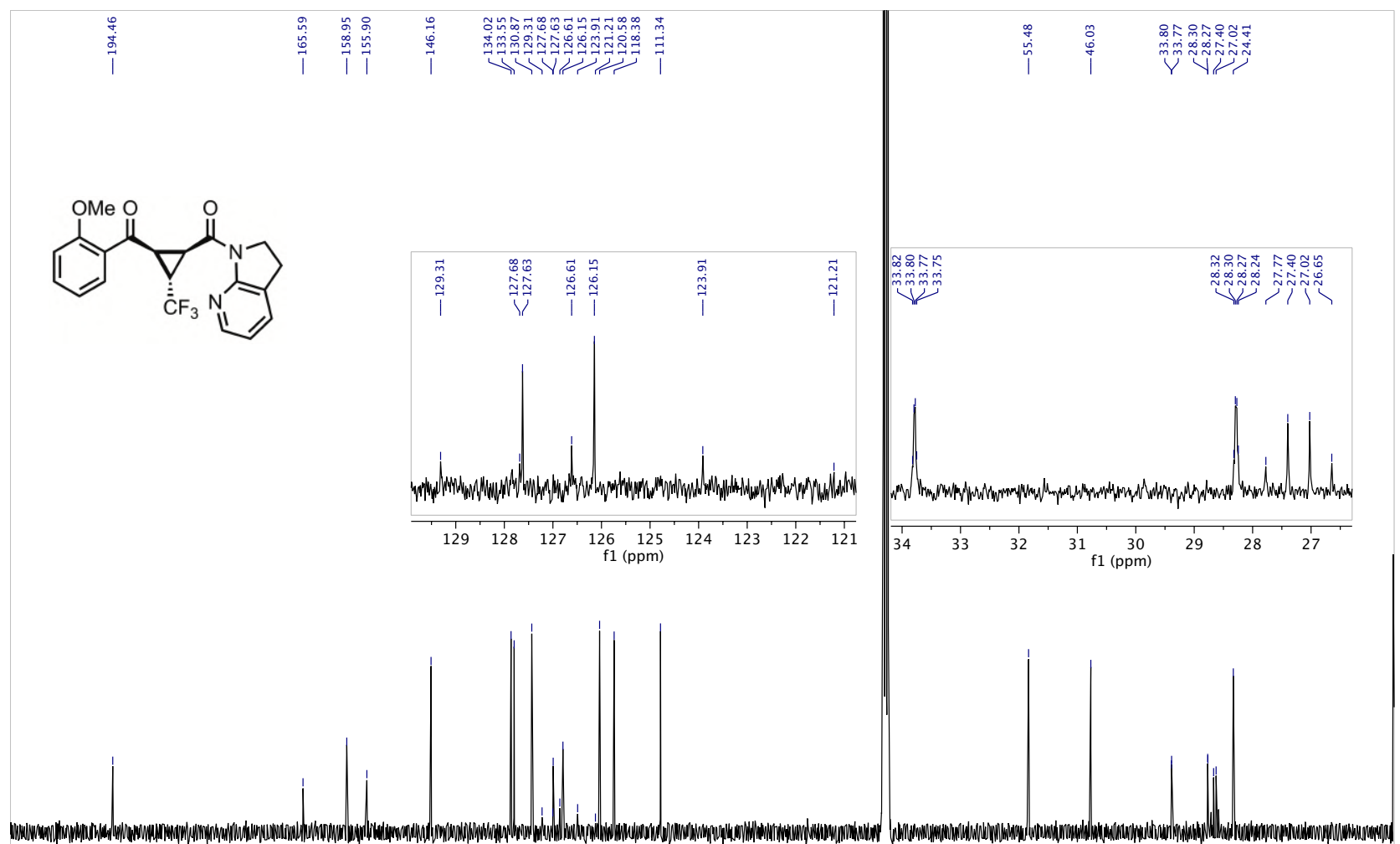

\begin{tabular}{|l|llllllllllllllllllllll}
\hline 10 & 200 & 190 & 180 & 170 & 160 & 150 & 140 & 130 & 120 & $\underset{\mathrm{f} 1(\mathrm{ppm})}{100}$ & 90 & 80 & 70 & 60 & 50 & 40 & 30 & 20 & 10 & 1
\end{tabular} 
${ }^{19} \mathrm{~F}$ NMR: 4ep

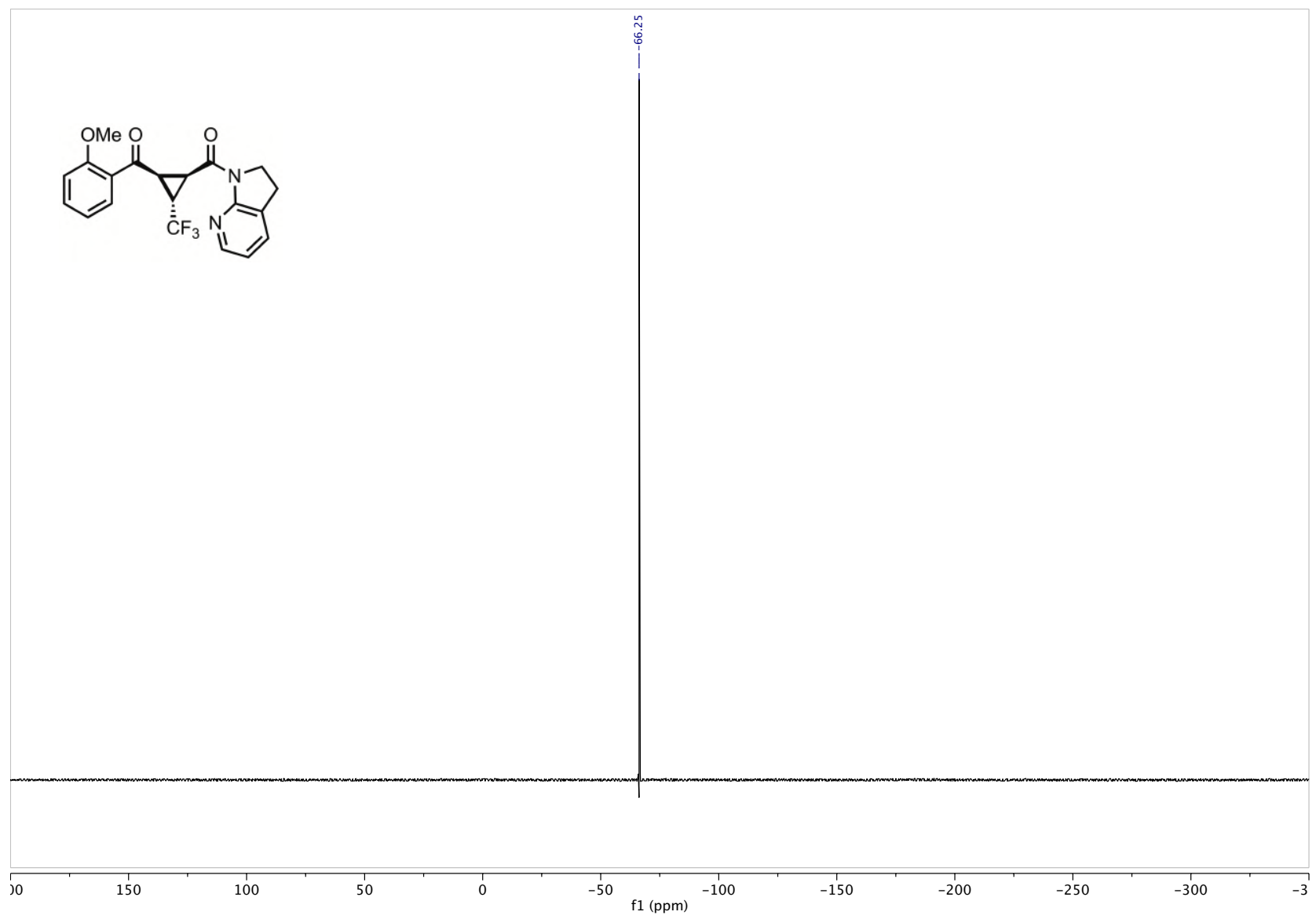


${ }^{1} \mathrm{H}$ NMR: 4fp

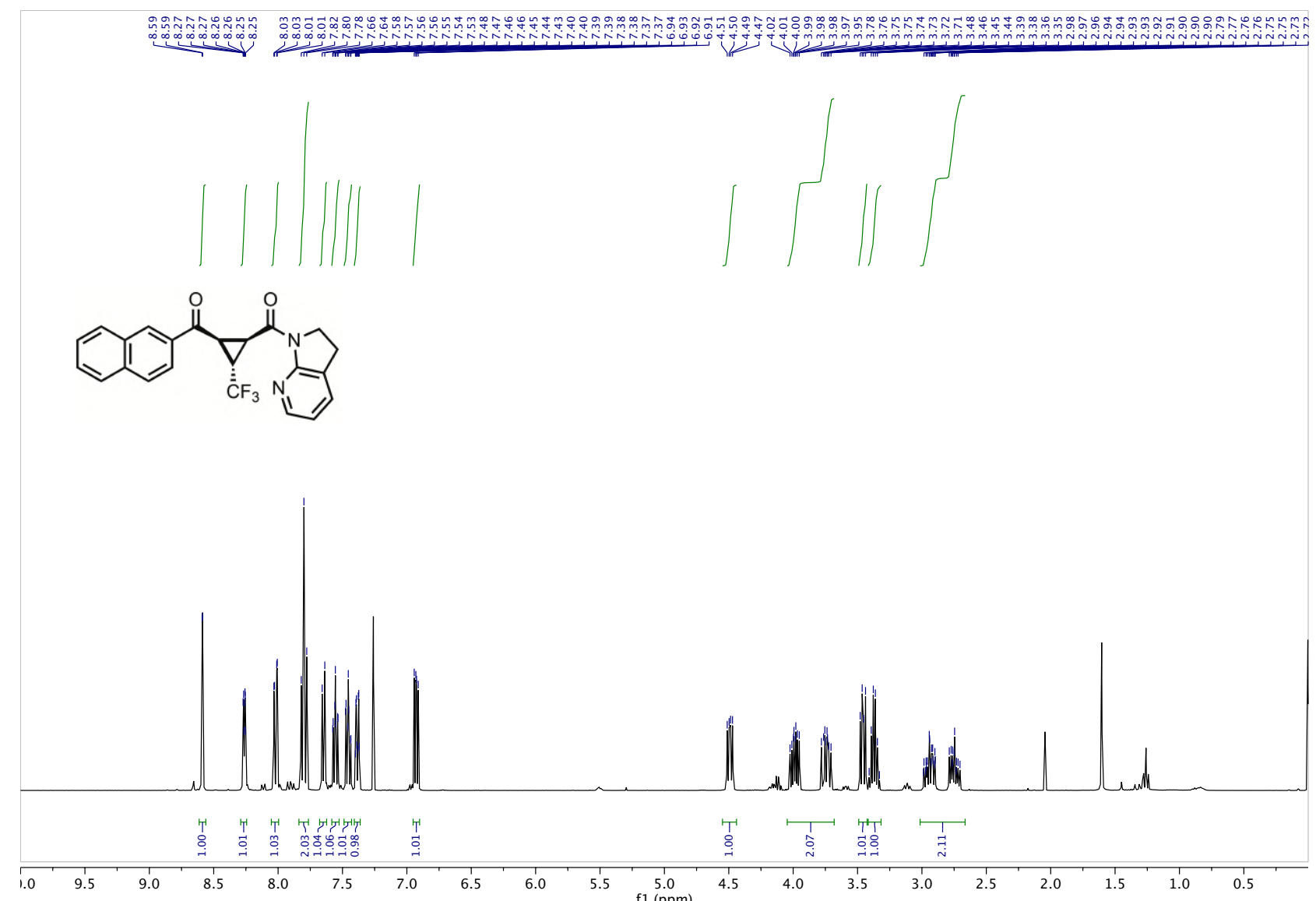

${ }^{13} \mathrm{C}$ NMR: $\mathbf{4 f p}$

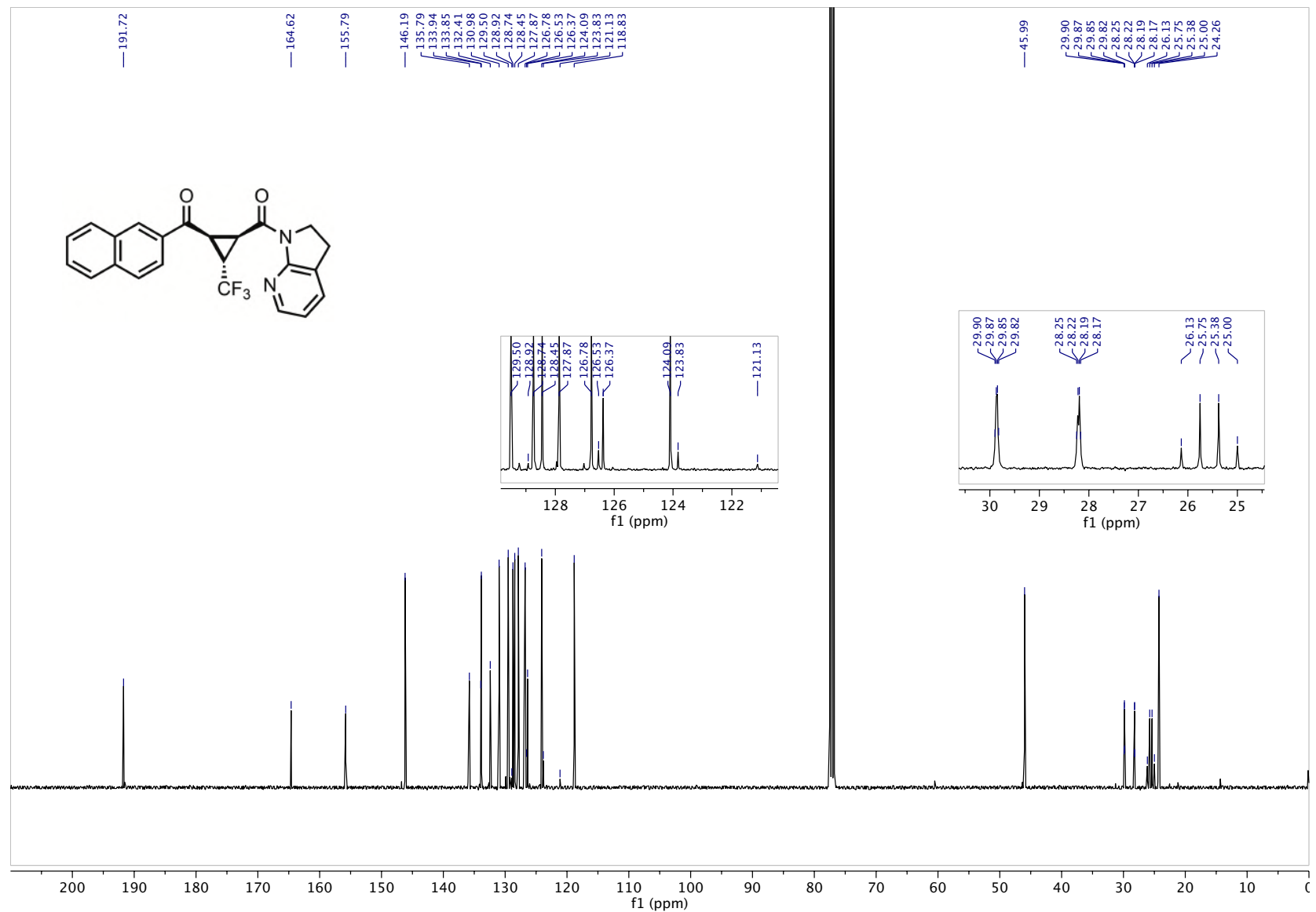


${ }^{19}$ F NMR: 4fp

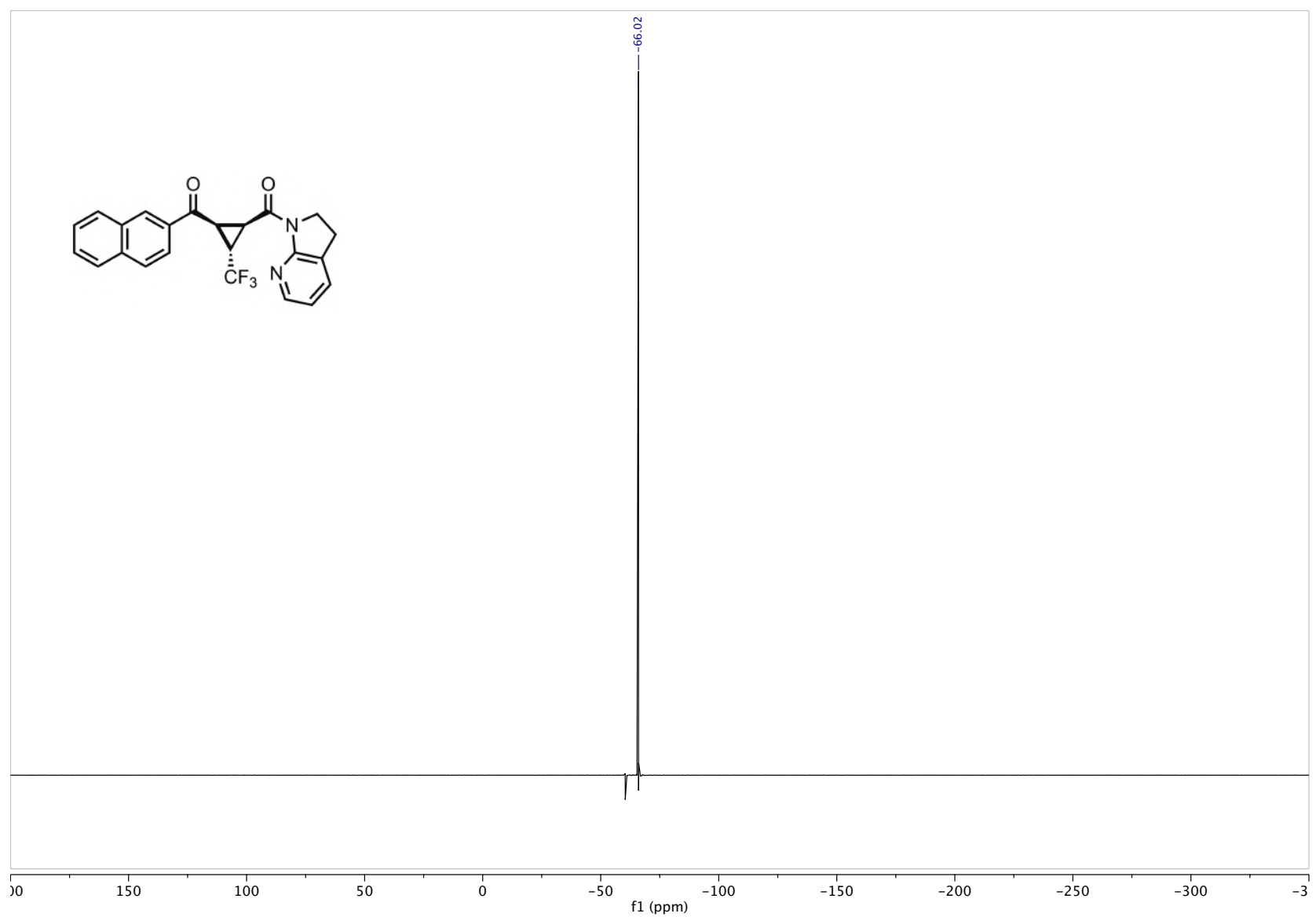


${ }^{1} \mathrm{H}$ NMR: 4gp

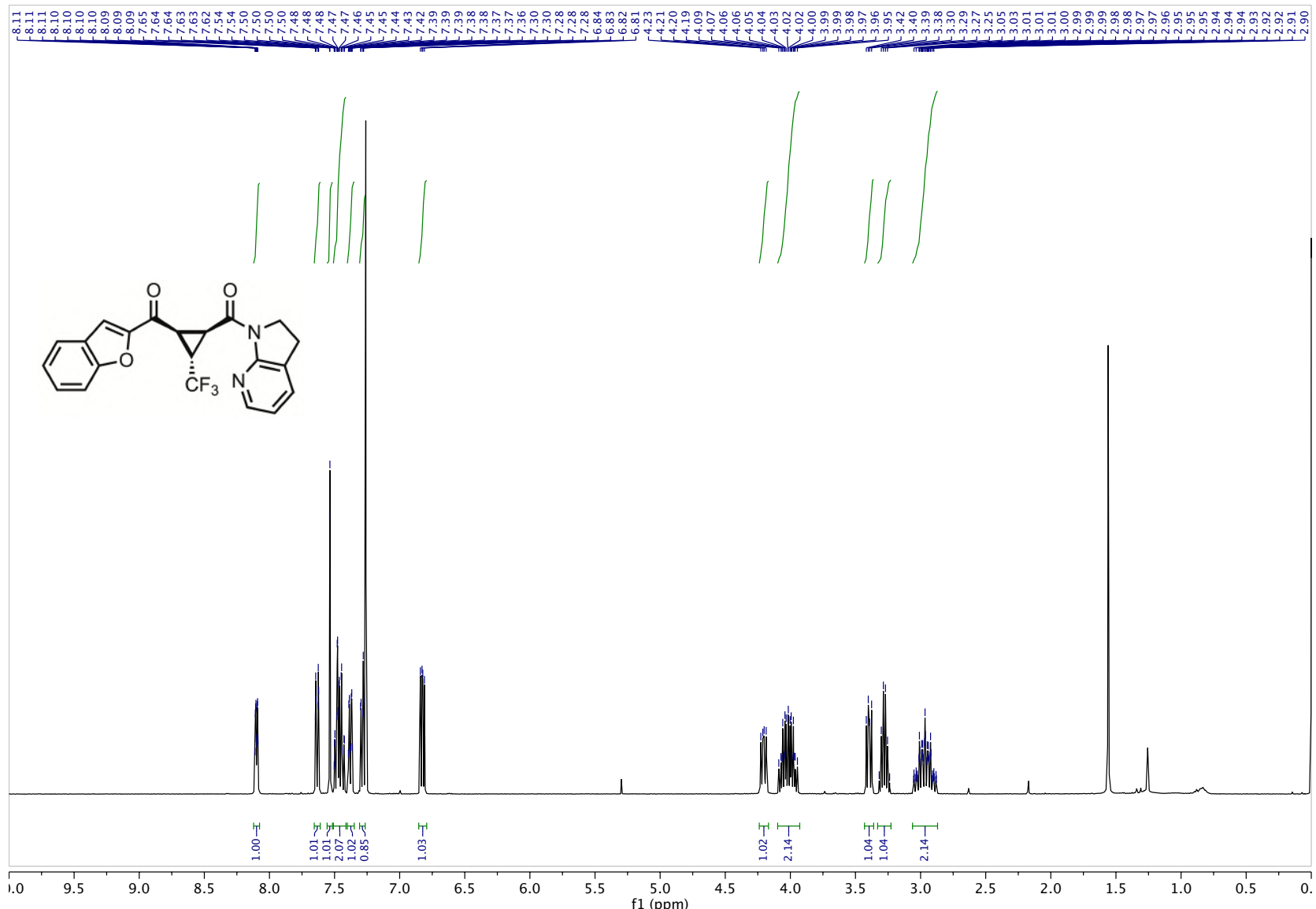

${ }^{13}$ C NMR: 4gp

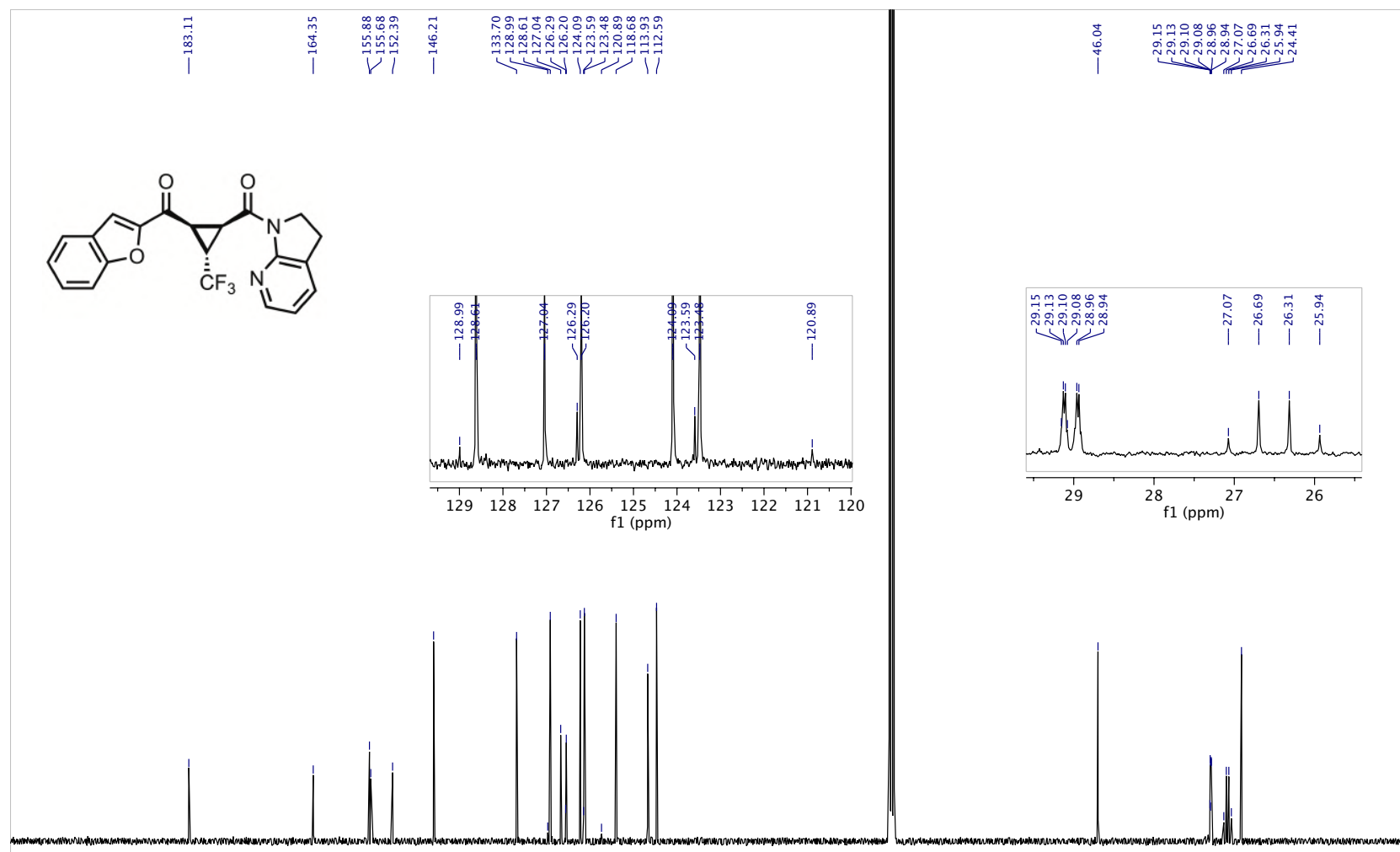

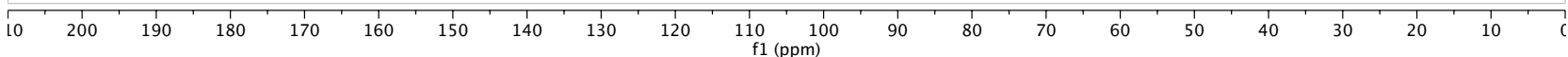


${ }^{19}$ F NMR: 4gp

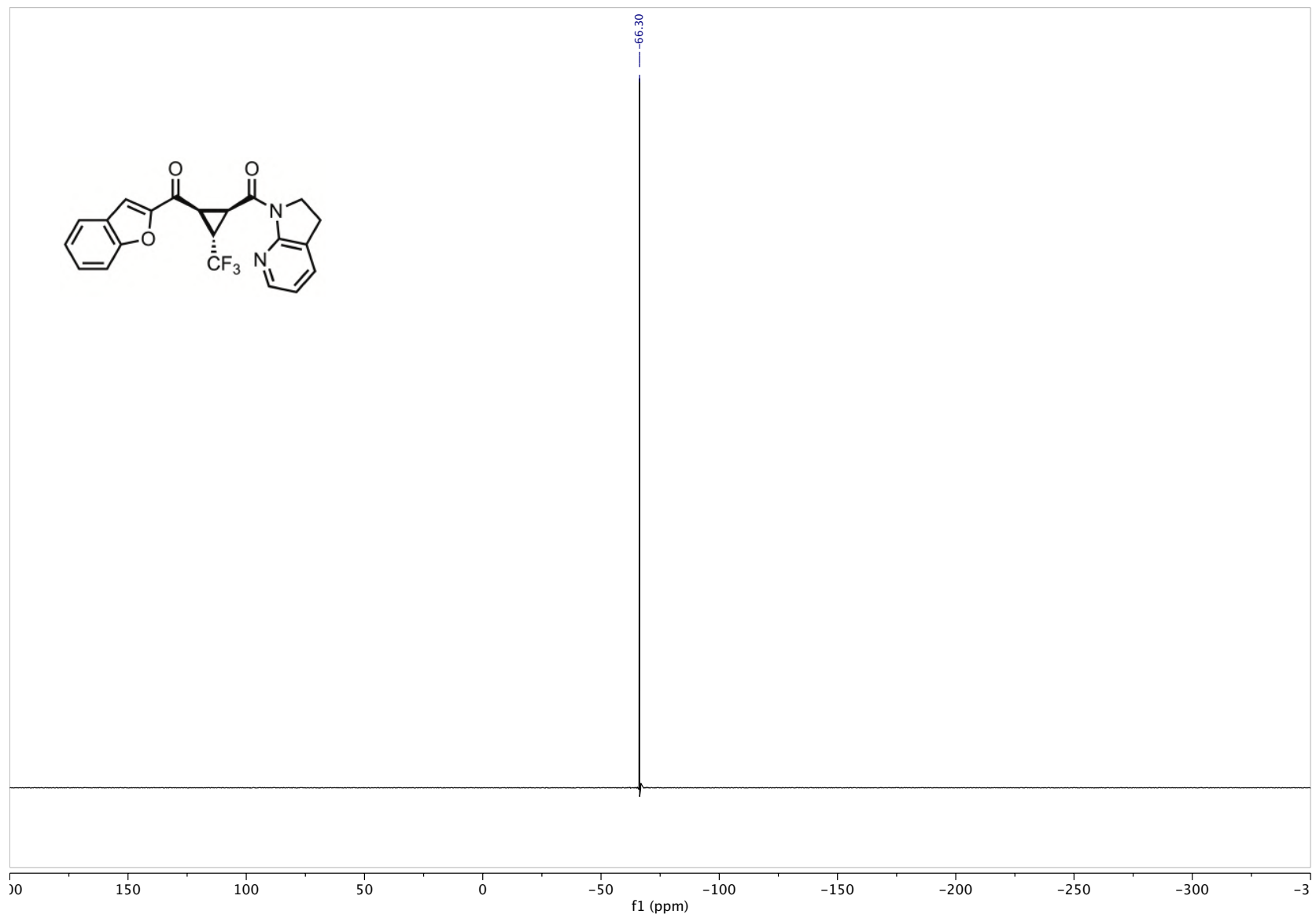


${ }^{1} \mathrm{H}$ NMR: $4 \mathrm{hp}$

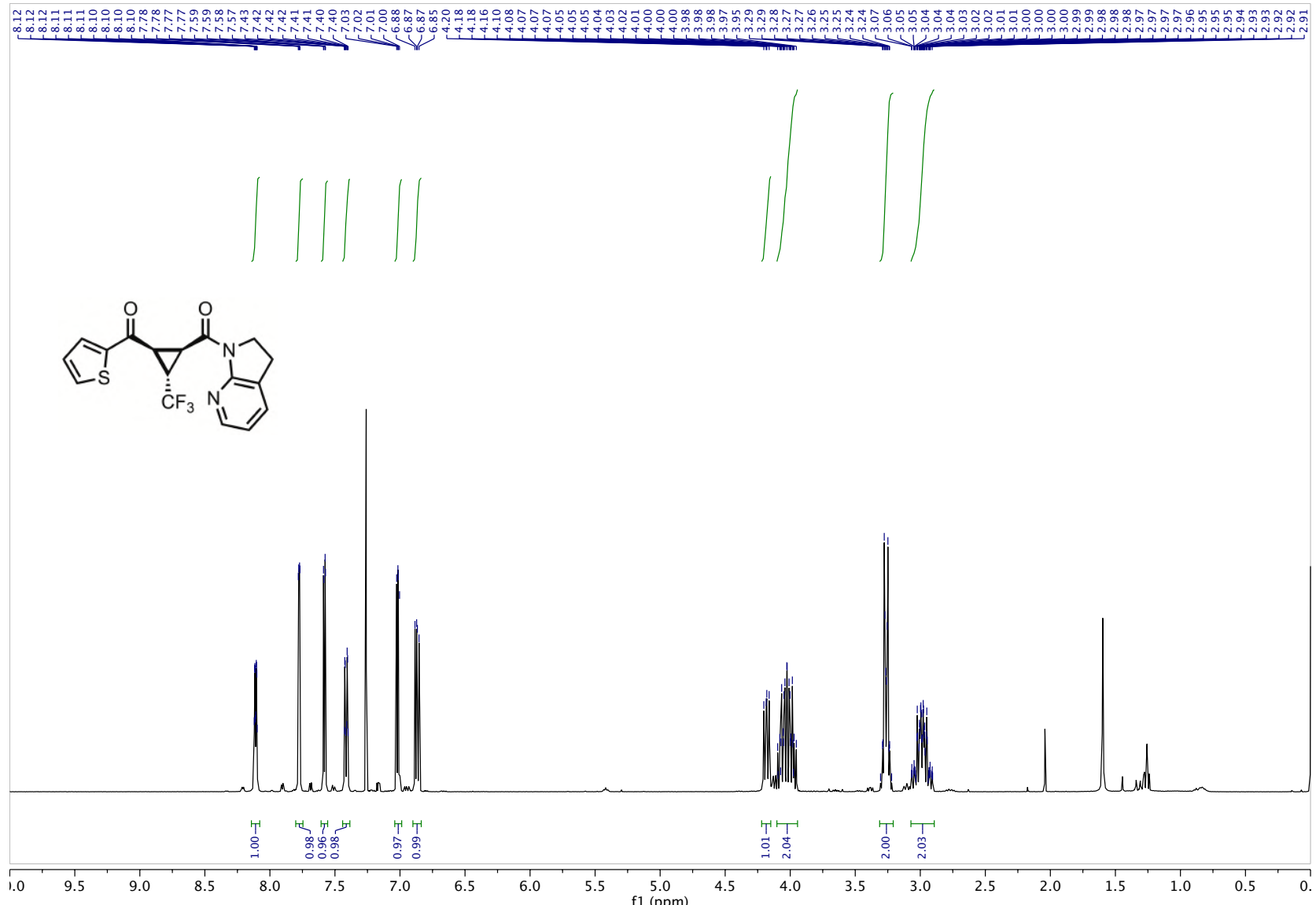

${ }^{13}$ C NMR: 4 hp

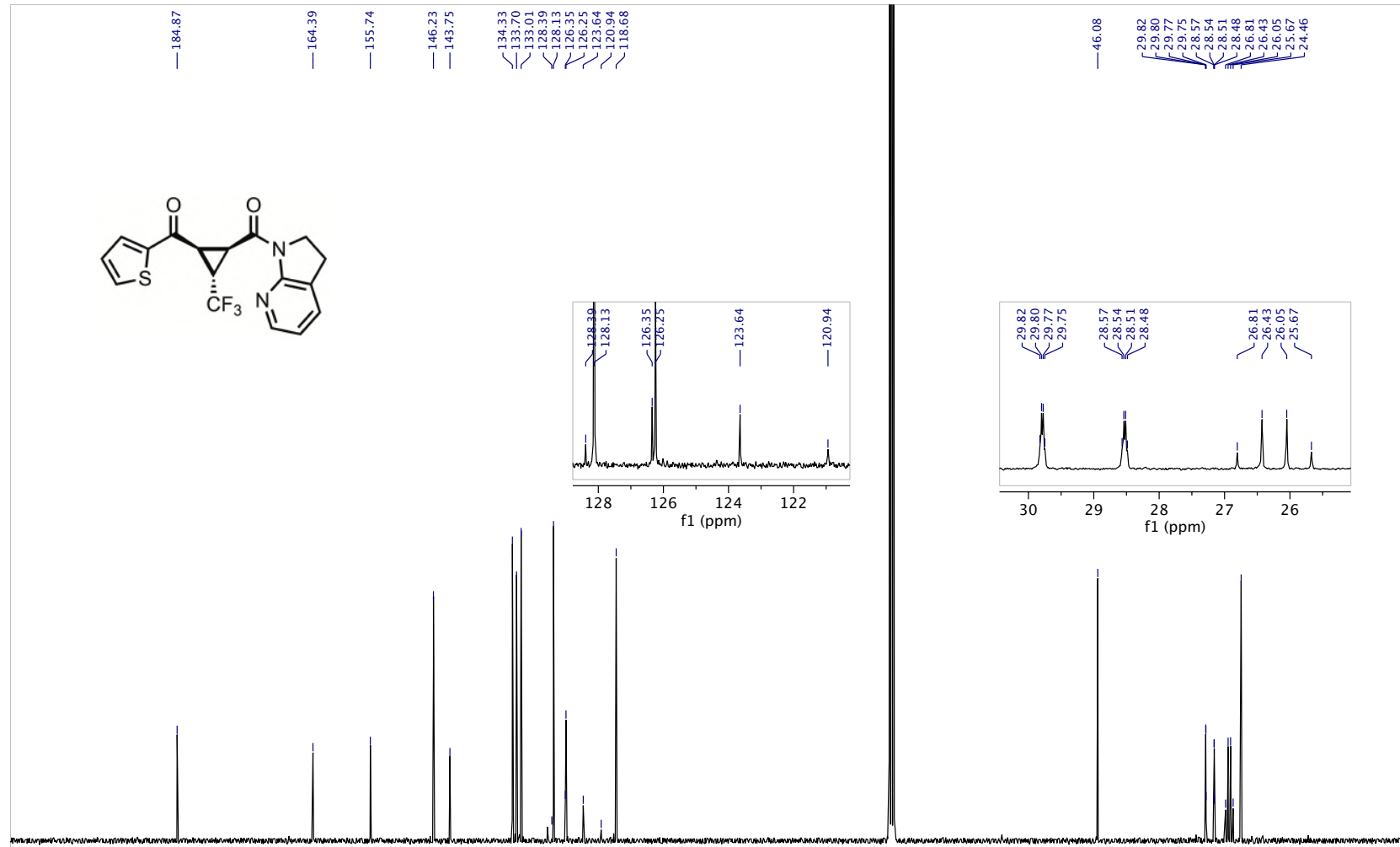

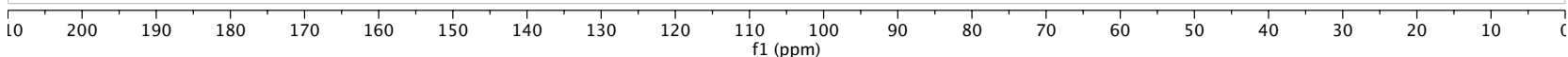


${ }^{19} \mathrm{~F}$ NMR: 4hp

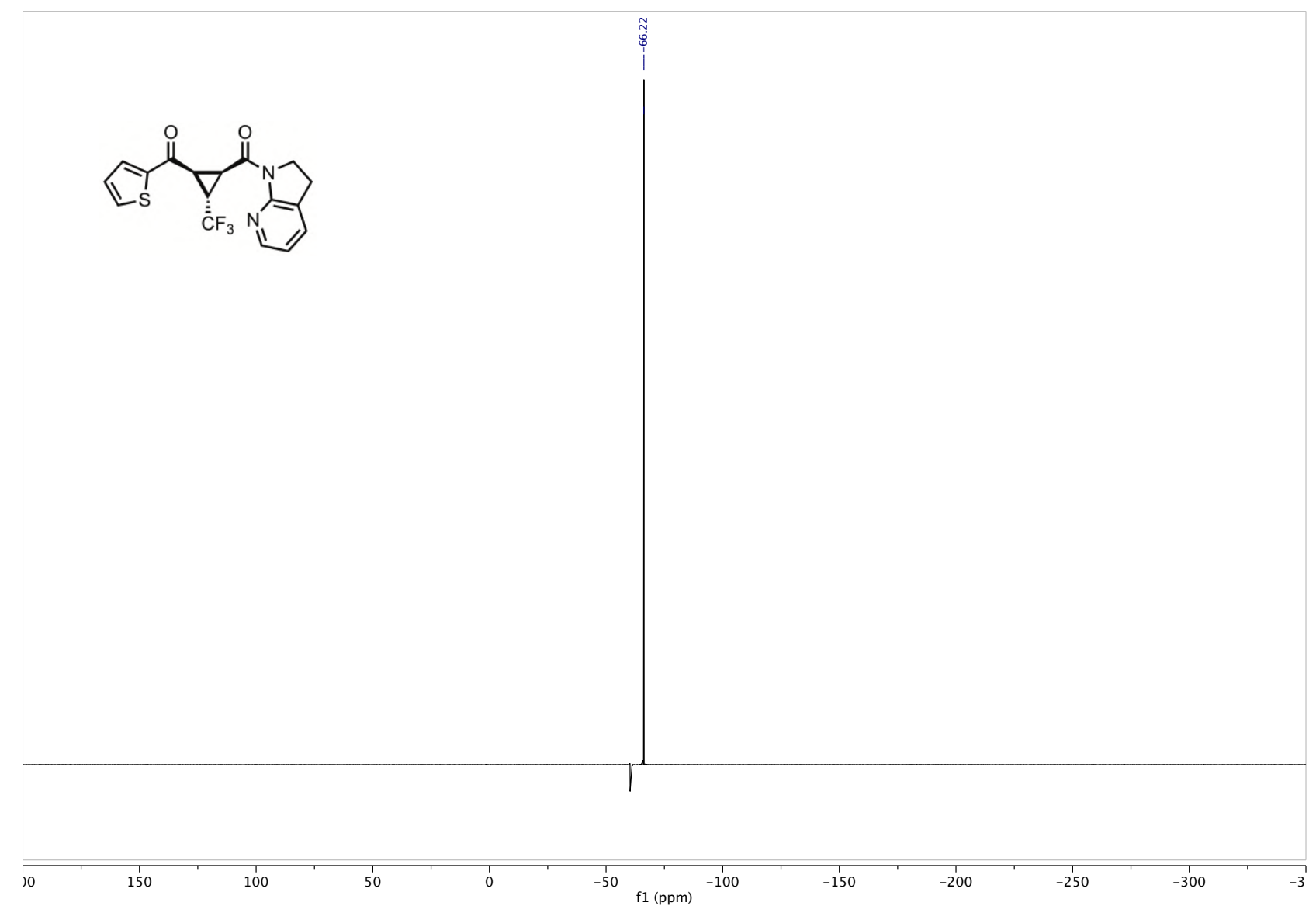


${ }^{1} \mathrm{H}$ NMR: 4ip

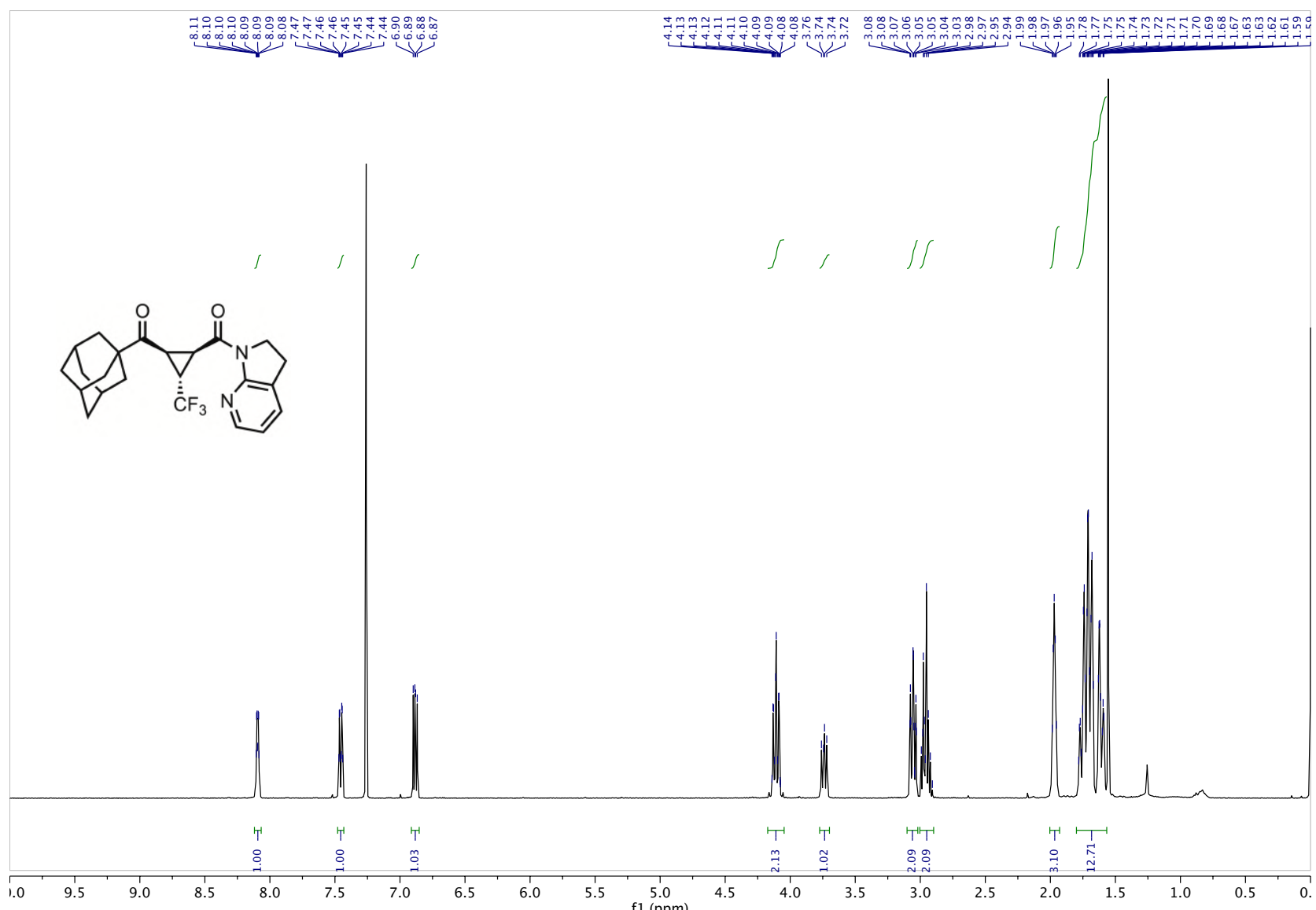

${ }^{13} \mathrm{C}$ NMR: 4ip

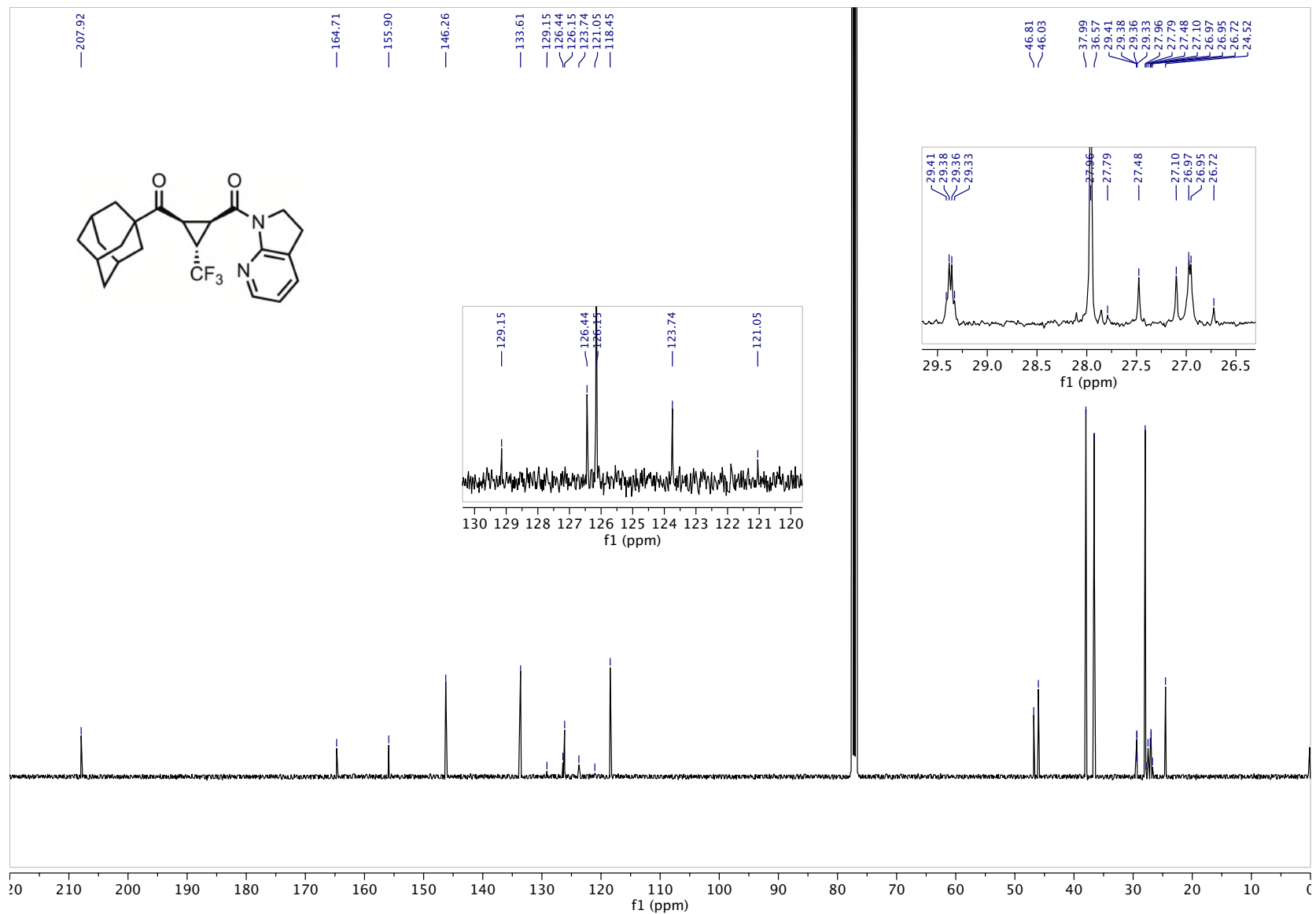


${ }^{19}$ F NMR: 4ip

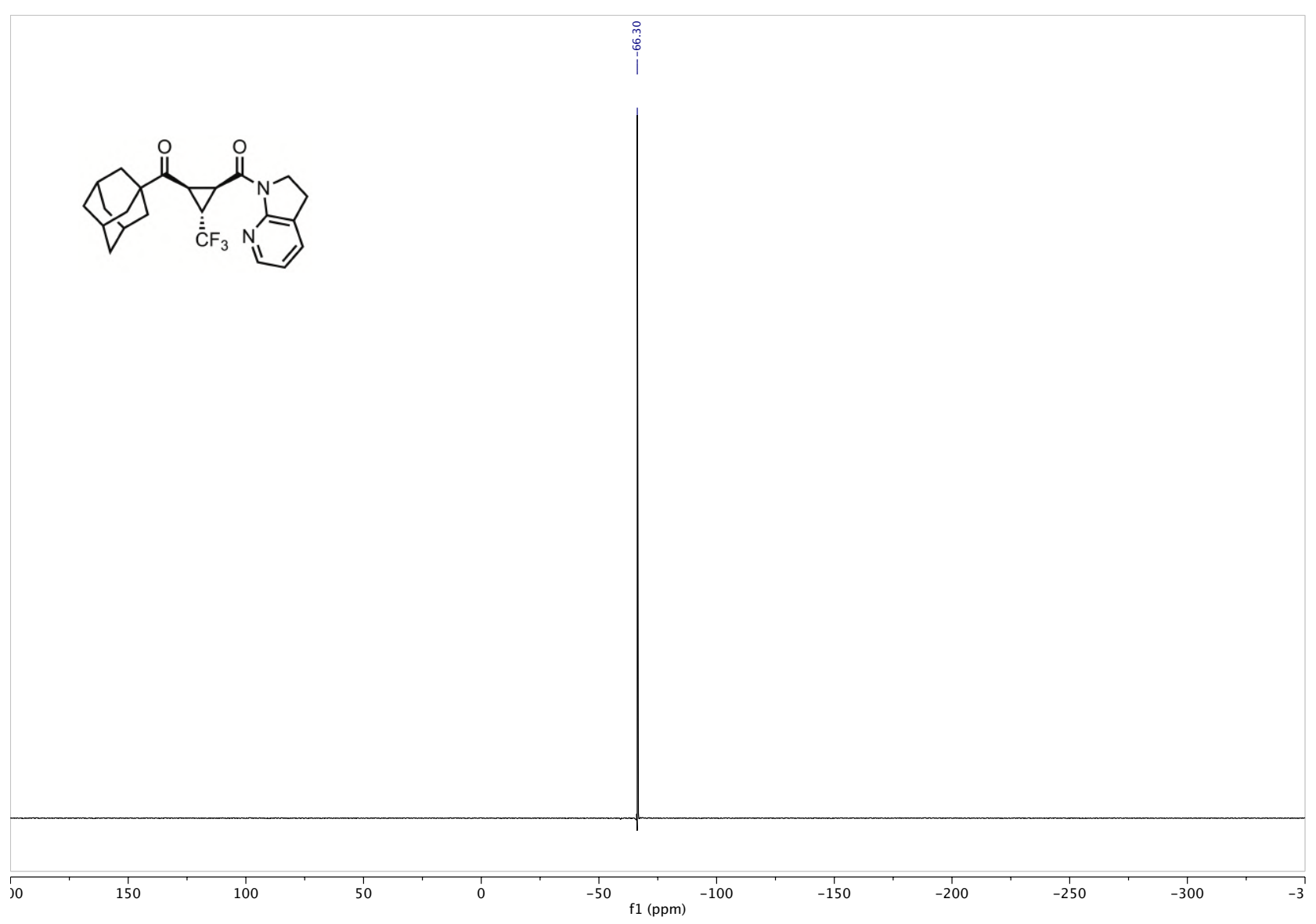




\section{${ }^{1} \mathrm{H}$ NMR: 4jp}

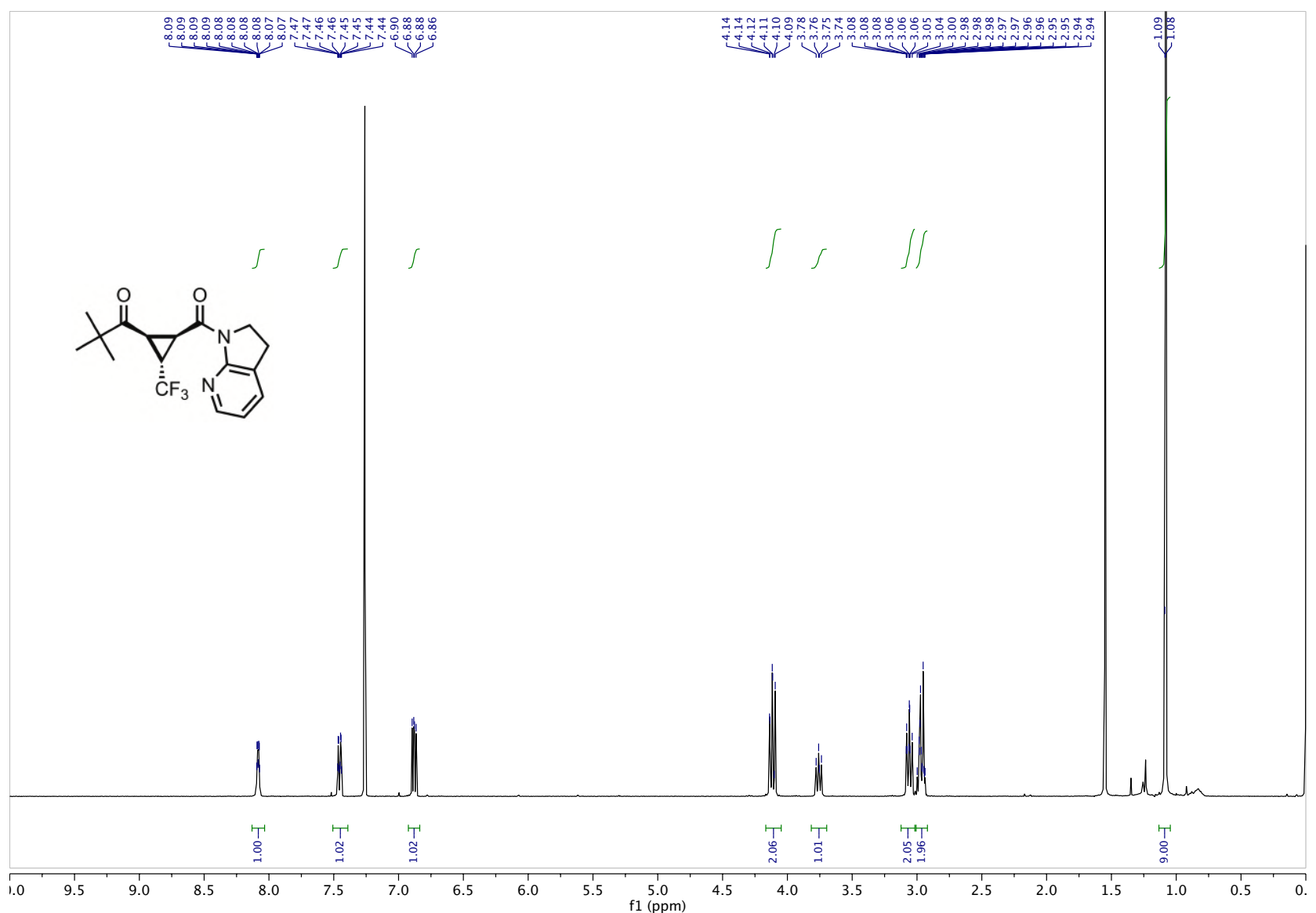

${ }^{13}$ C NMR: 4jp

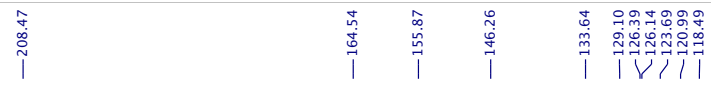<smiles>CC(C)(C)C(=O)C1C(C(=O)N2CCc3cccnc32)[C@H]1C(F)(F)F</smiles>
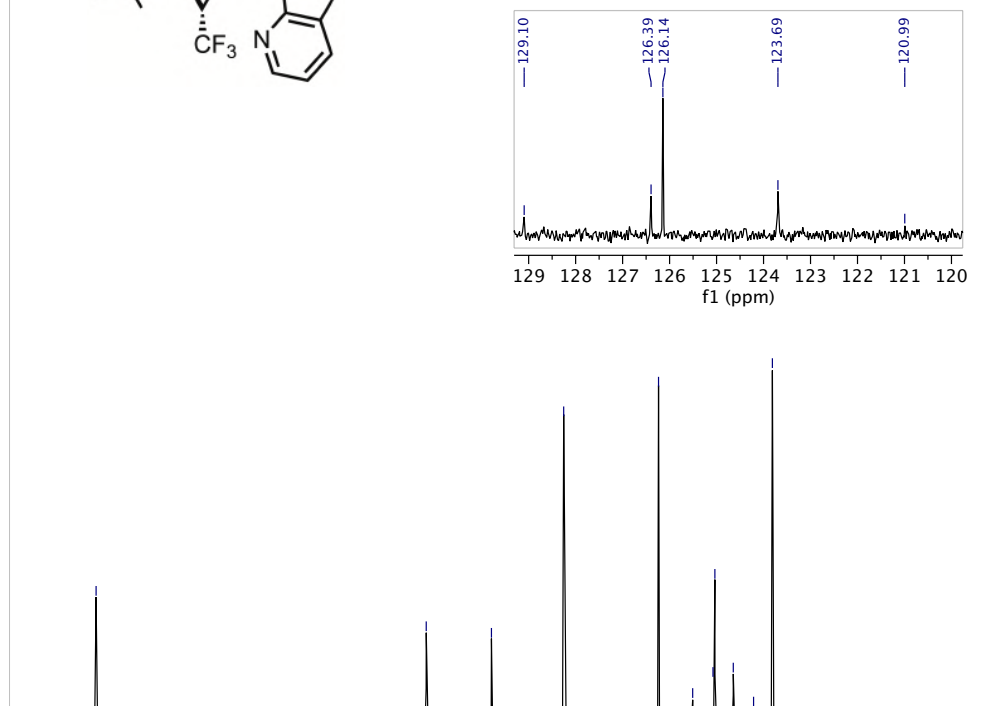

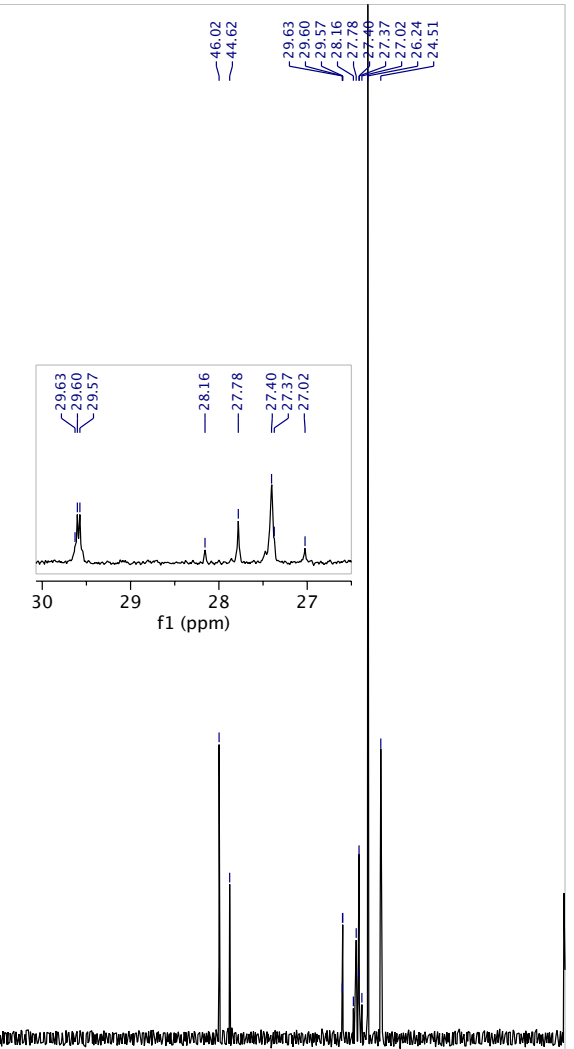

\begin{tabular}{llllllllllllllllllllllll}
\hline 0 & 210 & 200 & 190 & 180 & 170 & 160 & 150 & 140 & 130 & 120 & 110 & 100 & 90 & 80 & 70 & 60 & 50 & 40 & 30 & 20 & 10 & (
\end{tabular} 


\section{${ }^{19} \mathrm{~F}$ NMR: 4jp}

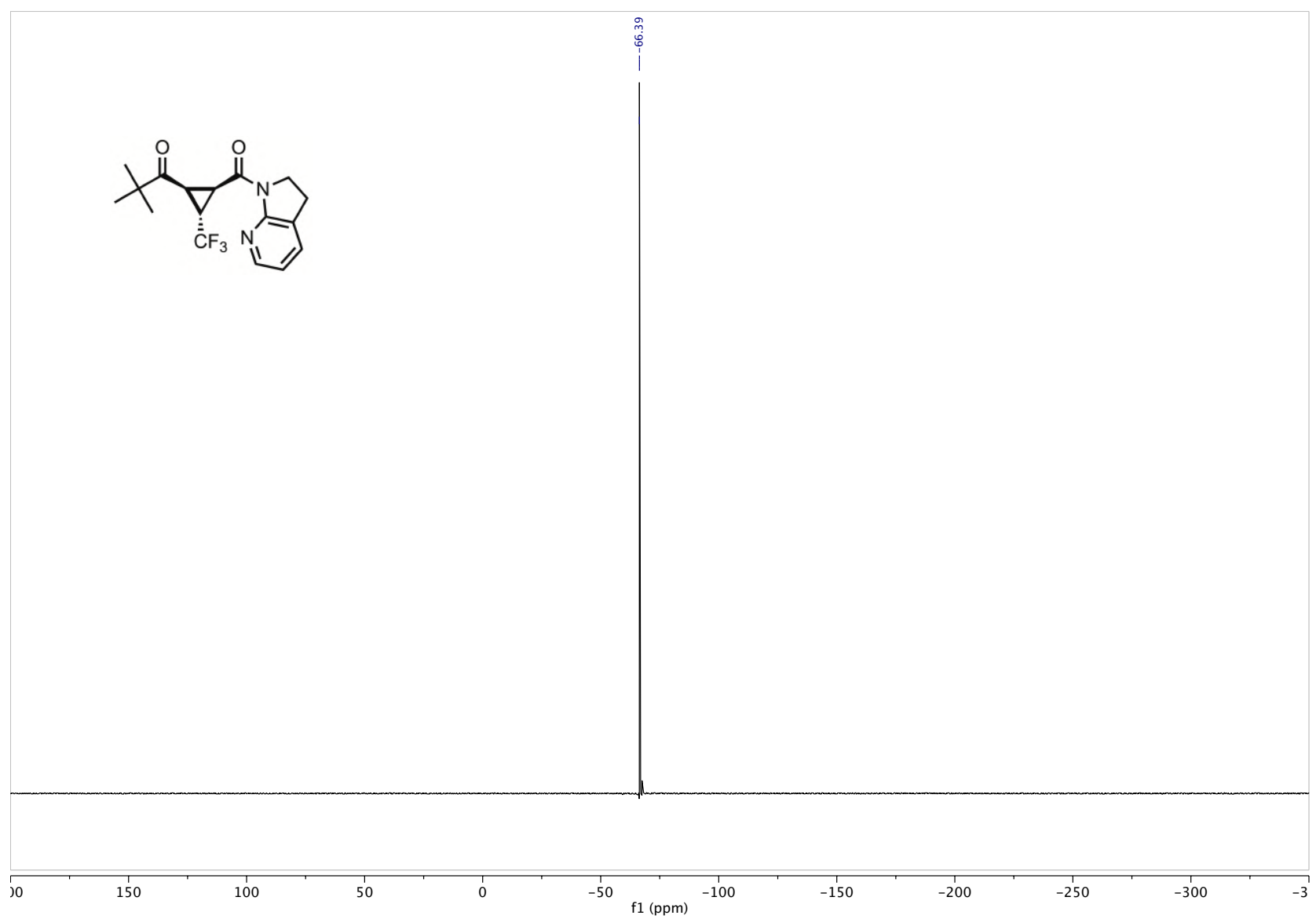


${ }^{1} \mathrm{H}$ NMR: 5aq

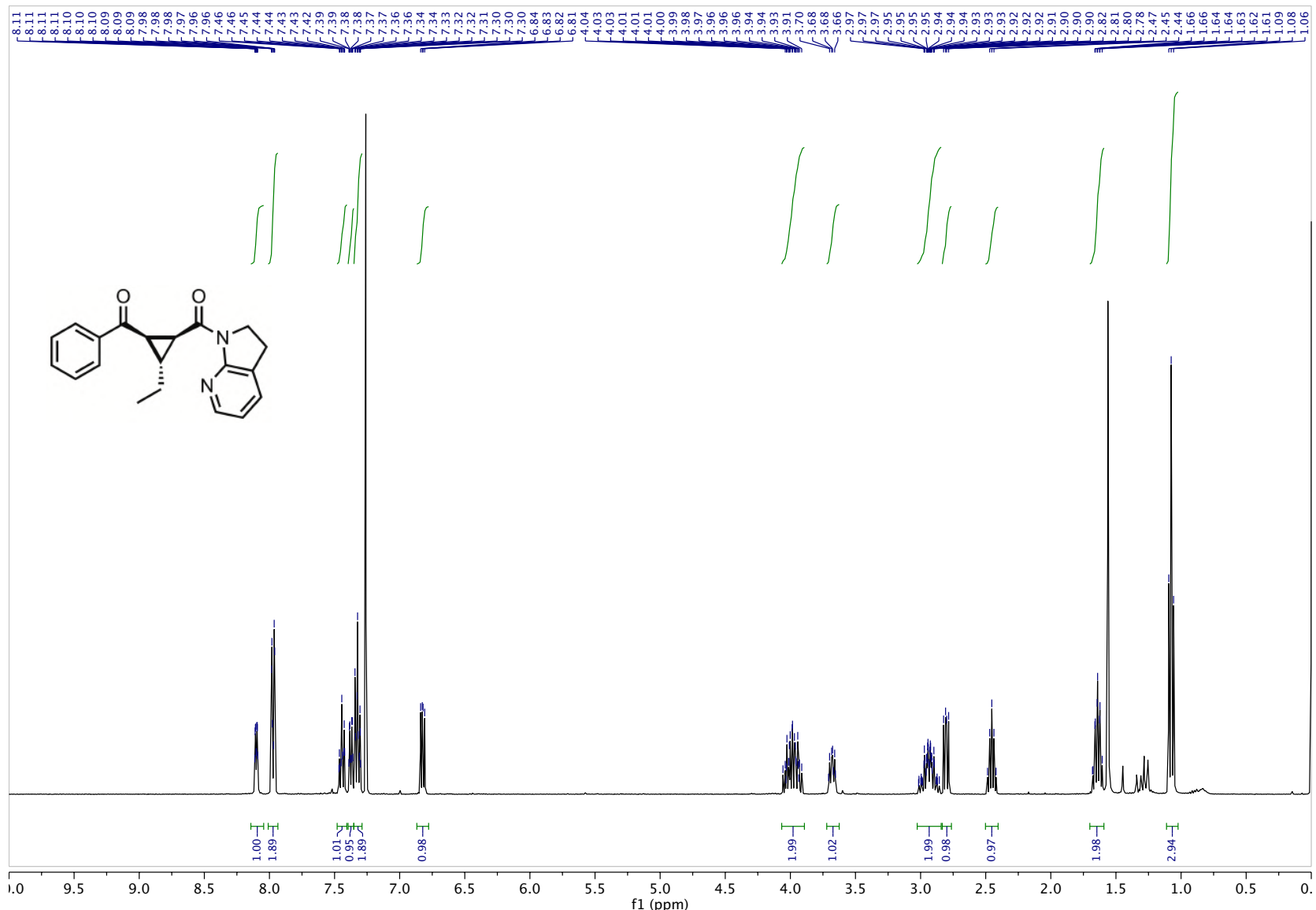

${ }^{13}$ C NMR: 5aq

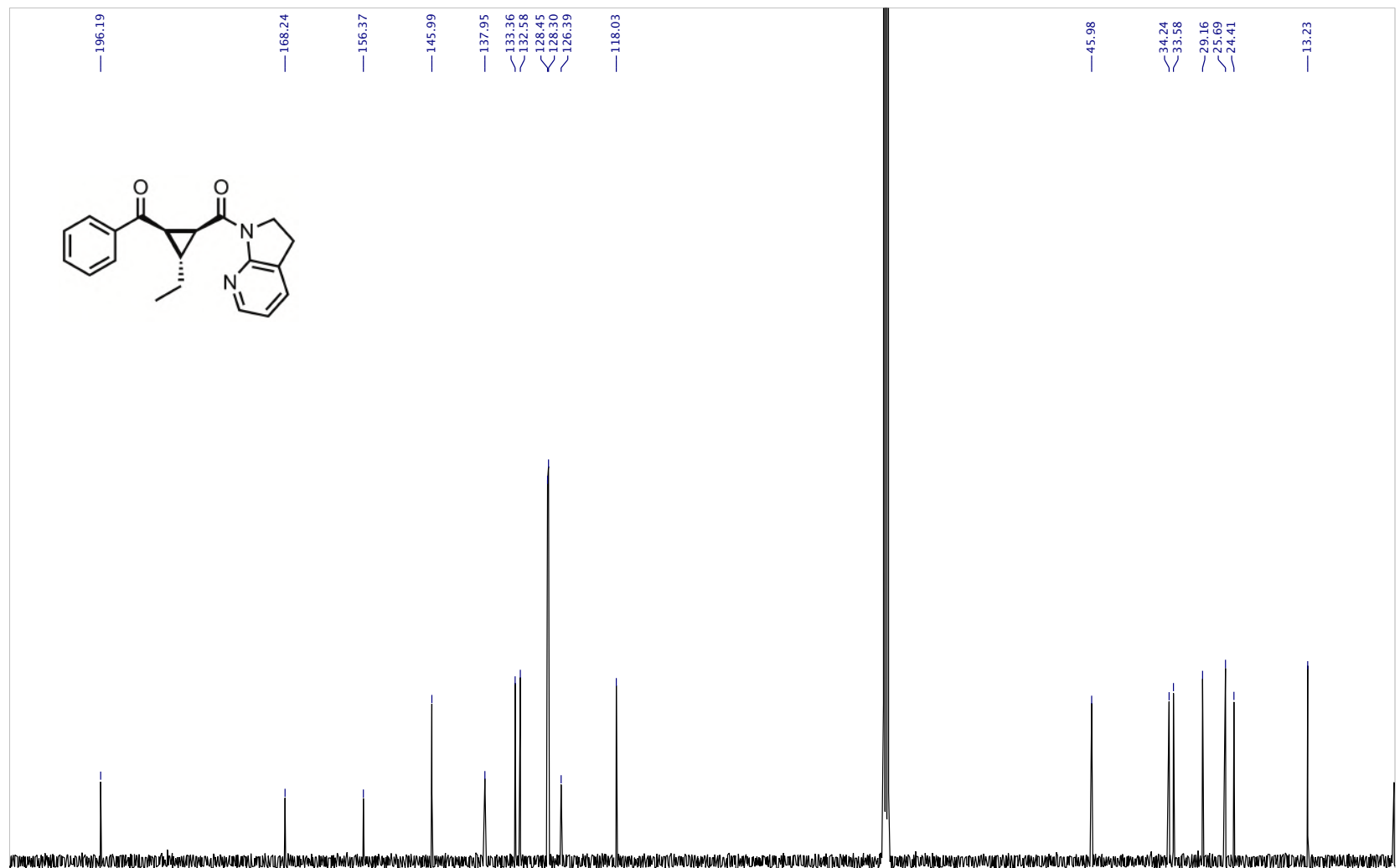

\begin{tabular}{|l|l|llllllllllllllllllll}
\hline 10 & 200 & 190 & 180 & 170 & 160 & 150 & 140 & 130 & 120 & $\underset{f}{1} 10$ & 100 & 90 & 80 & 70 & 60 & 50 & 40 & 30 & 20 & 10 & 1 \\
\hline
\end{tabular} 
${ }^{1} \mathrm{H}$ NMR: 5ar

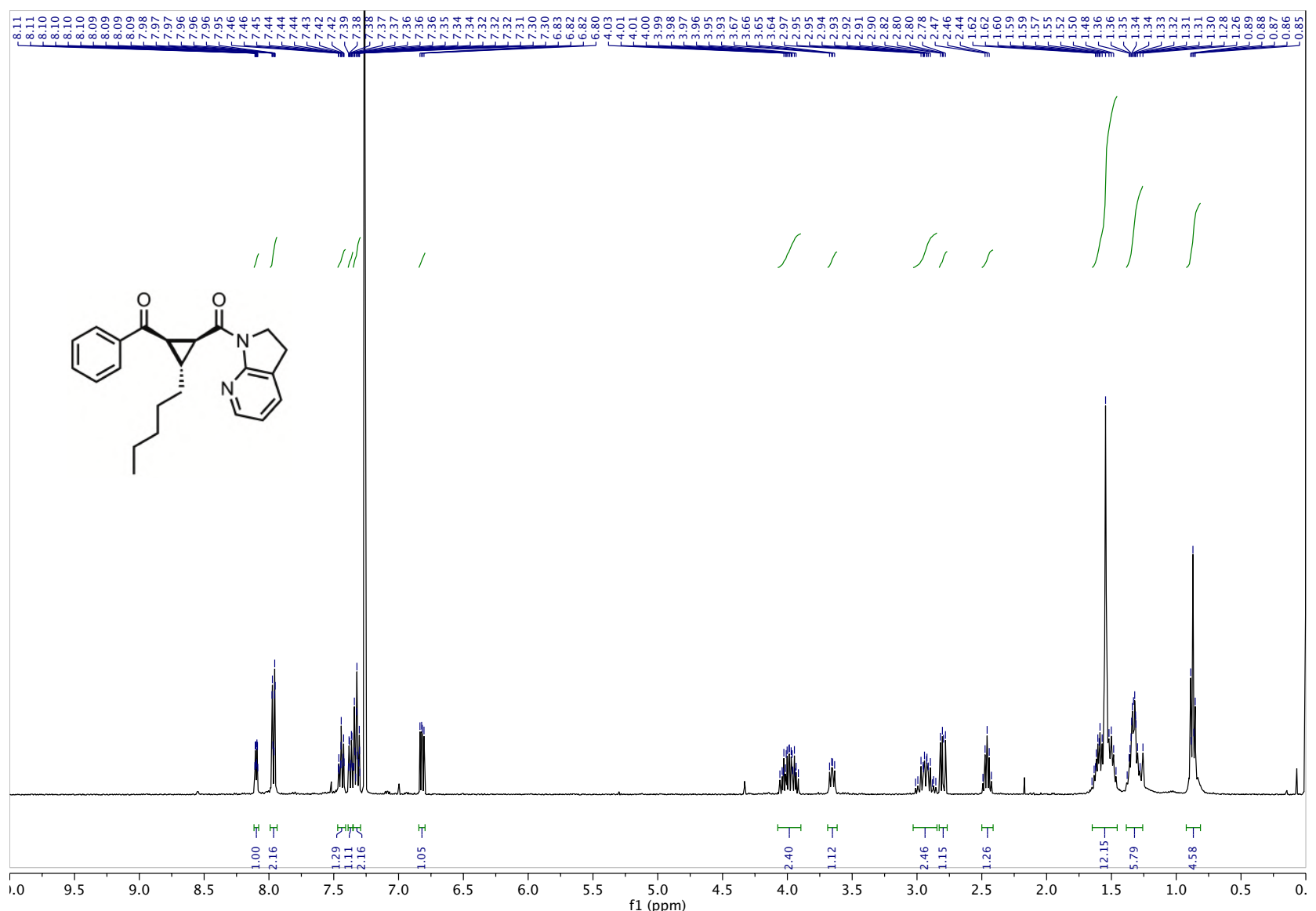

${ }^{13} \mathrm{C}$ NMR: 5 ar

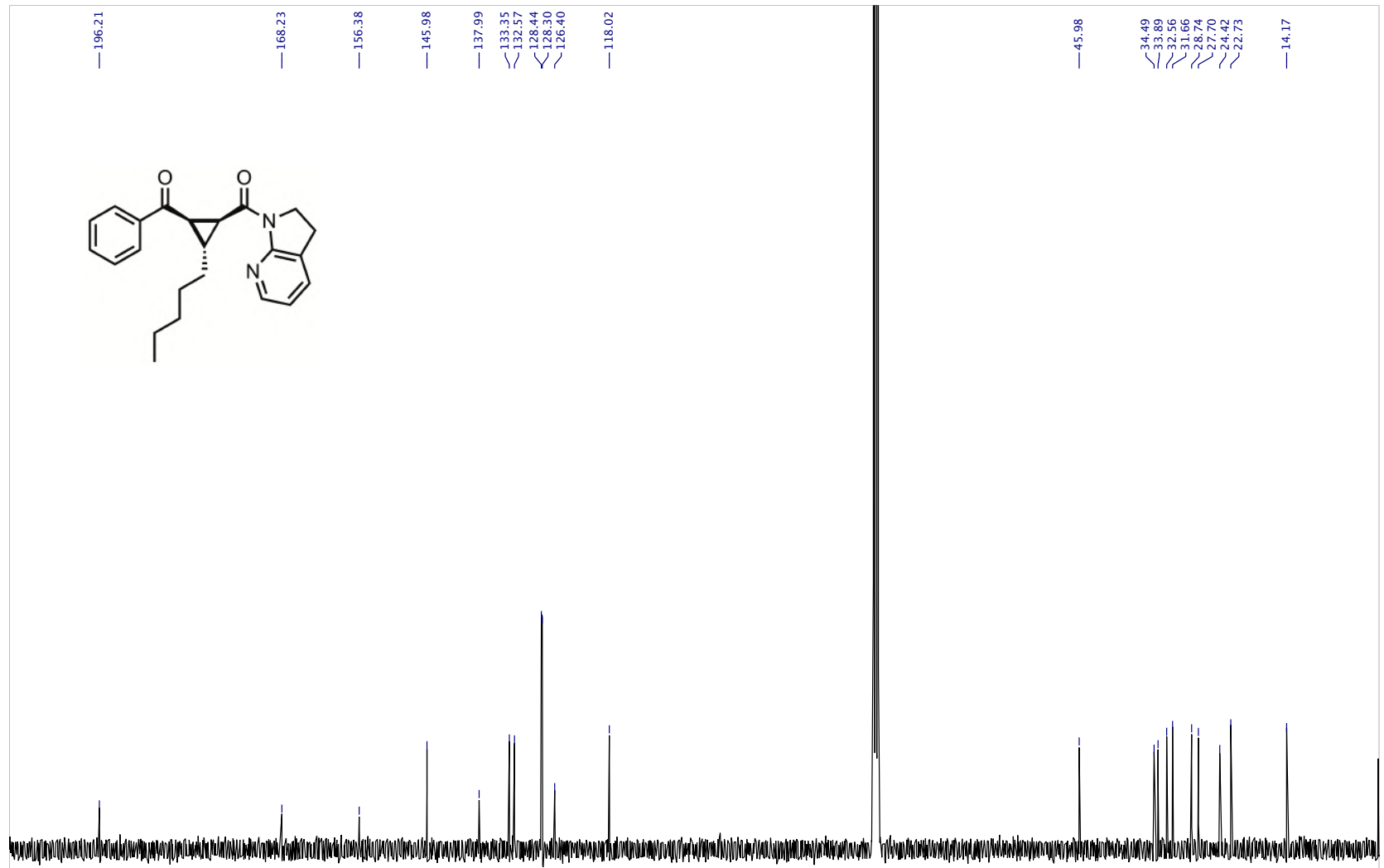

\begin{tabular}{rrrrrrrrrrr}
\hline 10 & 200 & 190 & 180 & 170 & 160 & 150 & 140 & 130 & 120 & 110 \\
$\mathrm{f} 1(\mathrm{ppm})$
\end{tabular} 
${ }^{1} \mathrm{H}$ NMR: 5as

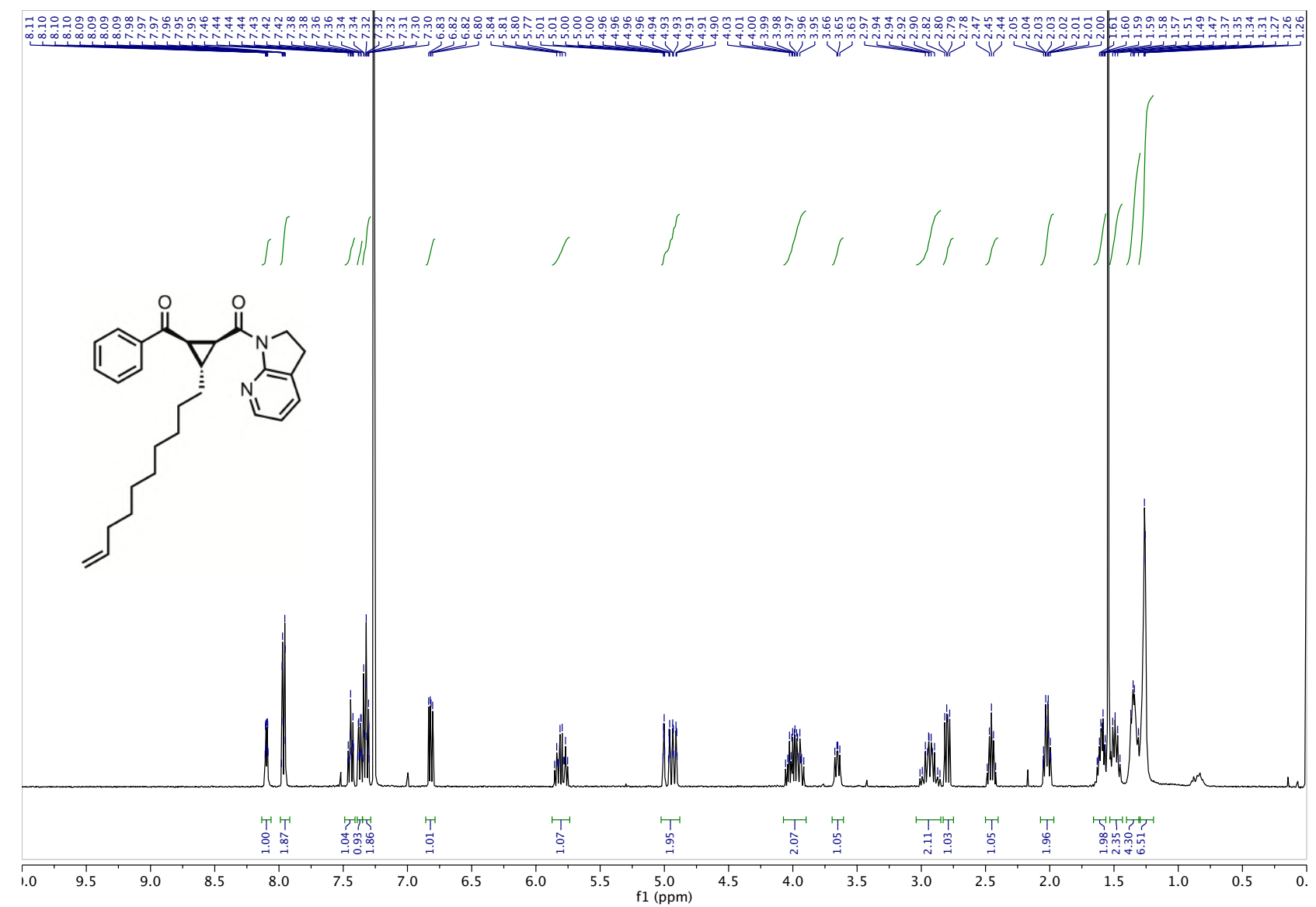

${ }^{13}$ C NMR: 5as

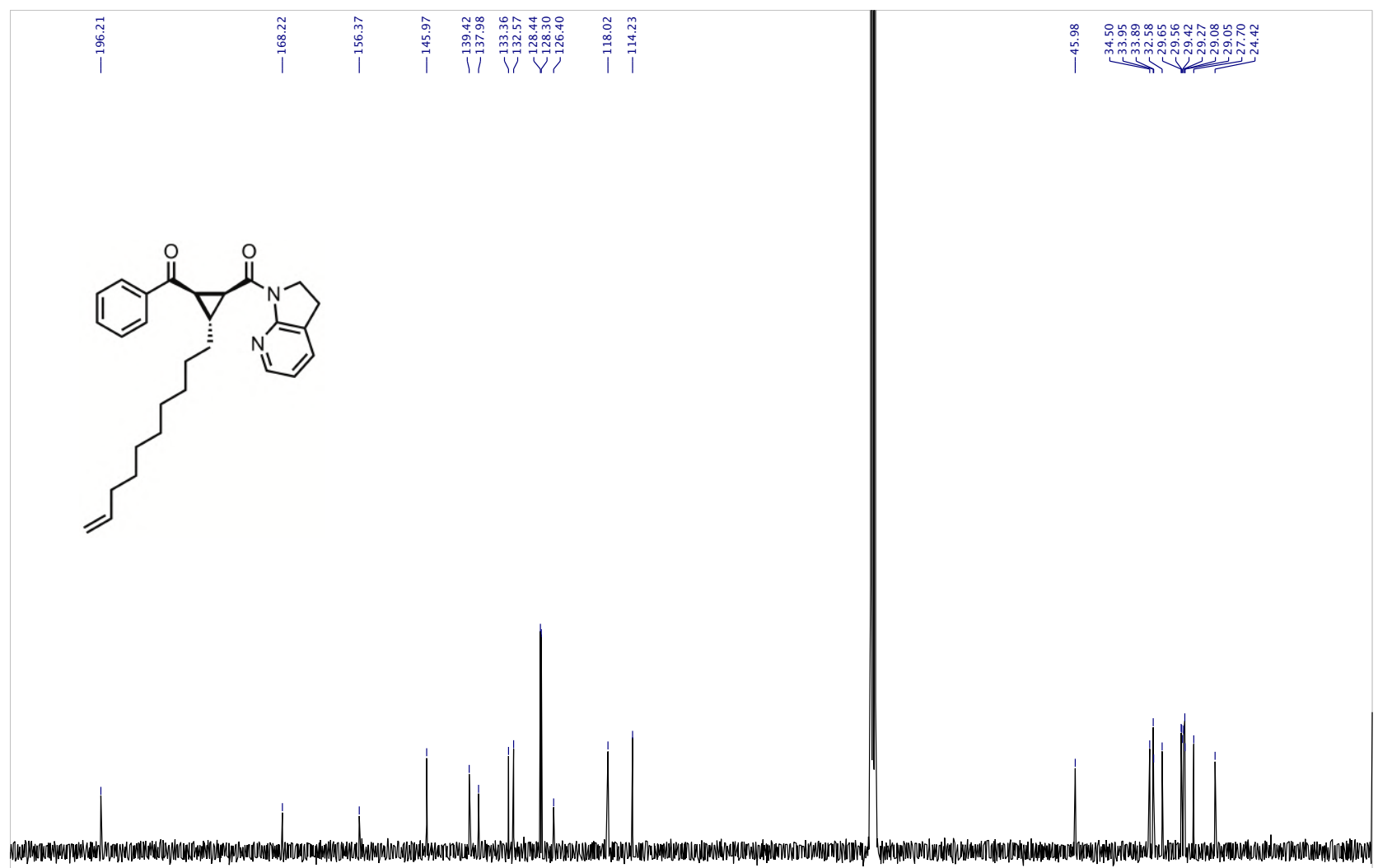

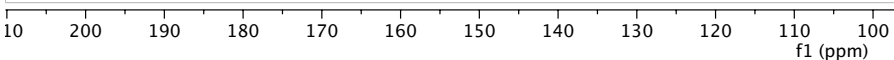


${ }^{1} \mathrm{H}$ NMR: 5at

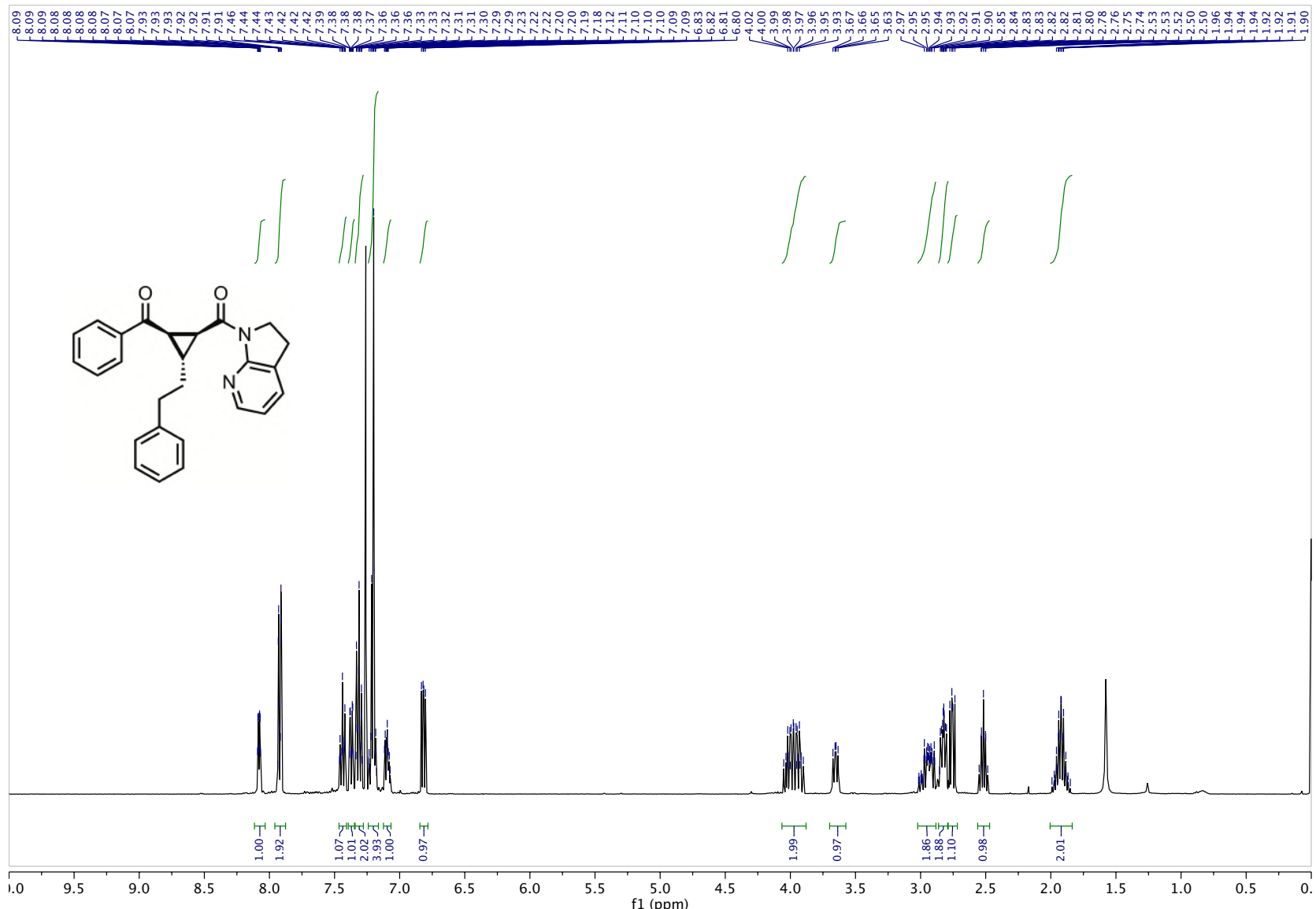

${ }^{13} \mathrm{C}$ NMR: 5at

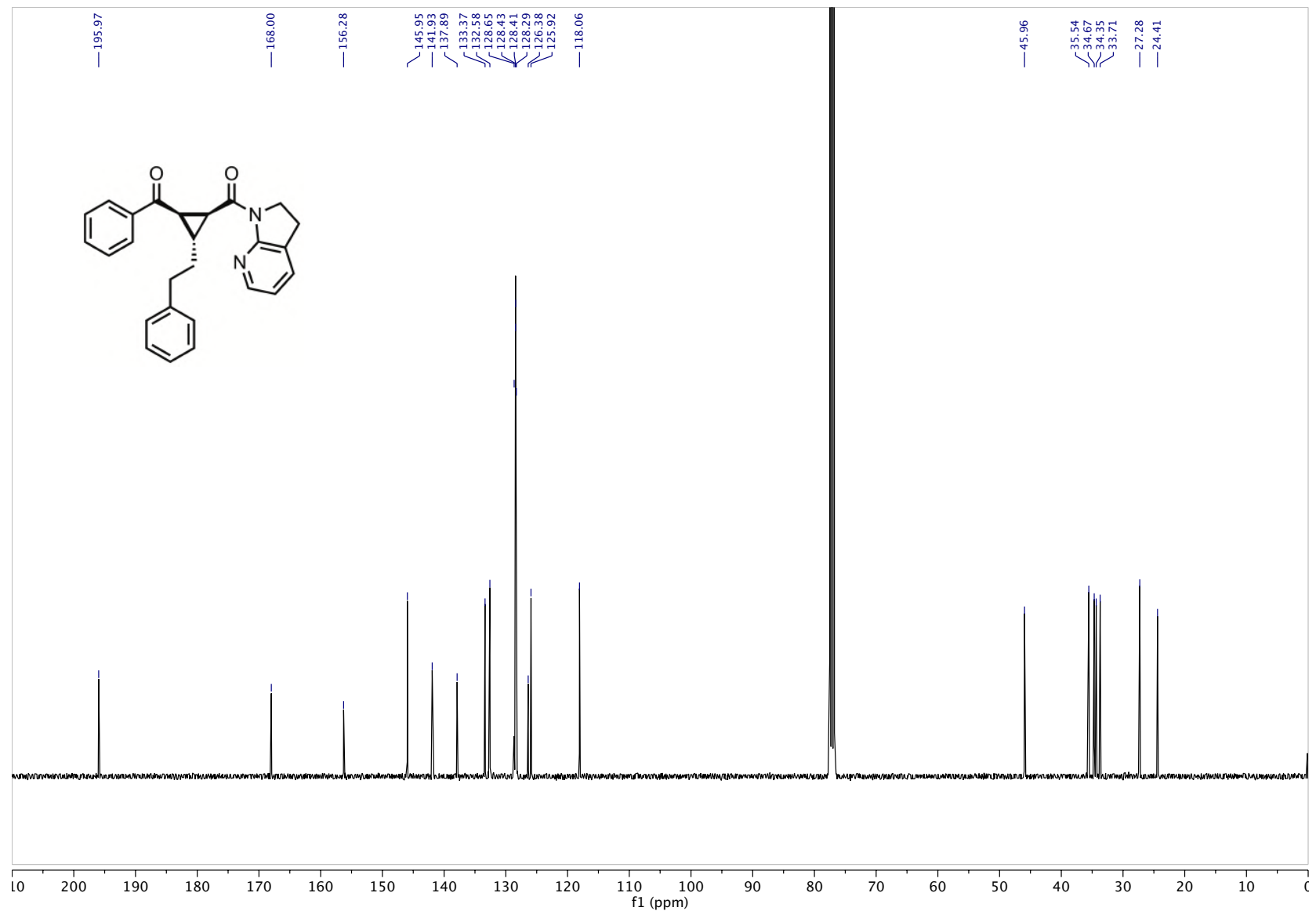


${ }^{1} \mathrm{H}$ NMR: $6 \mathrm{au}$

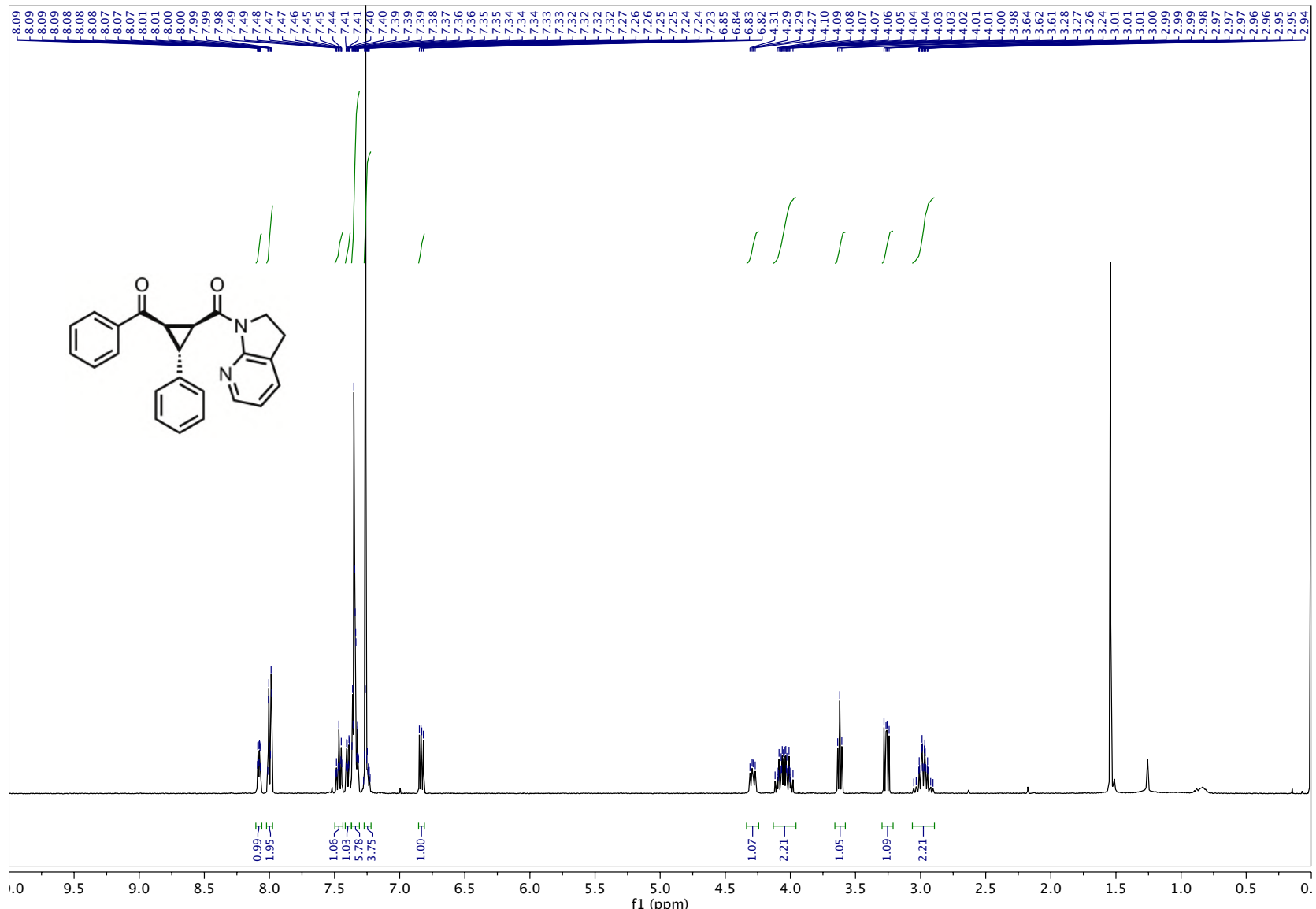

${ }^{13}$ C NMR: 6au

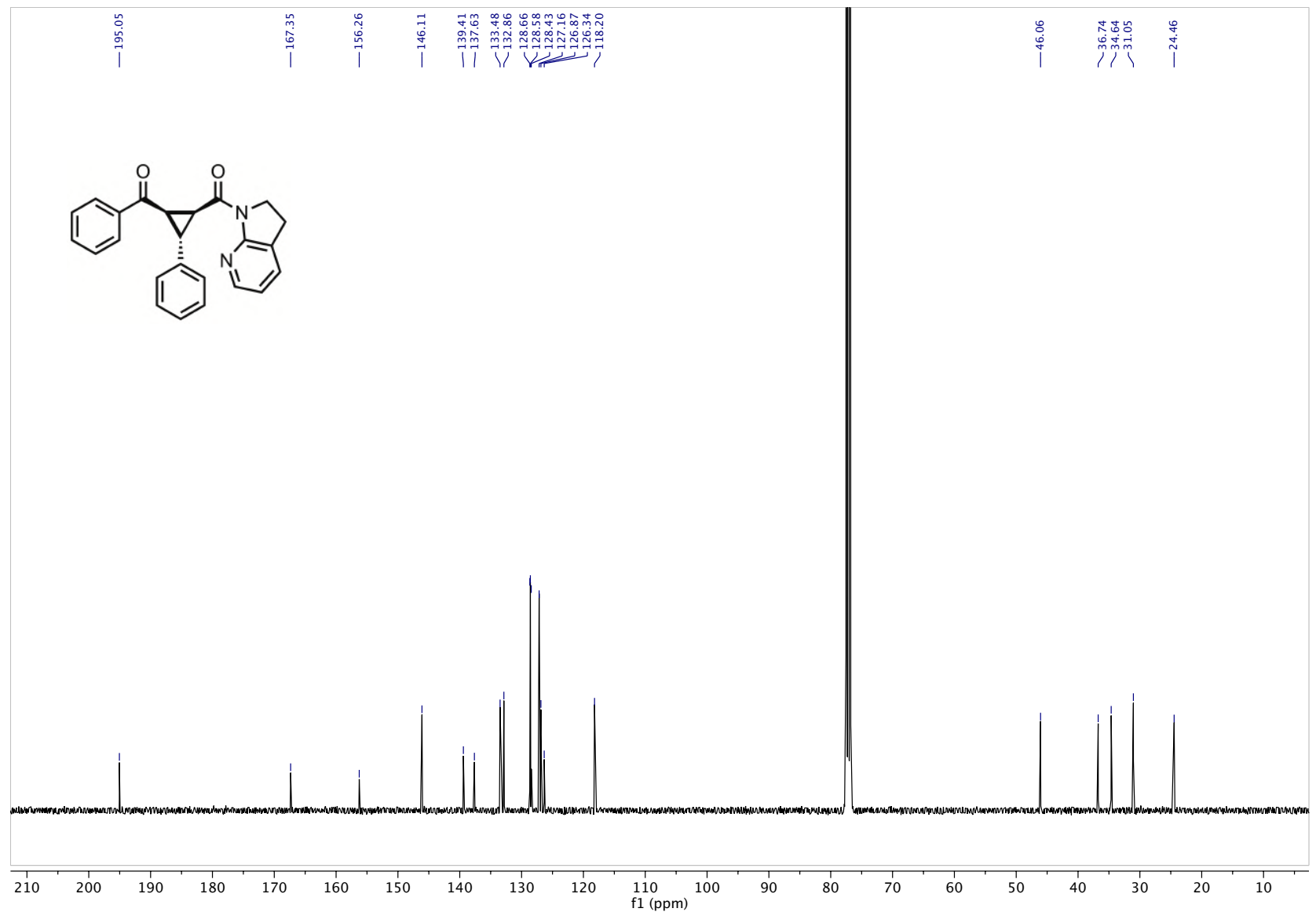


${ }^{1} \mathrm{H}$ NMR: 6av

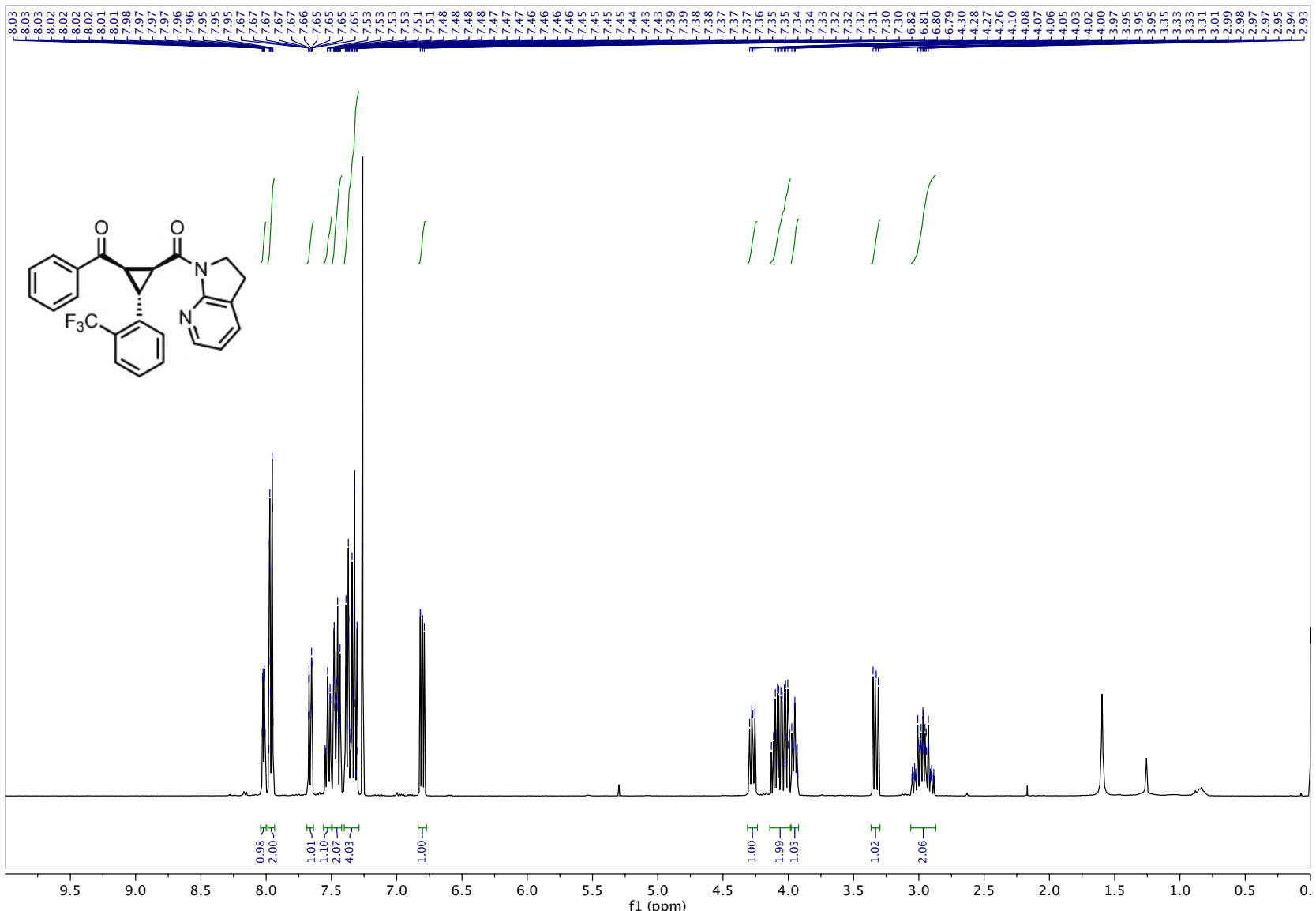

${ }^{13}$ C NMR: 6av

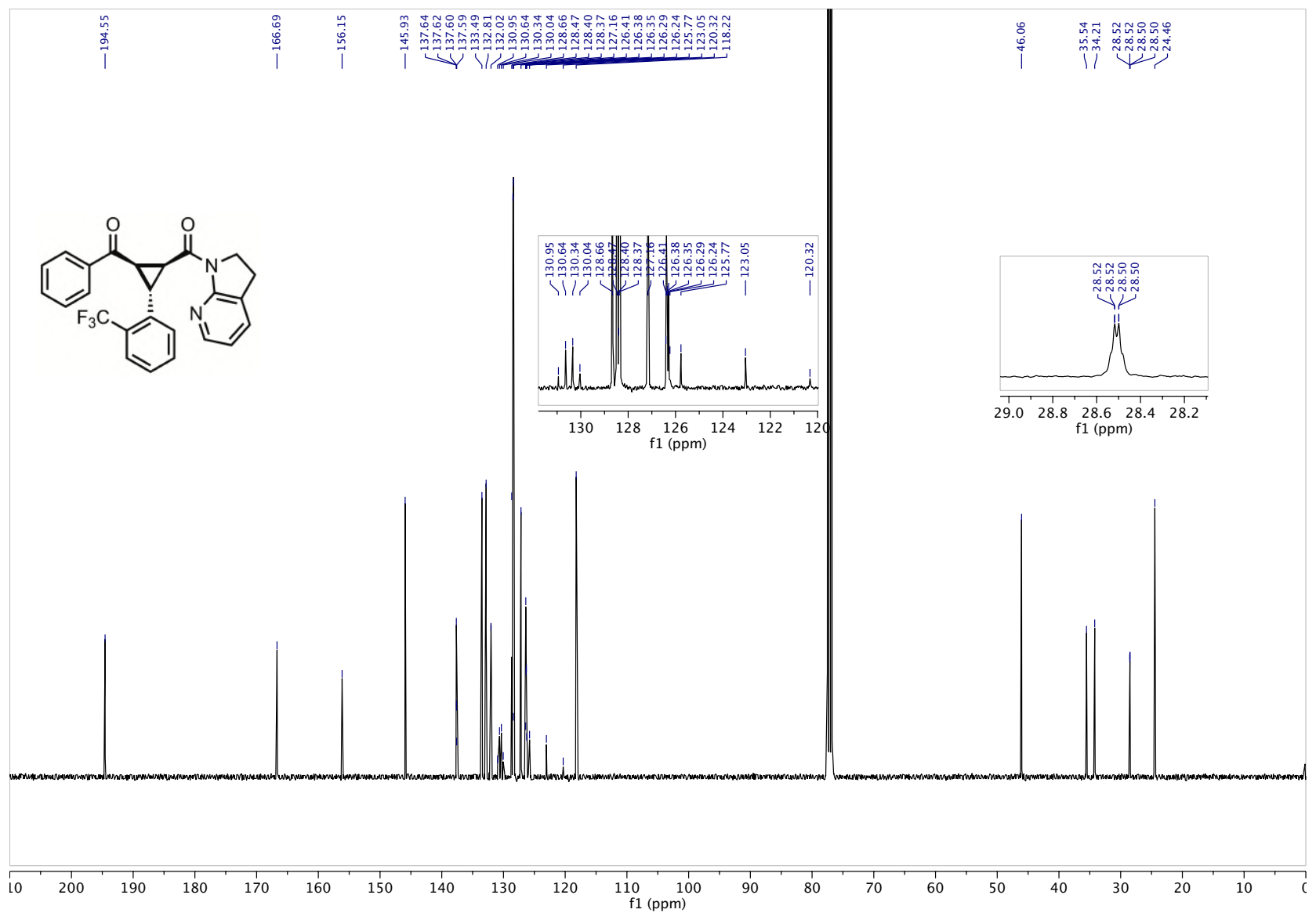


${ }^{19}$ F NMR: 6 av

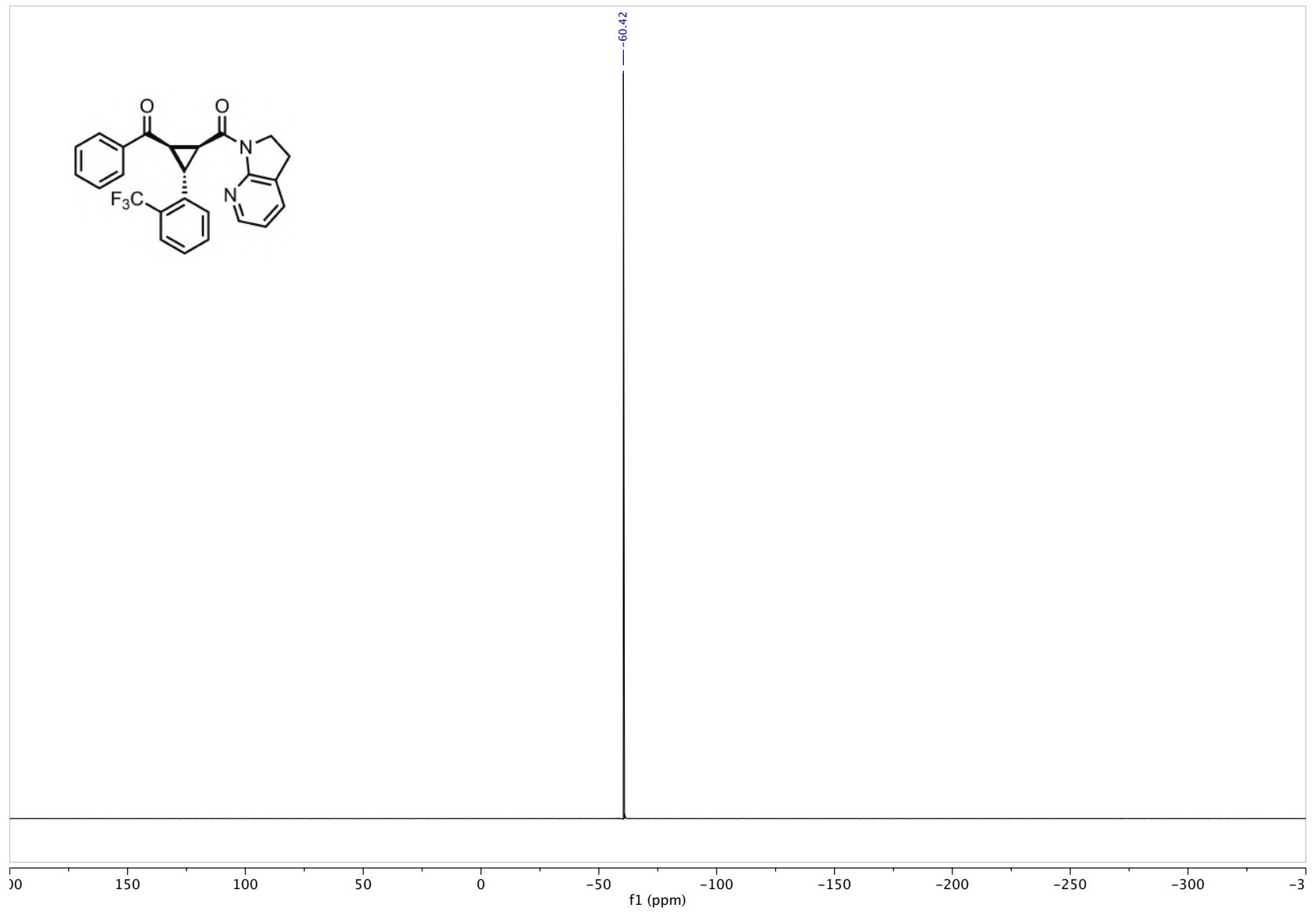


${ }^{1} \mathrm{H}$ NMR: 6aw

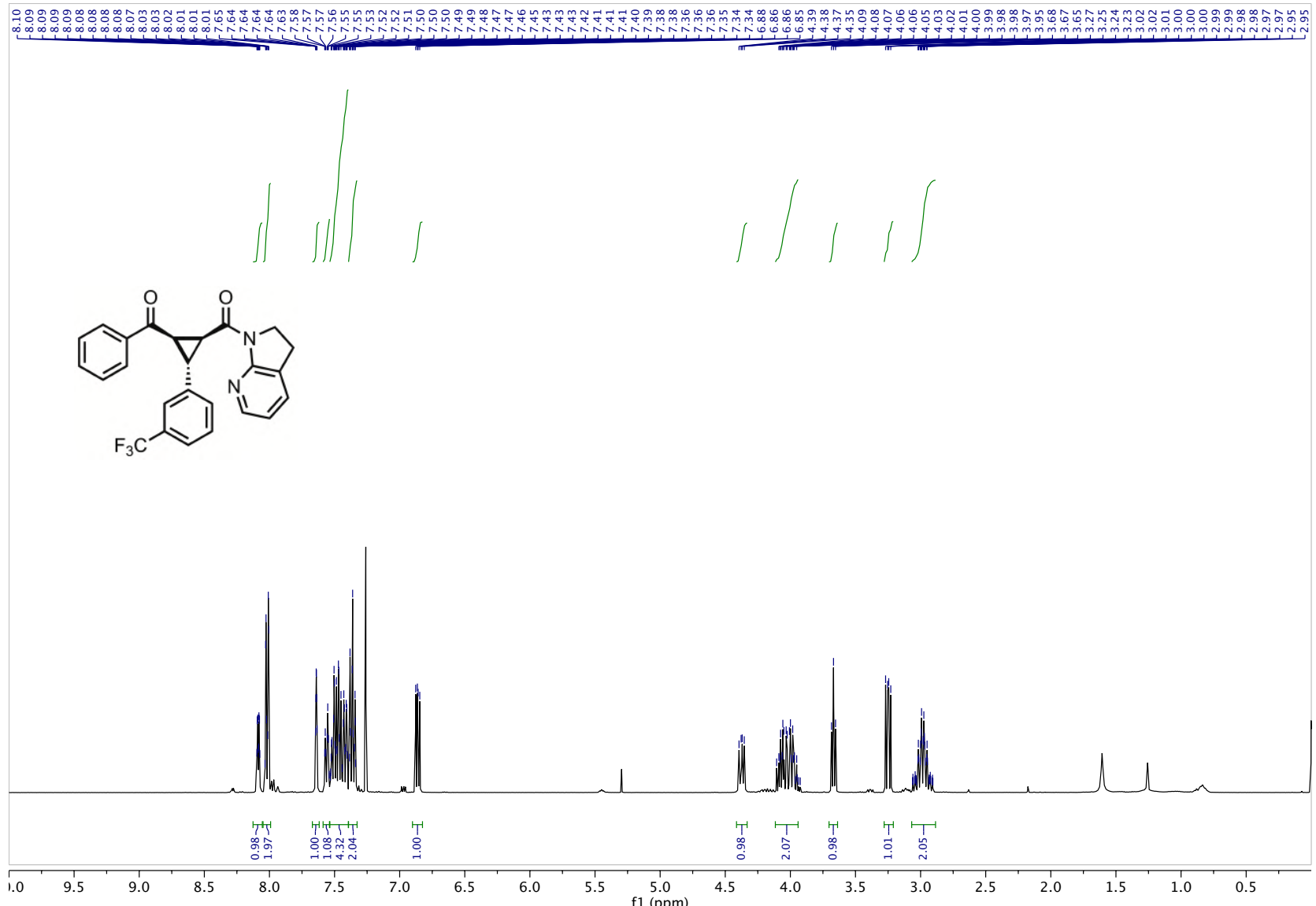

${ }^{13} \mathrm{C}$ NMR: 6aw

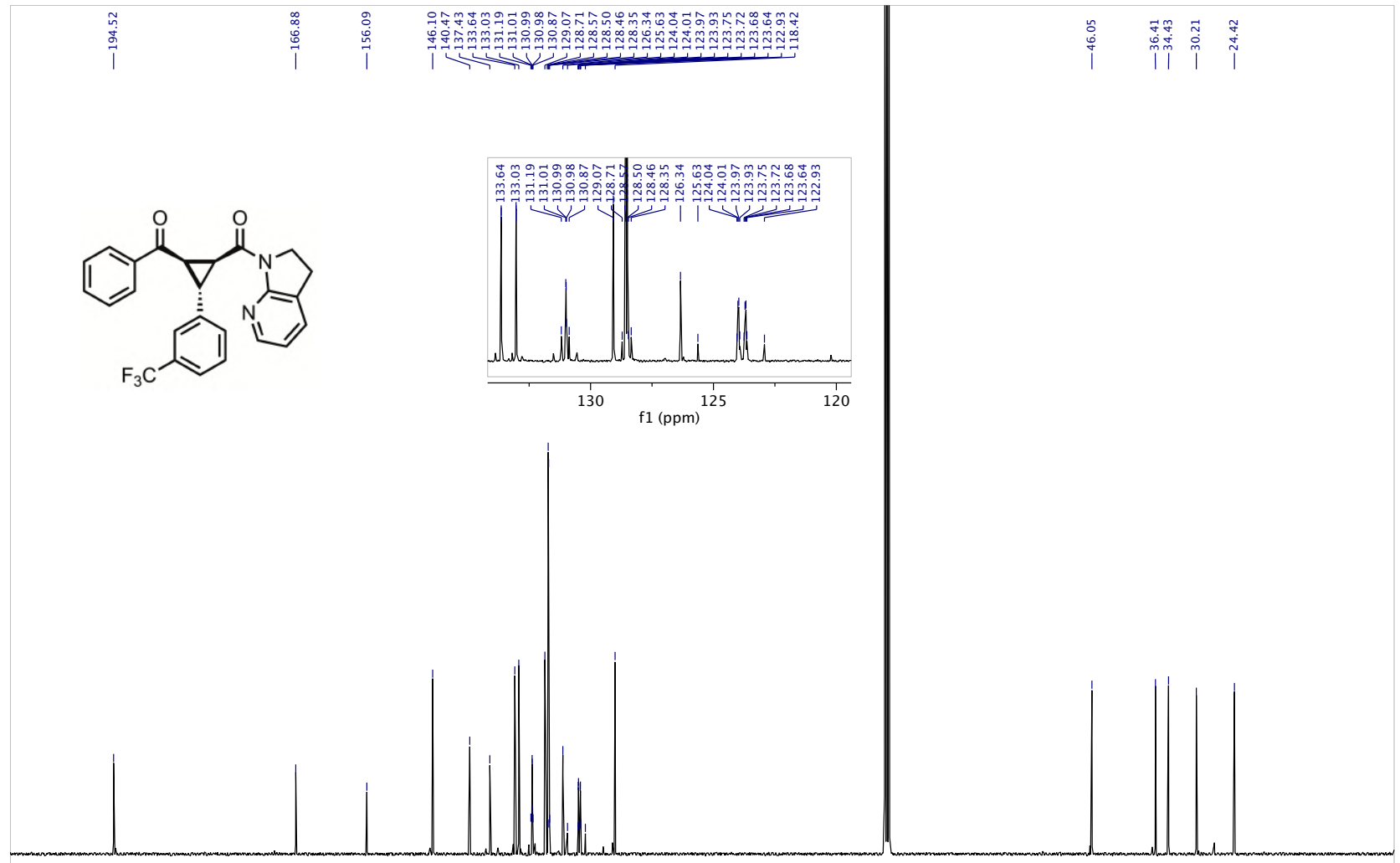

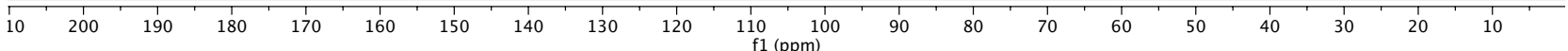




\section{${ }^{19} \mathrm{~F}$ NMR: 6aw}

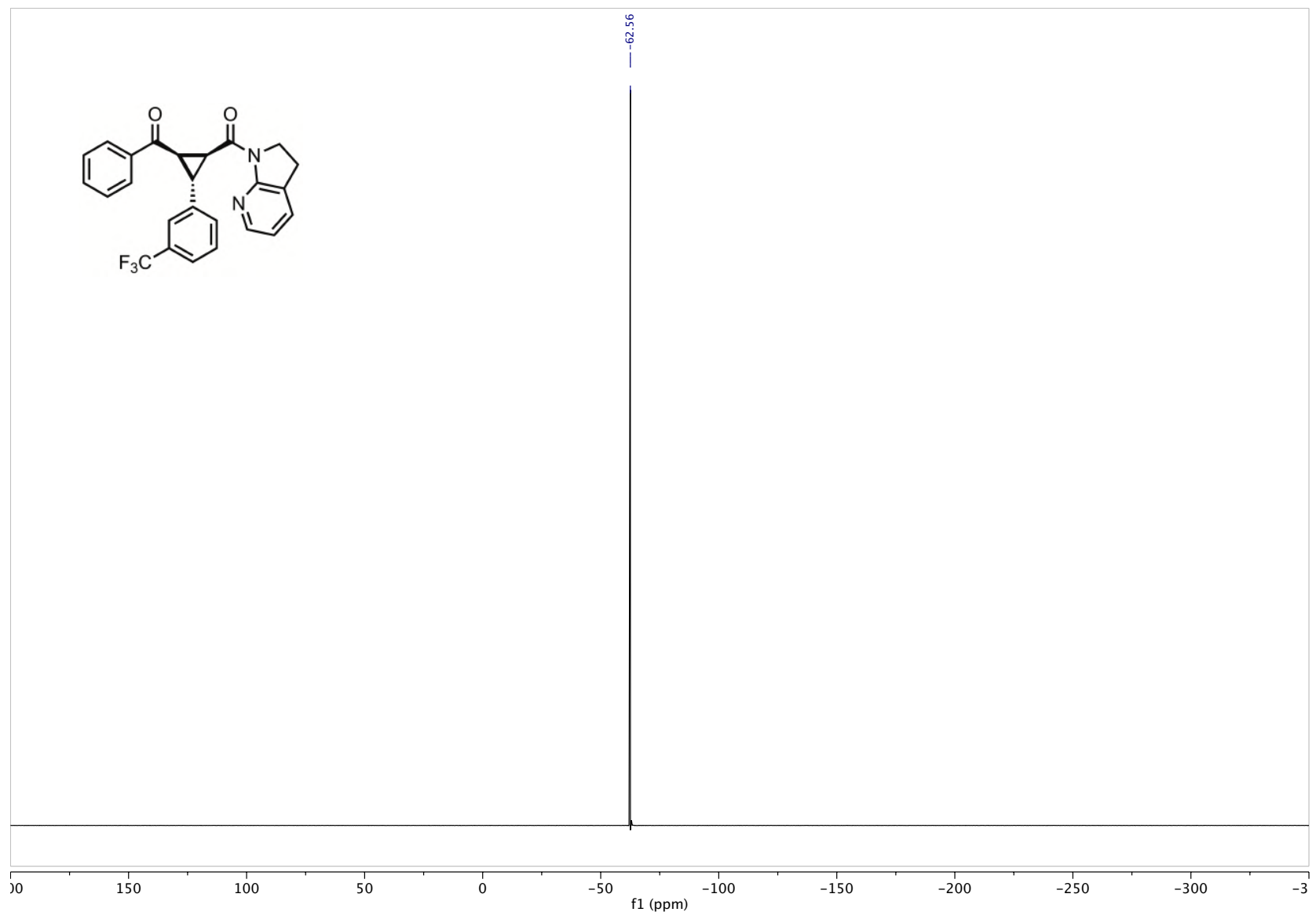


${ }^{1} \mathrm{H}$ NMR: $6 \mathbf{a x}$

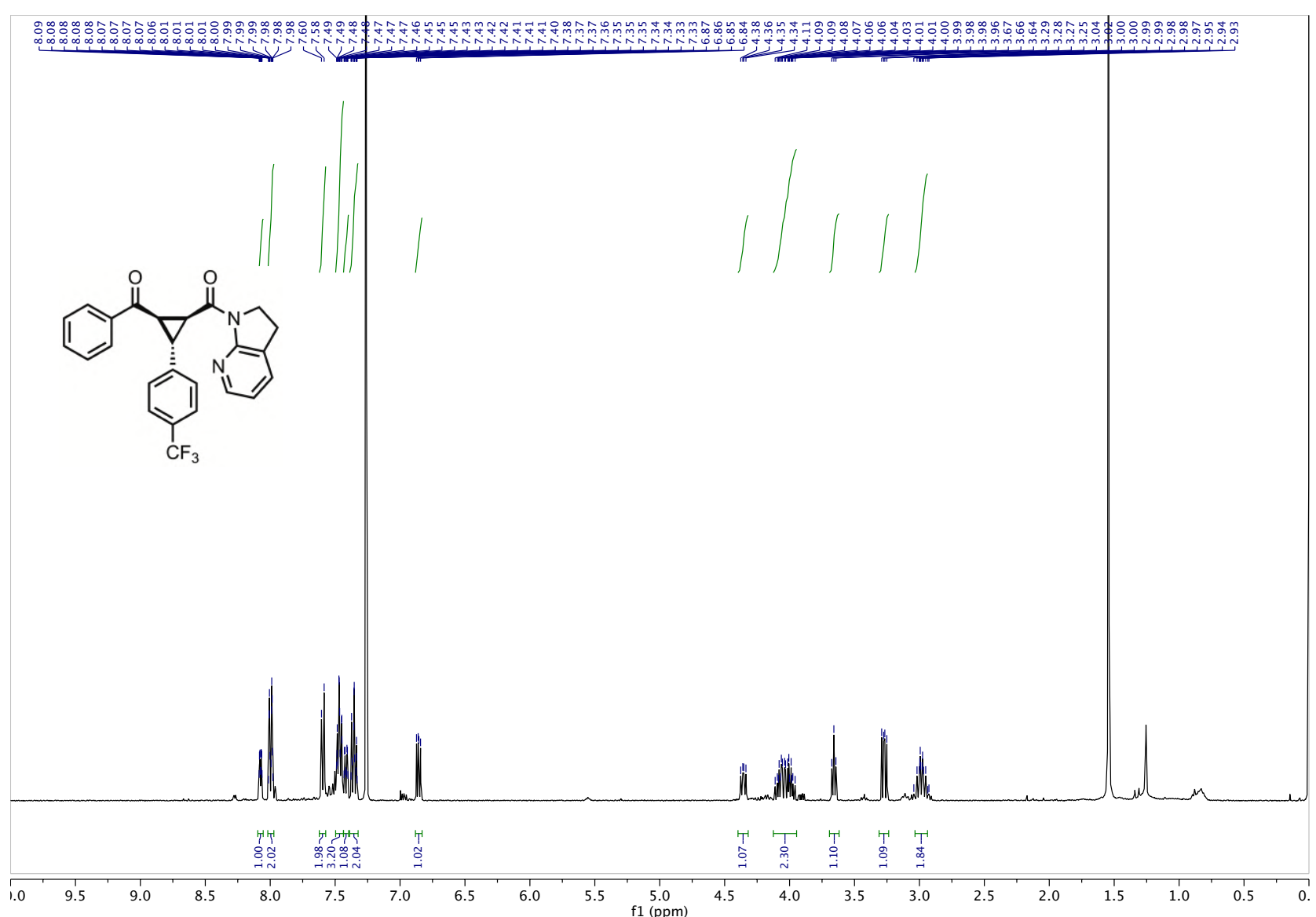

${ }^{13}$ C NMR: $6 a x$

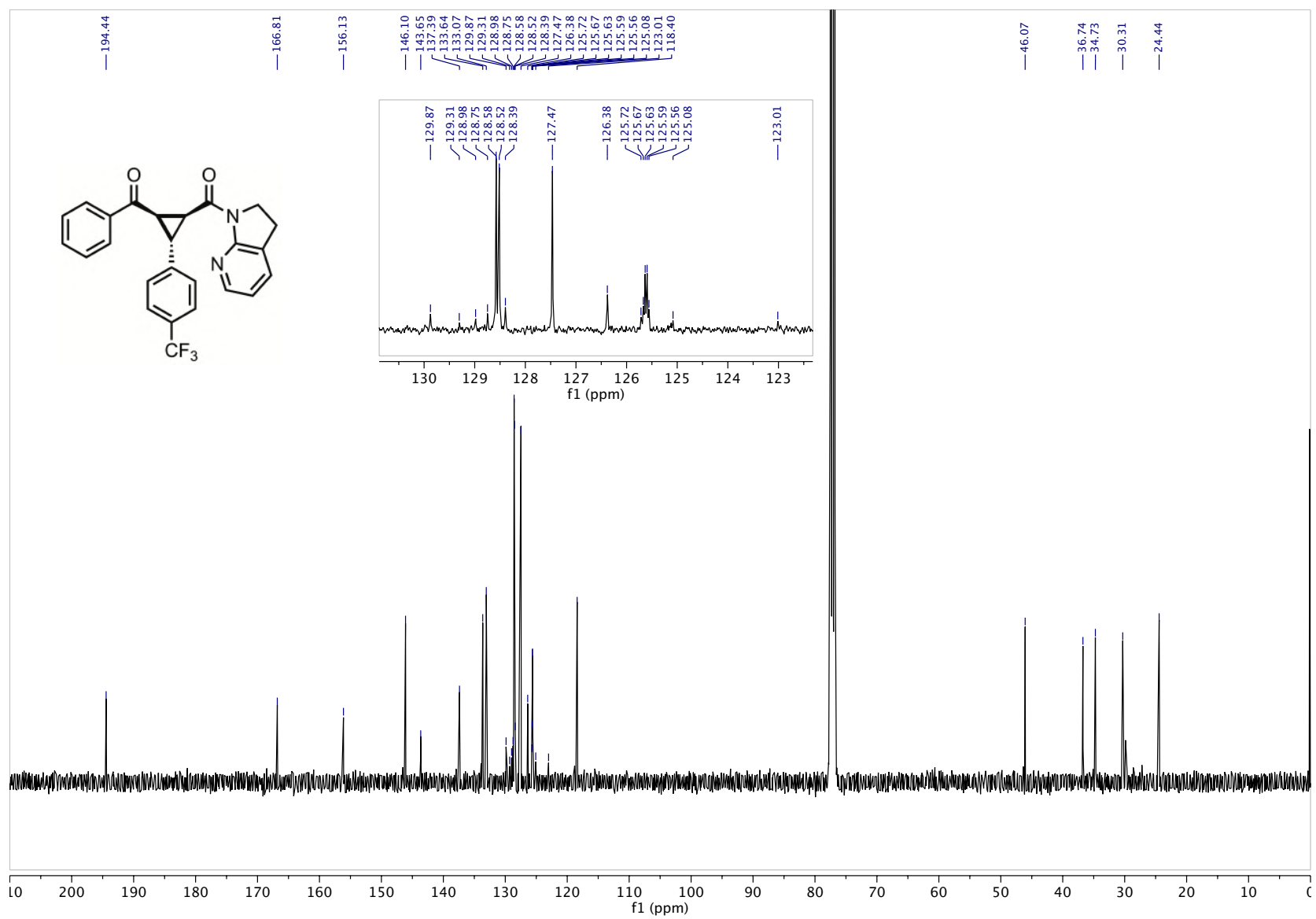


${ }^{19}$ F NMR: $6 \mathbf{a x}$

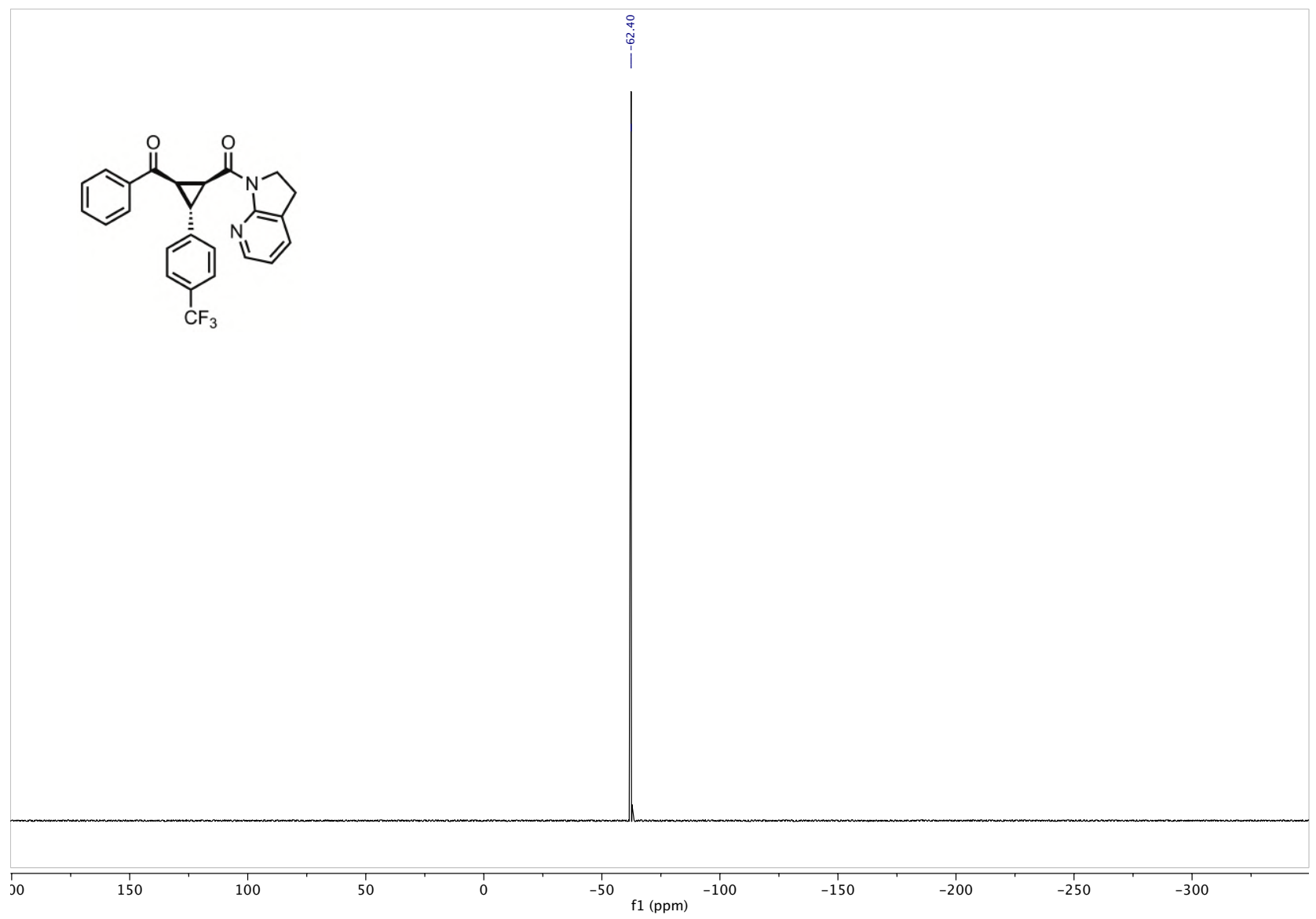


${ }^{1} \mathrm{H}$ NMR: 6ay

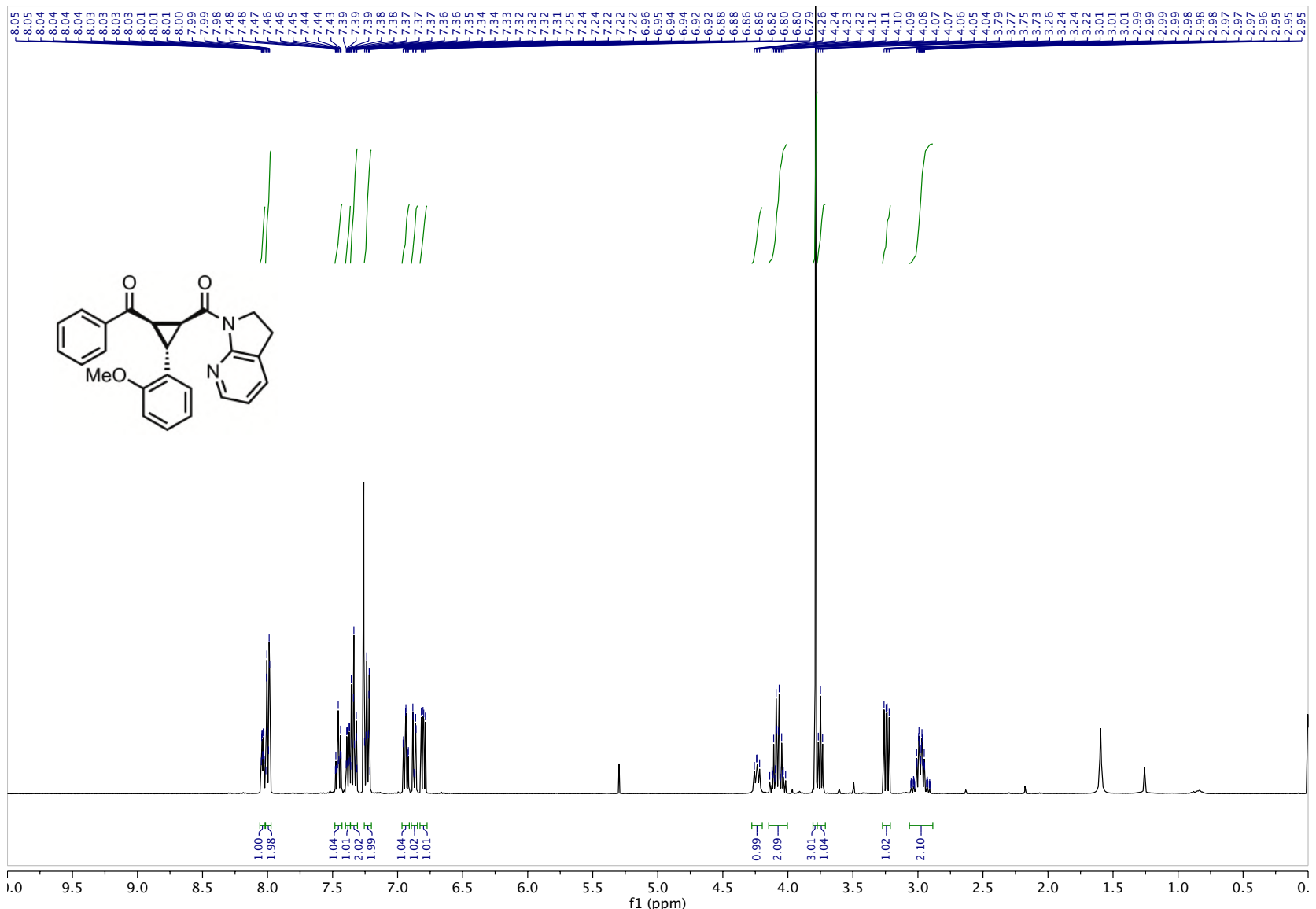

${ }^{13} \mathrm{C}$ NMR: 6ay

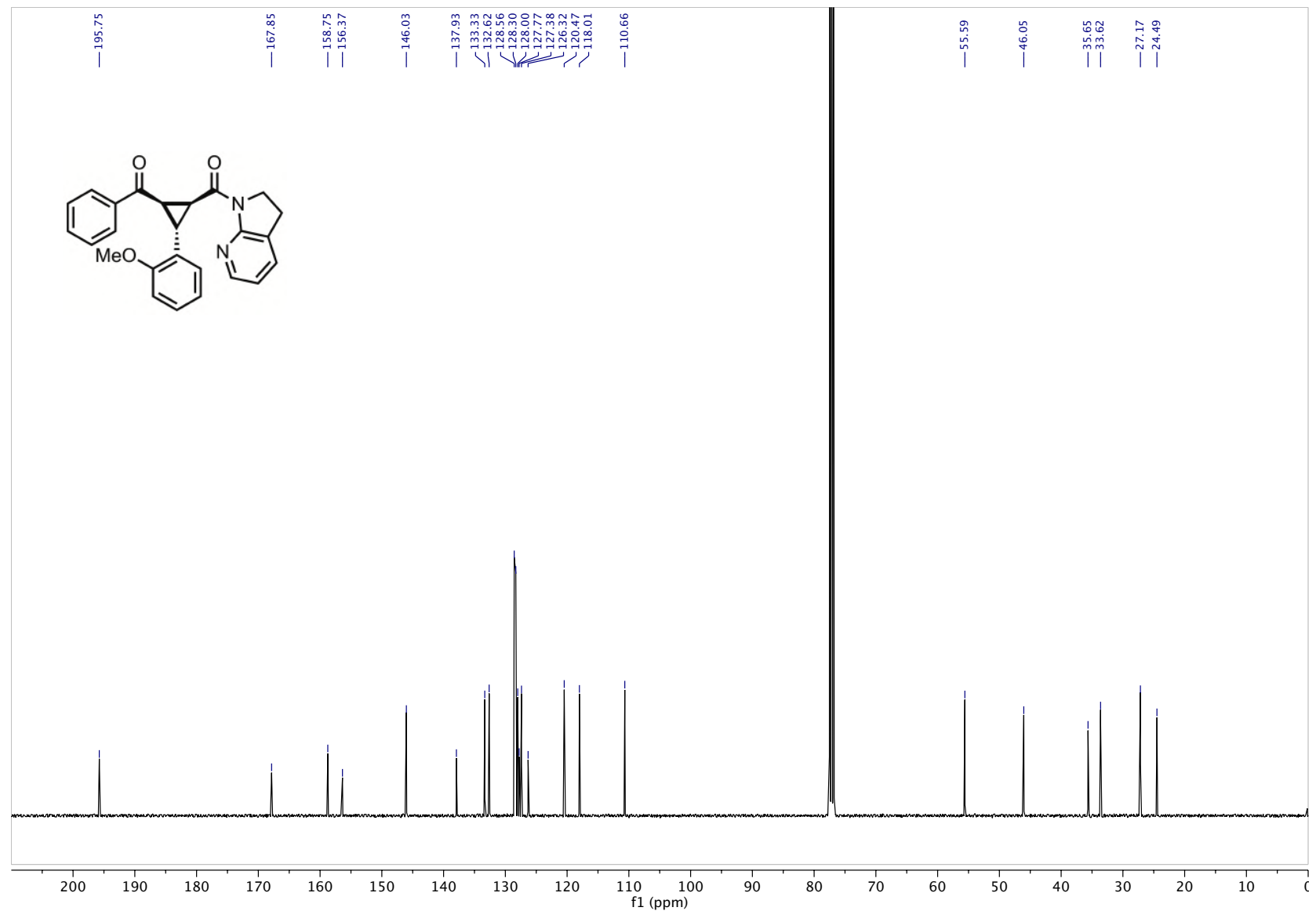


${ }^{1} \mathrm{H}$ NMR: 6az

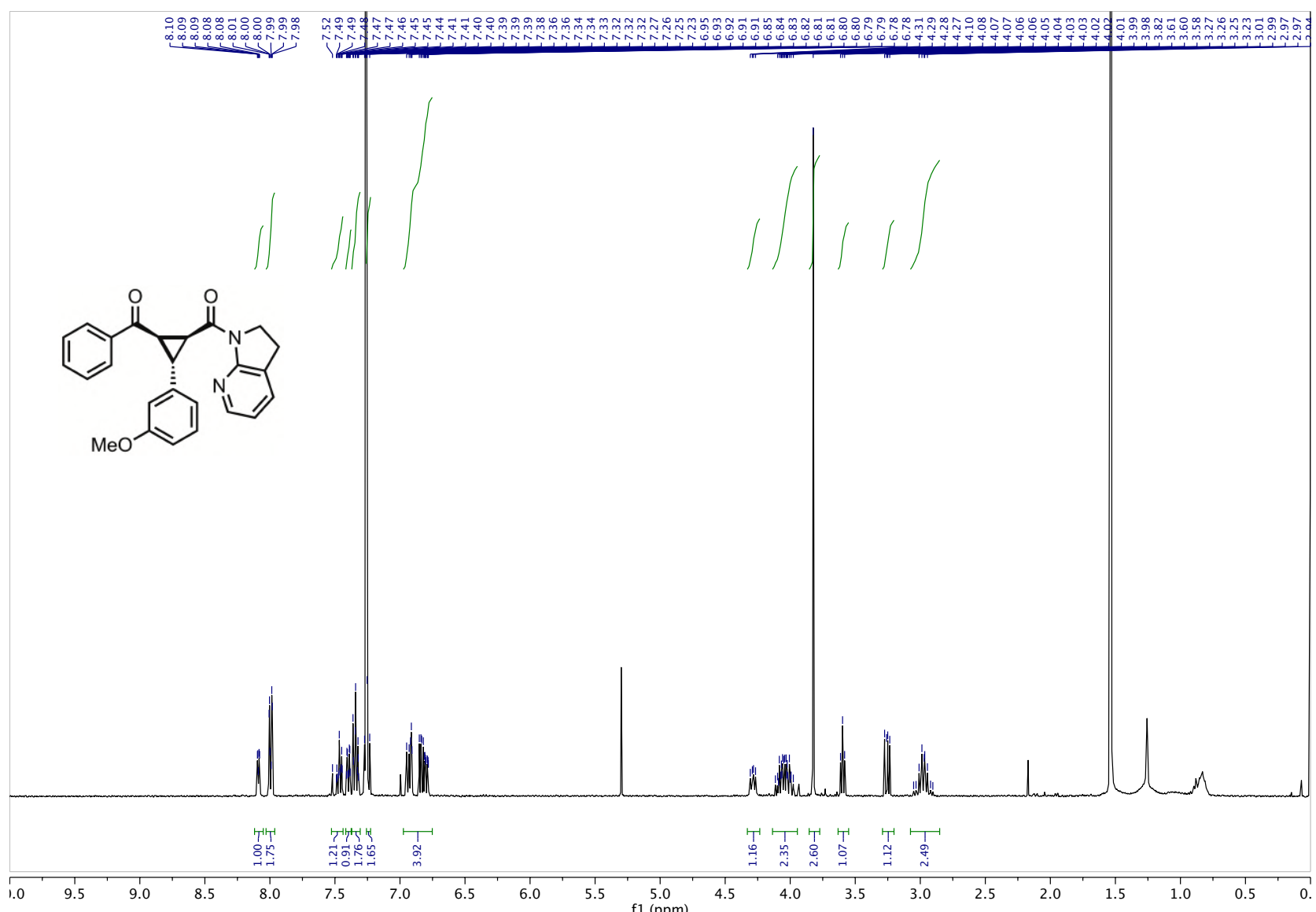

${ }^{13}$ C NMR: $6 \mathbf{a z}$

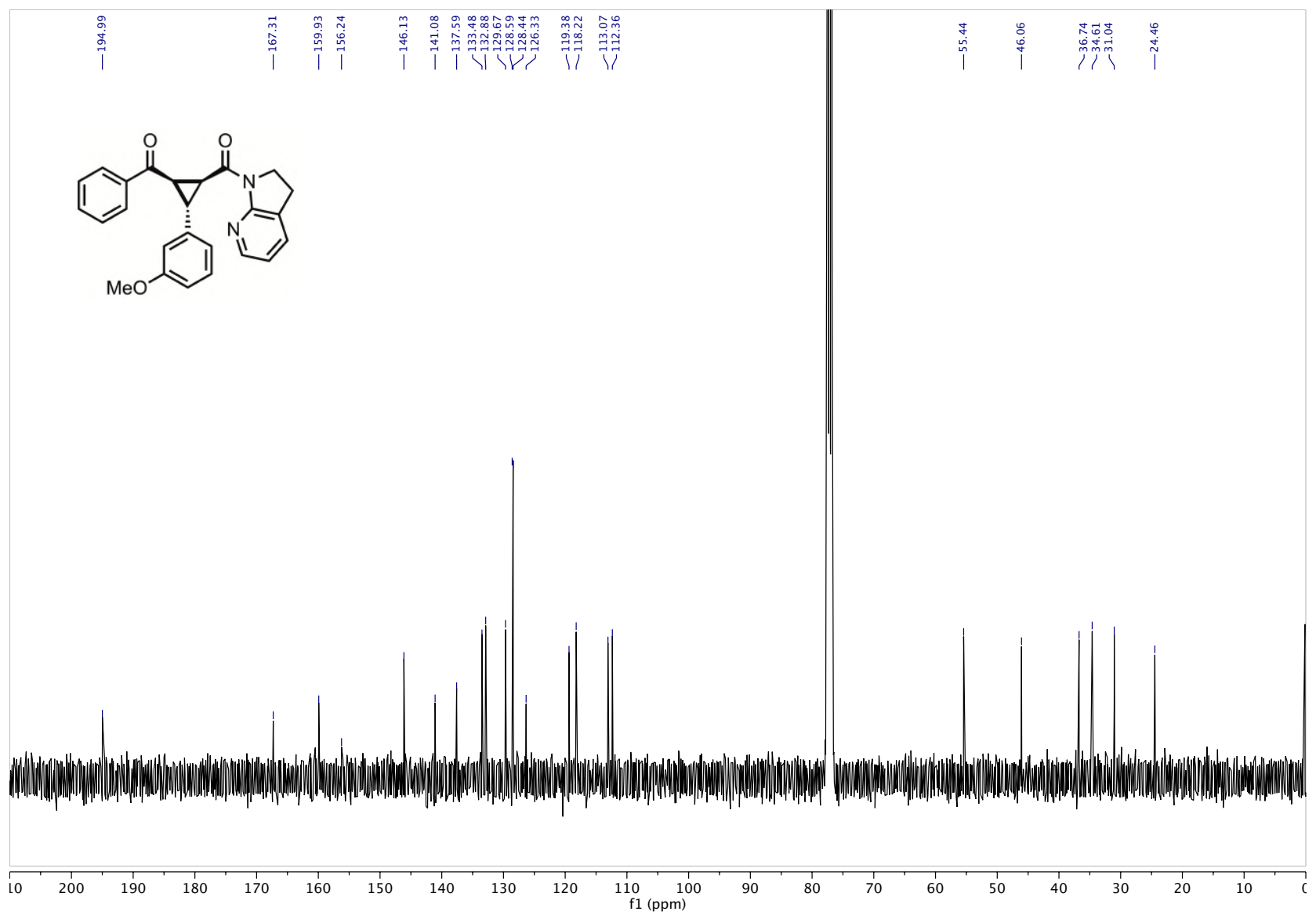


${ }^{1} \mathrm{H}$ NMR: 6aaa

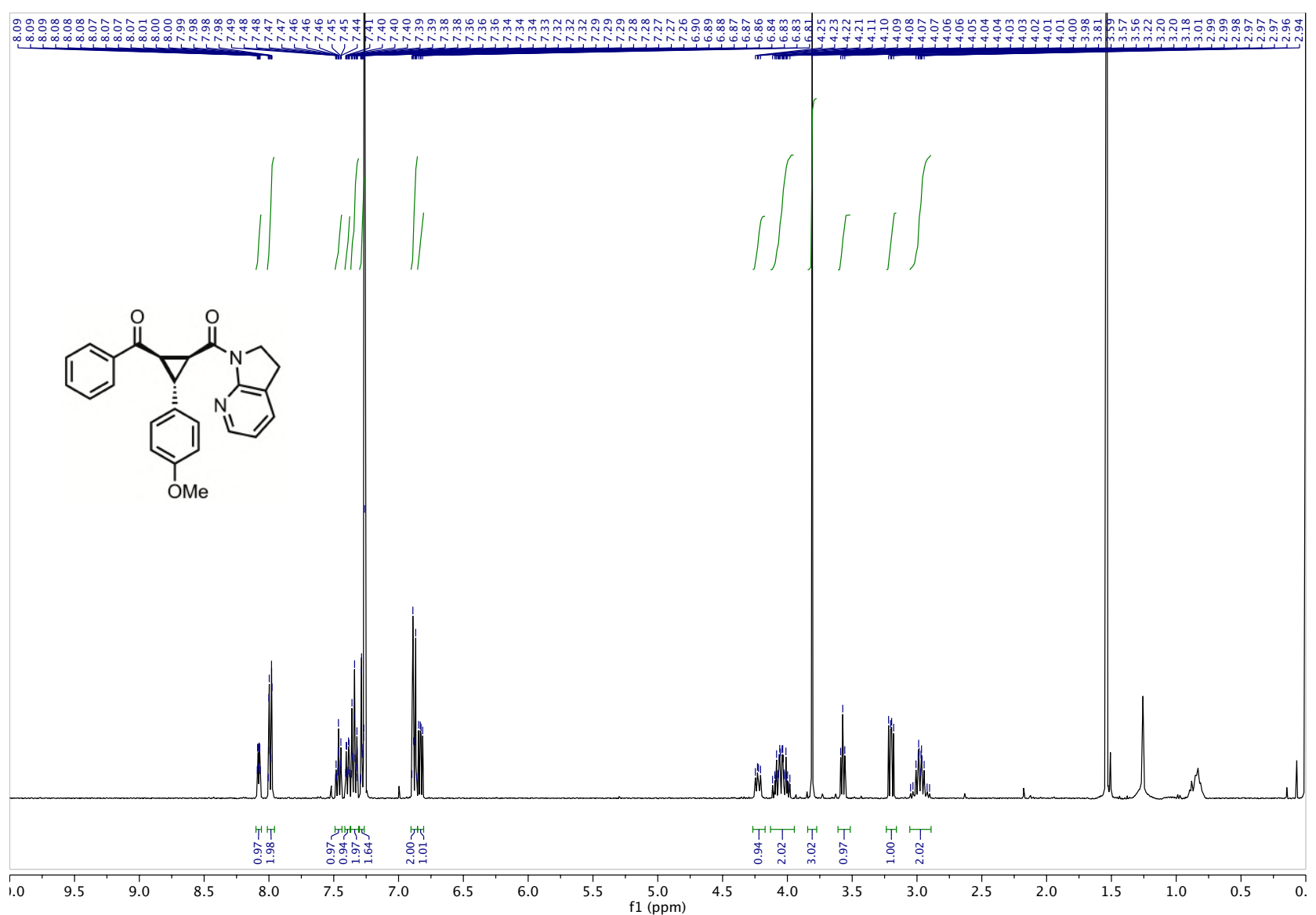

${ }^{13}$ C NMR: 6aaa

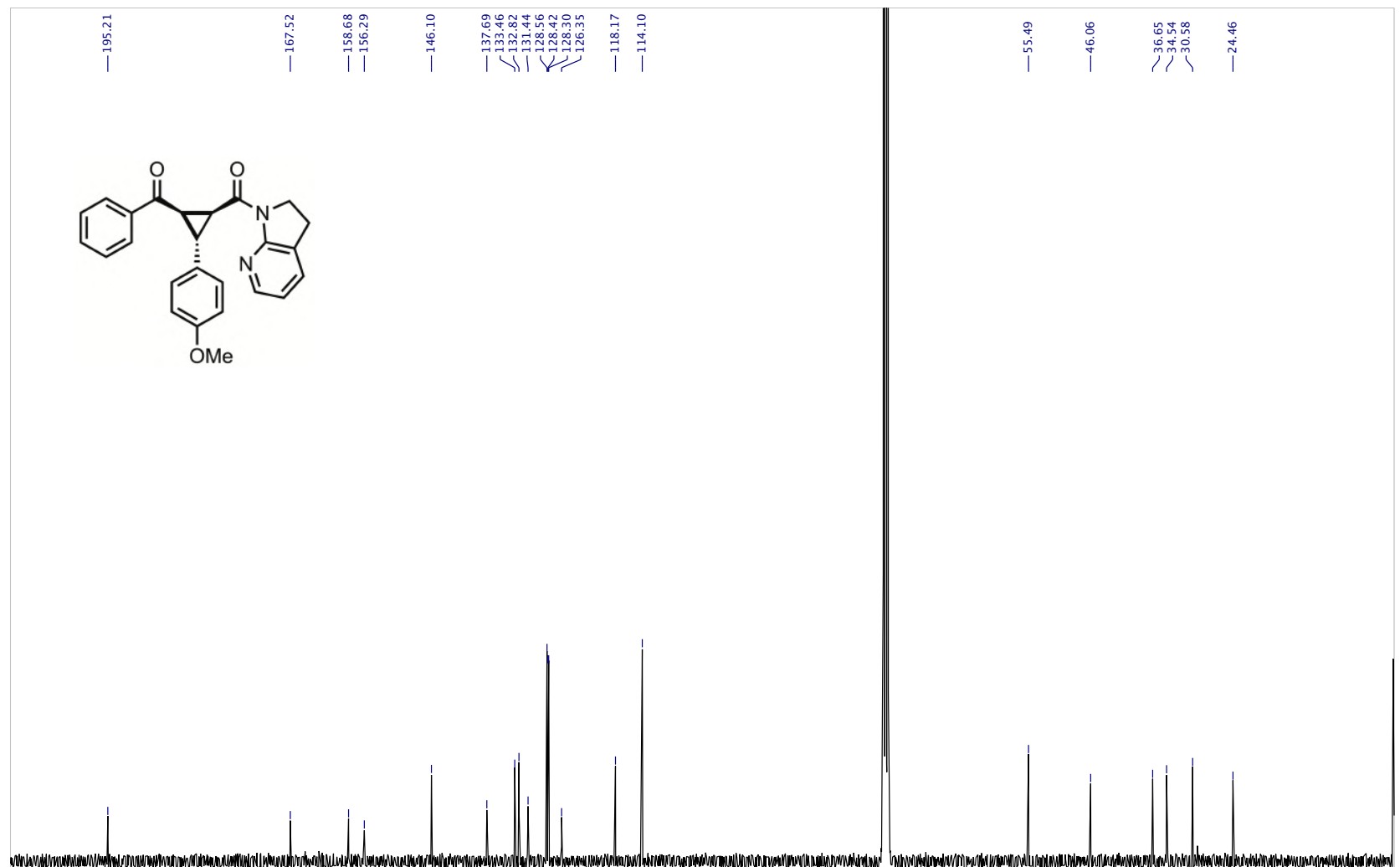

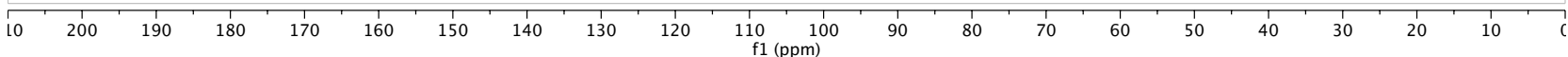


${ }^{1} \mathrm{H}$ NMR: $6 \mathbf{a a b}$

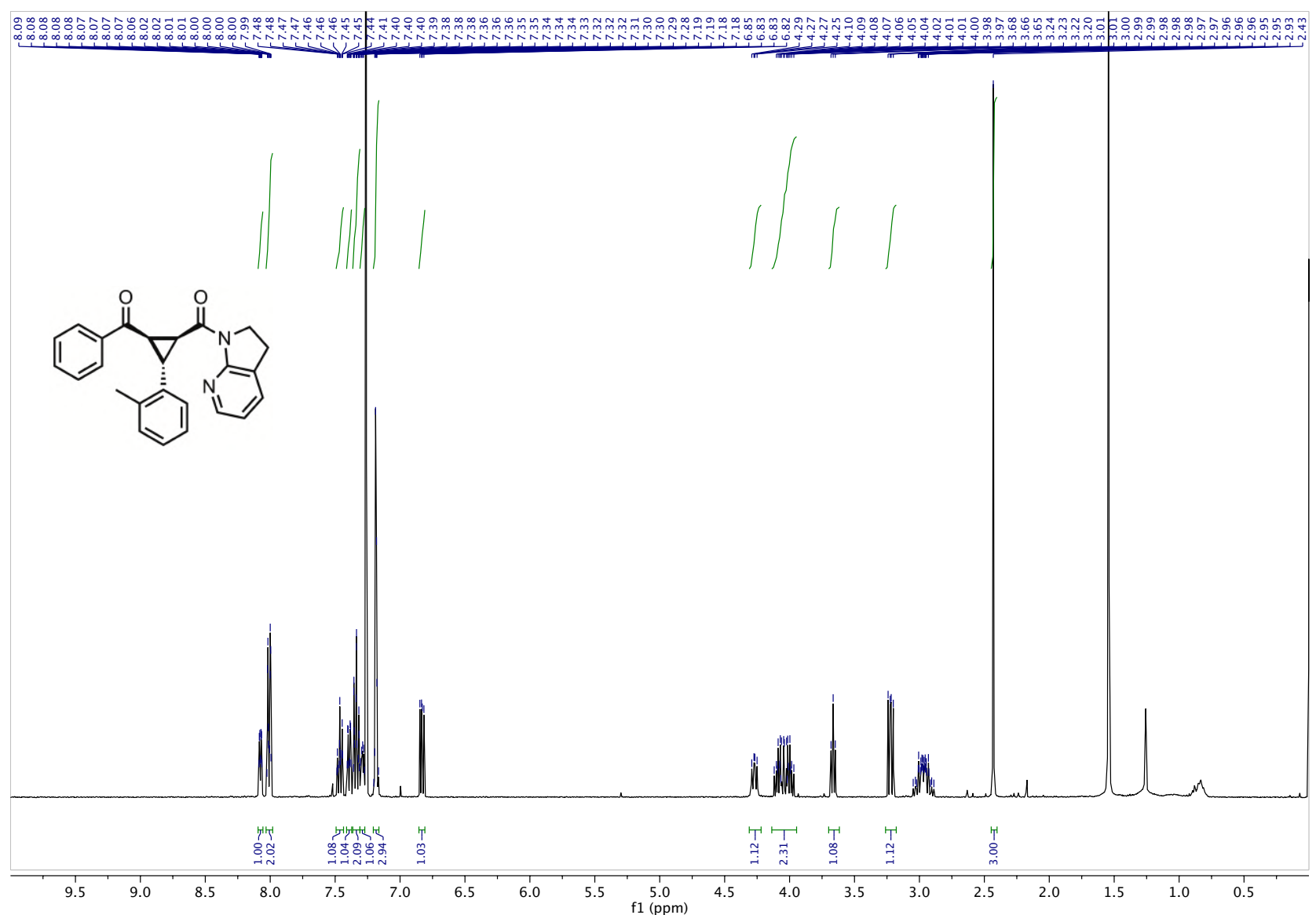

${ }^{13} \mathrm{C}$ NMR: $6 \mathbf{a a b}$

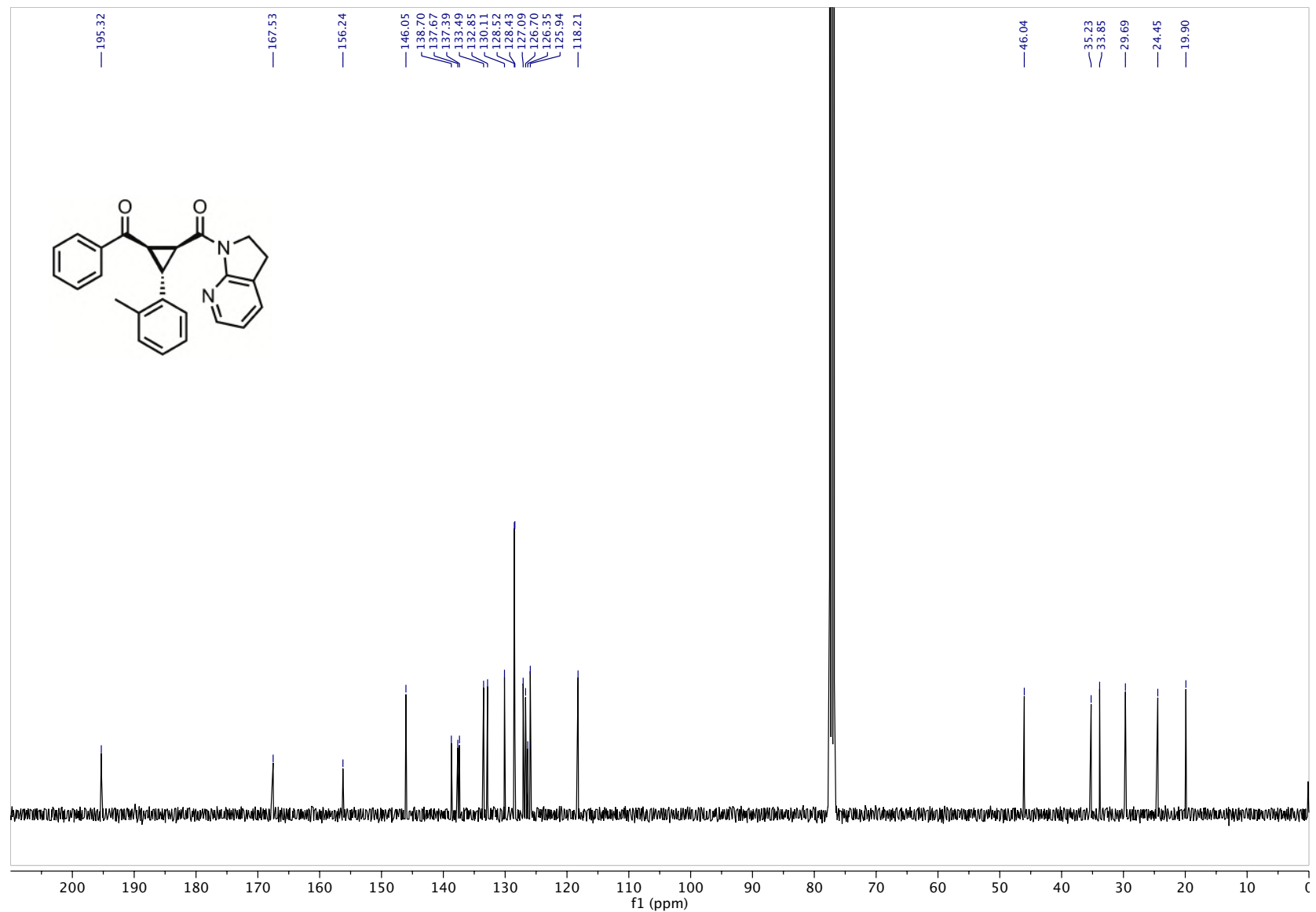


${ }^{1} \mathrm{H}$ NMR: 6aac

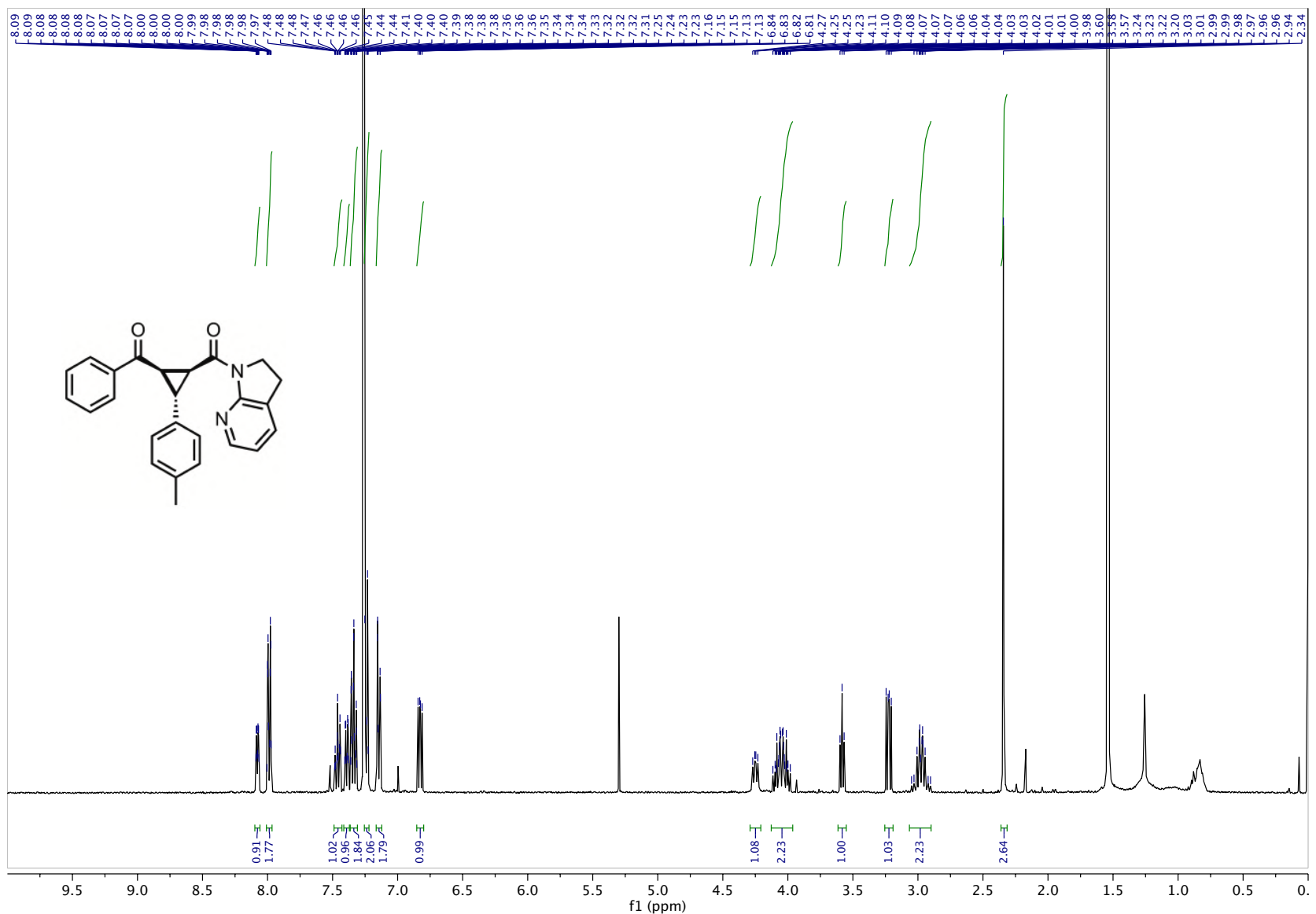

${ }^{13}$ C NMR: 6aac

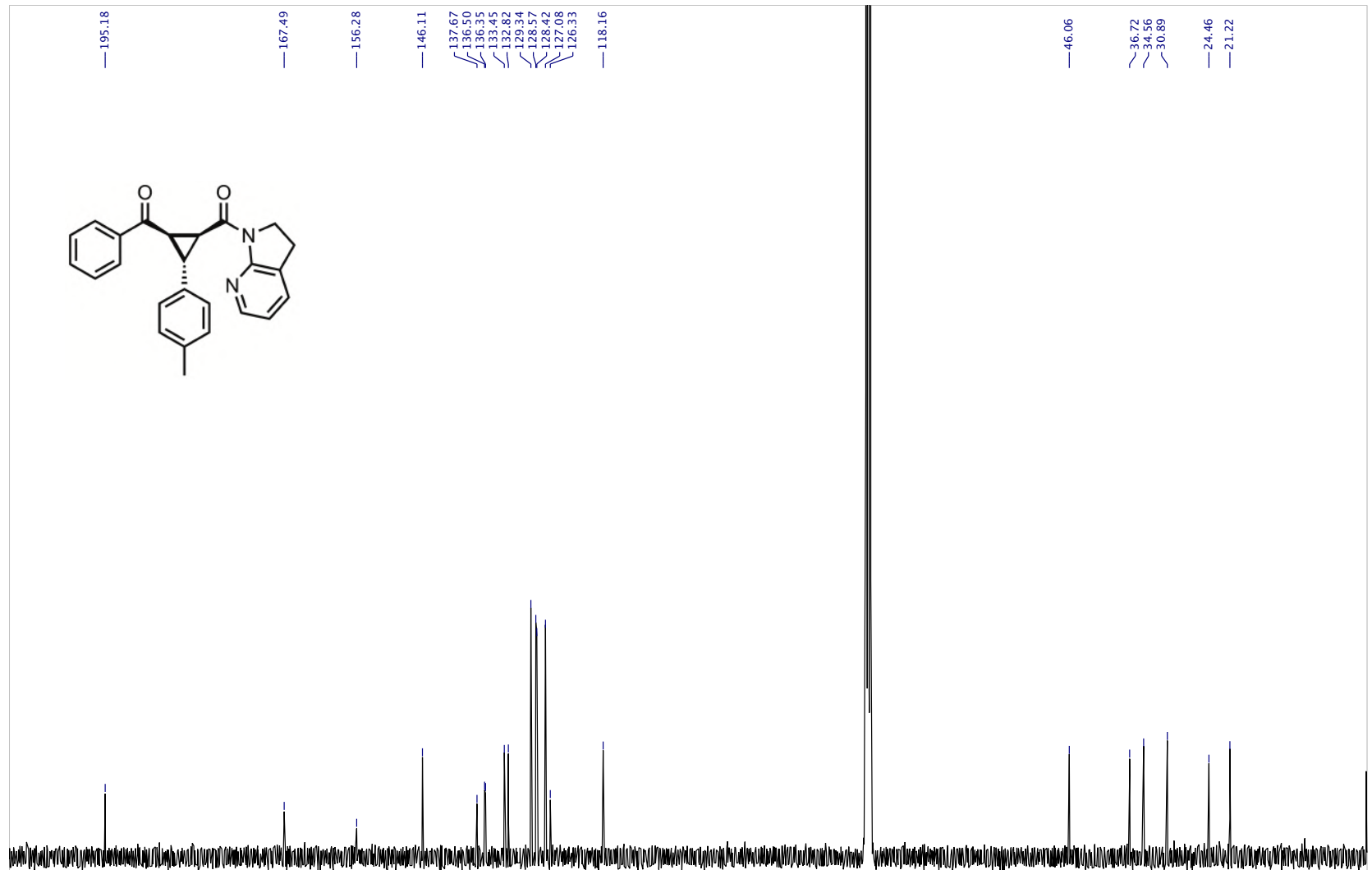

10

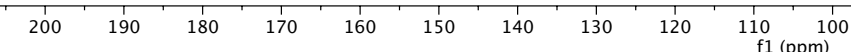


${ }^{1} \mathrm{H}$ NMR: 6aad

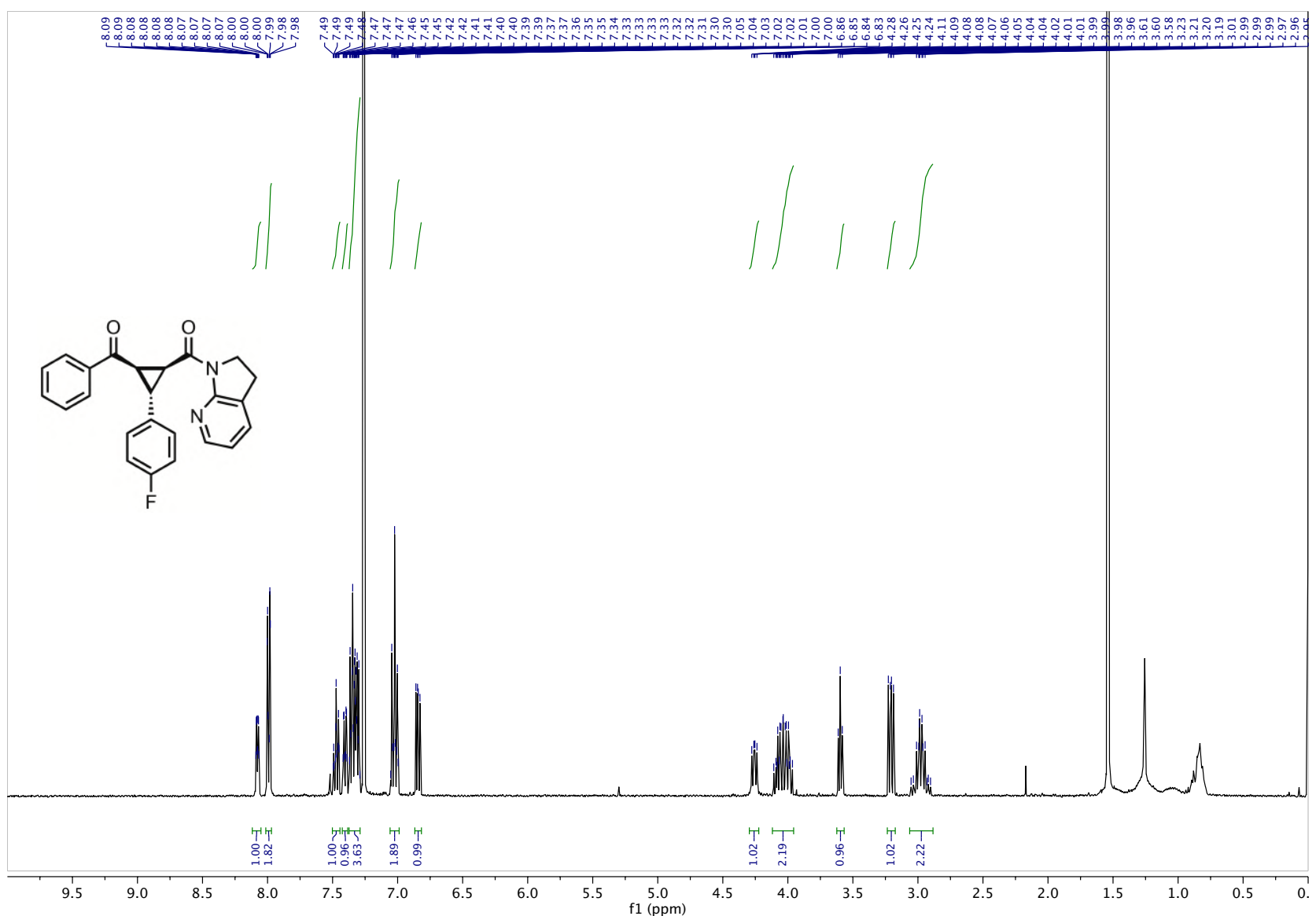

${ }^{13} \mathrm{C}$ NMR: 6aad

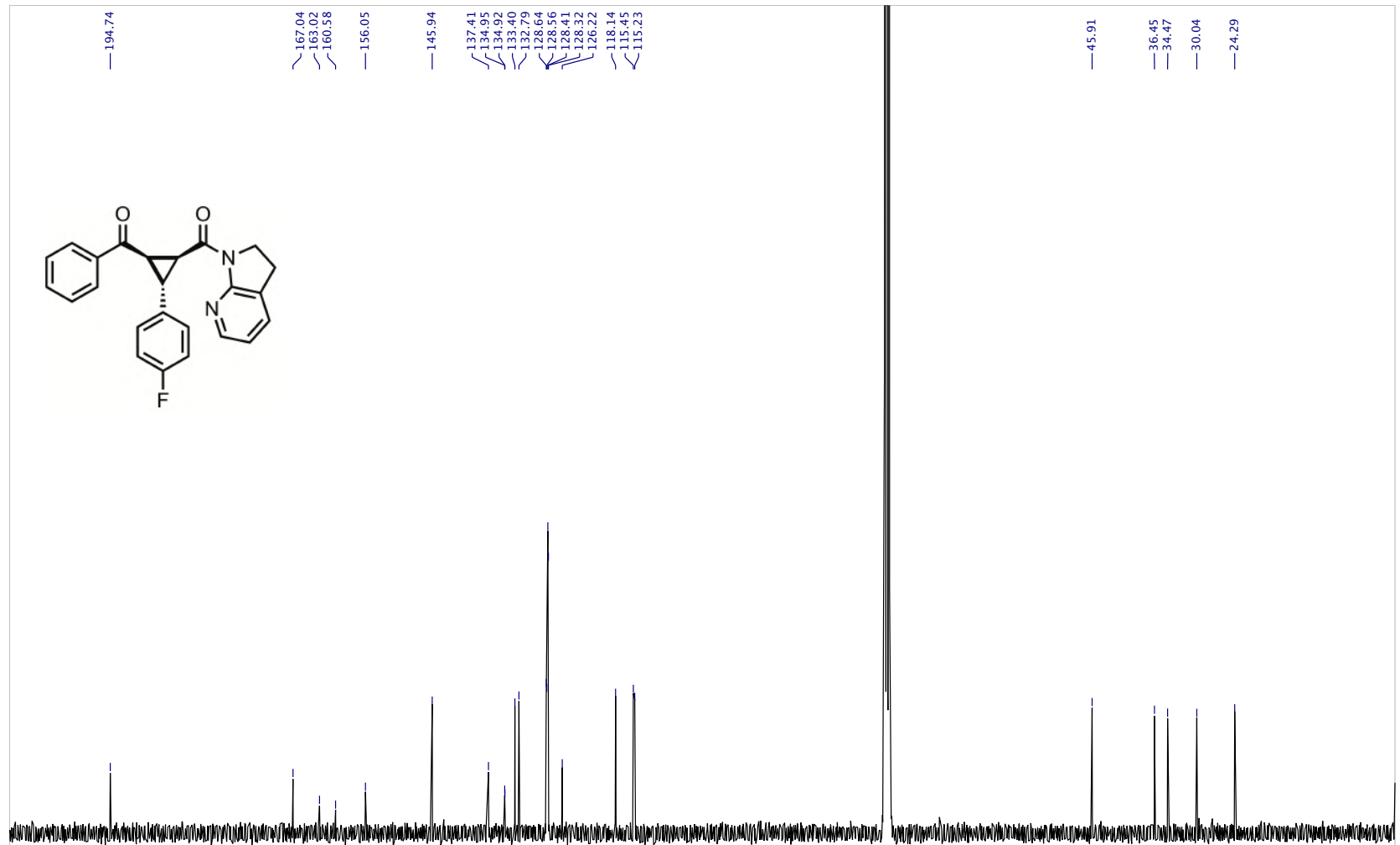

\begin{tabular}{rrrrrrrrrrr}
\hline 10 & 200 & 190 & 180 & 170 & 160 & 150 & 140 & 130 & 120 & 110 \\
$\mathrm{f} 1(\mathrm{ppm})$
\end{tabular} 
${ }^{19}$ F NMR: 6aad

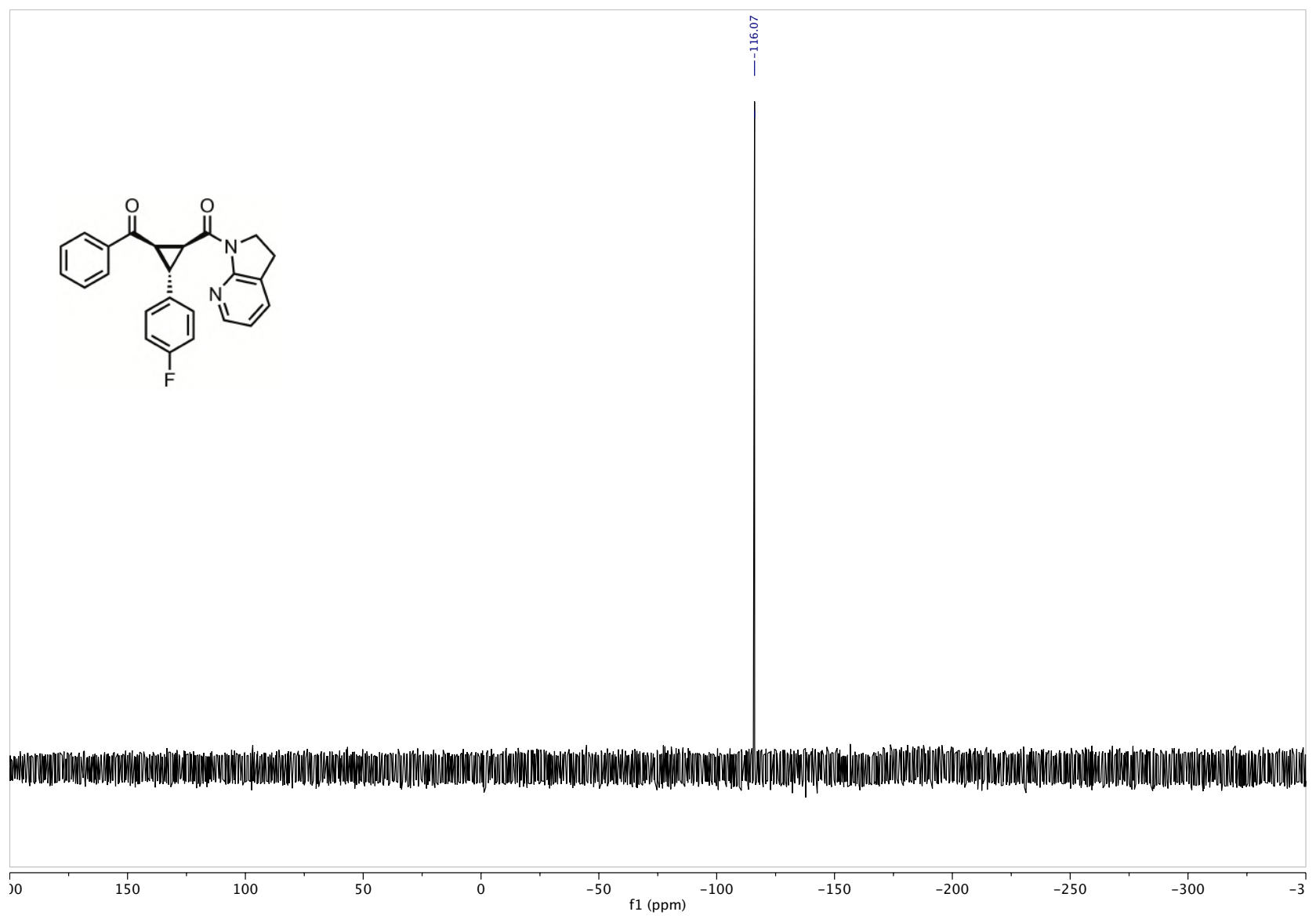


${ }^{1} \mathrm{H}$ NMR: 6aae

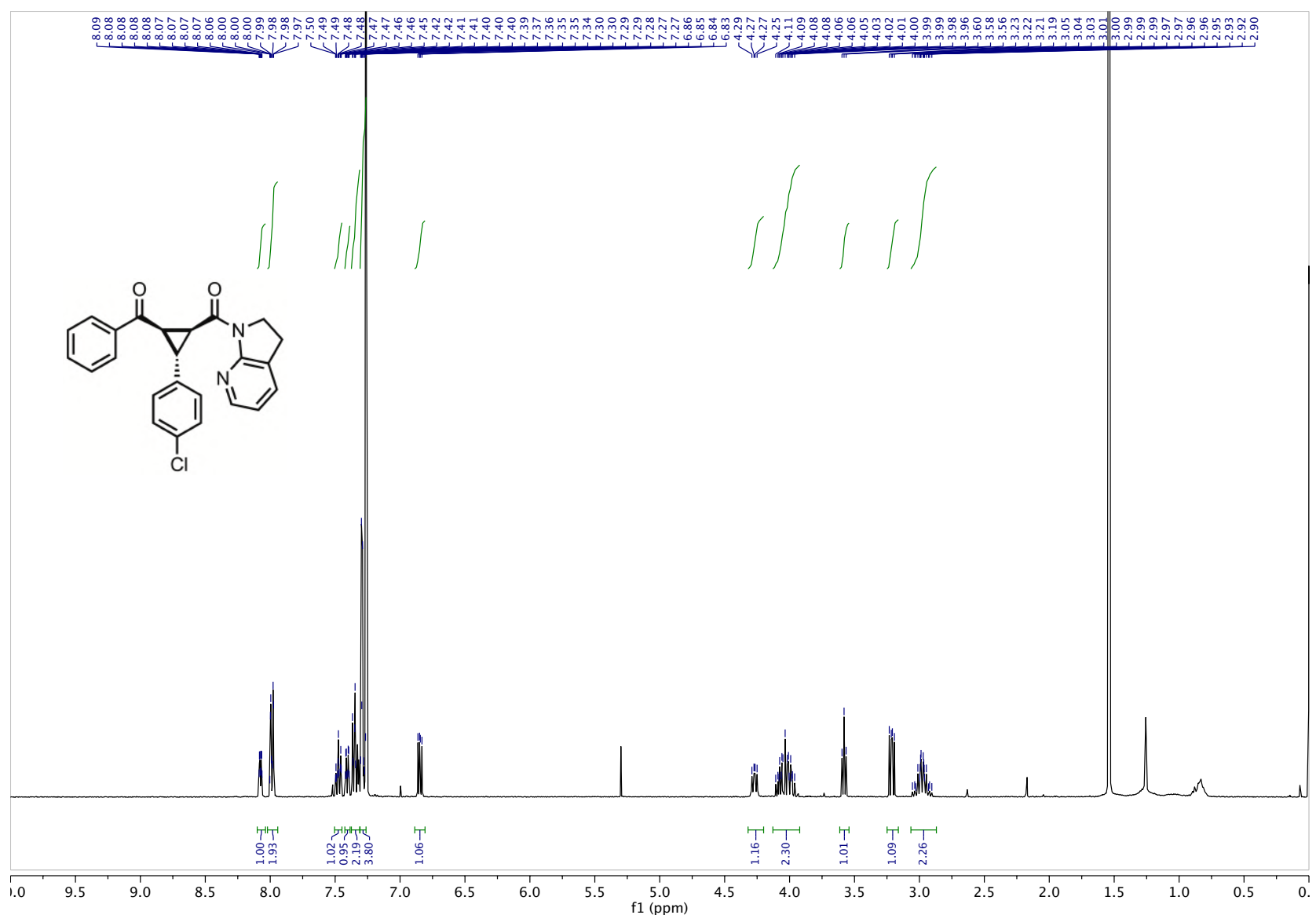

${ }^{13}$ C NMR: 6aae

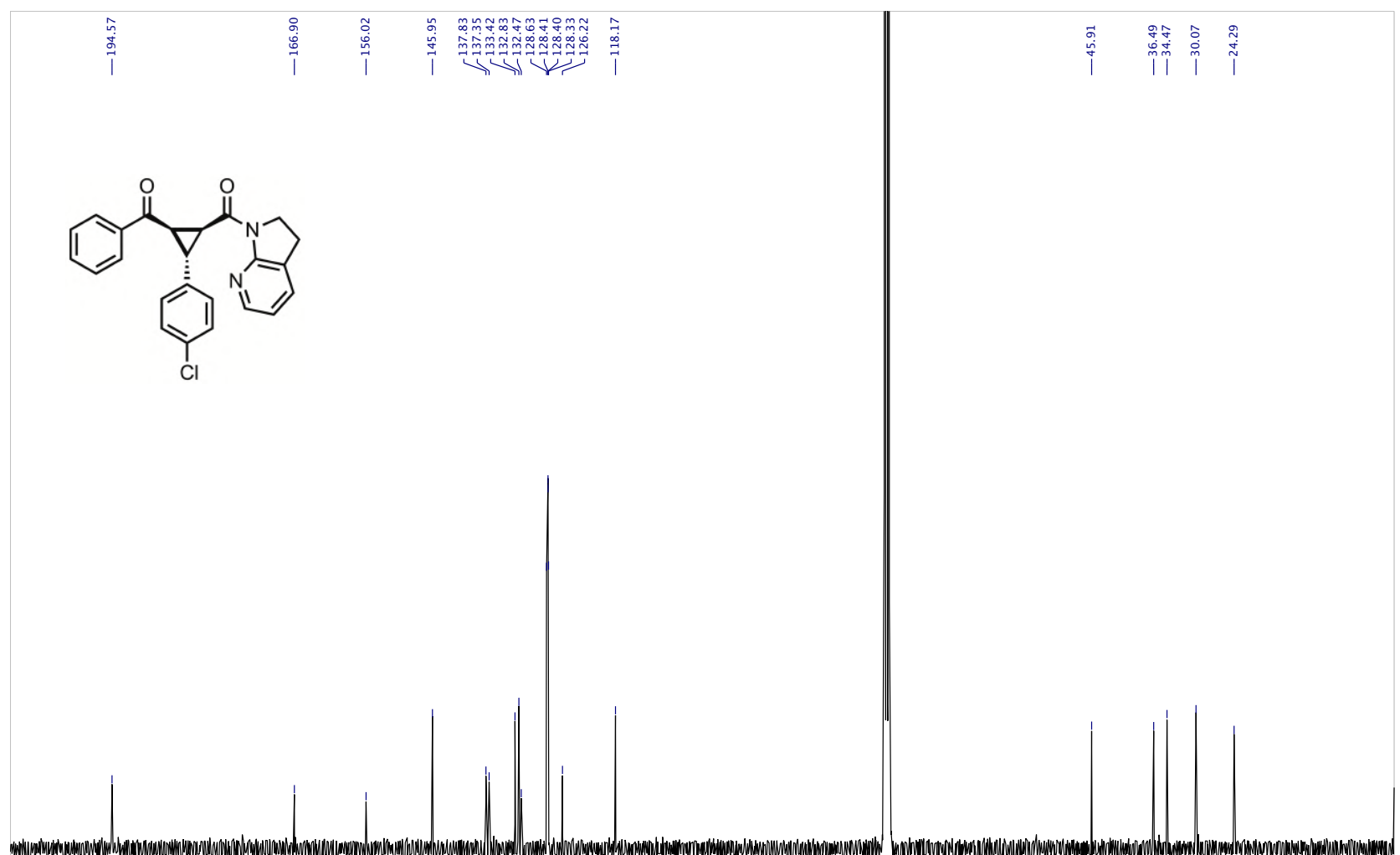

$\begin{array}{lllllllllll}10 & 200 & 190 & 180 & 170 & 160 & 150 & 140 & 130 & 120 & 110 \\ \mathrm{f} 1(\mathrm{ppm})\end{array}$ 
${ }^{1} \mathrm{H}$ NMR: 6aaf

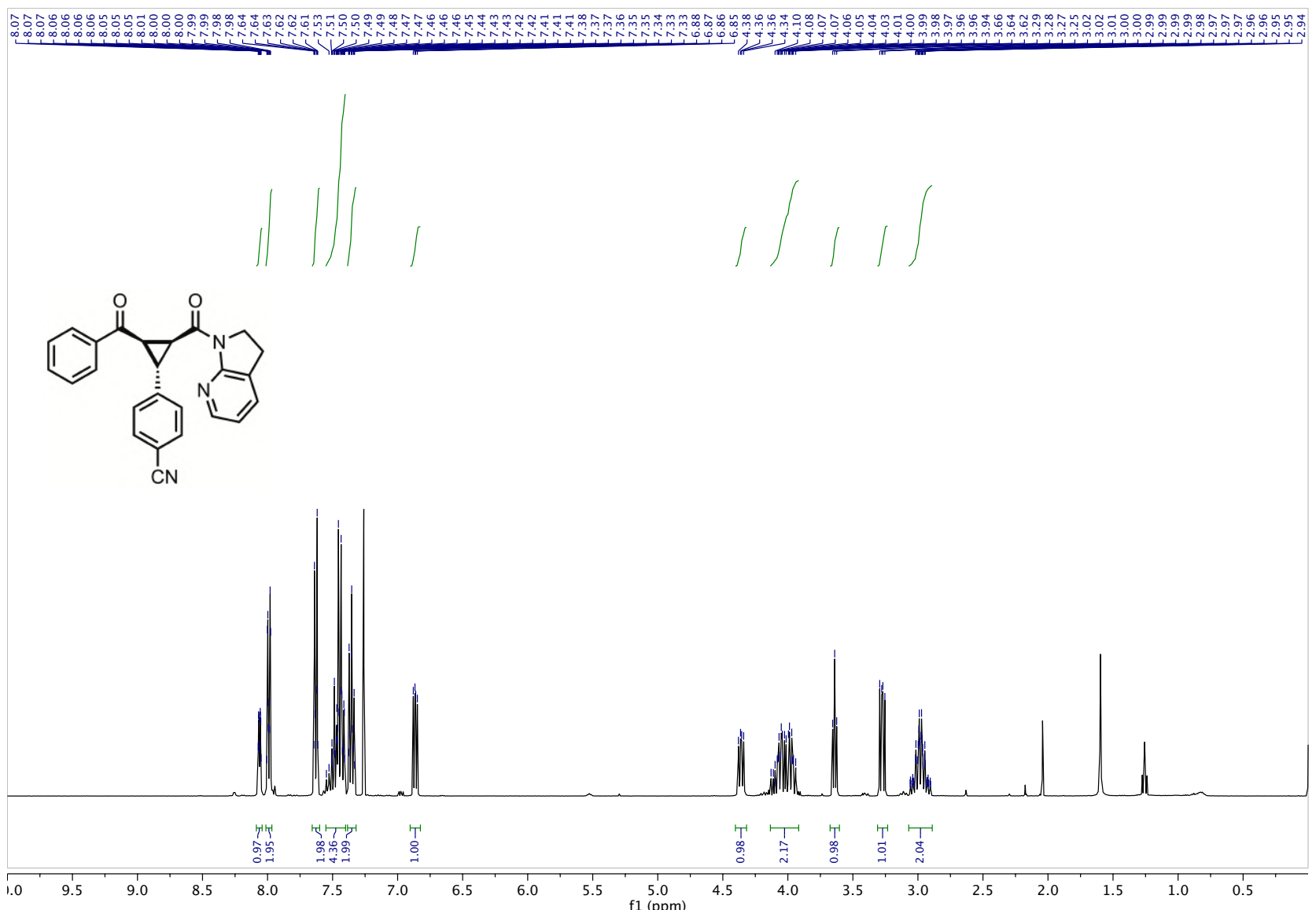

${ }^{13}$ C NMR: 6aaf

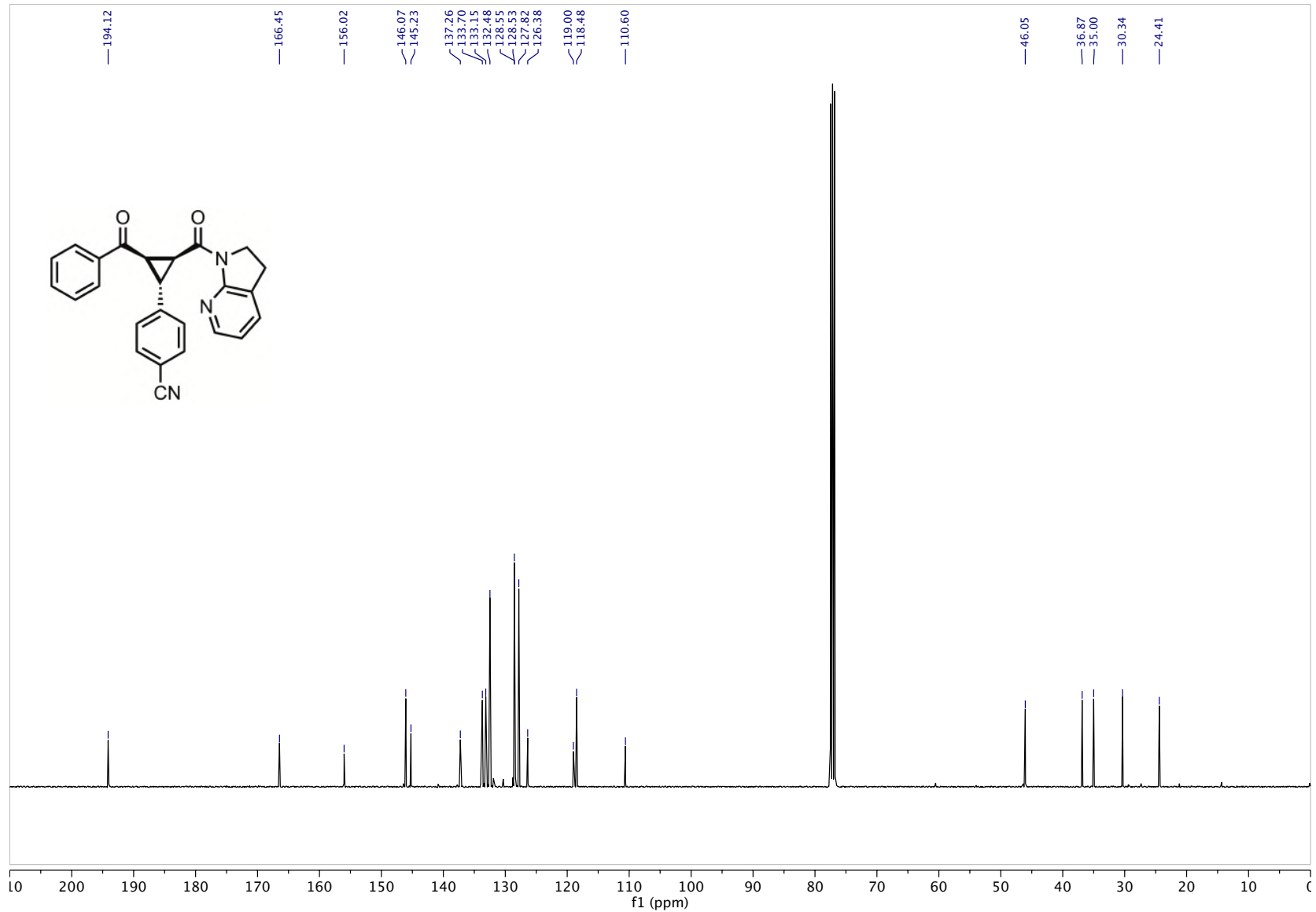


${ }^{1} \mathrm{H}$ NMR: 6aag

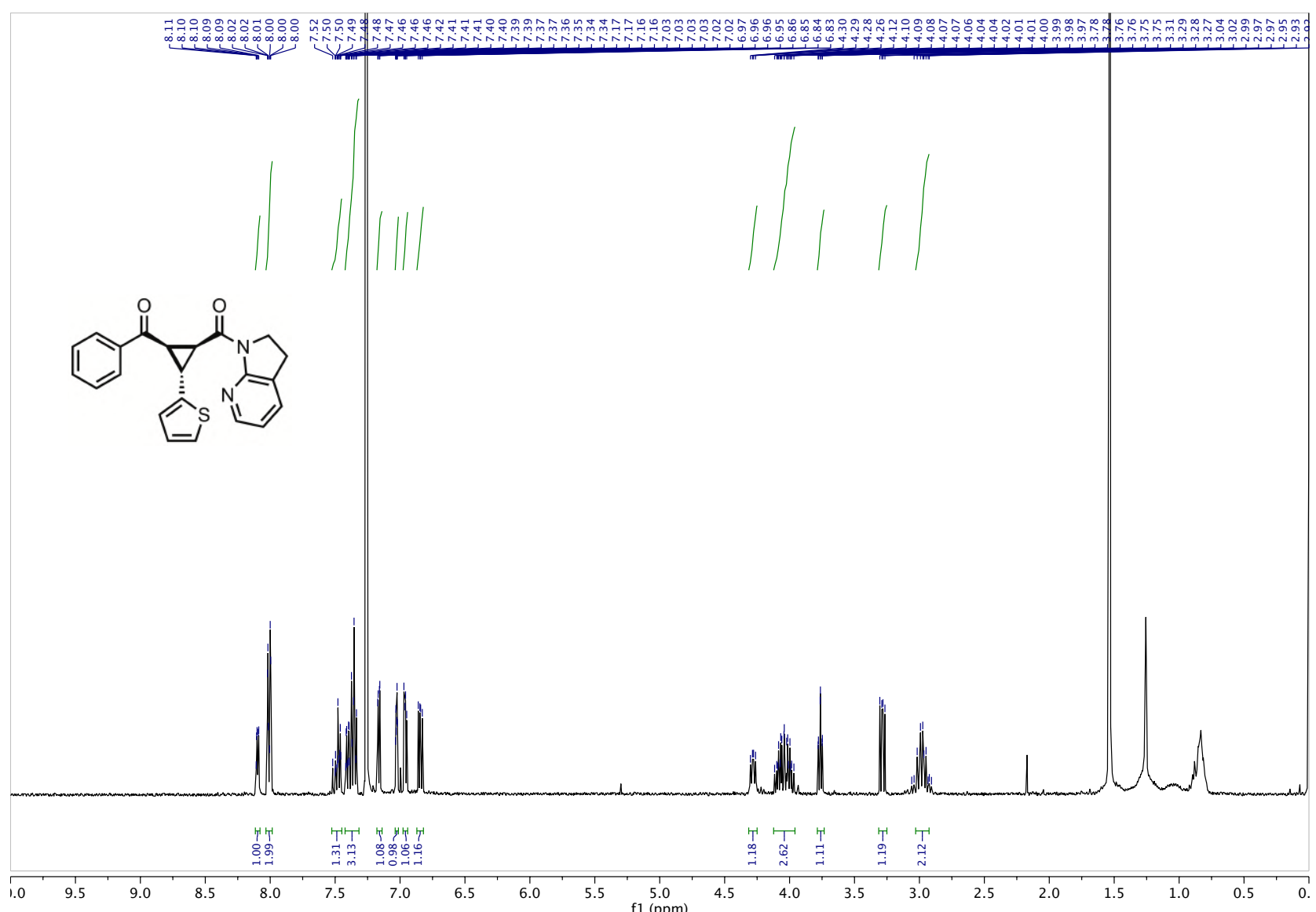

${ }^{13} \mathrm{C}$ NMR: 6aag

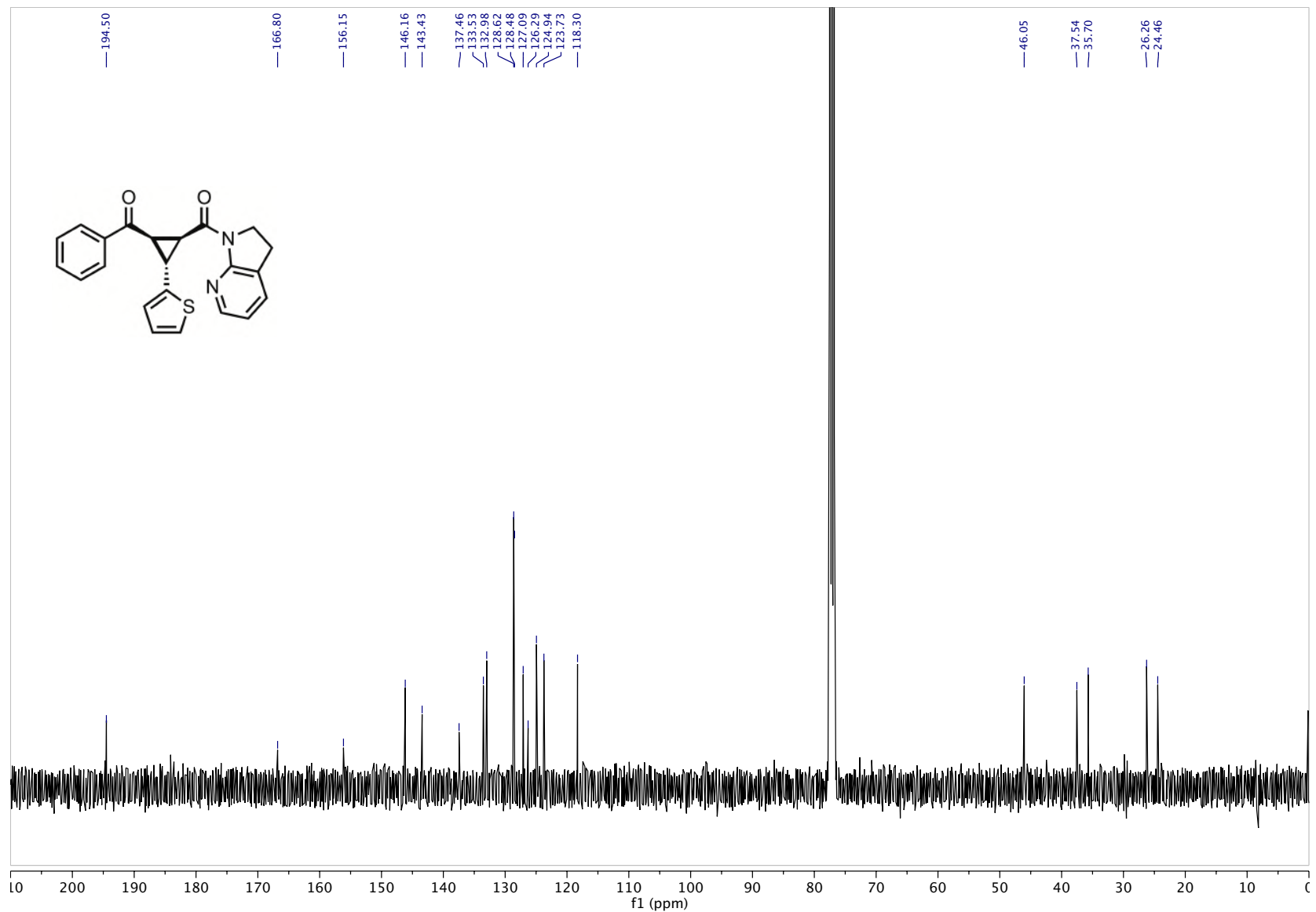


${ }^{1} \mathrm{H}$ NMR: 6aah

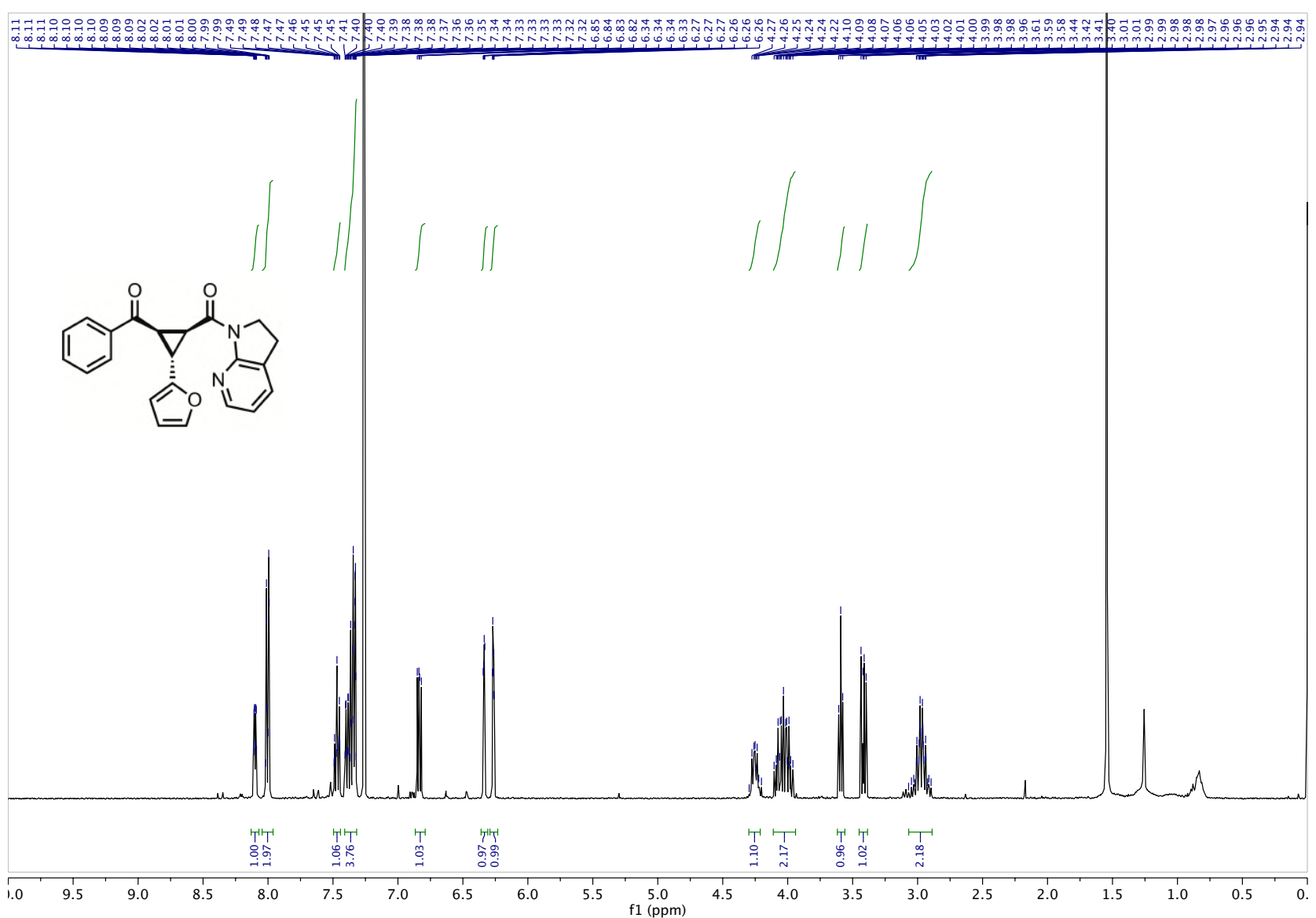

${ }^{13} \mathrm{C}$ NMR: 6aah

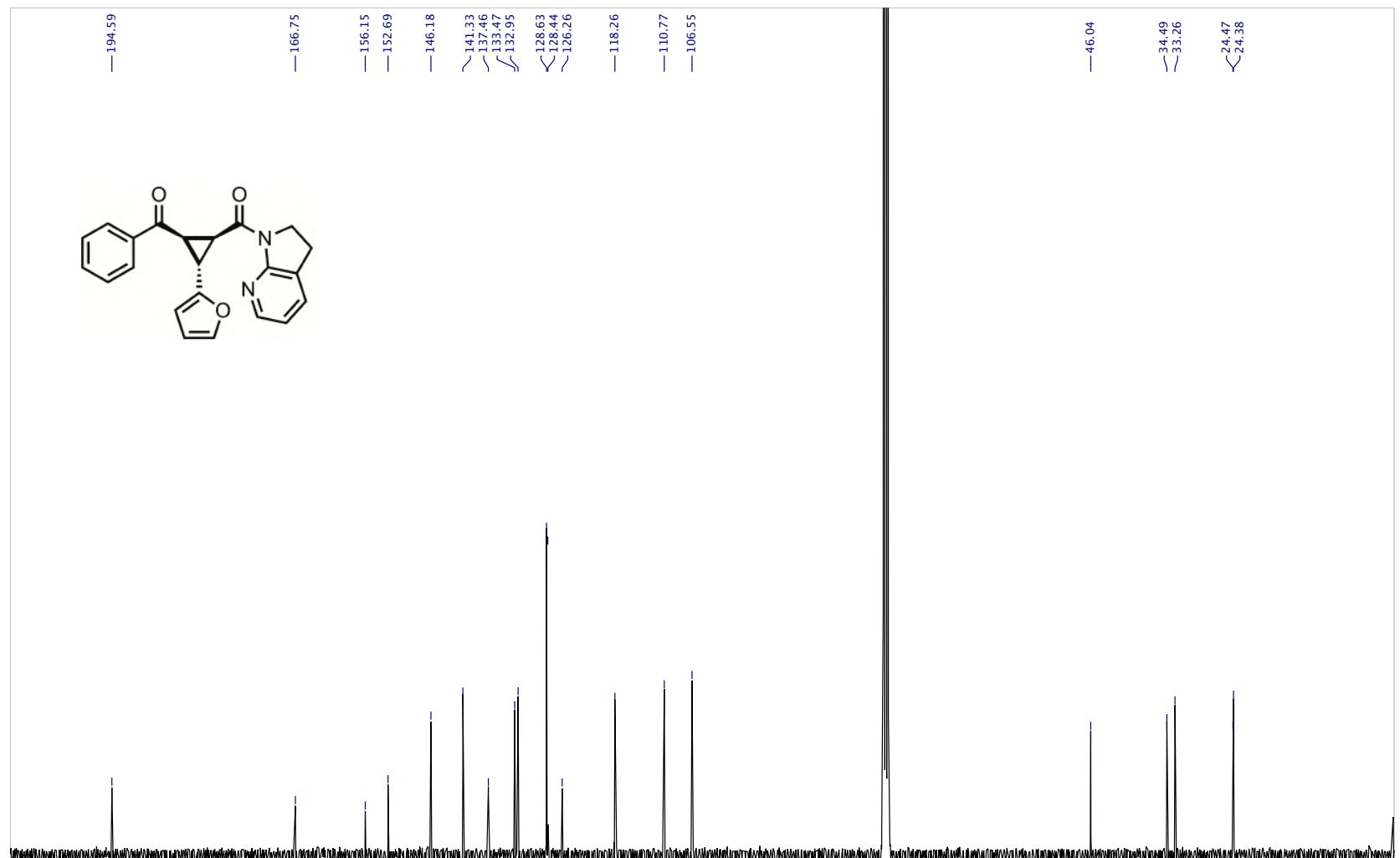

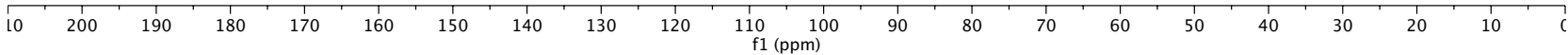


${ }^{1} \mathrm{H}$ NMR: 7a

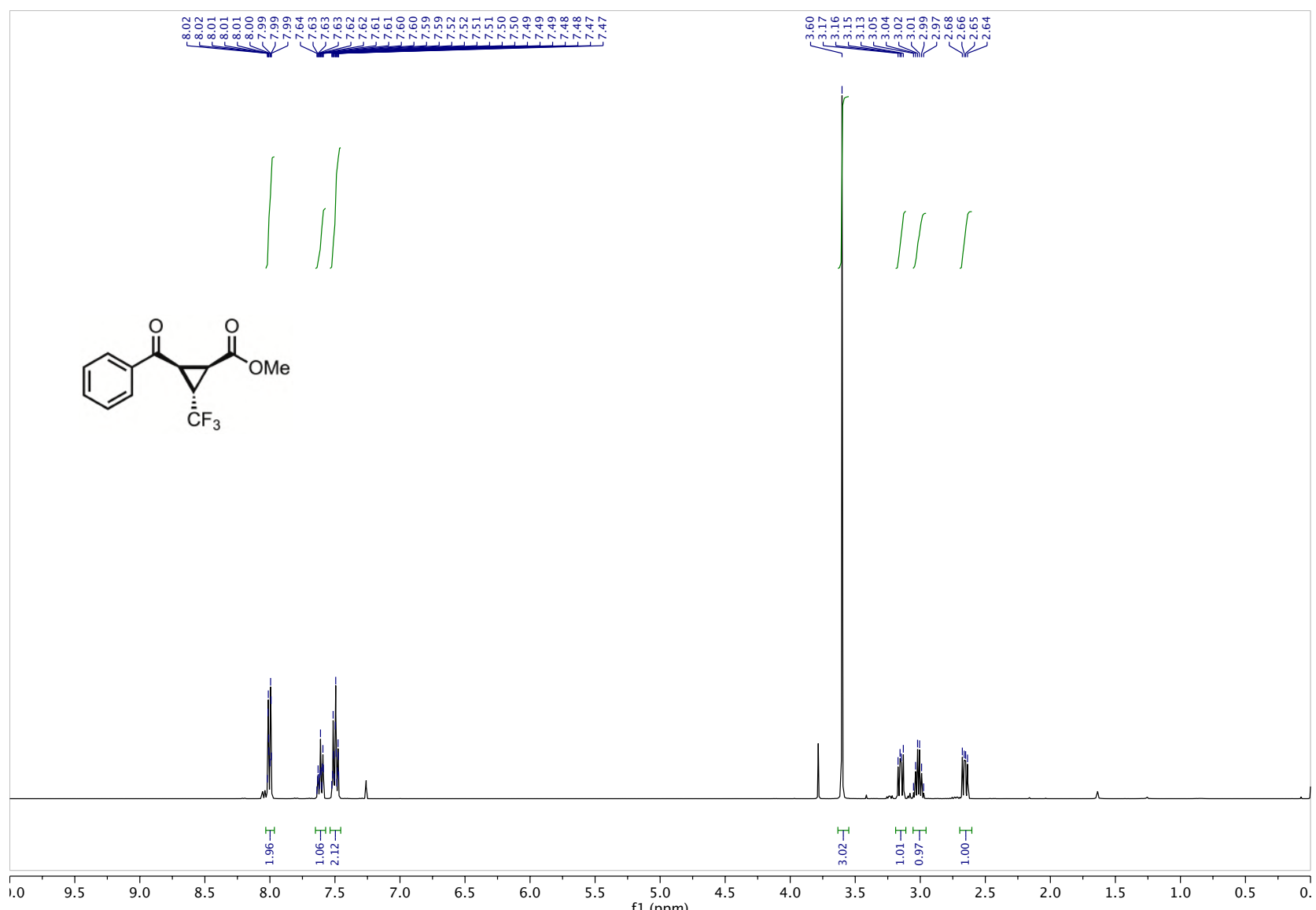

${ }^{13}$ C NMR: 7a

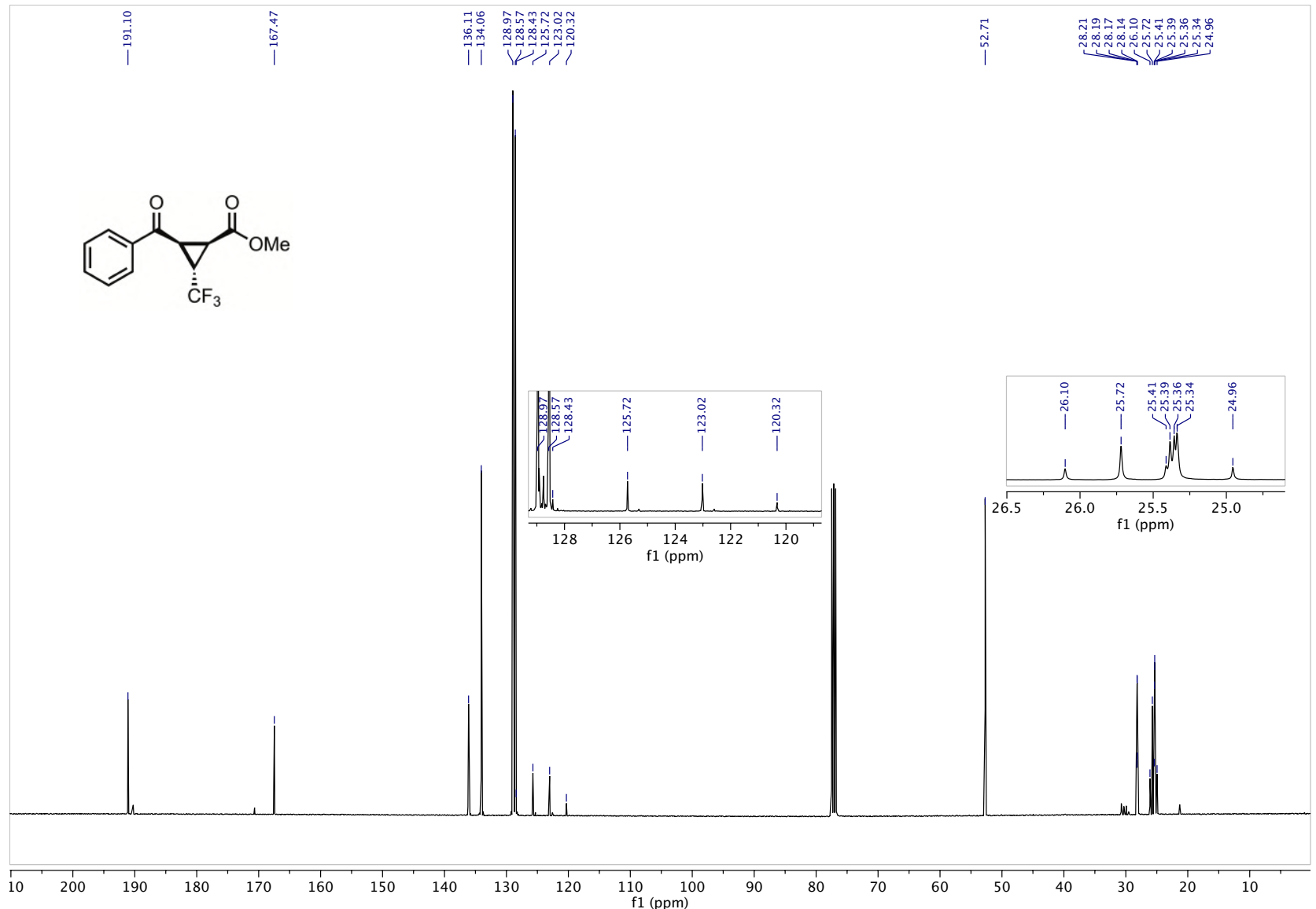


${ }^{19} \mathrm{~F}$ NMR: 7a

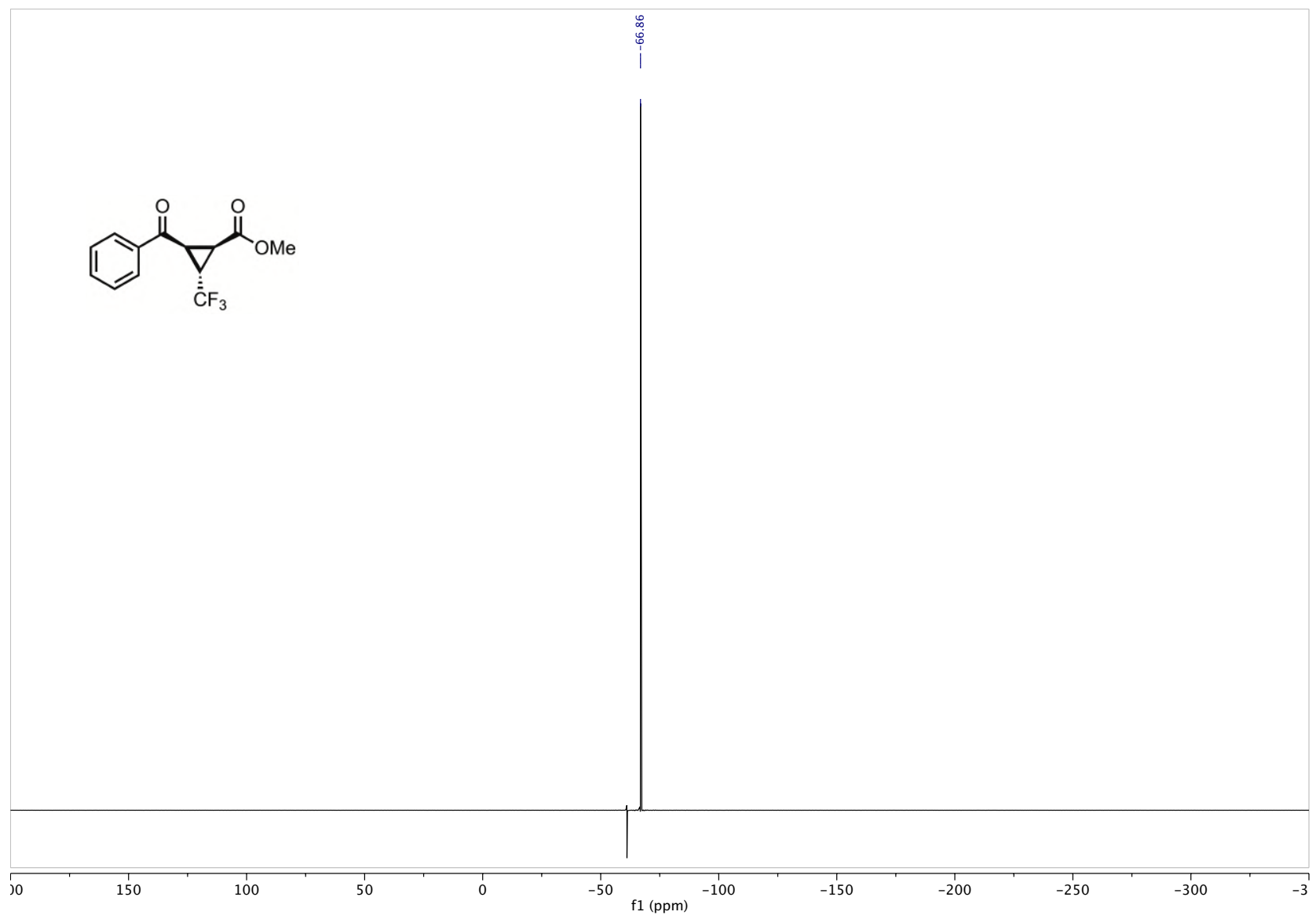


${ }^{1} \mathrm{H}$ NMR: 7b

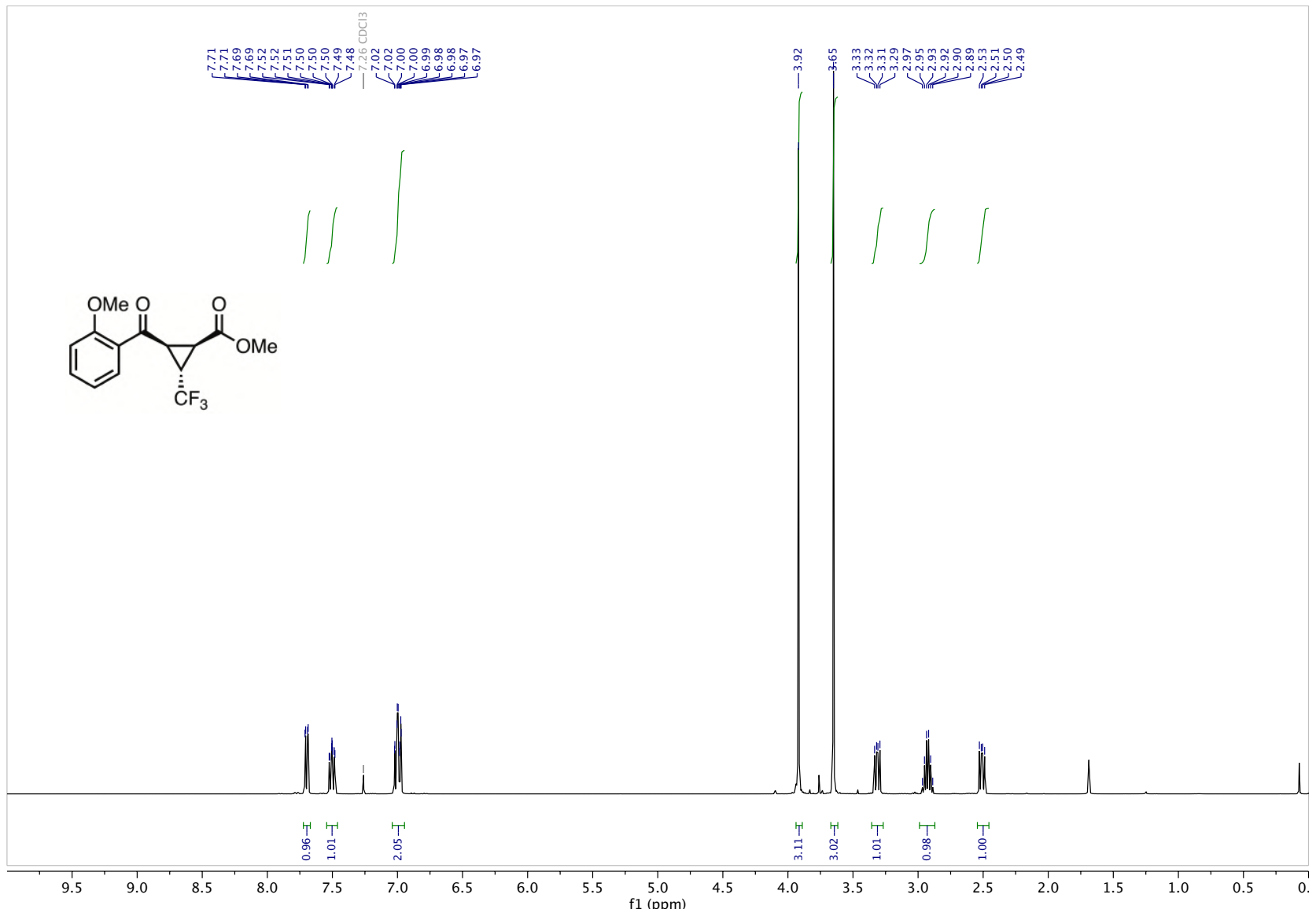

${ }^{13}$ C NMR: 7b

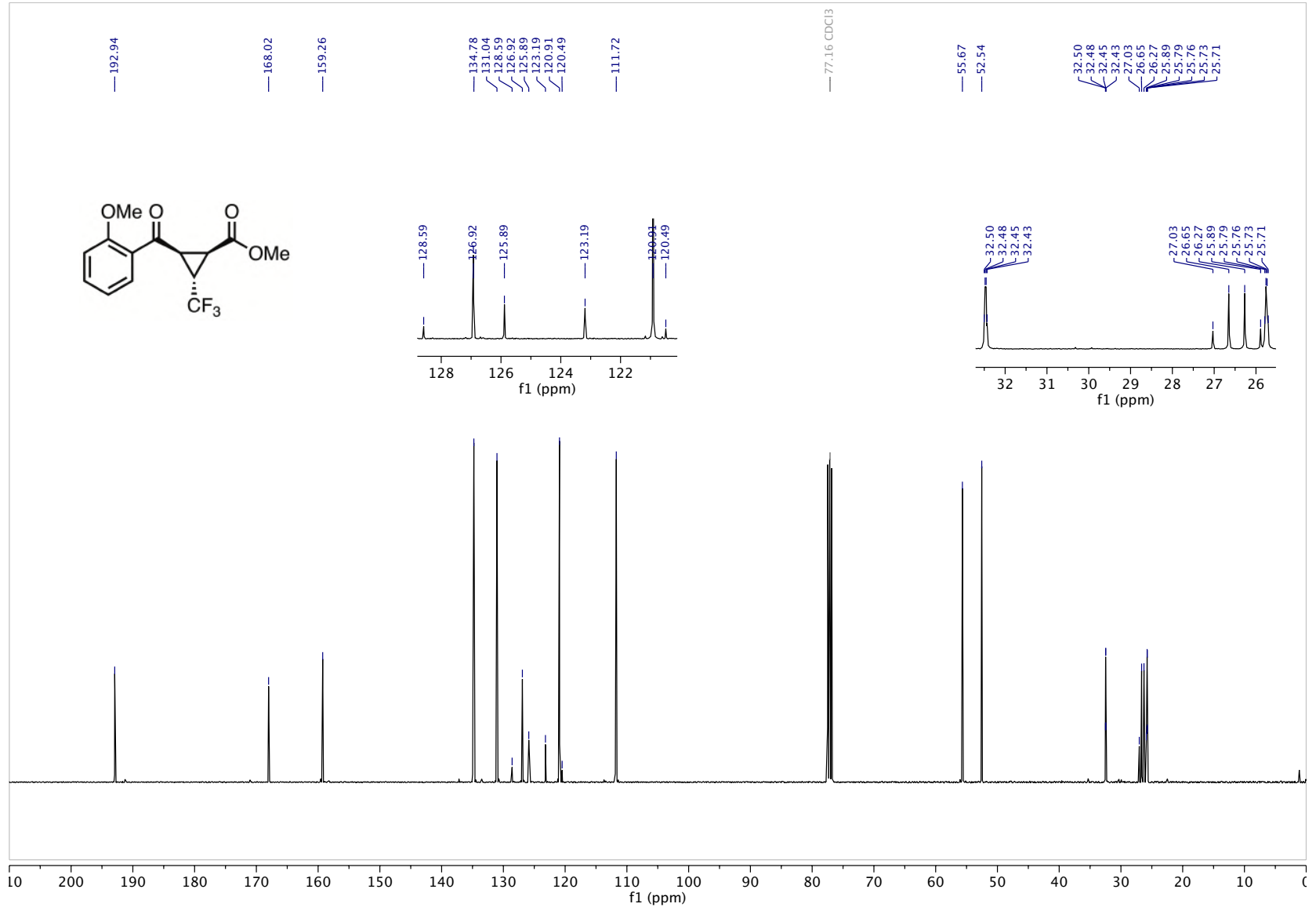


${ }^{19} \mathrm{~F}$ NMR: 7b

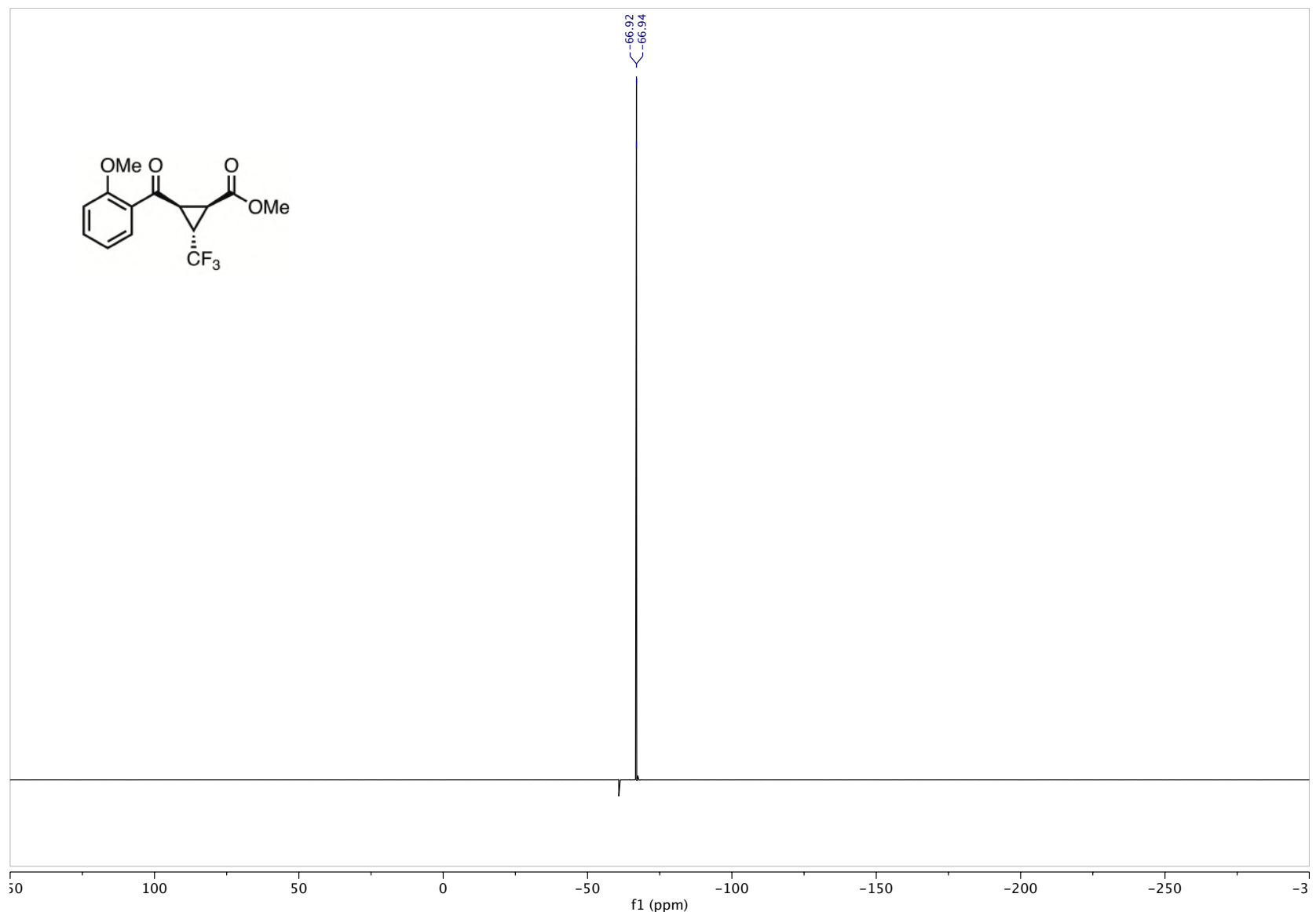


${ }^{1} \mathrm{H}$ NMR: 8a

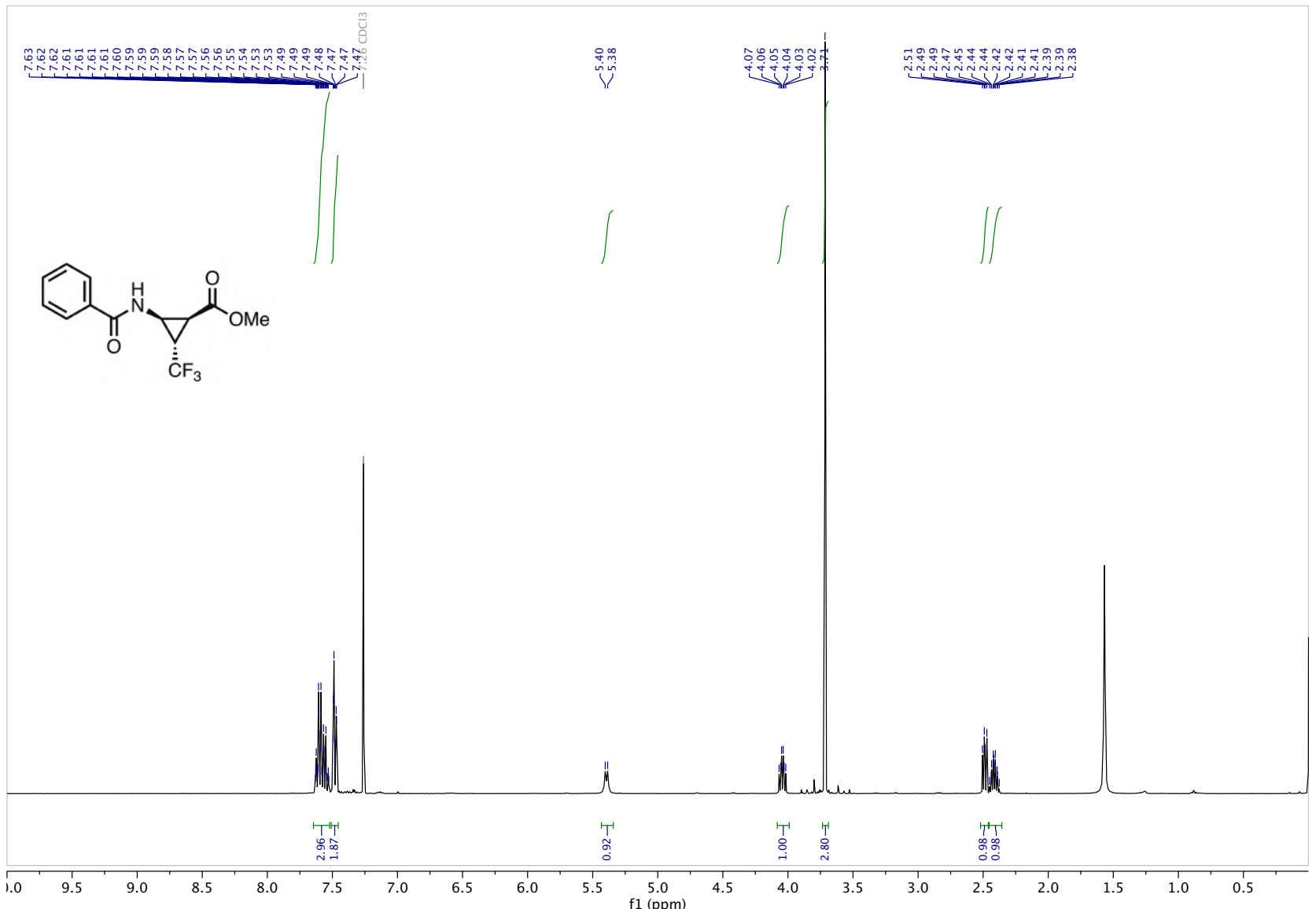

${ }^{13} \mathrm{C}$ NMR: 8a

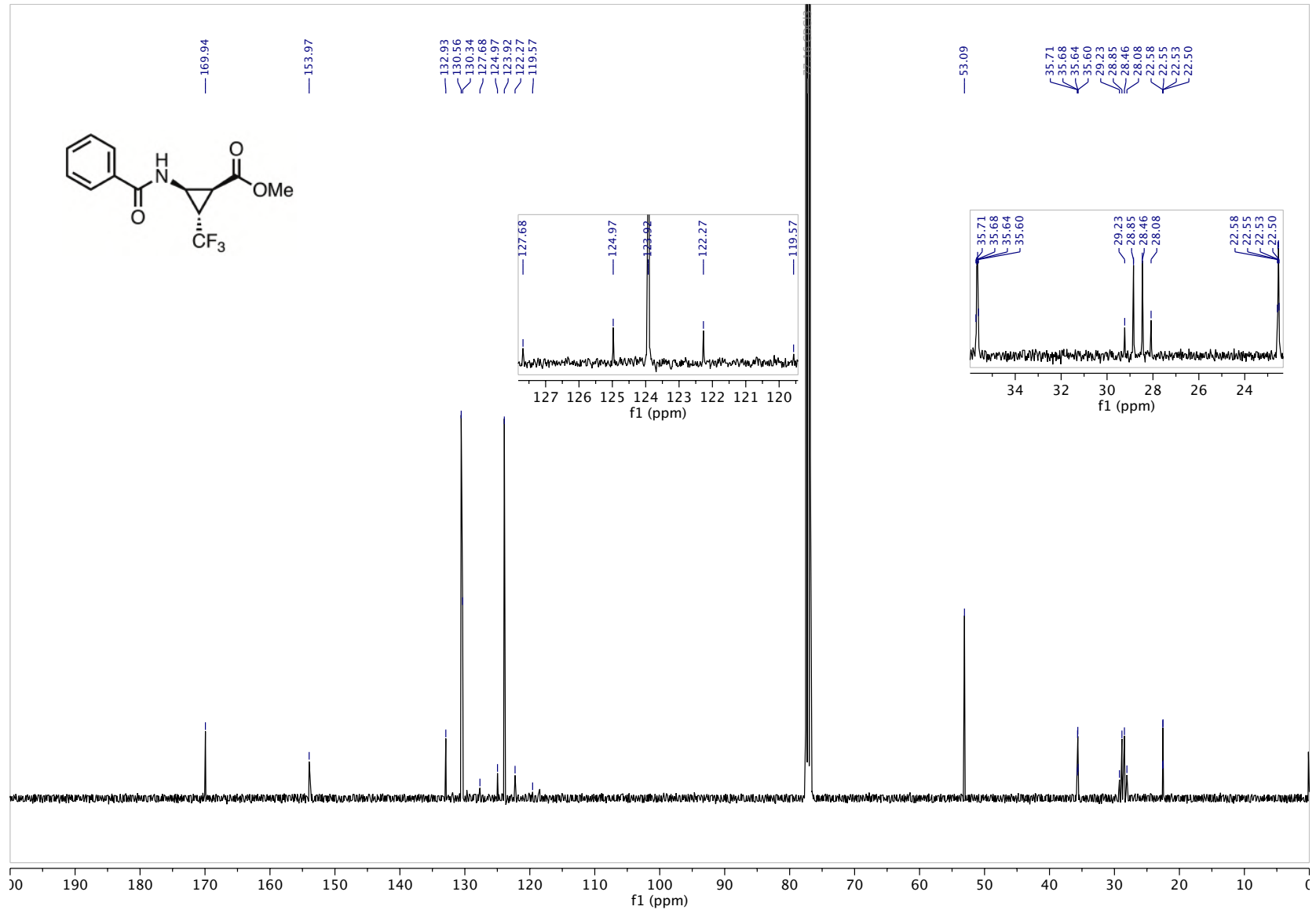


${ }^{1} \mathrm{H}$ NMR: 8a (COSY)

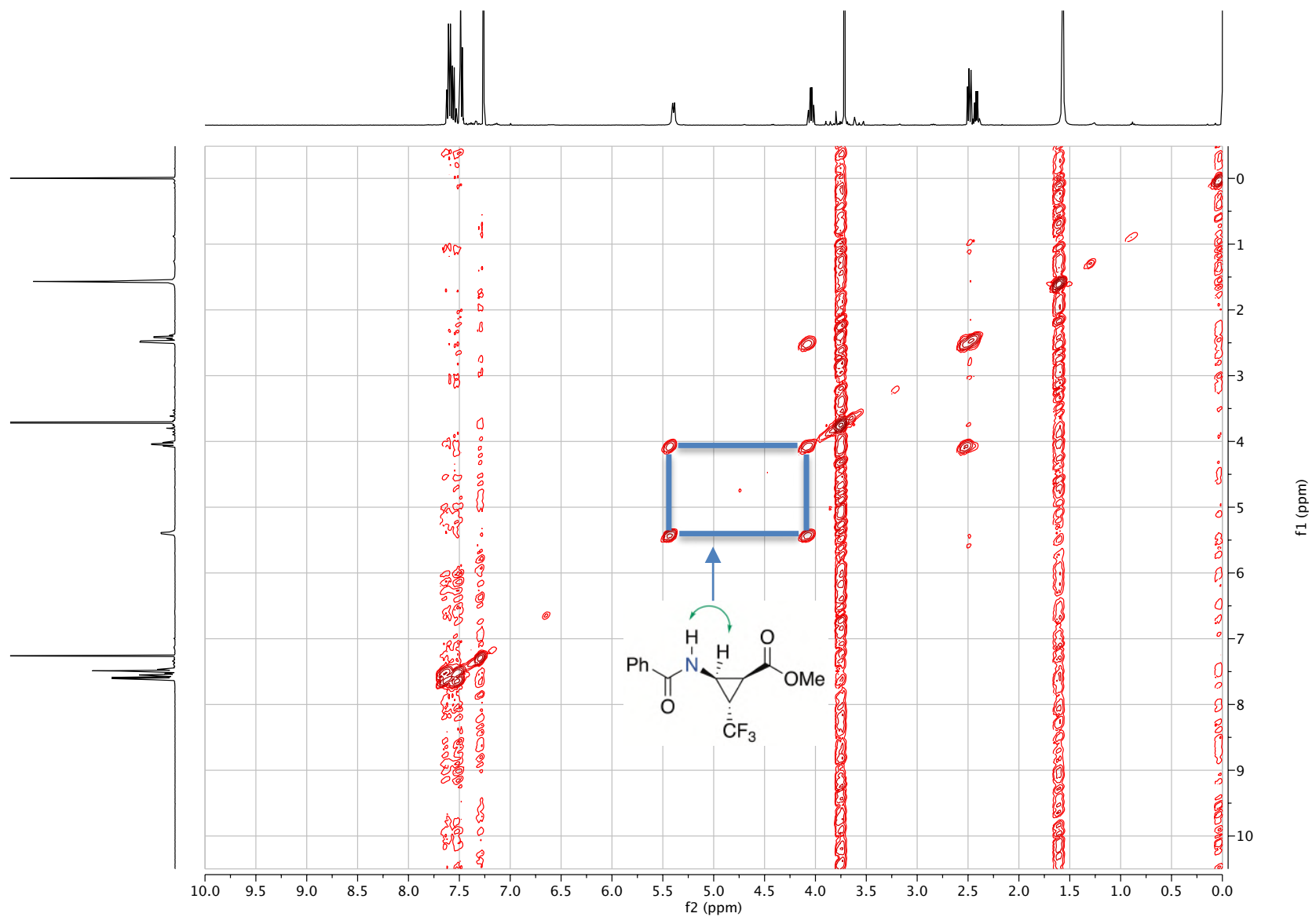

${ }^{19}$ F NMR: 8a

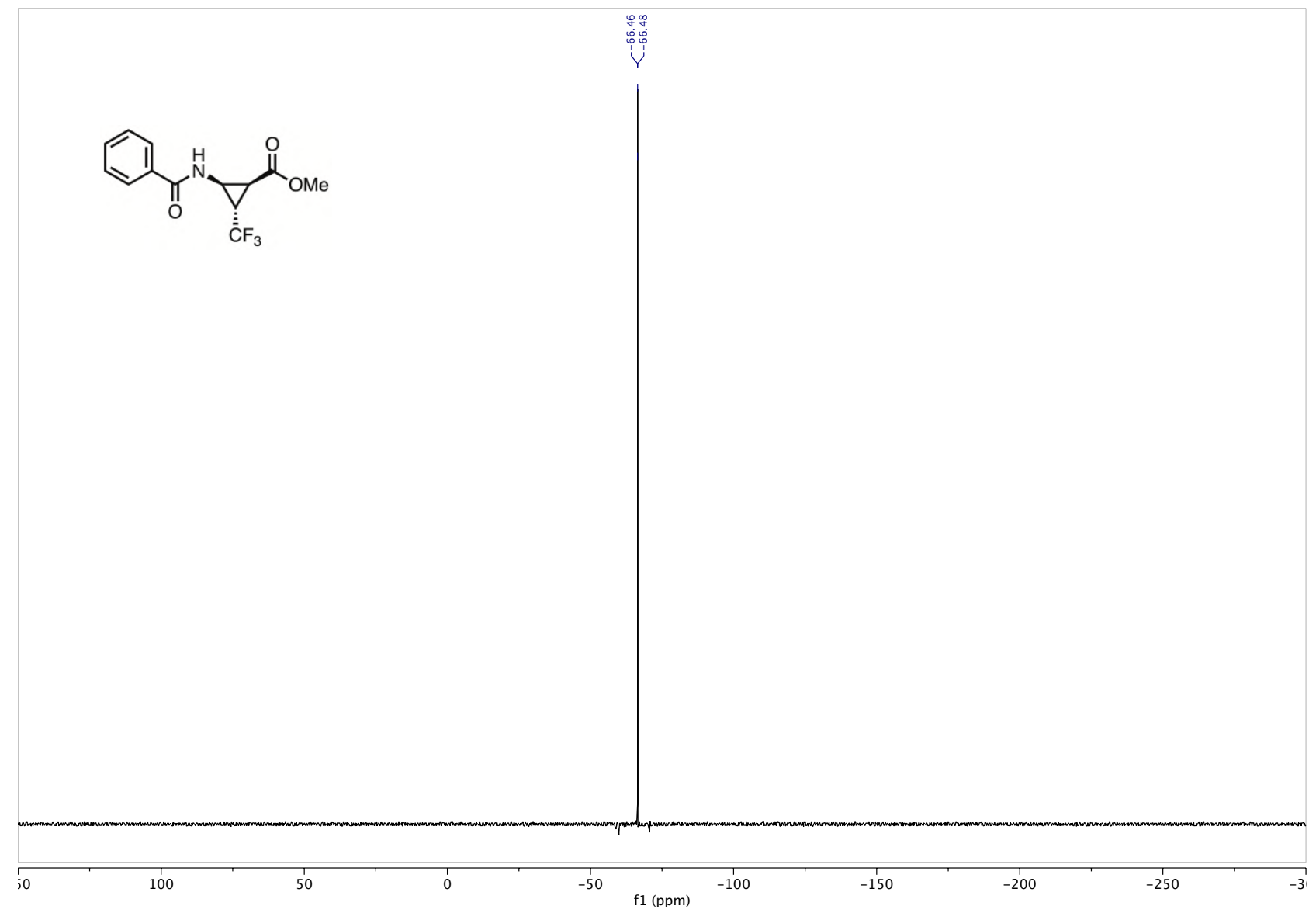


Supporting Information

${ }^{1} \mathrm{H}$ NMR: 9

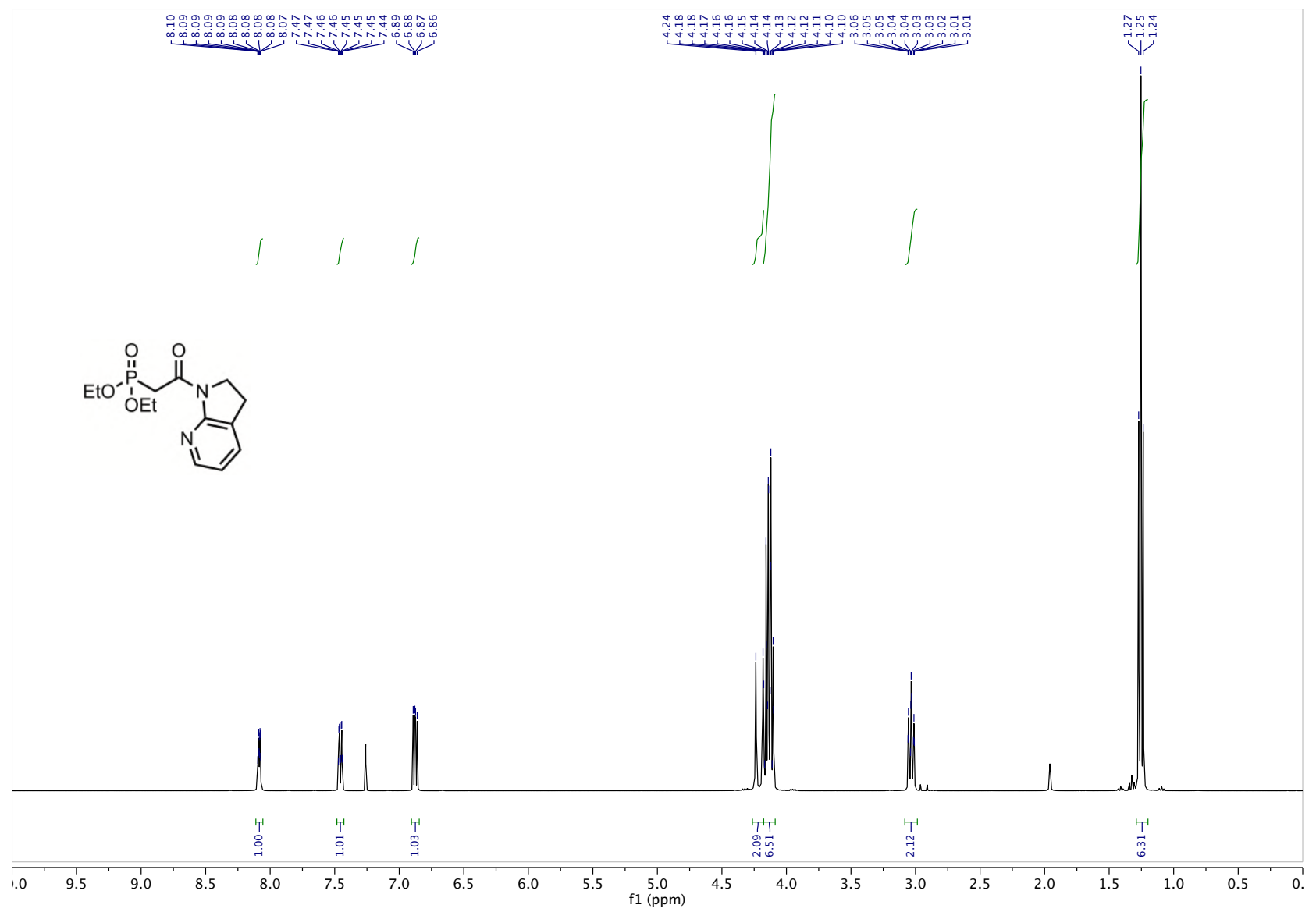

${ }^{13}$ C NMR: 9

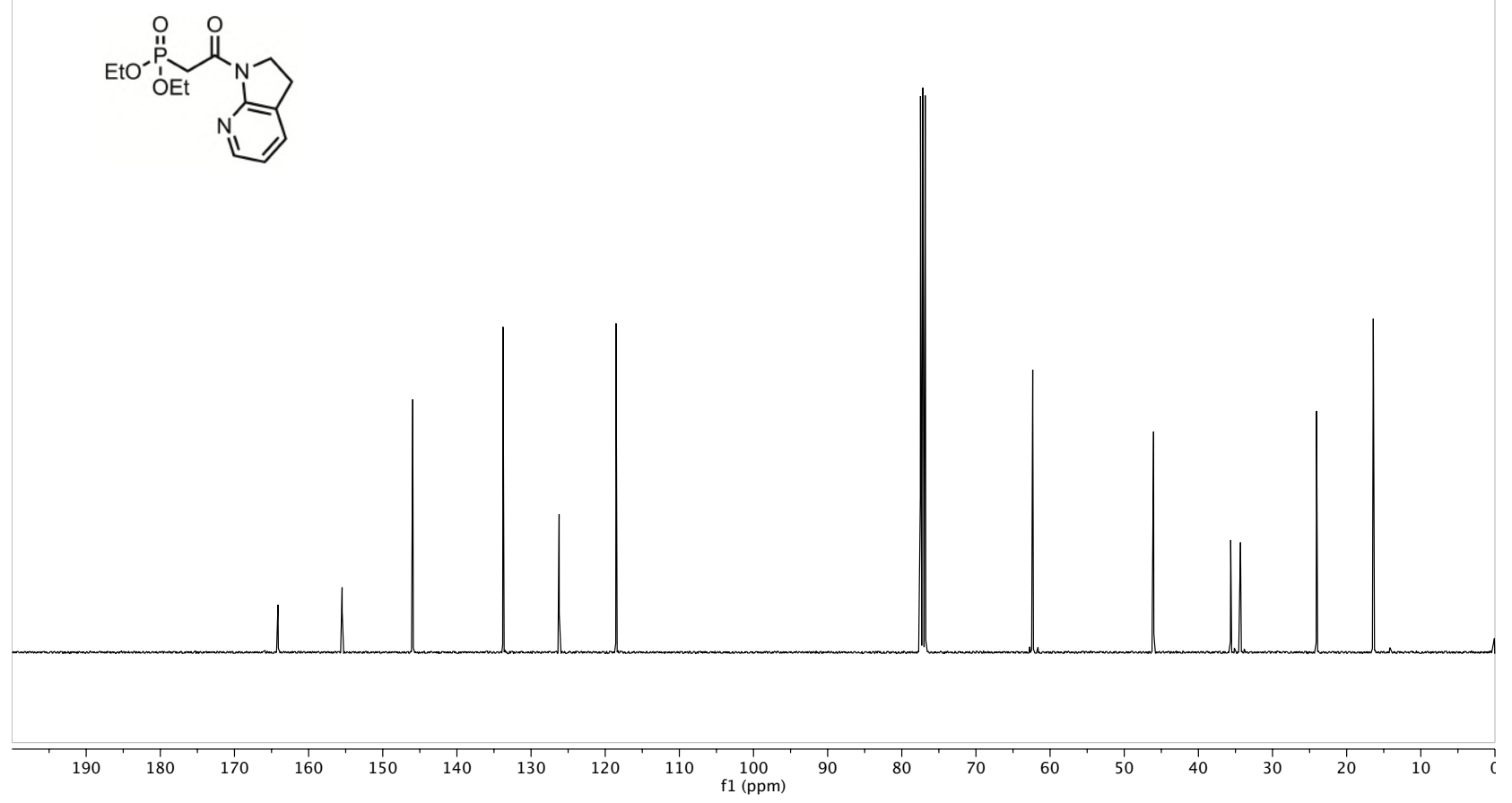

S189 
31P NMR: 9

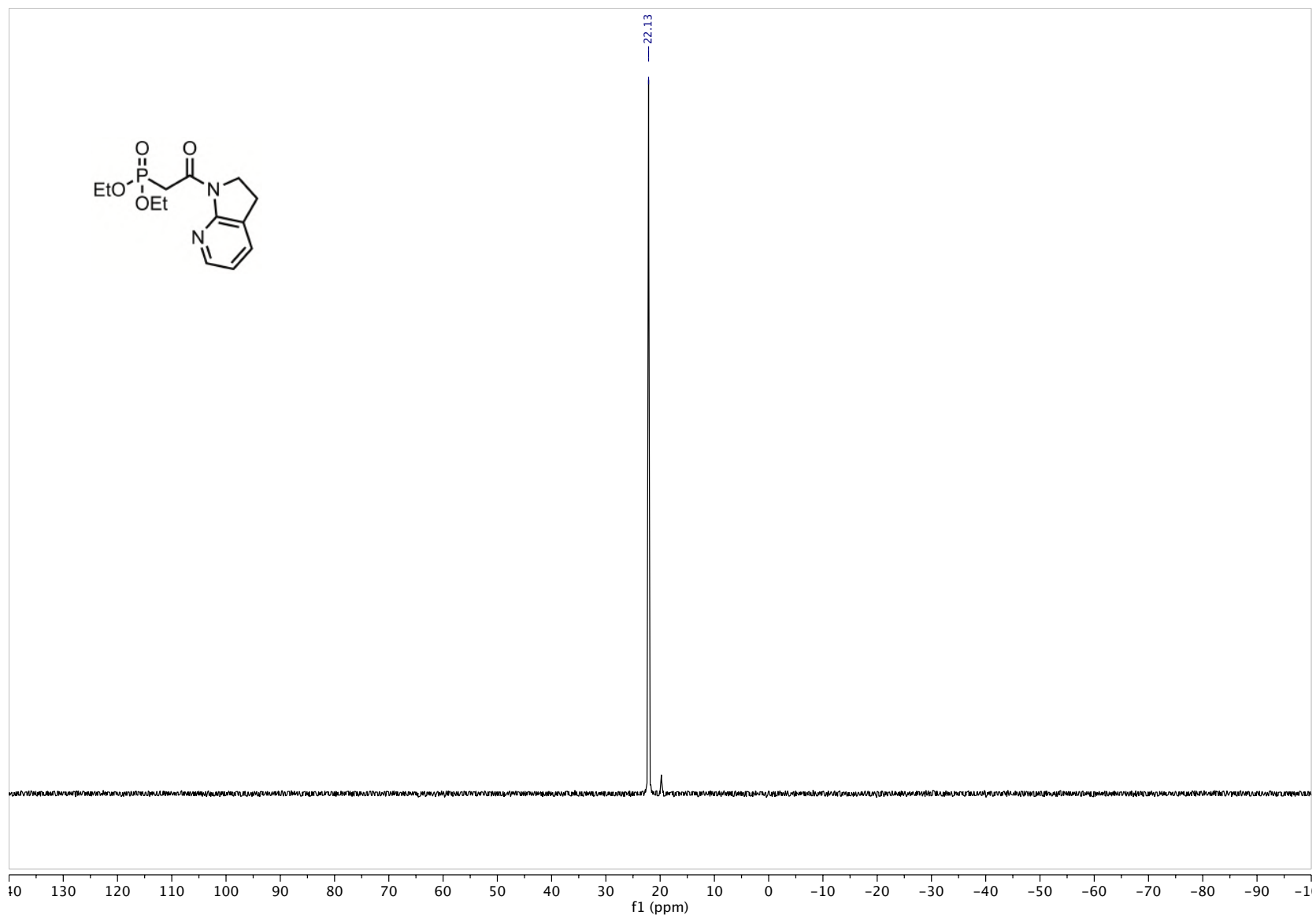

